\title{
Interpolation of Experimental Error Statistics for Mission Design of GPS Partial Attitude Determination
}

\author{
By \\ Michael Blois \\ Master of Applied Science \\ in \\ Aerospace Engineering \\ Carleton University \\ Ottawa, Ontario \\ (C) 2019 \\ Michael Blois
}

A thesis submitted to the Faculty of Graduate and Postdoctoral Affairs

in partial fulfillment of the requirements for the degree of 


\section{Abstract}

Attitude determination is a critical component of many navigation applications, including missions utilizing autonomous as well as piloted platforms. While Global Navigation Satellite Systems (GNSS) can be used to indirectly compute attitude angles, the cost of precise receivers and designing the antenna separation for a GNSS attitude system can pose significant design challenges. There is a need for a direct means of choosing the receiver quality as well as the necessary antenna separation distance, particularly for partial attitude determination systems using only 2 receivers.

This research presents an exhaustive simulation method for designing a GNSS pitch and heading determination system. A simulated environment, validated experimentally with 2 GNSS antennas, offset from each other along a front to rear platform axis, is used in conjunction with a fixed satellite geometry in order to generate pseudorange and carrier phase measurements from satellites to receiver antennas. Antenna separation as well as receiver quality are varied, with error statistics collected and tabulated for each variation. Experiments with real receivers are conducted in a rural environment as well as an urban environment to validate positioning algorithms and simulations. Raw Global Positioning System (GPS) data is collected in Receiver Independent Exchange Format (RINEX), then processed in post. Low cost GNSS viability for partial attitude determination is discussed based on tabulated simulation data and experimental data, especially in cases where dual frequency receivers and the carrier phase observable are not available. A method of directly computing minimum antenna separation or minimum receiver quality using interpolation of collected simulation data is proposed. 


\section{Acknowledgements}

I would like to first thank my supervisor, Dr Jurek Sasiadek. He trusted me and gave me the freedom to explore and research the topics that I determined to be interesting and important, giving guidance where it was needed.

I also want to give thanks to God, to my family, to my grandpa, to my mom and dad, to my brother Marty, to Dominique and to my close friends. They all helped to motivate me, they put my life into perspective and they gave me support when I needed it.

I also want to give thanks to my friends, classmates and professors from my undergraduate education at University of Calgary. The skills I gained and the friendships that I built in that time have been vital for my success here and I deeply appreciate having had the privilege of that experience. 


\section{List of Figures}

Figure 1: Illustration of the Earth-Centered, Earth-Fixed (ECEF) Coordinate System 16

Figure 2: Geographic Polar Coordinate References (Left), Geographic Height Above Sea Level (Right) 17

Figure 3: Illustration of a Local Vertical Compass-Based Reference Frame 18

Figure 4: Intersection of three spheres representing ranges from satellite to receiver antenna. A unique position solution exists when more than three satellites are in line of sight[34].

Figure 5: Explanation of the GNSS carrier phase measurement and integer ambiguity offset[37] 26

Figure 6:Illustration of Carrier Phase Ambiguity 27

Figure 7: RINEX Navigation Message Example 31

Table 1: Information Provided in RINEX GPS Navigation Messages 32

Figure 8: A Logged RINEX Observation File 33

Table 2: GNSS RINEX Mixed Observation Record Epoch Information 34

Table 3: Saastamoinen Model Input Values 36

Figure 9: TEC Map from Trimble GNSS 37

Figure 10: GNSS Double Difference Model and Setup 56

Figure 11: Definition of the Heading Angle on the North / East Horizontal Plane 62

Figure 12: Definition of a Bearing Between 2 Points as Projected on the North / East Horizontal Plane 63

Figure 13: Definition of Pitch Relative to the Horizontal Plane and Vertical Axis 64

Figure 14: Kalman Filter Iterative Cycle Flow Chart with Standard as well as Adapted Covariance Update $\begin{array}{ll}\text { Strategies } & 74\end{array}$

Figure 15: Horizontal and Vertical Errors for Simulation Set A

Figure 16: Horizontal 95 Percent Confidence Ellipses for Simulation Set A

Table 4: Mean Error Statistics for Set A 80

Figure 17: Horizontal and Vertical Errors for Simulation Set B 81

Figure 18: Horizontal 95 Percent Confidence Ellipses for Simulation Set B 82

Table 5: Mean Error Statistics for Set B 82 
Figure 19: Horizontal and Vertical Errors for Simulation Set C

Figure 20: Horizontal 95 Percent Confidence Ellipses for Simulation Set C

Table 6: Mean Error Statistics for Set C 85

Figure 21: Horizontal and Vertical Errors for Simulation Set D 87

Figure 22: Horizontal 95 Percent Confidence Ellipses for Simulation Set D 87

Table 7: Mean Error Statistics for Set D 88

Figure 23: Horizontal and Vertical Errors for Simulation Set E 89

Figure 24: Horizontal 95 Percent Confidence Ellipses for Simulation Set E 90

Table 8: Mean Error Statistics for Set E 91

Figure 25: Horizontal and Vertical Errors for Simulation Set F 92

Figure 26: Horizontal 95 Percent Confidence Ellipses for Simulation Set F 92

Table 9: Mean Error Statistics for Set F 93

Figure 27: Horizontal and Vertical Errors for Simulation Set F 94

Figure 28: Horizontal 95 Percent Confidence Ellipses for Simulation Set G 94

Table 10: Mean Error Statistics for Set G 95

Figure 29: Horizontal and Vertical Errors for Simulation Set H 96

Figure 30: Horizontal 95 Percent Confidence Ellipses for Simulation Set H 97

Table 11: Mean Error Statistics for Set H 97

Table 12: ASCM 764522 Information 99

Figure 31:Location of ASCM 764522 Outside of Didsbury, Alberta. Brass Cap (Left) With Precise Coordinates Used for Antenna Placement. Conditions are Ideal for Positioning in Open Sky (Right). 99

Figure 32: True Directional Error for Processed Rural ASCM Survey Data When Compared to Archived ASC

$\begin{array}{lr}\text { Coordinates } & 100\end{array}$

Table 13: Mean Error Statistics for Rural ASCM Survey Data Processing For Each Processing Method 101

Figure 33: Skyplot for Visualizing the Satellite Headings and Elevation Angles Relative to the Antenna Over the

Data Collection Period for Didsbury ASCM Survey 101

Table 14: Properties of Local Control Point ID 2003 From Civil Engineering Local Carleton Campus Survey 102 
Figure 34: Attitude Survey Control Point Locations. Rear Antenna Environment (Top Left), Rear Antenna Control Point in Concrete (Top Right), Front Antenna Environment (Bottom Left), Front Antenna Control Point in Concrete (Bottom Right)

Figure 35 True Directional Error for Processed Carleton Campus Control Point Survey Data When Compared to

Civil Engineering Department Control Coordinates

Table 15: Mean Error Statistics for Carleton University Survey Data Processing For Each Processing Method 104

Figure 36: Skyplot for Visualizing the Satellite Headings and Elevation Angles Relative to the Antenna Over the

Data Collection Period Carleton University Survey on February $1^{\text {st }}, 2019$

Figure 37: Computed and Measured Headings for Simulation Set A-1 107

Figure 38: Computed and Measured Pitch for Simulation Set A-1 108

Figure 39: Propagated 95\% Confidence Ellipses for Front and Rear GPS Antennas in Simulated Data Set A-1 109

Table 16: Mean Global Error Statistics of Unfused Pitch for Simulation Set A-1 110

Table 17: Mean Global Error Statistics of Unfused Heading for Simulation Set A-1 110

Table 18: Mean Global Error Statistics of GPS/IMU Fused Pitch for Simulation Set A-1 111

Table 19: Mean Global Error Statistics of GPS/IMU Fused Heading for Simulation Set A-1 111

Table 20: Success Rates of Reaching Angular Specifications of Unfused Pitch for Simulation Data Set A-1 112

Table 21: Success Rates of Reaching Angular Specifications of Unfused Heading for Simulation Data Set A-1 112

Table 22: Success Rates of Reaching Angular Specifications of Fused Pitch for Simulation Data Set A-1 113

Table 23: Success Rates of Reaching Angular Specifications of Fused Heading for Simulation Data Set A-1 113

Table 24: Pitch / Heading Correlation Coefficients for Each Computation Method in Simulation Data Set A-1 114

Table 25: Statistics from Selected Data Sets for Interpolation of Pseudorange Noise 115

Table 26: Statistics from Selected Data Sets for Interpolation of Antenna Separation 116

Figure 40: Locations of Control Points Used for Pitch and Heading Survey on Carleton University Campus 118

Figure 41: Attitude Experiment Location. Top Left: Rear GPS Environment. Top Right: Rear Control Point. Bottom Left: Front GPS Environment. Bottom Right: Front GPS Control Point

Table 27: Geographic Coordinates of Control Points in Attitude Survey, WGS84 Used as Ellipsoidal Datum for Height Reference 
Figure 42: True Error of Computed GPS Coordinates for the Front and Rear GPS Receivers Using Known Marker Locations as a Reference Position

Table 28: Global Mean Positioning Error Statistics for Each Receiver Across Carleton Pitch and Heading Survey

Figure 43: Sky Plot of Trajectories of Available GPS Satellites During Carleton Pitch and Heading Survey

Figure 44: Positioning Results in Horizontal Plane for Test Receivers 1 and 2 in Carleton Pitch and Heading Survey

Figure 45: True Pitch Error of Undedicated GPS Pitch and Roll System Using Test Receivers 1 and 2 in Carleton

Survey. Raw Solutions Using Interpolated Data as Well as Kalman Filtered Results are Shown

Figure 46: True Heading Error of Undedicated GPS Pitch and Roll System Using Test Receivers 1 and 2 in Carleton Survey. Raw Solutions Using Interpolated Data as Well as Kalman Filtered Results are Shown

Table 29: Global Mean Error Statistics of Undedicated GPS Pitch and Heading System During Carleton Survey 126

Table 30: Portion of Observations Obtaining Desired Specifications for Each Method and Metric During Carleton Survey

Figure 47: True Headings vs Extreme Error Computed Headings, Receiver Noise Properties Fixed at a Constant with Varying Antenna Separation. Long separation (Top), Medium Separation (Middle), Short Separation (Bottom)

Figure 48: Computed and Measured Headings for Simulation Set A-1

Figure 49: Computed and Measured Pitch for Simulation Set A-1

Figure 50: Propagated 95\% Confidence Ellipses for Front and Rear GPS Antennas in Simulated Data Set A-1 143

Table 31: Mean Global Error Statistics of Unfused Pitch for Simulation Set A-1 144

Table 32: Mean Global Error Statistics of Unfused Heading for Simulation Set A-1 144

Table 33: Mean Global Error Statistics of GPS/IMU Fused Pitch for Simulation Set A-1 145

Table 34: Mean Global Error Statistics of GPS/IMU Fused Heading for Simulation Set A-1 145

Table 35: Success Rates of Reaching Angular Specifications of Unfused Pitch for Simulation Data Set A-1 146

Table 36: Success Rates of Reaching Angular Specifications of Unfused Heading for Simulation Data Set A-1 146

Table 37: Success Rates of Reaching Angular Specifications of Fused Pitch for Simulation Data Set A-1 147 
Table 38: Success Rates of Reaching Angular Specifications of Fused Heading for Simulation Data Set A-1

Table 39: Pitch / Heading Correlation Coefficients for Each Computation Method in Simulation Data Set A-1 148

Figure 51: Computed and Measured Headings for Simulation Set A-2 148

Figure 52: Computed and Measured Pitch for Simulation Set A-2 149

Figure 53: Propagated 95\% Confidence Ellipses for Front and Rear GPS Antennas in Simulated Data Set A-2 149

Table 40: Mean Global Error Statistics of Unfused Pitch for Simulation Set A-2 150

Table 41: Mean Global Error Statistics of Unfused Heading for Simulation Set A-2 150

Table 42: Mean Global Error Statistics of Fused Pitch for Simulation Set A-2

Table 43: Mean Global Error Statistics of Fused Heading for Simulation Set A-2

Table 44: Success Rates of Reaching Angular Specifications of Unfused Pitch for Simulation Data Set A-2 152

Table 45: Success Rates of Reaching Angular Specifications of Unfused Heading for Simulation Data Set A-2 152

Table 46: Success Rates of Reaching Angular Specifications of Fused Pitch for Simulation Data Set A-2 153

Table 47: Success Rates of Reaching Angular Specifications of Fused Heading for Simulation Data Set A-2 153

Table 48: Pitch / Heading Correlation Coefficients for Each Computation Method in Simulation Data Set A-2 154

Figure 54: Computed and Measured Headings for Simulation Set A-3 154

Figure 55: Computed and Measured Pitch for Simulation Set A-3 155

Figure 56: Propagated 95\% Confidence Ellipses for Front and Rear GPS Antennas in Simulated Data Set A-3 155

Table 49: Mean Global Error Statistics of Unfused Pitch for Simulation Set A-3 156

Table 50: Mean Global Error Statistics of Unfused Heading for Simulation Set A-3 156

Table 51: Mean Global Error Statistics of Fused Pitch for Simulation Set A-3 157

Table 52: Mean Global Error Statistics of Fused Heading for Simulation Set A-3 157

Table 53: Success Rates of Reaching Angular Specifications of Unfused Pitch for Simulation Data Set A-3 158

Table 54: Success Rates of Reaching Angular Specifications of Unfused Heading for Simulation Data Set A-3 158

Table 55: Success Rates of Reaching Angular Specifications of Fused Pitch for Simulation Data Set A-3 159

Table 56: Success Rates of Reaching Angular Specifications of Fused Heading for Simulation Data Set A-3 159

Table 57: Pitch / Heading Correlation Coefficients for Each Computation Method in Simulation Data Set A-3 160

Figure 57: Computed and Measured Headings for Simulation Set A-4 160 
Figure 58: Computed and Measured Pitch for Simulation Set A-4

Figure 59: Propagated 95\% Confidence Ellipses for Front and Rear GPS Antennas in Simulated Data Set A-4 161

Table 58: Mean Global Error Statistics of Unfused Pitch for Simulation Set A-4

Table 59: Mean Global Error Statistics of Unfused Heading for Simulation Set A-4 162

Table 60: Mean Global Error Statistics of Fused Pitch for Simulation Set A-4 163

Table 61: Mean Global Error Statistics of Fused Heading for Simulation Set A-4 163

Table 62: Success Rates of Reaching Angular Specifications of Unfused Pitch for Simulation Data Set A-4 164

Table 63: Success Rates of Reaching Angular Specifications of Unfused Heading for Simulation Data Set A-4 164

Table 64: Success Rates of Reaching Angular Specifications of Fused Pitch for Simulation Data Set A-4 165

Table 65: Success Rates of Reaching Angular Specifications of Fused Heading for Simulation Data Set A-4 165

Table 66: Pitch / Heading Correlation Coefficients for Each Computation Method in Simulation Data Set A-4 166

Figure 60: Computed and Measured Headings for Simulation Set A-5 166

Figure 61: Computed and Measured Pitch for Simulation Set A-5 167

Figure 62: Propagated 95\% Confidence Ellipses for Front and Rear GPS Antennas in Simulated Data Set A-5 167

Table 67: Mean Global Error Statistics of Unfused Pitch for Simulation Set A-5 168

Table 68: Mean Global Error Statistics of Unfused Heading for Simulation Set A-5 168

Table 69: Mean Global Error Statistics of Fused Pitch for Simulation Set A-5

Table 70: Mean Global Error Statistics of Fused Heading for Simulation Set A-5 169

Table 71: Success Rates of Reaching Angular Specifications of Unfused Pitch for Simulation Data Set A-5 170

Table 72: Success Rates of Reaching Angular Specifications of Unfused Heading for Simulation Data Set A-5 170

Table 73: Success Rates of Reaching Angular Specifications of Fused Pitch for Simulation Data Set A-5 171

Table 74: Success Rates of Reaching Angular Specifications of Fused Heading for Simulation Data Set A-5 171

Table 75: Pitch / Heading Correlation Coefficients for Each Computation Method in Simulation Data Set A-5 172

Figure 63: Computed and Measured Headings for Simulation Set A-6 172

Figure 64: Computed and Measured Pitch for Simulation Set A-6 173

Figure 65: Propagated 95\% Confidence Ellipses for Front and Rear GPS Antennas in Simulated Data Set A-6 173

Table 76: Mean Global Error Statistics of Unfused Pitch for Simulation Set A-6 
Table 78: Mean Global Error Statistics of Fused Pitch for Simulation Set A-6

Table 79: Mean Global Error Statistics of Fused Heading for Simulation Set A-6

Table 80: Success Rates of Reaching Angular Specifications of Unfused Pitch for Simulation Data Set A-6

Table 81: Success Rates of Reaching Angular Specifications of Unfused Heading for Simulation Data Set A-6 176

Table 82: Success Rates of Reaching Angular Specifications of Fused Pitch for Simulation Data Set A-6

Table 83: Success Rates of Reaching Angular Specifications of Fused Heading for Simulation Data Set A-6

Table 84: Pitch / Heading Correlation Coefficients for Each Computation Method in Simulation Data Set A-6

Figure 68: Propagated 95\% Confidence Ellipses for Front and Rear GPS Antennas in Simulated Data Set A-7

Table 87: Mean Global Error Statistics of Fused Pitch for Simulation Set A-7

Table 88: Mean Global Error Statistics of Fused Heading for Simulation Set A-7

Table 89: Success Rates of Reaching Angular Specifications of Unfused Pitch for Simulation Data Set A-7

Table 90: Success Rates of Reaching Angular Specifications of Unfused Heading for Simulation Data Set A-7

Table 93: Pitch / Heading Correlation Coefficients for Each Computation Method in Simulation Data Set A-7 184 
Table 98: Success Rates of Reaching Angular Specifications of Unfused Pitch for Simulation Data Set A-8

Table 99: Success Rates of Reaching Angular Specifications of Unfused Heading for Simulation Data Set A-8 188

Table 100: Success Rates of Reaching Angular Specifications of Fused Pitch for Simulation Data Set A-8 189

Table 101: Success Rates of Reaching Angular Specifications of Fused Heading for Simulation Data Set A-8 189

Table 102: Pitch / Heading Correlation Coefficients for Each Computation Method in Simulation Data Set A-8 190

Figure 72: Computed and Measured Headings for Simulation Set B-1 190

Figure 73: Computed and Measured Pitch for Simulation Set B-1 191

Figure 74: Propagated 95\% Confidence Ellipses for Front and Rear GPS Antennas in Simulated Data Set B-1 191

Table 103: Mean Global Error Statistics of Unfused Pitch for Simulation Set B-1 192

Table 104: Mean Global Error Statistics of Unfused Heading for Simulation Set B-1 192

Table 105: Mean Global Error Statistics of Fused Pitch for Simulation Set B-1 193

Table 106: Mean Global Error Statistics of Fused Heading for Simulation Set B-1 193

Table 107: Success Rates of Reaching Angular Specifications of Unfused Pitch for Simulation Data Set B-1 194

Table 108: Success Rates of Reaching Angular Specifications of Unfused Heading for Simulation Data Set B-1 194

Table 109: Success Rates of Reaching Angular Specifications of Fused Pitch for Simulation Data Set B-1 195

Table 110: Success Rates of Reaching Angular Specifications of Fused Heading for Simulation Data Set B-1 195

Table 111: Pitch / Heading Correlation Coefficients for Each Computation Method in Simulation Data Set B-1 196

Figure 75: Computed and Measured Headings for Simulation Set B-2 196

Figure 76: Computed and Measured Pitch for Simulation Set B-2 197

Figure 77: Propagated 95\% Confidence Ellipses for Front and Rear GPS Antennas in Simulated Data Set B-2 198

Table 112: Mean Global Error Statistics of Unfused Pitch for Simulation Set B-2 198

Table 113: Mean Global Error Statistics of Unfused Heading for Simulation Set B-2 199

Table 114: Mean Global Error Statistics of Fused Pitch for Simulation Set B-2 199

Table 115: Mean Global Error Statistics of Fused Heading for Simulation Set B-2 200

Table 116: Success Rates of Reaching Angular Specifications of Unfused Pitch for Simulation Data Set B-2 200

Table 117: Success Rates of Reaching Angular Specifications of Unfused Heading for Simulation Data Set B-2 201

Table 118: Success Rates of Reaching Angular Specifications of Fused Pitch for Simulation Data Set B-2 201 
Table 119: Success Rates of Reaching Angular Specifications of Fused Heading for Simulation Data Set B-2 202

Table 120: Pitch / Heading Correlation Coefficients for Each Computation Method in Simulation Data Set B-2 202

Figure 78: Computed and Measured Headings for Simulation Set B-3 203

Figure 79: Computed and Measured Pitch for Simulation Set B-3 204

Figure 80: Propagated 95\% Confidence Ellipses for Front and Rear GPS Antennas in Simulated Data Set B-3 204

Table 121: Mean Global Error Statistics of Unfused Pitch for Simulation Set B-3 205

Table 122: Mean Global Error Statistics of Unfused Heading for Simulation Set B-3 205

Table 123: Mean Global Error Statistics of Fused Pitch for Simulation Set B-3 206

Table 124: Mean Global Error Statistics of Fused Heading for Simulation Set B-3 206

Table 125: Success Rates of Reaching Angular Specifications of Unfused Pitch for Simulation Data Set B-3 207

Table 126: Success Rates of Reaching Angular Specifications of Unfused Heading for Simulation Data Set B-3 207

Table 127: Success Rates of Reaching Angular Specifications of Fused Pitch for Simulation Data Set B-3 208

Table 128: Success Rates of Reaching Angular Specifications of Fused Heading for Simulation Data Set B-3 208

Table 129: Pitch / Heading Correlation Coefficients for Each Computation Method in Simulation Data Set B-3 209

Figure 81: Computed and Measured Headings for Simulation Set B-4 209

Figure 82: Computed and Measured Pitch for Simulation Set B-4 210

Figure 83: Propagated 95\% Confidence Ellipses for Front and Rear GPS Antennas in Simulated Data Set B-4 210

Table 130: Mean Global Error Statistics of Unfused Pitch for Simulation Set B-4 211

Table 131: Mean Global Error Statistics of Unfused Heading for Simulation Set B-4 211

Table 132: Mean Global Error Statistics of Fused Pitch for Simulation Set B-4 212

Table 133: Mean Global Error Statistics of Fused Heading for Simulation Set B-4 212

Table 134: Success Rates of Reaching Angular Specifications of Unfused Pitch for Simulation Data Set B-4 213

Table 135: Success Rates of Reaching Angular Specifications of Unfused Heading for Simulation Data Set B-4 213

Table 136: Success Rates of Reaching Angular Specifications of Fused Pitch for Simulation Data Set B-4 214

Table 137: Success Rates of Reaching Angular Specifications of Fused Heading for Simulation Data Set B-4 214

Table 138: Pitch / Heading Correlation Coefficients for Each Computation Method in Simulation Data Set B-4 215

Figure 84: Computed and Measured Headings for Simulation Set B-5 215 
Figure 85: Computed and Measured Pitch for Simulation Set B-5

Figure 86: Propagated 95\% Confidence Ellipses for Front and Rear GPS Antennas in Simulated Data Set B-5 216

Table 139: Mean Global Error Statistics of Unfused Pitch for Simulation Set B-5 217

Table 140: Mean Global Error Statistics of Unfused Heading for Simulation Set B-5 217

Table 141: Mean Global Error Statistics of Fused Pitch for Simulation Set B-5 218

Table 142: Mean Global Error Statistics of Fused Heading for Simulation Set B-5 218

Table 143: Success Rates of Reaching Angular Specifications of Unfused Pitch for Simulation Data Set B-5 219

Table 144: Success Rates of Reaching Angular Specifications of Unfused Heading for Simulation Data Set B-5 219

Table 145: Success Rates of Reaching Angular Specifications of Fused Pitch for Simulation Data Set B-5 220

Table 146: Success Rates of Reaching Angular Specifications of Fused Heading for Simulation Data Set B-5 220

Table 147: Pitch / Heading Correlation Coefficients for Each Computation Method in Simulation Data Set B-5 221

Figure 87: Computed and Measured Headings for Simulation Set B-6 221

Figure 88: Computed and Measured Pitch for Simulation Set B-6 222

Figure 89: Propagated 95\% Confidence Ellipses for Front and Rear GPS Antennas in Simulated Data Set B-6 223

Table 148: Mean Global Error Statistics of Unfused Pitch for Simulation Set B-6 223

Table 149: Mean Global Error Statistics of Unfused Heading for Simulation Set B-6 224

Table 150: Mean Global Error Statistics of Fused Pitch for Simulation Set B-6 224

Table 151: Mean Global Error Statistics of Fused Heading for Simulation Set B-6 225

Table 152: Success Rates of Reaching Angular Specifications of Unfused Pitch for Simulation Data Set B-6 225

Table 153: Success Rates of Reaching Angular Specifications of Unfused Heading for Simulation Data Set B-6 226

Table 154: Success Rates of Reaching Angular Specifications of Fused Pitch for Simulation Data Set B-6 226

Table 155: Success Rates of Reaching Angular Specifications of Fused Heading for Simulation Data Set B-6 227

Table 156: Pitch / Heading Correlation Coefficients for Each Computation Method in Simulation Data Set B-6 227

Figure 90: Computed and Measured Headings for Simulation Set B-7 228

Figure 91: Computed and Measured Pitch for Simulation Set B-7 229

Figure 92: Propagated 95\% Confidence Ellipses for Front and Rear GPS Antennas in Simulated Data Set B-7 229

Table 157: Mean Global Error Statistics of Unfused Pitch for Simulation Set B-7 230 
Table 158: Mean Global Error Statistics of Unfused Heading for Simulation Set B-7

Table 159: Mean Global Error Statistics of Fused Pitch for Simulation Set B-7

Table 160: Mean Global Error Statistics of Fused Heading for Simulation Set B-7

Table 161: Success Rates of Reaching Angular Specifications of Unfused Pitch for Simulation Data Set B-7

Table 162: Success Rates of Reaching Angular Specifications of Unfused Heading for Simulation Data Set B-7 232

Table 163: Success Rates of Reaching Angular Specifications of Fused Pitch for Simulation Data Set B-7

Table 164: Success Rates of Reaching Angular Specifications of Fused Heading for Simulation Data Set B-7

Table 165: Pitch / Heading Correlation Coefficients for Each Computation Method in Simulation Data Set B-7 234

Figure 93: Computed and Measured Headings for Simulation Set B-8

Figure 94: Computed and Measured Pitch for Simulation Set B-8

Figure 95: Propagated 95\% Confidence Ellipses for Front and Rear GPS Antennas in Simulated Data Set B-8

Table 166: Mean Global Error Statistics of Unfused Pitch for Simulation Set B-8

Table 167: Mean Global Error Statistics of Unfused Heading for Simulation Set B-8

Table 168: Mean Global Error Statistics of Fused Pitch for Simulation Set B-8

Table 169: Mean Global Error Statistics of Fused Heading for Simulation Set B-8

Table 170: Success Rates of Reaching Angular Specifications of Unfused Pitch for Simulation Data Set B-8

Table 171: Success Rates of Reaching Angular Specifications of Unfused Heading for Simulation Data Set B-8 238

Table 172: Success Rates of Reaching Angular Specifications of Fused Pitch for Simulation Data Set B-8

Table 173: Success Rates of Reaching Angular Specifications of Fused Heading for Simulation Data Set B-8

Table 174: Pitch / Heading Correlation Coefficients for Each Computation Method in Simulation Data Set B-8 240

Figure 96: Computed and Measured Headings for Simulation Set C-1

Figure 97: Computed and Measured Headings for Simulation Set C-1

Figure 98: Propagated 95\% Confidence Ellipses for Front and Rear GPS Antennas in Simulated Data Set C-1

Table 175: Mean Global Error Statistics of Unfused Pitch for Simulation Set C-1

Table 176: Mean Global Error Statistics of Unfused Heading for Simulation Set C-1

Table 177: Mean Global Error Statistics of Fused Pitch for Simulation Set C-1

Table 178: Mean Global Error Statistics of Fused Heading for Simulation Set C-1 
Table 179: Success Rates of Reaching Angular Specifications of Unfused Pitch for Simulation Data Set C-1

Table 180: Success Rates of Reaching Angular Specifications of Unfused Heading for Simulation Data Set C-1 245

Table 181: Success Rates of Reaching Angular Specifications of Fused Pitch for Simulation Data Set C-1 245

Table 182: Success Rates of Reaching Angular Specifications of Fused Heading for Simulation Data Set C-1 246

Table 183: Pitch / Heading Correlation Coefficients for Each Computation Method in Simulation Data Set C-1 246

Figure 99: Computed and Measured Headings for Simulation Set C-2 247

Figure 100: Computed and Measured Pitch for Simulation Set C-2 248

Figure 101: Propagated 95\% Confidence Ellipses for Front and Rear GPS Antennas in Simulated Data Set C-2 248

Table 184: Mean Global Error Statistics of Unfused Pitch for Simulation Set C-2 249

Table 185: Mean Global Error Statistics of Unfused Heading for Simulation Set C-2 249

Table 186: Mean Global Error Statistics of Fused Pitch for Simulation Set C-2 250

Table 187: Mean Global Error Statistics of Fused Heading for Simulation Set C-2 250

Table 188: Success Rates of Reaching Angular Specifications of Unfused Pitch for Simulation Data Set C-2 251

Table 189: Success Rates of Reaching Angular Specifications of Unfused Heading for Simulation Data Set C-2 251

Table 190: Success Rates of Reaching Angular Specifications of Fused Pitch for Simulation Data Set C-2 252

Table 191: Success Rates of Reaching Angular Specifications of Fused Heading for Simulation Data Set C-2 252

Table 192: Pitch / Heading Correlation Coefficients for Each Computation Method in Simulation Data Set C-2 253

Figure 102: Computed and Measured Headings for Simulation Set C-3 253

Figure 103: Computed and Measured Pitch for Simulation Set C-3 254

Figure 104: Propagated 95\% Confidence Ellipses for Front and Rear GPS Antennas in Simulated Data Set C-3 254

Table 193: Mean Global Error Statistics of Unfused Pitch for Simulation Set C-3 255

Table 194: Mean Global Error Statistics of Unfused Heading for Simulation Set C-3 255

Table 195: Mean Global Error Statistics of Fused Pitch for Simulation Set C-3 256

Table 196: Mean Global Error Statistics of Fused Heading for Simulation Set C-3 256

Table 197: Success Rates of Reaching Angular Specifications of Unfused Pitch for Simulation Data Set C-3 257

Table 198: Success Rates of Reaching Angular Specifications of Unfused Heading for Simulation Data Set C-3 257

Table 199: Success Rates of Reaching Angular Specifications of Fused Pitch for Simulation Data Set C-3 258 
Table 200: Success Rates of Reaching Angular Specifications of Fused Heading for Simulation Data Set C-3 258

Table 201: Pitch / Heading Correlation Coefficients for Each Computation Method in Simulation Data Set C-3 259

Figure 105: Computed and Measured Headings for Simulation Set C-4 259

Figure 106: Computed and Measured Pitch for Simulation Set C-4 260

Figure 107: Propagated 95\% Confidence Ellipses for Front and Rear GPS Antennas in Simulated Data Set C-4 260

Table 202: Mean Global Error Statistics of Unfused Pitch for Simulation Set C-4 261

Table 203: Mean Global Error Statistics of Unfused Heading for Simulation Set C-4 261

Table 204: Mean Global Error Statistics of Fused Pitch for Simulation Set C-4 262

Table 205: Mean Global Error Statistics of Fused Heading for Simulation Set C-4 262

Table 206: Success Rates of Reaching Angular Specifications of Unfused Pitch for Simulation Data Set C-4 263

Table 207: Success Rates of Reaching Angular Specifications of Unfused Heading for Simulation Data Set C-4 263

Table 208: Success Rates of Reaching Angular Specifications of Fused Pitch for Simulation Data Set C-5 264

Table 209: Success Rates of Reaching Angular Specifications of Fused Heading for Simulation Data Set C-5 264

Table 210: Pitch / Heading Correlation Coefficients for Each Computation Method in Simulation Data Set C-4 265

Figure 108: Computed and Measured Headings for Simulation Set C-5 265

Figure 109: Computed and Measured Pitch for Simulation Set C-5 266

Figure 110: Propagated 95\% Confidence Ellipses for Front and Rear GPS Antennas in Simulated Data Set C-5 266

Table 211: Mean Global Error Statistics of Unfused Pitch for Simulation Set C-5 267

Table 212: Mean Global Error Statistics of Unfused Heading for Simulation Set C-5 267

Table 213: Mean Global Error Statistics of Fused Pitch for Simulation Set C-5 268

Table 214: Mean Global Error Statistics of Fused Heading for Simulation Set C-5 268

Table 215: Success Rates of Reaching Angular Specifications of Unfused Pitch for Simulation Data Set C-5 269

Table 216: Success Rates of Reaching Angular Specifications of Unfused Heading for Simulation Data Set C-5 269

Table 217: Success Rates of Reaching Angular Specifications of Fused Pitch for Simulation Data Set C-5 270

Table 218: Success Rates of Reaching Angular Specifications of Fused Heading for Simulation Data Set C-5 270

Table 219: Pitch / Heading Correlation Coefficients for Each Computation Method in Simulation Data Set C-5 271

Figure 111: Computed and Measured Headings for Simulation Set C-6 271 
Figure 112: Computed and Measured Pitch for Simulation Set C-6

Figure 113: Propagated 95\% Confidence Ellipses for Front and Rear GPS Antennas in Simulated Data Set C-6 272

Table 220: Mean Global Error Statistics of Unfused Pitch for Simulation Set C-6

Table 221: Mean Global Error Statistics of Unfused Heading for Simulation Set C-6

Table 222: Mean Global Error Statistics of Fused Pitch for Simulation Set C-6

Table 223: Mean Global Error Statistics of Fused Heading for Simulation Set C-6

Table 224: Success Rates of Reaching Angular Specifications of Unfused Pitch for Simulation Data Set C-6

Table 225: Success Rates of Reaching Angular Specifications of Unfused Heading for Simulation Data Set C-6 275

Table 226: Success Rates of Reaching Angular Specifications of Fused Pitch for Simulation Data Set C-6 276

Table 227: Success Rates of Reaching Angular Specifications of Fused Heading for Simulation Data Set C-6 276

Table 228: Pitch / Heading Correlation Coefficients for Each Computation Method in Simulation Data Set C-6 277

Figure 114: Computed and Measured Headings for Simulation Set C-7

Figure 115: Computed and Measured Pitch for Simulation Set C-7

Figure 116: Propagated 95\% Confidence Ellipses for Front and Rear GPS Antennas in Simulated Data Set C-7 278

Table 229: Mean Global Error Statistics of Unfused Pitch for Simulation Set C-7

Table 230: Mean Global Error Statistics of Unfused Heading for Simulation Set C-7

Table 231: Mean Global Error Statistics of Fused Pitch for Simulation Set C-7

Table 232: Mean Global Error Statistics of Fused Heading for Simulation Set C-7

Table 233: Success Rates of Reaching Angular Specifications of Unfused Pitch for Simulation Data Set C-7

Table 234: Success Rates of Reaching Angular Specifications of Unfused Heading for Simulation Data Set C-7 281

Table 235: Success Rates of Reaching Angular Specifications of Fused Pitch for Simulation Data Set C-7 282

Table 236: Success Rates of Reaching Angular Specifications of Fused Heading for Simulation Data Set C-7 282

Table 237: Pitch / Heading Correlation Coefficients for Each Computation Method in Simulation Data Set C-7 283

Figure 117: Computed and Measured Headings for Simulation Set C-8 283

Figure 118: Computed and Measured Pitch for Simulation Set C-8 284

Figure 119: Propagated 95\% Confidence Ellipses for Front and Rear GPS Antennas in Simulated Data Set C-8 284

Table 238: Mean Global Error Statistics of Unfused Pitch for Simulation Set C-8 
Table 239: Mean Global Error Statistics of Unfused Heading for Simulation Set C-8

Table 240: Mean Global Error Statistics of Fused Pitch for Simulation Set C-8

Table 241: Mean Global Error Statistics of Fused Heading for Simulation Set C-8

Table 242: Success Rates of Reaching Angular Specifications of Unfused Pitch for Simulation Data Set C-8

Table 243: Success Rates of Reaching Angular Specifications of Unfused Heading for Simulation Data Set C-8 287

Table 244: Success Rates of Reaching Angular Specifications of Fused Pitch for Simulation Data Set C-8 288

Table 245: Success Rates of Reaching Angular Specifications of Fused Heading for Simulation Data Set C-8 288

Table 246: Pitch / Heading Correlation Coefficients for Each Computation Method in Simulation Data Set C-8 289

Figure 120: Global Error and Noise Trends for Pseudorange Solutions Across All Series A Simulation Data Sets 290

Figure 121: Series A Success Levels in Meeting Angular Specifications Between 1/2 Degree and 5 Degrees 291

Figure 122: Series A Success Levels in Meeting Angular Specifications Between 10 Degrees and 25 Degrees 291

Figure 123: Global Error and Noise Trends for Pseudorange Solutions Across All Series B Simulation Data Sets 292

Figure 124: Series B Success Levels in Meeting Angular Specifications Between 1/2 Degree and 5 Degrees 293

Figure 125: Series B Success Levels in Meeting Angular Specifications Between 10 Degrees and 25 Degrees 293

Figure 126: Global Error and Noise Trends for Pseudorange Solutions Across All Series C Simulation Data Sets 294

Figure 127: Series C Success Levels in Meeting Angular Specifications Between 1/2 Degree and 5 Degrees 295

Figure 128: Series C Success Levels in Meeting Angular Specifications Between 10 Degrees and 25 Degrees 295 


\section{Table of Contents}

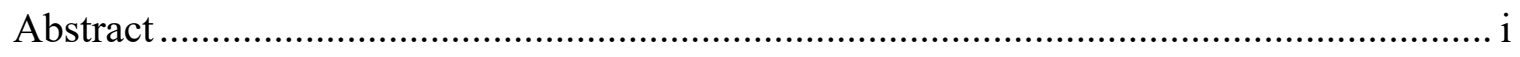

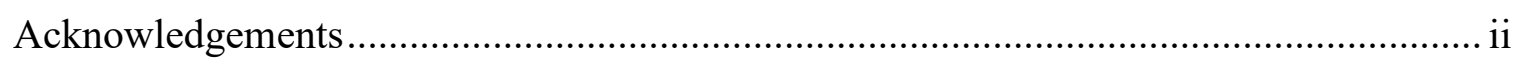

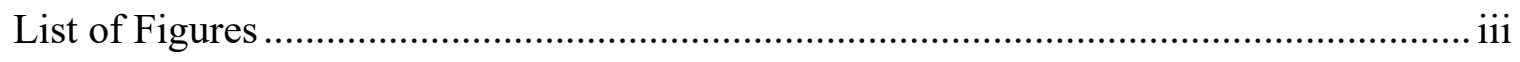

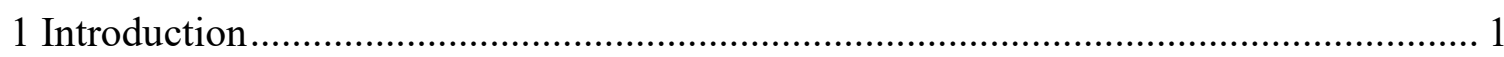

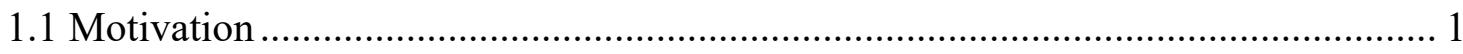

1.2 Problem Statement .................................................................................. 2

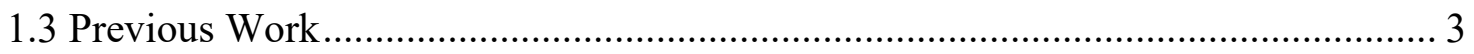

1.3.1 GNSS Error Mitigation................................................................................ 3

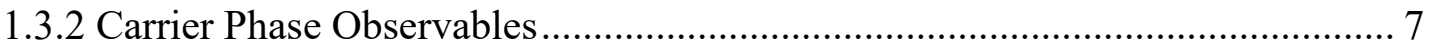

1.3.3 GPS for Pitch and Heading Determination............................................. 10

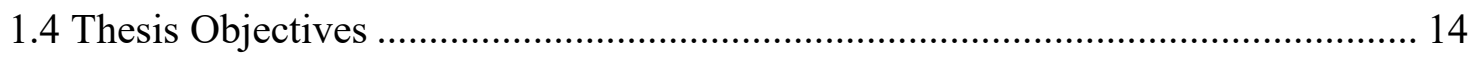

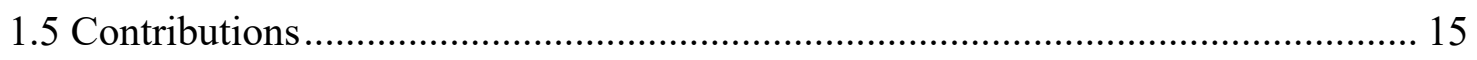

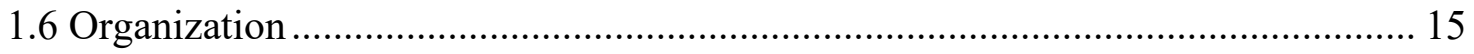

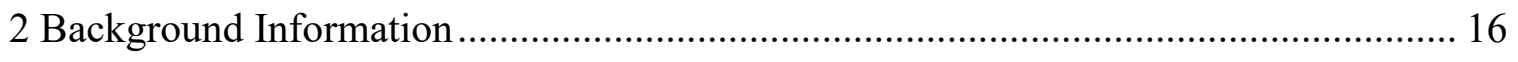

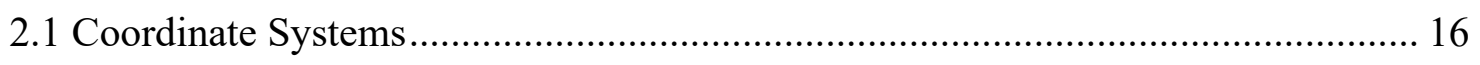

2.1.1 Earth-Centered, Earth-Fixed (ECEF) Coordinates ...................................... 16

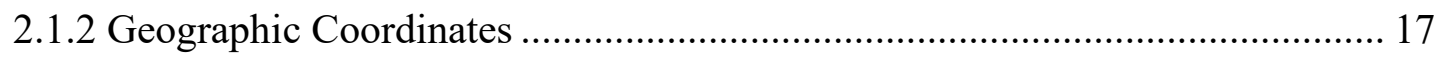


2.1.3 Local Vertical Compass Reference Frames................................................... 18

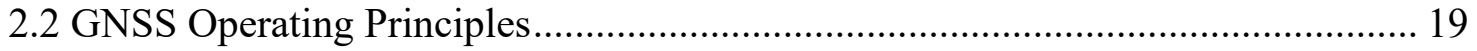

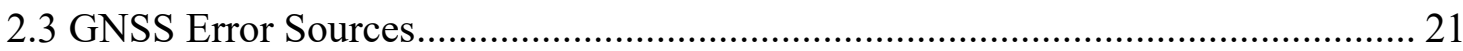

2.3.1 Pseudorange Error .............................................................................. 21

2.3.2 Carrier Phase Error ................................................................................ 25

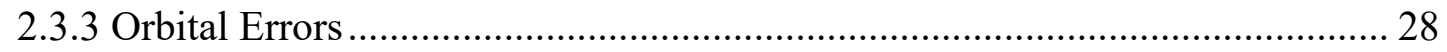

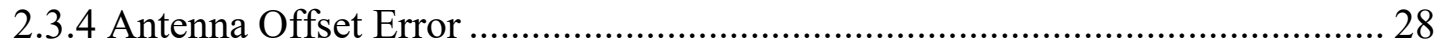

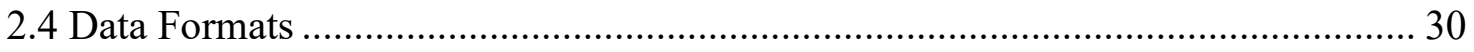

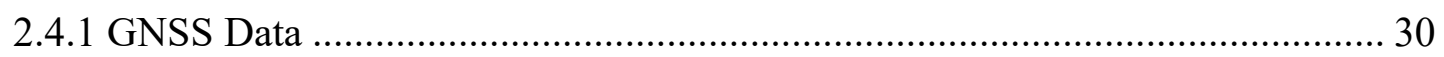

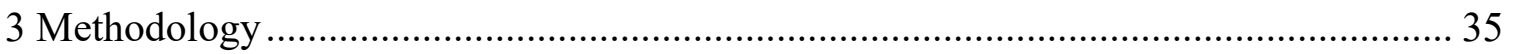

3.1 GNSS Pseudorange and Carrier Phase Corrections ........................................... 35

3.1.1 Pseudorange Corrections ........................................................................ 35

3.1.2 Carrier Phase Ambiguity Resolution ......................................................... 38

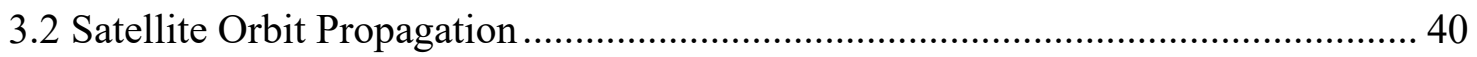

3.3 Parametric Least Squares Adjustments ............................................................. 46

3.3.1 Least Squares Adjustment in General .................................................... 46

3.3.2 Weighted Parametric Least Squares Adjustment ...................................... 48

3.4 Least Squares Mathematical and Stochastic Models ....................................... 49

3.4.1 Global Navigation Satellite Systems Positioning Models............................. 49

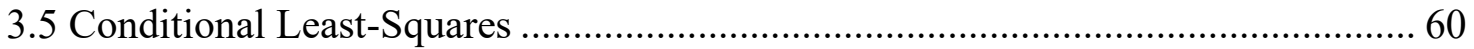




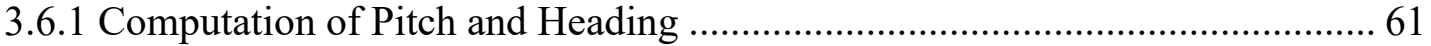

3.6.2 Static Kalman Filter.............................................................................. 71

3.7 Error and Uncertainty Metrics................................................................ 74

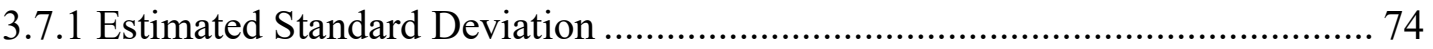

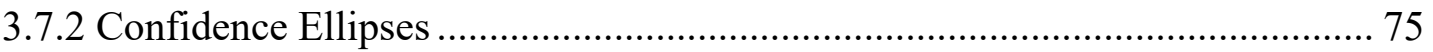

3.7.3 Root Mean Square (RMS) Error...................................................................... 76

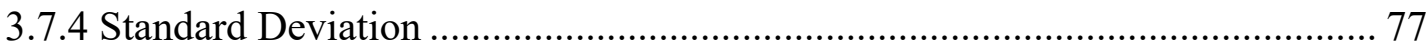

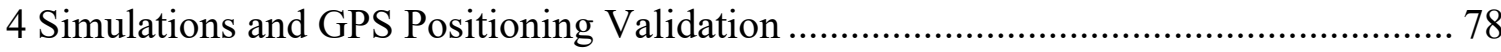

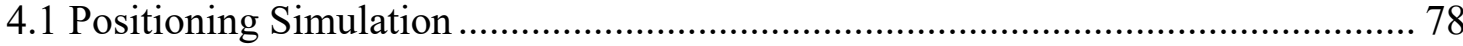

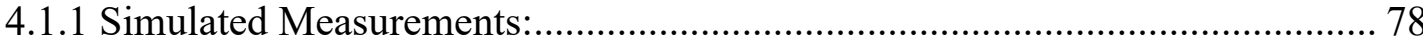

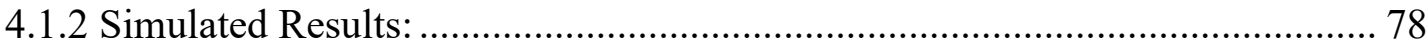

4.2 Didsbury Control Marker Survey ................................................................... 99

4.3 Carleton University Control Point Survey …........................................... 102

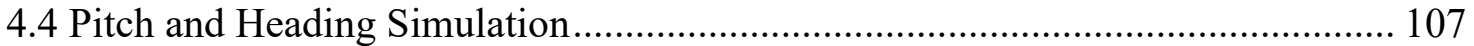

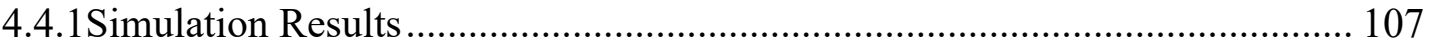

4.5 Mission Design Using Simulation Results ...................................................... 114

4.5.1 Example when Solving for Pseudorange Noise ...................................... 114

4.5.2 Example when Solving for Antenna Separation....................................... 116

5 GPS Pitch and Heading Experimental Control Point Survey ................................... 118 


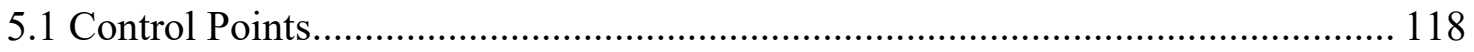

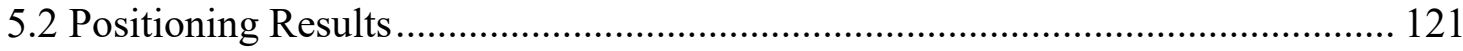

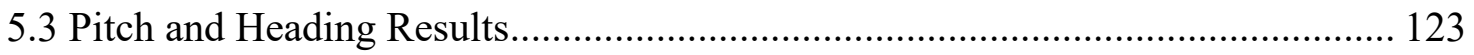

5.4 Effects of Antenna Separation on Pitch and Heading Accuracy........................ 127

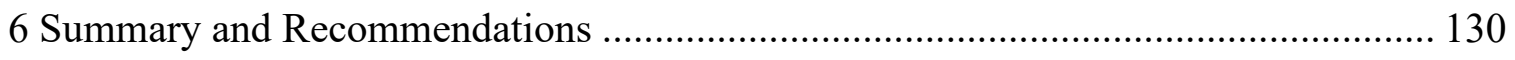

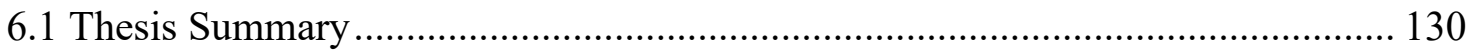

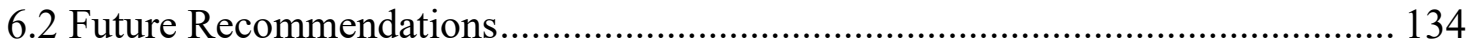

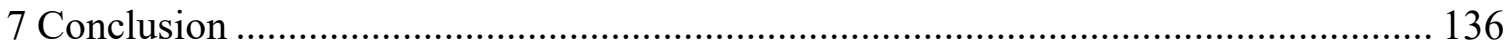

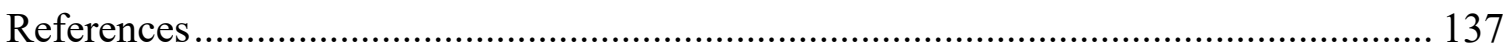

Appendix A: $10 \mathrm{~cm}$ Pseudorange Noise Standard Deviation Pitch and Heading Simulation Results ........................................................................................................ 142

Set A-1: 1 m Dual Antenna Separation ........................................................... 142

Set A-2: 3 m Dual Antenna Separation ............................................................ 148

Set A-3: 5 m Dual Antenna Separation ............................................................. 154

Set A-4: 10 m Dual Antenna Separation ........................................................... 160

Set A-5: 30 m Dual Antenna Separation .......................................................... 166

Set A-6: 50 m Dual Antenna Separation .............................................................. 172

Set A-7: 70 m Dual Antenna Separation ........................................................... 178

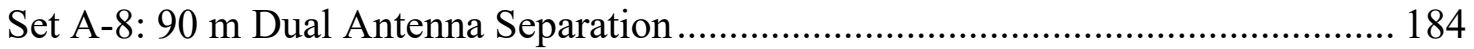


Appendix B: $1 \mathrm{~m}$ Pseudorange Noise Standard Deviation Pitch and Heading Simulation

Results 190

Set B-1: $1 \mathrm{~m}$ Dual Antenna Separation 190

Set B-2: 3 m Dual Antenna Separation 196

Set B-3: 5 m Dual Antenna Separation 203

Set B-4: 10 m Dual Antenna Separation 209

Set B-5: 30 m Dual Antenna Separation 215

Set B-6: 50 m Dual Antenna Separation 221

Set B-7: 70 m Dual Antenna Separation 228

Set B-8: 90 m Dual Antenna Separation 234

Appendix C: 3 m Pseudorange Noise Standard Deviation Pitch and Heading Simulation

Results 240

Set C-1 1 m Dual Antenna Separation 240

Set C-2 3 m Dual Antenna Separation 247

Set C-3 5 m Dual Antenna Separation 253

Set C-4 10 m Dual Antenna Separation 259

Set C-5 30 m Dual Antenna Separation 265

Set C-6 50 m Dual Antenna Separation 271

Set C-7 70 m Dual Antenna Separation 277

Set C-8 90 m Dual Antenna Separation 283 
Appendix D: Summary of Global Trends Across all Partial Attitude Simulation Data Sets

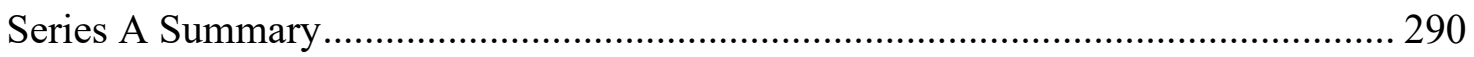

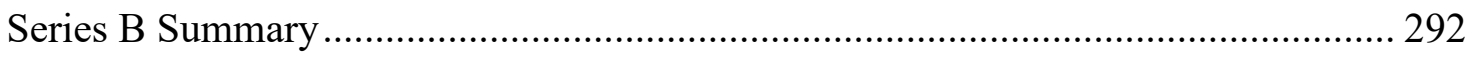

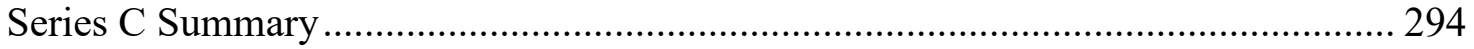




\section{Introduction}

\subsection{Motivation}

One major design consideration in mission planning for navigation is that it is highly desirable to minimize the budget for sensors while maximizing the robustness, accuracy and precision of the solutions that the sensors yield. One aspect of navigation is measurement of the platform position. Global Navigation Satellite Systems (GNSS) allow for determination of position in a global frame at any location on earth which has a line of sight to at least four satellites. Use of a second antenna on the same platform also allows the GNSS to double as a determiner of partial attitude. Specifically, a front to rear antenna setup allows for calculation of pitch and compass heading. In order to make a partial attitude determination system, an integrated Inertial Navigation System (INS) can be employed, which allows for the third attitude parameter, roll, to be computed along with the partial attitude. It also tends to offer higher precision attitude computation than a pseudorange-only GPS attitude solution with short antenna separation. Robustness of partial attitude determination can be increased by incorporating INS or using a high precision GPS system which incorporates the high precision carrier phase observables, or by increasing the separation between antennas. In the case of INS, it incurs an additional cost on top of positioning costs and it has a bias drift which increases over time due to the Inertial Measurement Unit (IMU) [1]. The use of high precision GPS requires a high grade of receiver, or a base station receiver at minimum so that the carrier phase can be utilized. Increasing antenna separation is only viable insofar as the geometry of the platform allows it. 
Therefore, it is pertinent to quantify the viability of a low-cost GPS partial attitude / positioning system for navigation in order to aid in mission design.

\subsection{Problem Statement}

One difficulty in designing a GPS partial attitude determination system is that using high quality sensors incurs costs which will not meet some budgets. The use of a GPS base station or dual frequency receiver on the platform, for instance, allows for a variety of strategies for solving the integer ambiguity problem of the carrier phase, which allows for high precision positioning[2]. That translates to higher precision of pitch and heading computations using GPS antenna positions. However, this incurs additional costs. A real time kinematic (RTK) GPS system with dual frequency capability currently ranges in price from approximately $\$ 3000 \mathrm{CDN}$ to $\$ 20,000 \mathrm{CDN}$. On the other hand, a single frequency, single point GPS system costs much less (approximately $\$ 100 \mathrm{CDN}$ for receiver boards with antennas, $\$ 500 \mathrm{CDN}$ for a system with a built-in interface). The main challenge of using the less expensive systems is that it is much more difficult to readily access the carrier phase. In fact, some systems such as the Huawei P20 do not support access to carrier phase measurement with raw data.

Therefore, it is important to determine what limitations a GPS partial attitude system may have when only pseudoranges are used. While it is well known that increasing antenna separation increases the precision of the pitch and heading computation, this effect has not been quantified in a thorough way to the author's knowledge. When designing a partial attitude system for navigation, it would be helpful to have tabulated statistics with varying antenna separation as well as varying pseudorange noise. This allows a designer to solve the problem of 
how far 2 antennas must be separated to meet their specifications, or the reverse problem of what maximum receiver noise standard deviation is required for a limited fixed separation. This thesis is intended to address both problems.

\subsection{Previous Work}

Use of satellite positioning systems for attitude determination is not a new concept. A great deal of research has been done regarding all steps which need to be taken in the attitude determination process using GPS. This section will discuss this research in 3 categories: GNSS error mitigation, use of the carrier phase observable and attitude determination strategies. Error mitigation and use of the carrier phase are important because computed positions from GPS solutions are used directly to compute attitude, while review of attitude determination strategies is important in order to evaluate available options in a low-cost system.

\subsubsection{GNSS Error Mitigation}

Mitigation of GPS errors entails isolating and attempting to remove the effects of ranging errors including atmospheric delays, multipath delay, receiver and satellite clock errors, satellite orbital errors and receiver noise. One of the simplest errors to mitigate is atmospheric bias.

Atmospheric delays generally come from two sources: the ionosphere and the troposphere. The ionosphere refracts a transiting signal due to ionized particles, while the troposphere has a refractive effect due to temperature and pressure changes. The most readily available way to mitigate these errors for a single point solution is to use models for the atmosphere. In order to model the troposphere, one could rely on the model developed by J. Saastamoinen[3] or utilize the Hopfield model[4]. Both models rely on weather information including pressure and temperature in conjunction with estimated geometry between satellites 
and the receiver antenna, which means that they can be utilized for any solution as long as weather parameters are available. A study by C. Satirapod and P. Chalermwattanachai[5] compared the effectiveness of these techniques and concluded that both methods yield very similar pseudorange positioning accuracy and that both yield optimal results for baseline positioning. Therefore, they recommended use of either method. Another study by Wielgosz et al.[6] compared the Hopfield model with more robust techniques which make use of reference networks, European Position Determination Systems (EUPOS) or precise point positioning based techniques. While these techniques were more effective in troposphere mitigation than the Hopfield model, the authors concluded that the Hopfield model still yielded results which were suitable for many positioning applications.

The other atmospheric delay for GPS pseudoranges is ionospheric delay. The most readily available method for modelling the ionosphere delay is the Total Electron Content (TEC) model outlined by the International GPS Service (IGS) Interface Control Document[7]. This is an important method because it only requires a measurement of the TEC in a particular geographic area at the time of acquisition, which can be acquired from a TEC map provided by various organizations. The method was evaluated by Jakowski et al.[8], who noted that the technique's ease of use and simplicity make it well-suited for use in near-real-time applications. Another method of ionosphere mitigation is using augmentation systems such as Wide Area Augmentation Systems (WAAS). This was examined by Paul Cannon[9], who suggested that while forecast based systems utilizing ground instruments could be improved by increasing the number of available ground stations for redundancy, these techniques simply will not be sufficient for some applications. A very effective way of mitigating ionosphere delay is by using dual frequency techniques, which use the dependency of ionosphere effects on signal frequency 
to make a linear combination of measurements from two signals which eliminates bias. Some methods using multiple frequencies were evaluated by P. Bolla and K. Borre[10], who noted that while first and second order ionosphere effects can be mitigated by dual frequency techniques, such receivers can consume considerable battery power. It is also important to note that dual frequency receivers are generally more expensive than single frequency receivers, making them a poor option for the purposes of this thesis.

Another error source which can induce significant biases is multipath error. Most methods for minimizing the impacts of multipath require improvements to antenna and receiver technology or planning missions in multipath-free environments. One such method examined by Jones et al[11] uses a pulse aperture correlator with narrow lane characteristics. The authors noted that while choke-ring ground planes can be used to optimize antenna gain patterns and site selection can also reduce multipath as well as noise effects, these are not necessarily practical for kinematic applications. The additional bulk of this equipment makes it impractical for many moving platforms. They concluded that the narrow correlation function in the receiver causes rejection of multipath signals at more than 1 chip length while having negligible influence from multipath in the 0.1 to 0.9 chip length range. Further, G. Seepersad and S. Bisnath[12] illustrated that modelling multipath temporal variability in order to mitigate it in static applications can improve pseudorange precision and decrease convergence time in precise point positioning applications. This temporal variability is a characteristic of most GNSS errors, not only multipath. M. Olynik[13] studied these effects in detail, concluding that using precise orbits, precise clocks, dual frequency receivers and a troposphere correction yields the best possible solution in a 10-50 second time interval. The narrow correlator incurs additional cost and the temporal variability modelling is restricted to static applications, which means that they cannot 
be used for this research, which will be dealing with low cost solutions which can be applied to kinematic applications. This suggests that noise and multipath would be expected as major factors to the effectiveness of a low-cost GPS attitude solution. 


\subsubsection{Carrier Phase Observables}

One ranging error which is difficult to eliminate from code pseudoranges is random noise. The second option for range measurement is the carrier phase range measurement. This is by far the less noisy of the two measurements, so there is a great deal of motivation to use the carrier phase when high precision positioning is required. However, the integer ambiguity problem prevents use of the measurement without isolating the ambiguities first. In particular, the carrier phase precisely measures a fractional number of partial cycles of the carrier signal. While the effects from noise on this measurement are minimal, there is an integer number of complete cycles which separates this measurement from the actual range from the satellite to the receiver antenna phase center. This is illustrated in Figure 4, found in section 2.3.2.3. This problem has been a significant topic for research. Techniques for ambiguity resolution can generally be placed in three different categories: geometric searches, dual frequency solutions and triple differencing.

Geometric searches are currently the best available ambiguity resolution option for applications where the antenna is not stationary over time and dual frequency measurements are not available. Many authors have investigated the use of such techniques, such as G. Teunissen[14] who developed the Least-Squares Ambiguity Decorrelation Adjustment (LAMBDA) technique, which uses a transformation based on the estimated variance-covariance matrix of the unknown coordinates to transform and decorrelate the double difference float ambiguities. This technique has been adopted and studied by many authors as well, such as D. Cox and J. Brading[15], who applied the method to a Kalman filtered spacecraft navigation solution. They computed a Kalman filtered state vector and its corresponding variancecovariance matrix at each epoch which were used as input to the LAMBDA algorithm, finding 
that the combination of a Kalman filter with LAMBDA for navigation yielded somewhat robust integer ambiguity selection success as well as reduced sensitivity to selection of incorrect integer combinations. The method was also used in Junjie Liu's thesis[16] on implementation and analysis of ambiguity resolution techniques in single and multiple reference station scenarios. Using the FLYKIN+ software package (which was developed using a C++ navigation lab library belonging to the University of Calgary Geomatics Engineering Department), he evaluated 8 different ambiguity resolution strategies, finding that double differenced ionosphere delay directly impacts the success of most strategies and that stochastic ionospheric modelling strategies generally performed better than ionosphere-free strategies in adverse ionosphere conditions.

While ionosphere-free solutions require use of a dual frequency receiver which generally imposes an increased cost to the GPS budget for a mission, they can nonetheless be very successful tools for ambiguity resolution. It has been analyzed in detail, such as the case with G. Blewitt[17], who applied the ionosphere free phase combination solution to baselines up to $2000 \mathrm{~km}$ apart and based on results, hypothesized that bias optimization was superior to bias fixing of the ionosphere free solution over some sparse networks, but could not conclusively say so with the available data to the study. It is also worth noting that this technique was using more than a single receiver for positioning, which is an additional cost for the mission. On the other hand, Collins et al[18] used a decoupled clock model as an alternative to the differential solution requirement. The authors noted that the ionosphere-free model cannot explicitly solve for the ambiguities because the correlation of the phase and code biases with the clock biases interferes with the isolation of the ambiguity. However, it was noted that by decoupling the code and phase oscillator parameters, the ambiguity can be more easily isolated and ambiguities can be 
successfully fixed to integer values. There is also an algorithm used by Bertiger et al[19] which works by first defining integer ambiguities in both L1 and L2 frequencies, then widelanes are computed and subsequently narrow lanes from a Kalman filter smoothed ionosphere free solution. The authors found that using IGS orbit products in conjunction with their modifications to the method, the precision in measured baselines could be significantly improved. Laurichesse et al[20] produced experimental results using undifferenced dual frequency measurements yielding positioning precision of less than $1 \mathrm{~cm}$, which does support use of both frequencies when available, even if a base receiver is not available.

Another method of resolving the integer ambiguity problem is using a triple difference of 2 double differences across 2 subsequent epochs. While this can be done by simply using the triple difference directly as an observable and assuming cancellation of the ambiguity term, this can be dangerous if cycle slip detection is not used such as the technique presented by Deng et al[21]. Some authors, such as L. Zhenkun and Huang Shunji[22] have done research on actually using the triple difference as an aid to solve for the ambiguity as opposed to just using the triple difference directly. In any case, the triple difference can only be utilized if the antenna coordinates remain constant over time, so that limitation makes it unsuitable for this thesis.

Other authors have done some useful evaluations of ambiguity resolution methods. D. Kim and R. Langley[23] noted that the focus research on ambiguity resolution has largely changed from computational efficiency to actual performance of various algorithms and asserted that many modern resolution techniques have sufficient computational efficiency for a wide variety of applications. Geng et al[24] compared fractional cycle bias determination with integerrecovery clock determination in the context of ambiguity-fixed position estimates, concluding that both approaches yield positioning precision and accuracy that are approximately equivalent 
due to having identical design matrices. For the purposes of this thesis, triple differencing and dual frequency techniques will not be used due to the assumed stationary geometry and availability of higher cost equipment, respectively. For any carrier phase ranges, a simple linear geometric ambiguity search will be used with the same search criteria that LAMBDA uses. This will ensure equivalent selection accuracy in a small region and does not require additional complexity for the ambiguity selection, which is not the primary focus of this thesis in any case. To attempt to fix ambiguities in simulation, the ratio test proposed in [25] will be implemented, which compares the smallest sum square of residuals combination with the combination producing the second smallest sum square of residuals.

\subsubsection{GPS for Pitch and Heading Determination}

Because the use of GNSS for attitude determination is contingent on access to robust positioning solutions, much of the research on the concept involves some degree of hardware sophistication and multi-sensor data fusion. An example of this is the multifrequency fusion of the GPS system with the Chinese Beidou system by Nadarajah et al[26]. In their paper they demonstrated the benefit of adding additional satellites to the positioning solution, which reduces dilution of precision and improved the heading computations. They also recommended that the theoretical framework for such a system be expanded in the future. Yang et al[27] proposed a method of fusing low-cost inertial and magnetic field sensors with GNSS to compute attitude. After using their fusion algorithm to smooth double difference carrier phase-based attitude measurements, they noted a significant decrease in the standard error of their pitch and yaw calculations compared to using raw measurements alone, which is to be expected. Xu et al[28] fused GPS, GLONASS and BEIDOU constellation satellites at high data rates in 3 Trimble R9 receivers to measure the pitch, roll and yaw as applied to a deformation analysis and used an 
IMU as a reference sensor to analyze the errors. They noted that, separately, the GPS solutions yielded the best results, followed by GLONASS and then Beidou having the noisiest solutions. They also interestingly noted that in some dynamic cases, the fusion of all 3 systems can perform slightly worse than the GPS only solution, suggesting that the other signals may not have been weighted optimally in their algorithm.

There has also been significant research on GPS-based attitude determination without high grade equipment being available. For example, Wang et al[29] developed a way of introducing constraints to the LAMBDA ambiguity resolution algorithm[14] in order to reduce ambiguity fixing time, including expansion of search space, as a way of using GPS to precisely compute attitudes in adverse error conditions. They found that introducing their constraints reduced the standard deviations of their pitch and heading computations to approximately one fifth that of the unconstrained solution. It is worth noting that a base station would still be required in order to obtain a solution sufficiently close to make the LAMBDA algorithm feasible with short ambiguity search periods. Chang et al[30], also utilizing a double difference measurement model, presented a vectorization approach to computing first estimates for the position and Euler angles in a GNSS attitude network. They showed that using this analytical approach can optimize the initial estimates for the iterative least squares estimation process, requiring fewer iterations while improving the precision of the solution. Sun et al[31] proposed a method of using GNSS multi-antenna baseline separations in conjunction with a Monte Carlo sampling strategy in order to establish a search space for the LAMBDA ambiguity resolution algorithm without the need of code pseudorange measurements to aid the algorithm. They found that, in a setup of 3 antennas with a baseline distance of approximately $0.5 \mathrm{~m}$ or less, the simultaneous computation of quaternion attitude as well as double difference ambiguities was 
nearly 100 percent successful. This was especially true when 7 or more satellites were available, and computation times for their algorithm ranged between $0.6 \mathrm{~s}$ and $4.3 \mathrm{~s}$.

To the author's knowledge, the most relevant research to that of this thesis is the work done by Jiun Han Keong[32] and Chaochao Wang[33]. Keong's thesis comprised the fusion of GPS with GLONASS signals in a non-dedicated, dual-antenna, external oscillator system with a single-difference measurement model for determining pitch and heading. It was determined that, while the code measurement noise from GLONASS was twice that of GPS, the carrier phase of each had approximately $1.1 \mathrm{~mm}$ standard deviation of random ranging error due to noise. Keong also noted that, for attitude determined using the carrier phase, multipath was a far more dominant error source than noise. It was also found that, while using the GLONASS system in conjunction with GPS increased attitude determination availability, but it decreased accuracy of the attitude solution due to the additional noise of the GLONASS signals. Keong recommended investigation of the effects of temperature on the error budget, expansion of the testing platform into additional environments such as urban canyons, testing with additional receiver types as well as using a double difference measurement model. The effects of temperature, additional receiver types and the double difference model are implicit in the simulations used in this thesis.

Wang's thesis focused on developing an attitude determination system at low cost. 3 CMC Allstar receivers were used in conjunction with 3 Murata rate gyros in order to measure the angular velocity of the platform. It was noted that, in absence of multipath, the low-cost system achieved similar accuracy to that of the high-performance Novatel OEM4 receivers integrated in a similar system. It was also asserted that the antenna had a significant effect on the attitude determination success due to multipath and its correlation with the antenna gain pattern. Interestingly, it was noted that ambiguity resolution success was severely degraded for the low- 
cost system due to increased noise and susceptibility to cycle slip occurrences. The author recommended increased data rates to solve this problem. The gyros were found to add significant benefits to the system, especially due to the Kalman filter coasting ability for the state vector during GPS outage periods. Some notable recommendations that Wang made were integration of doppler measurements for use in cycle slip detection as well as investigating the effects of increased inter-antenna separation which would theoretically improve the attitude calculation performance. A thorough simulation analysis using varied inter-antenna separation distances is a major focus of this thesis. 


\subsection{Thesis Objectives}

This work seeks to develop a method of solving two GPS partial attitude determination design problems. The method uses a collection of GPS error statistics with interpolation to solve for a minimum antenna separation at a given GPS receiver quality (specifically the noise characteristics), or to solve for maximum receiver noise specification given a specified GPS antenna separation length.

Experimental GPS control point surveys with a low-cost, wide correlator, L1-signal-only Emlid Reach M+ (hereon referred to as test receiver 1), a Huawei P20 cell phone with pseudorange only capability (hereon referred to as test receiver 2) and a VectorNav VN-300 wide correlator, L1 only integrated system (hereon referred to as test receiver 3 ) are to be conducted to validate positioning algorithms as well as to validate the partial attitude simulations.

The simulation results provide a potential design tool for navigation mission planning. Specifically, a designer could use the simulation results to design for a particular design criteria by fixing one of either the pseudorange noise or antenna separation, then interpolating the other parameter from the simulation results. 


\subsection{Contributions}

The contributions of this work to the field of positioning and navigation are as follows:

- A novel partial attitude simulation method using a fixed satellite constellation availability with pseudorange noise and GNSS antenna separation as input parameters

- Experimental control point surveys to validate simulated GPS data

- Novel quantification of the effects of antenna separation and noise on pitch and heading computation.

- Proposal of a mission design technique using interpolation from simulated data

\subsection{Organization}

The remainder of this work is organized into the following parts:

- Part 2: Background on coordinate systems, GPS positioning and partial attitude

- Part 3: Methodology for least squares estimation, satellite orbit propagation, positioning methods, Kalman filtering and partial attitude computation

- Part 4: Simulation for GPS positioning, validation of positioning algorithms by experimental survey, and partial attitude simulation explanation

- Part 5: Partial attitude control point survey, discussion and comparison with simulated results

- Part 6: Concludes the research, discusses the results and recommends future work for expansion of the concept. 


\section{Background Information}

\subsection{Coordinate Systems}

\subsubsection{Earth-Centered, Earth-Fixed (ECEF) Coordinates}

ECEF Coordinates use the center of the earth as the origin, with the $\mathrm{Z}$ axis being collinear with the earth rotation axis and positive being in the north direction. The $\mathrm{X}$ axis lies on the equatorial plane and is aligned with the prime meridian in Greenwich, UK. The Y axis completes a left-handed coordinate system, as illustrated in Figure 1:

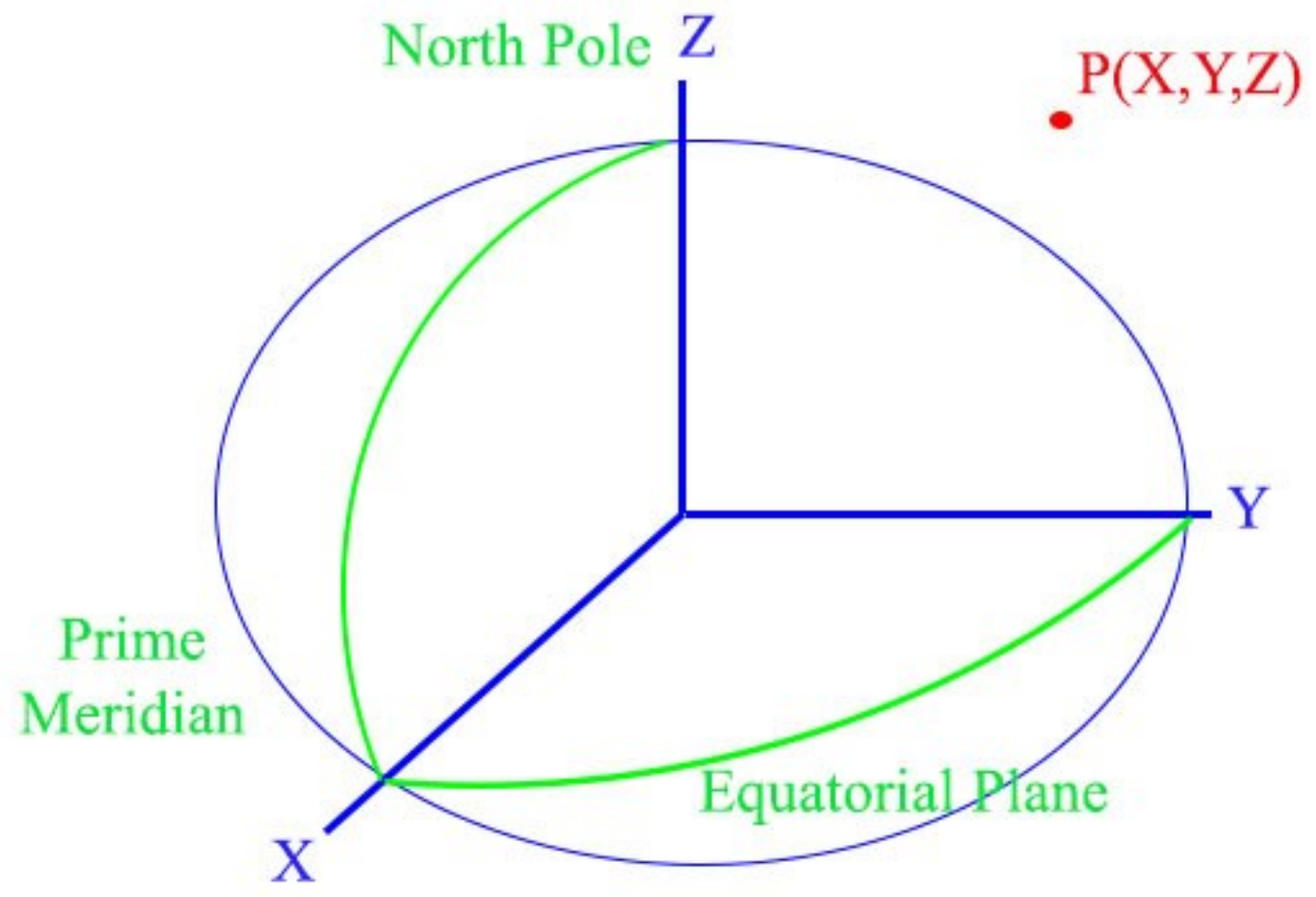

Figure 1: Illustration of the Earth-Centered, Earth-Fixed (ECEF) Coordinate System 
As shown in Figure 1, coordinates in this system are not referenced to the earth surface. This makes the system a good coordinate system to use when determining GPS receiver/antenna coordinates in terms of satellite coordinates and is therefore used in this research for GPS positioning computations.

\subsubsection{Geographic Coordinates}

Like ECEF coordinates, geographic coordinates use the earth's equator and prime meridian as references. However, geographic coordinates are polar in nature. The 3 coordinates associated are latitude, longitude and height, where latitude is an angle subtended by the point in question, the earth's center and the closest point on the equator which lies on the same meridian as the point in question. The height is collinear with the gravity vector and is measured using sea level as a reference datum. This sea level is modelled by an ellipsoid, as illustrated below:

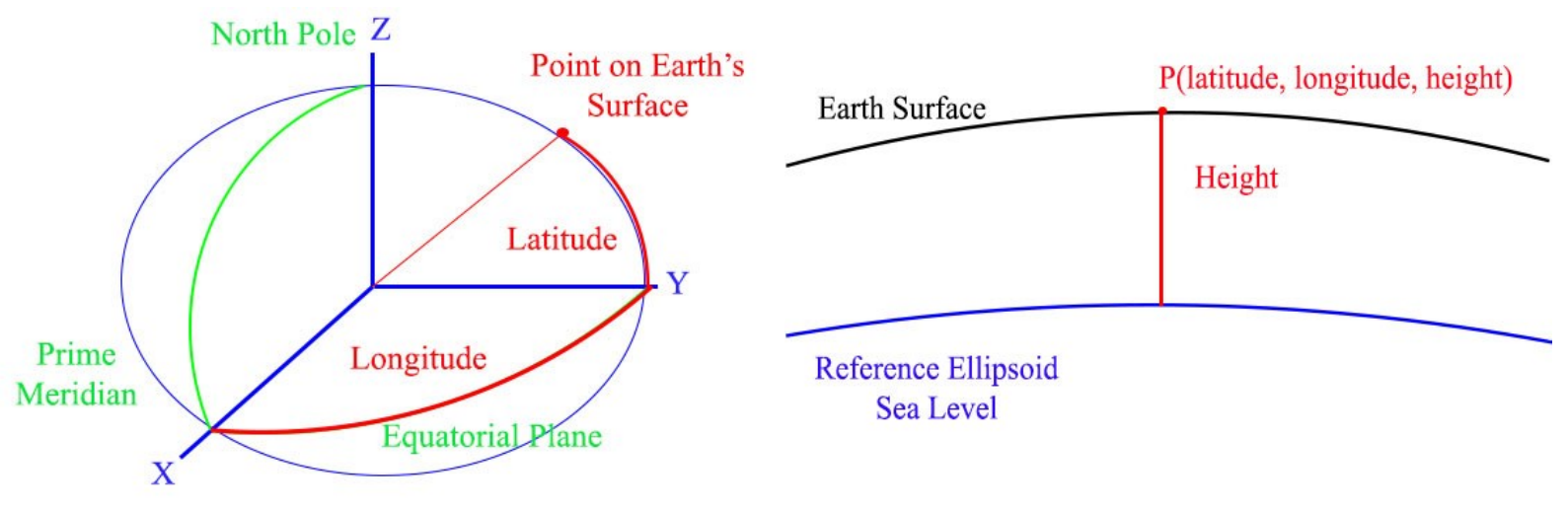

Figure 2: Geographic Polar Coordinate References (Left), Geographic Height Above Sea Level (Right)

As can be observed in Figure 2, the reference ellipsoid does not use a constant mean sea level because this would approximate the earth as a sphere, which is not as accurate a description as an ellipsoid. This research will use the WGS-84 ellipsoid as a reference ellipsoid for all purposes. 


\subsubsection{Local Vertical Compass Reference Frames}

A local vertical reference frame uses a nearby reference point as an origin and the $\mathrm{Z}$ axis is collinear with the gravity vector at the reference point and is referred to as the 'vertical' axis. The remaining 2 axis' lie on the horizontal plane at the reference point, with a 'north' axis being tangent to the local meridian at the reference point, positive to the north, and an 'east' axis being tangent to the local parallel at the reference point, positive to the east. This is illustrated below:

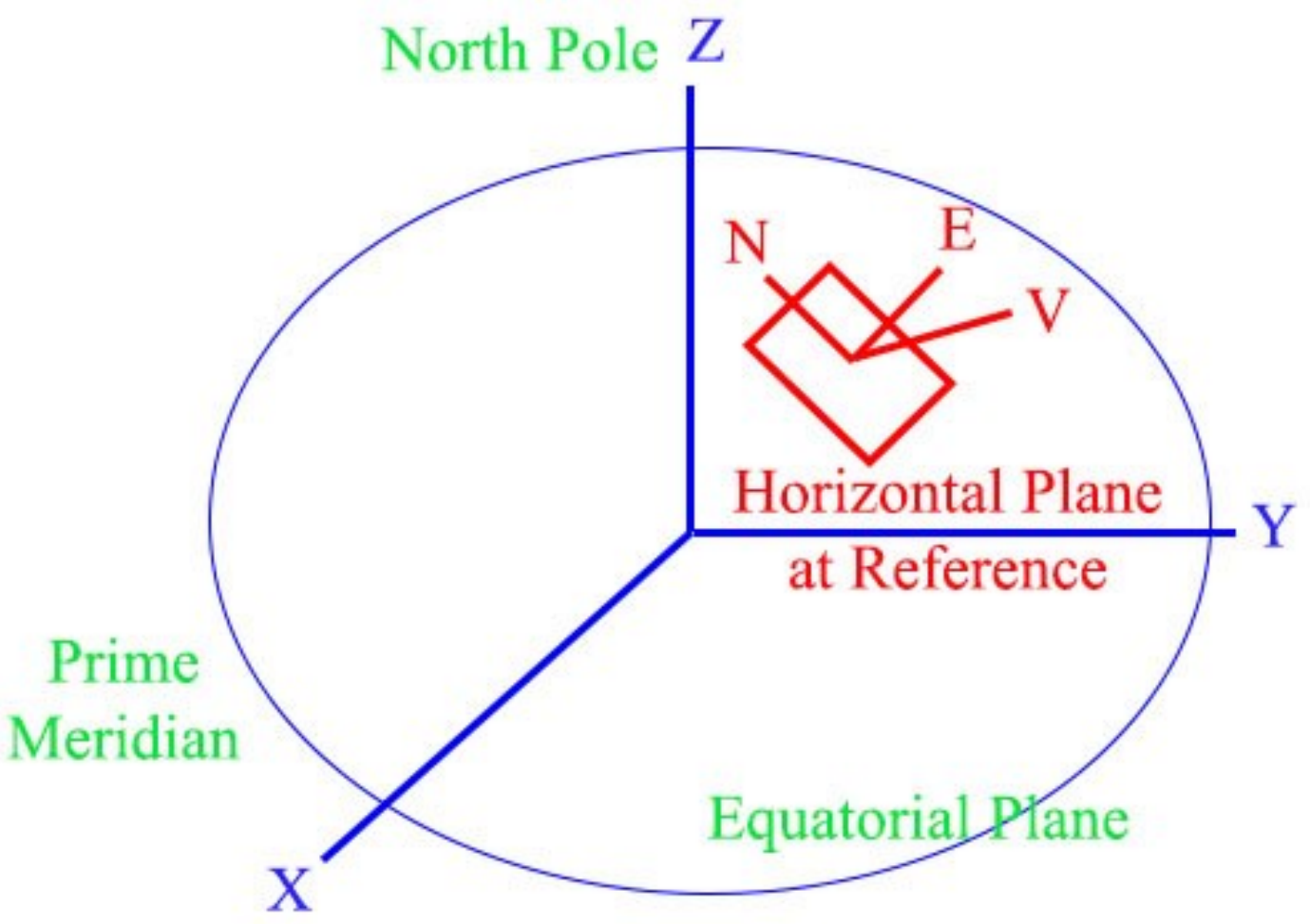

\section{Figure 3: Illustration of a Local Vertical Compass-Based Reference Frame}

Local vertical frames such as the one illustrated in Figure 3 have a distinct advantage over projected coordinate systems such as Mercator projections in that they allow for a good navigation frame (from one point on the earth's surface to the next) without suffering the same 
scaling distortions that projections do. This research will use local vertical frames extensively for error analysis as well as navigation and mapping.

\subsection{GNSS Operating Principles}

Global Navigation Satellite Systems (GNSS) is a time-of-flight positioning system. A constellation of satellites is maintained in varying orbits and each satellite constantly transduces a signal which can be received via antenna and interpreted by a receiver at the antenna location.

The fundamental GNSS measurement is the time taken for the signal to travel from the satellite to the receiver antenna. The message in the satellite signal contains a time of emission, measured by a precise atomic clock in a specific reference time system. The receiver records the time of arrival for the signal in its own time system which is offset from the satellite time reference. Because all electromagnetic radiation travels at the speed of light, the time-of-flight can be used to calculate a range between the satellite and receiver[2]:

$$
P_{\text {range }}=c \Delta t
$$

Where $c$ is the speed of light and $\Delta t$ is the travel time of the signal. If the position of the satellite is known, then this measurement yields a sphere of possible locations of the receiver in the GNSS coordinate frame:

$$
\begin{gathered}
P_{\text {range }}^{2}=\left(X_{\text {satellite }}-X_{\text {receiver }}\right)^{2}+\left(Y_{\text {satellite }}-Y_{\text {receiver }}\right)^{2} \\
+\left(Z_{\text {satellite }}-Z_{\text {receiver }}\right)^{2}
\end{gathered}
$$

If 3 of these ranges are simultaneously obtained from 3 different satellites with sufficiently optimized geometry relative to the receiver, then 3 spheres can be constructed. These 
spheres will all intersect at two points in space, one of which is the location of the receiver and the other only represents a geometric constraint of the three spheres. With additional ranges, a unique solution can be computed and the confidence in that solution can be improved. The intersection is illustrated below:

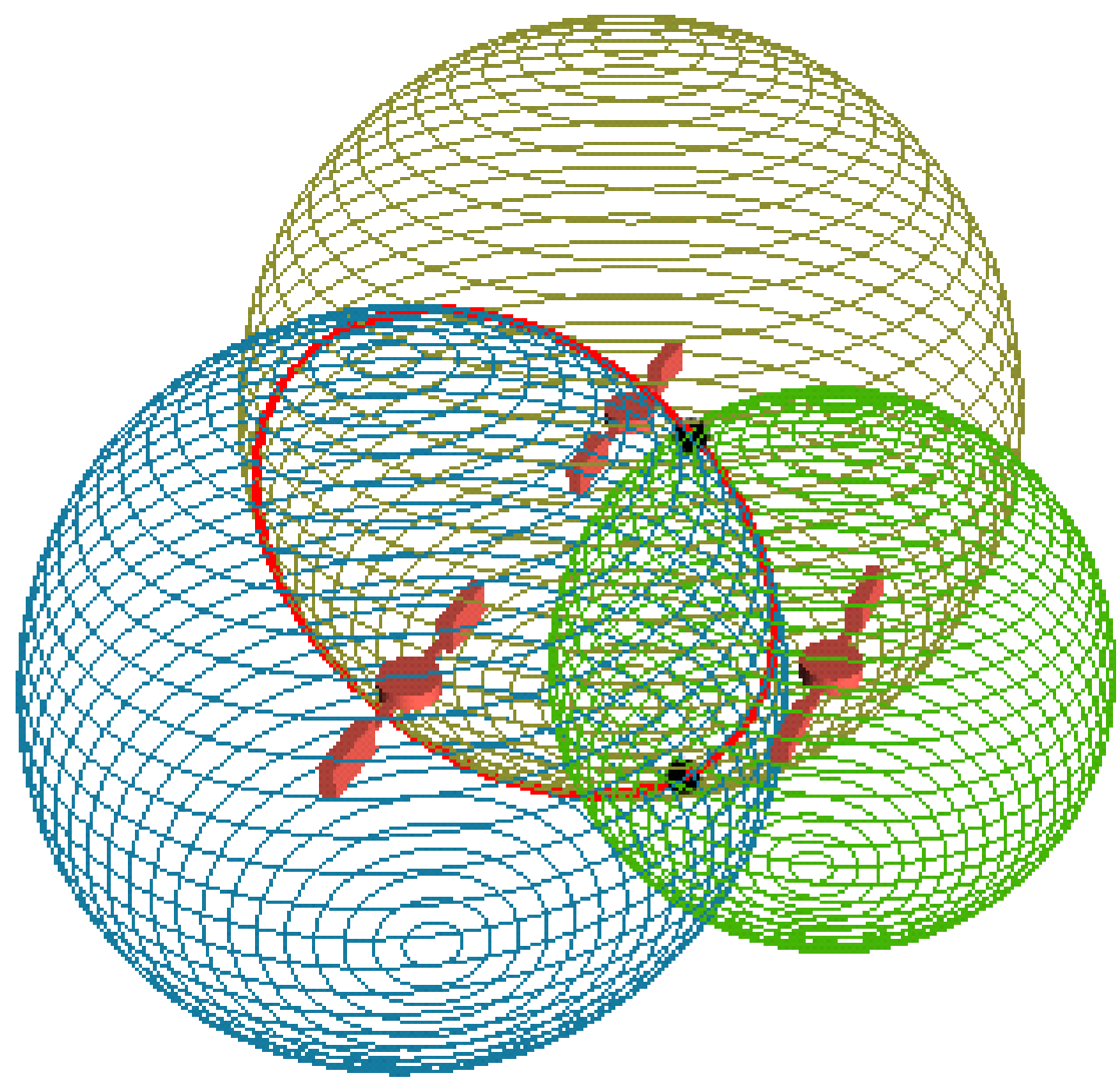

Figure 4: Intersection of three spheres representing ranges from satellite to receiver antenna. A unique position solution exists when more than three satellites are in line of sight [34].

However, computing this position is made more complicated by the fact that the measured time-of-flight is subject to delays and noise which induce uncertainty to the measurement. In addition, there are errors associated with the satellite position and the antenna 
setup by the user. The noise and biases must be modelled or avoided if an accurate and precise solution is to be computed.

\subsection{GNSS Error Sources}

\subsubsection{Pseudorange Error}

Generally, code pseudoranges are subject to errors in signal propagation as well as errors due to the receiver hardware quality. These include atmospheric errors, clock errors, multipath and noise.

\subsubsection{Receiver and Satellite Clock Error}

The satellite records a time of propagation while the receiver records a time of receiving the signal. However, the satellite and receiver have a non-zero error in recording these times. Part of this is measurement error, while part of it is manifest from the fact that the receiver and satellite each have their own offset from the system's reference time. This is due to imperfect synchronization of the clocks to the standard GPS reference time. Since both recorded times are used in the measurement of propagation time, it is useful to simply model this error as a single aggregate error due to the offsets and measurements in both instruments. The offset can be very significant. In fact, it is typically so large that it needs to be modelled as a separate unknown when computing a position[2].

\subsubsection{Ionosphere delay}

The Ionosphere extends from an elevation of approximately $50 \mathrm{~km}$ until greater than $1000 \mathrm{~km}$. It exists due to ultraviolet radiation from the sun and contains ionized gas molecules. These ionized particles will delay a GNSS signal by refracting it, and the amount of refraction depends on the frequency of the signal. The total ranging bias due to delay by the ionosphere can 
range from $1 \mathrm{~m}$ to about $100 \mathrm{~m}$, making it the most significant delay caused by the earth's atmosphere[13].

Since this error is caused by solar radiation, it is subject to temporal characteristics. It varies according to an 11-year solar cycle as well as daily variation due to the earth's rotation.

The ionosphere delay is also subject to spatial variability. It is most significant at the earth's poles and the geomagnetic equator. It is also typically more significant during winter time. The relative location of the satellite is also significant, as the delay is about 3 times greater for a satellite near the horizon compared to one at zenith. This is because the signal has a greater travel distance through the atmosphere and the refraction angle is greater.

The delay can be compensated by modelling according to the total electron content (TEC) at the time of recording, or with techniques using dual-frequency receivers to remove first-order effects.

\subsubsection{Troposphere Delay}

The troposphere is the layer of the atmosphere closest to the earth's surface. For GNSS purposes, the troposphere is the non-dispersive part of the atmosphere, which is to say that the delay of a GNSS signal is not correlated with the frequency of the signal. This extends from the earth's surface to approximately $60 \mathrm{~km}$ elevation.

Like the ionosphere, the troposphere delays a GNSS signal by refracting it. However, the troposphere has a much smaller effect than the delay caused by the ionosphere. The delay typically ranges between $2.3 \mathrm{~m}$ (at zenith) and $9.3 \mathrm{~m}$ at a 75 degree zenith angle[13].

Simple models which do not require dual frequency data can be used to estimate the delay and compensate it to within about $20 \mathrm{~cm}$ of the actual effect. Therefore This delay is not a 
problem for approximate positioning, though it can pose an obstacle to precise positioning applications, particularly when estimating height.

The delay is divided into a dry (hydrostatic) and wet component. The wet component is more difficult to model than the dry component. One way to mitigate the troposphere delay is via differential positioning with short baselines at similar heights.

There is some temporal variability in the troposphere delay, particularly in areas of high humidity and unstable weather.

\subsubsection{Multipath Error}

Multipath delay occurs when a GNSS signal reflects off of a surface before reaching the antenna, hence delaying the signal by the additional propagation time. GNSS multipath is particularly prone to occur in environments where there are smooth surfaces for reflection. In particular, smooth metallic surfaces, polished glass or water are all conducive to multipath effects[35].

GNSS receivers are designed with small chip length indicating higher noise and multipath mitigation properties, usually increasing the cost of the receiver. The delay from multipath can reach magnitudes of about half of a code chip length, which for the C/A code of a wide correlator receiver is $150 \mathrm{~m}$. This is very large, so it can be a limiting factor in positioning, especially for C/A code only, wide correlator receivers.

Multipath has a very high spatial variability, and it decorrelates very rapidly when the position changes. Two identical receiver/antenna combinations recording at the same time will yield very different results in an urban skyscraper environment near polished windows compared to an open sky environment with no reflective surfaces nearby. 
Multipath will also have temporal variability for a static position. Specifically, it will vary periodically according to the satellite orbital period. Unlike atmospheric errors, multipath is not generally mitigated by differential positioning. In fact, using differential techniques can amplify the effects.

\subsubsection{Code Noise}

In addition to biases due to signal delays during propagation, the pseudorange is also subject to random, gaussian distributed noise. This noise is a receiver-based error, not an atmospheric or environmental delay.

In general, higher quality receivers tend to have more options for limiting noise. The lowest quality receivers are generally wide-correlator, single-frequency receivers which can only access the $\mathrm{C} / \mathrm{A}$ code, not the $\mathrm{P}$ code.

The C/A code has significantly higher noise characteristics than the $\mathrm{P}$ code, so it is generally desirable to have access to the $\mathrm{P}$ code. That is generally a determining factor for the price of a receiver.

The noise on a receiver can be as large as about $3 \mathrm{~m}$ for low-quality receivers, down to the $\mathrm{dm}$ level for high-quality receivers[12].

Random noise is difficult to compensate with a model, but it can be modelled by connecting 2 receivers to the same antenna and recording data, since that allows for isolation of all other error sources. Once that is modelled, a standard deviation of the noise can be used in stochastic models for least squares adjustments. 


\subsubsection{Carrier Phase Error}

\subsubsection{Multipath}

Like the pseudorange, the carrier phase is also subject to multipath delays. However, due to the fact that the carrier phase measures fractional cycles rather than an entire range, the multipath delay is limited to a quarter wavelength in principle (though it is typically no more than a tenth of a wavelength in practice). Because this delay is much smaller than multipath effects on pseudoranges, this gives the carrier phase a significant advantage in precision positioning applications when multipath conditions are not favorable[24].

\subsubsection{Noise}

The carrier phase is also subject to gaussian random receiver noise. However, this effect is very small for the carrier phase (less than $1 \mathrm{~mm}$ typically)[36]. This makes the carrier phase essential for high-precision applications.

\subsubsection{Integer Ambiguity}

The carrier phase measurement is a measurement of the phase angle of the carrier signal on time of receipt. This angle corresponds to the fraction of a full cycle of the signal from reference to point and time of receipt. This is illustrated in the figure below: 


\section{CARRIER-PHASE MEASUREMENTS}
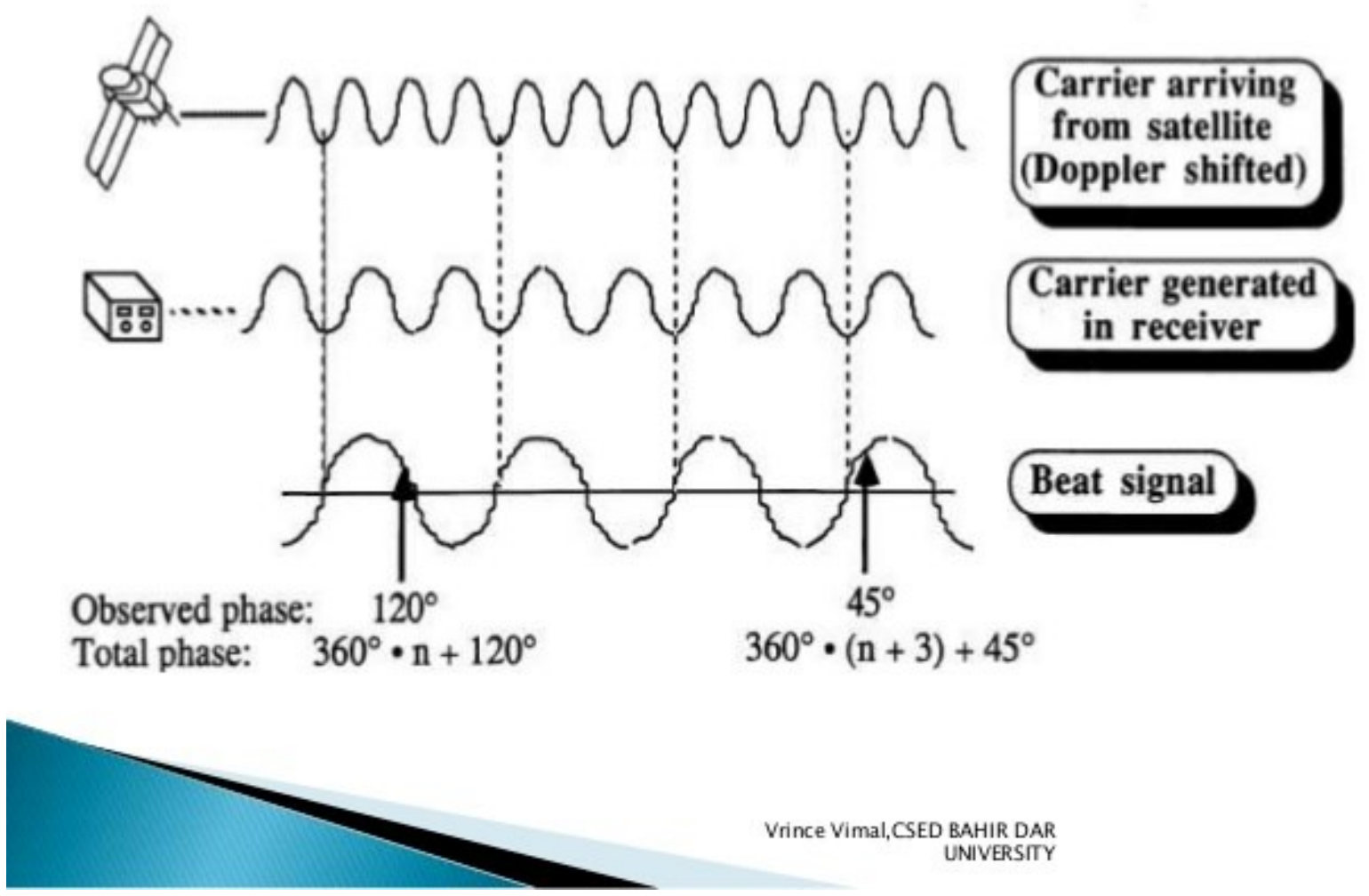

Vrince VImal,CSED BAHIR DAR UNIVERSITY

Figure 5: Explanation of the GNSS carrier phase measurement and integer ambiguity offset [37]

Because what the carrier phase measurement yields is a fractional number of cycles plus an arbitrary integer, there is an unknown remaining distance between the signal and satellite constituting an ambiguous integer number of cycles: 


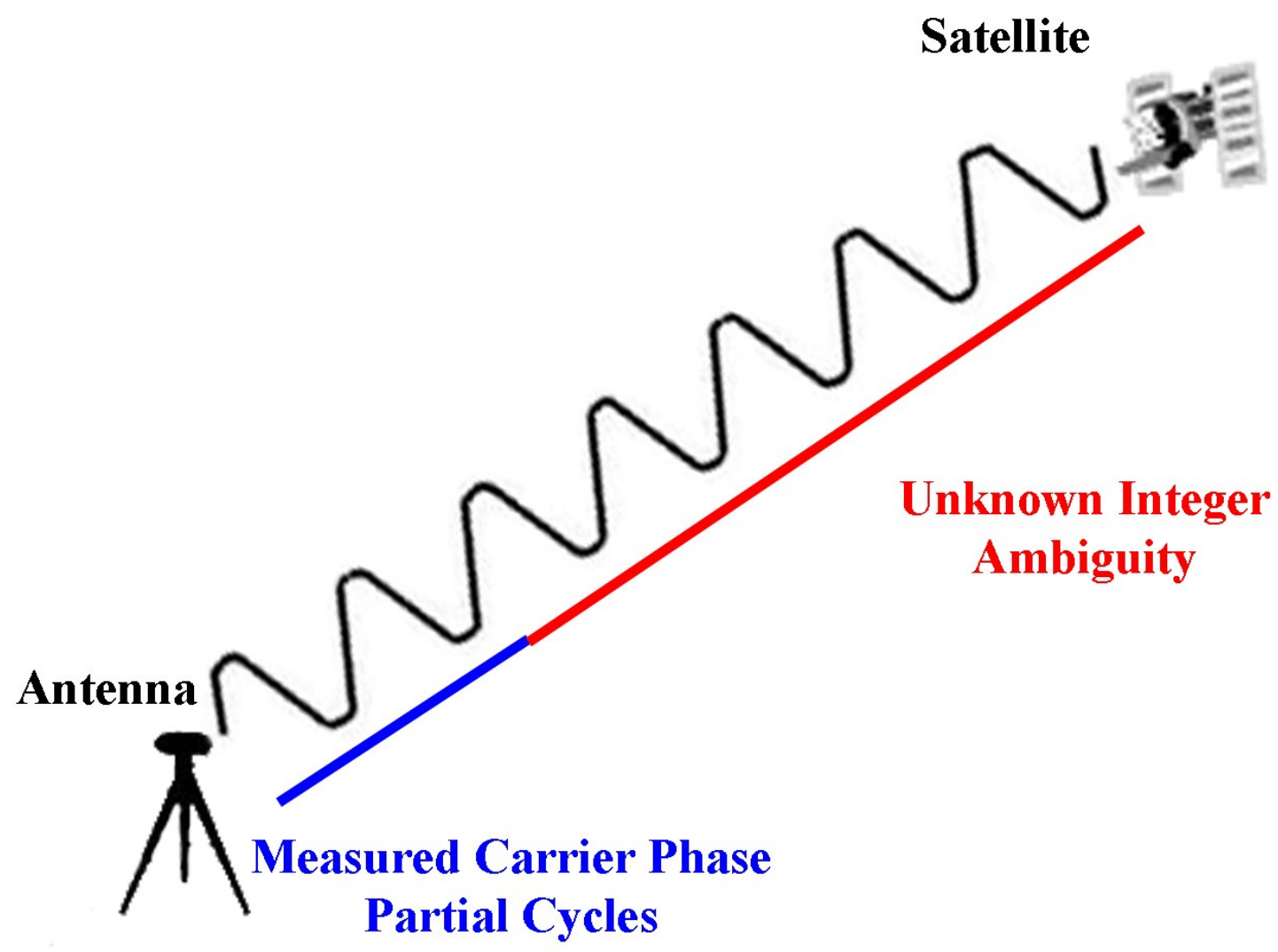

Figure 6:Illustration of Carrier Phase Ambiguity

It can be observed from the above figure that, as long as no cycle slips occur, the integer ambiguity will remain constant over time despite movement of satellites and the antenna. This is accounted for by the integer portion of the measurement itself. Therefore, the ambiguity can be fixed at one value and then used in the carrier phase measurement model to construct a precise pseudorange[23].

However, resolving the ambiguity is not simple, and in fact most techniques for ambiguity resolution require triple-differences or dual frequency capabilities for the receiver. This study will employ a technique which does not require triple-differencing or dual frequency observations in order to eliminate the need for additional costs and equipment. Namely, it will utilize an integer search-based technique. 


\subsubsection{Orbital Errors}

Orbital errors are not errors which affect the range measurement, but instead they refer to the fact that at any given time, a satellite's position cannot be known with certainty. Along with the observations, a satellite also broadcasts orbital characteristics in the navigation message. However, there are two reasons that this does not perfectly indicate a satellite's motion at the time of signal transduction.

First, the orbital elements broadcast in the navigation message are not perfectly accurate. They are observed with limited precision.

Second, the broadcast orbits are only transmitted for a single time before logging of observations began. Therefore, they must be propagated in order to obtain orbital elements at the time of signal transduction. There is a loss of precision associated with the nonlinear propagation algorithm used to do this which depends on the step size as well as how many perturbations are accounted for in the computation.

One way to mitigate this error is to use precise International GPS Service (IGS) orbits[7] instead of the broadcast orbits, though this can only be done in post-processing and not real-time applications since the IGS orbits are only updated periodically.

\subsubsection{Antenna Offset Error}

This error refers to the antenna being set up in a position other than the one which is recorded. This is usually caused by either human error, non-GNSS related measurement errors or physical limitations of the setup itself. 
For instance, when using a GNSS to measure the position of a point where coordinates are already known, it might be the case that the antenna center was not placed vertically on top of the point with sufficient precision, which would manifest in an apparent horizontal error bias relative to the known position. This is independent of any error in the sensor itself and is caused by the user.

The vertical offset of the antenna relative to the point in question may also be difficult to measure due to the need of levels to ensure vertical measurement as well as errors inherent to the measuring tape or laser used to measure the distance. Errors in this will manifest in an apparent vertical error bias in the solution. This is caused by a combination of lack of user skill and limitation of the levelling and distance measurement instruments used.

This error is especially important to mitigate for when a control point is being used as a base station for differential positioning. The technique works on the assumption that the antenna position of the base is known, so deviations from the correct setup will adversely affect the position solution for the rover antenna.

It is also very important to avoid setup errors for any GNSS antennas that are being used to compute attitude. The attitude of the vehicle is aligned to driving, upward and lateral directions, so deviations from those vectors in setup will induce a bias to the angles computed. 


\subsection{Data Formats}

In application and validation of the various techniques used in this thesis, data was collected using multiple instruments including GPS receivers, and an Inertial Measurement Unit. In order to fuse data from each of these sensors, the raw data must be collected and then processed separately. This section will discuss the form that this data takes as well as what processing needs to be done before the data can be used.

\subsubsection{GNSS Data}

Raw GNSS data can take a number of different forms, but for the purposes of this thesis, the Receiver Independent Exchange Format (RINEX) will be used, precisely because the receiver used to collect data will not affect a software's ability to read and store raw data (IGS 2015). The data comes in 2 separate files: The navigation message and the observation files containing epoch-by-epoch measurements from each visible satellite. 


\subsubsection{GPS RINEX Navigation Message}

The navigation message contains information about satellite orbits for discrete

timestamps which are not concurrent with the observation file timestamps. An example of a GPS

navigation message file is depicted in figure 5:

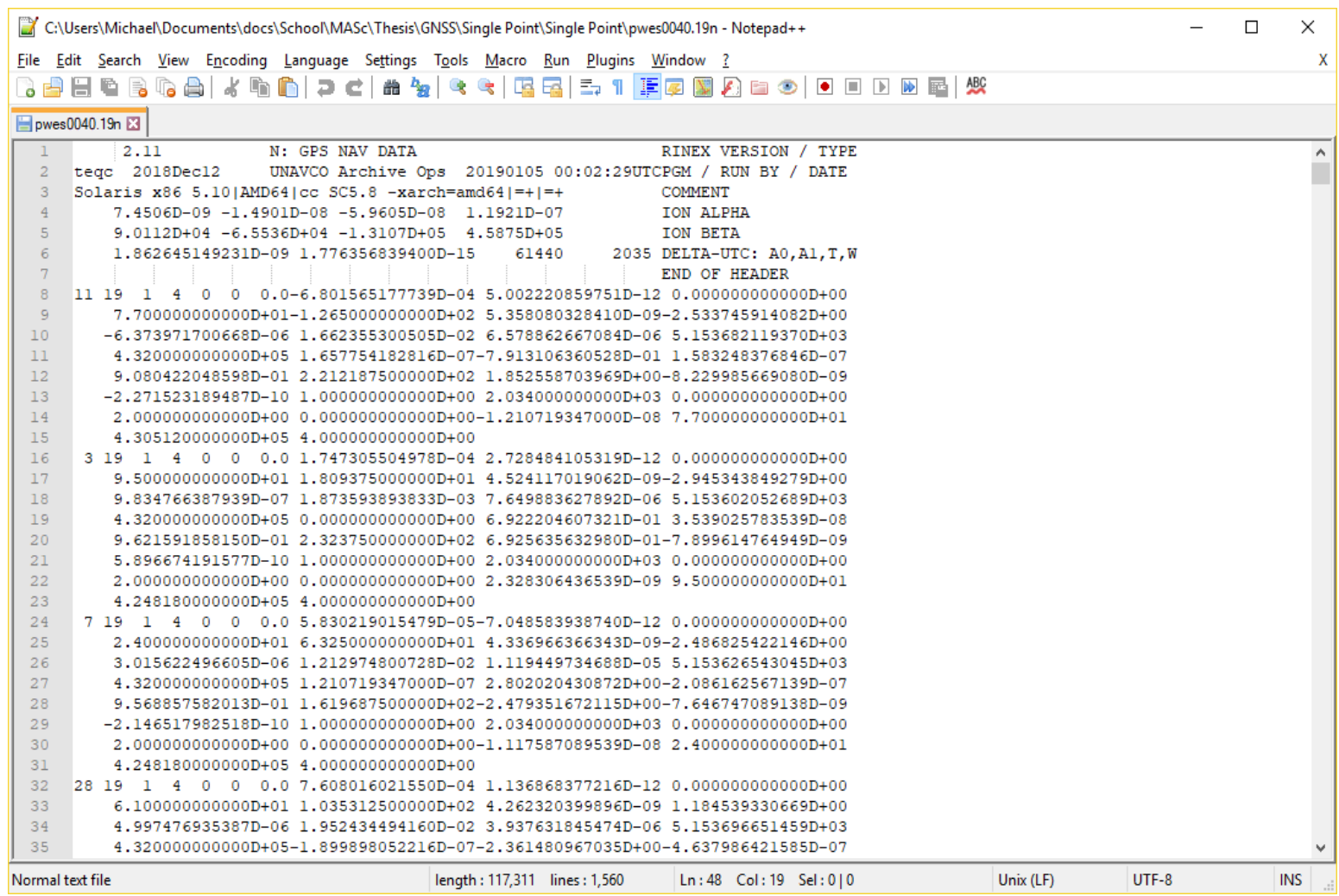

Figure 7: RINEX Navigation Message Example

In general, this file will begin with a header, and the header always finishes with the

phrase "END OF HEADER". Beyond this, the file begins to list navigation information for each satellite at a given time. It gives values in this order[7]: 
Table 1: Information Provided in RINEX GPS Navigation Messages

\begin{tabular}{|c|c|}
\hline Value & Units \\
\hline Satellite PRN Number & \\
\hline Time of Clock & \\
\hline \multicolumn{2}{|l|}{ Year (last 2 digits) } \\
\hline Month & 1 through 12 \\
\hline \multicolumn{2}{|l|}{ Day } \\
\hline Hour & Hours \\
\hline Minute & Minutes \\
\hline Second & Seconds \\
\hline SV Clock Bias & Seconds \\
\hline \multirow[t]{2}{*}{ SV Clock Drift } & Seconds \\
\hline & second \\
\hline SV Clock Drift Rate & $\frac{\text { Seconds }}{\text { Second }^{2}}$ \\
\hline \multicolumn{2}{|l|}{ IODE Issue of Data, Ephemeris } \\
\hline Crs & meters \\
\hline \multirow[t]{2}{*}{$\Delta n$} & radians \\
\hline & second \\
\hline$M_{0}$ & radians \\
\hline $\mathrm{Cuc}$ & radians \\
\hline \multicolumn{2}{|l|}{ Eccentricity $e$} \\
\hline Cus & radians \\
\hline$\sqrt{A}$ & $\sqrt{\mathrm{m}}$ \\
\hline Time of Ephemeris & Seconds of GPS week \\
\hline $\mathrm{Cic}$ & $\begin{array}{l}\text { radians } \\
\end{array}$ \\
\hline$\Omega$ & radians \\
\hline CIS & radians \\
\hline$i_{0}$ & radians \\
\hline $\mathrm{Crc}$ & $\mathrm{m}$ \\
\hline$\omega$ & radians \\
\hline \multirow[t]{2}{*}{$\dot{\Omega}$} & $\underline{\text { radians }}$ \\
\hline & $\begin{array}{l}\text { second } \\
\text { radians }\end{array}$ \\
\hline$\dot{I}$ & $\frac{\text { radians }}{\text { second }}$ \\
\hline \multicolumn{2}{|l|}{ Codes on L2 Channel } \\
\hline & \\
\hline \multicolumn{2}{|l|}{$\begin{array}{c}\text { GPS Week Number } \\
\text { L2 P Data Flag }\end{array}$} \\
\hline SV Accuracy & $\mathrm{m}$ \\
\hline \multicolumn{2}{|l|}{ SV Health } \\
\hline TGD & Seconds \\
\hline \multicolumn{2}{|l|}{ Issue of Data, Clock } \\
\hline Transmission Time of Message & Seconds of GPS Week \\
\hline Fit Interval & Hours \\
\hline
\end{tabular}


The navigation message entries will always come in the same order, so reading of the data files can be written assuming that. Since the classical orbital elements are given at discrete times, they cannot be used directly to calculate satellite positions at time of observation. Instead they must be propagated.

\subsubsection{GNSS RINEX Observation file}

The mixed RINEX observation file contains all of the logged raw data from every visible satellite, from every satellite system that the receiver is capable of transponding signals from, at each epoch. An example of a RINEX mixed observation file is depicted below:

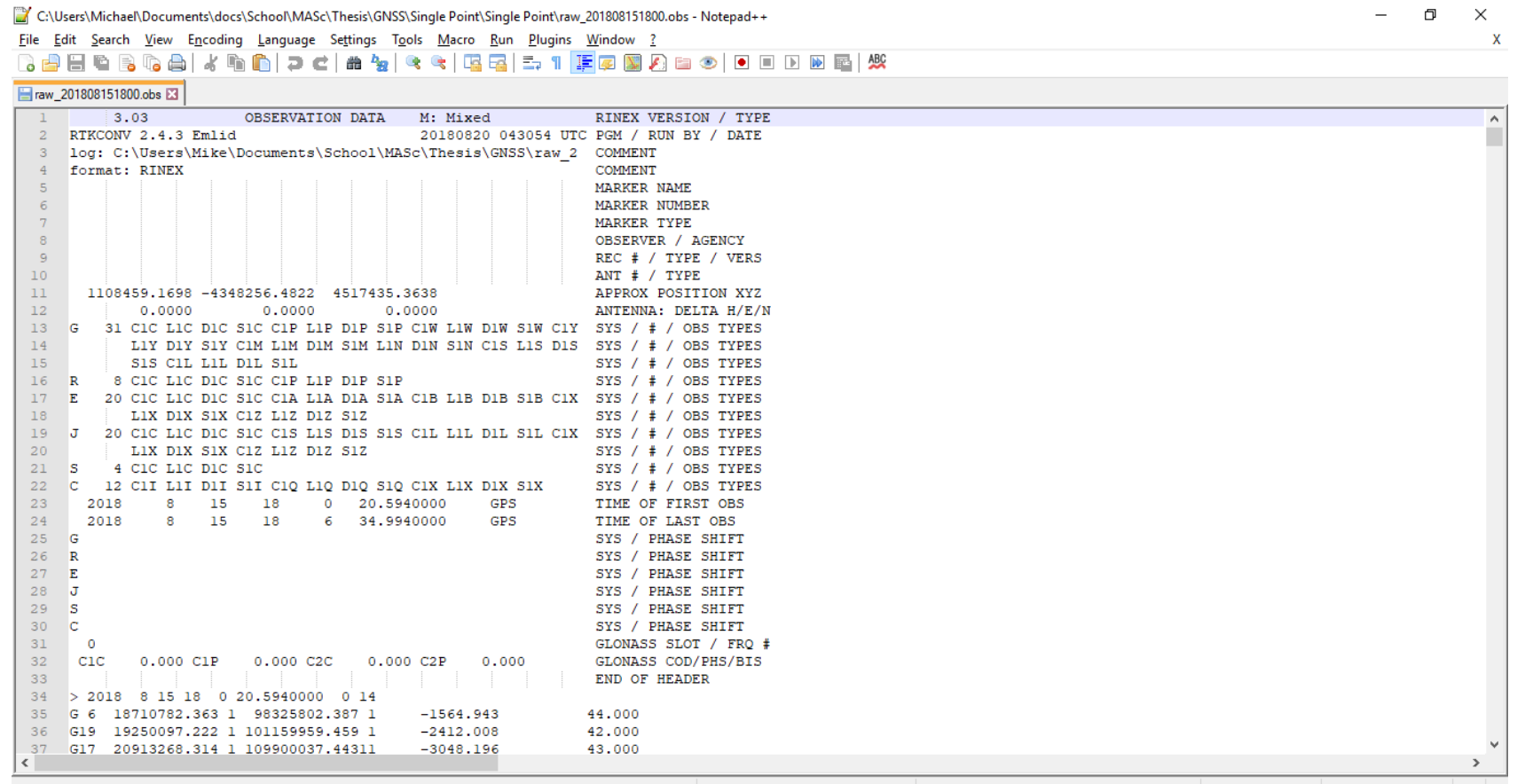

Figure 8: A Logged RINEX Observation File

Just as is the case for navigation message files, a RINEX observation file will always begin with a header which is terminated by the phrase "END OF HEADER". After this, the 
observation records begin. Each record begins with a line of values in the following order for the associated epoch[7]:

Table 2: GNSS RINEX Mixed Observation Record Epoch Information

\begin{tabular}{|c|c|}
\hline Value & Units \\
\hline Year & $01-12$ \\
\hline Month & $01-31$ \\
\hline Day & $00-23$ \\
\hline Hour & $00-59$ \\
\hline Minute & $00-59$ \\
\hline Second & 0 or 1 \\
\hline Event Flag & Total from all systems \\
\hline Number of Satellites
\end{tabular}

After each epoch record, the measurements for each satellite are listed. The format of each individual satellite observation record is dependent on what system the satellite belongs to, and each system's format is contained in the header of the file. 


\section{Methodology}

\subsection{GNSS Pseudorange and Carrier Phase Corrections}

\subsubsection{Pseudorange Corrections}

Because delays to GNSS pseudoranges can be quite substantial, the raw pseudoranges from logged RINEX files must be corrected before they can be used in a least squares adjustment.

\subsubsection{Troposphere Correction}

To correct for the troposphere delay, the Saastamoinen model [3] was implemented. Based on literature, this model tends to yield the greatest baseline accuracy possible and is statistically comparable to the Hopfield model. The Saastamoinen model computes the troposphere correction as follows:

$$
\begin{gathered}
\delta_{\text {trop }}=\frac{0.002277}{\cos (z)}\left(P+\left(\frac{1255}{T}+0.05\right) p p-B \tan ^{2}(z)\right)+\delta R \\
\text { where } p p=R_{h} e^{-37.2465+0.213166 T-0.000256908 T^{2}} \\
T(K)=T(\text { Celsius })+273.16 \\
P=P_{0}\left(1-0.000226\left(H-H_{0}\right)\right)^{5.225} \\
R_{h}=\rho e^{-0.0006396\left(H-H_{0}\right)}
\end{gathered}
$$

Where $z$ is the zenith angle of the satellite and $\delta R$ is a parameter depending on $z$ which is interpolated from an array of calibrated values documented in [3]. The model requires some input parameters, so the following parameters were used, including some standard atmospheric values: 
Table 3: Saastamoinen Model Input Values

\begin{tabular}{|c|c|}
\hline Variable & Value \\
\hline Reference Elevation $H_{0}(\mathrm{~m})$ & 0 \\
\hline Reference Pressure $P_{0}(\mathrm{mbar})$ & 1013.25 \\
\hline Temperature $T(\mathrm{~K})$ & 265 \\
\hline Reference Humidity $R_{h}$ & 0.5 \\
\hline
\end{tabular}

\subsubsection{Ionosphere Correction}

To correct for the ionosphere delay, the International GPS Service (IGS) ionosphere model will be used. This is because dual frequency methods are not applicable to this study since only single frequency receivers are being utilized. The IGS model uses a mapped value of the Total Electron Content in the ionosphere on a regional basis. In order to compute an ionosphere correction, a mapping function must first be computed based on the satellite geometry:

$$
F_{\text {map }}=\frac{1}{\sqrt{1-\left(\frac{6371}{6371-H_{\text {shell }}} \cos \left(\alpha_{\text {Elevation }}\right)\right)^{2}}}
$$

Where $H_{\text {shell }}$ is an estimate of the outer shell height of the ionosphere and $\alpha_{\text {Elevation }}$ is the elevation angle of the satellite for which the pseudorange is being corrected. With the mapping function computed, now the ionosphere correction can be computed:

$$
\delta_{\text {iono }}=F_{\text {map }}(T E C)(0.163)
$$


Where 0.163 is a constant calibrated to the L1 signal and TEC is a value for the total electron content in the region. Typically, this can be obtained from freely available maps which are updated frequently, such as the following from Trimble GNSS:

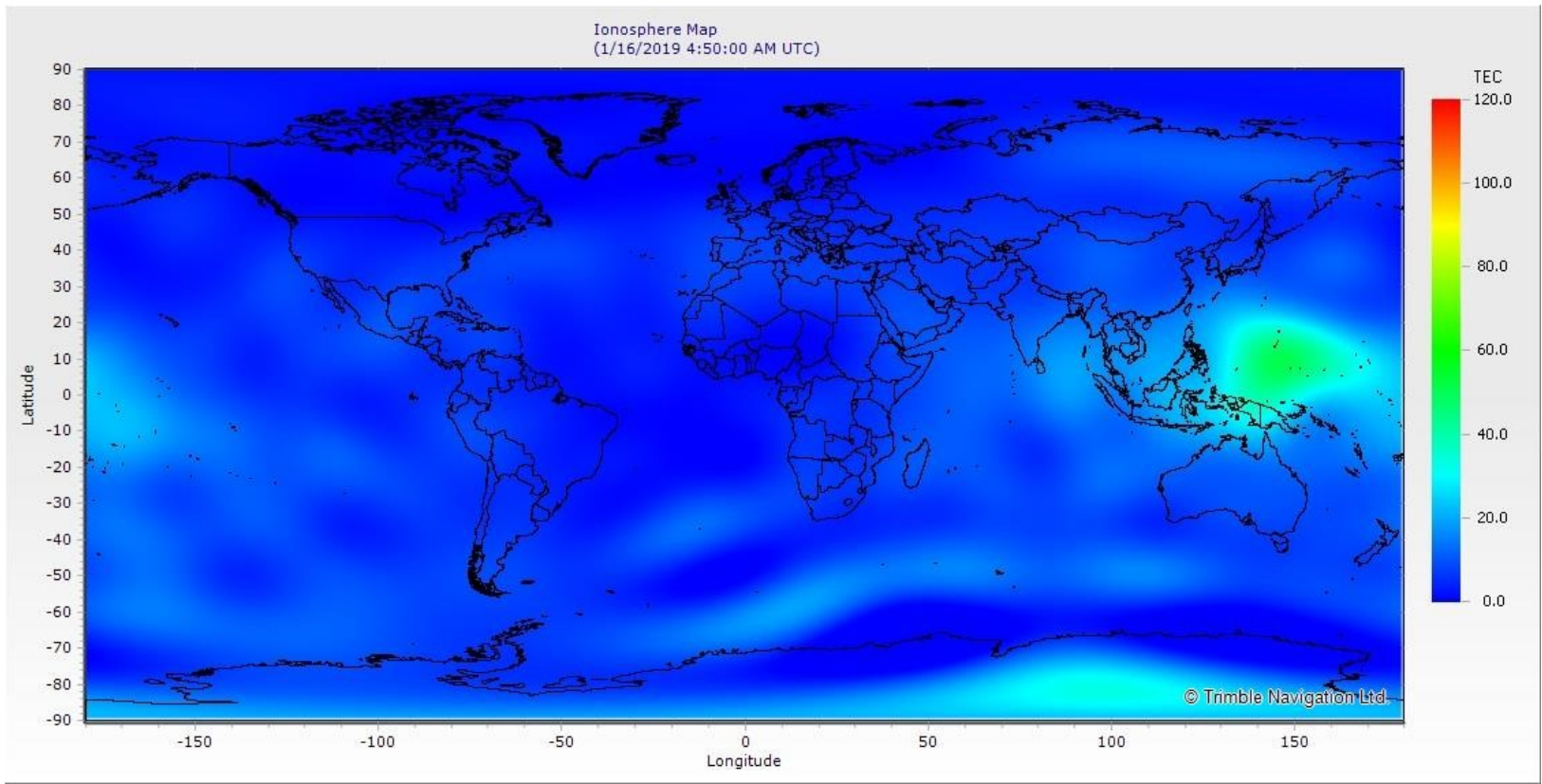

Figure 9: TEC Map from Trimble GNSS

Reading from these maps allows for interpolation by eye in order to acquire an approximate value of the TEC content for the data collection period.

\subsubsection{Relativistic Effects Correction}

For all GPS related purposes an earth centered earth fixed (ECEF) inertial coordinate system at rest is assumed during computations. This necessitates taking into consideration relativistic effects such as path range effects and the Earth's rotation itself. Path range effects have a maximum effect on the pseudoranges of only about $2 \mathrm{~cm}$. For a wide correlator receiver this is much smaller than the receiver noise and has little chance of affecting ambiguity resolution, so it is not compensated for. The effects of the earth's rotation, however, can be up to 
$30 \mathrm{~m}$ which would have a substantial effect on positioning solutions. It will therefore be corrected as follows:

$$
\begin{array}{r}
\delta p=\frac{\left(r_{r}-r_{s}\right)\left(\vec{v}_{r}\right)}{c} \\
\vec{v}_{r}=\omega_{e} r_{r}+\vec{v}_{k}
\end{array}
$$

Where $\delta p$ is the position correction due to the earth's rotation, $r_{r}$ is the receiver position vector, $r_{s}$ is the satellite position vector, $\omega_{e}$ is the rotational velocity of the earth and $\vec{v}_{k}$ is the velocity of the receiver relative to the earth surface[2].

\subsubsection{Carrier Phase Ambiguity Resolution}

Carrier phase measurements cannot be used without first resolving their ambiguities. Techniques which are frequently used involve dual frequency or triple-differences as the observation model. However, this is not viable if only one frequency is available, and tripledifferences are less viable for mobile applications than static ones, so neither of those concepts will be used. Instead, a vector-based search technique will be implemented.

First, a solution must be computed using pseudoranges. Refer to section 2.2 for pseudorange positioning techniques. Since this solution represents the current best estimate of the antenna position, a best estimate for each 3D geometric range from satellite to receiver can be calculated:

$$
\begin{aligned}
& \text { range }_{\text {geo }} \\
& =\sqrt{\left(X_{\text {est }}-X_{\text {satellite }}\right)^{2}+\left(Y_{\text {est }}-Y_{\text {satellite }}\right)^{2}+\left(Z_{\text {est }}-Z_{\text {satellite }}\right)^{2}}
\end{aligned}
$$

Next, this range can be used to calculate a float ambiguity: 


$$
N_{\text {float }}=\frac{\text { range }_{\text {geo }}-\phi_{\text {cycles }} * \lambda_{L 1}}{\lambda_{L 1}}
$$

Where $\phi_{\text {cycles }}$ is the fractional number of cycles measured and $\lambda_{L 1}$ is the wavelength of the L1 signal. Since it is known that the ambiguity must be an integer, this float ambiguity is then rounded. Now, a search area needs to be defined. In order to be thorough and maximize the chances of finding the correct integer for each satellite, the search will be done by adding an integer to the float ambiguity and then testing it.

The number of integers to test will determine the required processing time as well as the likelihood of finding the correct integer. For the purposes of this study, the integers $-2,-1,0,1$ and 2 are tested. Since there are 5 integers to be tested and $m$ satellites contributing measurements, there are $5^{m}$ possible combinations of integer ambiguities to be tested. In order to organize these combinations, a $5^{m} \times m$ matrix of combinations is built:

$$
\text { Combinations }_{5}{ }^{m} \times m\left[\begin{array}{cccccc}
-2 & -2 & \ldots & \ldots & -2 & -2 \\
-2 & -2 & \ldots & \ldots & -2 & -1 \\
\ldots & \ldots & \ldots & \ldots & \ldots & \ldots \\
\ldots & \ldots & \ldots & \ldots & \ldots & \ldots \\
2 & 2 & \ldots & \ldots & 2 & 1 \\
2 & 2 & \ldots & \ldots & 2 & 2
\end{array}\right]
$$

Next, every combination is tested, one at a time, in a least squares adjustment. The integer choice is used with the float ambiguity to construct a carrier phase-based range measurement:

$$
\Phi_{\text {measured }}=\left(N_{\text {float }_{(\text {rounded })}}+N_{\text {tested }}+\phi_{\text {cycles }}\right) \lambda_{L 1}
$$

Where $\Phi_{\text {measured }}$ is the measured range using the total number of cycles calculated and $N_{\text {tested }}$ is the tested integer taken from the matrix of cycle combinations. These measured ranges are used to build the least squares measurement vector: 


$$
l_{\text {observed }_{m x 1}}=\left[\begin{array}{c}
\Phi_{\text {measured }_{1}} \\
\Phi_{\text {measured }_{2}} \\
\ldots \\
\Phi_{\text {measured }_{m}}
\end{array}\right]
$$

This is used to compute $\hat{\delta}$ in the same process used in section 2.2. This can be done using the single point model or the differential model. Afterward, the vector of residuals $\hat{v}$ is computed using the stochastic model techniques from section 2.3. One way to select the optimal combination of integer ambiguities is to choose the combination yielding the smallest sum square of the residuals:

$$
\text { Total }=\sum v_{i}^{2}
$$

Once the optimal combination is chosen, a full iterative least squares adjustment can be done using the measurements derived from the optimal combination of integer ambiguities.

It is important to note that if the solution computed from the pseudoranges is not sufficiently close to the true coordinates of the antenna, the true geometric range will not fall within the search region and the ambiguity selected will be biased by a minimum of one full wavelength. This is especially important when using low cost, wide correlator receivers with single frequency and access only to the $\mathrm{P}$ code, since achieving high accuracy is much less likely with those receivers than with high quality equipment.

\subsection{Satellite Orbit Propagation}

In order to use a GNSS satellite position at any given epoch, an ephemeral set of classical orbital elements at a previous time must be propagated to the time of observation. These orbital elements must be acquired from the navigation message for real time use, while more precise 
orbits can be obtained from IGS products later on for post-processing. Some navigation files contain sets of orbital elements at multiple different times for the same satellite, giving an option for which record to use for propagation. Since this is a nonlinear process, there is loss of precision in propagation when the step size for propagation is not infinitely small. For this reason, the ephemeral set which is temporally closest to the observation times is used. First, the difference in time between the observation epoch and the ephemeral record is calculated:

$$
\Delta t_{e p h}=t_{o b s}-t_{e p h}
$$

Where $t_{o b s}$ is the time of observation in seconds and $t_{\text {eph }}$ is the time of ephemeris in seconds. Next, the rate of change of the mean anomaly is computed:

$$
\dot{M A}=\sqrt{\frac{\mu_{\text {earth }}}{a_{\text {semimajor }}^{3}}}+\Delta n
$$

Where $\mu_{\text {earth }}$ is the gravitational constant $\left(3.986005 \times 10^{14}\right), a_{\text {semimajor }}$ is the semimajor axis from the navigation message and $\Delta n$ is the difference in mean motion. Now the mean anomaly at the time of observation can be calculated:

$$
M A=M A_{0}+\dot{M A \Delta t_{e p h}}
$$

Where $M A_{0}$ is the initial mean anomaly from the navigation message. Next, the eccentric anomaly is set equal to the mean anomaly. Then, an adjustment to the eccentric anomaly is calculated iteratively:

$$
\begin{gathered}
d_{1_{\text {temp }}}=1-e_{\text {eccentricity }} \cos (E A) \\
\delta E A=\frac{M A-E A+e_{\text {eccentricity }} \sin (E A)}{d_{1_{\text {temp }}}} \\
E A_{\text {new }}=E A+\delta E A
\end{gathered}
$$


Where $e_{\text {eccentricity }}$ is the orbital eccentricity from the navigation message and $E A$ is the eccentric anomaly. The new eccentric anomaly is used again for processes 16-18 and the cycle is repeated until the value of $\delta E A$ falls below a desired threshold. Now the rate of change of eccentric anomaly and an intermediate value are calculated:

$$
\begin{gathered}
\dot{E A}=\frac{\dot{M A}}{d_{1_{\text {temp }}}} \\
E_{\text {ein }}=-4.442807633 \times 10^{-10} e_{\text {eccentricity } \sqrt{a_{\text {semimajor }}} \sin (E A)}
\end{gathered}
$$

Next, a satellite clock correction is calculated:

$$
\begin{aligned}
\Delta \text { cor }_{\text {satclock }}= & s v_{\text {clockbias }}+\Delta t_{\text {eph }}\left(s v_{\text {clockdrift }}+\Delta t_{\text {eph }} s v_{\text {clockdriftrate }}\right) \\
& +E_{\text {ein }}-T G D
\end{aligned}
$$

Where $s v_{\text {clockbias }}, s v_{\text {clockdrift }}, s v_{\text {clockdriftrate }}$ and $T G D$ (inter-signal correction term) are all taken from the navigation message. Now the satellite clock drift can be calculated:

$$
\text { clockdrift }=s v_{\text {clockdrift }}+2 \Delta t_{\text {eph }} s v_{\text {clockdriftrate }}
$$

Next, the argument of latitude and its rate of change are computed:

$$
\begin{gathered}
a_{\text {lat }}=\tan ^{-1} \frac{\left(1-e_{\text {eccentricity }}^{2}\right) \sin (E A)}{\cos (E A)-e_{\text {eccentricity }}}+\omega \\
\dot{a}_{\text {lat }}=\frac{\left(1-e_{\text {eccentricity }}^{2}\right) \dot{E A}}{d_{1 \text { temp }}}
\end{gathered}
$$

Where $\omega$ is the argument of perigee from the navigation message. Now corrections to these 2 terms are computed:

$$
a_{\text {lat }_{\text {corr }}}=a_{\text {lat }}+C_{\text {harm }_{a_{\text {lat }}}} \sin \left(2 a_{\text {lat }}\right)+C_{\text {harm }_{a_{\text {lat }}}} \cos \left(2 a_{\text {lat }}\right)
$$




$$
\dot{a}_{\text {lat }} \text { corr }=\dot{a}_{\text {lat }}\left(1+2\left(C_{\text {harm }_{a_{\text {lat }}}} \cos \left(2 a_{\text {lat }}\right)-C_{\text {harm }_{a_{\text {lat }}}} \sin \left(2 a_{\text {lat }}\right)\right)\right.
$$

Where $C_{\text {harm }_{a_{\text {lat }} c}}$ is the amplitude of the cosine harmonic term, taken from the navigation message, $C_{\text {harm }_{a_{l a t}}}$ is the amplitude of the sine harmonic term, taken from the navigation

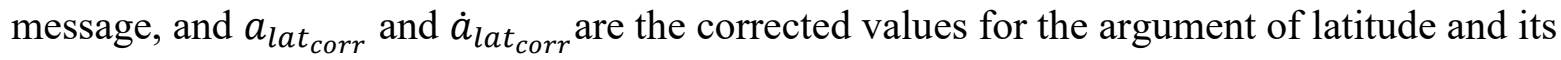
derivative, respectively. Next the orbital radius and its derivative are calculated:

$$
\begin{gathered}
R_{\text {orbit }}=a_{\text {semimajor }} d_{1_{\text {temp }}}+C_{\text {harm }_{R_{\text {orbit }}}} \cos \left(2 a_{\text {lat }}\right) \\
+C_{\text {harm }_{R_{\text {orbits }}} \sin \left(2 a_{\text {lat }}\right)} \\
\dot{R}_{\text {orbit }}=a_{\text {semimajor }} e_{\text {eccentricity }} \sin (E A) \dot{E A} \\
+2 \dot{a}_{\text {lat }}\left(C_{\text {harm }_{\text {orbit }^{s}}} \cos \left(2 a_{\text {lat }}\right)-C_{\text {harm }_{R_{\text {orbit }}}} \sin \left(2 a_{\text {lat }}\right)\right.
\end{gathered}
$$

Where $C_{\text {harm }_{R_{\text {orbit }}}}$ is the orbital radius cosine term amplitude and $C_{\text {harm }}$ Rorbit $^{S}$ is the orbital radius sine term amplitude. Now the satellite orbital inclination and its derivative are computed:

$$
\begin{aligned}
& i n c_{\text {orbit }}=i n c_{0}+\dot{I} \Delta t_{\text {eph }}+C_{\text {harm }_{\text {inc }} \text { orbit }^{c}} \cos \left(2 a_{\text {lat }}\right) \\
& +C_{\text {harm }_{\text {inc }} \text { orbit }} \sin \left(2 a_{\text {lat }}\right) \\
& i \dot{n} c_{\text {orbit }}=\dot{I}+2 \dot{a}_{\text {lat }}\left(C_{\text {harm }_{\text {inc }} \text { orbit }}{ }^{\text {s }} \cos \left(2 a_{\text {lat }}\right)\right. \\
& \left.-C_{\text {harm }_{\text {inc }} \text { orbit }} \sin \left(2 a_{\text {lat }}\right)\right)
\end{aligned}
$$

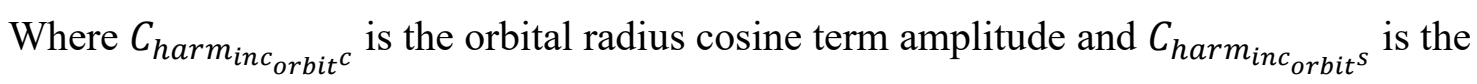
orbital radius sine term amplitude. Now the $2 \mathrm{D}$ position of the satellite as it lies in the orbital plane, as well as its partial rates of change, are calculated:

$$
\begin{aligned}
& x_{\text {orbit }_{\text {plane }}}=R_{\text {orbit }} \cos \left(a_{\text {lat }_{\text {corr }}}\right) \\
& y_{\text {orbit }_{\text {plane }}}=R_{\text {orbit }} \sin \left(a_{\text {lat }_{\text {corr }}}\right)
\end{aligned}
$$




$$
\begin{aligned}
& \dot{x}_{\text {orbit }_{\text {plane }}}=R_{\text {orbit }} \cos \left(a_{\text {lat }_{\text {corr }}}\right)-y_{\text {orbit }_{\text {plane }}} \dot{a}_{\text {lat }_{\text {corr }}} \\
& \dot{y}_{\text {orbit }_{\text {plane }}}=R_{\text {orbit }} \sin \left(a_{\text {lat }_{\text {corr }}}\right)+x_{\text {orbit }_{\text {plane }}} \dot{a}_{\text {lat }_{\text {corr }}}
\end{aligned}
$$

These coordinates and rates are only with respect to the orbital plane, so they will need to be transformed in order to obtain 3D coordinates in the earth-centered, earth-fixed frame. First, right ascension of the ascending node and its rate of change, adjusted for the earth rotation rate, need to be computed:

$$
\begin{gathered}
\dot{\omega}_{a d j}=\dot{\Omega}_{\text {long }}-\omega_{\text {earth }} \\
\omega_{a d j}=\Omega_{\text {long }}+\Delta t_{e p h} \dot{\omega}_{a d j}-\omega_{e a r t h} t_{e p h}
\end{gathered}
$$

Where $\Omega_{\text {long }}$ and $\dot{\Omega}_{\text {long }}$ are the longitude of the ascending node and its derivative, respectively and $\omega_{\text {earth }}$ is the earth rotation rate. Now the satellite's $3 \mathrm{D}$ coordinates can be calculated:

$$
\begin{gathered}
X_{\text {sat }}=x_{\text {orbit }_{\text {plane }}} \cos \left(\omega_{\text {adj }}\right)-y_{\text {orbit }_{\text {plane }}} \cos \left(i n c_{\text {orbit }}\right) \sin \left(\omega_{\text {adj }}\right) \\
Y_{\text {sat }}=x_{\text {orbit }_{\text {plane }}} \sin \left(\omega_{\text {adj }}\right)-y_{\text {orbit }_{\text {plane }}} \cos \left(i n c_{\text {orbit }}\right) \cos \left(\omega_{\text {adj }}\right) \\
Z_{\text {sat }}=y_{\text {orbit }_{\text {plane }}} \sin \left(i n c_{\text {orbit }}\right)
\end{gathered}
$$

Finally, an intermediate value as well as the satellite velocities can be computed:

$$
\begin{aligned}
& d_{2 \text { temp }}=\dot{y}_{\text {orbit }_{\text {plane }}} \cos \left(i n c_{\text {orbit }}\right)-\dot{y}_{\text {orbit }} \text { plane } \sin \left(i n c_{\text {orbit }}\right) i \dot{n} c_{\text {orbit }} \\
& \dot{X}_{\text {sat }}=-\dot{\omega}_{a d j} Y_{\text {sat }}+\dot{x}_{\text {orbit }} \text { plane } \cos \left(\omega_{a d j}\right)-d_{2 t e m p} \sin \left(\omega_{a d j}\right) \\
& \dot{Y}_{\text {sat }}=\dot{\omega}_{a d j} X_{\text {sat }}+\dot{x}_{\text {orbit }} \text { plane } \sin \left(\omega_{\text {adj }}\right)+d_{2_{\text {temp }}} \cos \left(\omega_{a d j}\right) \\
& \dot{Z}_{\text {sat }}=y_{\text {orbit }_{\text {plane }}} \cos \left(i n c_{\text {orbit }}\right) i \dot{n} c_{\text {orbit }}+\dot{y}_{\text {orbit }} \text { plane } \sin \left(i n c_{\text {orbit }}\right)
\end{aligned}
$$


Note that the coordinates and velocities for each satellite obtained from this process will be less accurate than the IGS produced values which can be obtained after all raw data is collected. 


\subsection{Parametric Least Squares Adjustments}

\subsubsection{Least Squares Adjustment in General}

In general, a least squares adjustment uses all available observations simultaneously to compute the most probable value for related unknowns in the presence of error and at any redundancy at or beyond the minimum to avoid singularities. This is done with a goal of minimizing the sum square value of all residuals between observed and modelled values. This algorithm will be utilized for most nonlinear, time-independent computations because it is widely used in geomatics networks, it is simple to implement, and it is a rigorous method with strong potential for precise results.

First, a mathematical and stochastic model must be selected. The mathematical could either be conditional (in which unknowns are given constraints in terms of observed values) or parametric (in which observations are constrained by the related unknowns). In this case, a parametric model is used because the pseudorange observable can easily be isolated without other measurements appearing in the equation. The derivatives of each observation with respect to the unknowns can be inserted into a design matrix as follows:

$$
A_{\text {design }_{m x n}}=\left[\begin{array}{cccc}
\frac{d_{l_{1}}}{d_{x_{1}}} & \frac{d_{l_{1}}}{d_{x_{2}}} & \ldots & \frac{d_{l_{1}}}{d_{x_{m}}} \\
\frac{d_{l_{2}}}{d_{x_{1}}} & \ldots & \ldots & \ldots \\
\ldots & \ldots & \ldots & \ldots \\
\frac{d_{l_{n}}}{d_{x_{1}}} & \ldots & \ldots & \frac{d_{l_{n}}}{d_{x_{m}}}
\end{array}\right]
$$

Since the derivatives of the observation equations in a nonlinear relationship will contain the estimations of the unknowns, this matrix will need to be updated after an adjustment is made. 
Using the same observation equation, we can populate a vector of modelled observations using our current estimate of the unknowns:

$$
l_{\text {estimated }_{n x 1}}=\left[\begin{array}{c}
l_{1}(x) \\
l_{2}(x) \\
\ldots \\
l_{n}(x)
\end{array}\right]
$$

For use with the stochastic model, it is also necessary to define the variance-covariance matrix of the observations, using the variances of each measurement to populate the diagonals:

$$
C_{l_{n x n}}=\left[\begin{array}{cccc}
\sigma_{l_{1}}^{2} & 0 & 0 & 0 \\
0 & \sigma_{l_{2}}^{2} & 0 & 0 \\
0 & 0 & \ldots & 0 \\
0 & 0 & 0 & \sigma_{l_{n}}^{2}
\end{array}\right]
$$

The least squares procedure operates based on minimizing the misclosure:

$$
w_{\text {misclosure }_{n \times 1}}=l_{\text {observed }}-l_{\text {estimated }}
$$

Where $l_{\text {observed }}$ is a vector containing all of the actual observed measurements corresponding to the observation equations used to construct $l_{\text {estimated }}$. In the case of an unweighted solution where no stochastic model is selected, constructing these matrices and vectors using initial estimates of the unknowns gives enough information to compute an adjustment to the unknowns as follows:

$$
\hat{\delta}_{m x 1}=N^{-1} A_{\text {design }}^{T} w_{\text {misclosure }}
$$

Where

$$
N=A_{\text {design }}^{T} A_{\text {design }}
$$

Now the adjustment $\hat{\delta}$ can be used to adjust the current estimate of the unknowns:

$$
\hat{x}_{m x 1}=x_{0_{m x 1}}+\hat{\delta}_{m x 1}
$$


Where $\hat{x}$ is the updated estimate of the unknowns and $x_{0}$ is the estimate of the unknowns used to compute $\hat{\delta}$. The new estimate of unknowns is used to compute the next iteration of $A_{\text {design }}, l_{\text {estimated }}$ and $\hat{\delta}$. It is important to not that if the model is nonlinear, more than one iteration of the algorithm may be required. In fact, the procedure will be repeated iteratively until the magnitude of $\hat{\delta}$ falls below a desired threshold.

Since many geometric mathematical models are non-linear, it is important to ensure that the initial estimates of the unknown values are sufficiently close to the optimal values before beginning the least-squares procedure. In addition, care must be taken to ensure that all measurements are done with biases eliminated or modeled as much as possible. If either of these conditions are not met, then the procedure may diverge from the optimal solution.

\subsubsection{Weighted Parametric Least Squares Adjustment}

It is often useful to use a stochastic model to assist in model optimization. The stochastic model is built by defining a weight matrix:

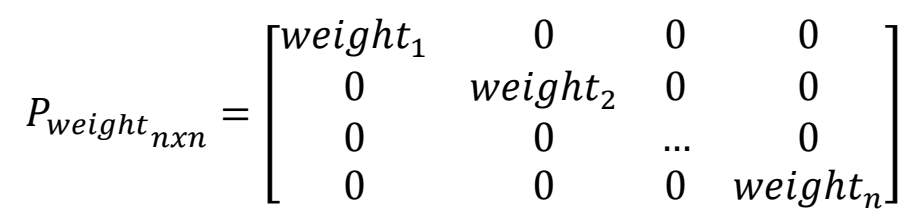

Where each weight corresponds to a measurement used in the adjustment. There are many ways to decide these weights, but as a general rule the goal is to give more weight to measurements which are trusted and less weight to measurements with less confidence. One way to select weights is using the standard deviations of each measurement. In that case, the weight matrix can be simply defined as: 


$$
P_{\text {weight }}=C_{l}^{-1}
$$

Now, this weight matrix can be utilized to augment the solution for computing the correction:

$$
\hat{\delta}_{m x 1}=N^{-1} A_{\text {design }}^{T} P_{\text {weight }} w_{\text {misclosure }}
$$

Where

$$
N=A_{\text {design }}^{T} P_{\text {weight }} A_{\text {design }}
$$

If weights are well-selected and the measurements are sufficiently free of bias, then this should yield a solution which more accurately reflects the physical reality of the estimated values. From the equations, it becomes apparent that when no stochastic model is selected, a model is implicitly imposed on the model where the weight matrix is simply equal to an identity matrix.

\subsection{Least Squares Mathematical and Stochastic Models}

\subsubsection{Global Navigation Satellite Systems Positioning}

\section{Models}

\subsubsection{Single Point Positioning}

In general, GNSS pseudorange measurements are modelled using the pseudorange equation:

$$
P_{\text {range }}=c \Delta t
$$




$$
\begin{gathered}
\text { pseudorange }= \\
P_{\text {range }}-c \delta T+\delta_{\text {troposphere }}+\delta_{\text {ionosphere }}+\delta_{\text {multipath }}+\text { noise }
\end{gathered}
$$

Where $\rho_{\text {pseudorange }}$ is the value directly extrapolated from the receiver's time of flight measurement from satellite to antenna, $P_{\text {range }}$ is the actual geometric range between the satellite and antenna, $c \delta T$ is the delay caused by the difference between the satellite clock offset and

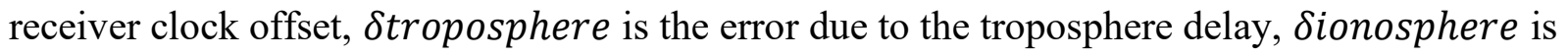
the error due to ionosphere delay, Smultipath is the multipath error and noise is the gaussian white noise of the signal.

While the least squares procedure is intended to compensate for the white noise and the multipath is highly spatially dependent requiring static testing to model its periodic behaviour, the atmospheric errors are very substantial and need to be modelled to mitigate their effects. Therefore, The vector of observables will be:

$$
l_{\text {obs }}=\left[\begin{array}{c}
\rho_{\text {pseudorange }} \text { satellite } 1 \text {-antenna } \\
\rho_{\text {pseudorange }} \text { satellite } 2 \text {-antenna } \\
\ldots \\
\rho_{\text {pseudorange }} \text { satellite } n \text {-antenna }
\end{array}\right]
$$

The clock delay can be very significant as well. Since there is not a similar way to model this delay (it is unknown before the adjustment), it will be one of the unknown values. Therefore the vector of unknowns will be:

$$
\hat{x}_{\text {single point }}=\left[\begin{array}{c}
X_{W G S 84_{\text {antenna }}} \\
Y_{\text {WGS84 }} \text { antenna } \\
Z_{W G S 84_{\text {antenna }}} \\
c \delta T
\end{array}\right]
$$


Where $X_{W G S 84_{\text {antenna }}}, Y_{W G S 84_{\text {antenna }}}$ and $Z_{W G S 84_{\text {antenna }}}$ are the coordinates of the antenna in the earth-centered, earth-fixed coordinate frame using the WGS84 datum. The observation equation relating the unknowns with the observations is as follows:

$$
\begin{aligned}
& \rho_{\text {pseudorange }}=l(x) \\
& =\sqrt{\left(X_{\text {antenna }}-X_{\text {satellite }}\right)^{2}+\left(Y_{\text {antenna }}-Y_{\text {satellite }}\right)^{2}+\left(Z_{\text {antenna }}-Z_{\text {satellite }}\right)^{2}} \\
& -c \delta T+\delta \text { troposphere }+ \text { Sionosphere }
\end{aligned}
$$

This is used to construct the vector of estimated observables:

$$
\begin{aligned}
& l_{\text {estimated }} \times 1 \\
& =\left[\begin{array}{c}
l(\text { antenna position, satellite } 1 \text { position, } \text { clock error }) \\
l(\text { antenna position, satellite } 2 \text { position, } \text { clock error }) \\
\ldots \\
l(\text { antenna position, satellite } n \text { position, clock error })
\end{array}\right]
\end{aligned}
$$

Due to the requirement of computing the clock error, it is important to note that a minimum of four satellites must be used to compute a unique solution, because there are 4 unknown quantities (X, Y and Z coordinates plus a clock offset) and each satellite provides one pseudorange measurement. When the derivative is computed of each pseudorange with respect to each unknown, the following is the result:

$$
\begin{gathered}
\frac{d \rho_{i}}{d X_{\text {antenna }_{\text {antena }}}-X_{\text {satellit }_{i}}} \\
\rho_{i} \\
\frac{d \rho_{i}}{d Y_{\text {antenna }}}=\frac{X_{\text {antenna }_{\text {ante }}-Y_{\text {satellite }_{i}}}}{\rho_{i}} \\
\frac{d \rho_{i}}{d Z_{\text {antenna }}}=\frac{Z_{\text {antenna }}-Z_{\text {satellite }_{i}}}{\rho_{i}} \\
\frac{d \rho_{i}}{d(c \delta T)}=-1
\end{gathered}
$$

These derivatives are used to construct the design matrix: 


$$
A_{\text {design }}=\left[\begin{array}{ccccc}
\frac{d \rho_{1}}{d X_{\text {antenna }}} & \frac{d \rho_{1}}{d Y_{\text {antenna }}} & \frac{d \rho_{1}}{d Z_{\text {antenna }}} & -1 \\
\frac{d \rho_{2}}{d X_{\text {antenna }}} & \frac{d \rho_{2}}{d Y_{\text {antenna }}} & \frac{d \rho_{2}}{d Z_{\text {antenna }}} & -1 \\
\frac{d \rho_{n}}{d X_{\text {antenna }}} & \frac{d \rho_{n}}{d Y_{\text {antenna }}} & \frac{d \rho_{n}}{d Z_{\text {antenna }}} & -1
\end{array}\right]
$$

It is important to note that the only way that the single point measurement model can compensate for pseudorange bias in the least squares procedure is with optimal satellite geometry. This is why atmospheric models must be modelled before they are used as observables.

In addition, while this is the least robust measurement model in terms of precision, it is also the least demanding model in terms of hardware requirements. Since it only requires access to a single antenna and receiver, it will still be used when a base station is not available.

Where the carrier phase is used instead of the pseudorange, the same procedure is used as with pseudoranges, but the carrier phase equation will be used as the fundamental parametric equation:

$$
\begin{gathered}
\phi_{\text {carrier }}= \\
P_{\text {range }}-c \delta T+\delta \text { troposphere }- \text { Sionosphere }+\delta \text { multipath }+ \text { noise }+\lambda N
\end{gathered}
$$

The primary differences here are that the equation contains an additional term $\lambda N$, which refers to the integer ambiguity term scaled by the wavelength of the carrier signal and that the ionosphere term is negative rather than positive. The design matrix is identical to the pseudorange design matrix, while the $l_{\text {estimated }}$ vector is constructed by subtracting the ionosphere term and adding the scaled ambiguity term.

\subsubsection{Between Receiver Single Difference}

The between receiver single difference is modelled as follows: 
$\Delta P-c \Delta T+\Delta$ troposphere $+\Delta$ ionosphere $+\Delta$ multipath $+\Delta$ noise

Where $\Delta P$ is the geometric difference in ranges between the satellite and each receiver, $\Delta$ troposphere is the residual troposphere error after differencing, $\Delta$ ionosphere is the residual ionosphere error after differencing, $\Delta$ multipath is the differential multipath delay and $\Delta$ noise is the measurement noise.

Since $\Delta$ troposphere and $\Delta$ ionosphere are atmospheric errors, the difference effectively mitigates those errors, especially for short baselines between the base and rover. This is because both signals will experience similar atmospheric delays when they travel through similar regions geometrically. Therefore, we can use this model to create differences from corrected ranges directly:

$$
l_{\text {obs differentialGNSS }}=\left[\begin{array}{c}
\rho_{r \times 1-s a t 1}-\rho_{r \times 2-s a t 1} \\
\rho_{r \times 1-s a t 2}-\rho_{r \times 2-s a t 2} \\
\ldots \\
\rho_{r \times 1-s a t n}-\rho_{r \times 2-s a t n}
\end{array}\right]
$$

The act of differencing eliminates the satellite clock error but not receiver clock.

Therefore, the vector of unknowns is the same as in single point positioning:

$$
\hat{x}_{\text {differential }}=\hat{x}_{\text {single point }}=\left[\begin{array}{c}
X_{W G S 84_{\text {antenna }}} \\
Y_{W G S 84_{\text {antenna }}} \\
Z_{W G S 84_{\text {antenna }}} \\
c \delta T
\end{array}\right]
$$

Where WGS84 is the reference frame and antenna indicates the antenna location on the platform. The observation equation for the differential solution is as follows:

$$
\begin{aligned}
& \Delta \rho_{\text {used }}=l(x) \\
& =\sqrt{\left(X_{\text {antenna } 1}-X_{\text {satellite }}\right)^{2}+\left(Y_{\text {antenna } 1}-Y_{\text {satellite }}\right)^{2}+\left(Z_{\text {antenna } 1}-Z_{\text {satellite }}\right)^{2}} \\
& -\sqrt{\left(X_{\text {antenna } 2}-X_{\text {satellite }}\right)^{2}+\left(Y_{\text {antenna } 2}-Y_{\text {satellite }}\right)^{2}+\left(Z_{\text {antenna } 2}-Z_{\text {satellite }}\right)^{2}}
\end{aligned}
$$

This is used to construct the vector of estimated observations: 


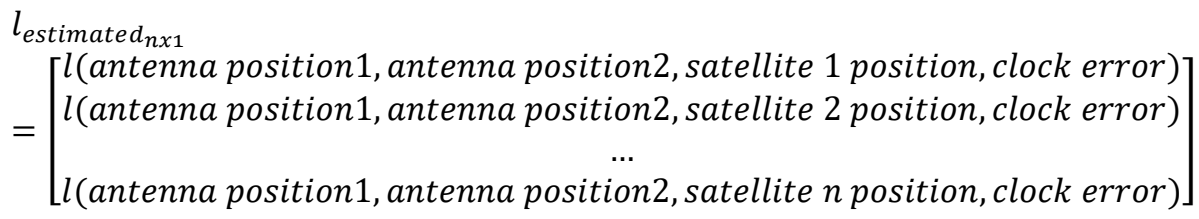

Next, the derivatives of the observation equation with respect to each unknown is computed for each observation:

$$
\begin{gathered}
\frac{d \Delta \rho_{i}}{d X_{\text {rover }}}=\frac{X_{\text {rover }}-X_{\text {satellite }_{i}}}{\rho_{i}} \\
\frac{d \Delta \rho_{i}}{d Y_{\text {rover }}}=\frac{Y_{\text {rover }}-Y_{\text {satellite }_{i}}}{\rho_{i}} \\
\frac{d \Delta \rho_{i}}{d Z_{\text {rover }}}=\frac{Z_{\text {rover }}-Z_{\text {satellite }_{i}}}{\rho_{i}} \\
\frac{d \rho_{i}}{d(c \Delta T)}=-1
\end{gathered}
$$

Where rover indicates that it is the non-base antenna location (at the platform). The derivatives are used to construct the design matrix:

$$
A_{\text {design }}=\left[\begin{array}{cccc}
\frac{d \Delta \rho_{1}}{d X_{\text {rover }}} & \frac{d \Delta \rho_{1}}{d Y_{\text {rover }}} & \frac{d \Delta \rho_{1}}{d Z_{\text {rover }}} & -1 \\
\frac{d \Delta \rho_{2}}{d X_{\text {rover }}} & \frac{d \Delta \rho_{2}}{d Y_{\text {rover }}} & \frac{d \Delta \rho_{2}}{d Z_{\text {rover }}} & -1 \\
\frac{d \Delta \rho_{n}}{d X_{\text {rover }}} & \frac{d \Delta \rho_{n}}{d Y_{\text {rover }}} & \frac{d \Delta \rho_{n}}{d Z_{\text {rover }}} & -1
\end{array}\right]
$$

It is interesting to note that the derivatives with respect to the unknowns are identical in this model compared to the single point model. This is because the term containing the base coordinates has a zero-derivative due to all variables being constant in that term.

It is also interesting to note that the property of mitigating atmospheric error makes the differential model preferable in almost all situations where a base is available. However, since 
the atmospheric error mitigation degrades at longer baselines and the differential model does not mitigate multipath errors, a single point model may be preferable to the differential one in some extreme situations, such as long baseline from the base or high multipath environments.

As is the case for single point positioning, the carrier phase merely uses a different equation in single differencing than the pseudorange solution does:

$$
\begin{gathered}
\Delta \phi= \\
\Delta P-c \Delta T+\Delta \text { troposphere }+\Delta \text { ionosphere }+\Delta \text { multipath }+\Delta \text { noise }+\Delta \lambda N
\end{gathered}
$$

\subsubsection{Double Differencing}

The double difference is a difference between satellites of 2 between receiver single differences, and is modelled as follows:

$$
\begin{gathered}
\Delta \nabla \rho= \\
\Delta \nabla P+\Delta \text { Vtroposphere }+\Delta \nabla \text { ionosphere }+\Delta \nabla \text { multipath }+\Delta \nabla \text { noise }
\end{gathered}
$$

Where $\Delta \nabla \rho$ is the measured, between receiver, between satellite double difference, $\Delta \nabla P$ is the true double difference based on the 2 satellite coordinates as well as the base and rover receiver coordinates, $\Delta \nabla$ troposphere and $\Delta \nabla$ ionosphere are the residual atmospheric biases after differencing, $\Delta \nabla$ multipath is the multipath bias and $\Delta \nabla$ noise is the double difference noise.

The same mitigative properties are true for double differences as are true for a between receiver single difference. In fact, for short baselines it eliminates even more of the atmospheric biases than the single difference does. It also has the property of completely removing the receiver and satellite clock biases. However, it does not remove multipath effects and noise effects can be significantly amplified compared to the single difference and single point models. 
In order to create a measurement vector with this model, a satellite must first be selected as a reference satellite, the single difference from which all other single differences are subtracted. In order to minimize the influence of the reference error on the whole solution, it is prudent to select a satellite which is expected to have the lowest bias effects. For the purposes of this study, the satellite which has the greatest weight in the stochastic model is selected. To be clear, that is the satellite which has the highest elevation angle relative to the receivers. Figure 8 below illustrates all 3 models along with the setup and how they combine together to form double differences:

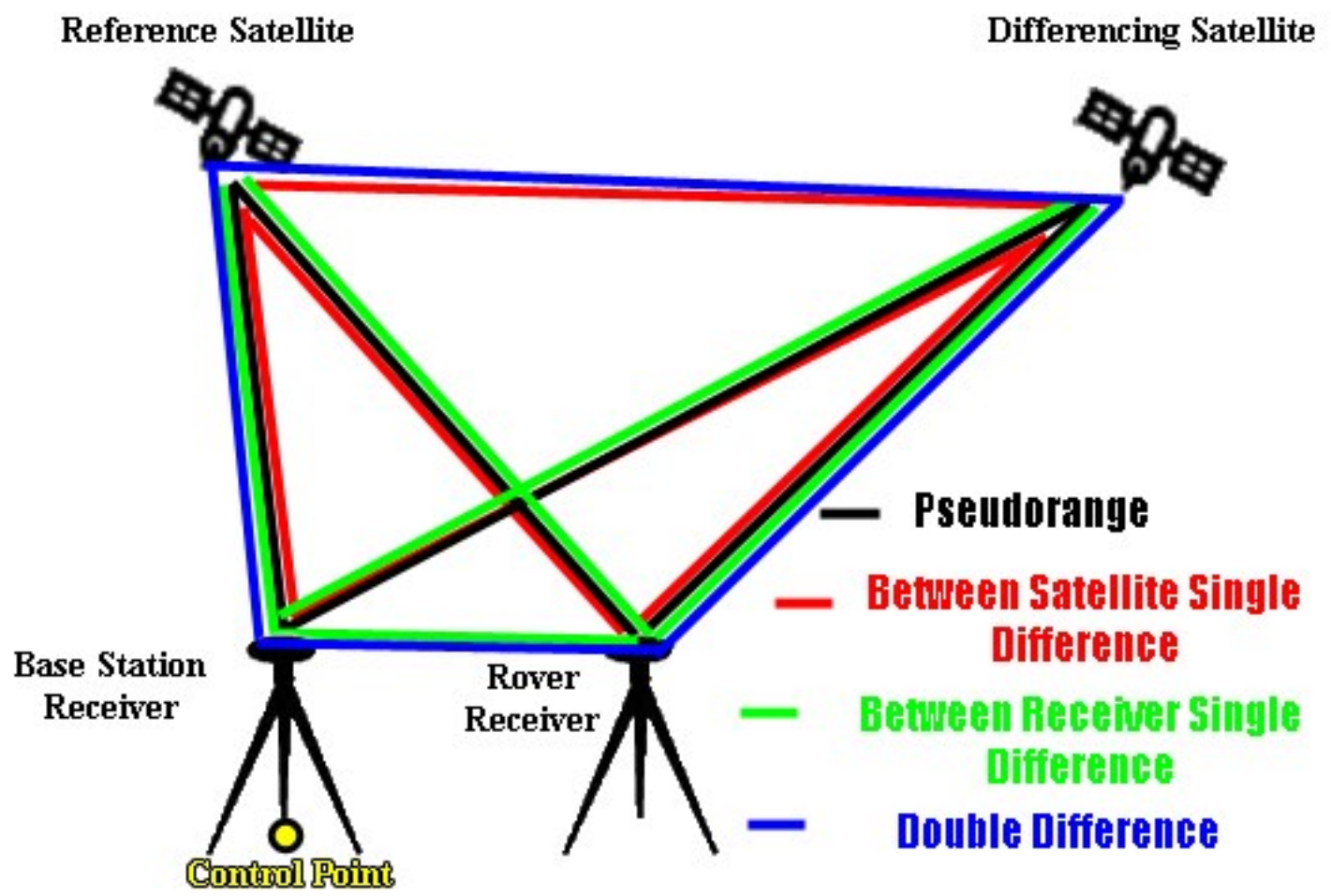

Figure 10: GNSS Double Difference Model and Setup

Once the reference satellite is selected, the vector of observed double differences can be constructed from all available between receiver single differences: 


$$
l_{\text {obs }_{\text {DoubleDifference }}(n-1) \times 1}=\left[\begin{array}{c}
\Delta \rho_{\text {reference }}-\Delta \rho_{\text {sat } 1} \\
\Delta \rho_{\text {reference }}-\Delta \rho_{\text {sat } 2} \\
\cdots \\
\Delta \rho_{\text {reference }}-\Delta \rho_{\text {satn }}
\end{array}\right]
$$

It is important to note that, although there are only 3 unknowns to be solved in this model, there is also 1 less observation available for redundancy. The vector of unknowns for the double difference model is as follows:

$$
\hat{x}_{\text {double difference }}=\left[\begin{array}{c}
X_{W G S 84_{\text {rover }}} \\
Y_{\text {WSS84 }_{\text {rover }}} \\
Z_{W G S 84_{\text {rover }}}
\end{array}\right]
$$

The observation equation for the double difference model is as follows:

$$
\begin{aligned}
& l(x)=\left(\text { range }_{\text {reference-rover }}-\text { range } e_{\text {reference-base }}\right) \\
& \text { - (range } \left.\text { differencer-rover }^{-} \text {range }_{\text {differencer-base }}\right)
\end{aligned}
$$

Where all ranges are constructed using the coordinates of the satellites, known base coordinates and estimates of the rover coordinates. These double differences are used to construct the vector of estimated measurements:

$$
\begin{aligned}
& l_{\text {estimated }}(n-1) x 1 \\
& {\left[\text { (reference position, differencer }{ }_{1}\right. \text { position, rover position, base position) }} \\
& =\left[\begin{array}{c}
l\left(\text { reference position, differencer } r_{2} \text { position, rover position, base position }\right) \\
\text { l(reference position, differencer } \left.r_{n} \text { position, rover position, base position }\right)
\end{array}\right]
\end{aligned}
$$

Now the derivatives are computed with respect to each unknown:

$$
\begin{gathered}
\frac{d \Delta \nabla \rho_{i}}{d X_{\text {rover }}}=\frac{X_{\text {rover }}-X_{\text {reference }}}{\rho_{\text {rover-reference }}}-\frac{X_{\text {rover }}-X_{\text {differencer }_{i}}}{\rho_{\text {rover-differencer }}} \\
\frac{d \Delta \nabla \rho_{i}}{d Y_{\text {rover }}}=\frac{Y_{\text {rover }}-Y_{\text {reference }}}{\rho_{\text {rover-reference }}}-\frac{Y_{\text {rover }}-Y_{\text {differencer }}}{\rho_{\text {rover-differencer }}} \\
\frac{d \Delta \nabla \rho_{i}}{d Z_{\text {rover }}}=\frac{Z_{\text {rover }}-Z_{\text {reference }}}{\rho_{\text {rover-reference }}}-\frac{Z_{\text {rover }}-Z_{\text {differencer }}}{\rho_{\text {rover-differencer }}}
\end{gathered}
$$


The derivatives are used to construct the design matrix:

$$
A_{\text {design }_{(n-1) \times 3}}=\left[\begin{array}{lll}
\frac{d \Delta \nabla \rho_{1}}{d X_{\text {rover }}} & \frac{d \Delta \nabla \rho_{1}}{d Y_{\text {rover }}} & \frac{d \Delta \nabla \rho_{1}}{d Z_{\text {rover }}} \\
\frac{d \Delta \nabla \rho_{2}}{d X_{\text {rover }}} & \frac{d \Delta \nabla \rho_{2}}{d Y_{\text {rover }}} & \frac{d \Delta \nabla \rho_{2}}{d Z_{\text {rover }}} \\
\frac{d \Delta \nabla \rho_{n}}{d X_{\text {rover }}} & \frac{d \Delta \nabla \rho_{n}}{d Y_{\text {rover }}} & \frac{d \Delta \nabla \rho_{n}}{d Z_{\text {rover }}}
\end{array}\right]
$$

It is interesting to note that, unlike the single difference model, the double difference derivatives are not identical to the single point model derivatives.

The double difference essentially amplifies the mitigative properties of the single difference. Since this can be quite effective at mitigating atmospheric errors at short baselines, this model is quite commonly used. However, multipath and noise effects could still render the single point model preferable in some situations since the double difference can amplify both errors.

As is the case with other measurement models, the carrier phase simply uses a different parametric equation than pseudoranges in double differencing:

$$
\begin{gathered}
\Delta \nabla \rho= \\
\Delta \nabla P+\Delta \nabla \text { troposphere }+\Delta \nabla \text { ionosphere }+\Delta \nabla \text { multipath }+\Delta \nabla \text { noise }+\Delta \nabla \lambda N
\end{gathered}
$$

As can be seen, the carrier phase can greatly benefit from differential positioning models because it is still subject to atmospheric error. Differential carrier phase solutions can mitigate bias while retaining the robust noise characteristics of the carrier phase.

\subsubsection{GNSS Stochastic Models}

Typically, the least squares stochastic model is one which is used to construct the weight matrix. One very common model uses the variance-covariance matrix of the measurements: 


$$
C l_{n x n}=\left[\begin{array}{cccc}
\sigma_{1}^{2} & 0 & 0 & 0 \\
0 & \sigma_{2}^{2} & 0 & 0 \\
0 & 0 & \ldots & 0 \\
0 & 0 & 0 & \sigma_{n}^{2}
\end{array}\right]
$$

Where $\sigma_{i}^{2}$ is the expected variance of the individual measurement. This value is typically taken from a manufacturer's specification or a value observed by experiment. In the case of a GNSS pseudorange, this refers to the noise variance, which will be the same for each pseudorange. This means that using this matrix will not impact the solution compared to using no stochastic model, since all measurements would be weighted equally regardless. Therefore, this stochastic model will not be used for the purpose of this study in the GNSS context.

One other method of weighting observations is to use the elevation angle of the satellite. When a satellite is directly overhead at an elevation angle of 90 degrees, it has optimal propagation geometry since it has the smallest possible distance to cover and the least refractive effects due to the atmosphere. At the horizon, satellites have the poorest propagation geometry, since the signal takes the most curved path possible, through the longest possible stretch of atmosphere, before reaching the antenna.

For this reason, it can be useful to use the elevation angle in the stochastic model:

$$
\text { weight }_{i}=\cos \left(90-\alpha_{\text {elevation }_{i}}\right)
$$

Where $\alpha_{\text {elevation }}$ is the elevation angle of each satellite. This ensures that satellites with poor geometry are given smaller weights relative to optimal satellites in the solution. These weights are used to build the weight matrix:

$$
P_{\text {weight }_{n x n}}=\left[\begin{array}{cccc}
\text { weight }_{1} & 0 & 0 & 0 \\
0 & \text { weight }_{2} & 0 & 0 \\
0 & 0 & \ldots & 0 \\
0 & 0 & 0 & \text { weight }_{n}
\end{array}\right]
$$


This weight matrix is used to augment the solution as follows:

$$
\begin{gathered}
N=A^{T} P A \\
\hat{\delta}=N^{-1} A_{\text {design }}^{T} P_{\text {weight }} w_{\text {misclosure }} \\
C_{\hat{x}}=N^{-1}=\left[\begin{array}{cccc}
\sigma_{x}^{2} & \sigma_{x y} & \sigma_{x z} & \sigma_{x \delta T} \\
\sigma_{x y} & \sigma_{y}^{2} & \sigma_{y z} & \sigma_{y \delta T} \\
\sigma_{x z} & \sigma_{y z} & \sigma_{z}^{2} & \sigma_{z \delta T} \\
\sigma_{x \delta T} & \sigma_{y \delta T} & \sigma_{z \delta T} & \sigma_{\delta T}^{2}
\end{array}\right]
\end{gathered}
$$

Where $C_{\hat{x}}$ is the variance-covariance matrix of the unknowns. This provides a measure of estimated precision in the calculated coordinates based on the estimated measurement noise. It does not indicate accuracy since the least squares procedure does not take bias into account and the measurement noise is not perfectly known. Accuracy can only be determined by comparing computed coordinates with known values (if available).

\subsection{Conditional Least-Squares}

Also discussed in [38] is the conditional least-squares model. The difference between this model and the parametric model is that this model uses unknown parameters in terms of measured quantities. This can be convenient for error propagation when this derivative is less complex than the parametric model. In general, the design matrix for the conditional model is:

$$
B_{\text {design }_{m \times n}}=\left[\begin{array}{ccc}
\frac{d x_{1}}{d l 1} & \ldots & \frac{d x_{1}}{d l_{n}} \\
\ldots & \ldots & \ldots \\
\frac{d x_{m}}{d l_{1}} & \ldots & \frac{d x_{m}}{d l_{n}}
\end{array}\right]
$$

In the conditional model, the measurement covariance matrix and weight matrix are the same as in the parametric model. In order to propagate the uncertainty, the inverse of the normal matrix is computed: 


$$
\begin{gathered}
N_{m \times m}=B P B^{T} \\
C_{x}=N^{-1}
\end{gathered}
$$

For the purposes of this research, this relationship will only be used to propagate uncertainty, and only in some select cases where it is convenient to do so. The reason for using this method of uncertainty propagation is that it preserves information about covariance between different observables and unknowns.

\subsection{Pitch and Heading Determination Using Dual}

\section{Antenna GNSS}

\subsubsection{Computation of Pitch and Heading}

\subsubsection{Definition of Heading}

For the purposes of this research, a heading will be defined as the direction of platform orientation, given as the clockwise angle between the geodetic north to the unit vector of platform orientation, projected onto the horizontal plane. This is illustrated below: 


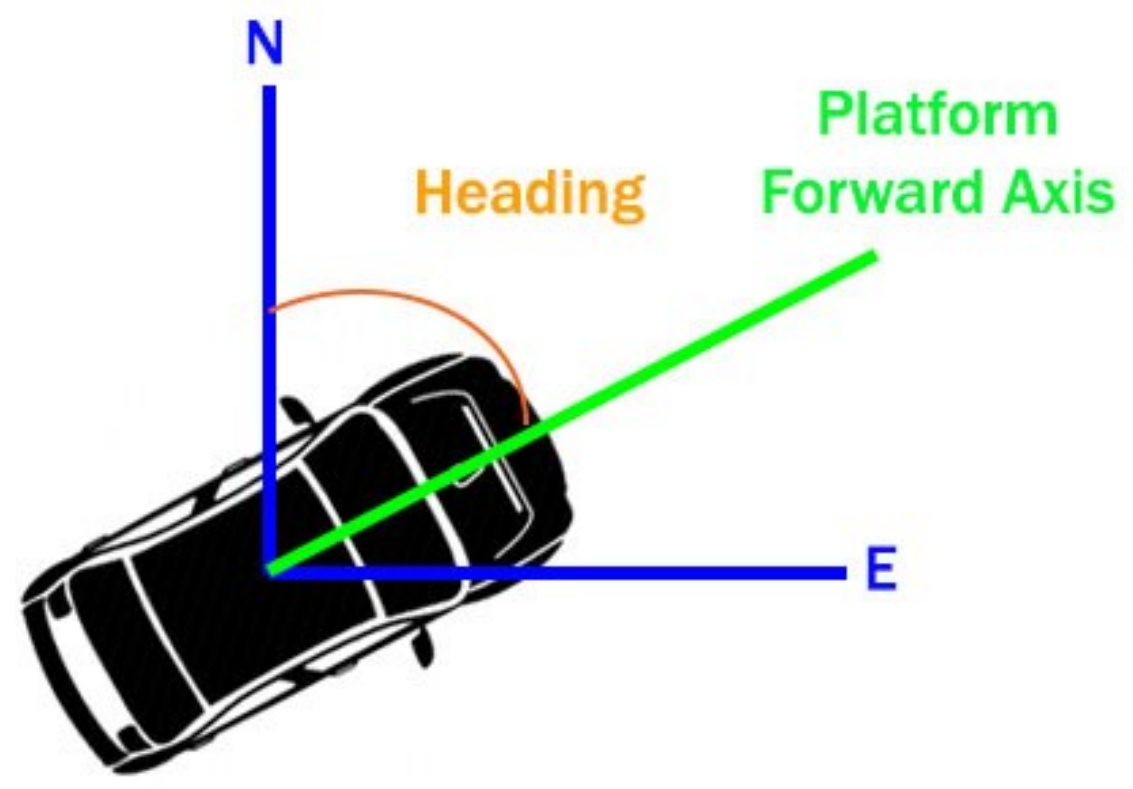

Figure 11: Definition of the Heading Angle on the North / East Horizontal Plane

Figure 9 illustrates the meaning of the heading angle as it relates to a moving car, but the heading holds the same definition regardless of the platform. There simply needs to be a reference defining the platform forward axis. An angle which holds a similar meaning to the heading angle is a bearing between 2 points, as illustrated below: 


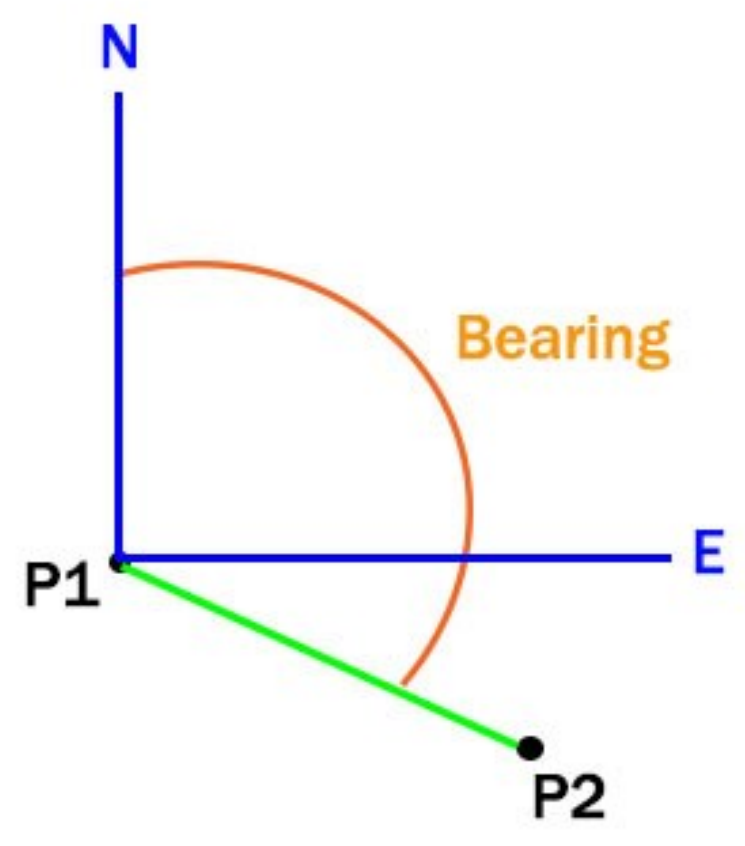

Figure 12: Definition of a Bearing Between 2 Points as Projected on the North / East Horizontal Plane

The bearing in Figure 10 and the heading in Figure 6 can each take on a positive value between 0 and 360 degrees for the purposes of this research. The heading angle is an angle critical to navigation because navigating pertains traversing from one location to an objective, and projected coordinate systems are very useful for terrestrial applications. The heading defines the direction of travel, so any adjustments to trajectories will need to take this angle into account.

\subsubsection{Definition of Pitch}

For the purposes of this research, pitch will be defined as the inclination of the platform plane, given as the counter-clockwise angle between the horizontal plane (perpendicular to the gravity vector) and the platform frontal axis. The pitch will be a rotation about the platform lateral axis by definition when using a zero-roll assumption. This is illustrated in Figure 11: 


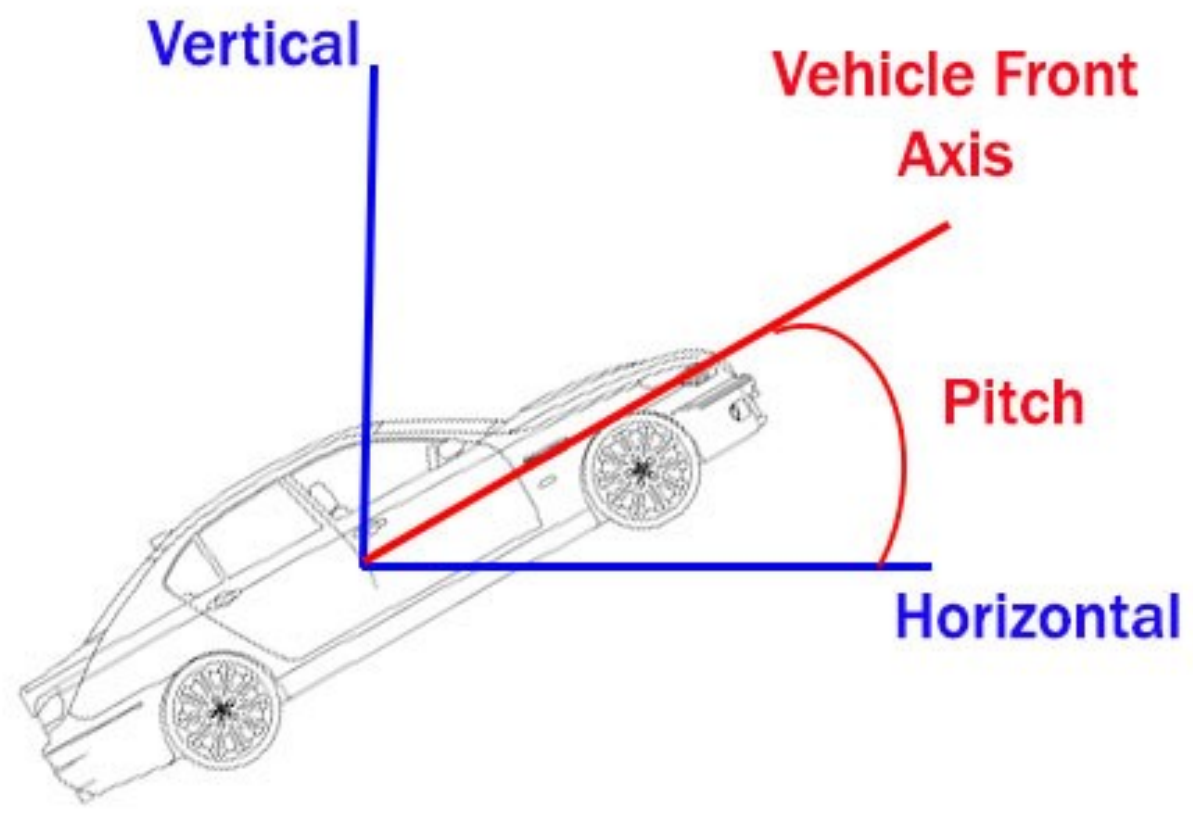

Figure 13: Definition of Pitch Relative to the Horizontal Plane and Vertical Axis

The pitch angle uses the horizontal plane as a reference, which does not have a fixed direction. Therefore, unlike the heading which uses a fixed north axis as a reference, pitch can take a value between -90 and 90 degrees. Pitch is an angle that could have safety implications for many applications, including marine and land navigation. High pitch angle indicates a sharp incline, which can compromise safety in some adverse weather conditions and on some surfaces.

\subsubsection{Computation and Error Propagation of Pitch and Heading Using GPS}

Suppose that the unit vector representing the platform direction of travel is defined by 2 points. If the coordinates of those points are known in a local vertical frame. The heading and pitch can then both be computed by triangle geometry: 


$$
\begin{gathered}
\Delta E=E_{\text {front }}-E_{\text {rear }} \\
\Delta N=N_{\text {front }}-N_{\text {rear }} \\
\Delta U=U_{\text {front }}-U_{\text {rear }} \\
\theta_{\text {pitch }}=\tan ^{-1} \frac{\Delta U}{\sqrt{\Delta E^{2}+\Delta N^{2}}} \\
\alpha_{\text {heading }}=\tan ^{-1} \frac{\Delta E}{\Delta N}
\end{gathered}
$$

Where $E_{\text {front }}$ and $E_{\text {rear }}$ are easting coordinates of the front and rear point, $N_{\text {front }}$ and $N_{\text {rear }}$ are northing coordinates of the front and rear point and $U_{\text {front }}$ and $U_{\text {rear }}$ are vertical coordinates of the front and rear point in a local vertical reference frame. It is important to note that the arctangent in (134) is a quadrant-dependent function. Therefore, the sign of the numerator and denominator must both be taken into account as follows:

$$
\begin{array}{cc}
\text { if } \Delta E>0 \text { and } \Delta N>0: & 0<\alpha<90 \\
\text { if } \Delta E>0 \text { and } \Delta N<0: & 90<\alpha<180 \\
\text { if } \Delta E<0 \text { and } \Delta N<0: & 180<\alpha<270 \\
\text { if } \Delta E<0 \text { and } \Delta N>0: & 270<\alpha<360
\end{array}
$$

For the purposes of this research, the coordinates computed for the first epoch of the rear GPS antenna will be used for the reference point in the coordinate transformation from the earthcentered, earth-fixed (ECEF) coordinate frame to the local vertical frame. In order to propagate the uncertainty from the computed ECEF coordinates to the local vertical equivalents, the parametric least-squares method will be used. The estimated covariance matrixes of the 
coordinates computed using GPS will be used to construct a new measurement covariance matrix:

$$
C l_{\text {transform }}=\left[\begin{array}{lll}
C_{x_{G P S_{1,1}}} & C_{x_{G P S_{1,2}}} & C_{x_{G P S_{1,3}}} \\
C_{x_{G P S_{2,1}}} & C_{x_{G P S_{2,2}}} & C_{x_{G P S_{2,3}}} \\
C_{x_{G P S_{3,1}}} & C_{x_{G P S_{3,2}}} & C_{x_{G P S_{3,3}}}
\end{array}\right]
$$

As in (132), only the positional elements are extracted from the covariance matrix of unknowns from the least-squares position solution, whether or not a combined clock offset was solved for in the solution. This is because only the geometry is being rotated in the transformation, not a pseudorange bias. The weight matrix for the transformation will be the inverse of this matrix. As for the design matrix, the rotation matrix from the ECEF frame to the local vertical frame can conveniently be used as the design matrix in a conditional least squares model instead of a parametric model. The rotation matrix will be a combination of two rotations, depending on the computed geodetic latitude and longitude of the GPS antenna:

$$
\begin{gathered}
R_{\lambda}=\left[\begin{array}{ccc}
\cos \left(\lambda_{\text {long }}\right) & -\sin \left(\lambda_{\text {long }}\right) & 0 \\
\sin \left(\lambda_{\text {long }}\right) & \cos \left(\lambda_{\text {long }}\right) & 0 \\
0 & 0 & 1
\end{array}\right] \\
R_{\phi}=\left[\begin{array}{ccc}
\cos \left(\phi_{\text {lat }}\right) & 0 & -\sin \left(\phi_{\text {lat }}\right) \\
0 & 1 & 0 \\
\sin \left(\phi_{\text {lat }}\right) & 0 & \cos \left(\phi_{\text {lat }}\right)
\end{array}\right] \\
R_{\text {transform }}=R_{\phi} R_{\lambda}
\end{gathered}
$$

Where $\lambda_{\text {long }}$ is the geodetic longitude and $\phi_{\text {lat }}$ is the geodetic latitude. With a weight matrix and rotation matrix computed, the uncertainty can be propagated using the conditional model:

$$
C_{x_{\text {transform }}}=\left(R_{\text {transform }} C l_{\text {transform }}^{-1} R_{\text {transform }}^{T}\right)^{-1}
$$


This will represent a covariance matrix of computed coordinates in a local vertical frame.

If this uncertainty is propagated for both antenna solutions, each individual covariance matrix can be used to build a new measurement covariance matrix for the pitch and heading computation:

$$
\begin{aligned}
& C l_{\text {GPS Attitude }}
\end{aligned}
$$

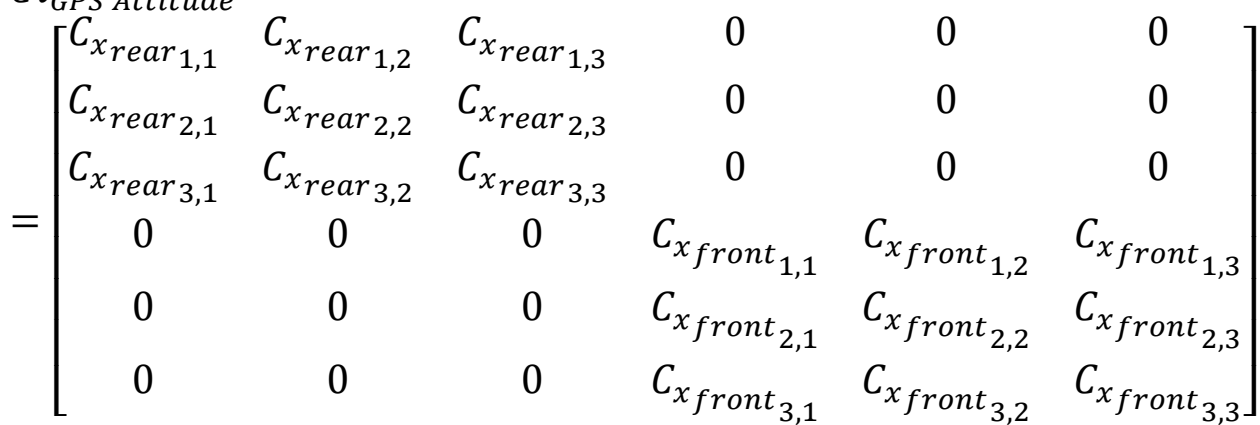

It is worth noting that, while some covariance information is retained through

propagation, this matrix assumes that the front and rear GPS antenna are uncorrelated. This is likely not true for this research, because the front and rear antenna share a common receiver.

With 2 antennas being utilized, (133) and (134) yield a unique solution, eliminating the need for a least-squares solution for the unknowns. However, the least-squares stochastic model is still useful for propagating the uncertainty from the previous step. Therefore, parametric equations and their partial derivatives are computed:

$$
\begin{gathered}
U_{\text {front }}=\tan \left(\theta_{\text {pitch }}\right) \sqrt{\Delta E^{2}+\Delta N^{2}}+U_{\text {rear }} \\
U_{\text {rear }}=-\tan \left(\theta_{\text {pitch }}\right) \sqrt{\Delta E^{2}+\Delta N^{2}}-U_{\text {front }} \\
E_{\text {front }}=\sqrt{\frac{\Delta U^{2}}{\tan ^{2} \theta_{\text {pitch }}}-\Delta N^{2}}+E_{\text {rear }} \\
E_{\text {rear }}=-\sqrt{\frac{\Delta U^{2}}{\tan ^{2} \theta_{\text {pitch }}}-\Delta N^{2}}-E_{\text {front }}
\end{gathered}
$$




$$
\begin{aligned}
& N_{\text {front }}=\sqrt{\frac{\Delta U^{2}}{\tan ^{2} \theta_{\text {pitch }}}-\Delta E^{2}}+N_{\text {rear }} \\
& N_{\text {rear }}=-\sqrt{\frac{\Delta U^{2}}{\tan ^{2} \theta_{\text {pitch }}}-\Delta E^{2}}-N_{\text {front }} \\
& E_{\text {front }}=\Delta N \tan \left(\alpha_{\text {heading }}\right)+E_{\text {rear }} \\
& E_{\text {rear }}=-\Delta N \tan \left(\alpha_{\text {heading }}\right)-E_{\text {front }} \\
& N_{\text {front }}=\frac{\Delta E}{\tan \left(\alpha_{\text {heading }}\right)}+N_{\text {rear }} \\
& N_{\text {rear }}=-\frac{\Delta E}{\tan \left(\alpha_{\text {heading }}\right)}-N_{\text {front }} \\
& \frac{d U_{\text {front }}}{d \theta_{\text {pitch }}}=\sec ^{2} \theta_{\text {pitch }} \sqrt{\Delta E^{2}+\Delta N^{2}} \\
& \frac{d U_{\text {rear }}}{d \theta_{\text {pitch }}}=-\frac{d U_{\text {front }}}{d \theta_{\text {pitch }}} \\
& \frac{d E_{\text {front }}}{d \theta_{\text {pitch }}}=\frac{-\cot \left(\theta_{\text {pitch }}\right) \csc ^{2} \theta_{\text {pitch }} \Delta U^{2}}{\sqrt{\frac{\Delta U^{2}}{\tan ^{2} \theta_{\text {pitch }}}-\Delta N^{2}}} \\
& \frac{d E_{\text {rear }}}{d \theta_{\text {pitch }}}=-\frac{d E_{\text {front }}}{d \theta_{\text {pitch }}} \\
& \frac{d N_{\text {front }}}{d \theta_{\text {pitch }}}=\frac{-\cot \left(\theta_{\text {pitch }}\right) \csc ^{2} \theta_{\text {pitch }} \Delta U^{2}}{\sqrt{\frac{\Delta U^{2}}{\tan ^{2} \theta_{\text {pitch }}}-\Delta E^{2}}} \\
& \frac{d N_{\text {rear }}}{d \theta_{\text {pitch }}}=-\frac{d N_{\text {front }}}{d \theta_{\text {pitch }}} \\
& \frac{d E_{\text {front }}}{d \alpha_{\text {heading }}}=\sec ^{2}\left(\alpha_{\text {heading }}\right) \Delta \mathrm{N}
\end{aligned}
$$




$$
\begin{gathered}
\frac{d E_{\text {rear }}}{d \alpha_{\text {heading }}}=-\frac{d E_{\text {front }}}{d \alpha_{\text {heading }}} \\
\frac{d N_{\text {front }}}{d \alpha_{\text {heading }}}=-\csc ^{2}\left(\alpha_{\text {heading }}\right) \Delta E \\
\frac{d N_{\text {rear }}}{d \alpha_{\text {heading }}}=-\frac{d N_{\text {front }}}{d \alpha_{\text {heading }}}
\end{gathered}
$$

Where computed coordinates and headings will be used to substitute into the derivatives. These derivatives can now be used to fill the design matrix:

$$
A_{\text {pitch,heading }}=\left[\begin{array}{cc}
\frac{d E_{\text {rear }}}{d \theta_{\text {pitch }}} & \frac{d E_{\text {rear }}}{d \alpha_{\text {heading }}} \\
\frac{d N_{\text {rear }}}{d \theta_{\text {pitch }}} & \frac{d N_{\text {rear }}}{d \alpha_{\text {heading }}} \\
\frac{d U_{\text {rear }}}{d \theta_{\text {pitch }}} & 0 \\
\frac{d E_{\text {front }}}{d \theta_{\text {pitch }}} & \frac{d E_{\text {front }}}{d \alpha_{\text {heading }}} \\
\frac{d N_{\text {front }}}{d \theta_{\text {pitch }}} & \frac{d N_{\text {front }}}{d \alpha_{\text {heading }}} \\
\frac{d U_{\text {front }}}{d \theta_{\text {pitch }}} & 0
\end{array}\right]
$$

With a covariance matrix and design matrix constructed, the uncertainty can be propagated:

$$
C_{x_{\text {pitch,heading }}}=\left(A_{\text {pitch,heading }}^{T} C l_{G P S_{\text {attitude }}} A_{\text {pitch,heading }}\right)^{-1}
$$




\subsubsection{Cropping and Linear Interpolation of GPS Data Sets for an Undedicated \\ Pitch and Heading Determination System}

Computation of pitch and heading requires simultaneous positions from 2 separate GPS antennas. However, the physical system used in this research is an undedicated system in which 1 receiver has a different data rate and is not synced with the other receiver. The receivers also begin recording data at different times. Therefore, before pitch and heading can be computed in post processing, data must be cropped to give both datasets the same duration, then data from the set with the lower data rate must be interpolated to fill a position at all times where a position is available in the set with the higher data rate.

Because the lower data rate set is to be interpolated, the longer dataset will be cropped to be equal in duration to the shorter dataset such that:

- The first and last epochs of each dataset share the same GPS time stamp if possible, or if not possible

- (If the high data rate set is longer) The first time stamp in the high data rate set which is later than the first time stamp in the low data rate set becomes the new first time stamp in both sets. In addition, the last time stamp in the high data rate set which is earlier than the last time stamp in the low data rate set becomes the new last time stamp in both sets.

- (If the low data rate set is longer) The first time stamp in the high data rate set becomes the new first time stamp in both sets. In addition, the last time stamp in the high data rate set becomes the new last time stamp in both sets. 
Once the data has been cropped to the appropriate size, the time stamps from the high data rate set will be used in conjunction the time stamps and positions in the low data rate set in order to interpolate a data set with the same data rate as the high data rate set:

$$
\begin{aligned}
& x_{\text {new }}(t)=\frac{t-t_{1}}{t_{2}-t_{1}}\left(x_{2}-x_{1}\right) \\
& y_{\text {new }}(t)=\frac{t-t_{1}}{t_{2}-t_{1}}\left(y_{2}-y_{1}\right) \\
& z_{\text {new }}(t)=\frac{t-t_{1}}{t_{2}-t_{1}}\left(z_{2}-z_{1}\right)
\end{aligned}
$$

Where $t_{1}$ is the nearest GPS time stamp in the low rate data set preceding time $t$ in the high data rate set, $t_{2}$ is the nearest GPS time stamp in the low data rate set after time $t$ and $x_{\text {new }}(t), x_{1}$ and $x_{2}$ are the interpolated coordinate, preceding coordinate in the low rate set and next coordinate in the low rate set, respectively. This interpolation yields 2 GPS positioning datasets which can be used directly to compute pitch and heading.

\subsubsection{Static Kalman Filter}

For the purposes of exploring how the results for pitch and heading computation in a static setup can be improved, a simple Kalman filter will be applied to computed angles from raw data in post processing. The algorithm is derived in [39] and it is a recommended technique for fusion of different sensors [40] as is the case for the undedicated GPS pitch and heading system implemented in this thesis. In order to utilize the recursive Kalman filter algorithm, some key matrices must be defined: 


$$
\begin{aligned}
& x_{k}=\left[\begin{array}{c}
\theta_{\text {pitch }_{k}} \\
\theta_{\text {heading }_{k}}
\end{array}\right](\text { state vector at time } k) \\
& \Phi_{k}=\left[\begin{array}{ll}
1 & 0 \\
0 & 1
\end{array}\right] \text { (state transition matrix) } \\
& z_{k}=\left[\begin{array}{c}
\theta_{\text {pitch }} k_{\text {measured }} \\
\theta_{\text {heading }} \\
k_{\text {measured }}
\end{array}\right] \text { (vector measurement update) } \\
& H_{k}=\left[\begin{array}{ll}
1 & 0 \\
0 & 1
\end{array}\right] \text { (connection between the measurements and state vector) } \\
& P_{k}=\left[\begin{array}{cc}
\sigma_{\theta}^{2} \text { pitch } & 0 \\
0 & \sigma_{\theta}^{2} \\
\text { heading }
\end{array}\right] \text { (covariance matrix of the measurements) } \\
& R_{k}=\left[\begin{array}{cc}
0.0001 & 0 \\
0 & 0.0005
\end{array}\right] \text { (process noise matrix) }
\end{aligned}
$$

Where the process noise matrix will be kept constant for this implementation. For $\sigma_{\theta}^{2}$ pitch and $\sigma_{\theta}^{2}$ heading , the diagonals of the estimated covariance matrix of the pitch and heading from GPS error propagation will be used. Only the diagonals are used because this implementation is assuming no correlation between pitch and heading. While this may not reflect reality, the uncorrelated model produced superior results compared to the correlated model in practice. $\theta_{\text {pitch }} k_{\text {measured }}$ and $\theta_{\text {heading }} k_{\text {measured }}$ are the computed pitch and roll values at time $\mathrm{k}$ using raw interpolated GPS coordinates from the front and rear GPS receiver. Process noise was tuned until optimal results were obtained, yielding the values in Equation 142. A trial/error method was used to tune the process noise in this case. Values were iteratively chosen until the pitch and heading errors were minimized.

In general, Kalman filtering is an iterative process. In the first epoch, the state vector is equal to the measurement update because no time has passed for propagation. In all proceeding steps, the state vector is a result of the filter cycle. First, Kalman gain $K_{k}$ is calculated: 


$$
K_{k}=P_{k}^{-} H_{k}^{T}\left(H_{k} P_{k}^{-} H_{k}^{T}+R_{k}\right)^{-1}
$$

Where $P_{k}^{-}$is either the propagated covariance matrix from the previous epoch's filter (if using Kalman propagation for the noise) or the covariance matrix propagated from all measurements at time $k$ (if using the least squares covariance matrix at each time instead). The Kalman gain is used to fuse the predicted state and measured state:

$$
x_{k}=x_{k}^{-}+K_{k}\left(z_{k}-H_{k} x_{k}^{-}\right)
$$

Where $x_{k}^{-}$is the predicted state based on the system dynamics and previous state. Next, if Kalman gain is used to propagate covariance, 2 steps will be undertaken:

$$
\begin{gathered}
P_{k}=\left(I-K_{k} H_{k}\right) P_{k}^{-} \\
P_{k+1}=\Phi_{k} P_{k} \Phi_{k}+P_{k}
\end{gathered}
$$

Where $P_{k}^{-}$is the previously propagated covariance matrix. If the alternative least squares adaptation is used (as in this thesis), $P_{k}^{-}$will simply be updated with a computed covariance matrix propagated from measurement geometry in the next epoch. Finally, the prediction for the next epoch is calculated:

$$
x_{k+1}^{-}=\Phi_{k} x_{k}
$$

This procedure is repeated for all available measurement updates. The iterative cycle is illustrated in the flow chart below: 


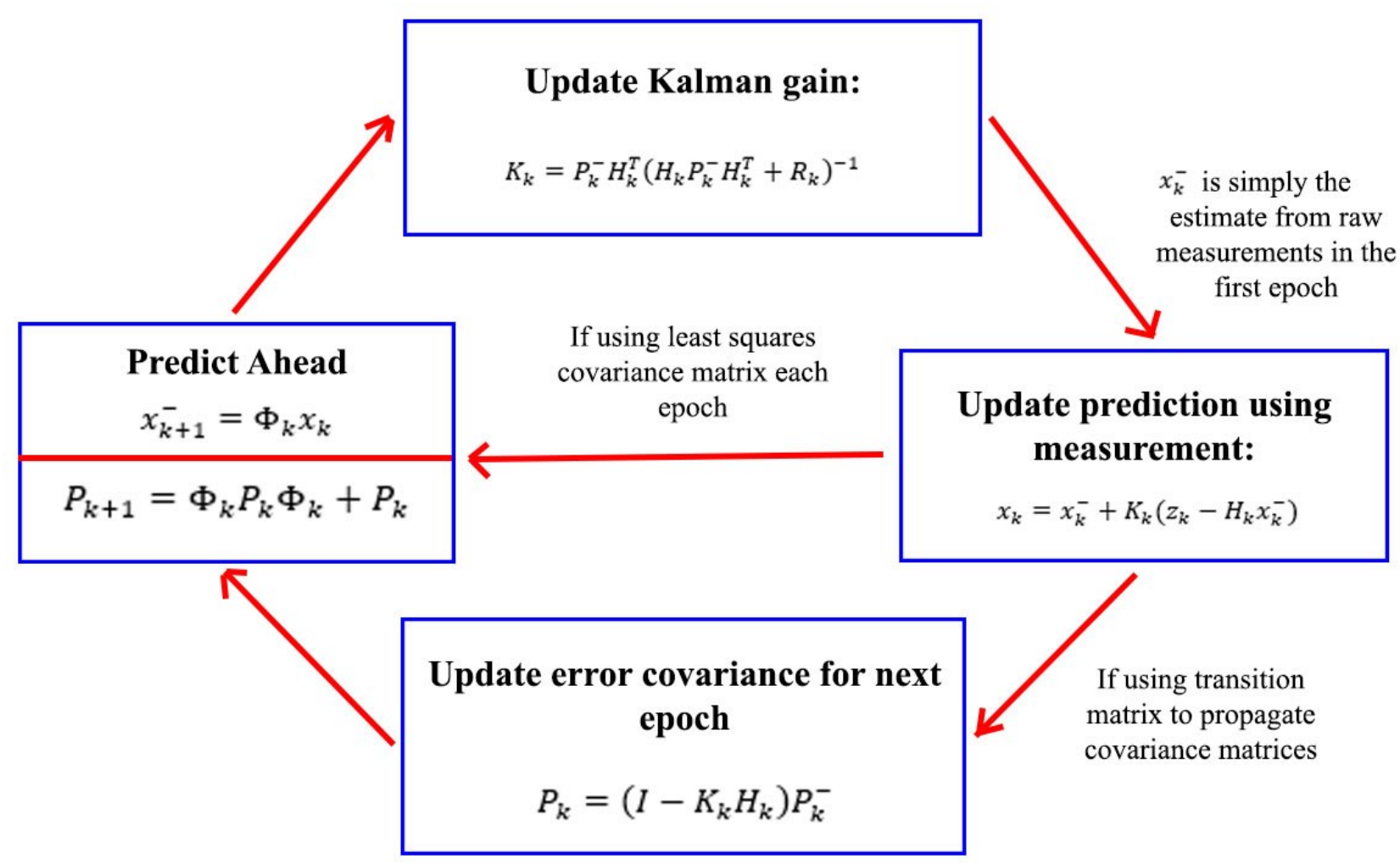

Figure 14: Kalman Filter Iterative Cycle Flow Chart with Standard as well as Adapted Covariance Update Strategies

\subsection{Error and Uncertainty Metrics}

In order to assess the validity of a measured or computed result, metrics for the accuracy and precision are necessary. Precision refers to the repeatability of a result from measured quantities, while accuracy refers to the difference between observed results and true quantities if they are known.

\subsubsection{Estimated Standard Deviation}

In the case of a GPS single point position solution, the covariance matrix is as in (110), while it is the result of (133) in the case of the pitch and heading. The diagonals of the covariance matrix represent the estimated variance of the corresponding variance. Therefore, the 
estimated standard deviation used to construct error ellipses as well as reported in simulation and surveys for pitch and heading is the square root of the variance:

$$
\sigma_{e s t_{i}}=\sqrt{\sigma_{e s t_{i}}^{2}}=\sqrt{C_{x}(i, i)}
$$

This is also known as dilution of precision (DOP) in the GNSS industry. It is perhaps a better term than standard deviation, because while it often does not reflect observed standard deviation, it does give a relative measure of precision with respect to geometry and provides a basis for construction of a confidence ellipse.

\subsubsection{Confidence Ellipses}

An error ellipse, otherwise called a confidence ellipse, will be used in this research to determine a region where $95 \%$ of positioning results are expected to lie if the coordinates are projected onto the horizontal plane at the true location of the antenna. In order to construct this

ellipse, the components defining the ellipse must be defined. The procedure outlined in [38] will be used to construct the ellipses. First, the semi-eastings and semi-northings lengths are defined by the estimated standard deviations in the eastings and northings directions, respectively. The larger of the 2 values will represent the semimajor axis of the ellipse with approximately $68 \%$ confidence. In order to draw an ellipse at a different confidence level, the semi-eastings and semi-northings must be scaled:

$$
\begin{aligned}
& a_{E}=S_{0} \sqrt{C x(1,1)} \\
& a_{N}=S_{0} \sqrt{C x(2,2)}
\end{aligned}
$$


Where $S_{0}$ is the scale factor (in this case a factor of 2.447 is used for the $95 \%$ confidence ellipse). Due to correlation between the eastings and northings, a rotation about the vertical axis is computed:

$$
\tan \left(2 \theta_{\text {ellipse }}\right)=\frac{2 C_{x}(1,2)}{C_{x}(2,2)-C_{x}(1,1)}
$$

Where $\theta_{\text {ellipse }}$ is the rotation angle about the vertical axis. The equation of the unrotated ellipse is defined by:

$$
\frac{\left(E-E_{0}\right)^{2}}{a_{E}^{2}}+\frac{\left(N-N_{0}\right)^{2}}{a_{N}^{2}}=1
$$

Where $E_{0}$ and $N_{0}$ are the true eastings and northings of the antenna. The ellipse is generated by entering a selection of eastings from $E_{0}-a_{E}$ to $E_{0}+a_{E}$, taking both the positive square root and negative square root solutions for $\mathrm{N}$. The coordinates are then rotated by $\theta_{\text {ellipse }}$ about the vertical axis. The rotated ellipse coordinates in the north and east directions are used for the confidence ellipse plots in this research.

\subsubsection{Root Mean Square (RMS) Error}

The RMS Error is used to give a measure of true accuracy of a solution on average. In order to compute this error, the true value must be known. In general, it can be defined as follows:

$$
R M S=\sqrt{\frac{\sum_{i=1}^{n}(\text { observed }- \text { true })^{2}}{n}}
$$

Where $n$ is the number of samples. The advantage that the RMS error has as a statistic is that it can aid in quantifying the effects of biases which are not random noise effects. While this 
is used to quantify errors on individual variables, it is also useful to have a broader positioning accuracy metric for computed GPS positions. One such metric used in this thesis is the 2DRMS:

$$
2 D R M S=\sqrt{\frac{\sum_{i=1}^{n}\left(\left(E_{\text {observed }}-E_{\text {true }}\right)^{2}+\left(N_{\text {observed }}-N_{\text {true }}\right)^{2}\right)}{n}}
$$

Where $E$ denotes eastings and $N$ denotes northings. This research uses 2DRMS to compare horizontal and vertical accuracy of GPS positioning solutions.

\subsubsection{Standard Deviation}

The standard deviation is a measure of precision of a data set. That is, it is the quantity, sharing units of the data set, such that approximately $68 \%$ of all data points in a normally distributed set fall within the mean plus or minus one standard deviation. It is computed as follows:

$$
\sigma=\sqrt{\frac{\sum_{i=1}^{n}(\text { observed }- \text { mean })^{2}}{n-1}}
$$

Where $n$ is the number of samples. The advantage of the standard deviation is that it gives a better measure of noise effects than the RMS error does. This can be adversely affected by temporal variability of measurement biases, however. It will be used as the primary measure of observed precision in this research. 


\section{Simulations and GPS Positioning Validation}

\subsection{Positioning Simulation}

Real GPS data is susceptible to multipath bias which is difficult to model. Therefore, a MALAB simulation will be done to evaluate computation methods under a variety of environmental conditions and based on the experimental control marker survey done in Didsbury, Alberta in which all errors will be controlled as an input parameter. The location was selected for its open sky environment as well as for easy access to precisely surveyed control monuments, each having coordinates and revision history available to the public.

\subsubsection{Simulated Measurements:}

In order to simulate an L1 pseudorange measurement, first the true geometric range is computed between the antenna location and satellite location. In this case, the known ASCM 764522 location is used as the antenna location and the satellites will take on identical geometry to the visible satellites in the first epoch of the actual logged data from the control marker survey in Didsbury.

\subsubsection{Simulated Results:}

Using satellite and marker geometry identical to that of the first epoch of the Didsbury ASCM survey, collections of results were simulated in a variety of bias, noise and differential baseline conditions. All positioning data collections are contained and discussed in this section. 


\subsubsection{Collection A: 0.1 ppm Bias, $10 \mathrm{~cm}$ Noise Standard Deviation, $100 \mathrm{~m}$ Differential Baseline}
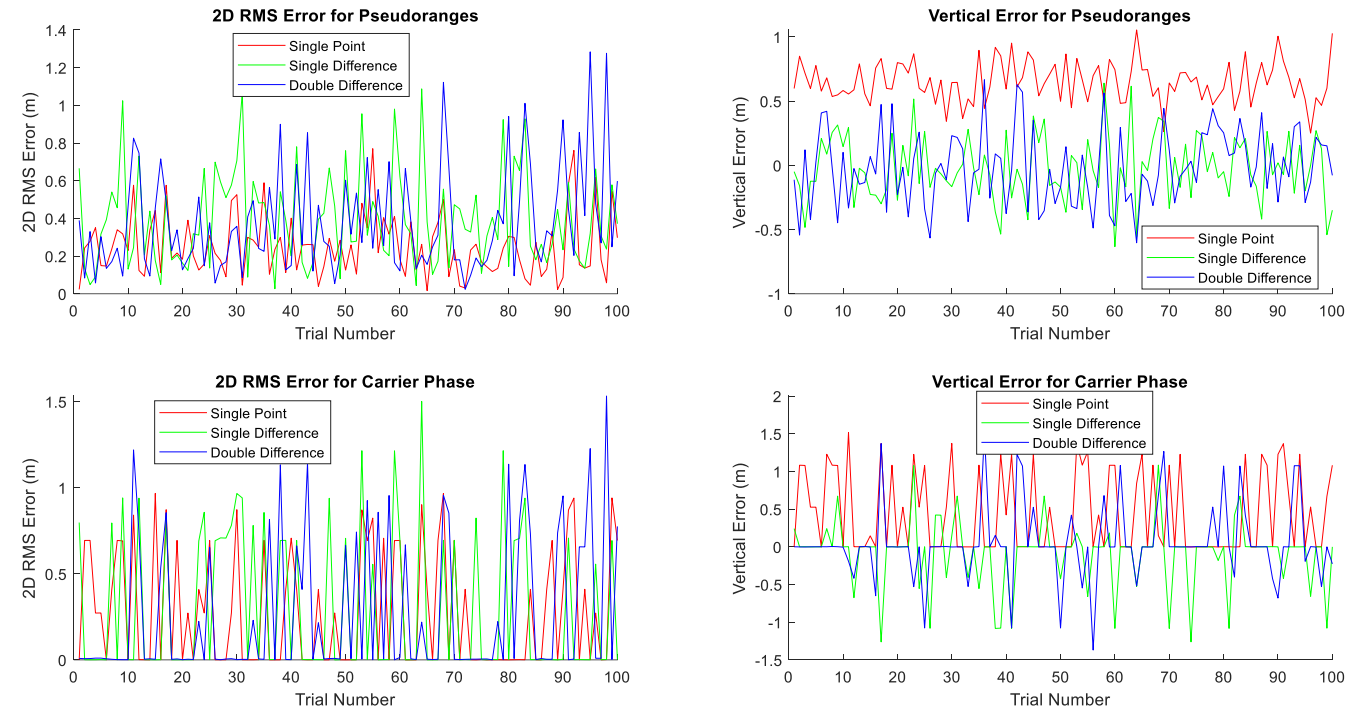

Figure 15: Horizontal and Vertical Errors for Simulation Set A

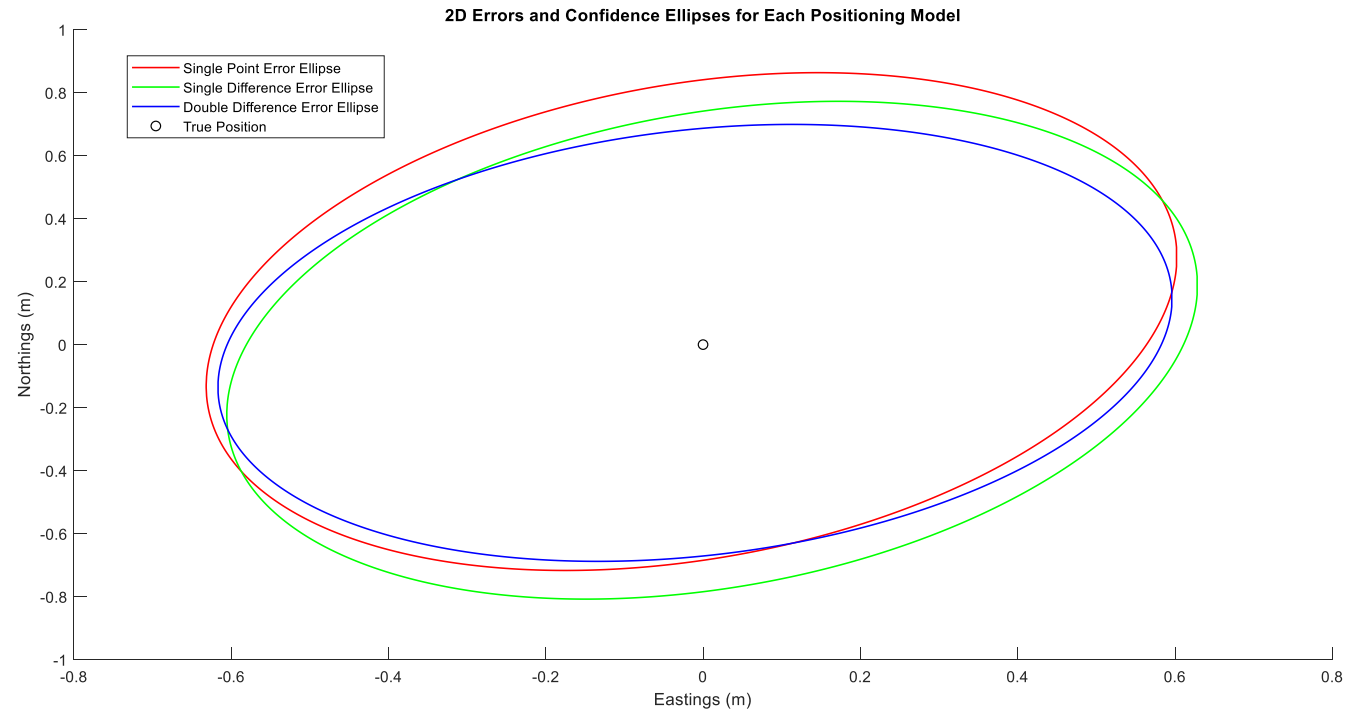

Figure 16: Horizontal 95 Percent Confidence Ellipses for Simulation Set A 
Table 4: Mean Error Statistics for Set A

\begin{tabular}{|c|c|c|c|c|}
\hline Technique & $\begin{array}{c}\text { 3DRMS Error } \\
(\mathrm{m})\end{array}$ & $\begin{array}{c}\text { Vertical Error } \\
(\mathrm{m})\end{array}$ & $\begin{array}{c}\text { Error Standard } \\
\text { deviation }(\mathrm{m})\end{array}$ & $\begin{array}{c}\text { Average } \\
\text { Ambiguity Error }\end{array}$ \\
\hline $\begin{array}{c}\text { Single Point: } \\
\text { Pseudoranges }\end{array}$ & 0.728 & 0.647 & 0.156 & - \\
\hline $\begin{array}{c}\text { Single Point: Carrier } \\
\text { Phase }\end{array}$ & 0.615 & 0.527 & 0.653 & 0.1486 \\
\hline $\begin{array}{c}\text { Single Difference: } \\
\text { Pseudoranges }\end{array}$ & 0.411 & -0.010 & 0.227 & 0.020 \\
\hline $\begin{array}{c}\text { Single Difference: } \\
\text { Carrier Phase }\end{array}$ & 0.250 & 0.019 & 0.480 & - \\
\hline $\begin{array}{c}\text { Double Difference: } \\
\text { Pseudoranges }\end{array}$ & 0.527 & -0.004 & 0.608 & 0.1814 \\
\hline $\begin{array}{c}\text { Double Difference: } \\
\text { Carrier Phase }\end{array}$ & 0.486 & 0.052 & 0.290 & \\
\hline
\end{tabular}

From Figure 13, it is apparent that the atmospheric biases have offset the vertical error whereas the differential solutions have a vertical mean closer to the true value, which is expected. It is also interesting to note that the differential solutions are noisier than the single point solution. This is also expected, since the noise from 2 receivers' signals will sometimes meet destructively but sometimes constructively in the difference.

From Figure 14, it is apparent that the true position falls within the confidence ellipse of the single point and single difference solutions are nearly circular while the double difference solution ellipse is elongated. This is expected since errors are highly correlated with the position of the reference satellite. 
From table 4 it is interesting to see that the single point solution has some advantages over the differential solution, particularly with regards to noise. This is because the error properties of Collection A are very optimistic. $10 \mathrm{~cm}$ pseudorange noise is what a high-precision P-code can yield if the equipment is high-quality, and only $0.1 \mathrm{ppm}$ of unmitigated atmospheric error is very difficult to achieve. Therefore, this set will provide a baseline for the ideal situation for positioning.

\subsubsection{Collection B: 0.1 ppm Bias, 1 m Noise Standard Deviation, $100 \mathrm{~m}$ Differential}

\section{Baseline}
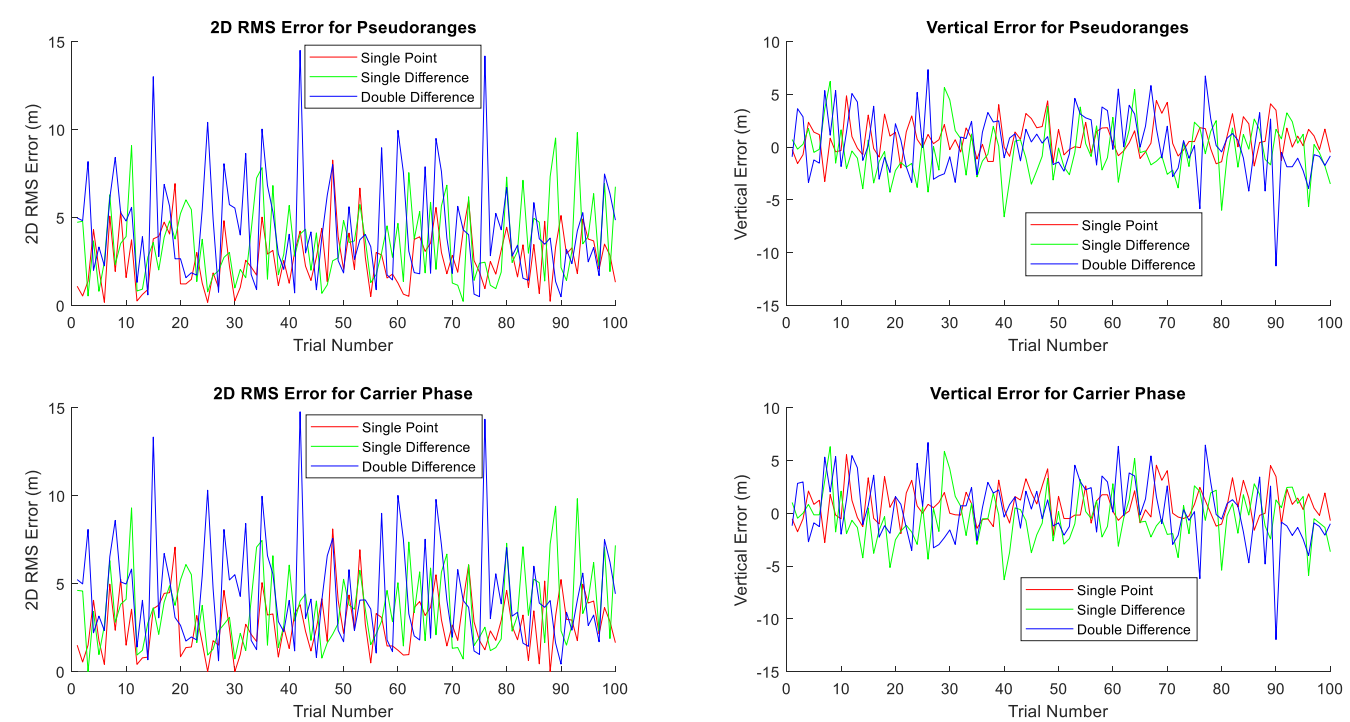

Figure 17: Horizontal and Vertical Errors for Simulation Set B

Collection B yields some interesting artifacts and trends which separate it from

Collection A. Regarding Figure 15, it is interesting that while noise has increased, the positioning solutions are not significantly offset from each other. There is a noticeable difference in the $3 \mathrm{D}$ positioning variation, however. A very extreme spike in positioning error is observed for the double difference solution near epoch 40 . This shows how strongly a constructive difference in signal noise can affect the solution. 


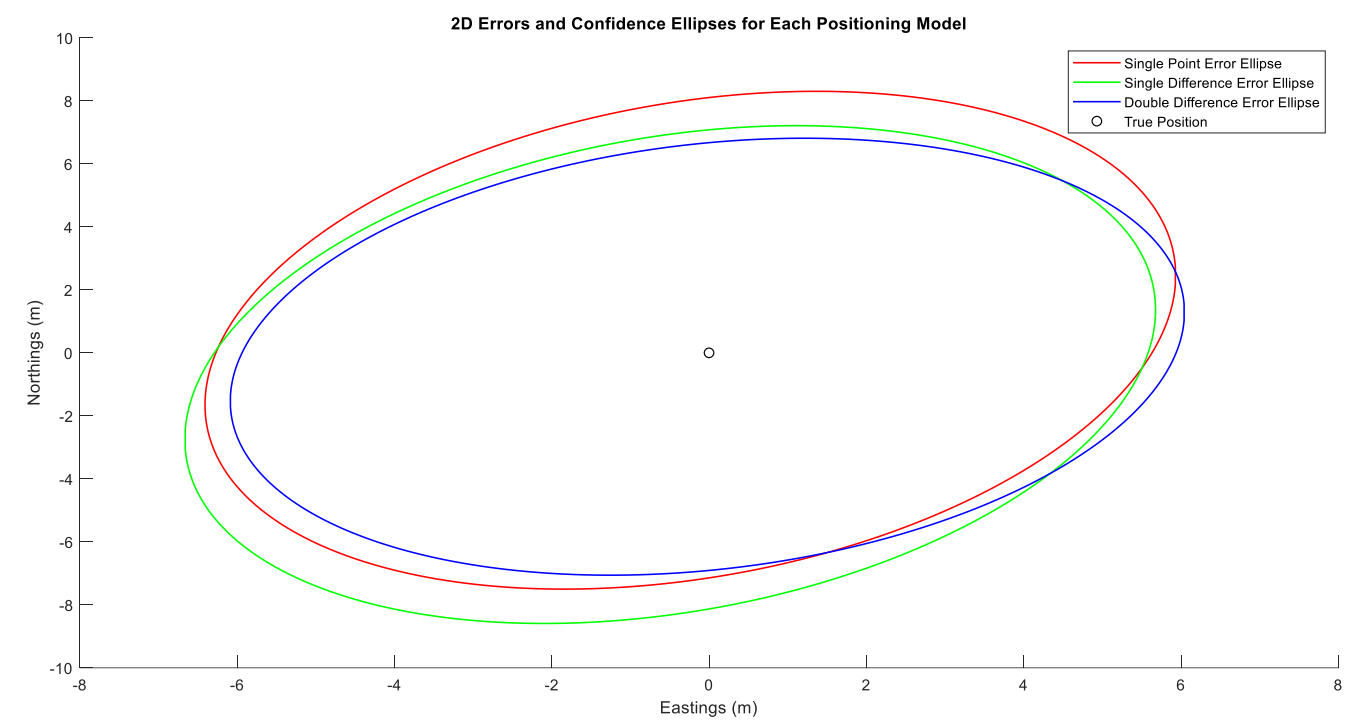

Figure 18: Horizontal 95 Percent Confidence Ellipses for Simulation Set B

Table 5: Mean Error Statistics for Set B

\begin{tabular}{|c|c|c|c|c|}
\hline Technique & $\begin{array}{c}\text { 3DRMS Error } \\
(\mathrm{m})\end{array}$ & $\begin{array}{c}\text { Vertical Error } \\
(\mathrm{m})\end{array}$ & $\begin{array}{c}\text { Error Standard } \\
\text { deviation }(\mathrm{m})\end{array}$ & $\begin{array}{c}\text { Average } \\
\text { Ambiguity Error }\end{array}$ \\
\hline $\begin{array}{c}\text { Single Point: } \\
\text { Pseudoranges }\end{array}$ & 3.233 & 0.591 & 1.646 & - \\
\hline $\begin{array}{c}\text { Single Point: Carrier } \\
\text { Phase }\end{array}$ & 3.231 & 0.571 & 1.640 & 1.23 \\
\hline $\begin{array}{c}\text { Single Difference: } \\
\text { Pseudoranges }\end{array}$ & 4.780 & -0.287 & 2.242 & 1.63 \\
\hline $\begin{array}{c}\text { Single Difference: } \\
\text { Carrier Phase }\end{array}$ & 4.827 & -0.336 & 2.304 & - \\
\hline $\begin{array}{c}\text { Double Difference: } \\
\text { Pseudoranges }\end{array}$ & 5.032 & -0.117 & 2.732 & 0.26 \\
\hline $\begin{array}{c}\text { Double Difference: } \\
\text { Carrier Phase }\end{array}$ & 5.031 & -0.111 & 2.746 & \\
\hline
\end{tabular}


Table 5 shows some interesting differences with Collection A. For instance, the correct integer ambiguity is not found as often, and since Collection B uses very optimistic atmospheric biases, there is only very marginal benefit overall to using the carrier phase, and in fact the pseudoranges performed better than the carrier phase when using the single difference measurement model.

One of the most significant statistics here which changed is the standard deviation of the error, being greater than $1.5 \mathrm{~m}$ in all cases. This is to be expected, since the control variable which was changed for this set is the measurement noise standard deviation. 


\subsubsection{Collection C: 0.1 ppm Bias, 3 m Noise Standard Deviation, $100 \mathrm{~m}$ Differential}

\section{Baseline}
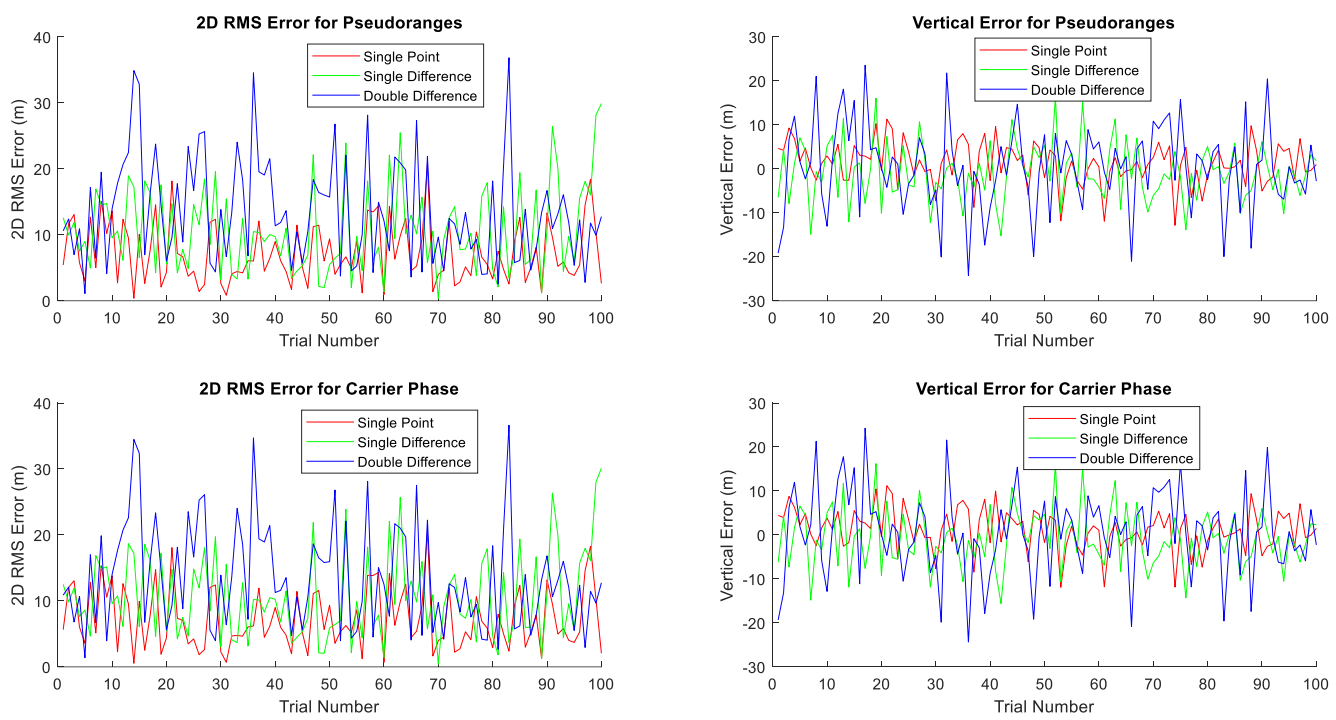

Figure 19: Horizontal and Vertical Errors for Simulation Set C

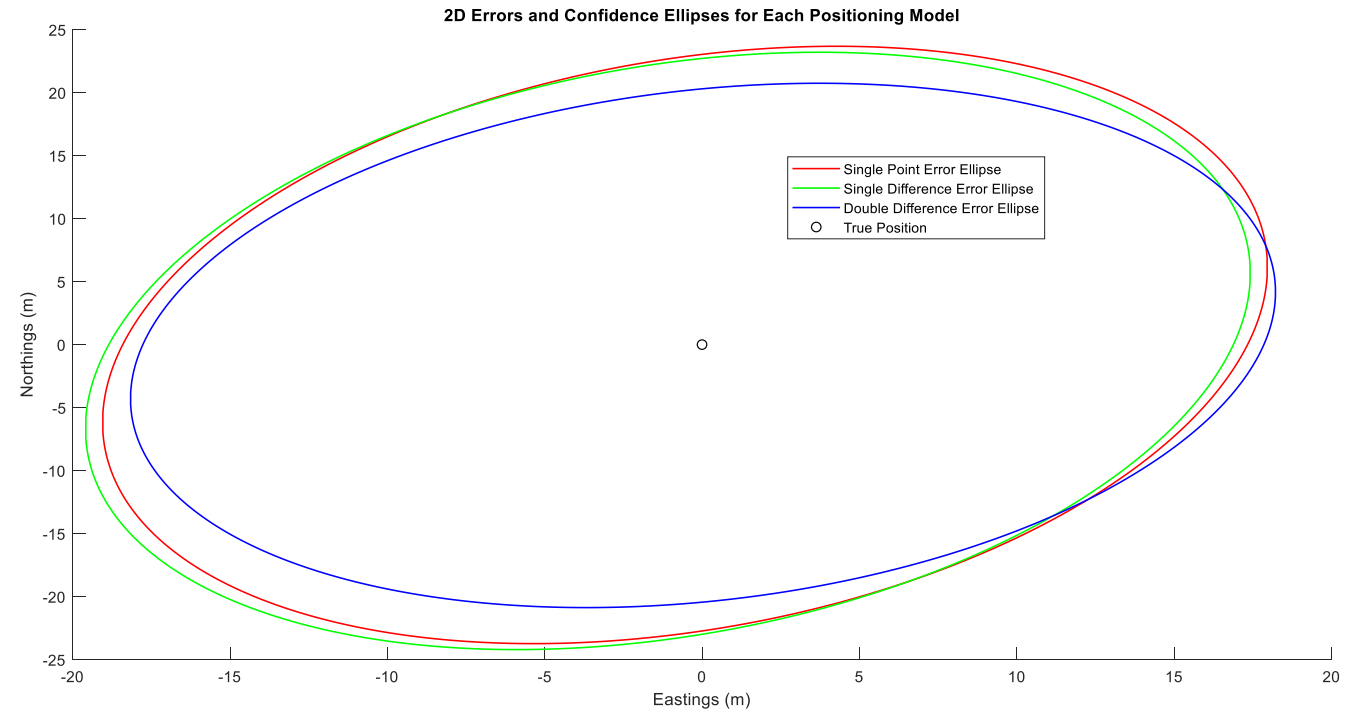

Figure 20: Horizontal 95 Percent Confidence Ellipses for Simulation Set C 
Table 6: Mean Error Statistics for Set C

\begin{tabular}{|c|c|c|c|c|}
\hline Technique & $\begin{array}{c}\text { 3DRMS Error } \\
(\mathrm{m})\end{array}$ & $\begin{array}{c}\text { Vertical Error } \\
(\mathrm{m})\end{array}$ & $\begin{array}{c}\text { Error Standard } \\
\text { deviation }(\mathrm{m})\end{array}$ & $\begin{array}{c}\text { Average Ambiguity } \\
\text { Error }\end{array}$ \\
\hline $\begin{array}{c}\text { Single Point: } \\
\text { Pseudoranges }\end{array}$ & 9.543 & 0.516 & 5.252 & - \\
\hline $\begin{array}{c}\text { Single Point: Carrier } \\
\text { Phase }\end{array}$ & 9.542 & 0.463 & 5.299 & -0.60 \\
\hline $\begin{array}{c}\text { Single Difference: } \\
\text { Pseudoranges }\end{array}$ & 12.480 & 0.556 & 6.837 & 1.29 \\
\hline $\begin{array}{c}\text { Single Difference: } \\
\text { Carrier Phase }\end{array}$ & 12.537 & 0.644 & 6.800 & - \\
\hline $\begin{array}{c}\text { Double Difference: } \\
\text { Pseudoranges }\end{array}$ & 16.236 & -0.388 & 8.269 & 4.87 \\
\hline $\begin{array}{c}\text { Double Difference: } \\
\text { Carrier Phase }\end{array}$ & 16.263 & -0.330 & 8.249 & \\
\hline
\end{tabular}

From Figure 17, a very pronounced difference is observed between differential and single point solutions is observed, though the mean position calculated for each is very similar. Collection C very clearly illustrates the amplified effect that differential measurement models have on noise.

Since the noise is so significant in this set, the pseudorange solution falls well outside of the linear search regions for the carrier phase ambiguities quite often. This results in discrete variations of carrier phase error in both directions, despite the carrier phase noise being modelled at only $1 \mathrm{~mm}$. This is to be expected. The pseudorange error is almost entirely due to noise in this set, so solutions are expected to fall anywhere inside of a 3D confidence ellipsoid defined by the $3 \mathrm{~m}$ measurement noise, with the mean being close to the true position. 
Benefits of the carrier phase are only marginal in collection $\mathrm{C}$, in fact the benefits are mostly random and inconsistent. However, there does seem to be a reasonable improvement to vertical positioning accuracy using the carrier phase in the single point model, possibly because that model has the least vulnerability to noise of the 3 models. 


\subsubsection{Collection D: 5 ppm Bias, $10 \mathrm{~cm}$ Noise Standard Deviation, $100 \mathrm{~m}$ Differential Baseline}
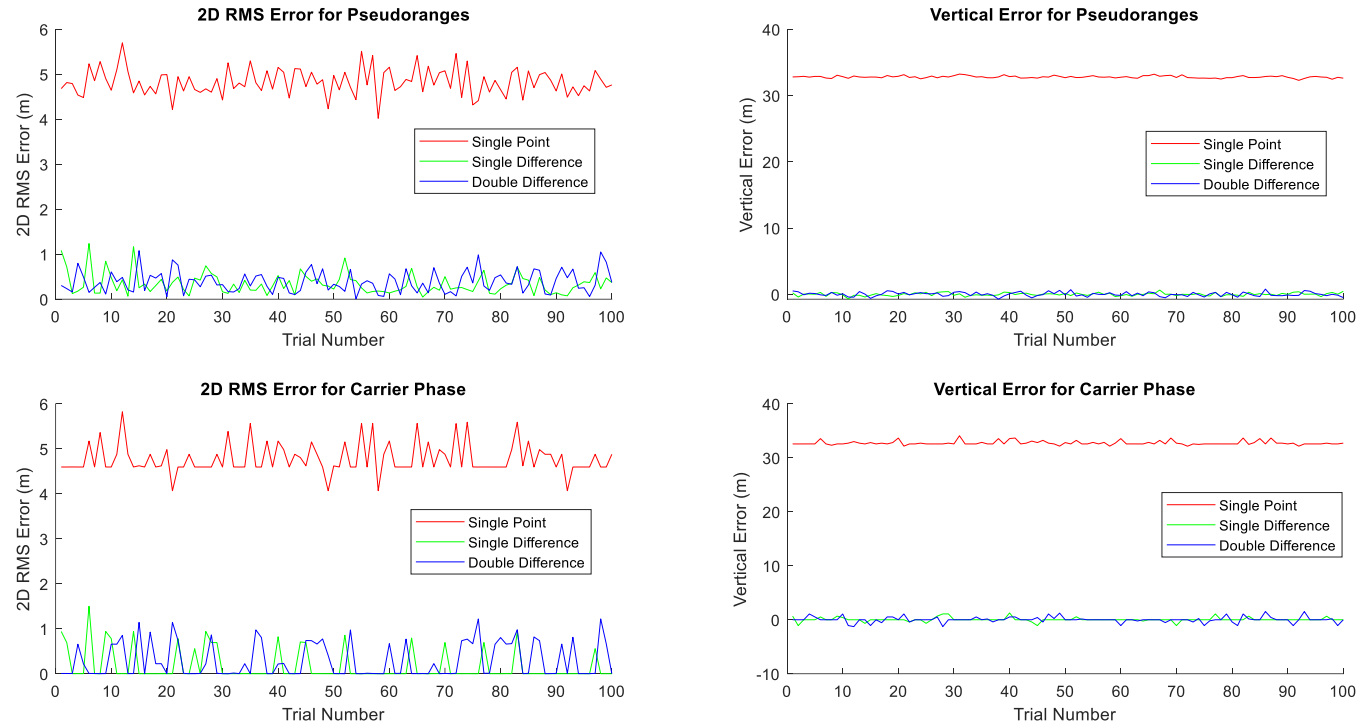

Figure 21: Horizontal and Vertical Errors for Simulation Set D

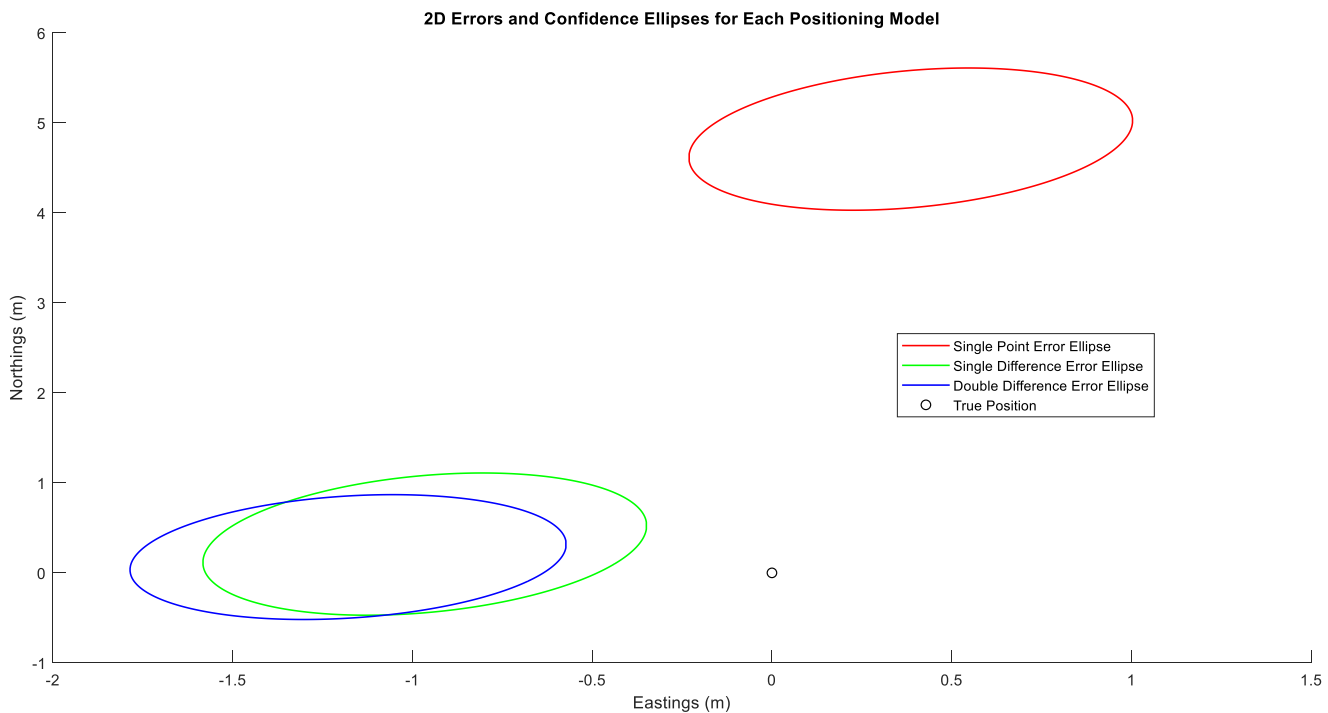

Figure 22: Horizontal 95 Percent Confidence Ellipses for Simulation Set D 
Table 7: Mean Error Statistics for Set D

\begin{tabular}{|c|c|c|c|c|}
\hline Technique & $\begin{array}{c}\text { 3DRMS Error } \\
(\mathrm{m})\end{array}$ & $\begin{array}{c}\text { Vertical Error } \\
(\mathrm{m})\end{array}$ & $\begin{array}{c}\text { Error Standard } \\
\text { deviation (m) }\end{array}$ & $\begin{array}{c}\text { Average } \\
\text { Ambiguity Error }\end{array}$ \\
\hline $\begin{array}{c}\text { Single Point: } \\
\text { Pseudoranges }\end{array}$ & 33.158 & 32.809 & 0.158 & - \\
\hline $\begin{array}{c}\text { Single Point: Carrier } \\
\text { Phase }\end{array}$ & 33.065 & 32.720 & 0.374 & -20.48 \\
\hline $\begin{array}{c}\text { Single Difference: } \\
\text { Pseudoranges }\end{array}$ & 0.418 & -0.005 & 0.192 & 0.31 \\
\hline $\begin{array}{c}\text { Single Difference: } \\
\text { Carrier Phase }\end{array}$ & 0.264 & -0.013 & 0.464 & - \\
\hline $\begin{array}{c}\text { Double Difference: } \\
\text { Pseudoranges }\end{array}$ & 0.592 & 0.017 & 0.302 & 0.34 \\
\hline $\begin{array}{c}\text { Double Difference: } \\
\text { Carrier Phase }\end{array}$ & 0.626 & -0.006 & 0.649 & \\
\hline
\end{tabular}

It is evident from looking at figures 19 and 20 that single point positioning solutions are strongly affected by the pseudorange biases. This is expected since the model has no way of mitigating residual atmospheric delays. Since the base to rover baseline is only $100 \mathrm{~m}$, the differential solutions do effectively mitigate the pseudorange biases, hence the solutions are far more accurate on average in the vertical and horizontal directions. It is possible that sufficiently optimal satellite geometry would improve the $2 \mathrm{D}$ positioning accuracy for single point solutions, but this would not significantly aid the vertical error, since this would require line of sight to satellites which are obscured by the earth itself. 
Collection D yields results which show use of the carrier phase improving the positioning accuracy with all 3 measurement models. This includes the single point model, which had a significant average error in estimating the integer ambiguity, so this is a good sign that use of carrier phase observables can be helpful in the presence of atmospheric biases.

\subsubsection{Collection E: 5 ppm Bias, $1 \mathrm{~m}$ noise Standard Deviation, $100 \mathrm{~m}$ Differential}

\section{Baseline}
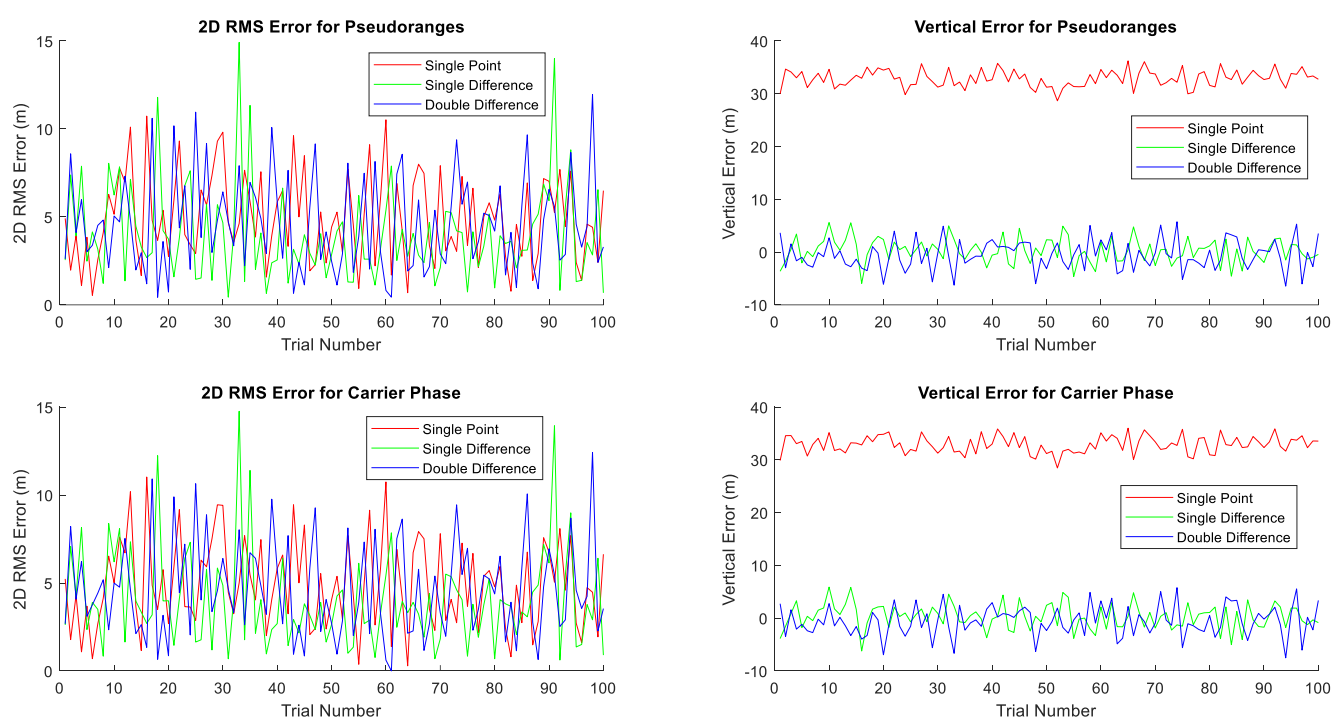

Figure 23: Horizontal and Vertical Errors for Simulation Set E 


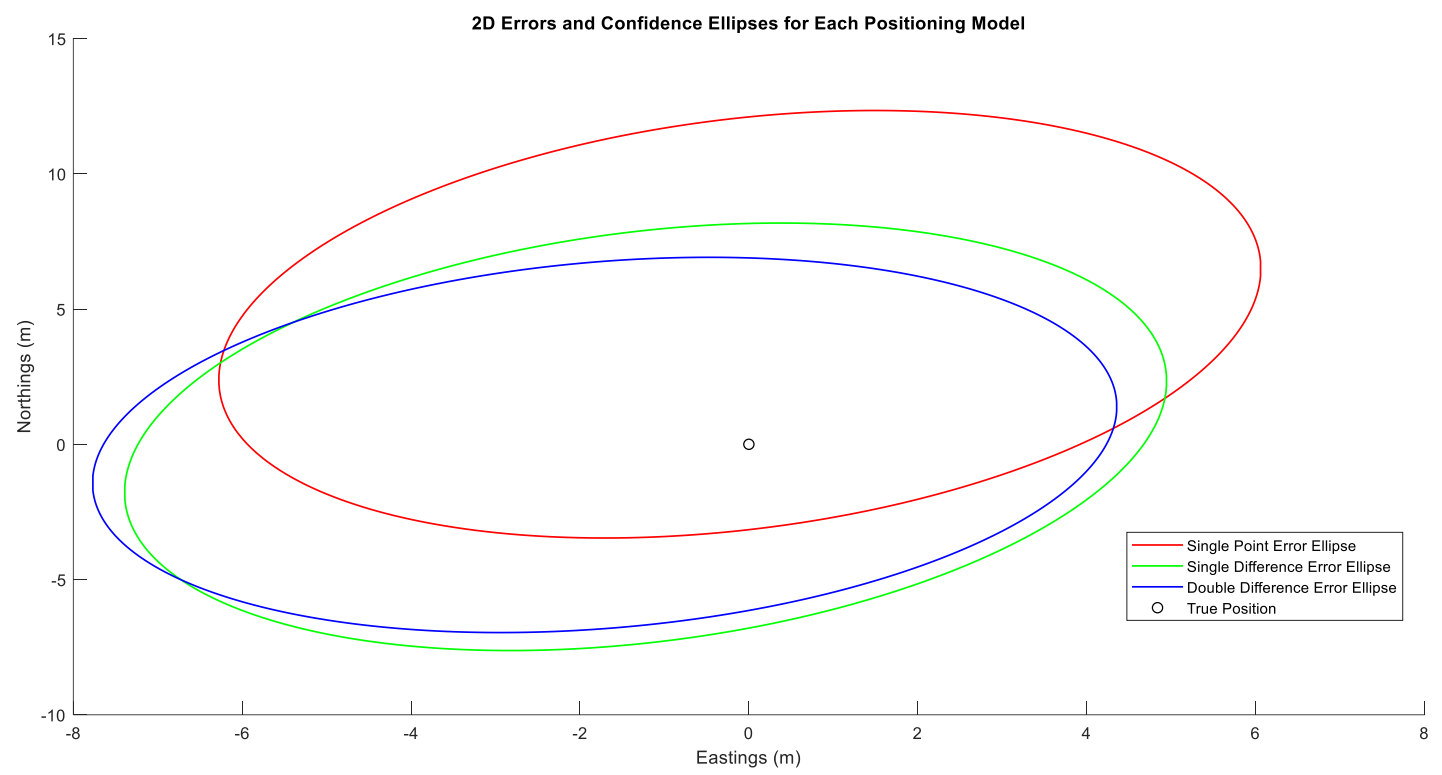

Figure 24: Horizontal 95 Percent Confidence Ellipses for Simulation Set E

Collection E yields results which do not support the use of carrier phase measurements in this scenario. The only method which yielded a better global error statistic using the carrier phase was the double difference solution, which had a slightly better error standard deviation. Even with that method, though, Table 8 shows that the other error statistics did not benefit from use of the carrier phase. It is possible that expanding the search region would improve this, but using this integer combination search, any extra integers would increase processing time exponentially. 
Table 8: Mean Error Statistics for Set E

\begin{tabular}{|l|c|c|c|c|}
\hline \multicolumn{1}{|c|}{ Technique } & $\begin{array}{c}\text { 3DRMS } \\
\text { Error (m) }\end{array}$ & $\begin{array}{c}\text { Vertical Error } \\
(\mathrm{m})\end{array}$ & $\begin{array}{r}\text { Standard } \\
\text { Deviation (m) }\end{array}$ & $\begin{array}{c}\text { Average } \\
\text { Ambiguity Error }\end{array}$ \\
\hline $\begin{array}{l}\text { Single Point: } \\
\text { Pseudoranges }\end{array}$ & 33.220 & 32.760 & 1.790 & -19.76 \\
\hline $\begin{array}{l}\text { Single Point: } \\
\text { Carrier Phase }\end{array}$ & 33.262 & 32.800 & 1.858 & - \\
\hline $\begin{array}{l}\text { Single Difference: } \\
\text { Pseudoranges }\end{array}$ & 4.628 & -0.318 & 2.521 & -2.05 \\
\hline $\begin{array}{l}\text { Single Difference: } \\
\text { Carrier Phase }\end{array}$ & 4.656 & -.273 & 2.529 & - \\
\hline $\begin{array}{l}\text { Double Difference: } \\
\text { Pseudoranges }\end{array}$ & 5.549 & 0.118 & 2.902 & 1.32 \\
\hline $\begin{array}{l}\text { Double Difference: } \\
\text { Carrier Phase }\end{array}$ & 5.593 & 0.156 & & 2.917 \\
\hline
\end{tabular}




\subsubsection{Collection F: 5 ppm Bias, 3 m Noise Standard Deviation, $100 \mathrm{~m}$ Differential}

\section{Baseline}
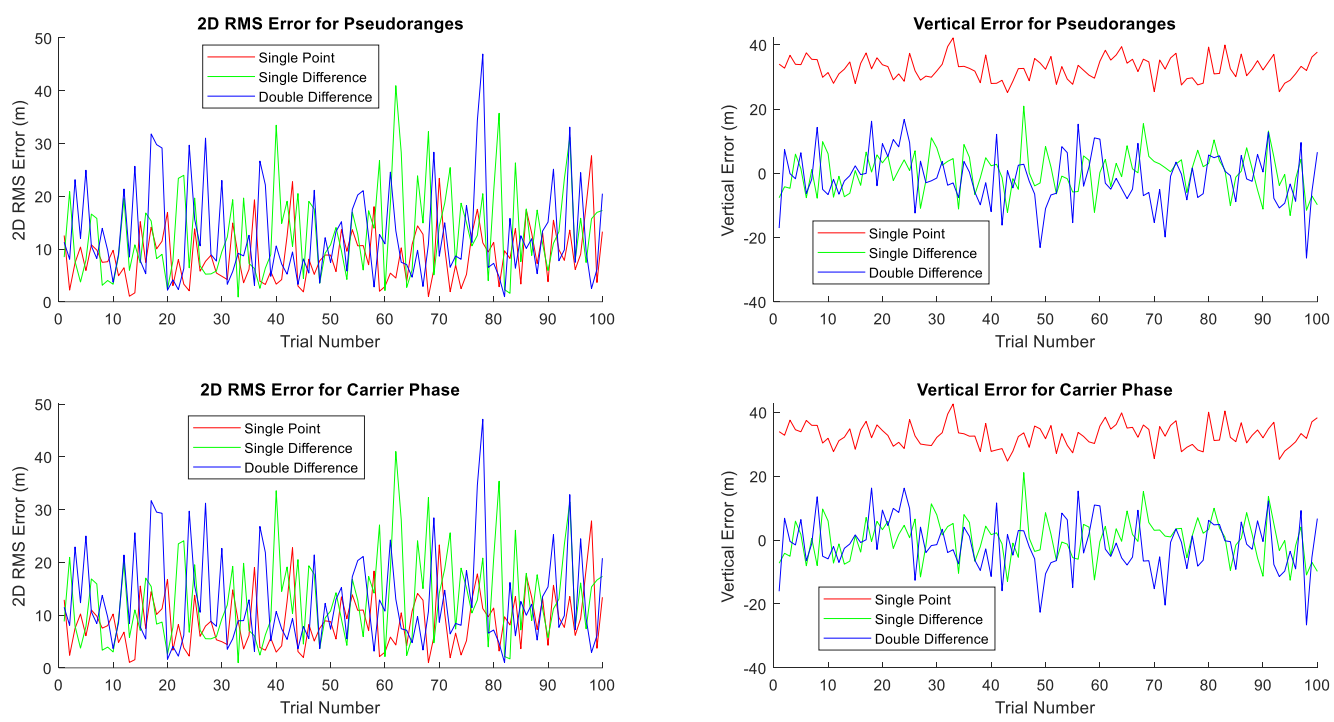

Figure 25: Horizontal and Vertical Errors for Simulation Set F

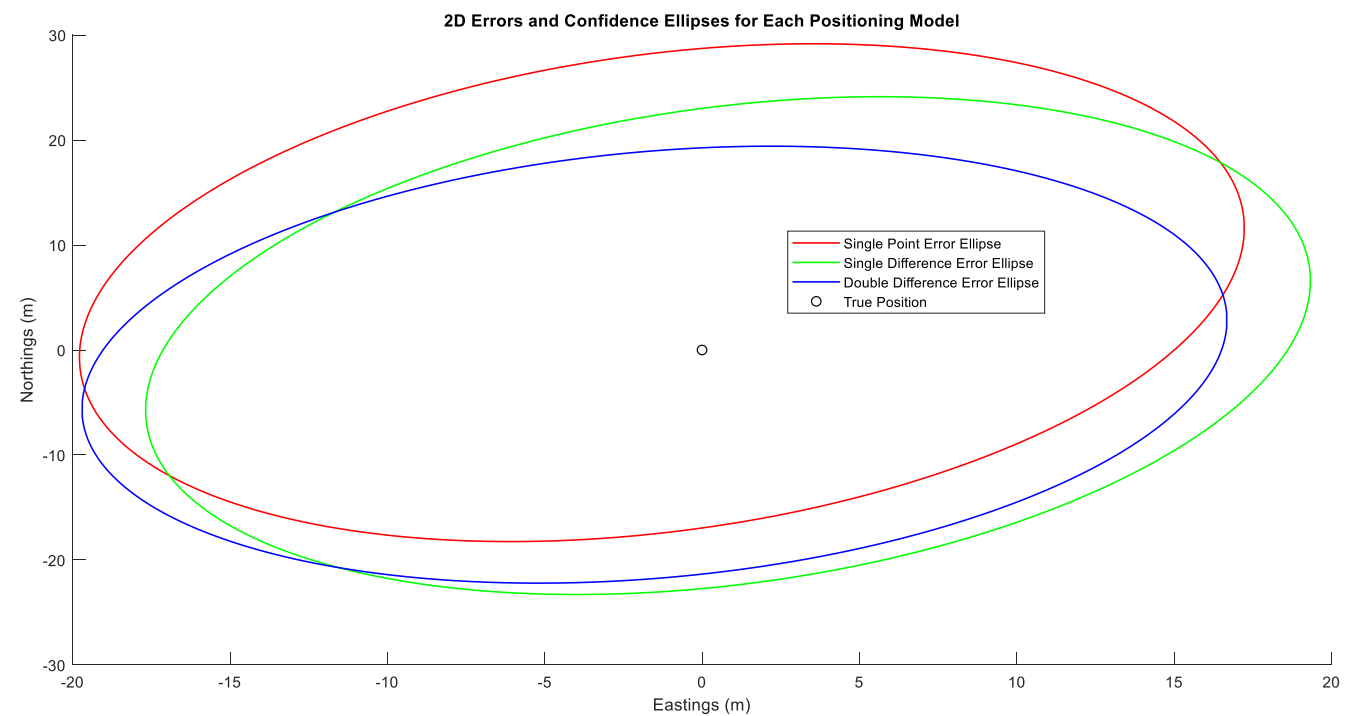

Figure 26: Horizontal 95 Percent Confidence Ellipses for Simulation Set F 
Table 9: Mean Error Statistics for Set F

\begin{tabular}{|c|c|c|c|c|}
\hline Technique & $\begin{array}{c}\text { 3DRMS Error } \\
(\mathrm{m})\end{array}$ & $\begin{array}{c}\text { Vertical Error } \\
(\mathrm{m})\end{array}$ & $\begin{array}{c}\text { Standard } \\
\text { Deviation }(\mathrm{m})\end{array}$ & $\begin{array}{c}\text { Average } \\
\text { Ambiguity Error }\end{array}$ \\
\hline $\begin{array}{c}\text { Single Point: } \\
\text { Pseudoranges }\end{array}$ & 34.438 & 32.662 & 4.708 & - \\
\hline $\begin{array}{c}\text { Single Point: Carrier } \\
\text { Phase }\end{array}$ & 34.381 & 32.610 & 4.792 & -20.74 \\
\hline $\begin{array}{c}\text { Single Difference: } \\
\text { Pseudoranges }\end{array}$ & 14.538 & -0.692 & 8.267 & - \\
\hline $\begin{array}{c}\text { Single Difference: } \\
\text { Carrier Phase }\end{array}$ & 14.550 & -0.626 & 8.229 & -3.62 \\
\hline $\begin{array}{c}\text { Double Difference: } \\
\text { Pseudoranges }\end{array}$ & 16.028 & 0.488 & 8.754 & 1.50 \\
\hline $\begin{array}{c}\text { Double Difference: } \\
\text { Carrier Phase }\end{array}$ & 16.024 & 0.431 & 8.798 & \\
\hline
\end{tabular}

Interestingly, Collection F yields results which reduced the mean error overall as well as the vertical error for every measurement model when the carrier phase is used, which is a stark contrast to the data from Collection E. This may suggest that the success rate for selection of optimal ambiguities depends on the geometry of the estimated pseudorange solution relative to both the true position and the satellites broadcasting measurements.

Since differential measurement models are being used here, it would also be interesting to examine results when the rover-base baseline distance is varied. 


\subsubsection{Collection G: 5 ppm Bias, 1 m Noise Standard Deviation, 1 km Differential}

\section{Baseline}
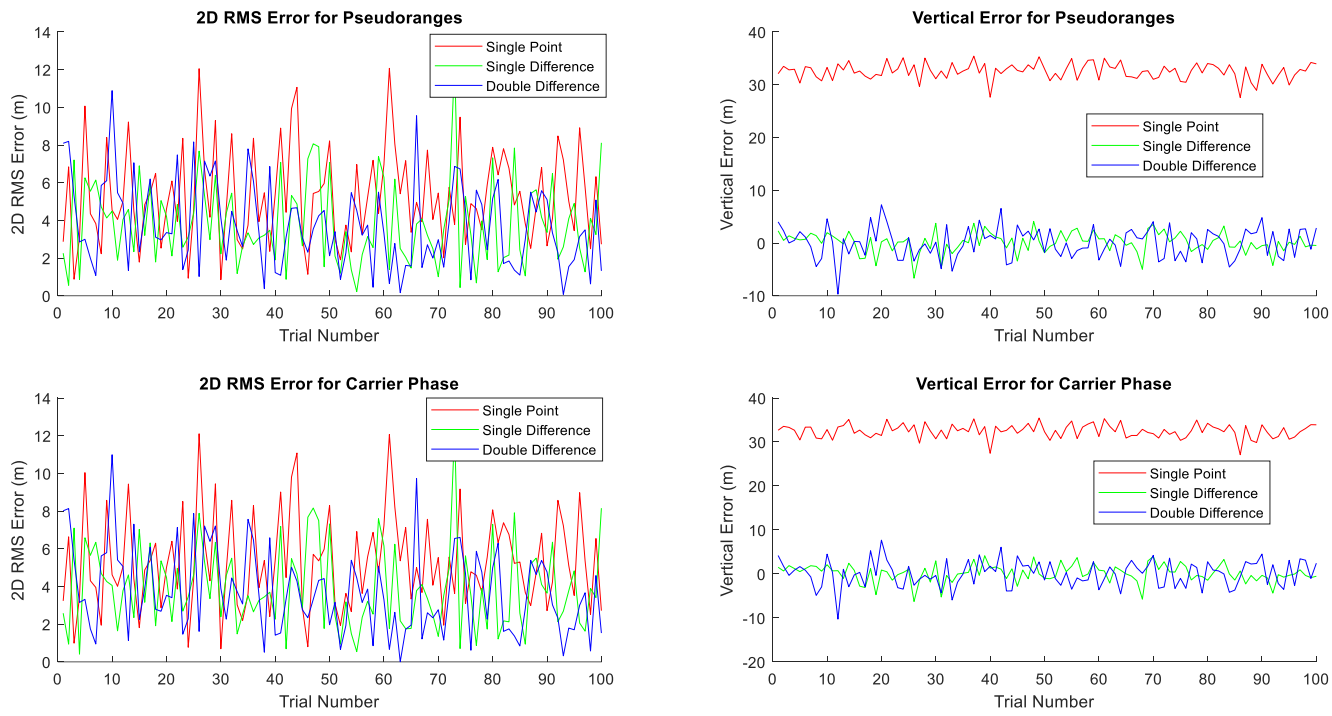

Figure 27: Horizontal and Vertical Errors for Simulation Set F

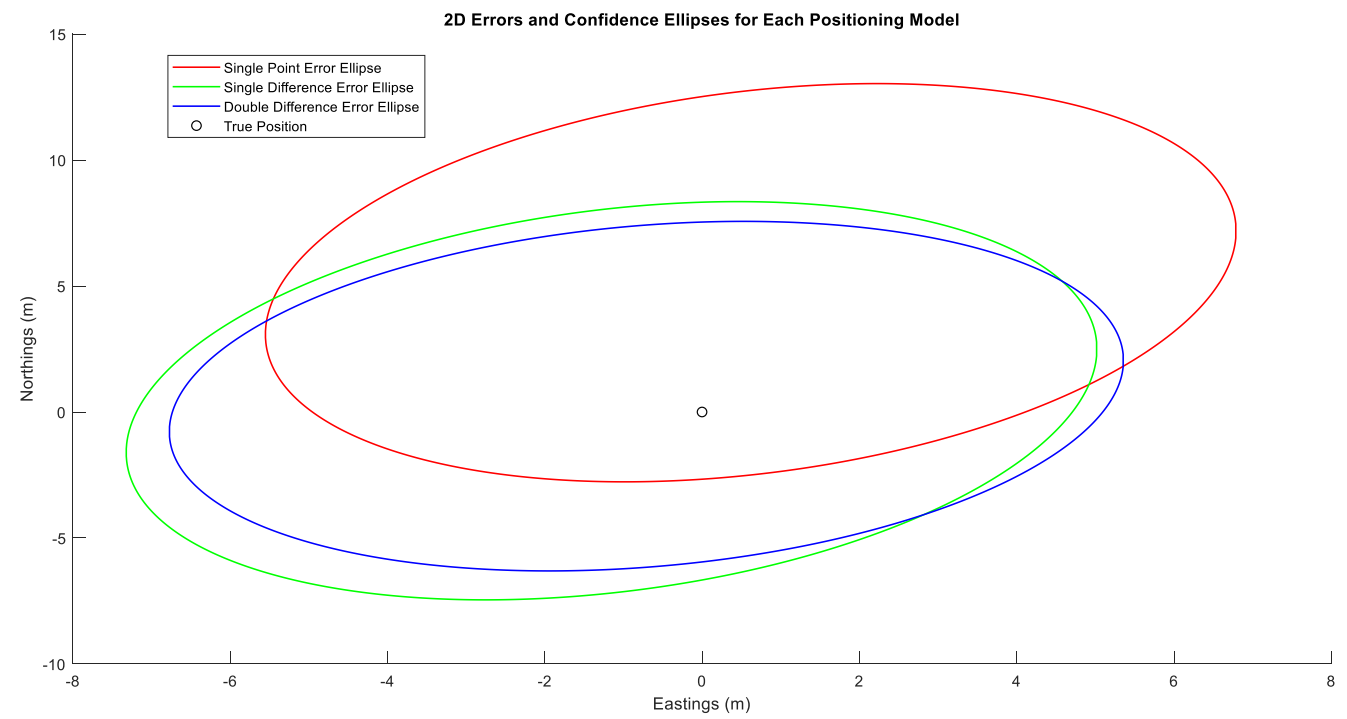

Figure 28: Horizontal 95 Percent Confidence Ellipses for Simulation Set G 
Table 10: Mean Error Statistics for Set G

\begin{tabular}{|c|c|c|c|c|}
\hline Technique & $\begin{array}{c}\text { 3DRMS Error } \\
(\mathrm{m})\end{array}$ & $\begin{array}{c}\text { Vertical Error } \\
(\mathrm{m})\end{array}$ & $\begin{array}{c}\text { Standard } \\
\text { Deviation }(\mathrm{m})\end{array}$ & $\begin{array}{c}\text { Average } \\
\text { Ambiguity Error }\end{array}$ \\
\hline $\begin{array}{c}\text { Single Point: } \\
\text { Pseudoranges }\end{array}$ & 32.220 & 32.760 & 1.790 & - \\
\hline $\begin{array}{c}\text { Single Point: Carrier } \\
\text { Phase }\end{array}$ & 32.262 & 32.799 & 1.858 & -19.76 \\
\hline $\begin{array}{c}\text { Single Difference: } \\
\text { Pseudoranges }\end{array}$ & 4.283 & -0.016 & 2.184 & - \\
\hline $\begin{array}{c}\text { Single Difference: } \\
\text { Carrier Phase }\end{array}$ & 4.345 & 0.055 & 2.185 & 2.52 \\
\hline $\begin{array}{c}\text { Double Difference: } \\
\text { Pseudoranges }\end{array}$ & 5.580 & 0.075 & 2.833 & - \\
\hline $\begin{array}{c}\text { Double Difference: } \\
\text { Carrier Phase }\end{array}$ & 5.622 & 0.083 & 2.185 & 1.03 \\
\hline
\end{tabular}

Collection $\mathrm{G}$ is yielding very similar results to those of Collection E, especially in the case of the single point solutions. This is expected, since the rover is simply shifted from its position in Collection E. This does, however, increase the error statistics for the differential solutions, particularly in the horizontal direction. This is because the separation of the baselines reduces the ability of the model to mitigate atmospheric errors and is reflected in the RMS error statistics in Table 10 .

It is important to note that the altitudes in both sets are assumed to be identical, which is potentially more ideal for the differential models depending on the satellite geometry, which 
could be reducing the effect of increasing the baseline. A set of data will also be produced varying the altitude difference between the rover and base antennas.

\subsubsection{Collection H: 5 ppm Bias, $1 \mathrm{~m}$ Noise Standard Deviation, $1 \mathrm{~km}$ Differential Baseline With 5m Vertical Offset}
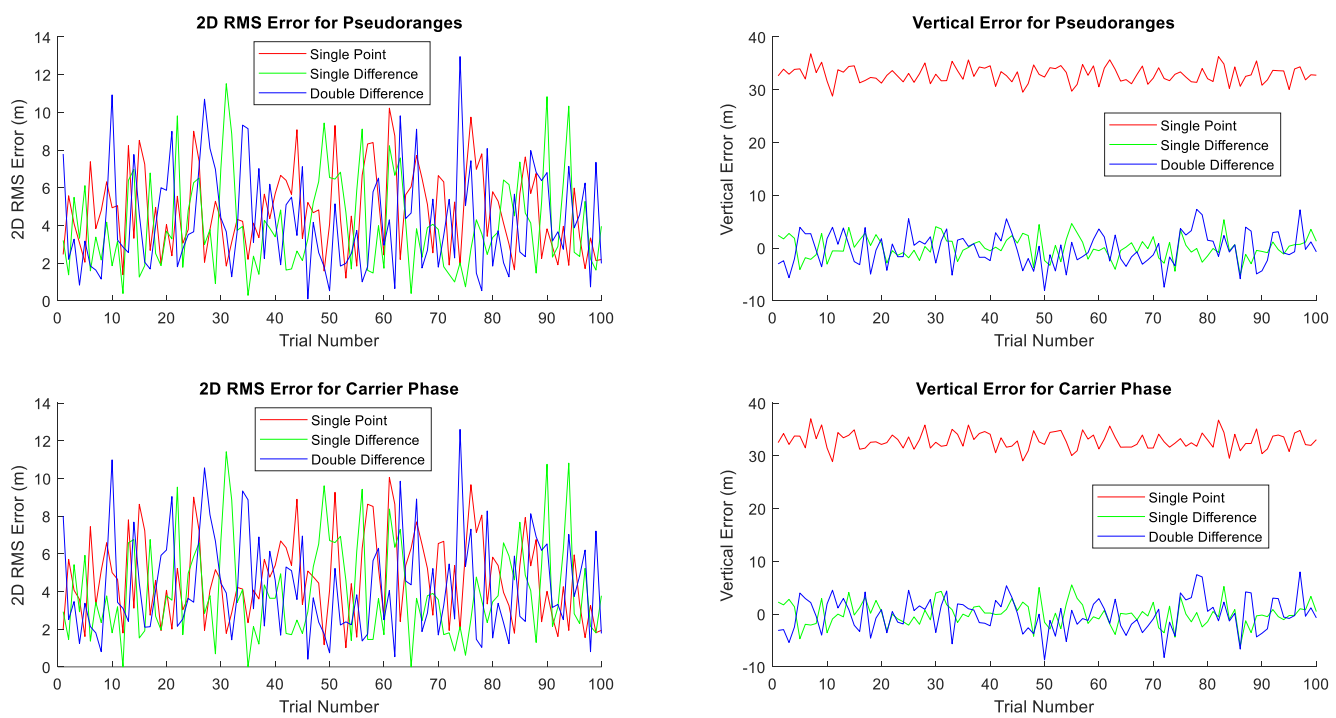

Figure 29: Horizontal and Vertical Errors for Simulation Set H 


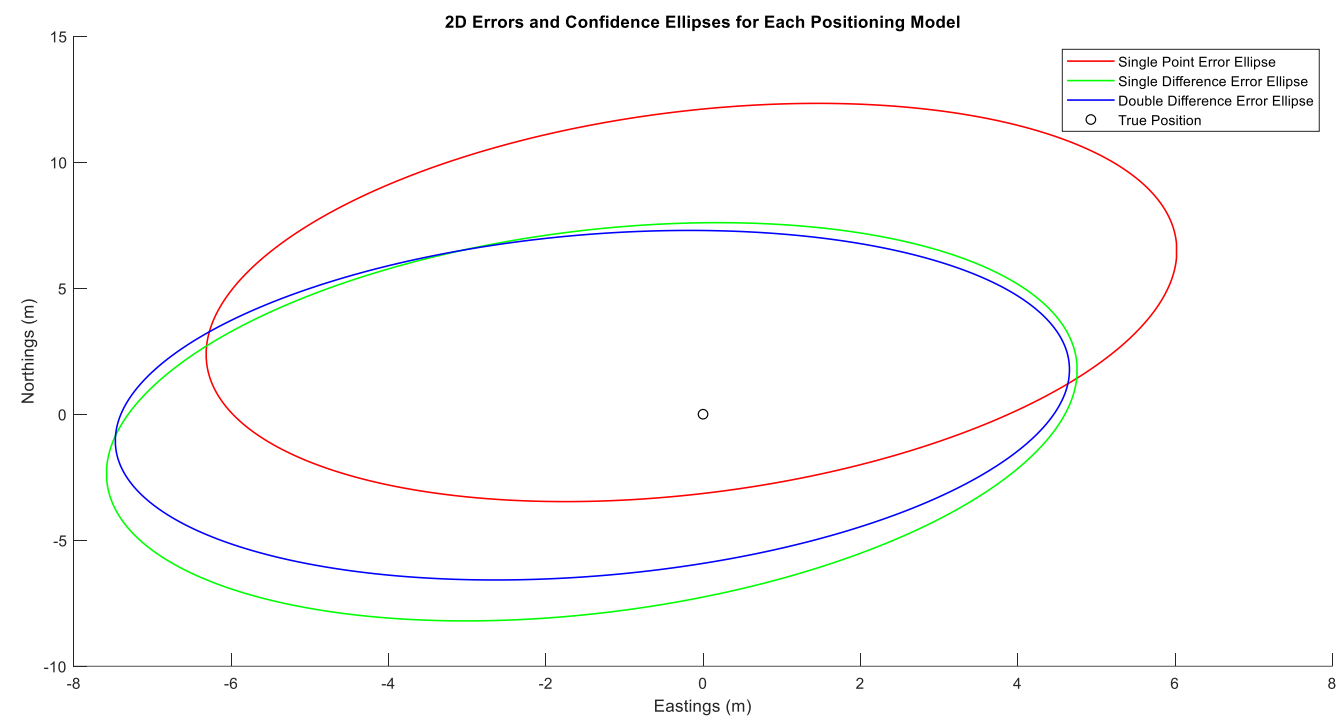

Figure 30: Horizontal 95 Percent Confidence Ellipses for Simulation Set H

Table 11: Mean Error Statistics for Set H

\begin{tabular}{|c|c|c|c|c|}
\hline Technique & $\begin{array}{c}\text { 3DRMS Error } \\
(\mathrm{m})\end{array}$ & $\begin{array}{c}\text { Vertical Error } \\
(\mathrm{m})\end{array}$ & $\begin{array}{c}\text { Error Standard } \\
\text { deviation }(\mathrm{m})\end{array}$ & $\begin{array}{c}\text { Average } \\
\text { Ambiguity Error }\end{array}$ \\
\hline $\begin{array}{c}\text { Single Point: } \\
\text { Pseudoranges }\end{array}$ & 33.450 & 32.954 & 1.824 & - \\
\hline $\begin{array}{c}\text { Single Point: Carrier } \\
\text { Phase }\end{array}$ & 33.476 & 32.977 & 1.897 & -19.71 \\
\hline $\begin{array}{c}\text { Single Difference: } \\
\text { Pseudoranges }\end{array}$ & 4.682 & 0.189 & 2.440 & 3.56 \\
\hline $\begin{array}{c}\text { Single Difference: } \\
\text { Carrier Phase }\end{array}$ & 4.749 & 0.191 & 2.514 & - \\
\hline $\begin{array}{c}\text { Double Difference: } \\
\text { Pseudoranges }\end{array}$ & 4.845 & 0.088 & 2.579 & -0.02 \\
\hline $\begin{array}{c}\text { Double Difference: } \\
\text { Carrier Phase }\end{array}$ & 4.841 & 0.097 & 2.597 & \\
\hline
\end{tabular}

From Table 11, it is apparent that the impact of the carrier phase on datasets with set $\mathrm{E}$ biases and noise was not strongly affected by the ground geometry of the receivers. Despite $1 \mathrm{~km}$ 
of separation as well as $5 \mathrm{~m}$ of vertical offset, no processing method had any significant benefit using the epoch-by-epoch integer ambiguity carrier phase measurements.

It is interesting to note that the double difference was more effective in mitigating the effect of the vertical offset on the vertical error than the single difference was.

\subsubsection{Positioning Simulation Summary}

When observing the results from simulation collections A through $\mathrm{H}$, some general patterns are evident. First, the pseudorange noise effect is proportional to the positioning error standard deviation. From the measurement model and from error propagation, this is expected. It is also reflected in the size of the confidence ellipse produced under different noise conditions. Larger confidence ellipses indicate more noise in the positioning solution.

Second, the amount of atmospheric bias added to the range to generate the pseudorange is proportional to the positioning bias, especially in the single point measurement model. This is expected, because the single point model has no inherent mechanism to isolate unmitigated atmospheric bias, while differential models do so with the differencing between base and rover ranges.

Another pattern is that the baseline distance between base and rover antennas is proportional to the positioning bias (especially in the vertical direction). This is also expected, because as the baseline distance increases, so does the difference between the base-to-satellite range versus the rover-to-satellite range. Hence, the bias is not cancelled as effectively by the differencing model when the baseline distance is large. 


\subsection{Didsbury Control Marker Survey}

To validate the single point positioning algorithms for pseudorange-only as well as carrier phase solutions, an Alberta Survey Control Marker (ASCM) was located in Didsbury Alberta which has the following characteristics:

Table 12: ASCM 764522 Information

\begin{tabular}{|c|c|}
\hline Latitude (DMS) & $52^{\circ} 40^{\prime} 02.81212^{\prime \prime}$ North \\
\hline Longitude (DMS) & $114^{\circ} 08^{\prime} 35.55293^{\prime \prime}$ West \\
\hline Elevation (m) & 1041.004 \\
\hline Type & ASC cap on $5 \mathrm{~cm}$ square heavy wall steel pipe \\
\hline
\end{tabular}

The cap in Table 12 is situated in a relatively ideal location outside of the rural town, minimizing the possibility of multipath effects:

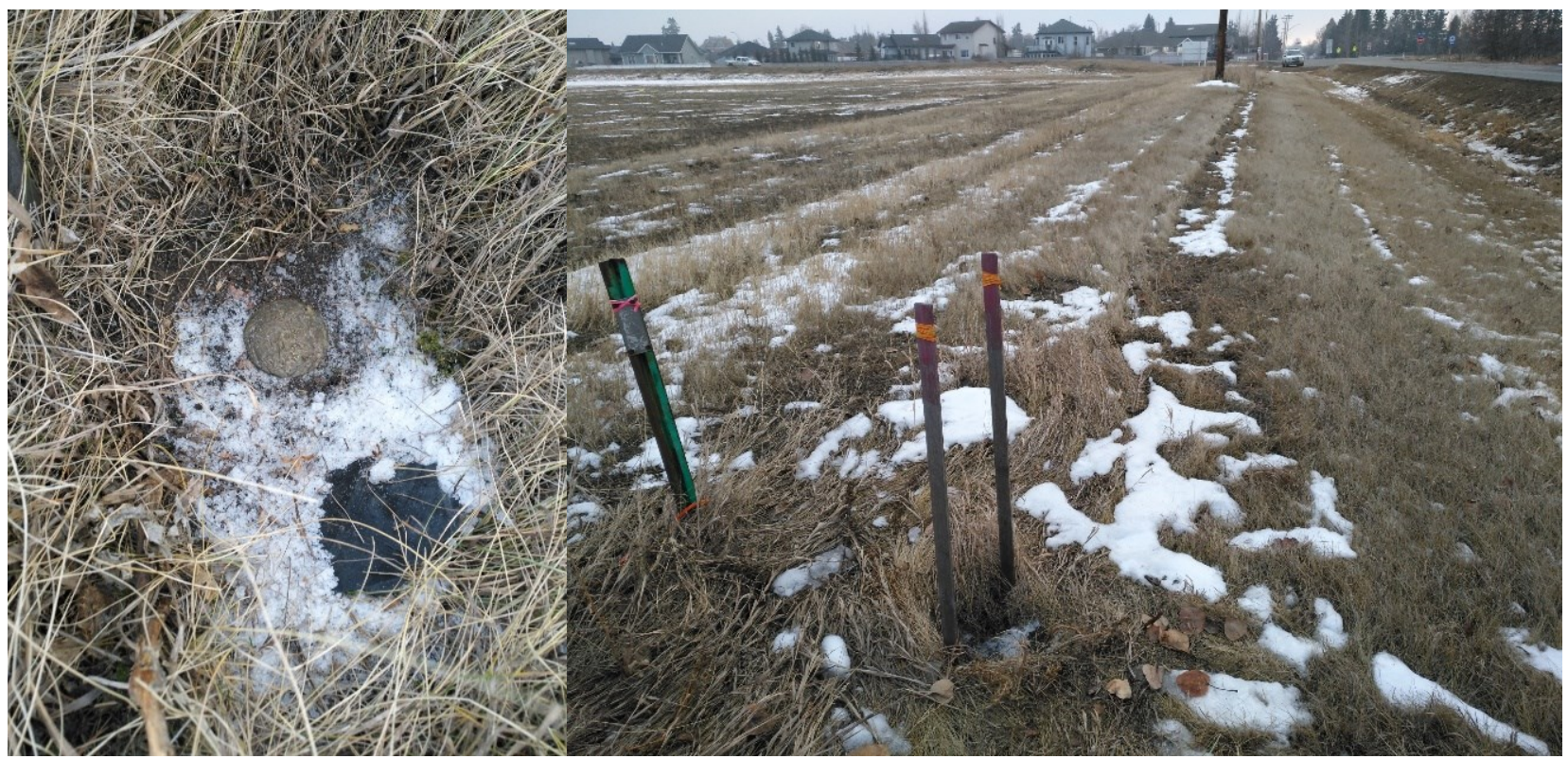

Figure 31:Location of ASCM 764522 Outside of Didsbury, Alberta. Brass Cap (Left) With Precise Coordinates Used for Antenna Placement. Conditions are Ideal for Positioning in Open Sky (Right).

As can be observed from Figure 30, the location of the ASCM has minimal multipath potential. The iron post east of the cap and the wooden stakes may cause some minimal 
multipath effects, but it is a superior setup to an urban setting when considering multipath and access to optimal satellite geometry.

In order to conduct an experimental survey of the marker, test receiver 1 was utilized. The antenna was centered on top of the cap as accurately as possible and left in a static position to log data via the Reachview Android application.

Data was logged in the RINEX format and processed using the $\mathrm{C}++$ software developed for the purposes of this research, with and without the use of the L1 carrier phase observable. The same data was also processed using RTKPost software using the Saastamoinen troposphere model and the broadcast ionosphere model for comparison. Results are as follows:
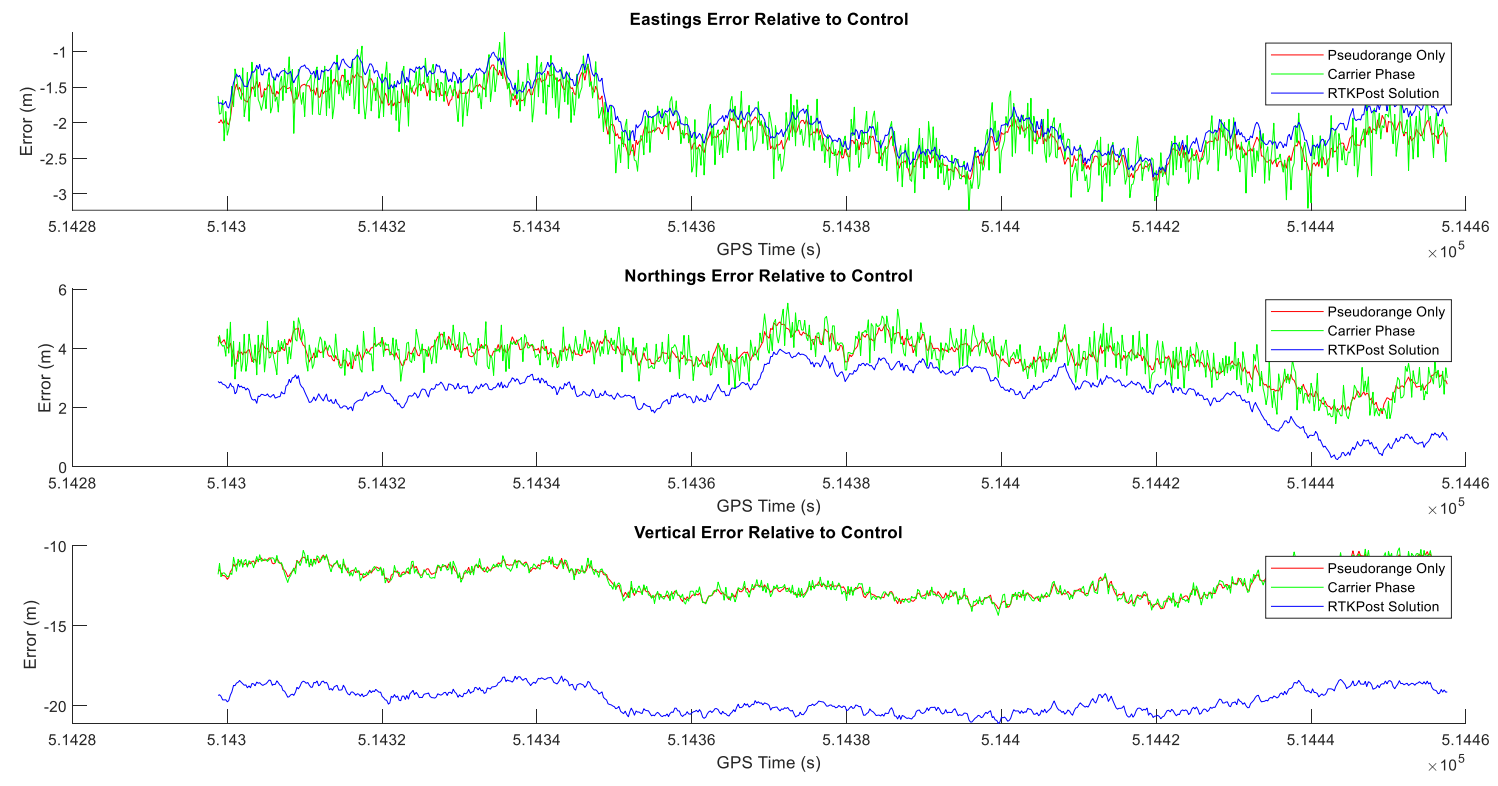

Figure 32: True Directional Error for Processed Rural ASCM Survey Data When Compared to Archived ASC Coordinates 
Table 13: Mean Error Statistics for Rural ASCM Survey Data Processing For Each Processing Method

\begin{tabular}{|c|c|c|c|}
\hline Processing Model & 3DRMS Error $(\mathrm{m})$ & Vertical Error $(\mathrm{m})$ & Standard Deviation $(\mathrm{m})$ \\
\hline Pseudoranges & 12.907 & -12.174 & 0.991 \\
\hline Carrier Phase & 12.921 & -12.176 & 1.011 \\
\hline RTKPost Software Solution & 19.898 & -19.635 & 0.833 \\
\hline
\end{tabular}

There are several interesting trends which immediately differentiate this real processed data from the processed simulated data. One such trend is that there is clear temporal variability in the error bias which was not observed in the simulations. One major reason for this is that satellite geometry was kept constant in simulations in order to isolate white noise effects while the satellite geometry changes over time in real data, illustrated in Figure 32:

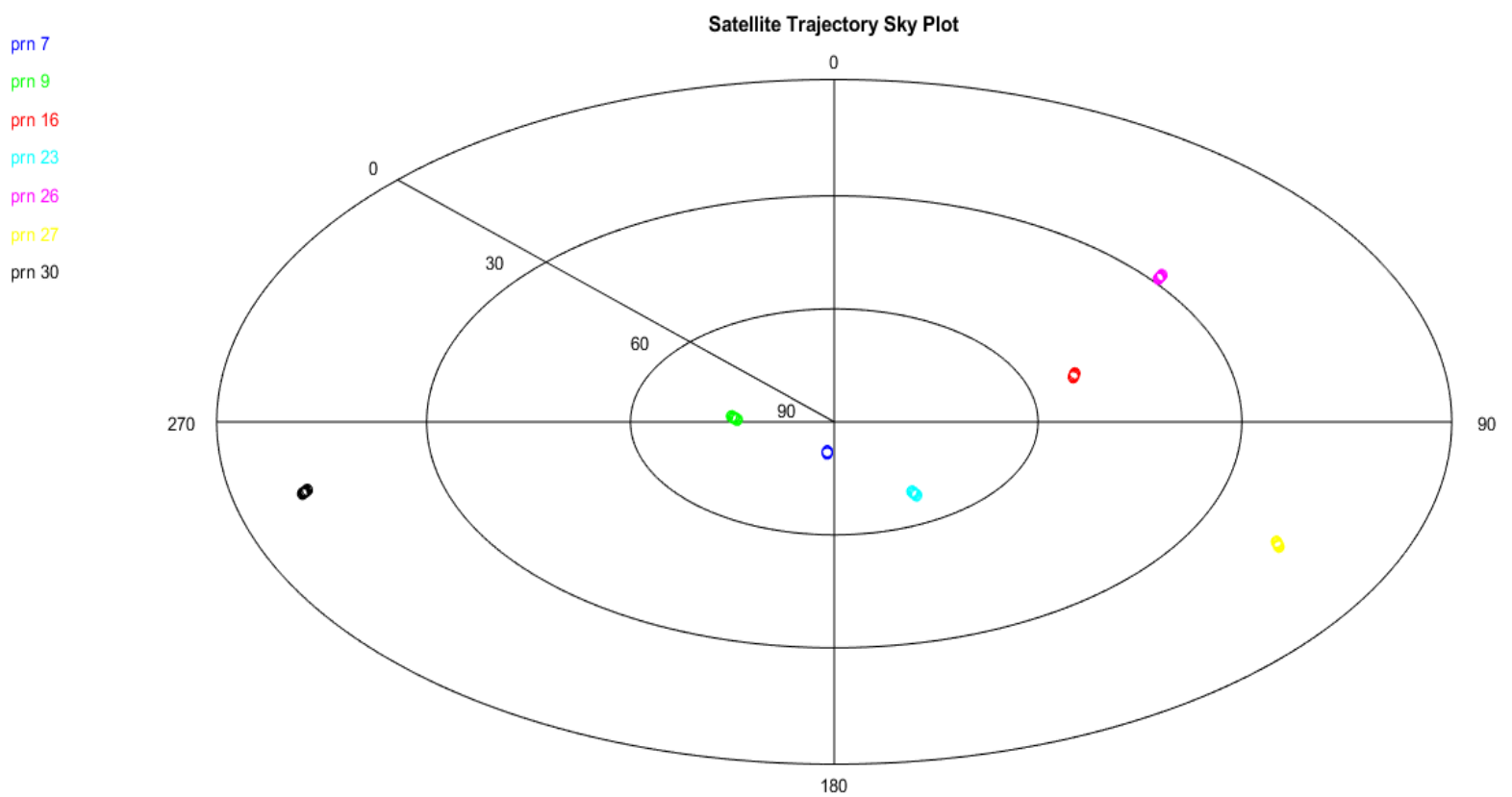

Figure 33: Skyplot for Visualizing the Satellite Headings and Elevation Angles Relative to the Antenna Over the Data Collection Period for Didsbury ASCM Survey 
In fact, the effect of changing satellite geometry is clearly evident in the overall trend curves in all directions in Figure 32. This makes it very difficult to directly measure the effect of white noise in this data. The standard deviations computed in Table 13 cannot be directly interpreted as the standard deviation of the white noise in the solution. In the absence of more information, this forces the user to rely only on the dilution of precision values from propagating an assumed pseudorange noise through the least squares solution. The standard deviation of the error in Table 13 suggests that test receiver 1's noise is consistent with a pseudorange noise of between $10 \mathrm{~cm}$ and $1 \mathrm{~m}$.

\subsection{Carleton University Control Point Survey}

In order to assess test receiver 3's positioning capabilities, raw RINEX data was collected with the rear antenna placed on top of a control point in Ottawa, Ontario which was surveyed in aggregate by the Carleton University Civil Engineering Department. Computed GPS positions from raw data were compared with coordinates provided by the Civil Engineering Department which were quoted as having a maximum estimated $6 \mathrm{~cm}$ of 3DRMS error.

Table 14: Properties of Local Control Point ID 2003 From Civil Engineering Local Carleton Campus Survey

\begin{tabular}{|c|c|}
\hline Latitude (DMS) & $45^{\circ} 23^{\prime} 02.55181^{\prime \prime}$ North \\
\hline Longitude (DMS) & $75^{\circ} 41^{\prime} 53.11418^{\prime \prime}$ West \\
\hline Elevation (m) & 64.393 \\
\hline Type & Bronze cap embedded in walking path \\
\hline
\end{tabular}




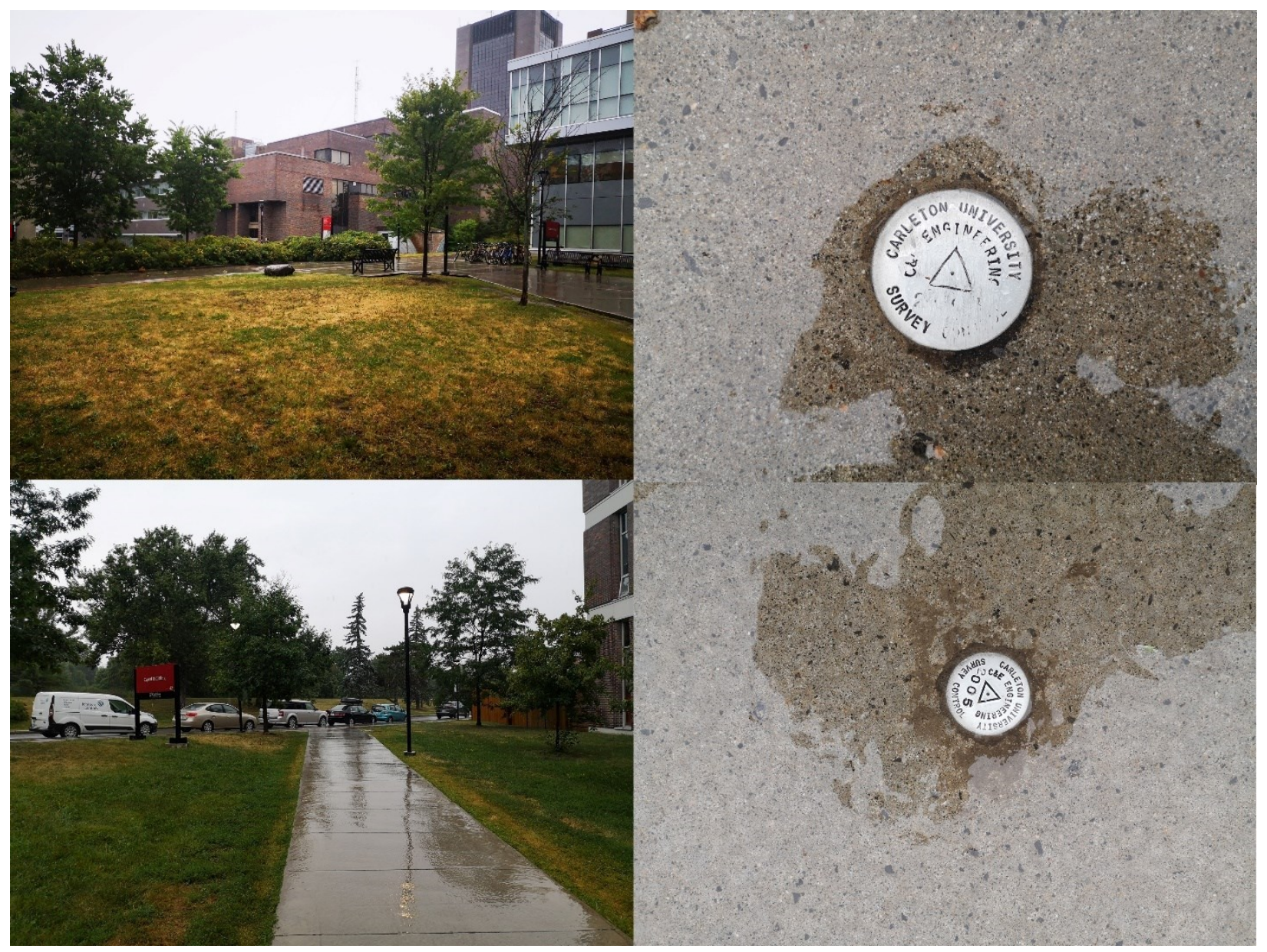

Figure 34: Attitude Survey Control Point Locations. Rear Antenna Environment (Top Left), Rear Antenna Control Point in Concrete (Top Right), Front Antenna Environment (Bottom Left), Front Antenna Control Point in Concrete (Bottom Right)

Figure 34 shows the location of the control point on which this experimental survey was conducted. The location of this control point has significantly greater multipath potential than the control point used in the rural Didsbury survey. There are tall permanent structures and numerous reflective surfaces nearby such as windows and smooth metal surfaces, which have a high potential for multipath, especially when paired with a wide correlator receiver, which is one attribute of test receiver 3 .

In order to conduct a survey of the control point, test receiver 3 was used, which is an integrated system with an IMU. The rear antenna/receiver was used un conjunction with a round 
steel plate to act as a ground plane. The antenna was centered on top of the cap as accurately as possible and left in a static position to log raw data via the VectorNav Control Center application.

Data was logged in a RINEX format and processed using the $\mathrm{C}++$ software developed for the purposes of this research, with the pseudorange-only single point model. The same data was also processed with the VN-300 real time filtered result for comparison. Results are as follows:
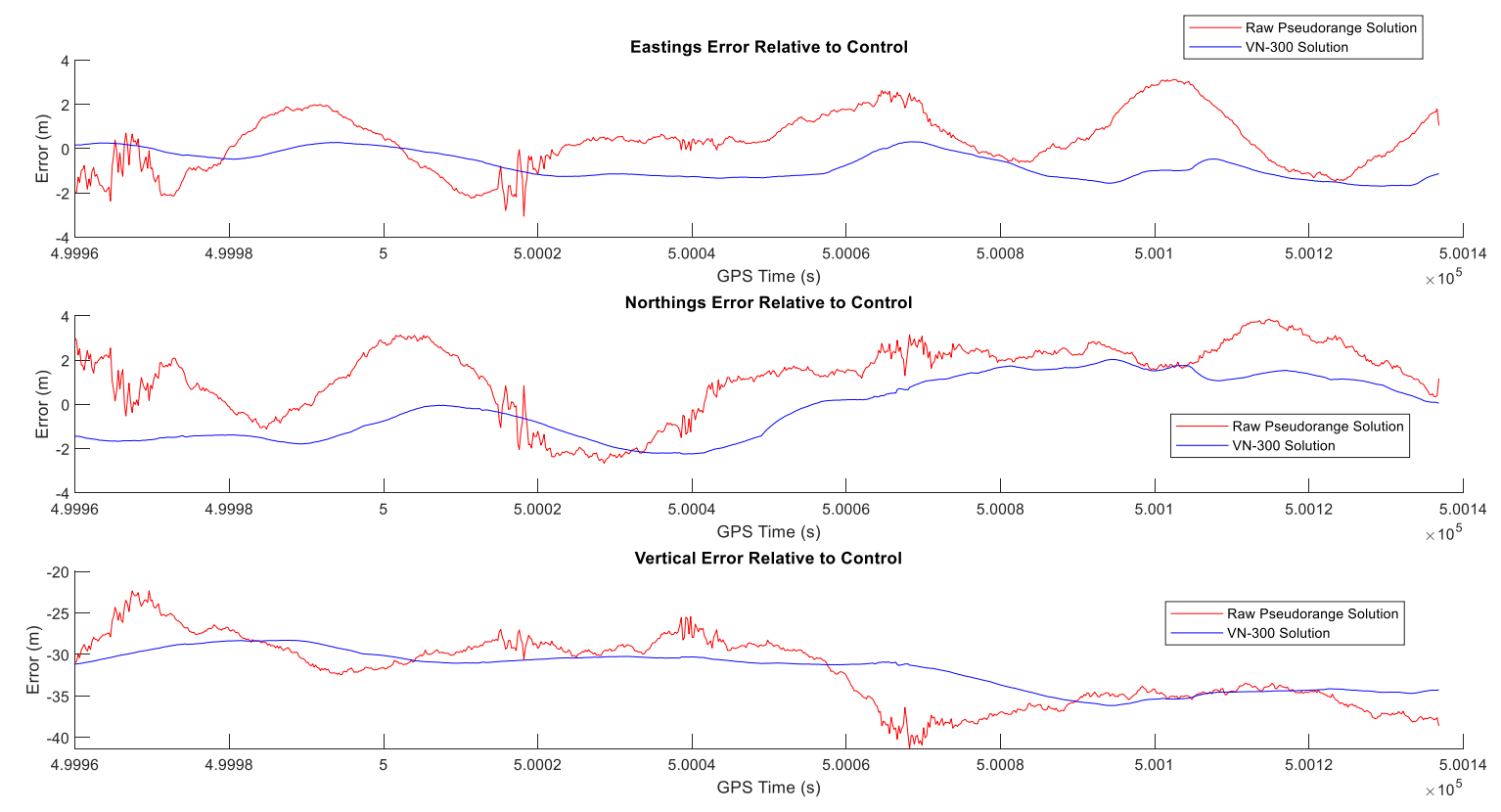

Figure 35 True Directional Error for Processed Carleton Campus Control Point Survey Data When Compared to Civil Engineering Department Control Coordinates

Table 15: Mean Error Statistics for Carleton University Survey Data Processing For Each Processing Method

\begin{tabular}{|c|c|c|c|}
\hline Processing Model & 3DRMS Error $(\mathrm{m})$ & Vertical Error $(\mathrm{m})$ & Standard Deviation $(\mathrm{m})$ \\
\hline $\begin{array}{c}\text { Pseudoranges } \\
\text { (Local Software) }\end{array}$ & 32.096 & -32.007 & 4.053 \\
\hline VN-300 Solution & 31.862 & -31.819 & 2.302 \\
\hline
\end{tabular}


As can be observed in Table 15 and Figure 33, there is much more temporal variability of coordinates computed in the Carleton experimental survey when compared with values in Figure 31 and Table 13. While there does appear to be more noise in these coordinates, it is not clear how much of the standard deviation can be accounted for by temporal variability in biases, especially multipath. The mean vertical error is much greater for the test receiver 3 survey than with the rural Alberta test receiver 1 survey, which suggests that the receiver was subject to more bias in general. Part of this can be accounted for by the lower elevation of the Carleton location, but models are used to mitigate those biases, so multipath is likely playing a significant factor in the error. The satellite geometry was in fact better in this survey compared to the rural survey, as seen in Figure 34:

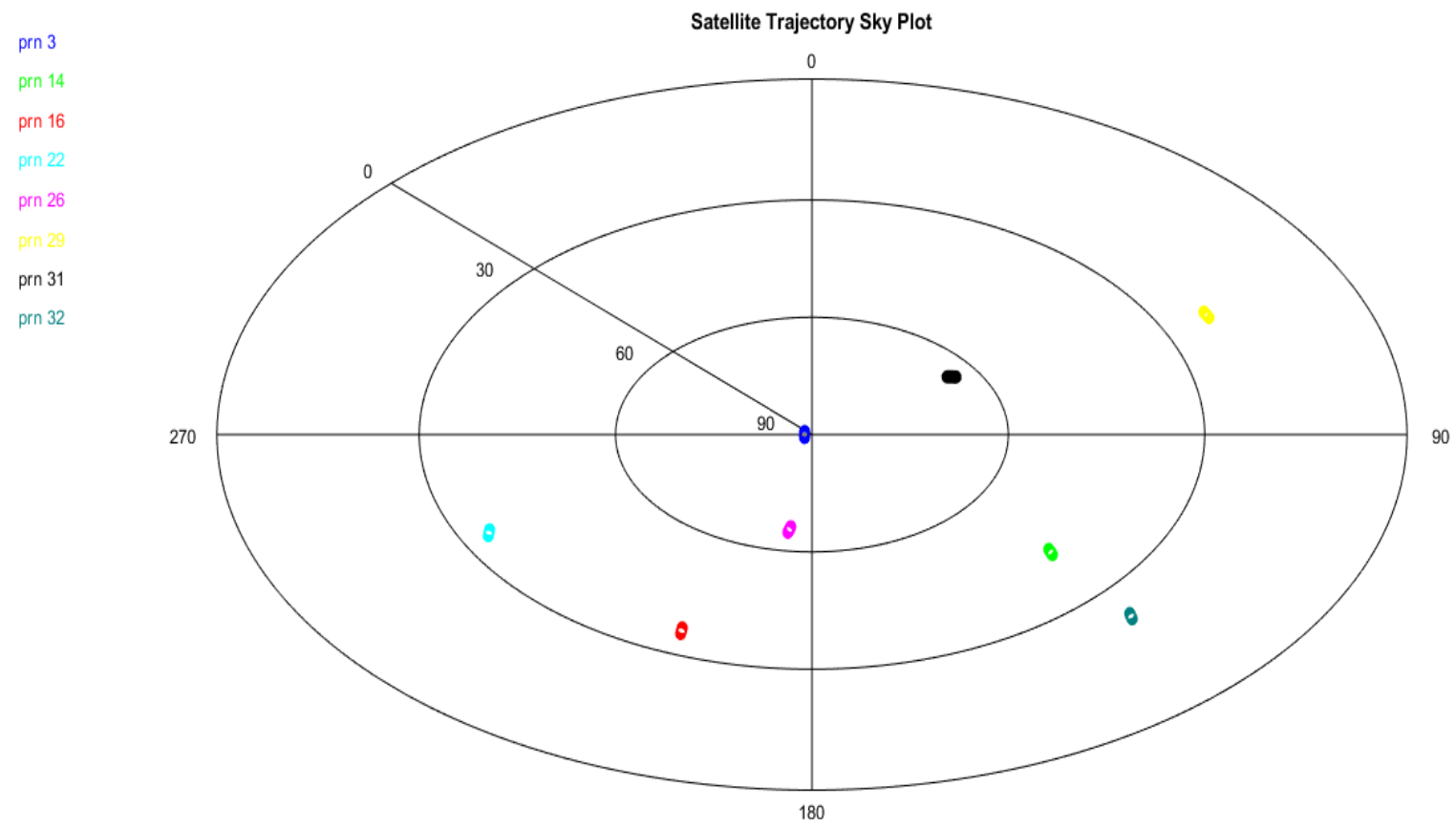

Figure 36: Skyplot for Visualizing the Satellite Headings and Elevation Angles Relative to the Antenna Over the Data Collection Period Carleton University Survey on February $1^{\text {st }}, 2019$ 
While there is an absence of satellite availability northwest of the antenna setup in Figure 34 (likely because they are obstructed by a nearby building), the solution has 1 more available satellite than the rural survey did, which should improve positioning precision. This further supports that the bias is being affected by multipath.

While the error standard deviation in Table 15 is consistent with simulation data with pseudorange noise between $1 \mathrm{~m}$ and $3 \mathrm{~m}$, the noise profile of the receiver is likely on the lower end, closer to $1 \mathrm{~m}$. 


\subsection{Pitch and Heading Simulation}

\subsubsection{Simulation Results}

Using satellite and marker geometry identical to that of the first epoch of the Didsbury ASCM experimental survey, sets of results were simulated in a variety of bias, noise and differential baseline conditions. In addition, an antenna is added in a location with a predetermined offset from the first rover to act as a frontal rover for partial attitude determination. For the pitch and attitude simulations, the base offset from the rear rover is fixed at $10 \mathrm{~km}$ and pseudorange bias is fixed at $3 \mathrm{ppm}$. All sets are found in Appendix A through C, with antenna separation being varied between $1 \mathrm{~m}$ and $90 \mathrm{~m}$, while receiver pseudorange noise being varied between $10 \mathrm{~cm}$ and $3 \mathrm{~m}$ standard deviation. Set A-1 is displayed in this section in order to explain the dataset layouts.

\section{Set A-1: 1 m Dual Antenna Separation}
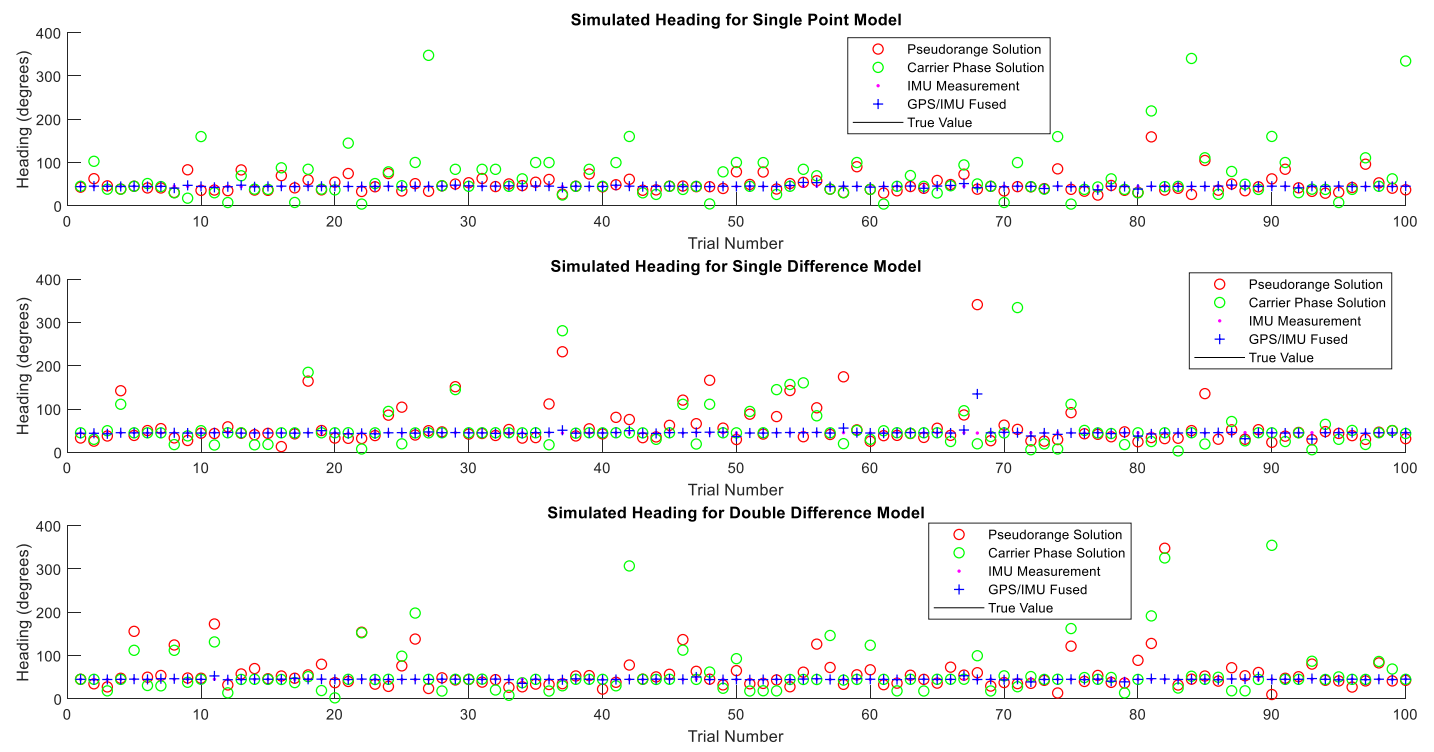

Figure 37: Computed and Measured Headings for Simulation Set A-1 

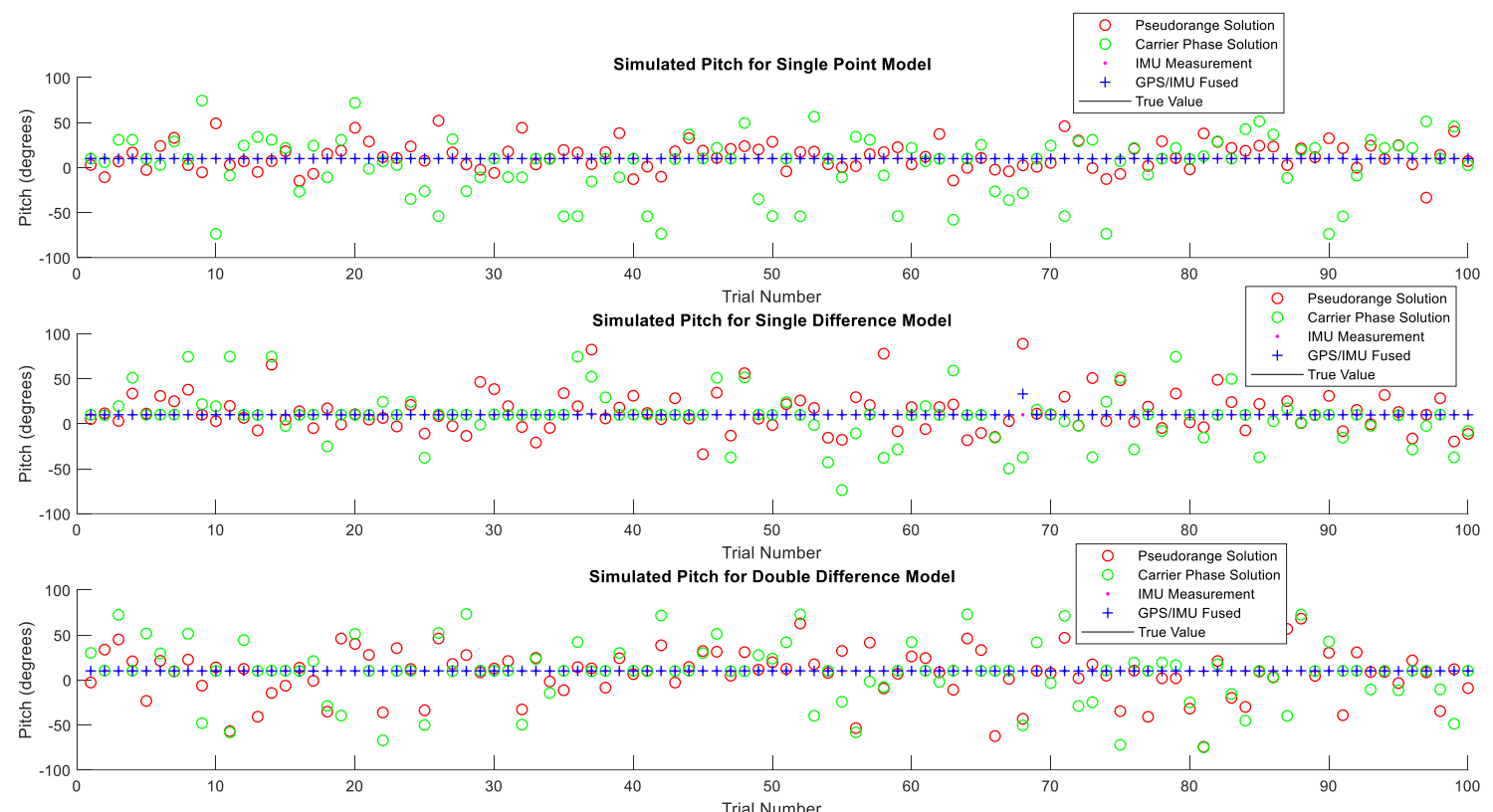

Figure 38: Computed and Measured Pitch for Simulation Set A-1

The first 2 figures presented for each dataset are computed and measured values for pitch and heading for each method and model, over the course of the 100-sample set. It is important to note that the true simulated value of heading is 45 degrees, while the true pitch is 0 degrees. It is also important to note that this does not represent a temporal analysis. The satellite geometry is constant and therefore all variation is due to noise. 


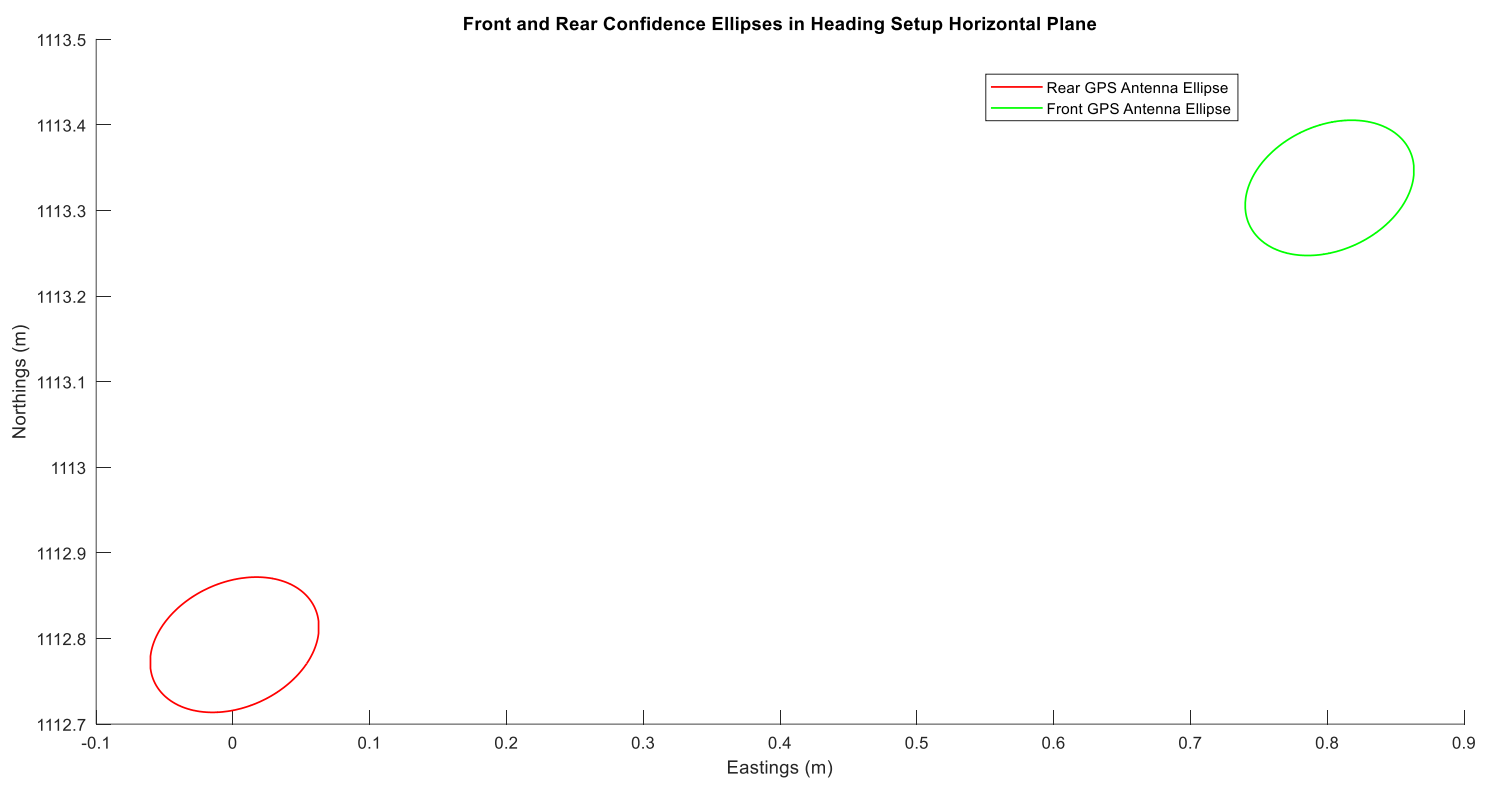

Figure 39: Propagated 95\% Confidence Ellipses for Front and Rear GPS Antennas in Simulated Data Set A-1

The next figure presented for each set is a pair of $95 \%$ confidence ellipses plotted in the horizontal plane. The red ellipse is centered on the true coordinates of the rear GPS antenna, while the green ellipse is centered on the true coordinates of the front antenna. The ellipse represents a horizontal region where $95 \%$ of all computed points would be expected to fall inside of if the propagated, expected standard deviations reflect the true standard deviation. 
Table 16: Mean Global Error Statistics of Unfused Pitch for Simulation Set A-1

\begin{tabular}{|c|c|c|c|}
\hline Method & $\begin{array}{c}\text { Measured Standard } \\
\text { Deviation } \\
\text { (degrees) }\end{array}$ & $\begin{array}{c}\text { Estimated } \\
\text { Standard } \\
\text { Deviation } \\
\text { (degrees) }\end{array}$ & $\begin{array}{c}\text { Mean RMS } \\
\text { Error } \\
\text { (degrees) }\end{array}$ \\
\hline Single Point GPS (Pseudoranges) & 15.769 & 12.779 & 9.117 \\
\hline Single Point GPS (Carrier Phase) & 33.264 & 24.640 & 0.050 \\
\hline $\begin{array}{c}\text { Single Difference GPS } \\
\text { (Pseudoranges) }\end{array}$ & 22.561 & 17.098 & 10.739 \\
\hline $\begin{array}{c}\text { Single Difference GPS (Carrier } \\
\text { Phase) }\end{array}$ & 27.603 & 17.057 & 0.038 \\
\hline $\begin{array}{c}\text { Double Difference GPS } \\
\text { (Pseudoranges) }\end{array}$ & 23.283 & 20.526 & 8.490 \\
\hline $\begin{array}{c}\text { Double Difference GPS (Carrier } \\
\text { Phase) }\end{array}$ & 30.602 & 22.521 & 0.061 \\
\hline IMU Measurement & 0.007 & - & 0.006 \\
\hline
\end{tabular}

Table 17: Mean Global Error Statistics of Unfused Heading for Simulation Set A-1

\begin{tabular}{|c|c|c|c|}
\hline Method & $\begin{array}{c}\text { Measured Standard } \\
\text { Deviation } \\
\text { (degrees) }\end{array}$ & $\begin{array}{c}\text { Estimated } \\
\text { Standard } \\
\text { Deviation } \\
\text { (degrees) }\end{array}$ & $\begin{array}{c}\text { Mean RMS } \\
\text { Error } \\
\text { (degrees) }\end{array}$ \\
\hline Single Point GPS (Pseudoranges) & 19.880 & 12.825 & 53.769 \\
\hline Single Point GPS (Carrier Phase) & 62.022 & 28.296 & 0.163 \\
\hline $\begin{array}{c}\text { Single Difference GPS } \\
\text { (Pseudoranges) }\end{array}$ & 47.757 & 22.831 & 35.088 \\
\hline $\begin{array}{c}\text { Single Difference GPS (Carrier } \\
\text { Phase) }\end{array}$ & 56.885 & 20.697 & 0.068 \\
\hline $\begin{array}{c}\text { Double Difference GPS } \\
\text { (Pseudoranges) }\end{array}$ & 42.724 & 19.831 & 28.739 \\
\hline $\begin{array}{c}\text { Double Difference GPS (Carrier } \\
\text { Phase) }\end{array}$ & 59.971 & 21.806 & 0.146 \\
\hline IMU Measurement & 0.320 & - & 0.269 \\
\hline
\end{tabular}


Table 18: Mean Global Error Statistics of GPS/IMU Fused Pitch for Simulation Set A-1

\begin{tabular}{|c|c|c|c|}
\hline Method & $\begin{array}{c}\text { Measured Standard } \\
\text { Deviation } \\
\text { (degrees) }\end{array}$ & $\begin{array}{c}\text { Estimated } \\
\text { Standard } \\
\text { Deviation } \\
\text { (degrees) }\end{array}$ & $\begin{array}{c}\text { Mean RMS } \\
\text { Error } \\
\text { (degrees) }\end{array}$ \\
\hline Single Point GPS (Pseudoranges) & 0.092 & 0.033 & 0.194 \\
\hline Single Point GPS (Carrier Phase) & 6.742 & 4.346 & 0.024 \\
\hline $\begin{array}{c}\text { Single Difference GPS } \\
\text { (Pseudoranges) }\end{array}$ & 2.316 & 0.266 & 0.194 \\
\hline $\begin{array}{c}\text { Single Difference GPS (Carrier } \\
\text { Phase) }\end{array}$ & 6.337 & 3.498 & 0.018 \\
\hline $\begin{array}{c}\text { Double Difference GPS } \\
\text { (Pseudoranges) }\end{array}$ & 0.049 & 0.028 & 0.198 \\
\hline $\begin{array}{c}\text { Double Difference GPS (Carrier } \\
\text { Phase) }\end{array}$ & 7.714 & 4.151 & 0.006 \\
\hline IMU Measurement & 0.007 & - & \\
\hline
\end{tabular}

Table 19: Mean Global Error Statistics of GPS/IMU Fused Heading for Simulation Set A-1

\begin{tabular}{|c|c|c|c|}
\hline Method & $\begin{array}{c}\text { Measured Standard } \\
\text { Deviation } \\
\text { (degrees) }\end{array}$ & $\begin{array}{c}\text { Estimated } \\
\text { Standard } \\
\text { Deviation } \\
\text { (degrees) }\end{array}$ & $\begin{array}{c}\text { Mean RMS } \\
\text { Error } \\
\text { (degrees) }\end{array}$ \\
\hline Single Point GPS (Pseudoranges) & 2.149 & 1.123 & 3.663 \\
\hline Single Point GPS (Carrier Phase) & 54.142 & 21.719 & 0.077 \\
\hline $\begin{array}{c}\text { Single Difference GPS } \\
\text { (Pseudoranges) }\end{array}$ & 9.489 & 2.301 & 3.828 \\
\hline $\begin{array}{c}\text { Single Difference GPS (Carrier } \\
\text { Phase) }\end{array}$ & 49.911 & 17.033 & 0.034 \\
\hline $\begin{array}{c}\text { Double Difference GPS } \\
\text { (Pseudoranges) }\end{array}$ & 2.036 & 0.971 & 4.181 \\
\hline $\begin{array}{c}\text { Double Difference GPS (Carrier } \\
\text { Phase) }\end{array}$ & 45.847 & 17.767 & 0.072 \\
\hline IMU Measurement & 0.320 & - & 0.269 \\
\hline
\end{tabular}

Next, each data set lists 4 tables of global mean error statistics for the heading and pitch. The first pair of tables lists statistics for the GPS only solutions, while the second pair lists the statistics for the solutions which fused the GPS with IMU solutions. 
Table 20: Success Rates of Reaching Angular Specifications of Unfused Pitch for Simulation Data Set A-1

\begin{tabular}{|c|c|c|c|c|c|c|c|c|}
\hline & \multicolumn{7}{|c|}{ Portion of Trials in Which Target Specification was reached } \\
\hline & $\pm 0.5^{\circ}$ & $\pm 1^{\circ}$ & $\pm 2^{\circ}$ & $\pm 5^{\circ}$ & $\pm 10^{\circ}$ & $\pm 15^{\circ}$ & $\pm 20^{\circ}$ & $\pm 25^{\circ}$ \\
\hline Method & 55 & 59 & 60 & 64 & 79 & 86 & 91 & 95 \\
\hline $\begin{array}{c}\text { Single Point GPS } \\
\text { (Pseudoranges) }\end{array}$ & 65 & 65 & 65 & 65 & 65 & 75 & 79 & 85 \\
\hline $\begin{array}{c}\text { Single Point GPS (Carrier } \\
\text { Phase) }\end{array}$ & 57 & 59 & 60 & 66 & 71 & 75 & 77 & 81 \\
\hline $\begin{array}{c}\text { Single Difference GPS } \\
\text { (Pseudoranges) }\end{array}$ & 77 & 77 & 77 & 77 & 81 & 88 & 91 & 92 \\
\hline $\begin{array}{c}\text { Single Difference GPS } \\
\text { (Carrier Phase) }\end{array}$ & 45 & 46 & 48 & 56 & 67 & 74 & 83 & 87 \\
\hline $\begin{array}{c}\text { Double Difference GPS } \\
\text { (Pseudoranges) }\end{array}$ & 74 & 74 & 74 & 74 & 75 & 81 & 85 & 90 \\
\hline $\begin{array}{c}\text { Double Difference GPS } \\
\text { (Carrier Phase) }\end{array}$ & 100 & 100 & 100 & 100 & 100 & 100 & 100 & 100 \\
\hline IMU Measured & & & & & & & & \\
\hline
\end{tabular}

Table 21: Success Rates of Reaching Angular Specifications of Unfused Heading for Simulation Data Set A-1

\begin{tabular}{|c|c|c|c|c|c|c|c|c|}
\hline & \multicolumn{7}{|c|}{ Portion of Trials in Which Target Specification was reached } \\
\hline Method & $\pm 0.5^{\circ}$ & $\pm 1^{\circ}$ & $\pm 2^{\circ}$ & $\pm 5^{\circ}$ & $\pm 10^{\circ}$ & $\pm 15^{\circ}$ & $\pm 20^{\circ}$ & $\pm 25^{\circ}$ \\
\hline $\begin{array}{c}\text { Single Point GPS } \\
\text { (Pseudoranges) }\end{array}$ & 45 & 46 & 51 & 56 & 71 & 81 & 85 & 89 \\
\hline $\begin{array}{c}\text { Single Point GPS (Carrier } \\
\text { Phase) }\end{array}$ & 58 & 58 & 66 & 66 & 71 & 71 & 78 & 81 \\
\hline $\begin{array}{c}\text { Single Difference GPS } \\
\text { (Pseudoranges) }\end{array}$ & 58 & 60 & 61 & 64 & 68 & 73 & 75 & 77 \\
\hline $\begin{array}{c}\text { Single Difference GPS } \\
\text { (Carrier Phase) }\end{array}$ & 70 & 70 & 71 & 75 & 82 & 82 & 82 & 82 \\
\hline $\begin{array}{c}\text { Double Difference GPS } \\
\text { (Pseudoranges) }\end{array}$ & 53 & 53 & 54 & 56 & 65 & 74 & 77 & 80 \\
\hline $\begin{array}{c}\text { Double Difference GPS } \\
\text { (Carrier Phase) }\end{array}$ & 71 & 72 & 72 & 74 & 79 & 79 & 79 & 79 \\
\hline IMU Measured & 91 & 100 & 100 & 100 & 100 & 100 & 100 & 100 \\
\hline
\end{tabular}


Table 22: Success Rates of Reaching Angular Specifications of Fused Pitch for Simulation Data Set A-1

\begin{tabular}{|c|c|c|c|c|c|c|c|c|}
\hline & \multicolumn{7}{|c|}{ Portion of Trials in Which Target Specification was reached } \\
\hline & $\pm 0.5^{\circ}$ & $\pm 1^{\circ}$ & $\pm 2^{\circ}$ & $\pm 5^{\circ}$ & $\pm 10^{\circ}$ & $\pm 15^{\circ}$ & $\pm 20^{\circ}$ & $\pm 25^{\circ}$ \\
\hline Method & 100 & 100 & 100 & 100 & 100 & 100 & 100 & 100 \\
\hline $\begin{array}{c}\text { Single Point GPS } \\
\text { (Pseudoranges) }\end{array}$ & 65 & 66 & 80 & 94 & 96 & 96 & 100 & 100 \\
\hline $\begin{array}{c}\text { Single Point GPS (Carrier } \\
\text { Phase) }\end{array}$ & 100 & 100 & 100 & 100 & 100 & 100 & 100 & 100 \\
\hline $\begin{array}{c}\text { Single Difference GPS } \\
\text { (Pseudoranges) }\end{array}$ & 77 & 77 & 84 & 93 & 95 & 95 & 99 & 99 \\
\hline $\begin{array}{c}\text { Single Difference GPS } \\
\text { (Carrier Phase) }\end{array}$ & 100 & 100 & 100 & 100 & 100 & 100 & 100 & 100 \\
\hline $\begin{array}{c}\text { Double Difference GPS } \\
\text { (Pseudoranges) }\end{array}$ & 74 & 74 & 83 & 97 & 97 & 97 & 100 & 100 \\
\hline $\begin{array}{c}\text { Double Difference GPS } \\
\text { (Carrier Phase) }\end{array}$ & 100 & 100 & 100 & 100 & 100 & 100 & 100 & 100 \\
\hline IMU Measured & & & & & & & & \\
\hline
\end{tabular}

Table 23: Success Rates of Reaching Angular Specifications of Fused Heading for Simulation Data Set A-1

\begin{tabular}{|c|c|c|c|c|c|c|c|c|}
\hline & \multicolumn{7}{|c|}{ Portion of Trials in Which Target Specification was reached } \\
\hline Method & $\pm 0.5^{\circ}$ & $\pm 1^{\circ}$ & $\pm 2^{\circ}$ & $\pm 5^{\circ}$ & $\pm 10^{\circ}$ & $\pm 15^{\circ}$ & $\pm 20^{\circ}$ & $\pm 25^{\circ}$ \\
\hline $\begin{array}{c}\text { Single Point GPS } \\
\text { (Pseudoranges) }\end{array}$ & 70 & 82 & 91 & 96 & 100 & 100 & 100 & 100 \\
\hline $\begin{array}{c}\text { Single Point GPS (Carrier } \\
\text { Phase) }\end{array}$ & 58 & 59 & 66 & 70 & 72 & 77 & 87 & 88 \\
\hline $\begin{array}{c}\text { Single Difference GPS } \\
\text { (Pseudoranges) }\end{array}$ & 79 & 86 & 90 & 94 & 97 & 97 & 98 & 98 \\
\hline $\begin{array}{c}\text { Single Difference GPS } \\
\text { (Carrier Phase) }\end{array}$ & 70 & 71 & 71 & 81 & 82 & 87 & 89 & 89 \\
\hline $\begin{array}{c}\text { Double Difference GPS } \\
\text { (Pseudoranges) }\end{array}$ & 74 & 83 & 92 & 96 & 99 & 100 & 100 & 100 \\
\hline $\begin{array}{c}\text { Double Difference GPS } \\
\text { (Carrier Phase) }\end{array}$ & 70 & 71 & 72 & 77 & 80 & 80 & 82 & 85 \\
\hline IMU Measured & 91 & 100 & 100 & 100 & 100 & 100 & 100 & 100 \\
\hline
\end{tabular}

Next, each data set lists 4 tables of specification success rates for the heading and pitch. The first pair of tables contains success rates for the GPS only solutions, while the second pair 
lists success rates for the solutions which fused the GPS with IMU solutions. Rates are listed for all specification categories.

Table 24: Pitch / Heading Correlation Coefficients for Each Computation Method in Simulation Data Set A-1

\begin{tabular}{|c|c|c|}
\hline & \multicolumn{2}{|c|}{ Pitch / Heading Correlation Coefficient } \\
\hline Method & Unfused & Fused \\
\hline $\begin{array}{c}\text { Single Point GPS } \\
\text { (Pseudoranges) }\end{array}$ & 0.858 & 0.107 \\
\hline $\begin{array}{c}\text { Single Difference GPS } \\
\text { (Pseudoranges) }\end{array}$ & 0.937 & 0.146 \\
\hline $\begin{array}{c}\text { Double Difference GPS } \\
\text { (Pseudoranges) }\end{array}$ & 0.573 & 0.046 \\
\hline
\end{tabular}

Finally, each dataset presents a table of correlation coefficients between the computed pitch and heading. These are taken from the off diagonals of the covariance matrix of the unknowns.

\subsection{Mission Design Using Simulation Results}

The simulation results collected can be used to solve design problems for GPS partial attitude determination systems. The 2 design parameters which can be solved for are one of either antenna separation required, or the pseudorange noise requirement for the receiver.

\subsubsection{Example when Solving for Pseudorange Noise}

Problem: Suppose that a navigation platform allows for a maximum $20 \mathrm{~m}$ of GPS antenna separation. What is the maximum allowable pseudorange noise on the receiver if $80 \%$ of the pitch measurements need to fall within a range of plus or minus 2 degrees of error? 
Solution: Each collected set has statistics tabulated for $10 \mathrm{~m}$ and $30 \mathrm{~m}$ separations. Therefore, endpoint values will be chosen from those sets in series' which have statistics bounding the $80 \%$ required specification. The following summarizes the values and sets used:

Table 25: Statistics from Selected Data Sets for Interpolation of Pseudorange Noise

\begin{tabular}{|c|c|c|c|}
\hline Simulation Set & $\begin{array}{c}\text { Antenna Separation } \\
(\mathrm{m})\end{array}$ & $\begin{array}{c}\text { Pseudorange Noise } \\
(\mathrm{m})\end{array}$ & $\begin{array}{c}\text { Success Rate of } \\
\text { Meeting } \pm 2 \text { Degree } \\
\text { Specification (\%) }\end{array}$ \\
\hline B-4 & 10 & 1 & 64 \\
\hline B-5 & 30 & 1 & 87 \\
\hline A-4 & 10 & 0.1 & 93 \\
\hline A-5 & 30 & 0.1 & 100 \\
\hline
\end{tabular}

First, interpolations will be done to compute 2-degree specification success rates for each series at $20 \mathrm{~m}$ separation:

$$
\begin{aligned}
\text { Series A Rate }(20 m)= & 93+\frac{(100-93)(20-10)}{(30-10)} \\
& =96.5 \% \\
\text { Series B Rate }(20 m)= & 64+\frac{(87-64)(20-10)}{(30-10)} \\
& =75.5 \%
\end{aligned}
$$

Now, a final interpolation is done to find a maximum pseudorange noise to meet an $80 \%$ specification:

$$
\begin{gathered}
\text { pseudorange noise }(80 \% \text { success })=0.1+\frac{(80-75.5)(1-0.1)}{(96.5-75.5)} \\
=0.19 \mathrm{~m}
\end{gathered}
$$

This suggests that at most $20 \mathrm{~cm}$ are allowable in meeting the required specifications. For the designer, this will indicate that a high-quality receiver with access to the precise $\mathrm{p}$ code is a minimum requirement here. 


\subsubsection{Example when Solving for Antenna Separation}

Problem: Suppose that 2 receivers with $2 \mathrm{~m}$ standard deviation of random error due to pseudorange noise are available for use in developing a low-cost partial attitude system. What is the minimum antenna separation required if the heading measurements must meet a required maximum of 2.5 degrees standard deviation?

Solution: Data series' are available with pseudorange noise of $1 \mathrm{~m}$ and $3 \mathrm{~m}$. The same strategy will be used as in the first problem, finding sets in Series B and Series C which encompass the required standard deviation. These sets are summarized below:

Table 26: Statistics from Selected Data Sets for Interpolation of Antenna Separation

\begin{tabular}{|c|c|c|c|}
\hline Simulation Set & $\begin{array}{c}\text { Antenna Separation } \\
(\mathrm{m})\end{array}$ & $\begin{array}{c}\text { Pseudorange Noise } \\
(\mathrm{m})\end{array}$ & $\begin{array}{c}\text { Heading Standard } \\
\text { Deviation (Degrees) }\end{array}$ \\
\hline B-4 & 10 & 1 & 6.45 \\
\hline B-5 & 30 & 1 & 1.98 \\
\hline C-6 & 50 & 3 & 2.91 \\
\hline C-7 & 70 & 3 & 1.63 \\
\hline
\end{tabular}

First, interpolations are done to compute the antenna separation at which each series meets the design requirement:

$$
\begin{aligned}
& \text { Separation }(B)=10+\frac{(2.5-6.45)(30-10)}{(1.98-6.45)} \\
& =27.673 \mathrm{~m} \\
& \text { Separation }(C)=50+\frac{(2.5-2.91)(70-50)}{(1.63-2.91)} \\
& =56.406 \mathrm{~m}
\end{aligned}
$$

Now the interpolated separations are used to interpolate the required separation with $2 \mathrm{~m}$ of pseudorange noise: 


$$
\text { Separation }(2 m \text { Noise })=27.673 \mathrm{~m}+\frac{(2-1)(56.406-27.673)}{(3-1)}
$$

This indicates that at least $42 \mathrm{~m}$ of separation is required to meet the 2.5 degree standard deviation specification. Most passenger aircraft meet this specification by their length. In fact, some aircraft meet this requirement with their wingspan, such as the Boeing 747 . This means such an aircraft could double as a roll determiner as well as meeting this heading requirement given this low-cost GPS specification. 


\section{GPS Pitch and Heading Experimental Control}

\section{Point Survey}

In order to validate the simulated results, an experimental survey was conducted at the Carleton University campus using 2 different GPS receivers of which the antennas were placed atop 2 control points previously surveyed by the Civil Engineering Department to high precision. This provides a large advantage for validation, as the known coordinates of the control points can be used to directly assess positioning accuracy as well as providing a true value of the pitch and heading defined by the 2 points.

\subsection{Control Points}

The 2 control points used for the survey are annotated in Figure 38:

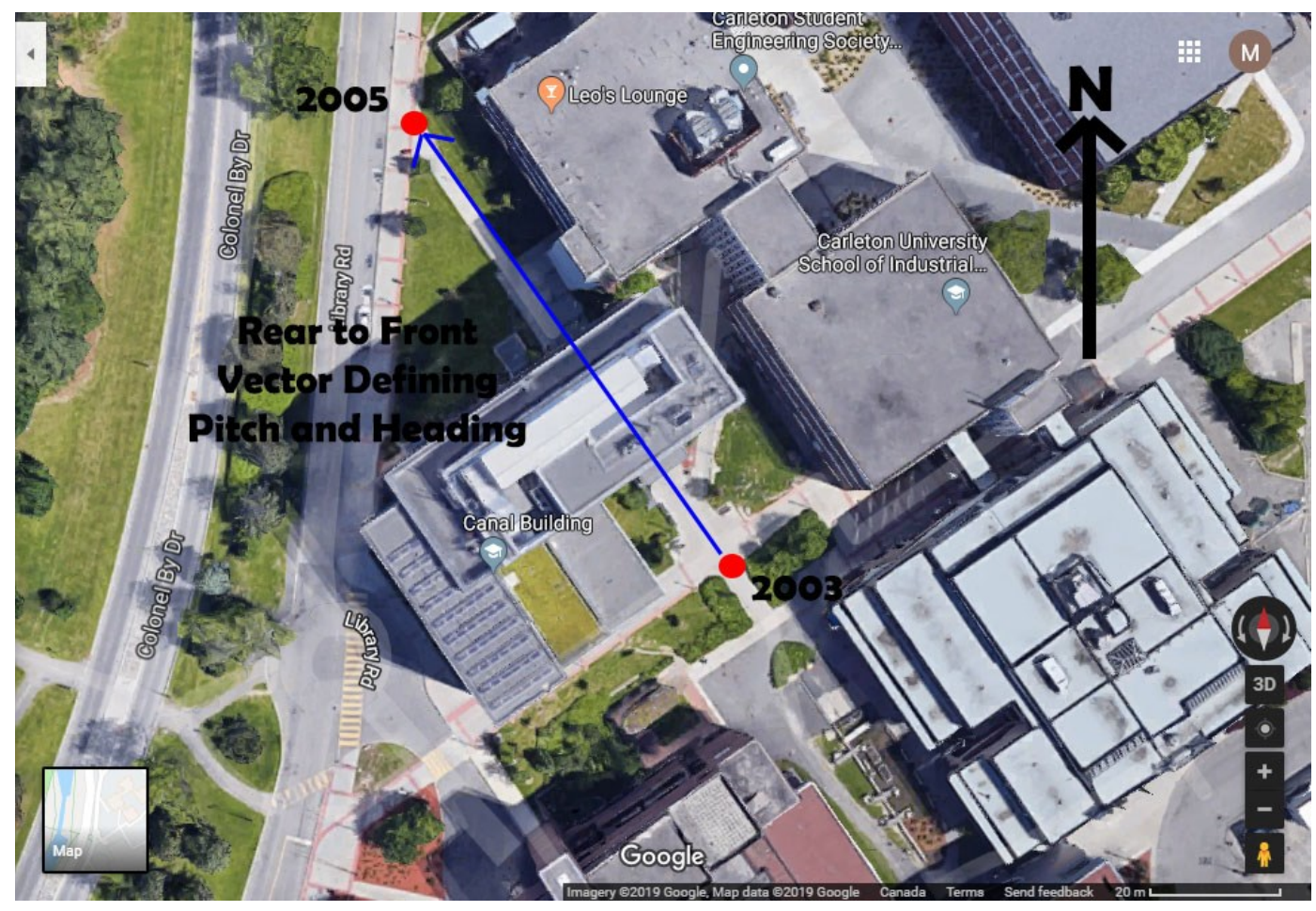

Figure 40: Locations of Control Points Used for Pitch and Heading Survey on Carleton University Campus 
As can be seen in Figure 115, the location of the 2 control points have a somewhat open view of the sky, but also have the potential for multipath hazards. Control point 2003 more likely will suffer from multipath due to window reflections as well as obscuring of satellites by the buildings themselves. Control point 2005 is situated very close to the roadside, and therefore the parked cars which were present at the time of the survey likely were the largest multipath contributors. This is shown in Figure 41:

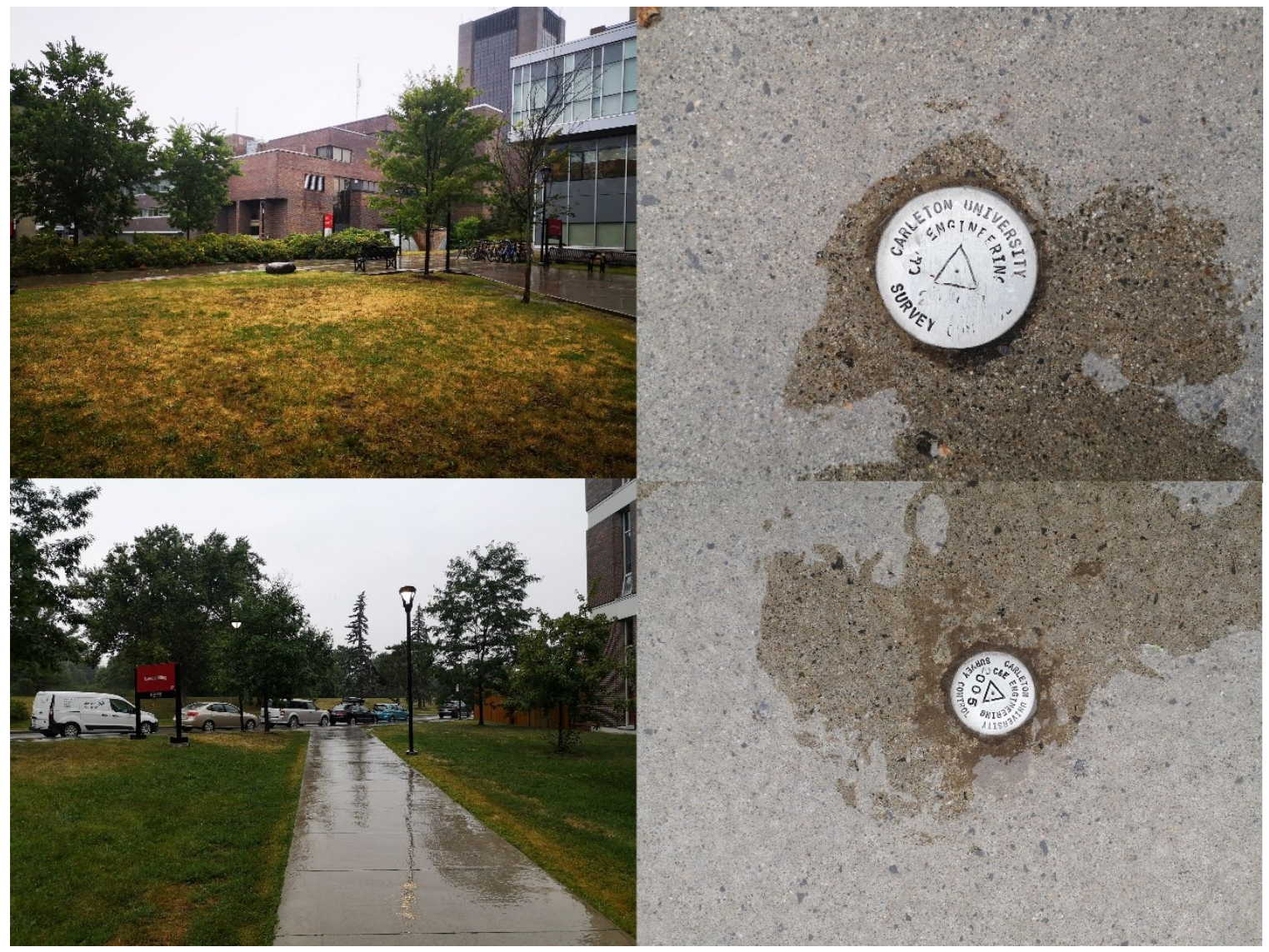

Figure 41: Attitude Experiment Location. Top Left: Rear GPS Environment. Top Right: Rear Control Point. Bottom Left: Front GPS Environment. Bottom Right: Front GPS Control Point 
The coordinates of the control points are listed below:

Table 27: Geographic Coordinates of Control Points in Attitude Survey, WGS84 Used as Ellipsoidal Datum for Height Reference

\begin{tabular}{|c|c|c|c|c|c|c|c|}
\hline Control & \multicolumn{3}{|c|}{ Latitude } & \multicolumn{3}{c|}{ Longitude } & Height \\
\cline { 2 - 8 } Point ID & Degrees & Minutes & Seconds & Degrees & Minutes & Seconds & $(\mathrm{m})$ \\
\hline 2003 & 45 & 23 & 2.552 & 75 & 41 & 53.114 & 64.393 \\
\hline 2005 & 45 & 23 & 4.891 & 75 & 41 & 55.567 & 64.158 \\
\hline
\end{tabular}

The control markers for both points were a bronze cap engraved with the point ID and embedded in the concrete walking paths at their locations. Test receiver 2 was placed on top of control point 2003 and the Geo++ RINEX Logger application was used to log raw GPS raw RINEX data captured by the internal GPS receiver of the cell phone. Unlike test receiver 1, test receiver 2 does not have the capacity to log carrier phase measurements, so the carrier phase will not be used in the pitch and heading analysis for this survey. The test receiver 1 antenna was placed on top of control point 2005, using the ReachView application to log raw RINEX data for it, just as was done in the Didsbury survey. 


\subsection{Positioning Results}

In order to assess the true positioning error for each receiver during this survey, the computed coordinates by each receiver were compared directly with the known coordinates of the markers by transforming computed coordinates into local vertical frames using their marker coordinates as reference points:
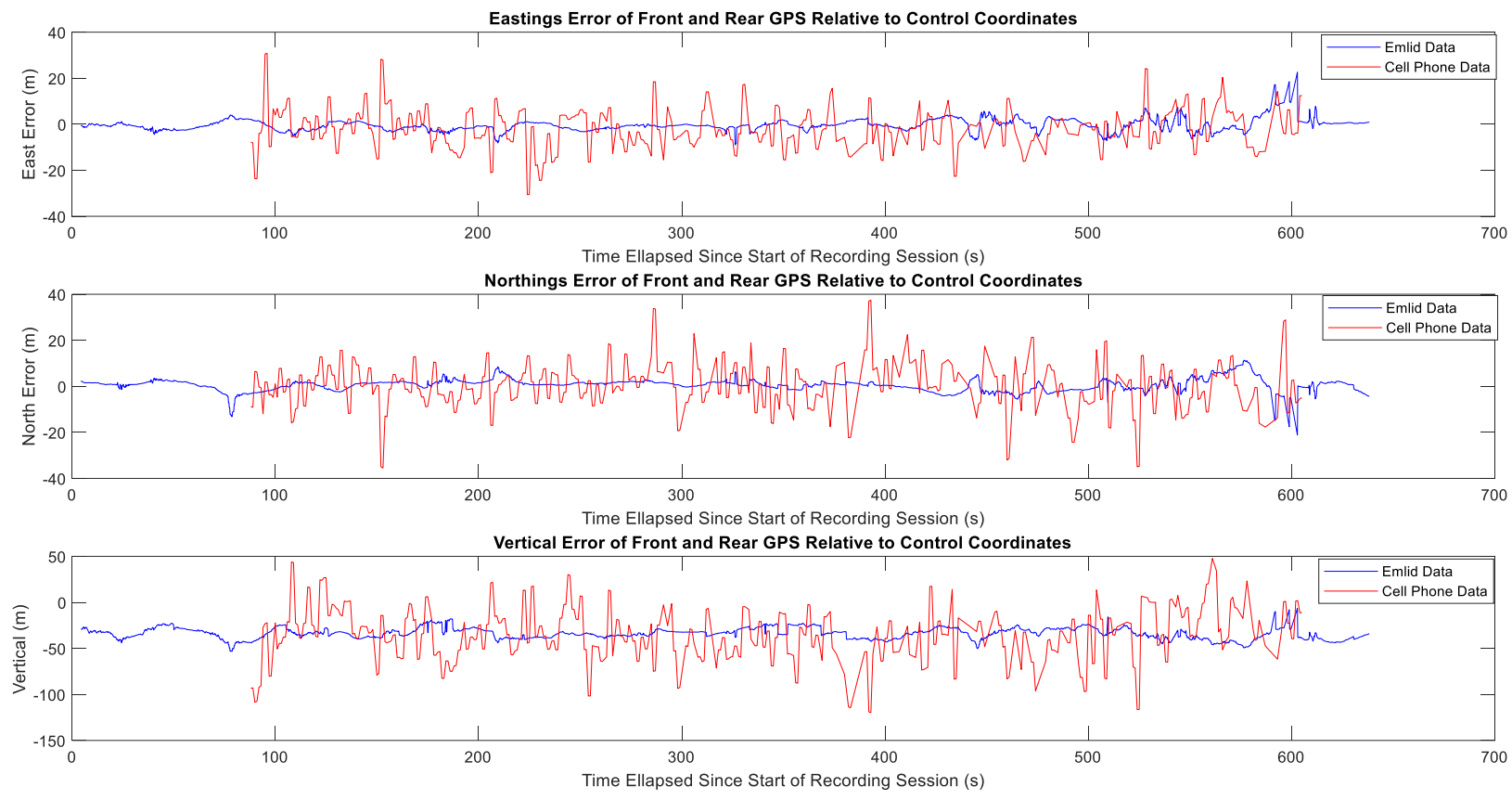

Figure 42: True Error of Computed GPS Coordinates for the Front and Rear GPS Receivers Using Known Marker Locations as a Reference Position

As can be observed in Figure 117, test receiver 1 was collecting data for a longer period than receiver 2 was. It is also important to note that the cell phone collected data at a rate of $1 \mathrm{~Hz}$ while receiver 1 collected data at a rate of $5 \mathrm{~Hz}$. Therefore, in order to use computed positions for pitch and heading computation, the cell phone data will be interpolated in order to obtain positions at times synced to receiver 1 data. It is also very interesting to note the noticeable difference in data quality between the two receivers. Receiver 2 was subject to a significantly higher amount of noise and likely multipath as well. Some of that can be explained by the 
location, but more of this disparity is likely explainable by the lower receiver quality in the cell phone. Global error statistics for each receiver are summarized below:

Table 28: Global Mean Positioning Error Statistics for Each Receiver Across Carleton Pitch and Heading Survey

\begin{tabular}{|c|c|c|c|}
\hline Receiver & 3DRMS Error $(\mathrm{m})$ & Vertical Error $(\mathrm{m})$ & Standard Deviation $(\mathrm{m})$ \\
\hline Test Receiver 1 & 34.095 & -33.890 & 5.873 \\
\hline Test Receiver 2 & 41.871 & -35.372 & 24.010 \\
\hline
\end{tabular}

Table 28 shows that, while the receiver 1 and receiver 2 yielded somewhat comparable mean true errors, the receiver 2 data was far noisier than that of receiver 1 . This may have implications in the comparison with simulation data, because all simulated data used an assumption that both receivers used to compute attitude have the same noise characteristics. Some of the trends in the data may be accounted for by changing satellite geometry, which is illustrated below:

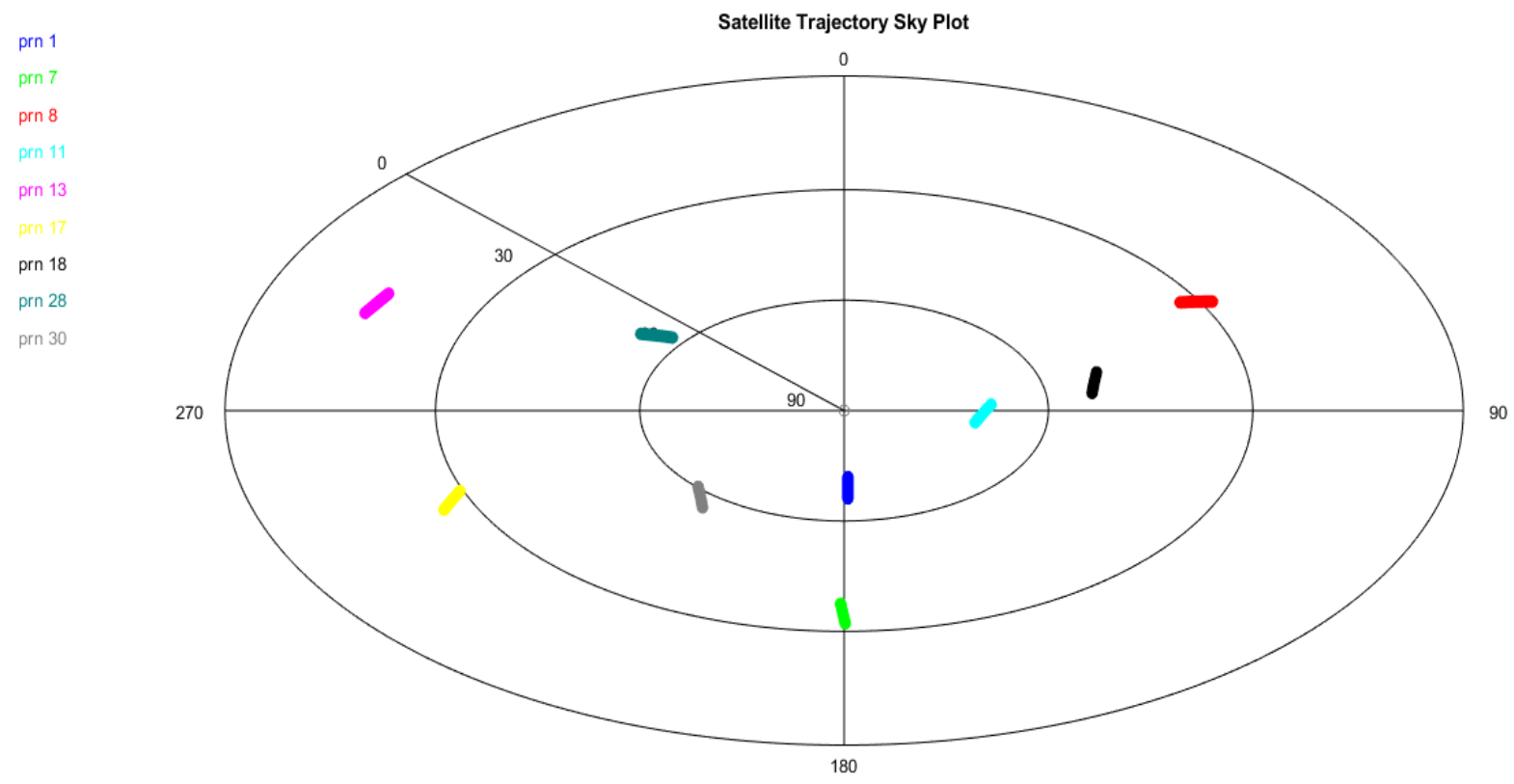

Figure 43: Sky Plot of Trajectories of Available GPS Satellites During Carleton Pitch and Heading Survey 
While not each of the satellites shown in Figure 118 are available to both receivers for the duration of the survey, it is still interesting to note that neither receiver had a line of sight to a GPS satellite directly north of it in the sky, which may affect the quality of positioning solutions in the north direction. In addition, solutions which have different available satellite geometry between satellites could induce some biases to the pitch and heading computations. This is because the 2 positions used for the angle computation would have slightly different biases in the solution caused by a disparity of satellite geometry. In an open sky environment this would not be a problem.

\subsection{Pitch and Heading Results}

To directly assess the accuracy of the pitch and heading results, coordinates were computed for control point 2005 relative to control point 2003 in a local vertical frame. Because control point 2003 represents the origin of this system, the northings, eastings and vertical coordinates of control point 2005 can be said to represent the change in eastings, northings and vertical of the front and rear points on a platform for orientation computation, with control point 2005 representing the front and control point 2003 representing the rear. This is also very convenient because the baseline distance between control points is $89.776 \mathrm{~m}$. This very close to the $90 \mathrm{~m}$ baseline, which was the longest baseline used in simulations, making a direct comparison more feasible. Positioning results laid out in the horizontal plane are shown below: 


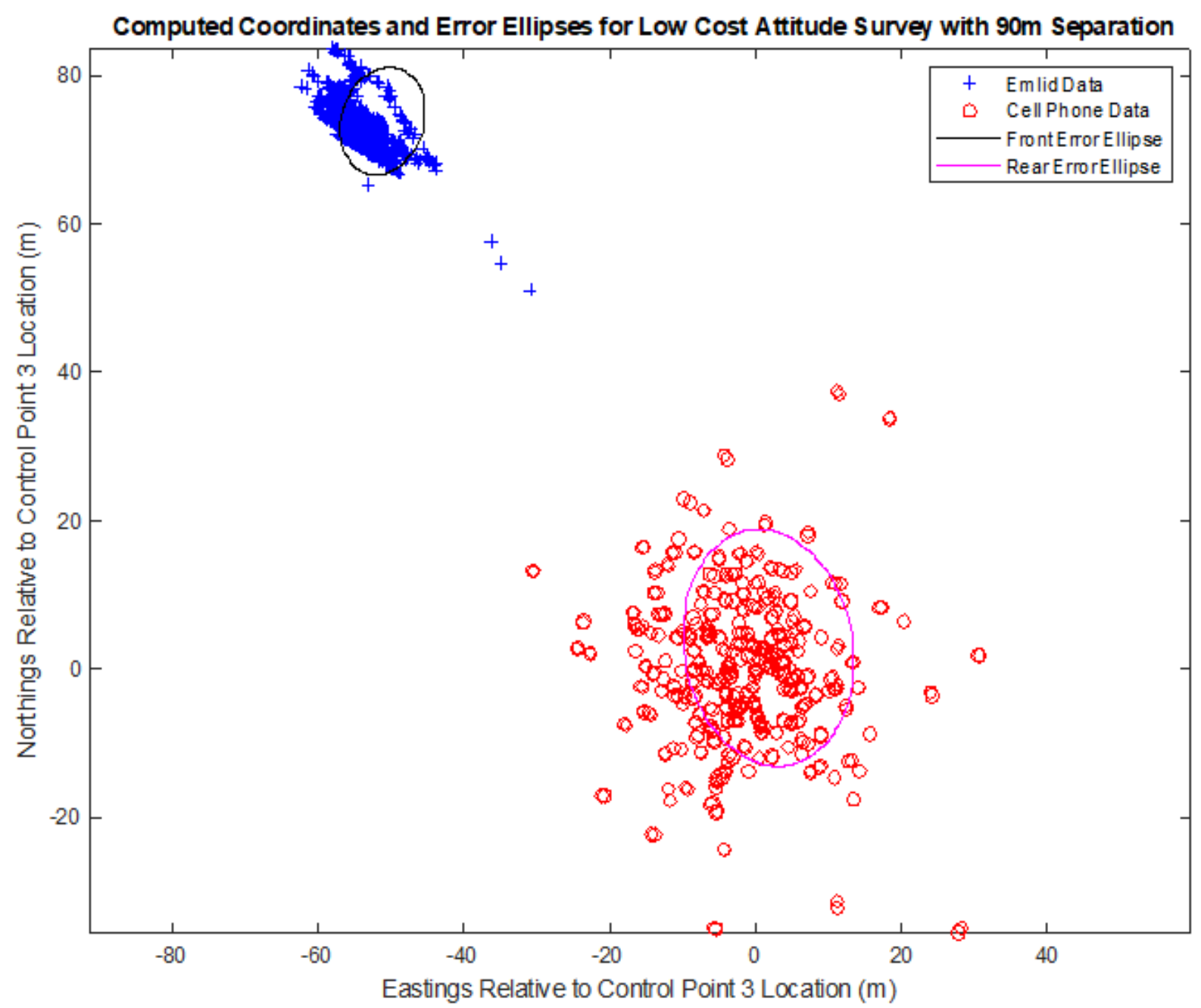

Figure 44: Positioning Results in Horizontal Plane for Test Receivers 1 and 2 in Carleton Pitch and Heading Survey

As can be seen in Figure 41, while the computed confidence ellipses somewhat effectively encompass the computed coordinates, they do not contain all of them. This is consistent with the simulation results, where $95 \%$ confidence ellipses were typically optimistic compared to the observed values. This was true even when pseudorange noise was forced to conform to a known standard deviation in simulation, so it is not likely caused to an optimistic choice of pseudorange noise in these results. Time series graphs of the pitch and heading errors are as follows: 


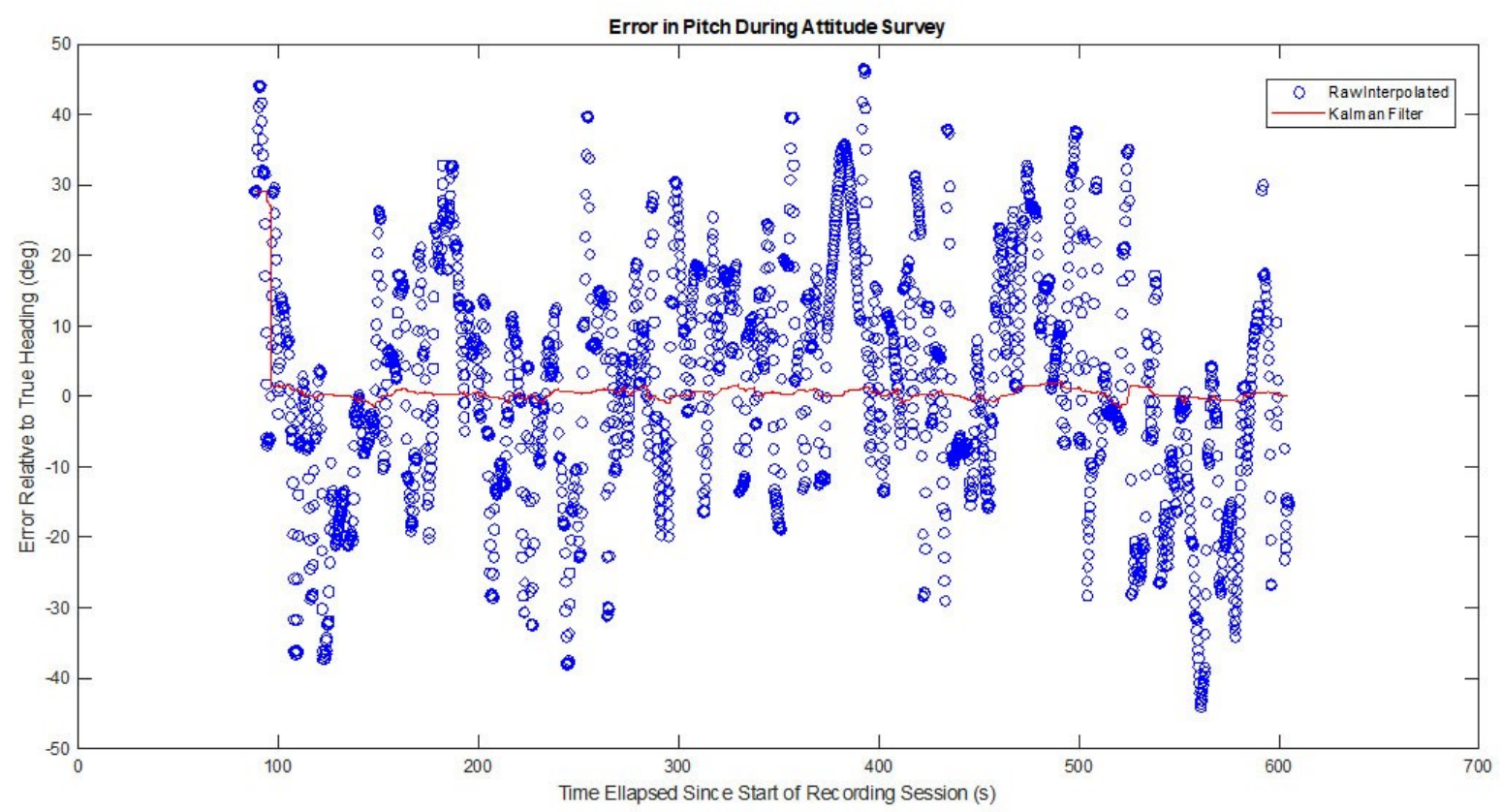

Figure 45: True Pitch Error of Undedicated GPS Pitch and Roll System Using Test Receivers 1 and 2 in Carleton Survey. Raw Solutions Using Interpolated Data as Well as Kalman Filtered Results are Shown

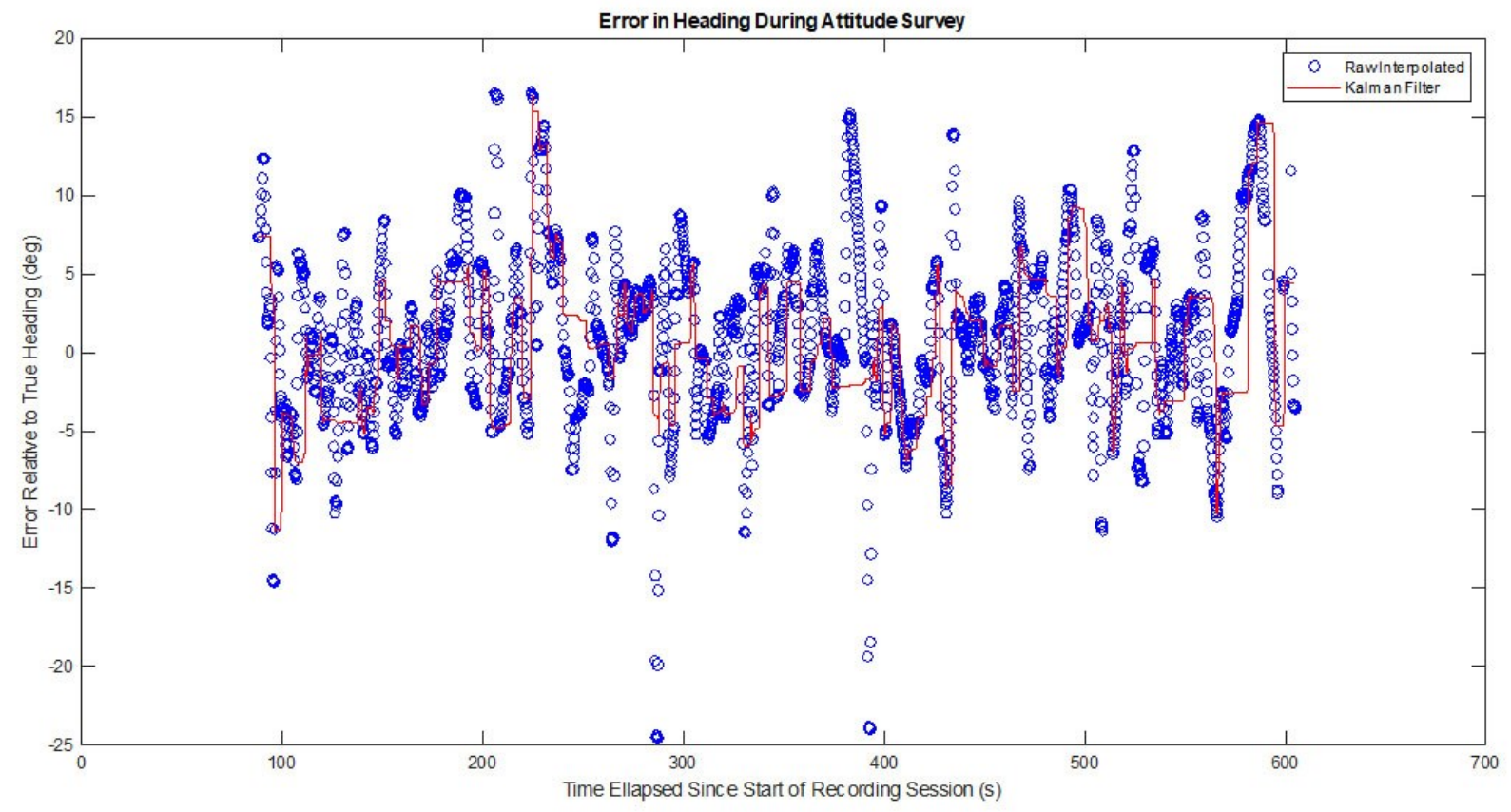

Figure 46: True Heading Error of Undedicated GPS Pitch and Roll System Using Test Receivers 1 and 2 in Carleton Survey. Raw Solutions Using Interpolated Data as Well as Kalman Filtered Results are Shown 
There are some interesting trends to be observed in Figures 42 and 43. First, it appears that the interpolation of the cell phone data has had a false smoothing effect on both the pitch and heading results. While this does not improve the solution where receiver 1 and receiver 2 positions are chronologically synced naturally, it does appear to induce solutions which are clustered closer to the true value than the random data points alone. It is also interesting that the pitch had larger peaks in error than the heading did. This is possibly due to the large variation in vertical error between and within datasets, which could be caused in part by multipath effects. The vertical coordinates are not used in the heading computation, so the heading is not affected by that. Global error statistics are summarized below:

Table 29: Global Mean Error Statistics of Undedicated GPS Pitch and Heading System During Carleton Survey

\begin{tabular}{|c|c|c|c|c|}
\hline Method & & $\begin{array}{c}\text { Observed } \\
\text { Standard } \\
\text { Deviation } \\
\text { (degrees) }\end{array}$ & $\begin{array}{c}\text { Estimated } \\
\text { Standard } \\
\text { Deviation } \\
\text { (degrees) }\end{array}$ & $\begin{array}{c}\text { Mean } \\
\text { RMS Error } \\
\text { (degrees) }\end{array}$ \\
\hline Raw Interpolation & Pitch & 26.991 & 0.647 & 13.881 \\
\cline { 2 - 5 } & Heading & 5.567 & 1.446 & 4.287 \\
\hline Kalman Filter & Pitch & 3.609 & - & 0.714 \\
\cline { 2 - 5 } & Heading & 4.449 & - & 3.539 \\
\hline
\end{tabular}

Table 30: Portion of Observations Obtaining Desired Specifications for Each Method and Metric During Carleton Survey

\begin{tabular}{|c|c|c|c|c|c|c|c|c|}
\hline & \multicolumn{8}{|c|}{ Portion of Trials in Which Target Specification was reached (\%) } \\
\hline Metric / Method & $\pm 0.5^{\circ}$ & $\pm 1^{\circ}$ & $\pm 2^{\circ}$ & $\pm 5^{\circ}$ & $\pm 10^{\circ}$ & $\pm 15^{\circ}$ & $\pm 20^{\circ}$ & $\pm 25^{\circ}$ \\
\hline Pitch (Interpolation) & 47 & 48 & 49 & 56 & 68 & 77 & 85 & 90 \\
\hline Heading (Interpolation) & 47 & 50 & 59 & 78 & 94 & 99 & 100 & 100 \\
\hline Pitch (Kalman Filter) & 88 & 96 & 98 & 98 & 98 & 98 & 98 & 98 \\
\hline Heading (Kalman Filter) & 45 & 54 & 63 & 87 & 96 & 99 & 100 & 100 \\
\hline
\end{tabular}

Using simulation results with a $90 \mathrm{~m}$ inter-receiver baseline as a basis for comparison, it is interesting to note that the results in Table 29 most closely resemble simulated dataset C-6 in magnitude, while the fact that heading was more successful in reaching specifications than pitch 
is more in keeping with simulation dataset C-8. Dataset C-6 had a baseline separation of only $50 \mathrm{~m}$ compared to this dataset having approximately $90 \mathrm{~m}$ of separation. This suggests that more error is present in the survey than would be expected from a $3 \mathrm{~m}$ pseudorange noise solution with noise alone. The standard deviation of the receiver 2 data solutions is much higher than any simulated solutions having $3 \mathrm{~m}$ of pseudorange noise, which suggests that receiver 2 likely has a combination of more pseudorange noise as well as more multipath at play here. It is likely that simulations modelled with noise greater than $3 \mathrm{~m}$ would yield pitch and heading computation error statistics comparable to those found in this survey.

One important thing to extrapolate from these results is that, for a given platform which has at least $90 \mathrm{~m}$ of front to rear separation, a GPS pitch and heading determination system could be constructed at low cost and still achieve desired specifications in some applications. In fact, this system benefitted significantly from a Kalman filter of the raw data. This is especially true for the pitch. The only specification category which the filtered pitch struggled to achieve was \pm $0.5^{\circ}$. Depending on the application, this could be very acceptable. This includes nautical applications where the pitch and heading are important for navigation purposes.

\subsection{Effects of Antenna Separation on Pitch and Heading}

\section{Accuracy}

Heading and pitch are angular quantities confined to a 180-degree range and 360-degree range, respectively. This means that the standard deviation is not a sufficient metric for assessing the effectiveness of a GPS attitude system. This is especially true at short antenna separation distances, based on the simulated results in Appendix A through C. The estimated standard deviations of heading exceed more than 360 degrees when noise is high and antenna separation 
is small. This does not make sense due to the cyclical nature of the angular quantities, because that standard deviation could never be observed in reality. It is geometrically understandable why this happens, because the general trend yields increasing partial attitude noise with decreasing antenna separation and increasing pseudorange noise. Suppose that two receivers with equal pseudorange noise are utilized in a pitch and heading system. Also suppose that a confidence ellipse can be drawn which contains $100 \%$ of possible computed positions for each receiver. The following figure illustrates the nature of the pitch and heading computation as it varies with antenna separation: 


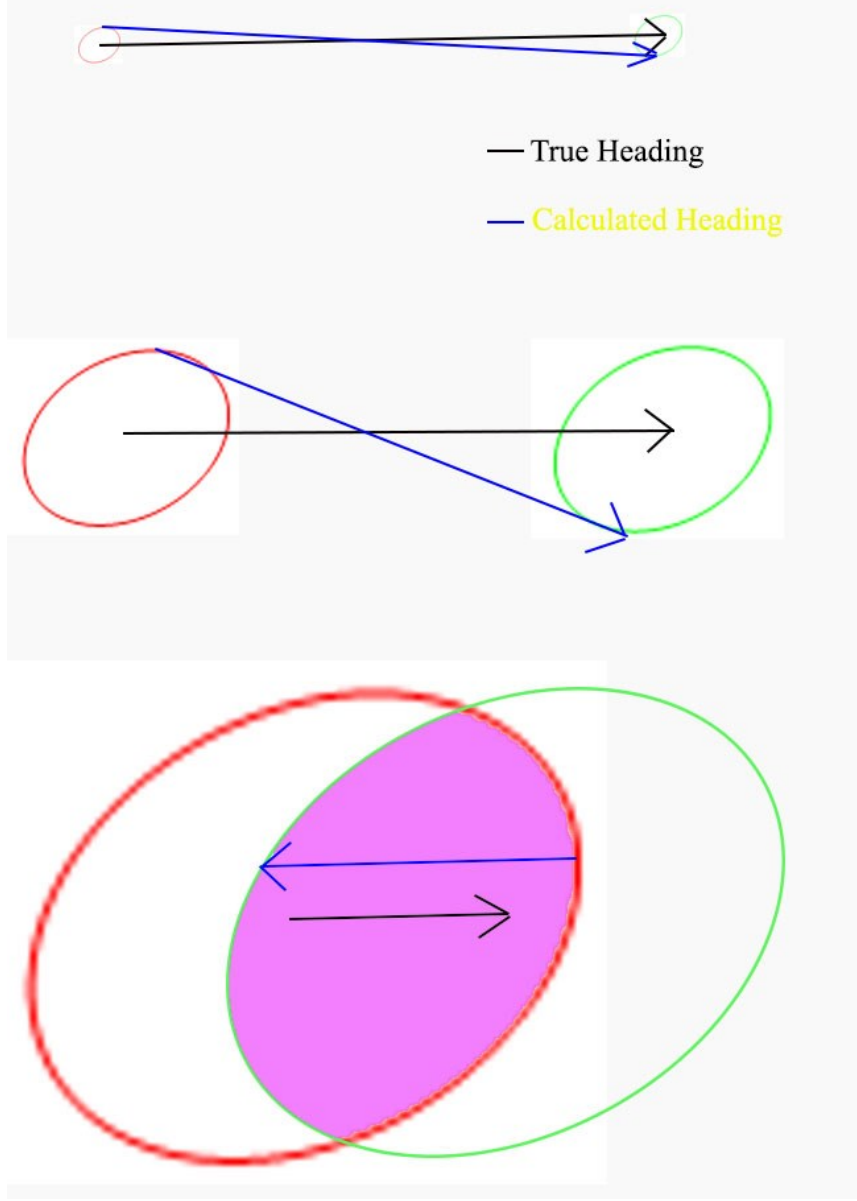

Figure 47: True Headings vs Extreme Error Computed Headings, Receiver Noise Properties Fixed at a Constant with Varying Antenna Separation. Long separation (Top), Medium Separation (Middle), Short Separation (Bottom)

As can be observed in Figure 44, far enough separation between antennas minimizes the maximum error in computed attitude. This is true regardless of how much noise a receiver has in the position solution. A noisier receiver simply requires a longer separation to achieve the same heading and pitch computation precision. On the other hand, at close distances, there is a region in which solutions for the two receiver positions overlap (magenta). This is a critical region in which solutions for heading could be in any quadrant. In order to avoid this problem, the separation must be increased or a higher quality receiver must be used with less pseudorange noise. The simulations produced in this thesis will aid in solving this design problem. 


\section{Summary and Recommendations}

This section will reiterate some of the motivation of this work as well as summarize the findings and contributions of the author's research to the field of positioning and navigation. Subsequently, it will lay out possibilities for future expansion of the thesis.

\subsection{Thesis Summary}

Positioning and navigation are vital components of any moving platform system, whether they are autonomous or manually driven. Small platforms such as autonomous rovers or autonomous vehicles, or large platforms such as offshore seismic or cargo vessels, or passenger aircraft, all rely on sensors to aid in this task. As with any design problem, there is always also a need to minimize cost while still meeting specifications. Such is the case for GNSS sensors, particularly if they are used for attitude or partial attitude determination. While the current status quo accepts use of the carrier phase observable as a necessity to have sufficient precision for most missions, the author's knowledge the feasibility of low-cost receivers, especially using pseudoranges only, has not been quantified in a way that is easily accessible to designers.

This thesis approached the design problem using an exhaustive simulation approach, validating those simulations using control point surveys with low-cost sensors. First a positioning simulation was conducted by generating biased pseudorange and carrier phase measurements from satellites with known positions to receivers with known positions. The effects of atmospheric errors, multipath and noise were quantified using 3 different positioning models: single point positioning, between receiver single differences and double differences. Differential solutions were shown to mitigate atmospheric biases, while not mitigating multipath effects and increasing noise effects relative to single point solutions. 
The positioning simulation was validated by conducting an experimental control point survey in Didsbury, Alberta on an Alberta Survey Control Marker (ASCM) with known coordinates. Data was collected using test receiver 1, and positions were computed in single point mode using pseudoranges as well as using an integer search to optimize carrier phase ambiguities. The carrier phase was found to not be helpful to the position calculation due to the search space being much too small to encompass the bias caused by atmospheric effects as well as due to only one frequency of observations being available on the low-cost receiver. The author's positioning methods were compared to results obtained by processing the same raw data with RTK Post software and while the author yielded higher error in the horizontal, the result was much closer to the true coordinate in the horizontal overall. Temporal variability of satellite geometry was observed to cause trends in the positioning results which were not simply caused by random noise. While the standard deviation was used to estimate a noise category for the receiver, more study would be required if the receiver noise characteristics are to be determined with precision. This is true for the VN-300 system (test receiver 3) as well as the Huawei P20 (test receiver 2) which were also utilized in this research.

The simulation framework was expanded into a thorough pitch and heading simulation. Raw measurements were generated just as was done with the positioning simulation, but 2 rover antennas with known positions were utilized instead of 1 . With the known pitch and heading of the system, a simulated partial attitude measurement was generated by adding gaussian noise with the standard deviation observed with the VN-300 recording pitch and heading in a static environment for approximately 10 minutes. The simulation computed pitch and heading using GPS coordinates only, IMU only and also a GPS/IMU fused solution using a weighted average approach with the estimated standard deviation of the GPS solution and the known standard 
deviation of the IMU. Interestingly the GPS seldom improved the IMU solution in fusion, but this might be due to lack of access to a Kalman filter because the simulation only repeated measurements with static satellite geometry rather than using temporally variable constellations. It is also important to note that single point solutions were found to perform better than differential solutions due to cancellation of bias effects and reduced noise by comparison. This may be counterintuitive to many designers, because in general, using more sensors is correlated with higher accuracy and precision.

The partial attitude simulation was consisted of data sets and data series'. A set determines the dual antenna separation distance, while a series determines the pseudorange noise (and by extension the receiver quality) of the data set. Pseudorange noise was varied at $10 \mathrm{~cm}$, $1 \mathrm{~m}$ and $3 \mathrm{~m}$ to yield 3 series', and antenna separation was varied from $1 \mathrm{~m}$ up to $90 \mathrm{~m}$ for a total of 8 data sets per series. A double interpolation method was proposed which could aid a mission designer in receiver selection or in setting out the necessary antenna separation distance for receivers with known noise characteristics. This was found to give a novel method of directly quantifying the necessary mission design criteria rather than reverting to higher cost receivers, differential solutions to access the carrier phase or buying an IMU because of known noise characteristics. This has potential to save costs as well as make mission designing an easier task on the whole.

The partial attitude simulation was validated with a control point survey on Carleton University campus in Ottawa, Ontario. Test receivers 1 and 2 were placed on 2 control points with known coordinates. Receiver 2 had a much noisier position solution than the receiver 1 , which makes direct comparison to the simulation difficult. However, several findings from the survey validated the simulations. For instance, the control points were $90 \mathrm{~m}$ apart, as was the 
case with the $8^{\text {th }}$ simulation set. Just as was observed in simulation, the data collected yielded pitch and heading with very little bias due to cancellation of atmospheric effects, which supports the earlier contention that single point solutions are preferable to differential solutions. In addition, many angular specification categories were met with high success rates due to the significant separation of the antennas. While this does not indicate that low-cost undedicated systems are feasible for small platforms, it does hold implications for larger platforms. For example, many marine seismic vessels meet this $90 \mathrm{~m}$ length description as a platform, so this suggests that a low-cost solution for pitch and heading could be considered by a ship designer, assuming a safety factor is used to consider possible multipath effects from the water surface. A simple static Kalman filter was also shown to drastically improve the partial attitude solution when propagated covariance information from the least squares solution was utilized.

It is also interesting to note that Chaochao Wang [33] did not factor in a specific attitude precision into his design as a constraint, while this research was directed toward solving the problem of maximizing quality and minimizing cost quantitatively. Instead, Wang decided to integrate gyroscopic sensors onto the design platform in order to achieve a relative improvement to the attitude solution while sacrificing cost. In the case of JiunHang Keong's [32] research, the relationship between inter-antenna distance and heading accuracy is discussed qualitatively but is not directly factored into the design parameters. Instead, the farthest possible distance allowable while maintaining the platform heading parallel to GNSS vector heading was used for a combination of accuracy maximization and computation convenience. To the author's knowledge, the interpolation technique presented in this thesis is currently the only quantitative technique which directly addresses the precision and accuracy design constraint problem. 


\subsection{Future Recommendations}

While the author has made some novel contributions to the field of partial attitude determination, some steps could be taken to expand on the research:

- The LAMBDA ambiguity resolution technique could be implemented in order to increase viability of the carrier phase. This would likely allow for ambiguity resolution when a base station is utilized

- The simulation could be expanded to include three-receiver platforms. This would allow for computation of all orientation angles, accurately representing a full attitude determination system and would add complexity to the angle computations.

- The simulation could be expanded to a temporal analysis with changing satellite geometry. While the author predicts that the direct effects of satellite geometry would be minimal due to the close proximity of antennas to each other, there could be multipath implications due to its dependence on satellite geometry

- A different fusion technique could be used to improve the result from the simple weighted average used between the IMU and GPS solutions in simulation. This technique rarely improved the IMU-only solution; therefore, it would be worth testing a Kalman filter in a temporal analysis as an alternative

- A pair of receivers could be tested to determine the pseudorange noise properties and allow for a direct validation of the simulation. This would require connecting two receivers of the same quality to the same antenna, then logging simultaneous data. The difference in measured pseudoranges could be used to compute a standard deviation over the data set. 
- A dedicated system of two GPS receivers sharing an oscillator could be built where the budget allows. This would allow for partial attitude computation without interpolating or extrapolating data from 1 receiver. 


\section{Conclusion}

To conclude, the author has successfully developed a novel, quantitative method for lowcost, GNSS partial attitude mission design using interpolation of simulation data. Key findings are as follows:

- Pseudorange noise is proportional to pitch and heading noise but inversely proportional to success rates of meeting specifications. This can be used as a design requirement and minimum receiver quality (maximum noise) can be computed by interpolating the author's simulation results.

- Antenna separation is inversely proportional to pitch and heading noise but proportional to success rates of meeting specifications. This can be used to interpolate minimum antenna separation for a system with known receiver noise.

- Single point positioning solutions are preferable to differential solutions when being used to compute attitude, unless the differential solution is used to optimize the search space for carrier phase ambiguity resolution.

- If high noise receivers have a sufficiently small antenna separation (approximately $3 \mathrm{~m}$ for a system with $1 \mathrm{~m}$ receiver noise standard deviation $10 \mathrm{~m}$ for a system with $3 \mathrm{~m}$ receiver noise standard deviation), there is a potential for heading solutions in any quadrant, which should be mitigated against strongly as it could have adverse effects on navigation.

- Kalman filtering can significantly improve the solutions from GPS-only partial attitude systems when the least squares covariance matrix is used to construct the measurement noise. 


\section{References}

[1] "IMU Errors and Their Effects," Novatel GNSS, 2014. [Online]. Available: https://www.novatel.com/assets/Documents/Bulletins/APN064.pdf. [Accessed: 21-May2019].

[2] G. Xu, GPS Theory, Algorithms and Applications, 2nd ed. Potsdam, Germany, 2007.

[3] J. Saastamoinen, "Contributions To The Theory Of Atmospheric Refraction (1)," Bull. Geod., vol. 105, no. 1, pp. 279-298, 1972.

[4] H. S. Hopfield, "Tropospheric Effect on Electromagnetically Measured Range: Prediction from Surface Weather Data," Radio Sci., vol. 6, no. 3, pp. 357-367, 1971.

[5] C. Satirapod and P. Chalermwattanachai, "Impact of Different Tropospheric Models on GPS Baseline Accuracy: Case Study in Thailand," J. Glob. Position. Syst., vol. 4, no. 1, pp. 36-40, 2005.

[6] P. Wielgosz, J. Paziewski, A. Krankowski, K. Kroszczyński, and M. Figurski, "Results of the application of tropospheric corrections from different troposphere models for precise GPS rapid static positioning," Acta Geophys., vol. 60, no. 4, pp. 1236-1257, 2012.

[7] M. J. Dunn, NAVSTAR GPS Space Segment/Navigation User Segment Interfaces IS-GPS200. National Coordination Office for Space-Based Positioning, Navigation, and Timing, 2018.

[8] N. Jakowski, C. Mayer, M. M. Hoque, V. Wilken, and P. S. Cannon, "Total electron content models and their use in ionosphere monitoring," Radio Sci., vol. 46, no. 5, pp. 1$11,2011$. 
[9] P. S. Cannon, "Mitigation and exploitation of the ionosphere: A military perspective," Radio Sci., vol. 44, no. 1, p. n/a-n/a, 2009.

[10] P. Bolla and K. Borre, "Performance analysis of dual-frequency receiver using combinations of GPS L1, L5, and L2 civil signals," J. Geod., vol. 93, no. 3, pp. 437-447, 2019.

[11] J. Jones and P. Fenton, "Theory and Performance of the Pulse Aperture Correlator," pp. $1-13,2004$.

[12] G. Seepersad and S. Bisnath, "Reduction of PPP convergence period through pseudorange multipath and noise mitigation," GPS Solut., vol. 19, no. 3, pp. 369-379, 2015.

[13] M. Olynik, M. G. Petovello, M. E. Cannon, and G. Lachapelle, “Temporal Variability of GPS Error Sources and Their Effect on Relative Positioning Accuracy," Inst. Navig. Natl. Tech. Meet., pp. 1-12, 2002.

[14] P. J. G. Teunissen, “The least-squares ambiguity decorrelation adjustment: a method for fast GPS integer ambiguity estimation,” J. Geod., pp. 65-82, 1995.

[15] D. B. Cox and J. D. W. Brading, "Integration of LAMBDA ambiguity resolution with Kalman filter for relative navigation of spacecraft," Navig. J. Inst. Navig., vol. 47, no. 3, pp. 205-210, 2000.

[16] Junjie Liu, "Implementation and Analysis of GPS Ambiguity Resolution Strategies in Single and Multiple Reference Station Scenarios,” no. 20168, 2003.

[17] G. Blewitt, "Carrier phase ambiguity resolution for the Global Positioning System applied to geodetic baselines up to $2000 \mathrm{~km}$," J. Geophys. Res. Solid Earth, vol. 94, no. B8, pp. 
$10187-10203,2008$.

[18] P. Collins, O. I. S. Lahaye, and P. He, "Undifferenced GPS Ambiguity Resolution Using the Decoupled Clock Model and Ambiguity Datum Fixing,”pp. 123-135, 2010.

[19] S. Owen et al., "Single receiver phase ambiguity resolution with GPS data," J. Geod., vol. 84, no. 5, pp. 327-337, 2010.

[20] D. Laurichesse, F. Mercier, and J. P. Berthias, "Real-time PPP with undifferenced integer ambiguity resolution, experimental results," Proc. 23rd Int. Tech. Meet. Satell. Div. Inst. Navig. (ION GNSS 2010), pp. 2534-2544, 2010.

[21] C. Deng, J. Cui, W. Tang, X. Zou, and L. Shu, "Reliable real-time triple-frequency cycle slip detection and recovery with adaptive detection thresholds," Meas. Sci. Technol., 2019.

[22] L. Zhenkun and H. Shunji, "Research on ambiguity resolution aided with triple difference,” J. Syst. Eng. Electron., vol. 19, no. 6, pp. 1090-1096, 2008.

[23] Kim, Donghyun and R. B. Langley, "GPS Ambiguity Resolution and Validation: Methodologies, Trends and Issues," J. Navig., vol. 61, no. 03, pp. 592-602, 2000.

[24] J. Geng, X. Meng, A. H. Dodson, and F. N. Teferle, "Integer ambiguity resolution in precise point positioning: Method comparison," J. Geod., vol. 84, no. 9, pp. 569-581, 2010.

[25] S. Verhagen and P. J. G. Teunissen, "The ratio test for future GNSS ambiguity resolution,” GPS Solut., vol. 17, no. 4, pp. 535-548, 2013.

[26] N. Nadarajah, P. J. G. Teunissen, and N. Raziq, "Instantaneous BeiDou-GPS attitude determination: A performance analysis," Adv. Sp. Res., vol. 54, no. 5, pp. 851-862, 2014. 
[27] Y. Yang, X. Mao, and W. Tian, "A novel method for low-cost MIMU aiding GNSS attitude determination,” Meas. Sci. Technol., vol. 27, no. 7, p. 75003, 2016.

[28] J. Liu, Q. Chen, Y. Shu, W. Yao, X. Niu, and P. Xu, "High-rate multi-GNSS attitude determination: experiments, comparisons with inertial measurement units and applications of GNSS rotational seismology to the 2011 Tohoku Mw9.0 earthquake," Meas. Sci. Technol., vol. 30, no. 2, p. 024003, 2018.

[29] B. Wang, L. Miao, S. Wang, and J. Shen, "A constrained LAMBDA method for GPS attitude determination," GPS Solut., vol. 13, no. 2, pp. 97-107, 2009.

[30] S. Li, T. Xu, Q. Wang, K. Deng, and G. Chang, "GNSS attitude determination method through vectorisation approach," IET Radar, Sonar Navig., vol. 11, no. 10, pp. 1477$1482,2017$.

[31] X. Sun, C. Han, and P. Chen, "Instantaneous GNSS attitude determination: A Monte Carlo sampling approach,” Acta Astronaut., vol. 133, no. January, pp. 24-29, 2017.

[32] Jiun Han Keong and J. Keong, "Determining Heading and Pitch Using a Single Difference GPS / GLONASS Approach by,” no. 20134, p. 144, 1999.

[33] C. Wang, "Development of a Low-cost GPS-based Attitude Determination System," University of Calgary, 2003.

[34] "3 Spheres for 3 Letters," ABC Maths, 2008. [Online]. Available: http://abcmathsblog.blogspot.com/2008/10/trois-sphres-pour-trois-lettres.html. [Accessed: 17-Aug-2019].

[35] S. Miller, X. Zhang, and A. Spanias, Multipath Effects in GPS Receivers, vol. 8, no. 1. 
Morgan \& Claypool Publishers, 2016.

[36] C. Erickson, "UCGE Reports Investigations of C / A Code and Carrier Measurements and Techniques for Rapid Static GPS Surveys,” University of Calgary, 1992.

[37] V. Vimal, “GPS Processing Techniques \& Some Applications,” 2014. [Online]. Available: https://www.slideshare.net/vrincevimal/gps-processing-techniques-someapplications. [Accessed: 17-Aug-2019].

[38] P. R. Wolf and C. D. Ghilani, Adjustment Computations: Statistics and Least Squares in Surveying and GIS. New York, NY: John Wiley \& Sons, Inc., 1997.

[39] R. G. Brown and P. Y. C. Hwang, Introduction to Random Signals and Applied Kalman Filtering, 4th ed. Hoboken, NJ: John Wiley \& Sons, Inc., 2012.

[40] H. B. Mitchell, Multi-Sensor Data Fusion. Berlin: Springer Berlin Heidelberg, 2010. 


\section{Appendix A: $10 \mathrm{~cm}$ Pseudorange Noise Standard}

\section{Deviation Pitch and Heading Simulation Results}

\section{Set A-1: 1 m Dual Antenna Separation}
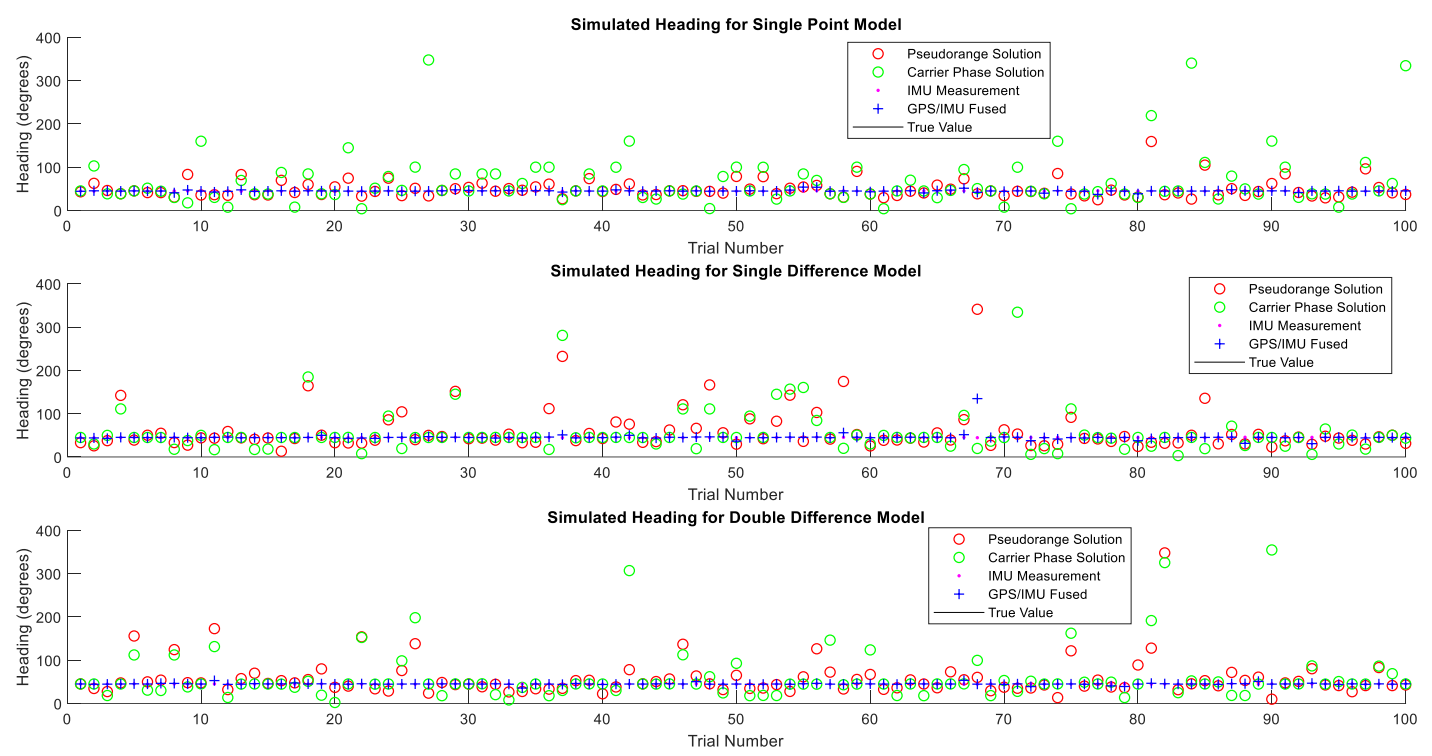

Figure 48: Computed and Measured Headings for Simulation Set A-1 


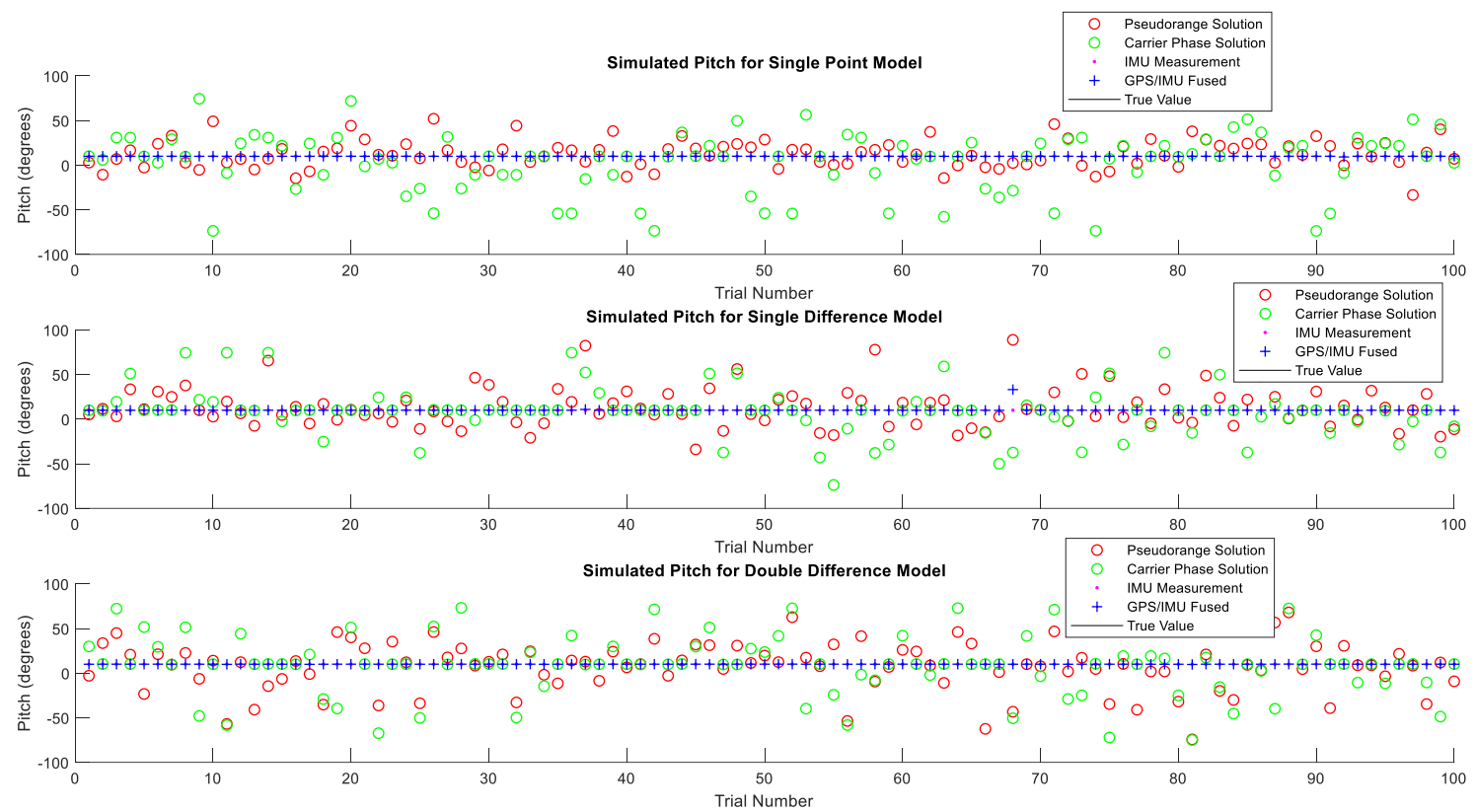

Figure 49: Computed and Measured Pitch for Simulation Set A-1

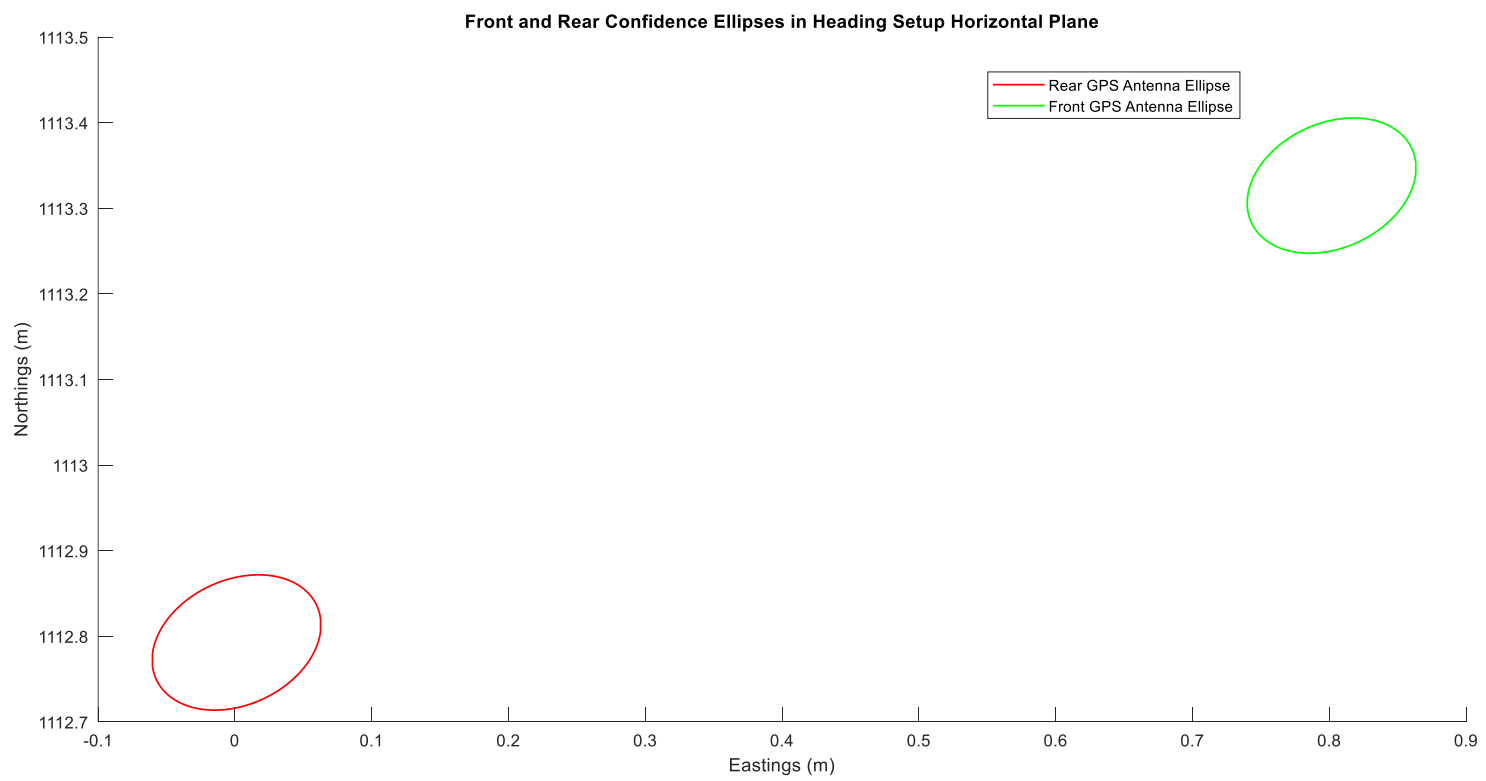

Figure 50: Propagated 95\% Confidence Ellipses for Front and Rear GPS Antennas in Simulated Data Set A-1 
Table 31: Mean Global Error Statistics of Unfused Pitch for Simulation Set A-1

\begin{tabular}{|c|c|c|c|}
\hline Method & $\begin{array}{c}\text { Measured Standard } \\
\text { Deviation } \\
\text { (degrees) }\end{array}$ & $\begin{array}{c}\text { Estimated } \\
\text { Standard } \\
\text { Deviation } \\
\text { (degrees) }\end{array}$ & $\begin{array}{c}\text { Mean RMS } \\
\text { Error } \\
\text { (degrees) }\end{array}$ \\
\hline Single Point GPS (Pseudoranges) & 15.769 & 12.779 & 9.117 \\
\hline Single Point GPS (Carrier Phase) & 33.264 & 24.640 & 0.050 \\
\hline $\begin{array}{c}\text { Single Difference GPS } \\
\text { (Pseudoranges) }\end{array}$ & 22.561 & 17.098 & 10.739 \\
\hline $\begin{array}{c}\text { Single Difference GPS (Carrier } \\
\text { Phase) }\end{array}$ & 27.603 & 17.057 & 0.038 \\
\hline $\begin{array}{c}\text { Double Difference GPS } \\
\text { (Pseudoranges) }\end{array}$ & 23.283 & 20.526 & 8.490 \\
\hline $\begin{array}{c}\text { Double Difference GPS (Carrier } \\
\text { Phase) }\end{array}$ & 30.602 & 22.521 & 0.061 \\
\hline IMU Measurement & 0.007 & - & 0.006 \\
\hline
\end{tabular}

Table 32: Mean Global Error Statistics of Unfused Heading for Simulation Set A-1

\begin{tabular}{|c|c|c|c|}
\hline Method & $\begin{array}{c}\text { Measured Standard } \\
\text { Deviation } \\
\text { (degrees) }\end{array}$ & $\begin{array}{c}\text { Estimated } \\
\text { Standard } \\
\text { Deviation } \\
\text { (degrees) }\end{array}$ & $\begin{array}{c}\text { Mean RMS } \\
\text { Error } \\
\text { (degrees) }\end{array}$ \\
\hline Single Point GPS (Pseudoranges) & 19.880 & 12.825 & 53.769 \\
\hline Single Point GPS (Carrier Phase) & 62.022 & 28.296 & 0.163 \\
\hline $\begin{array}{c}\text { Single Difference GPS } \\
\text { (Pseudoranges) }\end{array}$ & 47.757 & 22.831 & 35.088 \\
\hline $\begin{array}{c}\text { Single Difference GPS (Carrier } \\
\text { Phase) }\end{array}$ & 56.885 & 20.697 & 0.068 \\
\hline $\begin{array}{c}\text { Double Difference GPS } \\
\text { (Pseudoranges) }\end{array}$ & 42.724 & 19.831 & 28.739 \\
\hline $\begin{array}{c}\text { Double Difference GPS (Carrier } \\
\text { Phase) }\end{array}$ & 59.971 & 21.806 & 0.146 \\
\hline IMU Measurement & 0.320 & - & 0.269 \\
\hline
\end{tabular}


Table 33: Mean Global Error Statistics of GPS/IMU Fused Pitch for Simulation Set A-1

\begin{tabular}{|c|c|c|c|}
\hline Method & $\begin{array}{c}\text { Measured Standard } \\
\text { Deviation } \\
\text { (degrees) }\end{array}$ & $\begin{array}{c}\text { Estimated } \\
\text { Standard } \\
\text { Deviation } \\
\text { (degrees) }\end{array}$ & $\begin{array}{c}\text { Mean RMS } \\
\text { Error } \\
\text { (degrees) }\end{array}$ \\
\hline Single Point GPS (Pseudoranges) & 0.092 & 0.033 & 0.194 \\
\hline Single Point GPS (Carrier Phase) & 6.742 & 4.346 & 0.024 \\
\hline $\begin{array}{c}\text { Single Difference GPS } \\
\text { (Pseudoranges) }\end{array}$ & 2.316 & 0.266 & 0.194 \\
\hline $\begin{array}{c}\text { Single Difference GPS (Carrier } \\
\text { Phase) }\end{array}$ & 6.337 & 3.498 & 0.018 \\
\hline $\begin{array}{c}\text { Double Difference GPS } \\
\text { (Pseudoranges) }\end{array}$ & 0.049 & 0.028 & 0.198 \\
\hline $\begin{array}{c}\text { Double Difference GPS (Carrier } \\
\text { Phase) }\end{array}$ & 7.714 & 4.151 & 0.006 \\
\hline IMU Measurement & 0.007 & - & \\
\hline
\end{tabular}

Table 34: Mean Global Error Statistics of GPS/IMU Fused Heading for Simulation Set A-1

\begin{tabular}{|c|c|c|c|}
\hline Method & $\begin{array}{c}\text { Measured Standard } \\
\text { Deviation } \\
\text { (degrees) }\end{array}$ & $\begin{array}{c}\text { Estimated } \\
\text { Standard } \\
\text { Deviation } \\
\text { (degrees) }\end{array}$ & $\begin{array}{c}\text { Mean RMS } \\
\text { Error } \\
\text { (degrees) }\end{array}$ \\
\hline Single Point GPS (Pseudoranges) & 2.149 & 1.123 & 3.663 \\
\hline Single Point GPS (Carrier Phase) & 54.142 & 21.719 & 0.077 \\
\hline $\begin{array}{c}\text { Single Difference GPS } \\
\text { (Pseudoranges) }\end{array}$ & 9.489 & 2.301 & 3.828 \\
\hline $\begin{array}{c}\text { Single Difference GPS (Carrier } \\
\text { Phase) }\end{array}$ & 49.911 & 17.033 & 0.034 \\
\hline $\begin{array}{c}\text { Double Difference GPS } \\
\text { (Pseudoranges) }\end{array}$ & 2.036 & 0.971 & 4.181 \\
\hline $\begin{array}{c}\text { Double Difference GPS (Carrier } \\
\text { Phase) }\end{array}$ & 45.847 & 17.767 & 0.072 \\
\hline IMU Measurement & 0.320 & - & 0.269 \\
\hline
\end{tabular}


Table 35: Success Rates of Reaching Angular Specifications of Unfused Pitch for Simulation Data Set A-1

\begin{tabular}{|c|c|c|c|c|c|c|c|c|}
\hline & \multicolumn{7}{|c|}{ Portion of Trials in Which Target Specification was reached } \\
\hline & $\pm 0.5^{\circ}$ & $\pm 1^{\circ}$ & $\pm 2^{\circ}$ & $\pm 5^{\circ}$ & $\pm 10^{\circ}$ & $\pm 15^{\circ}$ & $\pm 20^{\circ}$ & $\pm 25^{\circ}$ \\
\hline Method & 55 & 59 & 60 & 64 & 79 & 86 & 91 & 95 \\
\hline $\begin{array}{c}\text { Single Point GPS } \\
\text { (Pseudoranges) }\end{array}$ & 65 & 65 & 65 & 65 & 65 & 75 & 79 & 85 \\
\hline $\begin{array}{c}\text { Single Point GPS (Carrier } \\
\text { Phase) }\end{array}$ & 57 & 59 & 60 & 66 & 71 & 75 & 77 & 81 \\
\hline $\begin{array}{c}\text { Single Difference GPS } \\
\text { (Pseudoranges) }\end{array}$ & 77 & 77 & 77 & 77 & 81 & 88 & 91 & 92 \\
\hline $\begin{array}{c}\text { Single Difference GPS } \\
\text { (Carrier Phase) }\end{array}$ & 45 & 46 & 48 & 56 & 67 & 74 & 83 & 87 \\
\hline $\begin{array}{c}\text { Double Difference GPS } \\
\text { (Pseudoranges) }\end{array}$ & 74 & 74 & 74 & 74 & 75 & 81 & 85 & 90 \\
\hline $\begin{array}{c}\text { Double Difference GPS } \\
\text { (Carrier Phase) }\end{array}$ & 100 & 100 & 100 & 100 & 100 & 100 & 100 & 100 \\
\hline IMU Measured & & & & & & & & \\
\hline
\end{tabular}

Table 36: Success Rates of Reaching Angular Specifications of Unfused Heading for Simulation Data Set A-1

\begin{tabular}{|c|c|c|c|c|c|c|c|c|}
\hline & \multicolumn{7}{|c|}{ Portion of Trials in Which Target Specification was reached } \\
\hline Method & $\pm 0.5^{\circ}$ & $\pm 1^{\circ}$ & $\pm 2^{\circ}$ & $\pm 5^{\circ}$ & $\pm 10^{\circ}$ & $\pm 15^{\circ}$ & $\pm 20^{\circ}$ & $\pm 25^{\circ}$ \\
\hline $\begin{array}{c}\text { Single Point GPS } \\
\text { (Pseudoranges) }\end{array}$ & 45 & 46 & 51 & 56 & 71 & 81 & 85 & 89 \\
\hline $\begin{array}{c}\text { Single Point GPS (Carrier } \\
\text { Phase) }\end{array}$ & 58 & 58 & 66 & 66 & 71 & 71 & 78 & 81 \\
\hline $\begin{array}{c}\text { Single Difference GPS } \\
\text { (Pseudoranges) }\end{array}$ & 58 & 60 & 61 & 64 & 68 & 73 & 75 & 77 \\
\hline $\begin{array}{c}\text { Single Difference GPS } \\
\text { (Carrier Phase) }\end{array}$ & 70 & 70 & 71 & 75 & 82 & 82 & 82 & 82 \\
\hline $\begin{array}{c}\text { Double Difference GPS } \\
\text { (Pseudoranges) }\end{array}$ & 53 & 53 & 54 & 56 & 65 & 74 & 77 & 80 \\
\hline $\begin{array}{c}\text { Double Difference GPS } \\
\text { (Carrier Phase) }\end{array}$ & 71 & 72 & 72 & 74 & 79 & 79 & 79 & 79 \\
\hline IMU Measured & 91 & 100 & 100 & 100 & 100 & 100 & 100 & 100 \\
\hline
\end{tabular}


Table 37: Success Rates of Reaching Angular Specifications of Fused Pitch for Simulation Data Set A-1

\begin{tabular}{|c|c|c|c|c|c|c|c|c|}
\hline & \multicolumn{7}{|c|}{ Portion of Trials in Which Target Specification was reached } \\
\hline & $\pm 0.5^{\circ}$ & $\pm 1^{\circ}$ & $\pm 2^{\circ}$ & $\pm 5^{\circ}$ & $\pm 10^{\circ}$ & $\pm 15^{\circ}$ & $\pm 20^{\circ}$ & $\pm 25^{\circ}$ \\
\hline Method & 100 & 100 & 100 & 100 & 100 & 100 & 100 & 100 \\
\hline $\begin{array}{c}\text { Single Point GPS } \\
\text { (Pseudoranges) }\end{array}$ & 65 & 66 & 80 & 94 & 96 & 96 & 100 & 100 \\
\hline $\begin{array}{c}\text { Single Point GPS (Carrier } \\
\text { Phase) }\end{array}$ & 100 & 100 & 100 & 100 & 100 & 100 & 100 & 100 \\
\hline $\begin{array}{c}\text { Single Difference GPS } \\
\text { (Pseudoranges) }\end{array}$ & 77 & 77 & 84 & 93 & 95 & 95 & 99 & 99 \\
\hline $\begin{array}{c}\text { Single Difference GPS } \\
\text { (Carrier Phase) }\end{array}$ & 100 & 100 & 100 & 100 & 100 & 100 & 100 & 100 \\
\hline $\begin{array}{c}\text { Double Difference GPS } \\
\text { (Pseudoranges) }\end{array}$ & 74 & 74 & 83 & 97 & 97 & 97 & 100 & 100 \\
\hline $\begin{array}{c}\text { Double Difference GPS } \\
\text { (Carrier Phase) }\end{array}$ & 100 & 100 & 100 & 100 & 100 & 100 & 100 & 100 \\
\hline IMU Measured & & & & & & & & \\
\hline
\end{tabular}

Table 38: Success Rates of Reaching Angular Specifications of Fused Heading for Simulation Data Set A-1

\begin{tabular}{|c|c|c|c|c|c|c|c|c|}
\hline & \multicolumn{7}{|c|}{ Portion of Trials in Which Target Specification was reached } \\
\hline & $\pm 0.5^{\circ}$ & $\pm 1^{\circ}$ & $\pm 2^{\circ}$ & $\pm 5^{\circ}$ & $\pm 10^{\circ}$ & $\pm 15^{\circ}$ & $\pm 20^{\circ}$ & $\pm 25^{\circ}$ \\
\hline Method & 70 & 82 & 91 & 96 & 100 & 100 & 100 & 100 \\
\hline $\begin{array}{c}\text { Single Point GPS } \\
\text { (Pseudoranges) }\end{array}$ & 58 & 59 & 66 & 70 & 72 & 77 & 87 & 88 \\
\hline $\begin{array}{c}\text { Single Point GPS (Carrier } \\
\text { Phase) }\end{array}$ & 79 & 86 & 90 & 94 & 97 & 97 & 98 & 98 \\
\hline $\begin{array}{c}\text { Single Difference GPS } \\
\text { (Pseudoranges) }\end{array}$ & 70 & 71 & 71 & 81 & 82 & 87 & 89 & 89 \\
\hline $\begin{array}{c}\text { Single Difference GPS } \\
\text { (Carrier Phase) }\end{array}$ & 74 & 83 & 92 & 96 & 99 & 100 & 100 & 100 \\
\hline $\begin{array}{c}\text { Double Difference GPS } \\
\text { (Pseudoranges) }\end{array}$ & 70 & 71 & 72 & 77 & 80 & 80 & 82 & 85 \\
\hline $\begin{array}{c}\text { Double Difference GPS } \\
\text { (Carrier Phase) }\end{array}$ & 91 & 100 & 100 & 100 & 100 & 100 & 100 & 100 \\
\hline IMU Measured & & & & & & & & \\
\hline
\end{tabular}


Table 39: Pitch / Heading Correlation Coefficients for Each Computation Method in Simulation Data Set A-1

\begin{tabular}{|c|c|c|}
\hline & \multicolumn{2}{|c|}{ Pitch / Heading Correlation Coefficient } \\
\hline Method & Unfused & Fused \\
\hline $\begin{array}{c}\text { Single Point GPS } \\
\text { (Pseudoranges) }\end{array}$ & 0.858 & 0.107 \\
\hline $\begin{array}{c}\text { Single Difference GPS } \\
\text { (Pseudoranges) }\end{array}$ & 0.937 & 0.146 \\
\hline $\begin{array}{c}\text { Double Difference GPS } \\
\text { (Pseudoranges) }\end{array}$ & 0.573 & 0.046 \\
\hline
\end{tabular}

\section{Set A-2: 3 m Dual Antenna Separation}

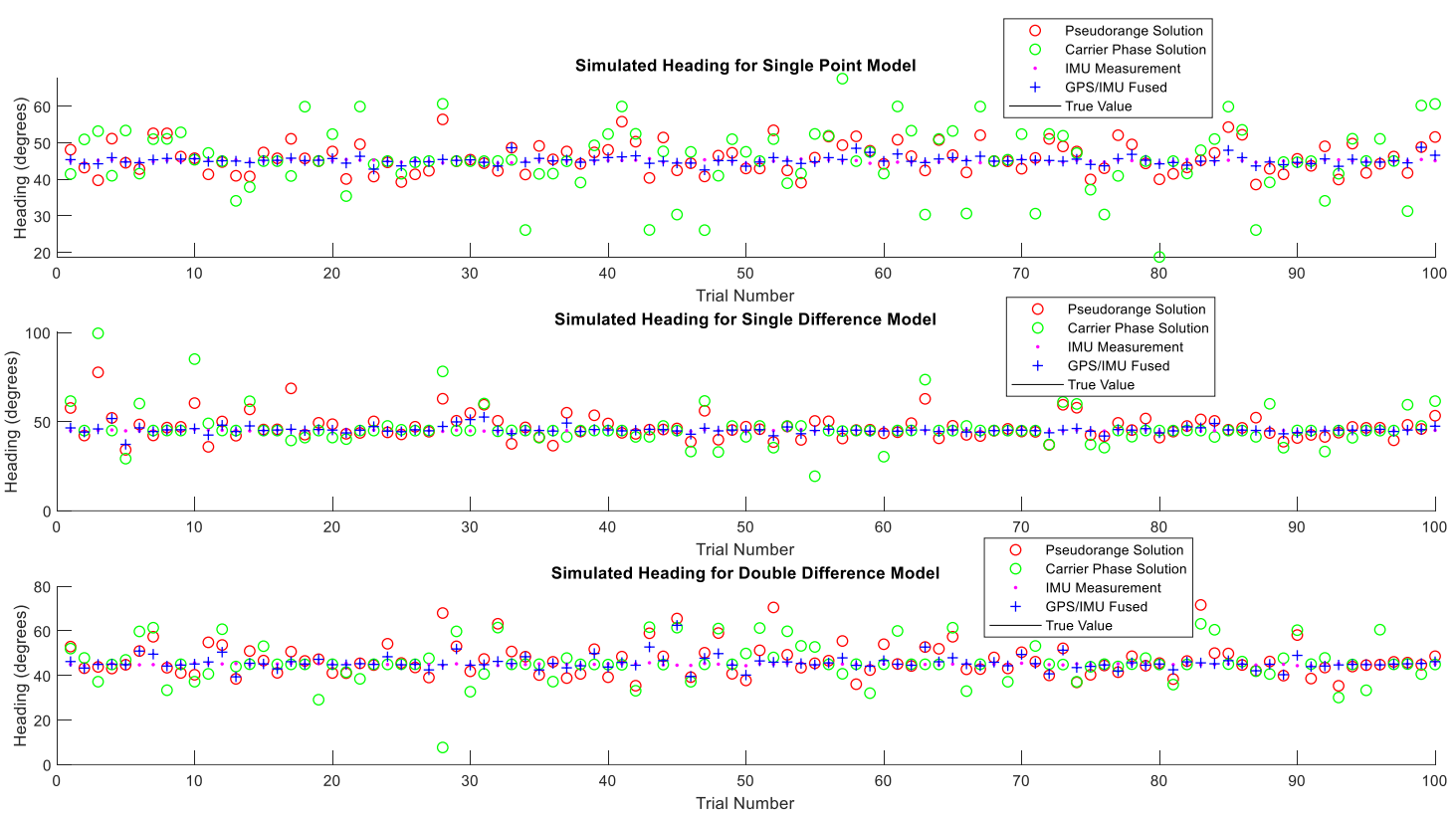

Figure 51: Computed and Measured Headings for Simulation Set A-2 


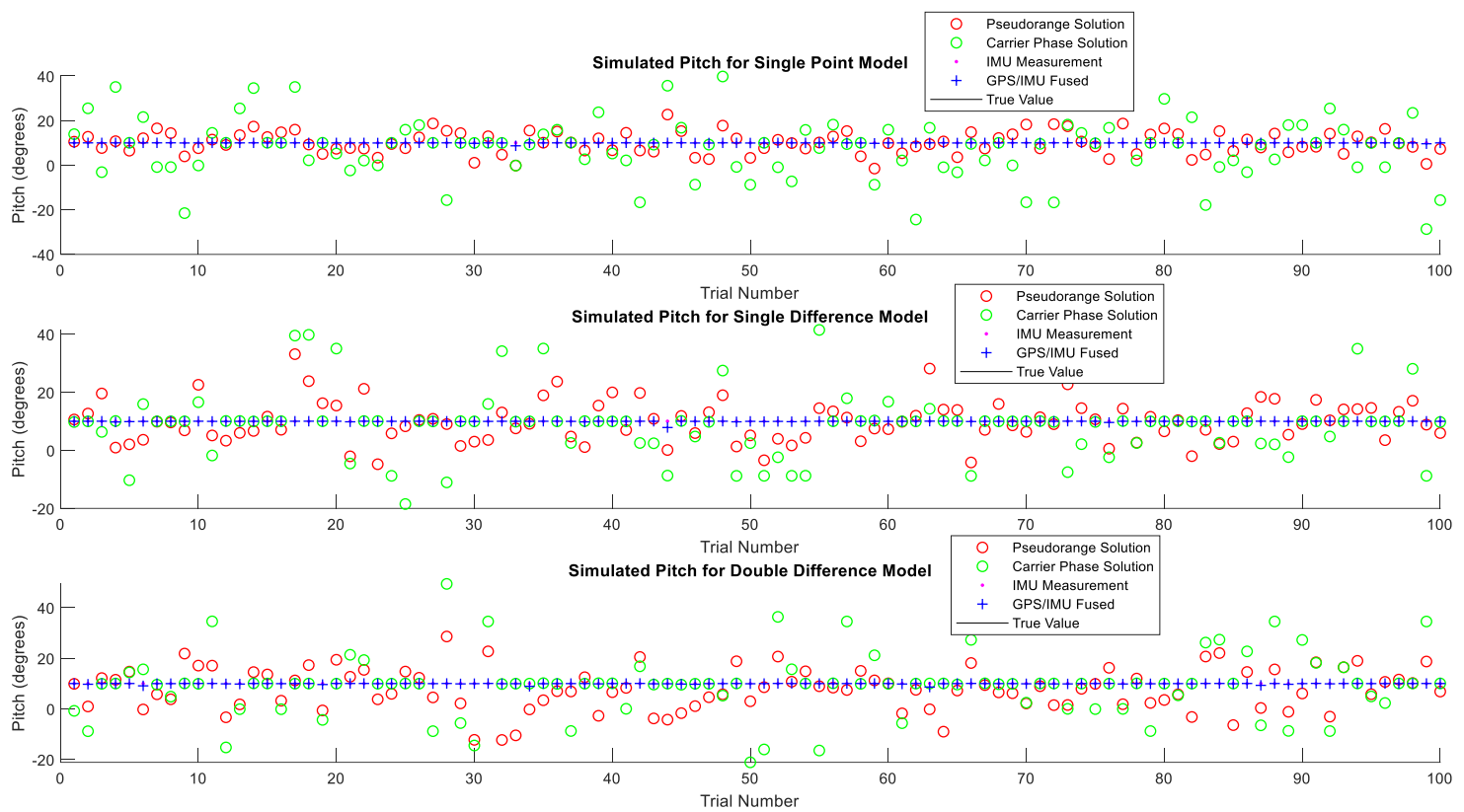

Figure 52: Computed and Measured Pitch for Simulation Set A-2

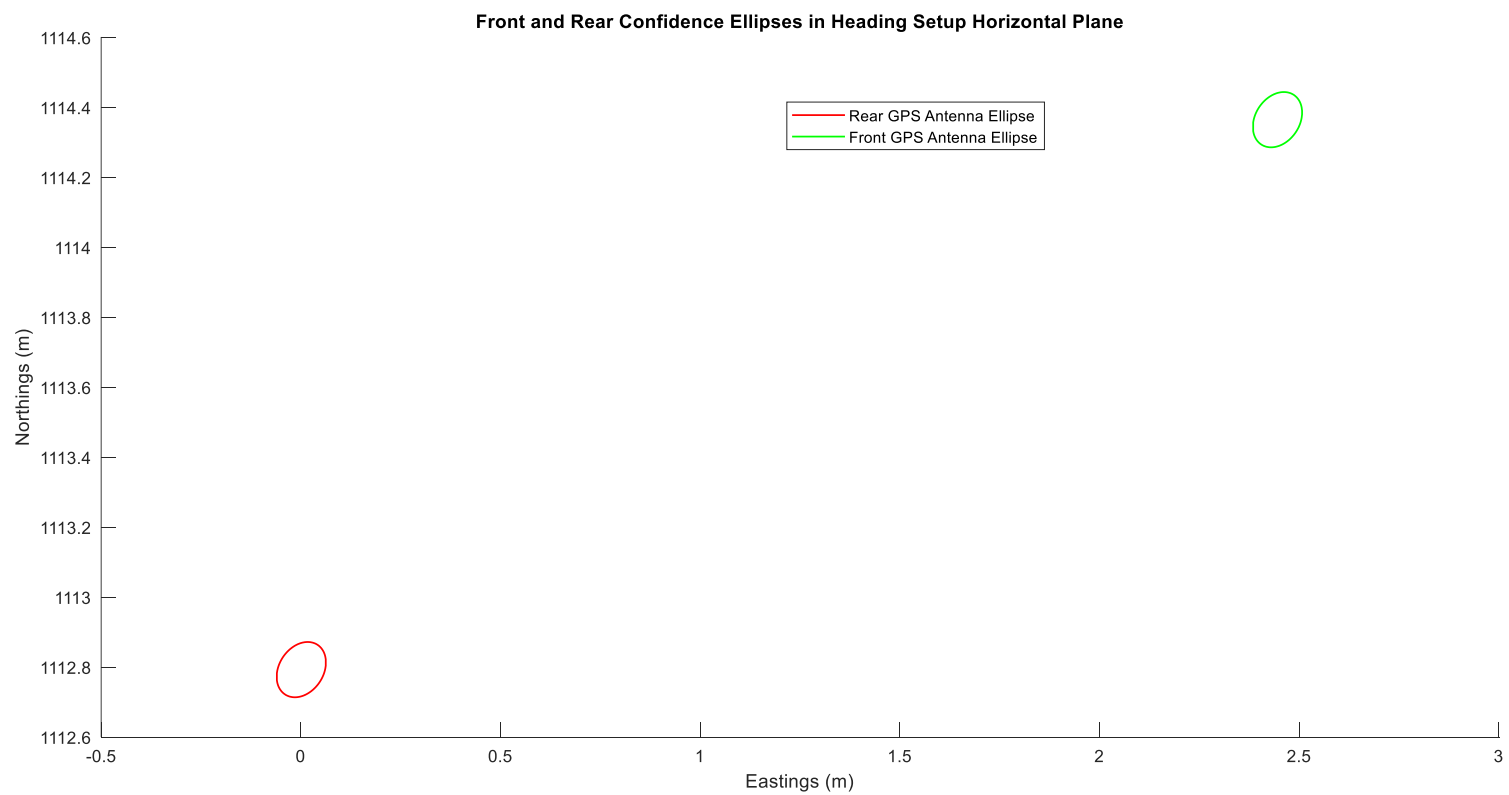

Figure 53: Propagated 95\% Confidence Ellipses for Front and Rear GPS Antennas in Simulated Data Set A-2 
Table 40: Mean Global Error Statistics of Unfused Pitch for Simulation Set A-2

\begin{tabular}{|c|c|c|c|}
\hline Method & $\begin{array}{c}\text { Measured Standard } \\
\text { Deviation } \\
\text { (degrees) }\end{array}$ & $\begin{array}{c}\text { Estimated } \\
\text { Standard } \\
\text { Deviation } \\
\text { (degrees) }\end{array}$ & $\begin{array}{c}\text { Mean RMS } \\
\text { Error } \\
\text { (degrees) }\end{array}$ \\
\hline Single Point GPS (Pseudoranges) & 4.881 & 2.547 & 4.017 \\
\hline Single Point GPS (Carrier Phase) & 13.424 & 0.012 & 9.816 \\
\hline $\begin{array}{c}\text { Single Difference GPS } \\
\text { (Pseudoranges) }\end{array}$ & 7.115 & 2.476 & 5.611 \\
\hline $\begin{array}{c}\text { Single Difference GPS (Carrier } \\
\text { Phase) }\end{array}$ & 11.071 & 0.012 & 6.506 \\
\hline $\begin{array}{c}\text { Double Difference GPS } \\
\text { (Pseudoranges) }\end{array}$ & 8.269 & 1.82 & 6.893 \\
\hline $\begin{array}{c}\text { Double Difference GPS (Carrier } \\
\text { Phase) }\end{array}$ & 12.179 & 0.022 & 7.567 \\
\hline IMU Measurement & 0.007 & - & 0.005 \\
\hline
\end{tabular}

Table 41: Mean Global Error Statistics of Unfused Heading for Simulation Set A-2

\begin{tabular}{|c|c|c|c|}
\hline Method & $\begin{array}{c}\text { Measured Standard } \\
\text { Deviation } \\
\text { (degrees) }\end{array}$ & $\begin{array}{c}\text { Estimated } \\
\text { Standard } \\
\text { Deviation } \\
\text { (degrees) }\end{array}$ & $\begin{array}{c}\text { Mean RMS } \\
\text { Error } \\
\text { (degrees) }\end{array}$ \\
\hline Single Point GPS (Pseudoranges) & 4.164 & 2.43 & 3.427 \\
\hline Single Point GPS (Carrier Phase) & 9.022 & 0.01 & 6.601 \\
\hline $\begin{array}{c}\text { Single Difference GPS } \\
\text { (Pseudoranges) }\end{array}$ & 6.941 & 2.869 & 4.893 \\
\hline $\begin{array}{c}\text { Single Difference GPS (Carrier } \\
\text { Phase) }\end{array}$ & 10.51 & 0.01 & 5.265 \\
\hline $\begin{array}{c}\text { Double Difference GPS } \\
\text { (Pseudoranges) }\end{array}$ & 7.275 & 1.95 & 5.339 \\
\hline $\begin{array}{c}\text { Double Difference GPS (Carrier } \\
\text { Phase) }\end{array}$ & 9.088 & 0.026 & 5.902 \\
\hline IMU Measurement & 0.301 & - & 0.245 \\
\hline
\end{tabular}


Table 42: Mean Global Error Statistics of Fused Pitch for Simulation Set A-2

\begin{tabular}{|c|c|c|c|}
\hline Method & $\begin{array}{c}\text { Measured Standard } \\
\text { Deviation } \\
\text { (degrees) }\end{array}$ & $\begin{array}{c}\text { Estimated } \\
\text { Standard } \\
\text { Deviation } \\
\text { (degrees) }\end{array}$ & $\begin{array}{c}\text { Mean RMS } \\
\text { Error } \\
\text { (degrees) }\end{array}$ \\
\hline Single Point GPS (Pseudoranges) & 0.14 & 0.192 & 0.036 \\
\hline Single Point GPS (Carrier Phase) & 5.133 & 0.006 & 4.255 \\
\hline $\begin{array}{c}\text { Single Difference GPS } \\
\text { (Pseudoranges) }\end{array}$ & 0.222 & 0.188 & 0.057 \\
\hline $\begin{array}{c}\text { Single Difference GPS (Carrier } \\
\text { Phase) }\end{array}$ & 4.383 & 0.006 & 2.8 \\
\hline $\begin{array}{c}\text { Double Difference GPS } \\
\text { (Pseudoranges) }\end{array}$ & 0.217 & 0.179 & 0.091 \\
\hline $\begin{array}{c}\text { Double Difference GPS (Carrier } \\
\text { Phase) }\end{array}$ & 3.46 & 0.011 & 2.181 \\
\hline IMU Measurement & 0.007 & - & 0.005 \\
\hline
\end{tabular}

Table 43: Mean Global Error Statistics of Fused Heading for Simulation Set A-2

\begin{tabular}{|c|c|c|c|}
\hline Method & $\begin{array}{c}\text { Measured Standard } \\
\text { Deviation } \\
\text { (degrees) }\end{array}$ & $\begin{array}{c}\text { Estimated } \\
\text { Standard } \\
\text { Deviation } \\
\text { (degrees) }\end{array}$ & $\begin{array}{c}\text { Mean RMS } \\
\text { Error } \\
\text { (degrees) }\end{array}$ \\
\hline Single Point GPS (Pseudoranges) & 1.052 & 0.69 & 0.723 \\
\hline Single Point GPS (Carrier Phase) & 8.716 & 0.005 & 6.385 \\
\hline $\begin{array}{c}\text { Single Difference GPS } \\
\text { (Pseudoranges) }\end{array}$ & 1.893 & 0.885 & 1.123 \\
\hline $\begin{array}{c}\text { Single Difference GPS (Carrier } \\
\text { Phase) }\end{array}$ & 9.652 & 0.005 & 4.941 \\
\hline $\begin{array}{c}\text { Double Difference GPS } \\
\text { (Pseudoranges) }\end{array}$ & 3.013 & 0.68 & 1.799 \\
\hline $\begin{array}{c}\text { Double Difference GPS (Carrier } \\
\text { Phase) }\end{array}$ & 8.192 & 0.013 & 5.368 \\
\hline IMU Measurement & 0.301 & - & 0.245 \\
\hline
\end{tabular}


Table 44: Success Rates of Reaching Angular Specifications of Unfused Pitch for Simulation Data Set A-2

\begin{tabular}{|c|c|c|c|c|c|c|c|c|}
\hline & \multicolumn{7}{|c|}{ Portion of Trials in Which Target Specification was reached } \\
\hline & $\pm 0.5^{\circ}$ & $\pm 1^{\circ}$ & $\pm 2^{\circ}$ & $\pm 5^{\circ}$ & $\pm 10^{\circ}$ & $\pm 15^{\circ}$ & $\pm 20^{\circ}$ & $\pm 25^{\circ}$ \\
\hline Method & 54 & 58 & 62 & 82 & 99 & 100 & 100 & 100 \\
\hline $\begin{array}{c}\text { Single Point GPS } \\
\text { (Pseudoranges) }\end{array}$ & 69 & 69 & 69 & 73 & 86 & 90 & 94 & 95 \\
\hline $\begin{array}{c}\text { Single Point GPS (Carrier } \\
\text { Phase) }\end{array}$ & 56 & 60 & 66 & 80 & 93 & 98 & 99 & 100 \\
\hline $\begin{array}{c}\text { Single Difference GPS } \\
\text { (Pseudoranges) }\end{array}$ & 85 & 85 & 85 & 86 & 91 & 91 & 93 & 95 \\
\hline $\begin{array}{c}\text { Single Difference GPS } \\
\text { (Carrier Phase) }\end{array}$ & 62 & 64 & 69 & 80 & 93 & 99 & 100 & 100 \\
\hline $\begin{array}{c}\text { Double Difference GPS } \\
\text { (Pseudoranges) }\end{array}$ & 79 & 79 & 79 & 80 & 86 & 89 & 93 & 98 \\
\hline $\begin{array}{c}\text { Double Difference GPS } \\
\text { (Carrier Phase) }\end{array}$ & 100 & 100 & 100 & 100 & 100 & 100 & 100 & 100 \\
\hline IMU Measured & & & & & & & & \\
\hline
\end{tabular}

Table 45: Success Rates of Reaching Angular Specifications of Unfused Heading for Simulation Data Set A-2

\begin{tabular}{|c|c|c|c|c|c|c|c|c|}
\hline & \multicolumn{7}{|c|}{ Portion of Trials in Which Target Specification was reached } \\
\hline & $\pm 0.5^{\circ}$ & $\pm 1^{\circ}$ & $\pm 2^{\circ}$ & $\pm 5^{\circ}$ & $\pm 10^{\circ}$ & $\pm 15^{\circ}$ & $\pm 20^{\circ}$ & $\pm 25^{\circ}$ \\
\hline Method & 51 & 57 & 62 & 81 & 98 & 100 & 100 & 100 \\
\hline $\begin{array}{c}\text { Single Point GPS } \\
\text { (Pseudoranges) }\end{array}$ & 58 & 58 & 58 & 66 & 89 & 96 & 99 & 100 \\
\hline $\begin{array}{c}\text { Single Point GPS (Carrier } \\
\text { Phase) }\end{array}$ & 47 & 54 & 60 & 74 & 89 & 95 & 98 & 99 \\
\hline $\begin{array}{c}\text { Single Difference GPS } \\
\text { (Pseudoranges) }\end{array}$ & 77 & 77 & 77 & 86 & 86 & 89 & 96 & 96 \\
\hline $\begin{array}{c}\text { Single Difference GPS } \\
\text { (Carrier Phase) }\end{array}$ & 50 & 53 & 61 & 71 & 89 & 95 & 96 & 98 \\
\hline $\begin{array}{c}\text { Double Difference GPS } \\
\text { (Pseudoranges) }\end{array}$ & 67 & 67 & 69 & 77 & 82 & 86 & 100 & 100 \\
\hline $\begin{array}{c}\text { Double Difference GPS } \\
\text { (Carrier Phase) }\end{array}$ & 96 & 100 & 100 & 100 & 100 & 100 & 100 & 100 \\
\hline IMU Measured & & & & & & & & \\
\hline
\end{tabular}


Table 46: Success Rates of Reaching Angular Specifications of Fused Pitch for Simulation Data Set A-2

\begin{tabular}{|c|c|c|c|c|c|c|c|c|}
\hline & \multicolumn{7}{|c|}{ Portion of Trials in Which Target Specification was reached } \\
\hline & $\pm 0.5^{\circ}$ & $\pm 1^{\circ}$ & $\pm 2^{\circ}$ & $\pm 5^{\circ}$ & $\pm 10^{\circ}$ & $\pm 15^{\circ}$ & $\pm 20^{\circ}$ & $\pm 25^{\circ}$ \\
\hline Method & 100 & 100 & 100 & 100 & 100 & 100 & 100 & 100 \\
\hline $\begin{array}{c}\text { Single Point GPS } \\
\text { (Pseudoranges) }\end{array}$ & 69 & 69 & 75 & 100 & 100 & 100 & 100 & 100 \\
\hline $\begin{array}{c}\text { Single Point GPS (Carrier } \\
\text { Phase) }\end{array}$ & 100 & 100 & 100 & 100 & 100 & 100 & 100 & 100 \\
\hline $\begin{array}{c}\text { Single Difference GPS } \\
\text { (Pseudoranges) }\end{array}$ & 85 & 85 & 88 & 100 & 100 & 100 & 100 & 100 \\
\hline $\begin{array}{c}\text { Single Difference GPS } \\
\text { (Carrier Phase) }\end{array}$ & 100 & 100 & 100 & 100 & 100 & 100 & 100 & 100 \\
\hline $\begin{array}{c}\text { Double Difference GPS } \\
\text { (Pseudoranges) }\end{array}$ & 79 & 79 & 87 & 99 & 100 & 100 & 100 & 100 \\
\hline $\begin{array}{c}\text { Double Difference GPS } \\
\text { (Carrier Phase) }\end{array}$ & 100 & 100 & 100 & 100 & 100 & 100 & 100 & 100 \\
\hline IMU Measured & & & & & & & & \\
\hline
\end{tabular}

Table 47: Success Rates of Reaching Angular Specifications of Fused Heading for Simulation Data Set A-2

\begin{tabular}{|c|c|c|c|c|c|c|c|c|}
\hline & \multicolumn{7}{|c|}{ Portion of Trials in Which Target Specification was reached } \\
\hline & $\pm 0.5^{\circ}$ & $\pm 1^{\circ}$ & $\pm 2^{\circ}$ & $\pm 5^{\circ}$ & $\pm 10^{\circ}$ & $\pm 15^{\circ}$ & $\pm 20^{\circ}$ & $\pm 25^{\circ}$ \\
\hline Method & 72 & 88 & 95 & 100 & 100 & 100 & 100 & 100 \\
\hline $\begin{array}{c}\text { Single Point GPS } \\
\text { (Pseudoranges) }\end{array}$ & 58 & 58 & 58 & 66 & 89 & 99 & 99 & 100 \\
\hline $\begin{array}{c}\text { Single Point GPS (Carrier } \\
\text { Phase) }\end{array}$ & 70 & 80 & 87 & 97 & 100 & 100 & 100 & 100 \\
\hline $\begin{array}{c}\text { Single Difference GPS } \\
\text { (Pseudoranges) }\end{array}$ & 77 & 77 & 77 & 86 & 86 & 91 & 96 & 96 \\
\hline $\begin{array}{c}\text { Single Difference GPS } \\
\text { (Carrier Phase) }\end{array}$ & 64 & 70 & 82 & 93 & 99 & 99 & 100 & 100 \\
\hline $\begin{array}{c}\text { Double Difference GPS } \\
\text { (Pseudoranges) }\end{array}$ & 67 & 67 & 69 & 77 & 82 & 91 & 100 & 100 \\
\hline $\begin{array}{c}\text { Double Difference GPS } \\
\text { (Carrier Phase) }\end{array}$ & 96 & 100 & 100 & 100 & 100 & 100 & 100 & 100 \\
\hline IMU Measured & & & & & & & & \\
\hline
\end{tabular}


Table 48: Pitch / Heading Correlation Coefficients for Each Computation Method in Simulation Data Set A-2

\begin{tabular}{|c|c|c|}
\hline & \multicolumn{2}{|c|}{ Pitch / Heading Correlation Coefficient } \\
\hline Method & Unfused & Fused \\
\hline $\begin{array}{c}\text { Single Point GPS } \\
\text { (Pseudoranges) }\end{array}$ & 0.839865 & 0.291616 \\
\hline $\begin{array}{c}\text { Single Difference GPS } \\
\text { (Pseudoranges) }\end{array}$ & 0.834911 & 0.304303 \\
\hline $\begin{array}{c}\text { Double Difference GPS } \\
\text { (Pseudoranges) }\end{array}$ & 0.779955 & 0.307236 \\
\hline
\end{tabular}

\section{Set A-3: 5 m Dual Antenna Separation}

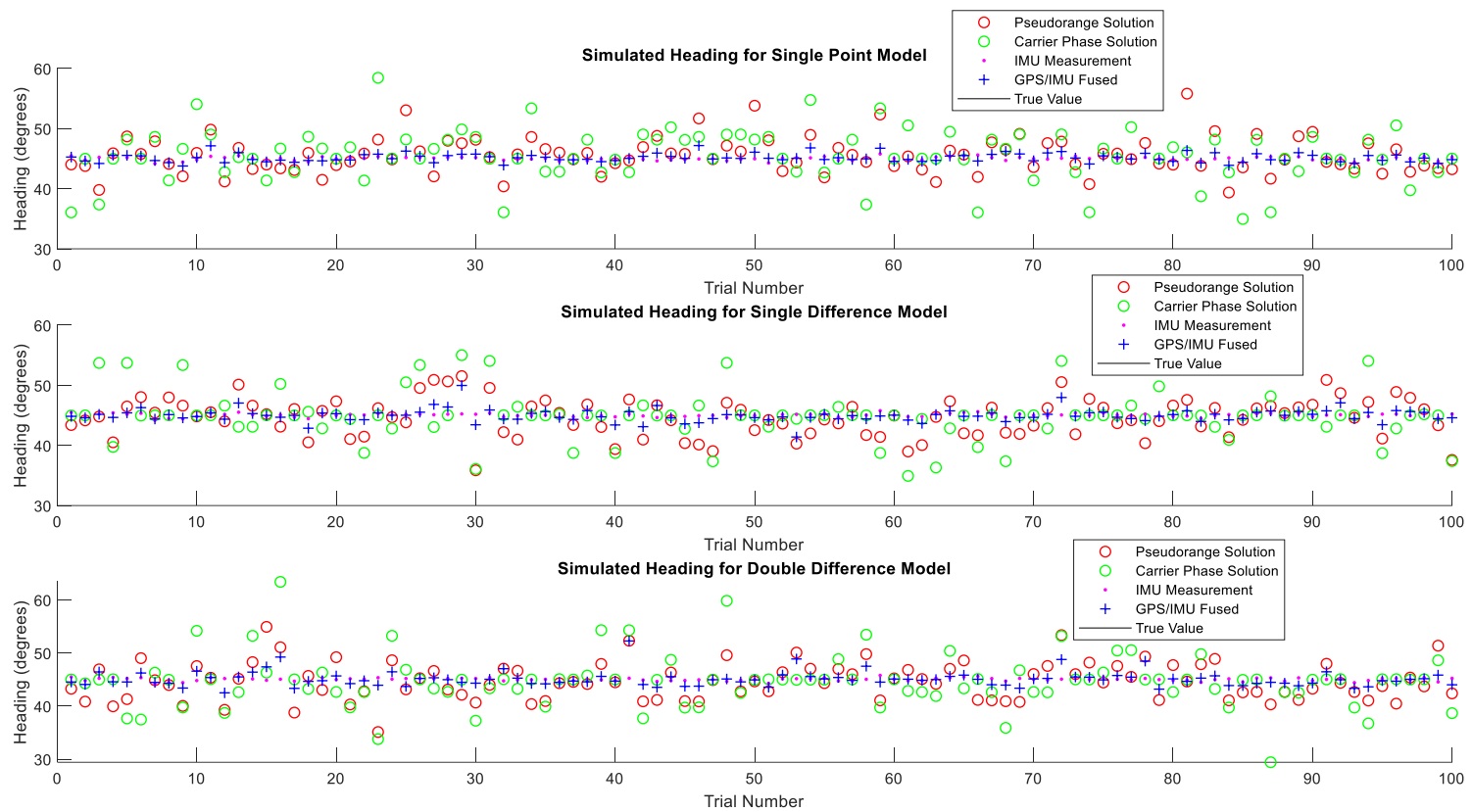

Figure 54: Computed and Measured Headings for Simulation Set A-3 


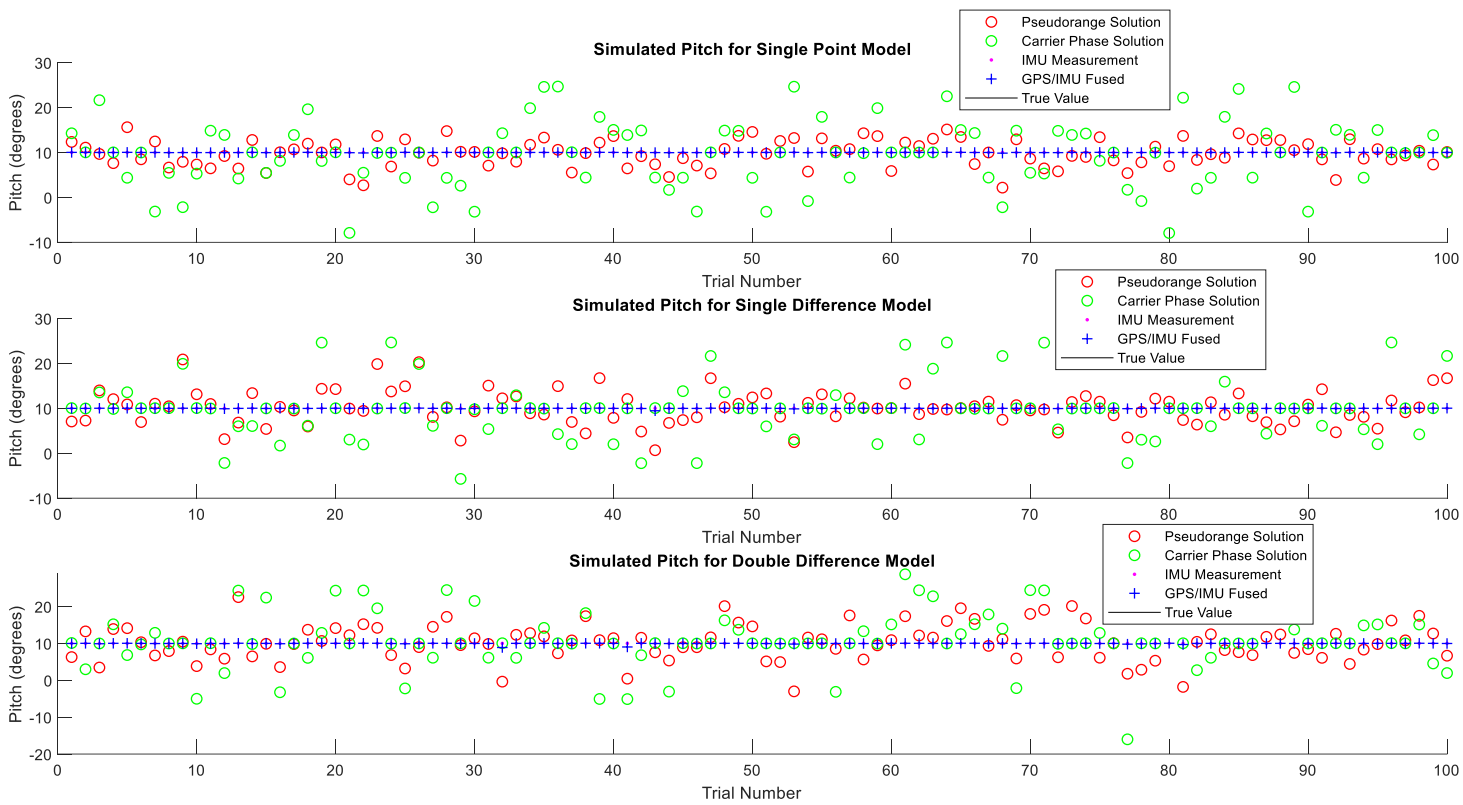

Figure 55: Computed and Measured Pitch for Simulation Set A-3

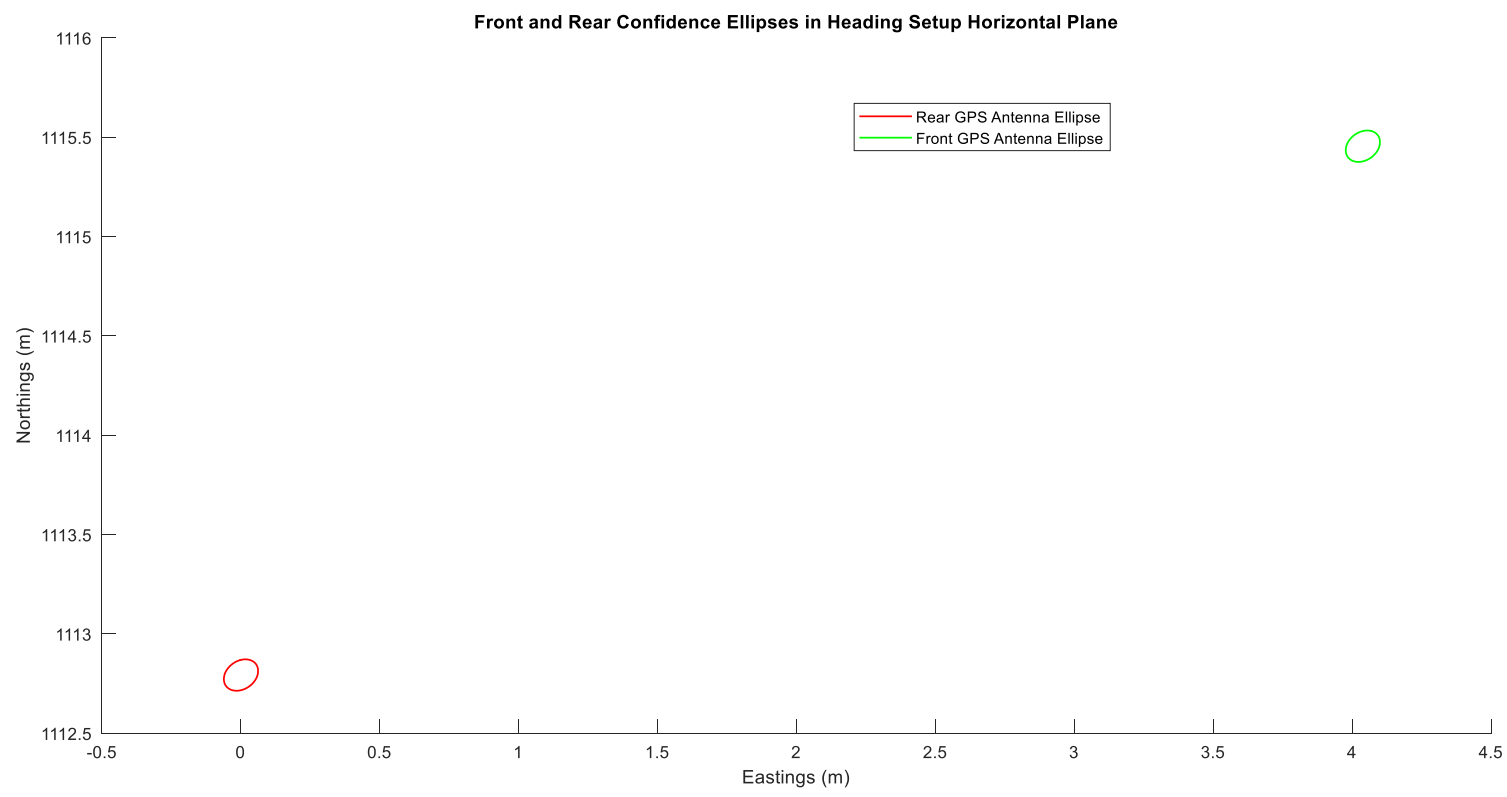

Figure 56: Propagated 95\% Confidence Ellipses for Front and Rear GPS Antennas in Simulated Data Set A-3 
Table 49: Mean Global Error Statistics of Unfused Pitch for Simulation Set A-3

\begin{tabular}{|c|c|c|c|}
\hline Method & $\begin{array}{c}\text { Measured Standard } \\
\text { Deviation } \\
\text { (degrees) }\end{array}$ & $\begin{array}{c}\text { Estimated } \\
\text { Standard } \\
\text { Deviation } \\
\text { (degrees) }\end{array}$ & $\begin{array}{c}\text { Mean RMS } \\
\text { Error } \\
\text { (degrees) }\end{array}$ \\
\hline Single Point GPS (Pseudoranges) & 3.01 & 1.539 & 2.474 \\
\hline Single Point GPS (Carrier Phase) & 7.364 & 0.006 & 5.549 \\
\hline $\begin{array}{c}\text { Single Difference GPS } \\
\text { (Pseudoranges) }\end{array}$ & 3.794 & 1.55 & 2.892 \\
\hline $\begin{array}{c}\text { Single Difference GPS (Carrier } \\
\text { Phase) }\end{array}$ & 6.192 & 0.006 & 3.856 \\
\hline $\begin{array}{c}\text { Double Difference GPS } \\
\text { (Pseudoranges) }\end{array}$ & 5.075 & 1.243 & 3.974 \\
\hline $\begin{array}{c}\text { Double Difference GPS (Carrier } \\
\text { Phase) }\end{array}$ & 7.555 & 0.013 & 4.821 \\
\hline IMU Measurement & 0.007 & - & 0.006 \\
\hline
\end{tabular}

Table 50: Mean Global Error Statistics of Unfused Heading for Simulation Set A-3

\begin{tabular}{|c|c|c|c|}
\hline Method & $\begin{array}{c}\text { Measured Standard } \\
\text { Deviation } \\
\text { (degrees) }\end{array}$ & $\begin{array}{c}\text { Estimated } \\
\text { Standard } \\
\text { Deviation } \\
\text { (degrees) }\end{array}$ & $\begin{array}{c}\text { Mean RMS } \\
\text { Error } \\
\text { (degrees) }\end{array}$ \\
\hline Single Point GPS (Pseudoranges) & 2.97 & 1.303 & 2.292 \\
\hline Single Point GPS (Carrier Phase) & 4.386 & 0.004 & 3.233 \\
\hline $\begin{array}{c}\text { Single Difference GPS } \\
\text { (Pseudoranges) }\end{array}$ & 3.155 & 1.416 & 2.559 \\
\hline $\begin{array}{c}\text { Single Difference GPS (Carrier } \\
\text { Phase) }\end{array}$ & 3.981 & 0.004 & 2.332 \\
\hline $\begin{array}{c}\text { Double Difference GPS } \\
\text { (Pseudoranges) }\end{array}$ & 3.506 & 1.137 & 2.852 \\
\hline $\begin{array}{c}\text { Double Difference GPS (Carrier } \\
\text { Phase) }\end{array}$ & 4.914 & 0.014 & 3.111 \\
\hline IMU Measurement & 0.306 & - & 0.245 \\
\hline
\end{tabular}


Table 51: Mean Global Error Statistics of Fused Pitch for Simulation Set A-3

\begin{tabular}{|c|c|c|c|}
\hline Method & $\begin{array}{c}\text { Measured Standard } \\
\text { Deviation } \\
\text { (degrees) }\end{array}$ & $\begin{array}{c}\text { Estimated } \\
\text { Standard } \\
\text { Deviation } \\
\text { (degrees) }\end{array}$ & $\begin{array}{c}\text { Mean RMS } \\
\text { Error } \\
\text { (degrees) }\end{array}$ \\
\hline Single Point GPS (Pseudoranges) & 0.026 & 0.192 & 0.017 \\
\hline Single Point GPS (Carrier Phase) & 4.45 & 0.003 & 3.298 \\
\hline $\begin{array}{c}\text { Single Difference GPS } \\
\text { (Pseudoranges) }\end{array}$ & 0.06 & 0.19 & 0.023 \\
\hline $\begin{array}{c}\text { Single Difference GPS (Carrier } \\
\text { Phase) }\end{array}$ & 3.638 & 0.003 & 2.297 \\
\hline $\begin{array}{c}\text { Double Difference GPS } \\
\text { (Pseudoranges) }\end{array}$ & 0.154 & 0.183 & 0.051 \\
\hline $\begin{array}{c}\text { Double Difference GPS (Carrier } \\
\text { Phase) }\end{array}$ & 2.973 & 0.007 & 1.789 \\
\hline IMU Measurement & 0.007 & - & 0.006 \\
\hline
\end{tabular}

Table 52: Mean Global Error Statistics of Fused Heading for Simulation Set A-3

\begin{tabular}{|c|c|c|c|}
\hline Method & $\begin{array}{c}\text { Measured Standard } \\
\text { Deviation } \\
\text { (degrees) }\end{array}$ & $\begin{array}{c}\text { Estimated } \\
\text { Standard } \\
\text { Deviation } \\
\text { (degrees) }\end{array}$ & $\begin{array}{c}\text { Mean RMS } \\
\text { Error } \\
\text { (degrees) }\end{array}$ \\
\hline Single Point GPS (Pseudoranges) & 0.685 & 0.365 & 0.536 \\
\hline Single Point GPS (Carrier Phase) & 4.317 & 0.002 & 3.187 \\
\hline $\begin{array}{c}\text { Single Difference GPS } \\
\text { (Pseudoranges) }\end{array}$ & 1.033 & 0.397 & 0.696 \\
\hline $\begin{array}{c}\text { Single Difference GPS (Carrier } \\
\text { Phase) }\end{array}$ & 3.915 & 0.002 & 2.296 \\
\hline $\begin{array}{c}\text { Double Difference GPS } \\
\text { (Pseudoranges) }\end{array}$ & 1.403 & 0.4 & 0.917 \\
\hline $\begin{array}{c}\text { Double Difference GPS (Carrier } \\
\text { Phase) }\end{array}$ & 4.694 & 0.007 & 2.974 \\
\hline IMU Measurement & 0.306 & - & 0.245 \\
\hline
\end{tabular}


Table 53: Success Rates of Reaching Angular Specifications of Unfused Pitch for Simulation Data Set A-3

\begin{tabular}{|c|c|c|c|c|c|c|c|c|}
\hline & \multicolumn{7}{|c|}{ Portion of Trials in Which Target Specification was reached } \\
\hline & $\pm 0.5^{\circ}$ & $\pm 1^{\circ}$ & $\pm 2^{\circ}$ & $\pm 5^{\circ}$ & $\pm 10^{\circ}$ & $\pm 15^{\circ}$ & $\pm 20^{\circ}$ & $\pm 25^{\circ}$ \\
\hline Method & 58 & 64 & 71 & 98 & 100 & 100 & 100 & 100 \\
\hline $\begin{array}{c}\text { Single Point GPS } \\
\text { (Pseudoranges) }\end{array}$ & 65 & 65 & 65 & 84 & 92 & 100 & 100 & 100 \\
\hline $\begin{array}{c}\text { Single Point GPS (Carrier } \\
\text { Phase) }\end{array}$ & 58 & 62 & 71 & 91 & 98 & 100 & 100 & 100 \\
\hline $\begin{array}{c}\text { Single Difference GPS } \\
\text { (Pseudoranges) }\end{array}$ & 81 & 81 & 81 & 87 & 91 & 100 & 100 & 100 \\
\hline $\begin{array}{c}\text { Single Difference GPS } \\
\text { (Carrier Phase) }\end{array}$ & 51 & 56 & 66 & 82 & 96 & 100 & 100 & 100 \\
\hline $\begin{array}{c}\text { Double Difference GPS } \\
\text { (Pseudoranges) }\end{array}$ & 67 & 67 & 67 & 77 & 87 & 99 & 100 & 100 \\
\hline $\begin{array}{c}\text { Double Difference GPS } \\
\text { (Carrier Phase) }\end{array}$ & 100 & 100 & 100 & 100 & 100 & 100 & 100 & 100 \\
\hline IMU Measured & & & & & & & & \\
\hline
\end{tabular}

Table 54: Success Rates of Reaching Angular Specifications of Unfused Heading for Simulation Data Set A-3

\begin{tabular}{|c|c|c|c|c|c|c|c|c|}
\hline & \multicolumn{7}{|c|}{ Portion of Trials in Which Target Specification was reached } \\
\hline & $\pm 0.5^{\circ}$ & $\pm 1^{\circ}$ & $\pm 2^{\circ}$ & $\pm 5^{\circ}$ & $\pm 10^{\circ}$ & $\pm 15^{\circ}$ & $\pm 20^{\circ}$ & $\pm 25^{\circ}$ \\
\hline Method & 50 & 63 & 73 & 95 & 99 & 100 & 100 & 100 \\
\hline $\begin{array}{c}\text { Single Point GPS } \\
\text { (Pseudoranges) }\end{array}$ & 54 & 54 & 63 & 89 & 99 & 100 & 100 & 100 \\
\hline $\begin{array}{c}\text { Single Point GPS (Carrier } \\
\text { Phase) }\end{array}$ & 57 & 60 & 79 & 94 & 100 & 100 & 100 & 100 \\
\hline $\begin{array}{c}\text { Single Difference GPS } \\
\text { (Pseudoranges) }\end{array}$ & 80 & 81 & 87 & 89 & 99 & 100 & 100 & 100 \\
\hline $\begin{array}{c}\text { Single Difference GPS } \\
\text { (Carrier Phase) }\end{array}$ & 63 & 65 & 74 & 94 & 100 & 100 & 100 & 100 \\
\hline $\begin{array}{c}\text { Double Difference GPS } \\
\text { (Pseudoranges) }\end{array}$ & 77 & 78 & 84 & 88 & 98 & 99 & 100 & 100 \\
\hline $\begin{array}{c}\text { Double Difference GPS } \\
\text { (Carrier Phase) }\end{array}$ & 97 & 100 & 100 & 100 & 100 & 100 & 100 & 100 \\
\hline IMU Measured & & & & & & & & \\
\hline
\end{tabular}


Table 55: Success Rates of Reaching Angular Specifications of Fused Pitch for Simulation Data Set A-3

\begin{tabular}{|c|c|c|c|c|c|c|c|c|}
\hline & \multicolumn{7}{|c|}{ Portion of Trials in Which Target Specification was reached } \\
\hline & $\pm 0.5^{\circ}$ & $\pm 1^{\circ}$ & $\pm 2^{\circ}$ & $\pm 5^{\circ}$ & $\pm 10^{\circ}$ & $\pm 15^{\circ}$ & $\pm 20^{\circ}$ & $\pm 25^{\circ}$ \\
\hline Method & 100 & 100 & 100 & 100 & 100 & 100 & 100 & 100 \\
\hline $\begin{array}{c}\text { Single Point GPS } \\
\text { (Pseudoranges) }\end{array}$ & 65 & 65 & 76 & 100 & 100 & 100 & 100 & 100 \\
\hline $\begin{array}{c}\text { Single Point GPS (Carrier } \\
\text { Phase) }\end{array}$ & 100 & 100 & 100 & 100 & 100 & 100 & 100 & 100 \\
\hline $\begin{array}{c}\text { Single Difference GPS } \\
\text { (Pseudoranges) }\end{array}$ & 81 & 81 & 87 & 100 & 100 & 100 & 100 & 100 \\
\hline $\begin{array}{c}\text { Single Difference GPS } \\
\text { (Carrier Phase) }\end{array}$ & 100 & 100 & 100 & 100 & 100 & 100 & 100 & 100 \\
\hline $\begin{array}{c}\text { Double Difference GPS } \\
\text { (Pseudoranges) }\end{array}$ & 67 & 71 & 83 & 100 & 100 & 100 & 100 & 100 \\
\hline $\begin{array}{c}\text { Double Difference GPS } \\
\text { (Carrier Phase) }\end{array}$ & 100 & 100 & 100 & 100 & 100 & 100 & 100 & 100 \\
\hline IMU Measured & & & & & & & & \\
\hline
\end{tabular}

Table 56: Success Rates of Reaching Angular Specifications of Fused Heading for Simulation Data Set A-3

\begin{tabular}{|c|c|c|c|c|c|c|c|c|}
\hline & \multicolumn{7}{|c|}{ Portion of Trials in Which Target Specification was reached } \\
\hline & $\pm 0.5^{\circ}$ & $\pm 1^{\circ}$ & $\pm 2^{\circ}$ & $\pm 5^{\circ}$ & $\pm 10^{\circ}$ & $\pm 15^{\circ}$ & $\pm 20^{\circ}$ & $\pm 25^{\circ}$ \\
\hline Method & 70 & 90 & 98 & 100 & 100 & 100 & 100 & 100 \\
\hline $\begin{array}{c}\text { Single Point GPS } \\
\text { (Pseudoranges) }\end{array}$ & 54 & 55 & 63 & 89 & 99 & 100 & 100 & 100 \\
\hline $\begin{array}{c}\text { Single Point GPS (Carrier } \\
\text { Phase) }\end{array}$ & 78 & 92 & 96 & 100 & 100 & 100 & 100 & 100 \\
\hline $\begin{array}{c}\text { Single Difference GPS } \\
\text { (Pseudoranges) }\end{array}$ & 80 & 81 & 87 & 89 & 100 & 100 & 100 & 100 \\
\hline $\begin{array}{c}\text { Single Difference GPS } \\
\text { (Carrier Phase) }\end{array}$ & 76 & 86 & 92 & 99 & 100 & 100 & 100 & 100 \\
\hline $\begin{array}{c}\text { Double Difference GPS } \\
\text { (Pseudoranges) }\end{array}$ & 77 & 78 & 84 & 88 & 98 & 99 & 100 & 100 \\
\hline $\begin{array}{c}\text { Double Difference GPS } \\
\text { (Carrier Phase) }\end{array}$ & 97 & 100 & 100 & 100 & 100 & 100 & 100 & 100 \\
\hline IMU Measured & & & & & & & & \\
\hline
\end{tabular}


Table 57: Pitch / Heading Correlation Coefficients for Each Computation Method in Simulation Data Set A-3

\begin{tabular}{|c|c|c|}
\hline & \multicolumn{2}{|c|}{ Pitch / Heading Correlation Coefficient } \\
\hline Method & Unfused & Fused \\
\hline $\begin{array}{c}\text { Single Point GPS } \\
\text { (Pseudoranges) }\end{array}$ & 0.839865 & 0.291616 \\
\hline $\begin{array}{c}\text { Single Difference GPS } \\
\text { (Pseudoranges) }\end{array}$ & 0.834911 & 0.304303 \\
\hline $\begin{array}{c}\text { Double Difference GPS } \\
\text { (Pseudoranges) }\end{array}$ & 0.779955 & 0.307236 \\
\hline
\end{tabular}

\section{Set A-4: 10 m Dual Antenna Separation}
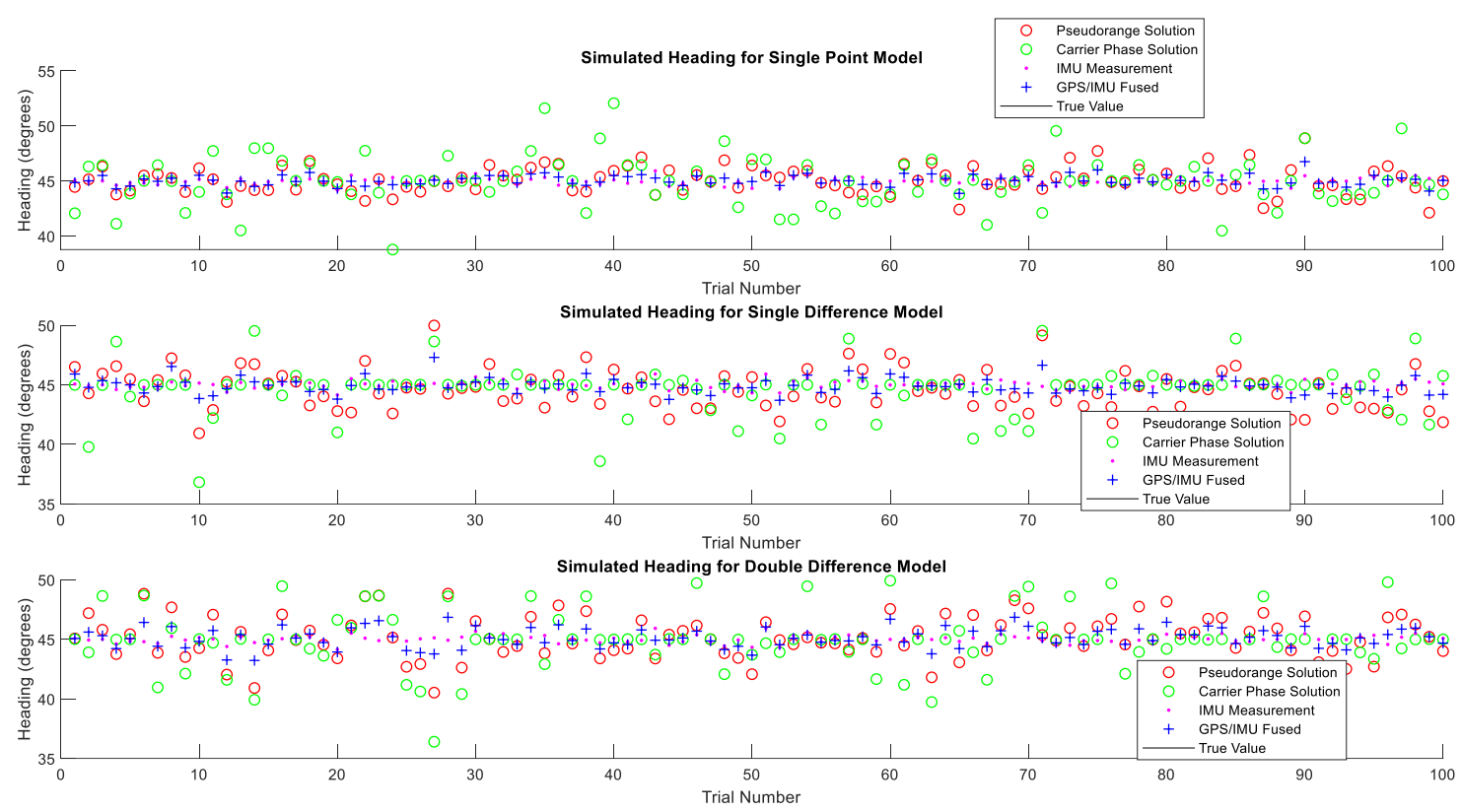

Figure 57: Computed and Measured Headings for Simulation Set A-4 


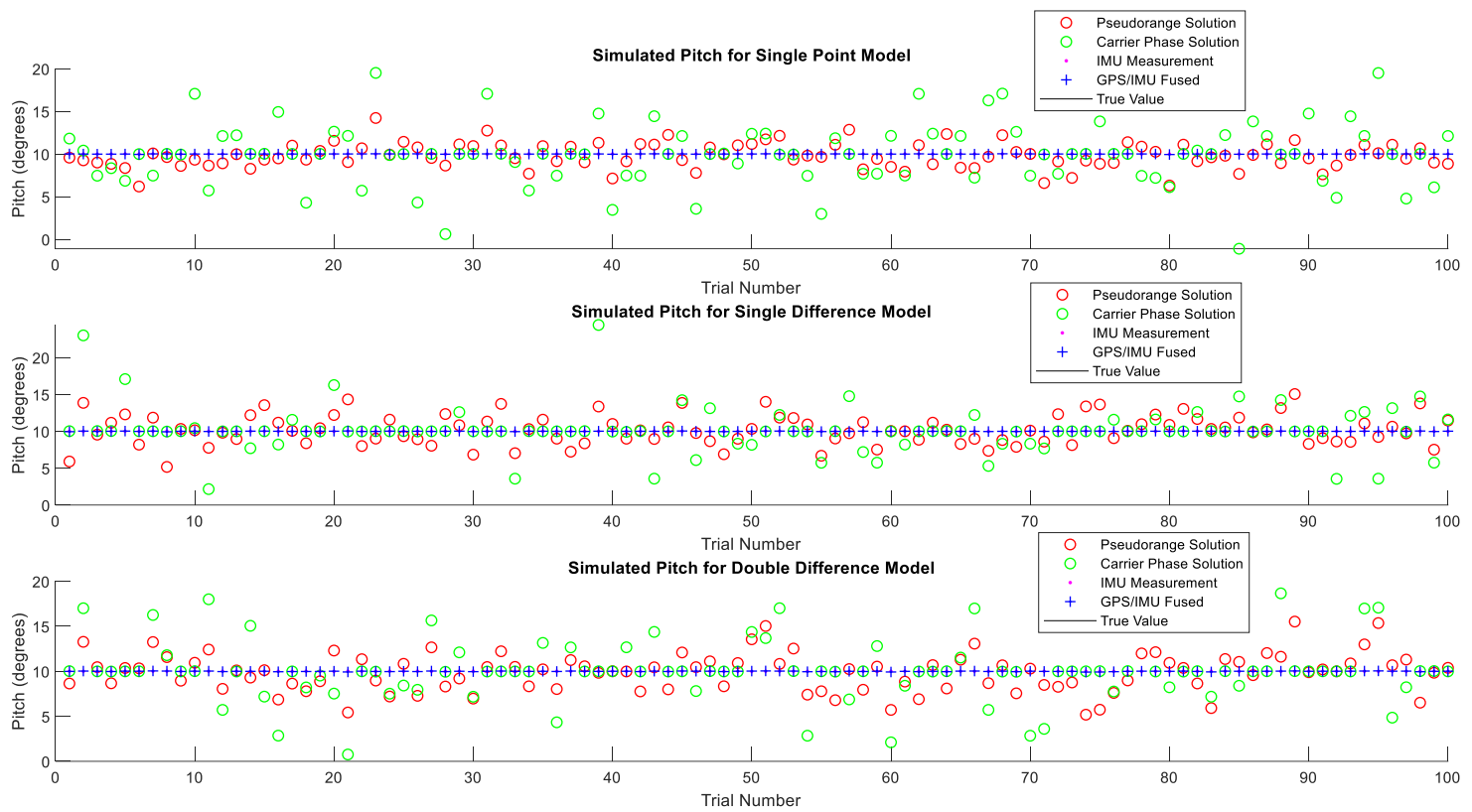

Figure 58: Computed and Measured Pitch for Simulation Set A-4

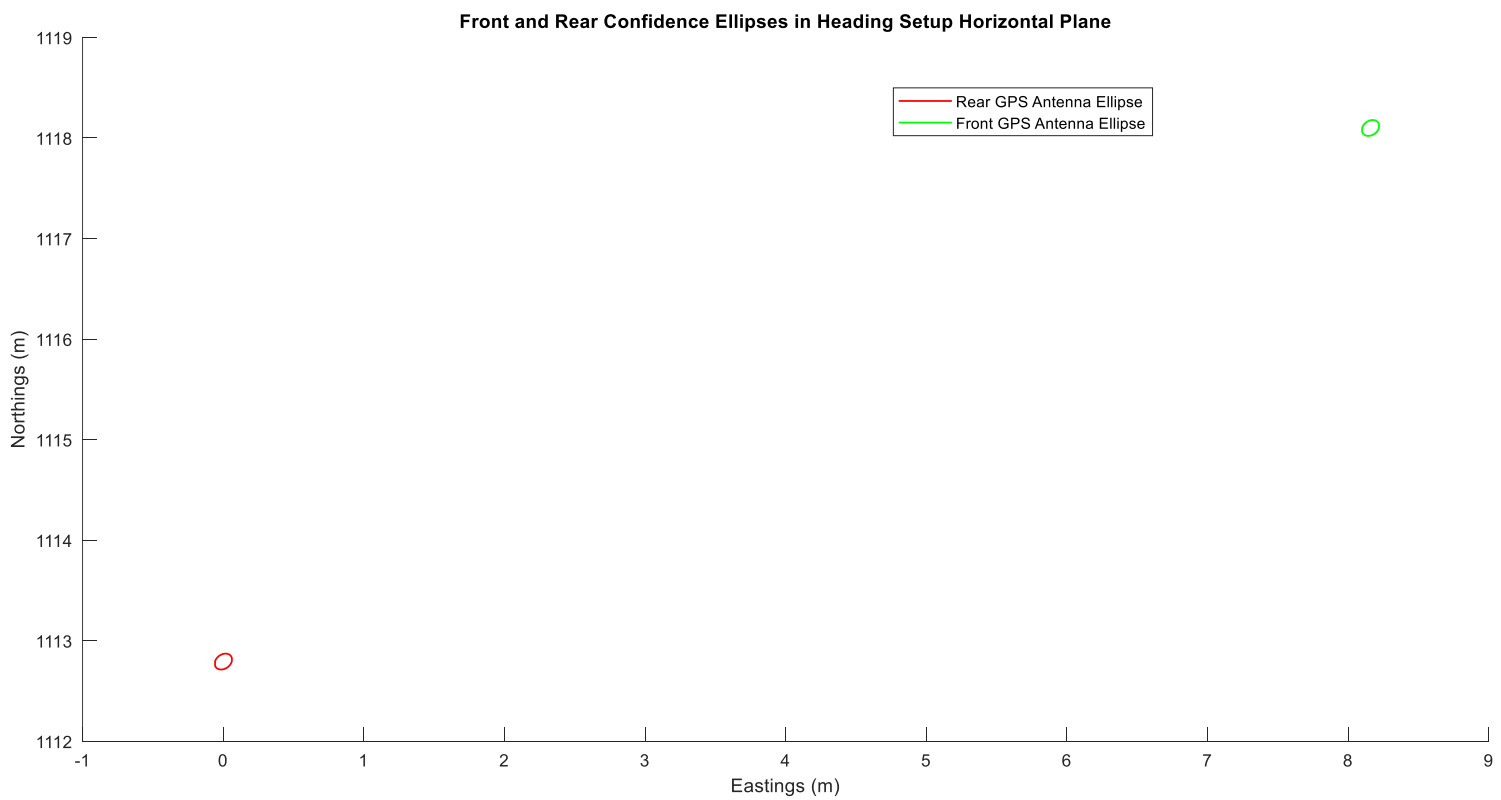

Figure 59: Propagated 95\% Confidence Ellipses for Front and Rear GPS Antennas in Simulated Data Set A-4 
Table 58: Mean Global Error Statistics of Unfused Pitch for Simulation Set A-4

\begin{tabular}{|c|c|c|c|}
\hline Method & $\begin{array}{c}\text { Measured Standard } \\
\text { Deviation } \\
\text { (degrees) }\end{array}$ & $\begin{array}{c}\text { Estimated } \\
\text { Standard } \\
\text { Deviation } \\
\text { (degrees) }\end{array}$ & $\begin{array}{c}\text { Mean RMS } \\
\text { Error } \\
\text { (degrees) }\end{array}$ \\
\hline Single Point GPS (Pseudoranges) & 1.46 & 0.777 & 1.185 \\
\hline Single Point GPS (Carrier Phase) & 3.63 & 0.003 & 2.518 \\
\hline $\begin{array}{c}\text { Single Difference GPS } \\
\text { (Pseudoranges) }\end{array}$ & 2.048 & 0.799 & 1.644 \\
\hline $\begin{array}{c}\text { Single Difference GPS (Carrier } \\
\text { Phase) }\end{array}$ & 3.156 & 0.003 & 1.647 \\
\hline $\begin{array}{c}\text { Double Difference GPS } \\
\text { (Pseudoranges) }\end{array}$ & 2.141 & 0.622 & 1.691 \\
\hline $\begin{array}{c}\text { Double Difference GPS (Carrier } \\
\text { Phase) }\end{array}$ & 3.391 & 0.006 & 2.037 \\
\hline IMU Measurement & 0.007 & - & 0.006 \\
\hline
\end{tabular}

Table 59: Mean Global Error Statistics of Unfused Heading for Simulation Set A-4

\begin{tabular}{|c|c|c|c|}
\hline Method & $\begin{array}{c}\text { Measured Standard } \\
\text { Deviation } \\
\text { (degrees) }\end{array}$ & $\begin{array}{c}\text { Estimated } \\
\text { Standard } \\
\text { Deviation } \\
\text { (degrees) }\end{array}$ & $\begin{array}{c}\text { Mean RMS } \\
\text { Error } \\
\text { (degrees) }\end{array}$ \\
\hline Single Point GPS (Pseudoranges) & 1.212 & 0.624 & 0.962 \\
\hline Single Point GPS (Carrier Phase) & 2.252 & 0.002 & 1.606 \\
\hline $\begin{array}{c}\text { Single Difference GPS } \\
\text { (Pseudoranges) }\end{array}$ & 1.648 & 0.684 & 1.376 \\
\hline $\begin{array}{c}\text { Single Difference GPS (Carrier } \\
\text { Phase) }\end{array}$ & 2.058 & 0.002 & 1.118 \\
\hline $\begin{array}{c}\text { Double Difference GPS } \\
\text { (Pseudoranges) }\end{array}$ & 1.778 & 0.477 & 1.433 \\
\hline $\begin{array}{c}\text { Double Difference GPS (Carrier } \\
\text { Phase) }\end{array}$ & 2.483 & 0.005 & 1.608 \\
\hline IMU Measurement & 0.329 & - & 0.253 \\
\hline
\end{tabular}


Table 60: Mean Global Error Statistics of Fused Pitch for Simulation Set A-4

\begin{tabular}{|c|c|c|c|}
\hline Method & $\begin{array}{c}\text { Measured Standard } \\
\text { Deviation } \\
\text { (degrees) }\end{array}$ & $\begin{array}{c}\text { Estimated } \\
\text { Standard } \\
\text { Deviation } \\
\text { (degrees) }\end{array}$ & $\begin{array}{c}\text { Mean RMS } \\
\text { Error } \\
\text { (degrees) }\end{array}$ \\
\hline Single Point GPS (Pseudoranges) & 0.015 & 0.177 & 0.012 \\
\hline Single Point GPS (Carrier Phase) & 2.709 & 0.001 & 1.836 \\
\hline $\begin{array}{c}\text { Single Difference GPS } \\
\text { (Pseudoranges) }\end{array}$ & 0.02 & 0.178 & 0.016 \\
\hline $\begin{array}{c}\text { Single Difference GPS (Carrier } \\
\text { Phase) }\end{array}$ & 2.129 & 0.001 & 1.152 \\
\hline $\begin{array}{c}\text { Double Difference GPS } \\
\text { (Pseudoranges) }\end{array}$ & 0.027 & 0.167 & 0.021 \\
\hline $\begin{array}{c}\text { Double Difference GPS (Carrier } \\
\text { Phase) }\end{array}$ & 2.043 & 0.003 & 1.155 \\
\hline IMU Measurement & 0.007 & - & 0.006 \\
\hline
\end{tabular}

Table 61: Mean Global Error Statistics of Fused Heading for Simulation Set A-4

\begin{tabular}{|c|c|c|c|}
\hline Method & $\begin{array}{c}\text { Measured Standard } \\
\text { Deviation } \\
\text { (degrees) }\end{array}$ & $\begin{array}{c}\text { Estimated } \\
\text { Standard } \\
\text { Deviation } \\
\text { (degrees) }\end{array}$ & $\begin{array}{c}\text { Mean RMS } \\
\text { Error } \\
\text { (degrees) }\end{array}$ \\
\hline Single Point GPS (Pseudoranges) & 0.477 & 0.198 & 0.371 \\
\hline Single Point GPS (Carrier Phase) & 2.24 & 0.001 & 1.596 \\
\hline $\begin{array}{c}\text { Single Difference GPS } \\
\text { (Pseudoranges) }\end{array}$ & 0.635 & 0.215 & 0.487 \\
\hline $\begin{array}{c}\text { Single Difference GPS (Carrier } \\
\text { Phase) }\end{array}$ & 2.042 & 0.001 & 1.11 \\
\hline $\begin{array}{c}\text { Double Difference GPS } \\
\text { (Pseudoranges) }\end{array}$ & 0.801 & 0.179 & 0.657 \\
\hline $\begin{array}{c}\text { Double Difference GPS (Carrier } \\
\text { Phase) }\end{array}$ & 2.443 & 0.003 & 1.584 \\
\hline IMU Measurement & 0.329 & - & 0.253 \\
\hline
\end{tabular}


Table 62: Success Rates of Reaching Angular Specifications of Unfused Pitch for Simulation Data Set A-4

\begin{tabular}{|c|c|c|c|c|c|c|c|c|}
\hline & \multicolumn{7}{|c|}{ Portion of Trials in Which Target Specification was reached } \\
\hline & $\pm 0.5^{\circ}$ & $\pm 1^{\circ}$ & $\pm 2^{\circ}$ & $\pm 5^{\circ}$ & $\pm 10^{\circ}$ & $\pm 15^{\circ}$ & $\pm 20^{\circ}$ & $\pm 25^{\circ}$ \\
\hline Method & 66 & 76 & 93 & 100 & 100 & 100 & 100 & 100 \\
\hline $\begin{array}{c}\text { Single Point GPS } \\
\text { (Pseudoranges) }\end{array}$ & 69 & 69 & 71 & 93 & 100 & 100 & 100 & 100 \\
\hline $\begin{array}{c}\text { Single Point GPS (Carrier } \\
\text { Phase) }\end{array}$ & 59 & 67 & 81 & 99 & 100 & 100 & 100 & 100 \\
\hline $\begin{array}{c}\text { Single Difference GPS } \\
\text { (Pseudoranges) }\end{array}$ & 79 & 79 & 83 & 96 & 98 & 100 & 100 & 100 \\
\hline $\begin{array}{c}\text { Single Difference GPS } \\
\text { (Carrier Phase) }\end{array}$ & 63 & 74 & 84 & 97 & 100 & 100 & 100 & 100 \\
\hline $\begin{array}{c}\text { Double Difference GPS } \\
\text { (Pseudoranges) }\end{array}$ & 79 & 79 & 81 & 89 & 100 & 100 & 100 & 100 \\
\hline $\begin{array}{c}\text { Double Difference GPS } \\
\text { (Carrier Phase) }\end{array}$ & 100 & 100 & 100 & 100 & 100 & 100 & 100 & 100 \\
\hline IMU Measured & & & & & & & & \\
\hline
\end{tabular}

Table 63: Success Rates of Reaching Angular Specifications of Unfused Heading for Simulation Data Set A-4

\begin{tabular}{|c|c|c|c|c|c|c|c|c|}
\hline & \multicolumn{7}{|c|}{ Portion of Trials in Which Target Specification was reached } \\
\hline & $\pm 0.5^{\circ}$ & $\pm 1^{\circ}$ & $\pm 2^{\circ}$ & $\pm 5^{\circ}$ & $\pm 10^{\circ}$ & $\pm 15^{\circ}$ & $\pm 20^{\circ}$ & $\pm 25^{\circ}$ \\
\hline Method & 66 & 78 & 94 & 100 & 100 & 100 & 100 & 100 \\
\hline $\begin{array}{c}\text { Single Point GPS } \\
\text { (Pseudoranges) }\end{array}$ & 66 & 69 & 87 & 98 & 100 & 100 & 100 & 100 \\
\hline $\begin{array}{c}\text { Single Point GPS (Carrier } \\
\text { Phase) }\end{array}$ & 72 & 79 & 93 & 100 & 100 & 100 & 100 & 100 \\
\hline $\begin{array}{c}\text { Single Difference GPS } \\
\text { (Pseudoranges) }\end{array}$ & 85 & 93 & 93 & 100 & 100 & 100 & 100 & 100 \\
\hline $\begin{array}{c}\text { Single Difference GPS } \\
\text { (Carrier Phase) }\end{array}$ & 58 & 67 & 81 & 100 & 100 & 100 & 100 & 100 \\
\hline $\begin{array}{c}\text { Double Difference GPS } \\
\text { (Pseudoranges) }\end{array}$ & 76 & 80 & 83 & 100 & 100 & 100 & 100 & 100 \\
\hline $\begin{array}{c}\text { Double Difference GPS } \\
\text { (Carrier Phase) }\end{array}$ & 95 & 100 & 100 & 100 & 100 & 100 & 100 & 100 \\
\hline IMU Measured & & & & & & & & \\
\hline
\end{tabular}


Table 64: Success Rates of Reaching Angular Specifications of Fused Pitch for Simulation Data Set A-4

\begin{tabular}{|c|c|c|c|c|c|c|c|c|}
\hline & \multicolumn{7}{|c|}{ Portion of Trials in Which Target Specification was reached } \\
\hline Method & $\pm 0.5^{\circ}$ & $\pm 1^{\circ}$ & $\pm 2^{\circ}$ & $\pm 5^{\circ}$ & $\pm 10^{\circ}$ & $\pm 15^{\circ}$ & $\pm 20^{\circ}$ & $\pm 25^{\circ}$ \\
\hline $\begin{array}{c}\text { Single Point GPS } \\
\text { (Pseudoranges) }\end{array}$ & 100 & 100 & 100 & 100 & 100 & 100 & 100 & 100 \\
\hline $\begin{array}{c}\text { Single Point GPS (Carrier } \\
\text { Phase) }\end{array}$ & 69 & 69 & 86 & 98 & 100 & 100 & 100 & 100 \\
\hline $\begin{array}{c}\text { Single Difference GPS } \\
\text { (Pseudoranges) }\end{array}$ & 100 & 100 & 100 & 100 & 100 & 100 & 100 & 100 \\
\hline $\begin{array}{c}\text { Single Difference GPS } \\
\text { (Carrier Phase) }\end{array}$ & 79 & 79 & 89 & 98 & 100 & 100 & 100 & 100 \\
\hline $\begin{array}{c}\text { Double Difference GPS } \\
\text { (Pseudoranges) }\end{array}$ & 100 & 100 & 100 & 100 & 100 & 100 & 100 & 100 \\
\hline $\begin{array}{c}\text { Double Difference GPS } \\
\text { (Carrier Phase) }\end{array}$ & 79 & 81 & 87 & 100 & 100 & 100 & 100 & 100 \\
\hline IMU Measured & 100 & 100 & 100 & 100 & 100 & 100 & 100 & 100 \\
\hline
\end{tabular}

Table 65: Success Rates of Reaching Angular Specifications of Fused Heading for Simulation Data Set A-4

\begin{tabular}{|c|c|c|c|c|c|c|c|c|}
\hline & \multicolumn{7}{|c|}{ Portion of Trials in Which Target Specification was reached } \\
\hline & $\pm 0.5^{\circ}$ & $\pm 1^{\circ}$ & $\pm 2^{\circ}$ & $\pm 5^{\circ}$ & $\pm 10^{\circ}$ & $\pm 15^{\circ}$ & $\pm 20^{\circ}$ & $\pm 25^{\circ}$ \\
\hline Method & 81 & 99 & 100 & 100 & 100 & 100 & 100 & 100 \\
\hline $\begin{array}{c}\text { Single Point GPS } \\
\text { (Pseudoranges) }\end{array}$ & 66 & 69 & 87 & 98 & 100 & 100 & 100 & 100 \\
\hline $\begin{array}{c}\text { Single Point GPS (Carrier } \\
\text { Phase) }\end{array}$ & 85 & 96 & 99 & 100 & 100 & 100 & 100 & 100 \\
\hline $\begin{array}{c}\text { Single Difference GPS } \\
\text { (Pseudoranges) }\end{array}$ & 85 & 93 & 93 & 100 & 100 & 100 & 100 & 100 \\
\hline $\begin{array}{c}\text { Single Difference GPS } \\
\text { (Carrier Phase) }\end{array}$ & 68 & 84 & 100 & 100 & 100 & 100 & 100 & 100 \\
\hline $\begin{array}{c}\text { Double Difference GPS } \\
\text { (Pseudoranges) }\end{array}$ & 76 & 80 & 83 & 100 & 100 & 100 & 100 & 100 \\
\hline $\begin{array}{c}\text { Double Difference GPS } \\
\text { (Carrier Phase) }\end{array}$ & 95 & 100 & 100 & 100 & 100 & 100 & 100 & 100 \\
\hline IMU Measured &
\end{tabular}


Table 66: Pitch / Heading Correlation Coefficients for Each Computation Method in Simulation Data Set A-4

\begin{tabular}{|c|c|c|}
\hline & \multicolumn{2}{|c|}{ Pitch / Heading Correlation Coefficient } \\
\hline Method & Unfused & Fused \\
\hline $\begin{array}{c}\text { Single Point GPS } \\
\text { (Pseudoranges) }\end{array}$ & 0.877546 & 0.678435 \\
\hline $\begin{array}{c}\text { Single Difference GPS } \\
\text { (Pseudoranges) }\end{array}$ & 0.881098 & 0.623469 \\
\hline $\begin{array}{c}\text { Double Difference GPS } \\
\text { (Pseudoranges) }\end{array}$ & 0.795612 & 0.557494 \\
\hline
\end{tabular}

\section{Set A-5: 30 m Dual Antenna Separation}

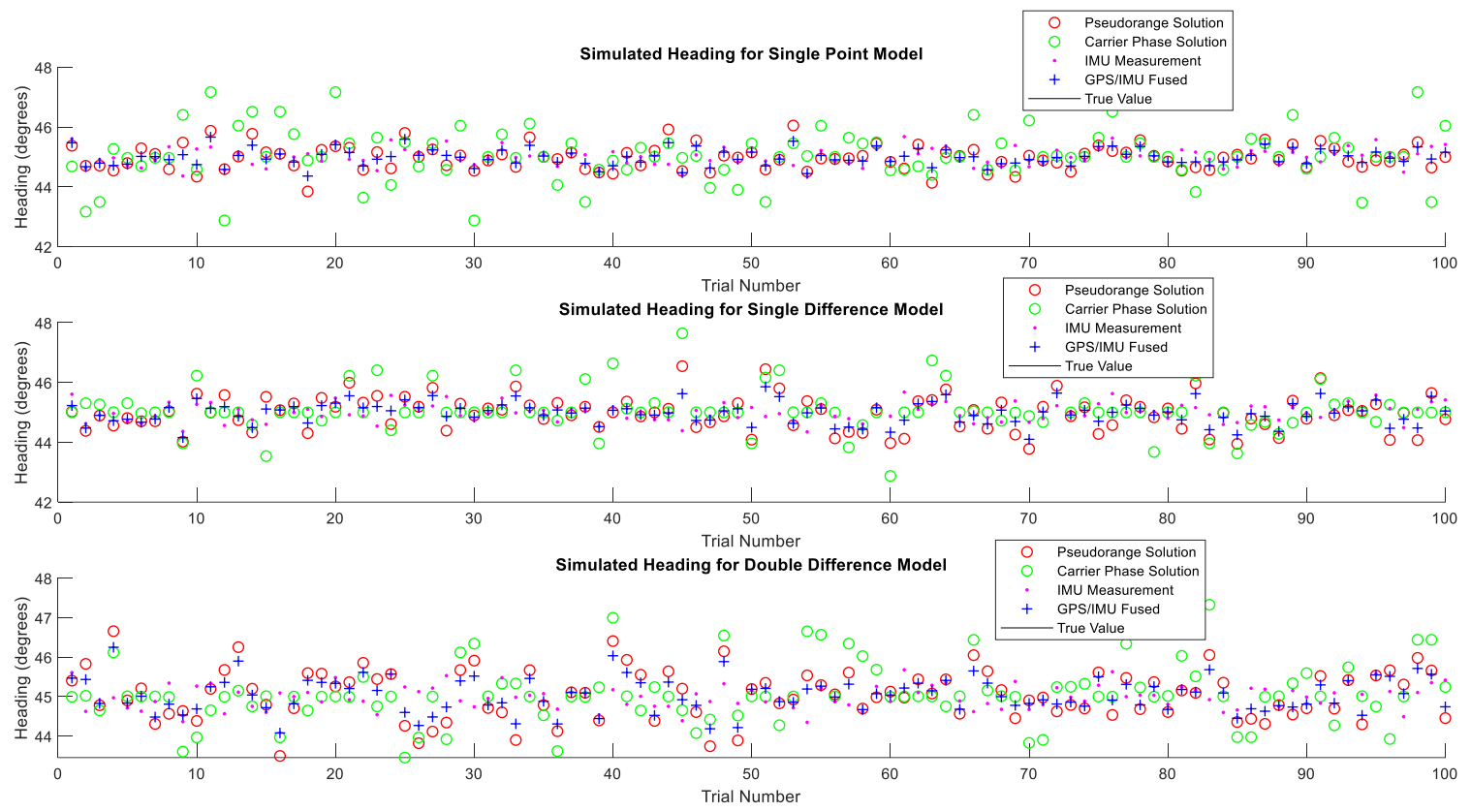

Figure 60: Computed and Measured Headings for Simulation Set A-5 


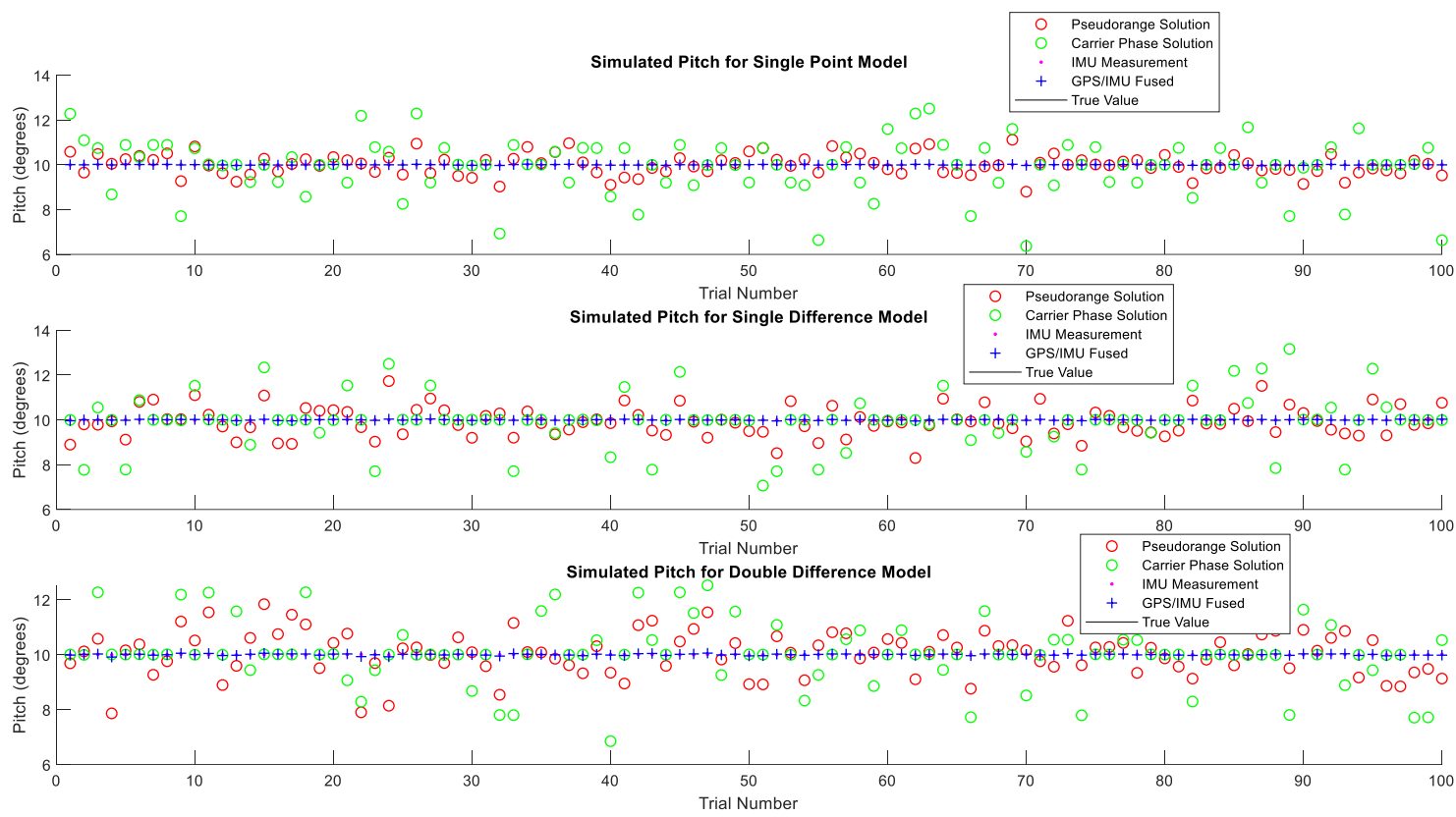

Figure 61: Computed and Measured Pitch for Simulation Set A-5

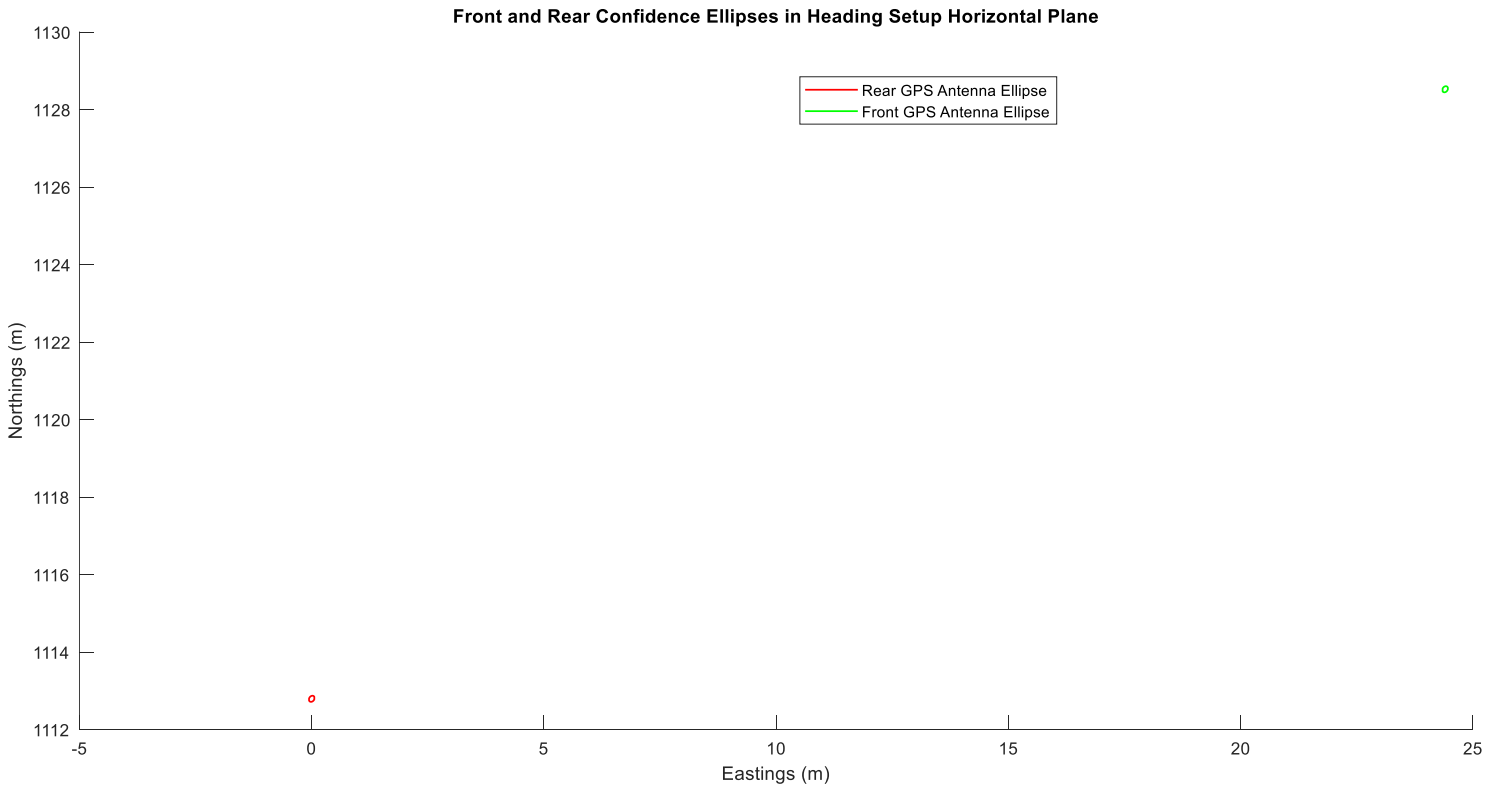

Figure 62: Propagated 95\% Confidence Ellipses for Front and Rear GPS Antennas in Simulated Data Set A-5 
Table 67: Mean Global Error Statistics of Unfused Pitch for Simulation Set A-5

\begin{tabular}{|c|c|c|c|}
\hline Method & $\begin{array}{c}\text { Measured Standard } \\
\text { Deviation } \\
\text { (degrees) }\end{array}$ & $\begin{array}{c}\text { Estimated } \\
\text { Standard } \\
\text { Deviation } \\
\text { (degrees) }\end{array}$ & $\begin{array}{c}\text { Mean RMS } \\
\text { Error } \\
\text { (degrees) }\end{array}$ \\
\hline Single Point GPS (Pseudoranges) & 0.467 & 0.264 & 0.372 \\
\hline Single Point GPS (Carrier Phase) & 1.23 & 0.001 & 0.874 \\
\hline $\begin{array}{c}\text { Single Difference GPS } \\
\text { (Pseudoranges) }\end{array}$ & 0.666 & 0.262 & 0.54 \\
\hline $\begin{array}{c}\text { Single Difference GPS (Carrier } \\
\text { Phase) }\end{array}$ & 1.159 & 0.001 & 0.679 \\
\hline $\begin{array}{c}\text { Double Difference GPS } \\
\text { (Pseudoranges) }\end{array}$ & 0.797 & 0.212 & 0.642 \\
\hline $\begin{array}{c}\text { Double Difference GPS (Carrier } \\
\text { Phase) }\end{array}$ & 1.13 & 0.002 & 0.706 \\
\hline IMU Measurement & 0.007 & - & 0.006 \\
\hline
\end{tabular}

Table 68: Mean Global Error Statistics of Unfused Heading for Simulation Set A-5

\begin{tabular}{|c|c|c|c|}
\hline Method & $\begin{array}{c}\text { Measured Standard } \\
\text { Deviation } \\
\text { (degrees) }\end{array}$ & $\begin{array}{c}\text { Estimated } \\
\text { Standard } \\
\text { Deviation } \\
\text { (degrees) }\end{array}$ & $\begin{array}{c}\text { Mean RMS } \\
\text { Error } \\
\text { (degrees) }\end{array}$ \\
\hline Single Point GPS (Pseudoranges) & 0.401 & 0.214 & 0.315 \\
\hline Single Point GPS (Carrier Phase) & 0.863 & 0.001 & 0.606 \\
\hline $\begin{array}{c}\text { Single Difference GPS } \\
\text { (Pseudoranges) }\end{array}$ & 0.569 & 0.211 & 0.449 \\
\hline $\begin{array}{c}\text { Single Difference GPS (Carrier } \\
\text { Phase) }\end{array}$ & 0.714 & 0.001 & 0.407 \\
\hline $\begin{array}{c}\text { Double Difference GPS } \\
\text { (Pseudoranges) }\end{array}$ & 0.622 & 0.161 & 0.515 \\
\hline $\begin{array}{c}\text { Double Difference GPS (Carrier } \\
\text { Phase) }\end{array}$ & 0.748 & 0.002 & 0.486 \\
\hline IMU Measurement & 0.299 & - & 0.242 \\
\hline
\end{tabular}


Table 69: Mean Global Error Statistics of Fused Pitch for Simulation Set A-5

\begin{tabular}{|c|c|c|c|}
\hline Method & $\begin{array}{c}\text { Measured Standard } \\
\text { Deviation } \\
\text { (degrees) }\end{array}$ & $\begin{array}{c}\text { Estimated } \\
\text { Standard } \\
\text { Deviation } \\
\text { (degrees) }\end{array}$ & $\begin{array}{c}\text { Mean RMS } \\
\text { Error } \\
\text { (degrees) }\end{array}$ \\
\hline Single Point GPS (Pseudoranges) & 0.013 & 0.11 & 0.01 \\
\hline Single Point GPS (Carrier Phase) & 1.099 & 0 & 0.775 \\
\hline $\begin{array}{c}\text { Single Difference GPS } \\
\text { (Pseudoranges) }\end{array}$ & 0.019 & 0.11 & 0.015 \\
\hline $\begin{array}{c}\text { Single Difference GPS (Carrier } \\
\text { Phase) }\end{array}$ & 1.024 & 0 & 0.6 \\
\hline $\begin{array}{c}\text { Double Difference GPS } \\
\text { (Pseudoranges) }\end{array}$ & 0.027 & 0.094 & 0.022 \\
\hline $\begin{array}{c}\text { Double Difference GPS (Carrier } \\
\text { Phase) }\end{array}$ & 0.874 & 0.001 & 0.544 \\
\hline IMU Measurement & 0.007 & - & 0.006 \\
\hline
\end{tabular}

Table 70: Mean Global Error Statistics of Fused Heading for Simulation Set A-5

\begin{tabular}{|c|c|c|c|}
\hline Method & $\begin{array}{c}\text { Measured Standard } \\
\text { Deviation } \\
\text { (degrees) }\end{array}$ & $\begin{array}{c}\text { Estimated } \\
\text { Standard } \\
\text { Deviation } \\
\text { (degrees) }\end{array}$ & $\begin{array}{c}\text { Mean RMS } \\
\text { Error } \\
\text { (degrees) }\end{array}$ \\
\hline Single Point GPS (Pseudoranges) & 0.267 & 0.094 & 0.213 \\
\hline Single Point GPS (Carrier Phase) & 0.861 & 0 & 0.605 \\
\hline $\begin{array}{c}\text { Single Difference GPS } \\
\text { (Pseudoranges) }\end{array}$ & 0.362 & 0.093 & 0.285 \\
\hline $\begin{array}{c}\text { Single Difference GPS (Carrier } \\
\text { Phase) }\end{array}$ & 0.713 & 0 & 0.406 \\
\hline $\begin{array}{c}\text { Double Difference GPS } \\
\text { (Pseudoranges) }\end{array}$ & 0.427 & 0.075 & 0.351 \\
\hline $\begin{array}{c}\text { Double Difference GPS (Carrier } \\
\text { Phase) }\end{array}$ & 0.744 & 0.001 & 0.483 \\
\hline IMU Measurement & 0.299 & - & 0.242 \\
\hline
\end{tabular}


Table 71: Success Rates of Reaching Angular Specifications of Unfused Pitch for Simulation Data Set A-5

\begin{tabular}{|c|c|c|c|c|c|c|c|c|}
\hline & \multicolumn{7}{|c|}{ Portion of Trials in Which Target Specification was reached } \\
\hline & $\pm 0.5^{\circ}$ & $\pm 1^{\circ}$ & $\pm 2^{\circ}$ & $\pm 5^{\circ}$ & $\pm 10^{\circ}$ & $\pm 15^{\circ}$ & $\pm 20^{\circ}$ & $\pm 25^{\circ}$ \\
\hline Method & 86 & 99 & 100 & 100 & 100 & 100 & 100 & 100 \\
\hline $\begin{array}{c}\text { Single Point GPS } \\
\text { (Pseudoranges) }\end{array}$ & 64 & 90 & 95 & 100 & 100 & 100 & 100 & 100 \\
\hline $\begin{array}{c}\text { Single Point GPS (Carrier } \\
\text { Phase) }\end{array}$ & 80 & 96 & 100 & 100 & 100 & 100 & 100 & 100 \\
\hline $\begin{array}{c}\text { Single Difference GPS } \\
\text { (Pseudoranges) }\end{array}$ & 80 & 86 & 92 & 100 & 100 & 100 & 100 & 100 \\
\hline $\begin{array}{c}\text { Single Difference GPS } \\
\text { (Carrier Phase) }\end{array}$ & 71 & 90 & 100 & 100 & 100 & 100 & 100 & 100 \\
\hline $\begin{array}{c}\text { Double Difference GPS } \\
\text { (Pseudoranges) }\end{array}$ & 73 & 84 & 92 & 100 & 100 & 100 & 100 & 100 \\
\hline $\begin{array}{c}\text { Double Difference GPS } \\
\text { (Carrier Phase) }\end{array}$ & 100 & 100 & 100 & 100 & 100 & 100 & 100 & 100 \\
\hline IMU Measured & & & & & & & & \\
\hline
\end{tabular}

Table 72: Success Rates of Reaching Angular Specifications of Unfused Heading for Simulation Data Set A-5

\begin{tabular}{|c|c|c|c|c|c|c|c|c|}
\hline & \multicolumn{7}{|c|}{ Portion of Trials in Which Target Specification was reached } \\
\hline & $\pm 0.5^{\circ}$ & $\pm 1^{\circ}$ & $\pm 2^{\circ}$ & $\pm 5^{\circ}$ & $\pm 10^{\circ}$ & $\pm 15^{\circ}$ & $\pm 20^{\circ}$ & $\pm 25^{\circ}$ \\
\hline Method & 90 & 99 & 100 & 100 & 100 & 100 & 100 & 100 \\
\hline $\begin{array}{c}\text { Single Point GPS } \\
\text { (Pseudoranges) }\end{array}$ & 78 & 85 & 97 & 100 & 100 & 100 & 100 & 100 \\
\hline $\begin{array}{c}\text { Single Point GPS (Carrier } \\
\text { Phase) }\end{array}$ & 84 & 97 & 100 & 100 & 100 & 100 & 100 & 100 \\
\hline $\begin{array}{c}\text { Single Difference GPS } \\
\text { (Pseudoranges) }\end{array}$ & 85 & 85 & 99 & 100 & 100 & 100 & 100 & 100 \\
\hline $\begin{array}{c}\text { Single Difference GPS } \\
\text { (Carrier Phase) }\end{array}$ & 73 & 94 & 100 & 100 & 100 & 100 & 100 & 100 \\
\hline $\begin{array}{c}\text { Double Difference GPS } \\
\text { (Pseudoranges) }\end{array}$ & 81 & 85 & 99 & 100 & 100 & 100 & 100 & 100 \\
\hline $\begin{array}{c}\text { Double Difference GPS } \\
\text { (Carrier Phase) }\end{array}$ & 94 & 100 & 100 & 100 & 100 & 100 & 100 & 100 \\
\hline IMU Measured & & & & & & & & \\
\hline
\end{tabular}


Table 73: Success Rates of Reaching Angular Specifications of Fused Pitch for Simulation Data Set A-5

\begin{tabular}{|c|c|c|c|c|c|c|c|c|}
\hline & \multicolumn{7}{|c|}{ Portion of Trials in Which Target Specification was reached } \\
\hline & $\pm 0.5^{\circ}$ & $\pm 1^{\circ}$ & $\pm 2^{\circ}$ & $\pm 5^{\circ}$ & $\pm 10^{\circ}$ & $\pm 15^{\circ}$ & $\pm 20^{\circ}$ & $\pm 25^{\circ}$ \\
\hline Method & 100 & 100 & 100 & 100 & 100 & 100 & 100 & 100 \\
\hline $\begin{array}{c}\text { Single Point GPS } \\
\text { (Pseudoranges) }\end{array}$ & 64 & 91 & 99 & 100 & 100 & 100 & 100 & 100 \\
\hline $\begin{array}{c}\text { Single Point GPS (Carrier } \\
\text { Phase) }\end{array}$ & 100 & 100 & 100 & 100 & 100 & 100 & 100 & 100 \\
\hline $\begin{array}{c}\text { Single Difference GPS } \\
\text { (Pseudoranges) }\end{array}$ & 83 & 86 & 98 & 100 & 100 & 100 & 100 & 100 \\
\hline $\begin{array}{c}\text { Single Difference GPS } \\
\text { (Carrier Phase) }\end{array}$ & 100 & 100 & 100 & 100 & 100 & 100 & 100 & 100 \\
\hline $\begin{array}{c}\text { Double Difference GPS } \\
\text { (Pseudoranges) }\end{array}$ & 81 & 86 & 100 & 100 & 100 & 100 & 100 & 100 \\
\hline $\begin{array}{c}\text { Double Difference GPS } \\
\text { (Carrier Phase) }\end{array}$ & 100 & 100 & 100 & 100 & 100 & 100 & 100 & 100 \\
\hline
\end{tabular}

Table 74: Success Rates of Reaching Angular Specifications of Fused Heading for Simulation Data Set A-5

\begin{tabular}{|c|c|c|c|c|c|c|c|c|}
\hline & \multicolumn{7}{|c|}{ Portion of Trials in Which Target Specification was reached } \\
\hline & $\pm 0.5^{\circ}$ & $\pm 1^{\circ}$ & $\pm 2^{\circ}$ & $\pm 5^{\circ}$ & $\pm 10^{\circ}$ & $\pm 15^{\circ}$ & $\pm 20^{\circ}$ & $\pm 25^{\circ}$ \\
\hline Method & 97 & 100 & 100 & 100 & 100 & 100 & 100 & 100 \\
\hline $\begin{array}{c}\text { Single Point GPS } \\
\text { (Pseudoranges) }\end{array}$ & 78 & 85 & 97 & 100 & 100 & 100 & 100 & 100 \\
\hline $\begin{array}{c}\text { Single Point GPS (Carrier } \\
\text { Phase) }\end{array}$ & 89 & 100 & 100 & 100 & 100 & 100 & 100 & 100 \\
\hline $\begin{array}{c}\text { Single Difference GPS } \\
\text { (Pseudoranges) }\end{array}$ & 85 & 85 & 99 & 100 & 100 & 100 & 100 & 100 \\
\hline $\begin{array}{c}\text { Single Difference GPS } \\
\text { (Carrier Phase) }\end{array}$ & 85 & 98 & 100 & 100 & 100 & 100 & 100 & 100 \\
\hline $\begin{array}{c}\text { Double Difference GPS } \\
\text { (Pseudoranges) }\end{array}$ & 81 & 85 & 99 & 100 & 100 & 100 & 100 & 100 \\
\hline $\begin{array}{c}\text { Double Difference GPS } \\
\text { (Carrier Phase) }\end{array}$ & 94 & 100 & 100 & 100 & 100 & 100 & 100 & 100 \\
\hline IMU Measured & & & & & & & & \\
\hline
\end{tabular}


Table 75: Pitch / Heading Correlation Coefficients for Each Computation Method in Simulation Data Set A-5

\begin{tabular}{|c|c|c|}
\hline & \multicolumn{2}{|c|}{ Pitch / Heading Correlation Coefficient } \\
\hline Method & Unfused & Fused \\
\hline $\begin{array}{c}\text { Single Point GPS } \\
\text { (Pseudoranges) }\end{array}$ & 0.873332 & 0.834883 \\
\hline $\begin{array}{c}\text { Single Difference GPS } \\
\text { (Pseudoranges) }\end{array}$ & 0.866496 & 0.818398 \\
\hline $\begin{array}{c}\text { Double Difference GPS } \\
\text { (Pseudoranges) }\end{array}$ & 0.803797 & 0.772162 \\
\hline
\end{tabular}

\section{Set A-6: 50 m Dual Antenna Separation}

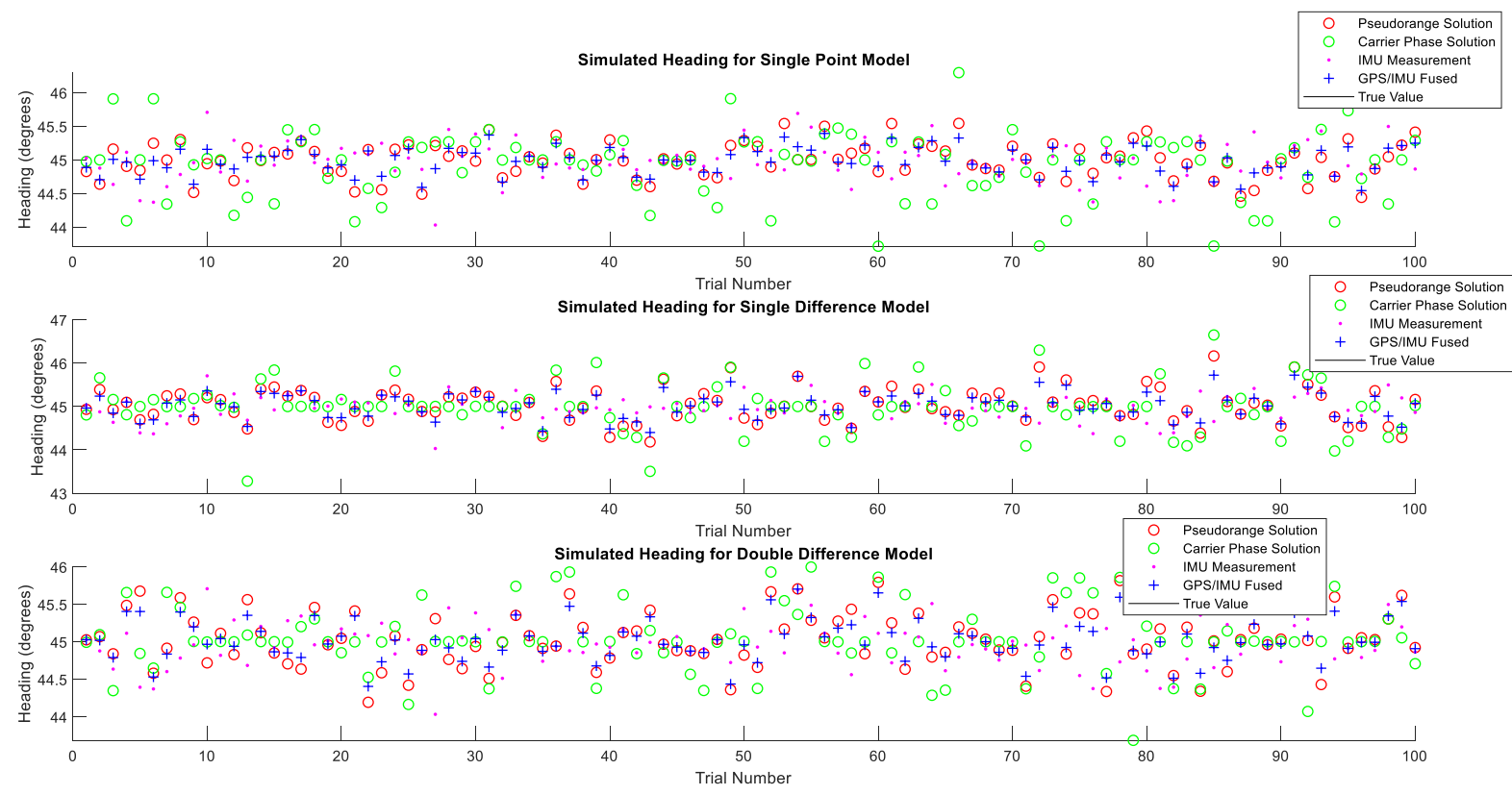

Figure 63: Computed and Measured Headings for Simulation Set A-6 


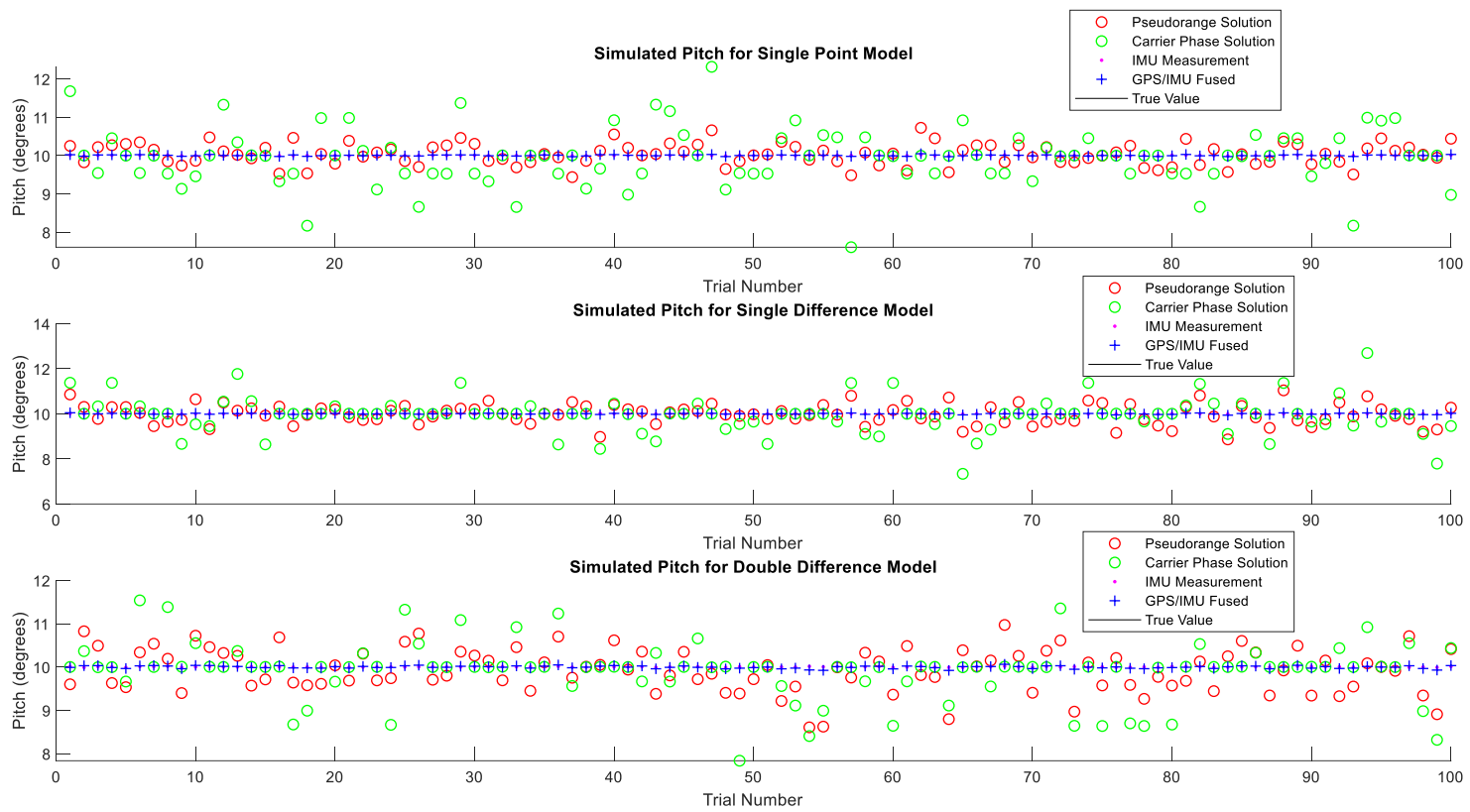

Figure 64: Computed and Measured Pitch for Simulation Set A-6

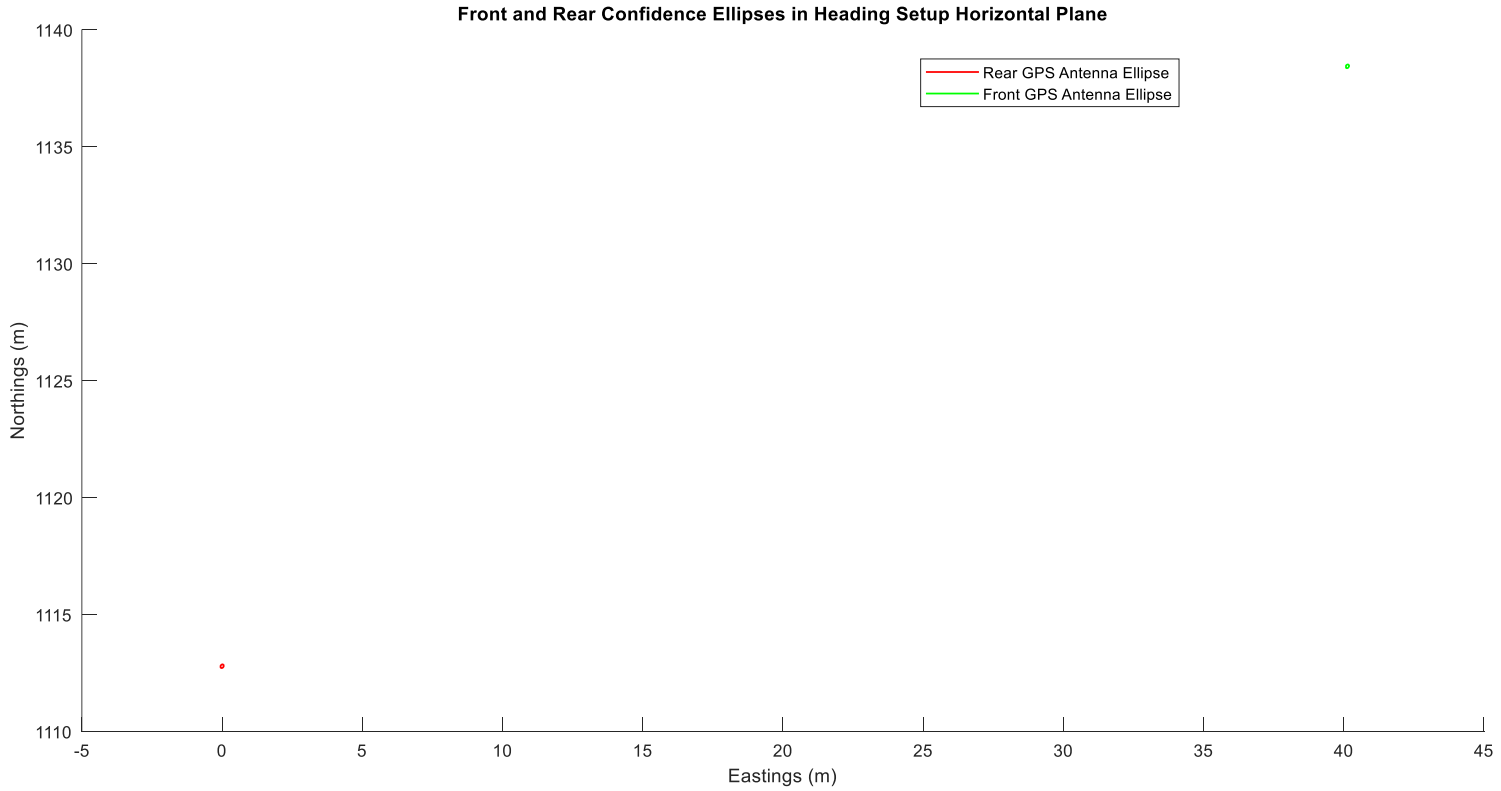

Figure 65: Propagated 95\% Confidence Ellipses for Front and Rear GPS Antennas in Simulated Data Set A-6 
Table 76: Mean Global Error Statistics of Unfused Pitch for Simulation Set A-6

\begin{tabular}{|c|c|c|c|}
\hline Method & $\begin{array}{c}\text { Measured Standard } \\
\text { Deviation } \\
\text { (degrees) }\end{array}$ & $\begin{array}{c}\text { Estimated } \\
\text { Standard } \\
\text { Deviation } \\
\text { (degrees) }\end{array}$ & $\begin{array}{c}\text { Mean RMS } \\
\text { Error } \\
\text { (degrees) }\end{array}$ \\
\hline Single Point GPS (Pseudoranges) & 0.28 & 0.159 & 0.233 \\
\hline Single Point GPS (Carrier Phase) & 0.742 & 0.001 & 0.526 \\
\hline $\begin{array}{c}\text { Single Difference GPS } \\
\text { (Pseudoranges) }\end{array}$ & 0.446 & 0.158 & 0.362 \\
\hline $\begin{array}{c}\text { Single Difference GPS (Carrier } \\
\text { Phase) }\end{array}$ & 0.793 & 0.001 & 0.487 \\
\hline $\begin{array}{c}\text { Double Difference GPS } \\
\text { (Pseudoranges) }\end{array}$ & 0.502 & 0.126 & 0.418 \\
\hline $\begin{array}{c}\text { Double Difference GPS (Carrier } \\
\text { Phase) }\end{array}$ & 0.67 & 0.001 & 0.397 \\
\hline IMU Measurement & 0.007 & - & 0.006 \\
\hline
\end{tabular}

Table 77: Mean Global Error Statistics of Unfused Heading for Simulation Set A-6

\begin{tabular}{|c|c|c|c|}
\hline Method & $\begin{array}{c}\text { Measured Standard } \\
\text { Deviation } \\
\text { (degrees) }\end{array}$ & $\begin{array}{c}\text { Estimated } \\
\text { Standard } \\
\text { Deviation } \\
\text { (degrees) }\end{array}$ & $\begin{array}{c}\text { Mean RMS } \\
\text { Error } \\
\text { (degrees) }\end{array}$ \\
\hline Single Point GPS (Pseudoranges) & 0.26 & 0.129 & 0.207 \\
\hline Single Point GPS (Carrier Phase) & 0.498 & 0 & 0.363 \\
\hline $\begin{array}{c}\text { Single Difference GPS } \\
\text { (Pseudoranges) }\end{array}$ & 0.387 & 0.127 & 0.31 \\
\hline $\begin{array}{c}\text { Single Difference GPS (Carrier } \\
\text { Phase) }\end{array}$ & 0.55 & 0 & 0.348 \\
\hline $\begin{array}{c}\text { Double Difference GPS } \\
\text { (Pseudoranges) }\end{array}$ & 0.355 & 0.094 & 0.279 \\
\hline $\begin{array}{c}\text { Double Difference GPS (Carrier } \\
\text { Phase) }\end{array}$ & 0.447 & 0.001 & 0.294 \\
\hline IMU Measurement & 0.305 & - & 0.239 \\
\hline
\end{tabular}


Table 78: Mean Global Error Statistics of Fused Pitch for Simulation Set A-6

\begin{tabular}{|c|c|c|c|}
\hline Method & $\begin{array}{c}\text { Measured Standard } \\
\text { Deviation } \\
\text { (degrees) }\end{array}$ & $\begin{array}{c}\text { Estimated } \\
\text { Standard } \\
\text { Deviation } \\
\text { (degrees) }\end{array}$ & $\begin{array}{c}\text { Mean RMS } \\
\text { Error } \\
\text { (degrees) }\end{array}$ \\
\hline Single Point GPS (Pseudoranges) & 0.015 & 0.074 & 0.012 \\
\hline Single Point GPS (Carrier Phase) & 0.687 & 0 & 0.487 \\
\hline $\begin{array}{c}\text { Single Difference GPS } \\
\text { (Pseudoranges) }\end{array}$ & 0.02 & 0.074 & 0.016 \\
\hline $\begin{array}{c}\text { Single Difference GPS (Carrier } \\
\text { Phase) }\end{array}$ & 0.735 & 0 & 0.451 \\
\hline $\begin{array}{c}\text { Double Difference GPS } \\
\text { (Pseudoranges) }\end{array}$ & 0.029 & 0.06 & 0.023 \\
\hline $\begin{array}{c}\text { Double Difference GPS (Carrier } \\
\text { Phase) }\end{array}$ & 0.572 & 0.001 & 0.338 \\
\hline IMU Measurement & 0.007 & - & 0.006 \\
\hline
\end{tabular}

Table 79: Mean Global Error Statistics of Fused Heading for Simulation Set A-6

\begin{tabular}{|c|c|c|c|}
\hline Method & $\begin{array}{c}\text { Measured Standard } \\
\text { Deviation } \\
\text { (degrees) }\end{array}$ & $\begin{array}{c}\text { Estimated } \\
\text { Standard } \\
\text { Deviation } \\
\text { (degrees) }\end{array}$ & $\begin{array}{c}\text { Mean RMS } \\
\text { Error } \\
\text { (degrees) }\end{array}$ \\
\hline Single Point GPS (Pseudoranges) & 0.203 & 0.061 & 0.167 \\
\hline Single Point GPS (Carrier Phase) & 0.497 & 0 & 0.363 \\
\hline $\begin{array}{c}\text { Single Difference GPS } \\
\text { (Pseudoranges) }\end{array}$ & 0.297 & 0.06 & 0.244 \\
\hline $\begin{array}{c}\text { Single Difference GPS (Carrier } \\
\text { Phase) }\end{array}$ & 0.549 & 0 & 0.348 \\
\hline $\begin{array}{c}\text { Double Difference GPS } \\
\text { (Pseudoranges) }\end{array}$ & 0.274 & 0.046 & 0.21 \\
\hline $\begin{array}{c}\text { Double Difference GPS (Carrier } \\
\text { Phase) }\end{array}$ & 0.445 & 0 & 0.293 \\
\hline IMU Measurement & 0.305 & - & 0.239 \\
\hline
\end{tabular}


Table 80: Success Rates of Reaching Angular Specifications of Unfused Pitch for Simulation Data Set A-6

\begin{tabular}{|c|c|c|c|c|c|c|c|c|}
\hline & \multicolumn{7}{|c|}{ Portion of Trials in Which Target Specification was reached } \\
\hline & $\pm 0.5^{\circ}$ & $\pm 1^{\circ}$ & $\pm 2^{\circ}$ & $\pm 5^{\circ}$ & $\pm 10^{\circ}$ & $\pm 15^{\circ}$ & $\pm 20^{\circ}$ & $\pm 25^{\circ}$ \\
\hline Method & 97 & 100 & 100 & 100 & 100 & 100 & 100 & 100 \\
\hline $\begin{array}{c}\text { Single Point GPS } \\
\text { (Pseudoranges) }\end{array}$ & 83 & 94 & 99 & 100 & 100 & 100 & 100 & 100 \\
\hline $\begin{array}{c}\text { Single Point GPS (Carrier } \\
\text { Phase) }\end{array}$ & 88 & 99 & 100 & 100 & 100 & 100 & 100 & 100 \\
\hline $\begin{array}{c}\text { Single Difference GPS } \\
\text { (Pseudoranges) }\end{array}$ & 87 & 90 & 99 & 100 & 100 & 100 & 100 & 100 \\
\hline $\begin{array}{c}\text { Single Difference GPS } \\
\text { (Carrier Phase) }\end{array}$ & 88 & 100 & 100 & 100 & 100 & 100 & 100 & 100 \\
\hline $\begin{array}{c}\text { Double Difference GPS } \\
\text { (Pseudoranges) }\end{array}$ & 87 & 94 & 100 & 100 & 100 & 100 & 100 & 100 \\
\hline $\begin{array}{c}\text { Double Difference GPS } \\
\text { (Carrier Phase) }\end{array}$ & 100 & 100 & 100 & 100 & 100 & 100 & 100 & 100 \\
\hline IMU Measured & & & & & & & & \\
\hline
\end{tabular}

Table 81: Success Rates of Reaching Angular Specifications of Unfused Heading for Simulation Data Set A-6

\begin{tabular}{|c|c|c|c|c|c|c|c|c|}
\hline & \multicolumn{7}{|c|}{ Portion of Trials in Which Target Specification was reached } \\
\hline & $\pm 0.5^{\circ}$ & $\pm 1^{\circ}$ & $\pm 2^{\circ}$ & $\pm 5^{\circ}$ & $\pm 10^{\circ}$ & $\pm 15^{\circ}$ & $\pm 20^{\circ}$ & $\pm 25^{\circ}$ \\
\hline Method & 97 & 100 & 100 & 100 & 100 & 100 & 100 & 100 \\
\hline $\begin{array}{c}\text { Single Point GPS } \\
\text { (Pseudoranges) }\end{array}$ & 95 & 99 & 100 & 100 & 100 & 100 & 100 & 100 \\
\hline $\begin{array}{c}\text { Single Point GPS (Carrier } \\
\text { Phase) }\end{array}$ & 90 & 99 & 100 & 100 & 100 & 100 & 100 & 100 \\
\hline $\begin{array}{c}\text { Single Difference GPS } \\
\text { (Pseudoranges) }\end{array}$ & 84 & 97 & 100 & 100 & 100 & 100 & 100 & 100 \\
\hline $\begin{array}{c}\text { Single Difference GPS } \\
\text { (Carrier Phase) }\end{array}$ & 89 & 100 & 100 & 100 & 100 & 100 & 100 & 100 \\
\hline $\begin{array}{c}\text { Double Difference GPS } \\
\text { (Pseudoranges) }\end{array}$ & 82 & 100 & 100 & 100 & 100 & 100 & 100 & 100 \\
\hline $\begin{array}{c}\text { Double Difference GPS } \\
\text { (Carrier Phase) }\end{array}$ & 97 & 100 & 100 & 100 & 100 & 100 & 100 & 100 \\
\hline IMU Measured & & & & & & & & \\
\hline
\end{tabular}


Table 82: Success Rates of Reaching Angular Specifications of Fused Pitch for Simulation Data Set A-6

\begin{tabular}{|c|c|c|c|c|c|c|c|c|}
\hline & \multicolumn{7}{|c|}{ Portion of Trials in Which Target Specification was reached } \\
\hline Method & $\pm 0.5^{\circ}$ & $\pm 1^{\circ}$ & $\pm 2^{\circ}$ & $\pm 5^{\circ}$ & $\pm 10^{\circ}$ & $\pm 15^{\circ}$ & $\pm 20^{\circ}$ & $\pm 25^{\circ}$ \\
\hline $\begin{array}{c}\text { Single Point GPS } \\
\text { (Pseudoranges) }\end{array}$ & 97 & 100 & 100 & 100 & 100 & 100 & 100 & 100 \\
\hline $\begin{array}{c}\text { Single Point GPS (Carrier } \\
\text { Phase) }\end{array}$ & 83 & 94 & 99 & 100 & 100 & 100 & 100 & 100 \\
\hline $\begin{array}{c}\text { Single Difference GPS } \\
\text { (Pseudoranges) }\end{array}$ & 88 & 99 & 100 & 100 & 100 & 100 & 100 & 100 \\
\hline $\begin{array}{c}\text { Single Difference GPS } \\
\text { (Carrier Phase) }\end{array}$ & 87 & 90 & 99 & 100 & 100 & 100 & 100 & 100 \\
\hline $\begin{array}{c}\text { Double Difference GPS } \\
\text { (Pseudoranges) }\end{array}$ & 88 & 100 & 100 & 100 & 100 & 100 & 100 & 100 \\
\hline $\begin{array}{c}\text { Double Difference GPS } \\
\text { (Carrier Phase) }\end{array}$ & 87 & 94 & 100 & 100 & 100 & 100 & 100 & 100 \\
\hline IMU Measured & 100 & 100 & 100 & 100 & 100 & 100 & 100 & 100 \\
\hline
\end{tabular}

Table 83: Success Rates of Reaching Angular Specifications of Fused Heading for Simulation Data Set A-6

\begin{tabular}{|c|c|c|c|c|c|c|c|c|}
\hline & \multicolumn{7}{|c|}{ Portion of Trials in Which Target Specification was reached } \\
\hline & $\pm 0.5^{\circ}$ & $\pm 1^{\circ}$ & $\pm 2^{\circ}$ & $\pm 5^{\circ}$ & $\pm 10^{\circ}$ & $\pm 15^{\circ}$ & $\pm 20^{\circ}$ & $\pm 25^{\circ}$ \\
\hline Method & 100 & 100 & 100 & 100 & 100 & 100 & 100 & 100 \\
\hline $\begin{array}{c}\text { Single Point GPS } \\
\text { (Pseudoranges) }\end{array}$ & 86 & 94 & 99 & 100 & 100 & 100 & 100 & 100 \\
\hline $\begin{array}{c}\text { Single Point GPS (Carrier } \\
\text { Phase) }\end{array}$ & 100 & 100 & 100 & 100 & 100 & 100 & 100 & 100 \\
\hline $\begin{array}{c}\text { Single Difference GPS } \\
\text { (Pseudoranges) }\end{array}$ & 88 & 90 & 99 & 100 & 100 & 100 & 100 & 100 \\
\hline $\begin{array}{c}\text { Single Difference GPS } \\
\text { (Carrier Phase) }\end{array}$ & 100 & 100 & 100 & 100 & 100 & 100 & 100 & 100 \\
\hline $\begin{array}{c}\text { Double Difference GPS } \\
\text { (Pseudoranges) }\end{array}$ & 91 & 95 & 100 & 100 & 100 & 100 & 100 & 100 \\
\hline $\begin{array}{c}\text { Double Difference GPS } \\
\text { (Carrier Phase) }\end{array}$ & 100 & 100 & 100 & 100 & 100 & 100 & 100 & 100 \\
\hline IMU Measured & & & & & & & & \\
\hline
\end{tabular}


Table 84: Pitch / Heading Correlation Coefficients for Each Computation Method in Simulation Data Set A-6

\begin{tabular}{|c|c|c|}
\hline & \multicolumn{2}{|c|}{ Pitch / Heading Correlation Coefficient } \\
\hline Method & Unfused & Fused \\
\hline $\begin{array}{c}\text { Single Point GPS } \\
\text { (Pseudoranges) }\end{array}$ & 0.86429 & 0.846041 \\
\hline $\begin{array}{c}\text { Single Difference GPS } \\
\text { (Pseudoranges) }\end{array}$ & 0.867223 & 0.849816 \\
\hline $\begin{array}{c}\text { Double Difference GPS } \\
\text { (Pseudoranges) }\end{array}$ & 0.784954 & 0.769789 \\
\hline
\end{tabular}

\section{Set A-7: 70 m Dual Antenna Separation}
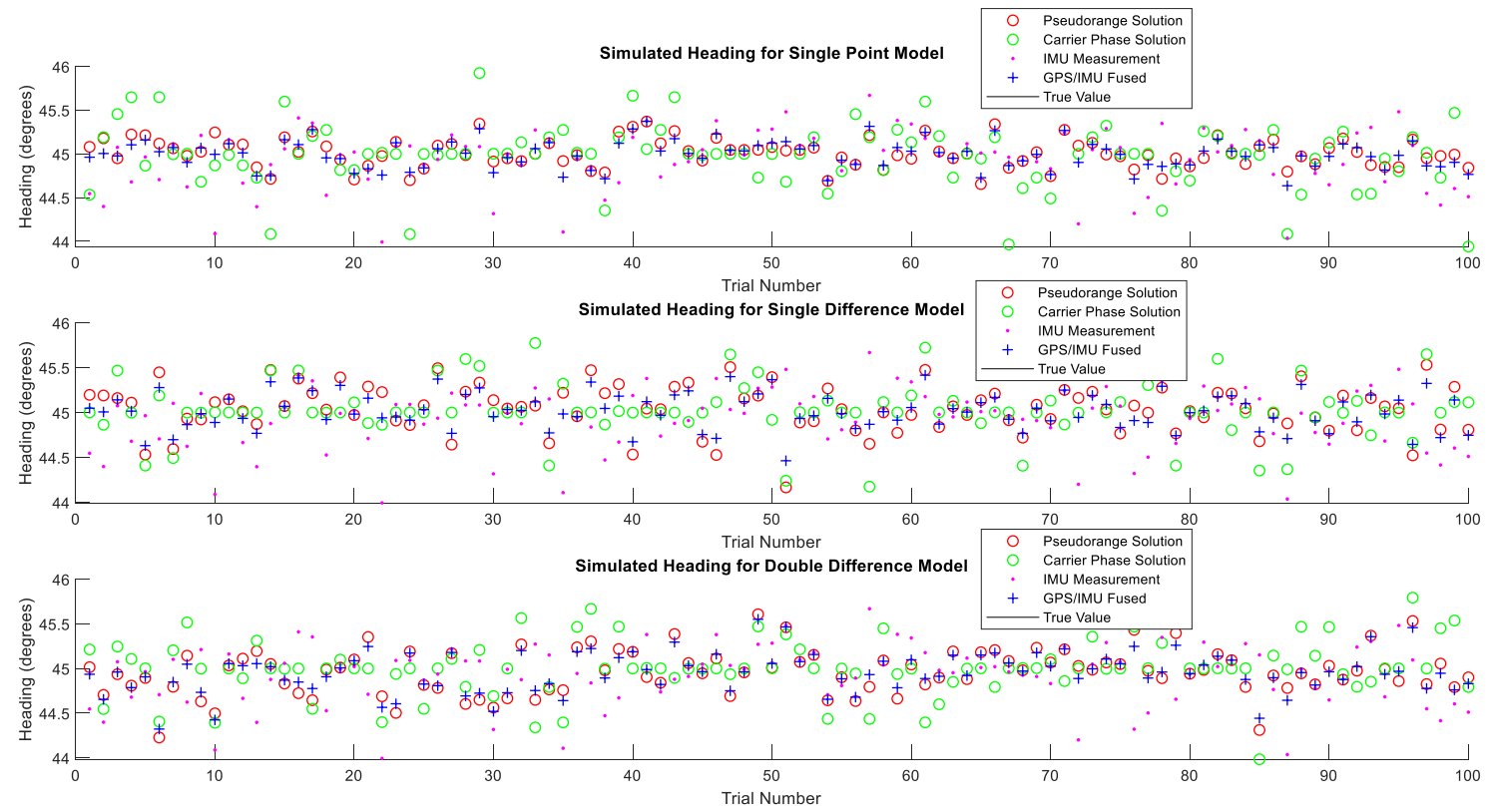

Figure 66: Computed and Measured Headings for Simulation Set A-7 


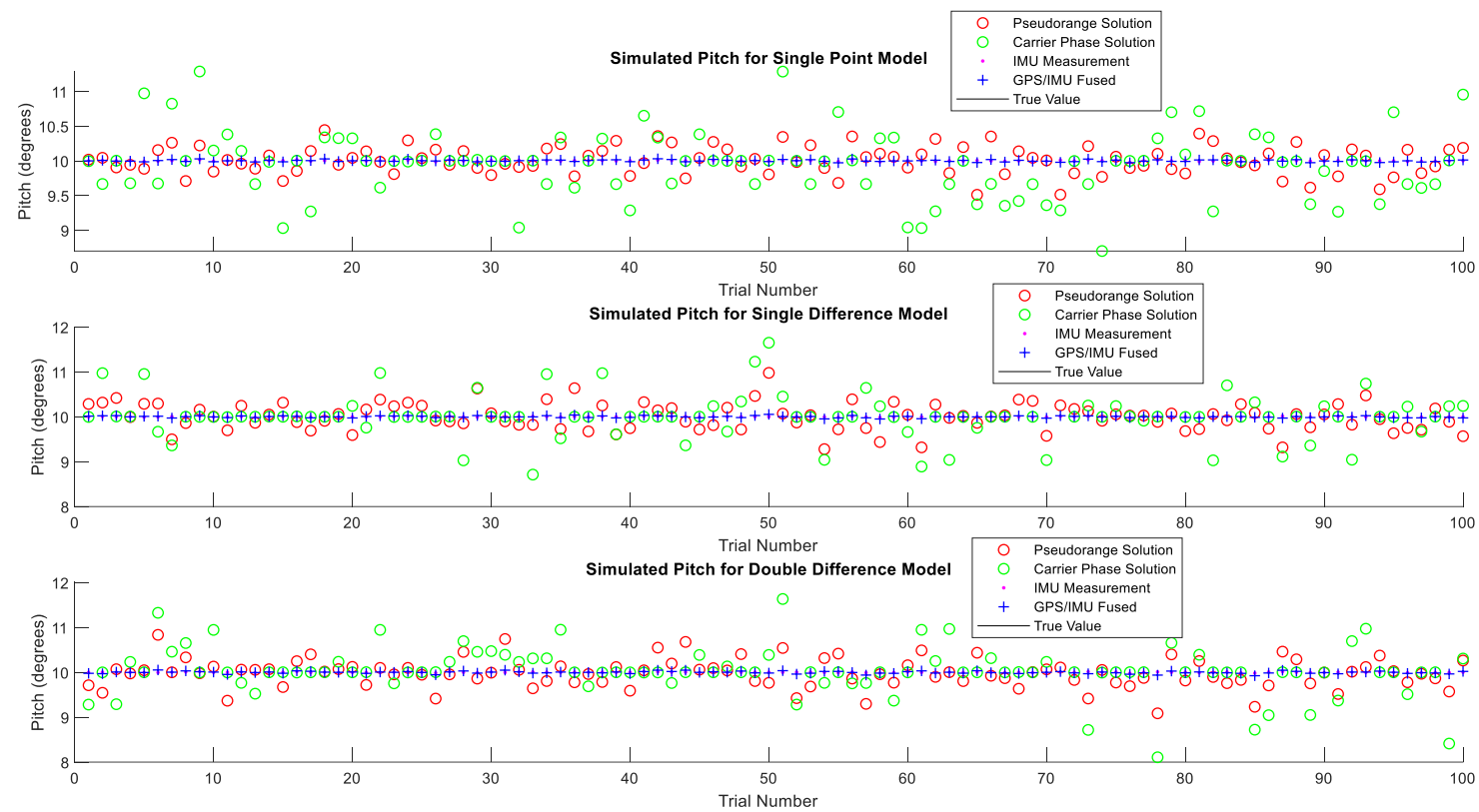

Figure 67: Computed and Measured Pitch for Simulation Set A-7

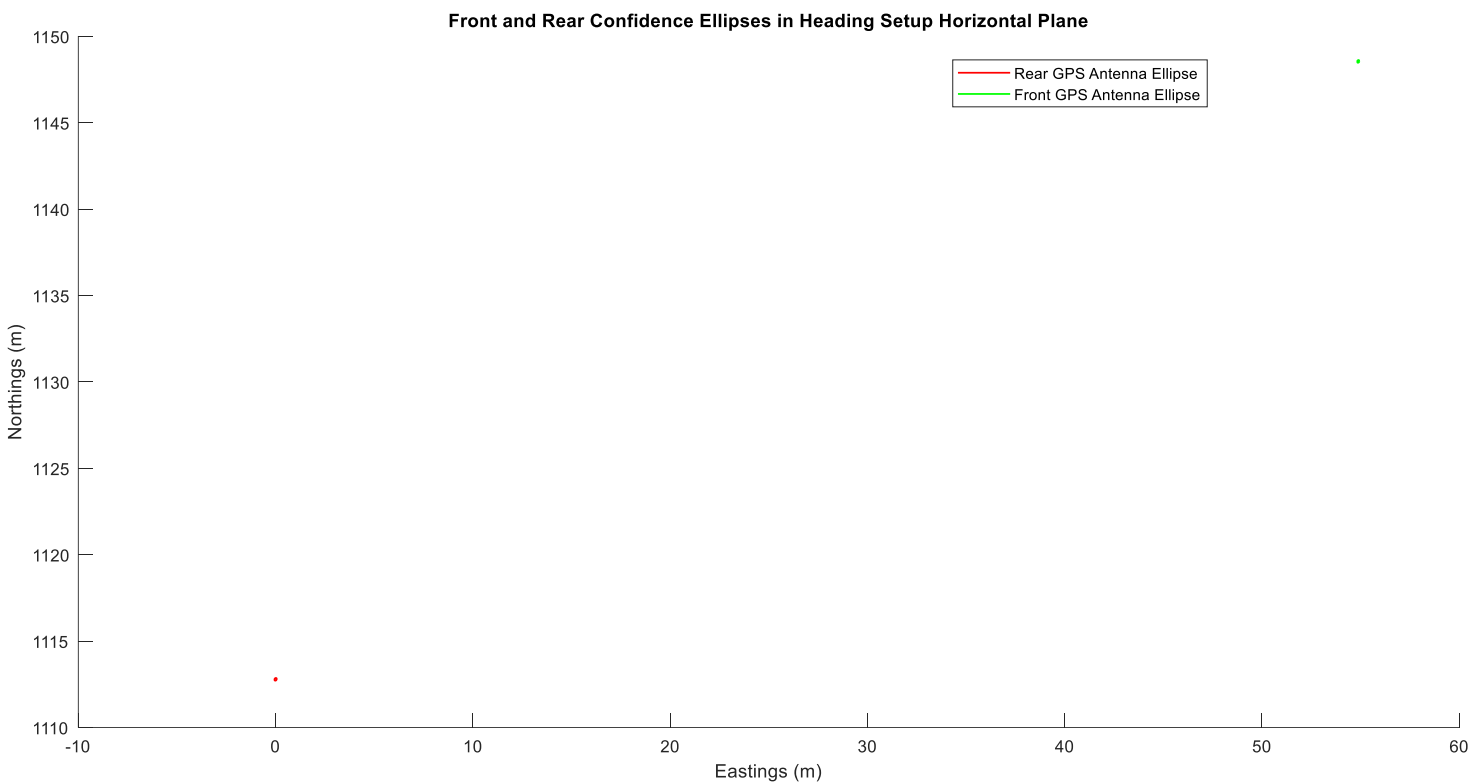

Figure 68: Propagated 95\% Confidence Ellipses for Front and Rear GPS Antennas in Simulated Data Set A-7 
Table 85: Mean Global Error Statistics of Unfused Pitch for Simulation Set A-7

\begin{tabular}{|c|c|c|c|}
\hline Method & $\begin{array}{c}\text { Measured Standard } \\
\text { Deviation } \\
\text { (degrees) }\end{array}$ & $\begin{array}{c}\text { Estimated } \\
\text { Standard } \\
\text { Deviation } \\
\text { (degrees) }\end{array}$ & $\begin{array}{c}\text { Mean RMS } \\
\text { Error } \\
\text { (degrees) }\end{array}$ \\
\hline Single Point GPS (Pseudoranges) & 0.203 & 0.113 & 0.166 \\
\hline Single Point GPS (Carrier Phase) & 0.48 & 0 & 0.34 \\
\hline $\begin{array}{c}\text { Single Difference GPS } \\
\text { (Pseudoranges) }\end{array}$ & 0.302 & 0.113 & 0.241 \\
\hline $\begin{array}{c}\text { Single Difference GPS (Carrier } \\
\text { Phase) }\end{array}$ & 0.51 & 0 & 0.304 \\
\hline $\begin{array}{c}\text { Double Difference GPS } \\
\text { (Pseudoranges) }\end{array}$ & 0.323 & 0.09 & 0.243 \\
\hline $\begin{array}{c}\text { Double Difference GPS (Carrier } \\
\text { Phase) }\end{array}$ & 0.54 & 0.001 & 0.323 \\
\hline IMU Measurement & 0.007 & - & 0.006 \\
\hline
\end{tabular}

Table 86: Mean Global Error Statistics of Unfused Heading for Simulation Set A-7

\begin{tabular}{|c|c|c|c|}
\hline Method & $\begin{array}{c}\text { Measured Standard } \\
\text { Deviation } \\
\text { (degrees) }\end{array}$ & $\begin{array}{c}\text { Estimated } \\
\text { Standard } \\
\text { Deviation } \\
\text { (degrees) }\end{array}$ & $\begin{array}{c}\text { Mean RMS } \\
\text { Error } \\
\text { (degrees) }\end{array}$ \\
\hline Single Point GPS (Pseudoranges) & 0.16 & 0.091 & 0.127 \\
\hline Single Point GPS (Carrier Phase) & 0.367 & 0 & 0.25 \\
\hline $\begin{array}{c}\text { Single Difference GPS } \\
\text { (Pseudoranges) }\end{array}$ & 0.258 & 0.091 & 0.208 \\
\hline $\begin{array}{c}\text { Single Difference GPS (Carrier } \\
\text { Phase) }\end{array}$ & 0.309 & 0 & 0.19 \\
\hline $\begin{array}{c}\text { Double Difference GPS } \\
\text { (Pseudoranges) }\end{array}$ & 0.251 & 0.068 & 0.197 \\
\hline $\begin{array}{c}\text { Double Difference GPS (Carrier } \\
\text { Phase) }\end{array}$ & 0.315 & 0.001 & 0.204 \\
\hline IMU Measurement & 0.342 & - & 0.263 \\
\hline
\end{tabular}


Table 87: Mean Global Error Statistics of Fused Pitch for Simulation Set A-7

\begin{tabular}{|c|c|c|c|}
\hline Method & $\begin{array}{c}\text { Measured Standard } \\
\text { Deviation } \\
\text { (degrees) }\end{array}$ & $\begin{array}{c}\text { Estimated } \\
\text { Standard } \\
\text { Deviation } \\
\text { (degrees) }\end{array}$ & $\begin{array}{c}\text { Mean RMS } \\
\text { Error } \\
\text { (degrees) }\end{array}$ \\
\hline Single Point GPS (Pseudoranges) & 0.013 & 0.055 & 0.011 \\
\hline Single Point GPS (Carrier Phase) & 0.454 & 0 & 0.322 \\
\hline $\begin{array}{c}\text { Single Difference GPS } \\
\text { (Pseudoranges) }\end{array}$ & 0.019 & 0.055 & 0.015 \\
\hline $\begin{array}{c}\text { Single Difference GPS (Carrier } \\
\text { Phase) }\end{array}$ & 0.482 & 0 & 0.288 \\
\hline $\begin{array}{c}\text { Double Difference GPS } \\
\text { (Pseudoranges) }\end{array}$ & 0.025 & 0.044 & 0.019 \\
\hline $\begin{array}{c}\text { Double Difference GPS (Carrier } \\
\text { Phase) }\end{array}$ & 0.48 & 0 & 0.286 \\
\hline IMU Measurement & 0.007 & - & 0.006 \\
\hline
\end{tabular}

Table 88: Mean Global Error Statistics of Fused Heading for Simulation Set A-7

\begin{tabular}{|c|c|c|c|}
\hline Method & $\begin{array}{c}\text { Measured Standard } \\
\text { Deviation } \\
\text { (degrees) }\end{array}$ & $\begin{array}{c}\text { Estimated } \\
\text { Standard } \\
\text { Deviation } \\
\text { (degrees) }\end{array}$ & $\begin{array}{c}\text { Mean RMS } \\
\text { Error } \\
\text { (degrees) }\end{array}$ \\
\hline Single Point GPS (Pseudoranges) & 0.157 & 0.044 & 0.125 \\
\hline Single Point GPS (Carrier Phase) & 0.367 & 0 & 0.25 \\
\hline $\begin{array}{c}\text { Single Difference GPS } \\
\text { (Pseudoranges) }\end{array}$ & 0.2 & 0.044 & 0.161 \\
\hline $\begin{array}{c}\text { Single Difference GPS (Carrier } \\
\text { Phase) }\end{array}$ & 0.308 & 0 & 0.19 \\
\hline $\begin{array}{c}\text { Double Difference GPS } \\
\text { (Pseudoranges) }\end{array}$ & 0.221 & 0.033 & 0.175 \\
\hline $\begin{array}{c}\text { Double Difference GPS (Carrier } \\
\text { Phase) }\end{array}$ & 0.314 & 0 & 0.203 \\
\hline IMU Measurement & 0.342 & - & 0.263 \\
\hline
\end{tabular}


Table 89: Success Rates of Reaching Angular Specifications of Unfused Pitch for Simulation Data Set A-7

\begin{tabular}{|c|c|c|c|c|c|c|c|c|}
\hline & \multicolumn{7}{|c|}{ Portion of Trials in Which Target Specification was reached } \\
\hline & $\pm 0.5^{\circ}$ & $\pm 1^{\circ}$ & $\pm 2^{\circ}$ & $\pm 5^{\circ}$ & $\pm 10^{\circ}$ & $\pm 15^{\circ}$ & $\pm 20^{\circ}$ & $\pm 25^{\circ}$ \\
\hline Method & 100 & 100 & 100 & 100 & 100 & 100 & 100 & 100 \\
\hline $\begin{array}{c}\text { Single Point GPS } \\
\text { (Pseudoranges) }\end{array}$ & 90 & 98 & 100 & 100 & 100 & 100 & 100 & 100 \\
\hline $\begin{array}{c}\text { Single Point GPS (Carrier } \\
\text { Phase) }\end{array}$ & 97 & 100 & 100 & 100 & 100 & 100 & 100 & 100 \\
\hline $\begin{array}{c}\text { Single Difference GPS } \\
\text { (Pseudoranges) }\end{array}$ & 86 & 98 & 100 & 100 & 100 & 100 & 100 & 100 \\
\hline $\begin{array}{c}\text { Single Difference GPS } \\
\text { (Carrier Phase) }\end{array}$ & 95 & 100 & 100 & 100 & 100 & 100 & 100 & 100 \\
\hline $\begin{array}{c}\text { Double Difference GPS } \\
\text { (Pseudoranges) }\end{array}$ & 87 & 98 & 100 & 100 & 100 & 100 & 100 & 100 \\
\hline $\begin{array}{c}\text { Double Difference GPS } \\
\text { (Carrier Phase) }\end{array}$ & 100 & 100 & 100 & 100 & 100 & 100 & 100 & 100 \\
\hline IMU Measured & & & & & & & & \\
\hline
\end{tabular}

Table 90: Success Rates of Reaching Angular Specifications of Unfused Heading for Simulation Data Set A-7

\begin{tabular}{|c|c|c|c|c|c|c|c|c|}
\hline & \multicolumn{7}{|c|}{ Portion of Trials in Which Target Specification was reached } \\
\hline & $\pm 0.5^{\circ}$ & $\pm 1^{\circ}$ & $\pm 2^{\circ}$ & $\pm 5^{\circ}$ & $\pm 10^{\circ}$ & $\pm 15^{\circ}$ & $\pm 20^{\circ}$ & $\pm 25^{\circ}$ \\
\hline Method & 100 & 100 & 100 & 100 & 100 & 100 & 100 & 100 \\
\hline $\begin{array}{c}\text { Single Point GPS } \\
\text { (Pseudoranges) }\end{array}$ & 92 & 100 & 100 & 100 & 100 & 100 & 100 & 100 \\
\hline $\begin{array}{c}\text { Single Point GPS (Carrier } \\
\text { Phase) }\end{array}$ & 98 & 100 & 100 & 100 & 100 & 100 & 100 & 100 \\
\hline $\begin{array}{c}\text { Single Difference GPS } \\
\text { (Pseudoranges) }\end{array}$ & 92 & 100 & 100 & 100 & 100 & 100 & 100 & 100 \\
\hline $\begin{array}{c}\text { Single Difference GPS } \\
\text { (Carrier Phase) }\end{array}$ & 98 & 100 & 100 & 100 & 100 & 100 & 100 & 100 \\
\hline $\begin{array}{c}\text { Double Difference GPS } \\
\text { (Pseudoranges) }\end{array}$ & 94 & 100 & 100 & 100 & 100 & 100 & 100 & 100 \\
\hline $\begin{array}{c}\text { Double Difference GPS } \\
\text { (Carrier Phase) }\end{array}$ & 99 & 100 & 100 & 100 & 100 & 100 & 100 & 100 \\
\hline IMU Measured & & & & & & & & \\
\hline
\end{tabular}


Table 91: Success Rates of Reaching Angular Specifications of Fused Pitch for Simulation Data Set A-7

\begin{tabular}{|c|c|c|c|c|c|c|c|c|}
\hline & \multicolumn{7}{|c|}{ Portion of Trials in Which Target Specification was reached } \\
\hline & $\pm 0.5^{\circ}$ & $\pm 1^{\circ}$ & $\pm 2^{\circ}$ & $\pm 5^{\circ}$ & $\pm 10^{\circ}$ & $\pm 15^{\circ}$ & $\pm 20^{\circ}$ & $\pm 25^{\circ}$ \\
\hline Method & 100 & 100 & 100 & 100 & 100 & 100 & 100 & 100 \\
\hline $\begin{array}{c}\text { Single Point GPS } \\
\text { (Pseudoranges) }\end{array}$ & 90 & 98 & 100 & 100 & 100 & 100 & 100 & 100 \\
\hline $\begin{array}{c}\text { Single Point GPS (Carrier } \\
\text { Phase) }\end{array}$ & 100 & 100 & 100 & 100 & 100 & 100 & 100 & 100 \\
\hline $\begin{array}{c}\text { Single Difference GPS } \\
\text { (Pseudoranges) }\end{array}$ & 86 & 98 & 100 & 100 & 100 & 100 & 100 & 100 \\
\hline $\begin{array}{c}\text { Single Difference GPS } \\
\text { (Carrier Phase) }\end{array}$ & 100 & 100 & 100 & 100 & 100 & 100 & 100 & 100 \\
\hline $\begin{array}{c}\text { Double Difference GPS } \\
\text { (Pseudoranges) }\end{array}$ & 87 & 98 & 100 & 100 & 100 & 100 & 100 & 100 \\
\hline $\begin{array}{c}\text { Double Difference GPS } \\
\text { (Carrier Phase) }\end{array}$ & 100 & 100 & 100 & 100 & 100 & 100 & 100 & 100 \\
\hline
\end{tabular}

Table 92: Success Rates of Reaching Angular Specifications of Fused Heading for Simulation Data Set A-7

\begin{tabular}{|c|c|c|c|c|c|c|c|c|}
\hline & \multicolumn{7}{|c|}{ Portion of Trials in Which Target Specification was reached } \\
\hline Method & $\pm 0.5^{\circ}$ & $\pm 1^{\circ}$ & $\pm 2^{\circ}$ & $\pm 5^{\circ}$ & $\pm 10^{\circ}$ & $\pm 15^{\circ}$ & $\pm 20^{\circ}$ & $\pm 25^{\circ}$ \\
\hline $\begin{array}{c}\text { Single Point GPS } \\
\text { (Pseudoranges) }\end{array}$ & 100 & 100 & 100 & 100 & 100 & 100 & 100 & 100 \\
\hline $\begin{array}{c}\text { Single Point GPS (Carrier } \\
\text { Phase) }\end{array}$ & 92 & 100 & 100 & 100 & 100 & 100 & 100 & 100 \\
\hline $\begin{array}{c}\text { Single Difference GPS } \\
\text { (Pseudoranges) }\end{array}$ & 100 & 100 & 100 & 100 & 100 & 100 & 100 & 100 \\
\hline $\begin{array}{c}\text { Single Difference GPS } \\
\text { (Carrier Phase) }\end{array}$ & 92 & 100 & 100 & 100 & 100 & 100 & 100 & 100 \\
\hline $\begin{array}{c}\text { Double Difference GPS } \\
\text { (Pseudoranges) }\end{array}$ & 99 & 100 & 100 & 100 & 100 & 100 & 100 & 100 \\
\hline $\begin{array}{c}\text { Double Difference GPS } \\
\text { (Carrier Phase) }\end{array}$ & 94 & 100 & 100 & 100 & 100 & 100 & 100 & 100 \\
\hline IMU Measured & 99 & 100 & 100 & 100 & 100 & 100 & 100 & 100 \\
\hline
\end{tabular}


Table 93: Pitch / Heading Correlation Coefficients for Each Computation Method in Simulation Data Set A-7

\begin{tabular}{|c|c|c|}
\hline & \multicolumn{2}{|c|}{ Pitch / Heading Correlation Coefficient } \\
\hline Method & Unfused & Fused \\
\hline $\begin{array}{c}\text { Single Point GPS } \\
\text { (Pseudoranges) }\end{array}$ & 0.869998 & 0.861423 \\
\hline $\begin{array}{c}\text { Single Difference GPS } \\
\text { (Pseudoranges) }\end{array}$ & 0.874294 & 0.866835 \\
\hline $\begin{array}{c}\text { Double Difference GPS } \\
\text { (Pseudoranges) }\end{array}$ & 0.786869 & 0.779073 \\
\hline
\end{tabular}

\section{Set A-8: 90 m Dual Antenna Separation}

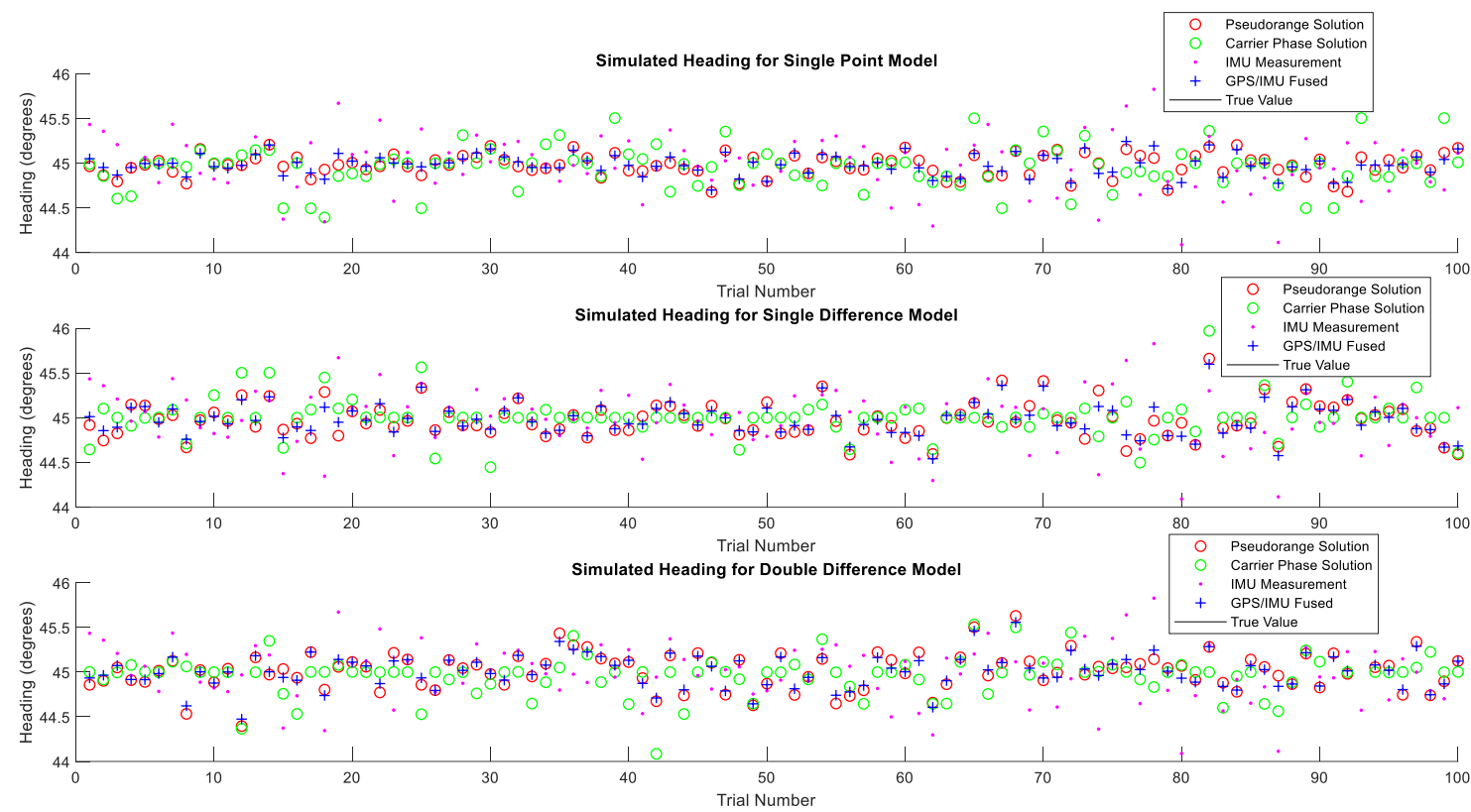

Figure 69: Computed and Measured Headings for Simulation Set A-8 


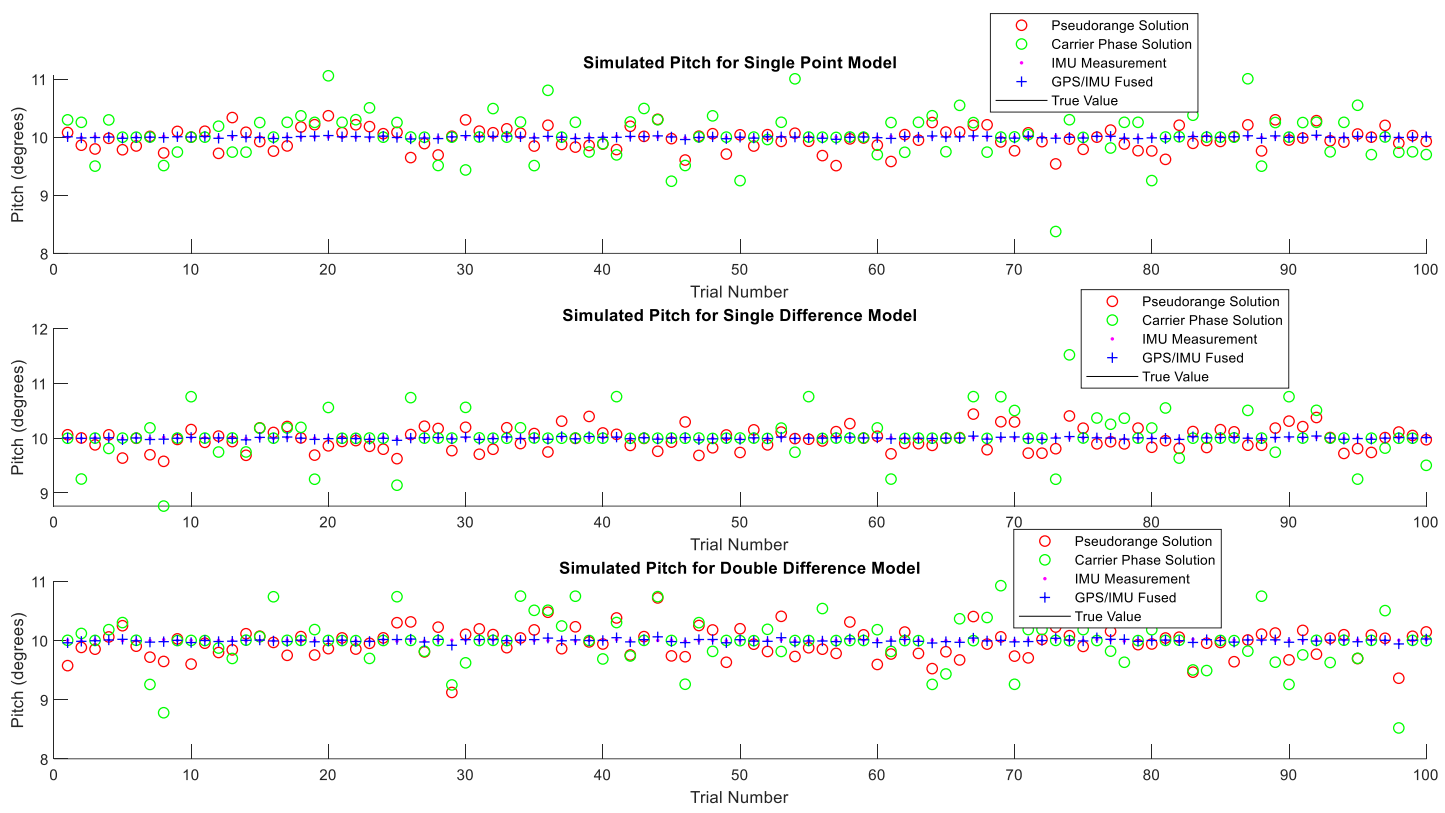

Figure 70: Computed and Measured Pitch for Simulation Set A-8

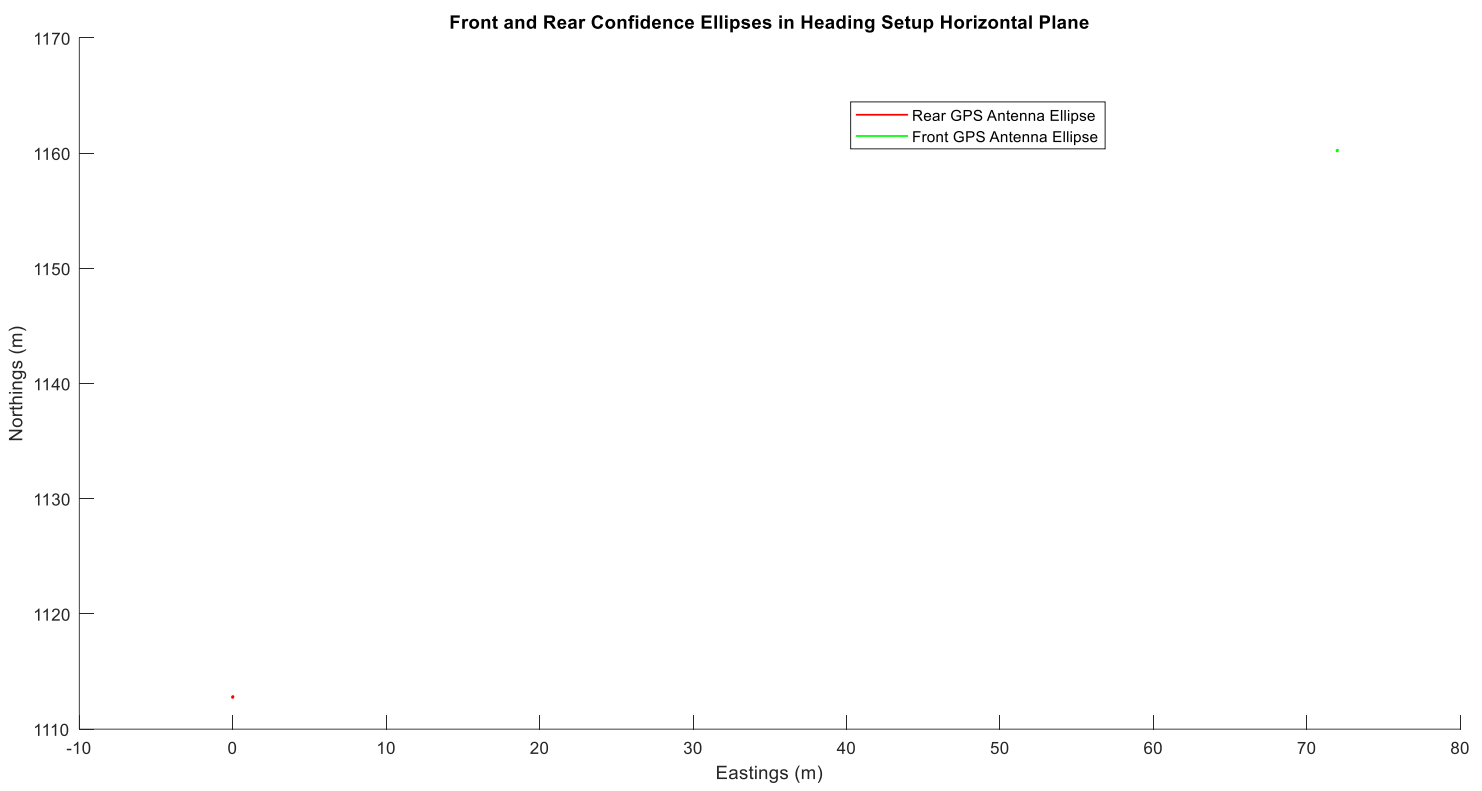

Figure 71: Propagated 95\% Confidence Ellipses for Front and Rear GPS Antennas in Simulated Data Set A-8 
Table 94: Mean Global Error Statistics of Unfused Pitch for Simulation Set A-8

\begin{tabular}{|c|c|c|c|}
\hline Method & $\begin{array}{c}\text { Measured Standard } \\
\text { Deviation } \\
\text { (degrees) }\end{array}$ & $\begin{array}{c}\text { Estimated } \\
\text { Standard } \\
\text { Deviation } \\
\text { (degrees) }\end{array}$ & $\begin{array}{c}\text { Mean RMS } \\
\text { Error } \\
\text { (degrees) }\end{array}$ \\
\hline Single Point GPS (Pseudoranges) & 0.187 & 0.088 & 0.15 \\
\hline Single Point GPS (Carrier Phase) & 0.395 & 0 & 0.275 \\
\hline $\begin{array}{c}\text { Single Difference GPS } \\
\text { (Pseudoranges) }\end{array}$ & 0.196 & 0.088 & 0.16 \\
\hline $\begin{array}{c}\text { Single Difference GPS (Carrier } \\
\text { Phase) }\end{array}$ & 0.379 & 0 & 0.207 \\
\hline $\begin{array}{c}\text { Double Difference GPS } \\
\text { (Pseudoranges) }\end{array}$ & 0.254 & 0.07 & 0.201 \\
\hline $\begin{array}{c}\text { Double Difference GPS (Carrier } \\
\text { Phase) }\end{array}$ & 0.392 & 0.001 & 0.243 \\
\hline IMU Measurement & 0.007 & - & 0.005 \\
\hline
\end{tabular}

Table 95: Mean Global Error Statistics of Unfused Heading for Simulation Set A-8

\begin{tabular}{|c|c|c|c|}
\hline Method & $\begin{array}{c}\text { Measured Standard } \\
\text { Deviation } \\
\text { (degrees) }\end{array}$ & $\begin{array}{c}\text { Estimated } \\
\text { Standard } \\
\text { Deviation } \\
\text { (degrees) }\end{array}$ & $\begin{array}{c}\text { Mean RMS } \\
\text { Error } \\
\text { (degrees) }\end{array}$ \\
\hline Single Point GPS (Pseudoranges) & 0.124 & 0.071 & 0.1 \\
\hline Single Point GPS (Carrier Phase) & 0.233 & 0 & 0.165 \\
\hline $\begin{array}{c}\text { Single Difference GPS } \\
\text { (Pseudoranges) }\end{array}$ & 0.197 & 0.071 & 0.155 \\
\hline $\begin{array}{c}\text { Single Difference GPS (Carrier } \\
\text { Phase) }\end{array}$ & 0.215 & 0 & 0.116 \\
\hline $\begin{array}{c}\text { Double Difference GPS } \\
\text { (Pseudoranges) }\end{array}$ & 0.212 & 0.053 & 0.165 \\
\hline $\begin{array}{c}\text { Double Difference GPS (Carrier } \\
\text { Phase) }\end{array}$ & 0.235 & 0.001 & 0.141 \\
\hline IMU Measurement & 0.326 & - & 0.258 \\
\hline
\end{tabular}


Table 96: Mean Global Error Statistics of Fused Pitch for Simulation Set A-8

\begin{tabular}{|c|c|c|c|}
\hline Method & $\begin{array}{c}\text { Measured Standard } \\
\text { Deviation } \\
\text { (degrees) }\end{array}$ & $\begin{array}{c}\text { Estimated } \\
\text { Standard } \\
\text { Deviation } \\
\text { (degrees) }\end{array}$ & $\begin{array}{c}\text { Mean RMS } \\
\text { Error } \\
\text { (degrees) }\end{array}$ \\
\hline Single Point GPS (Pseudoranges) & 0.015 & 0.043 & 0.012 \\
\hline Single Point GPS (Carrier Phase) & 0.379 & 0 & 0.263 \\
\hline $\begin{array}{c}\text { Single Difference GPS } \\
\text { (Pseudoranges) }\end{array}$ & 0.016 & 0.043 & 0.012 \\
\hline $\begin{array}{c}\text { Single Difference GPS (Carrier } \\
\text { Phase) }\end{array}$ & 0.362 & 0 & 0.198 \\
\hline $\begin{array}{c}\text { Double Difference GPS } \\
\text { (Pseudoranges) }\end{array}$ & 0.023 & 0.035 & 0.018 \\
\hline $\begin{array}{c}\text { Double Difference GPS (Carrier } \\
\text { Phase) }\end{array}$ & 0.357 & 0 & 0.221 \\
\hline IMU Measurement & 0.007 & - & 0.005 \\
\hline
\end{tabular}

Table 97: Mean Global Error Statistics of Fused Heading for Simulation Set A-8

\begin{tabular}{|c|c|c|c|}
\hline Method & $\begin{array}{c}\text { Measured Standard } \\
\text { Deviation } \\
\text { (degrees) }\end{array}$ & $\begin{array}{c}\text { Estimated } \\
\text { Standard } \\
\text { Deviation } \\
\text { (degrees) }\end{array}$ & $\begin{array}{c}\text { Mean RMS } \\
\text { Error } \\
\text { (degrees) }\end{array}$ \\
\hline Single Point GPS (Pseudoranges) & 0.116 & 0.035 & 0.092 \\
\hline Single Point GPS (Carrier Phase) & 0.233 & 0 & 0.165 \\
\hline $\begin{array}{c}\text { Single Difference GPS } \\
\text { (Pseudoranges) }\end{array}$ & 0.177 & 0.035 & 0.141 \\
\hline $\begin{array}{c}\text { Single Difference GPS (Carrier } \\
\text { Phase) }\end{array}$ & 0.215 & 0 & 0.116 \\
\hline $\begin{array}{c}\text { Double Difference GPS } \\
\text { (Pseudoranges) }\end{array}$ & 0.188 & 0.026 & 0.148 \\
\hline $\begin{array}{c}\text { Double Difference GPS (Carrier } \\
\text { Phase) }\end{array}$ & 0.234 & 0 & 0.141 \\
\hline IMU Measurement & 0.326 & - & 0.258 \\
\hline
\end{tabular}


Table 98: Success Rates of Reaching Angular Specifications of Unfused Pitch for Simulation Data Set A-8

\begin{tabular}{|c|c|c|c|c|c|c|c|c|}
\hline & \multicolumn{7}{|c|}{ Portion of Trials in Which Target Specification was reached } \\
\hline & $\pm 0.5^{\circ}$ & $\pm 1^{\circ}$ & $\pm 2^{\circ}$ & $\pm 5^{\circ}$ & $\pm 10^{\circ}$ & $\pm 15^{\circ}$ & $\pm 20^{\circ}$ & $\pm 25^{\circ}$ \\
\hline Method & 100 & 100 & 100 & 100 & 100 & 100 & 100 & 100 \\
\hline $\begin{array}{c}\text { Single Point GPS } \\
\text { (Pseudoranges) }\end{array}$ & 91 & 97 & 100 & 100 & 100 & 100 & 100 & 100 \\
\hline $\begin{array}{c}\text { Single Point GPS (Carrier } \\
\text { Phase) }\end{array}$ & 100 & 100 & 100 & 100 & 100 & 100 & 100 & 100 \\
\hline $\begin{array}{c}\text { Single Difference GPS } \\
\text { (Pseudoranges) }\end{array}$ & 86 & 99 & 100 & 100 & 100 & 100 & 100 & 100 \\
\hline $\begin{array}{c}\text { Single Difference GPS } \\
\text { (Carrier Phase) }\end{array}$ & 99 & 100 & 100 & 100 & 100 & 100 & 100 & 100 \\
\hline $\begin{array}{c}\text { Double Difference GPS } \\
\text { (Pseudoranges) }\end{array}$ & 89 & 100 & 100 & 100 & 100 & 100 & 100 & 100 \\
\hline $\begin{array}{c}\text { Double Difference GPS } \\
\text { (Carrier Phase) }\end{array}$ & 100 & 100 & 100 & 100 & 100 & 100 & 100 & 100 \\
\hline IMU Measured & & & & & & & & \\
\hline
\end{tabular}

Table 99: Success Rates of Reaching Angular Specifications of Unfused Heading for Simulation Data Set A-8

\begin{tabular}{|c|c|c|c|c|c|c|c|c|}
\hline & \multicolumn{7}{|c|}{ Portion of Trials in Which Target Specification was reached } \\
\hline & $\pm 0.5^{\circ}$ & $\pm 1^{\circ}$ & $\pm 2^{\circ}$ & $\pm 5^{\circ}$ & $\pm 10^{\circ}$ & $\pm 15^{\circ}$ & $\pm 20^{\circ}$ & $\pm 25^{\circ}$ \\
\hline Method & 100 & 100 & 100 & 100 & 100 & 100 & 100 & 100 \\
\hline $\begin{array}{c}\text { Single Point GPS } \\
\text { (Pseudoranges) }\end{array}$ & 96 & 100 & 100 & 100 & 100 & 100 & 100 & 100 \\
\hline $\begin{array}{c}\text { Single Point GPS (Carrier } \\
\text { Phase) }\end{array}$ & 99 & 100 & 100 & 100 & 100 & 100 & 100 & 100 \\
\hline $\begin{array}{c}\text { Single Difference GPS } \\
\text { (Pseudoranges) }\end{array}$ & 96 & 100 & 100 & 100 & 100 & 100 & 100 & 100 \\
\hline $\begin{array}{c}\text { Single Difference GPS } \\
\text { (Carrier Phase) }\end{array}$ & 97 & 100 & 100 & 100 & 100 & 100 & 100 & 100 \\
\hline $\begin{array}{c}\text { Double Difference GPS } \\
\text { (Pseudoranges) }\end{array}$ & 97 & 100 & 100 & 100 & 100 & 100 & 100 & 100 \\
\hline $\begin{array}{c}\text { Double Difference GPS } \\
\text { (Carrier Phase) }\end{array}$ & 97 & 100 & 100 & 100 & 100 & 100 & 100 & 100 \\
\hline IMU Measured & & & & & & & & \\
\hline
\end{tabular}


Table 100: Success Rates of Reaching Angular Specifications of Fused Pitch for Simulation Data Set A-8

\begin{tabular}{|c|c|c|c|c|c|c|c|c|}
\hline & \multicolumn{7}{|c|}{ Portion of Trials in Which Target Specification was reached } \\
\hline & $\pm 0.5^{\circ}$ & $\pm 1^{\circ}$ & $\pm 2^{\circ}$ & $\pm 5^{\circ}$ & $\pm 10^{\circ}$ & $\pm 15^{\circ}$ & $\pm 20^{\circ}$ & $\pm 25^{\circ}$ \\
\hline Method & 100 & 100 & 100 & 100 & 100 & 100 & 100 & 100 \\
\hline $\begin{array}{c}\text { Single Point GPS } \\
\text { (Pseudoranges) }\end{array}$ & 92 & 99 & 100 & 100 & 100 & 100 & 100 & 100 \\
\hline $\begin{array}{c}\text { Single Point GPS (Carrier } \\
\text { Phase) }\end{array}$ & 100 & 100 & 100 & 100 & 100 & 100 & 100 & 100 \\
\hline $\begin{array}{c}\text { Single Difference GPS } \\
\text { (Pseudoranges) }\end{array}$ & 89 & 99 & 100 & 100 & 100 & 100 & 100 & 100 \\
\hline $\begin{array}{c}\text { Single Difference GPS } \\
\text { (Carrier Phase) }\end{array}$ & 100 & 100 & 100 & 100 & 100 & 100 & 100 & 100 \\
\hline $\begin{array}{c}\text { Double Difference GPS } \\
\text { (Pseudoranges) }\end{array}$ & 93 & 100 & 100 & 100 & 100 & 100 & 100 & 100 \\
\hline $\begin{array}{c}\text { Double Difference GPS } \\
\text { (Carrier Phase) }\end{array}$ & 100 & 100 & 100 & 100 & 100 & 100 & 100 & 100 \\
\hline IMU Measured & & & & & & & & \\
\hline
\end{tabular}

Table 101: Success Rates of Reaching Angular Specifications of Fused Heading for Simulation Data Set A-8

\begin{tabular}{|c|c|c|c|c|c|c|c|c|}
\hline & \multicolumn{7}{|c|}{ Portion of Trials in Which Target Specification was reached } \\
\hline & $\pm 0.5^{\circ}$ & $\pm 1^{\circ}$ & $\pm 2^{\circ}$ & $\pm 5^{\circ}$ & $\pm 10^{\circ}$ & $\pm 15^{\circ}$ & $\pm 20^{\circ}$ & $\pm 25^{\circ}$ \\
\hline Method & 100 & 100 & 100 & 100 & 100 & 100 & 100 & 100 \\
\hline $\begin{array}{c}\text { Single Point GPS } \\
\text { (Pseudoranges) }\end{array}$ & 96 & 100 & 100 & 100 & 100 & 100 & 100 & 100 \\
\hline $\begin{array}{c}\text { Single Point GPS (Carrier } \\
\text { Phase) }\end{array}$ & 99 & 100 & 100 & 100 & 100 & 100 & 100 & 100 \\
\hline $\begin{array}{c}\text { Single Difference GPS } \\
\text { (Pseudoranges) }\end{array}$ & 96 & 100 & 100 & 100 & 100 & 100 & 100 & 100 \\
\hline $\begin{array}{c}\text { Single Difference GPS } \\
\text { (Carrier Phase) }\end{array}$ & 98 & 100 & 100 & 100 & 100 & 100 & 100 & 100 \\
\hline $\begin{array}{c}\text { Double Difference GPS } \\
\text { (Pseudoranges) }\end{array}$ & 97 & 100 & 100 & 100 & 100 & 100 & 100 & 100 \\
\hline $\begin{array}{c}\text { Double Difference GPS } \\
\text { (Carrier Phase) }\end{array}$ & 97 & 100 & 100 & 100 & 100 & 100 & 100 & 100 \\
\hline IMU Measured & & & & & & & & \\
\hline
\end{tabular}


Table 102: Pitch / Heading Correlation Coefficients for Each Computation Method in Simulation Data Set A-8

\begin{tabular}{|c|c|c|}
\hline & \multicolumn{2}{|c|}{ Pitch / Heading Correlation Coefficient } \\
\hline Method & Unfused & Fused \\
\hline $\begin{array}{c}\text { Single Point GPS } \\
\text { (Pseudoranges) }\end{array}$ & 0.869504 & 0.86444 \\
\hline $\begin{array}{c}\text { Single Difference GPS } \\
\text { (Pseudoranges) }\end{array}$ & 0.873157 & 0.86824 \\
\hline $\begin{array}{c}\text { Double Difference GPS } \\
\text { (Pseudoranges) }\end{array}$ & 0.78564 & 0.780952 \\
\hline
\end{tabular}

\section{Appendix B: 1 m Pseudorange Noise Standard}

\section{Deviation Pitch and Heading Simulation Results}

\section{Set B-1: 1 m Dual Antenna Separation}

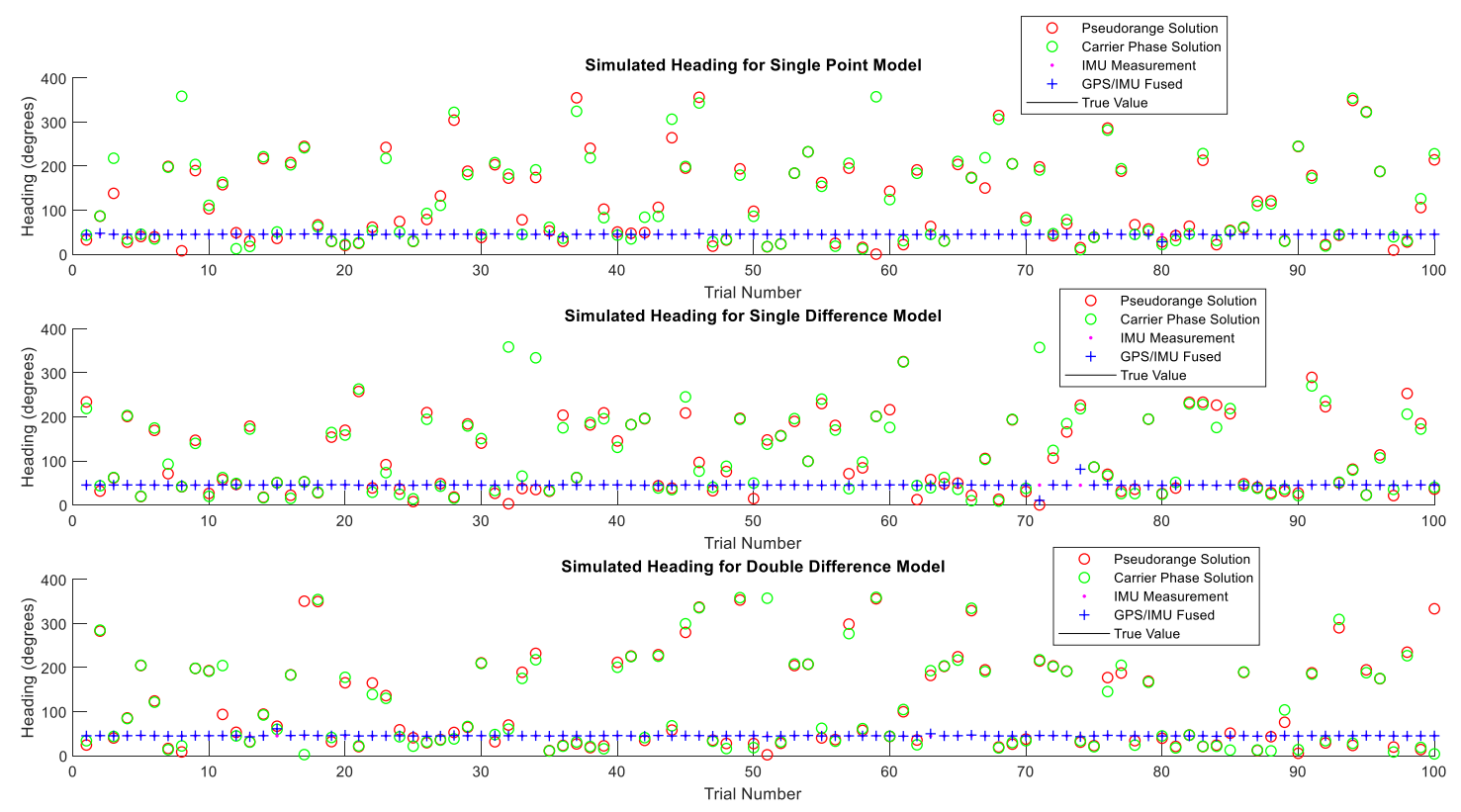

Figure 72: Computed and Measured Headings for Simulation Set B-1 


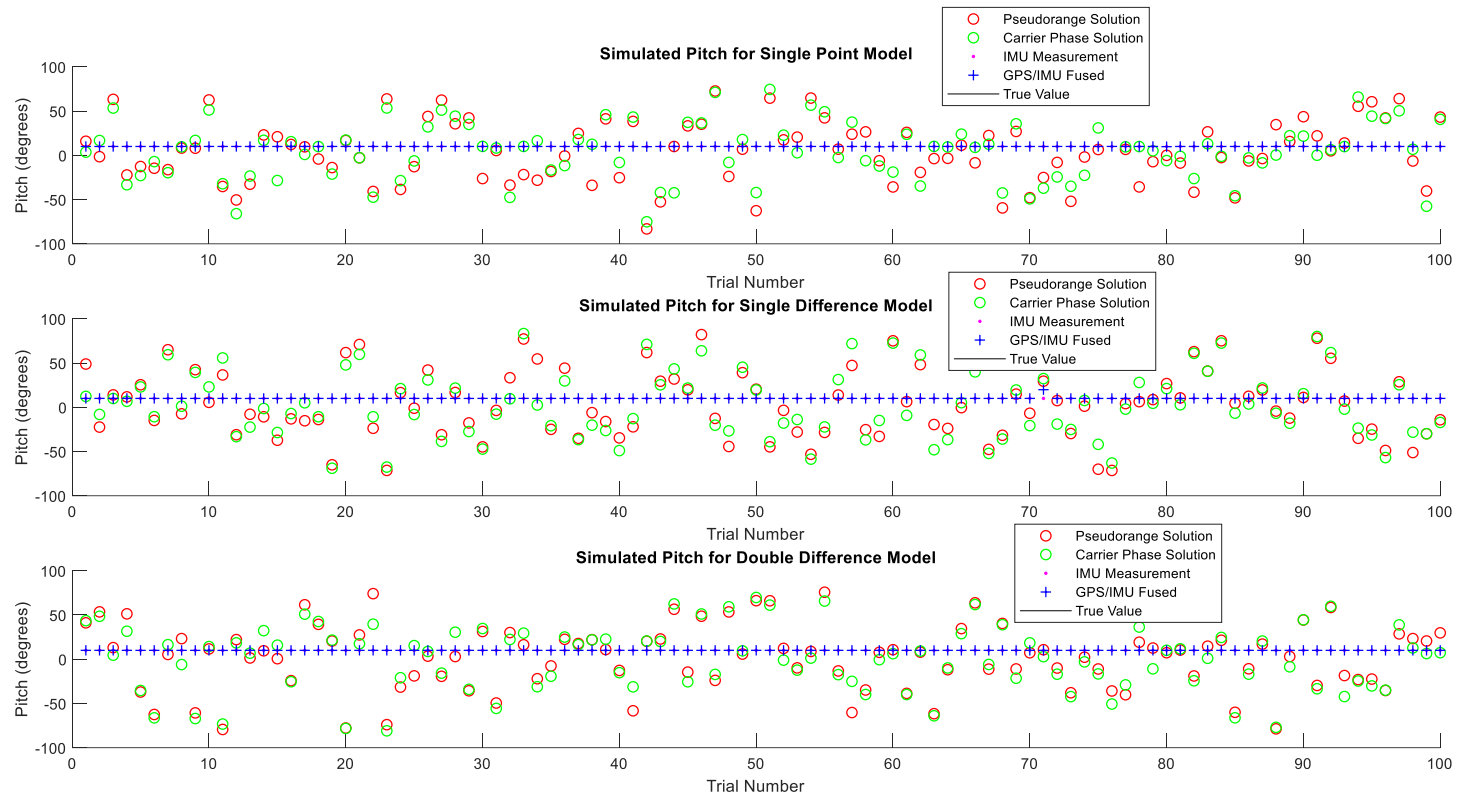

Figure 73: Computed and Measured Pitch for Simulation Set B-1

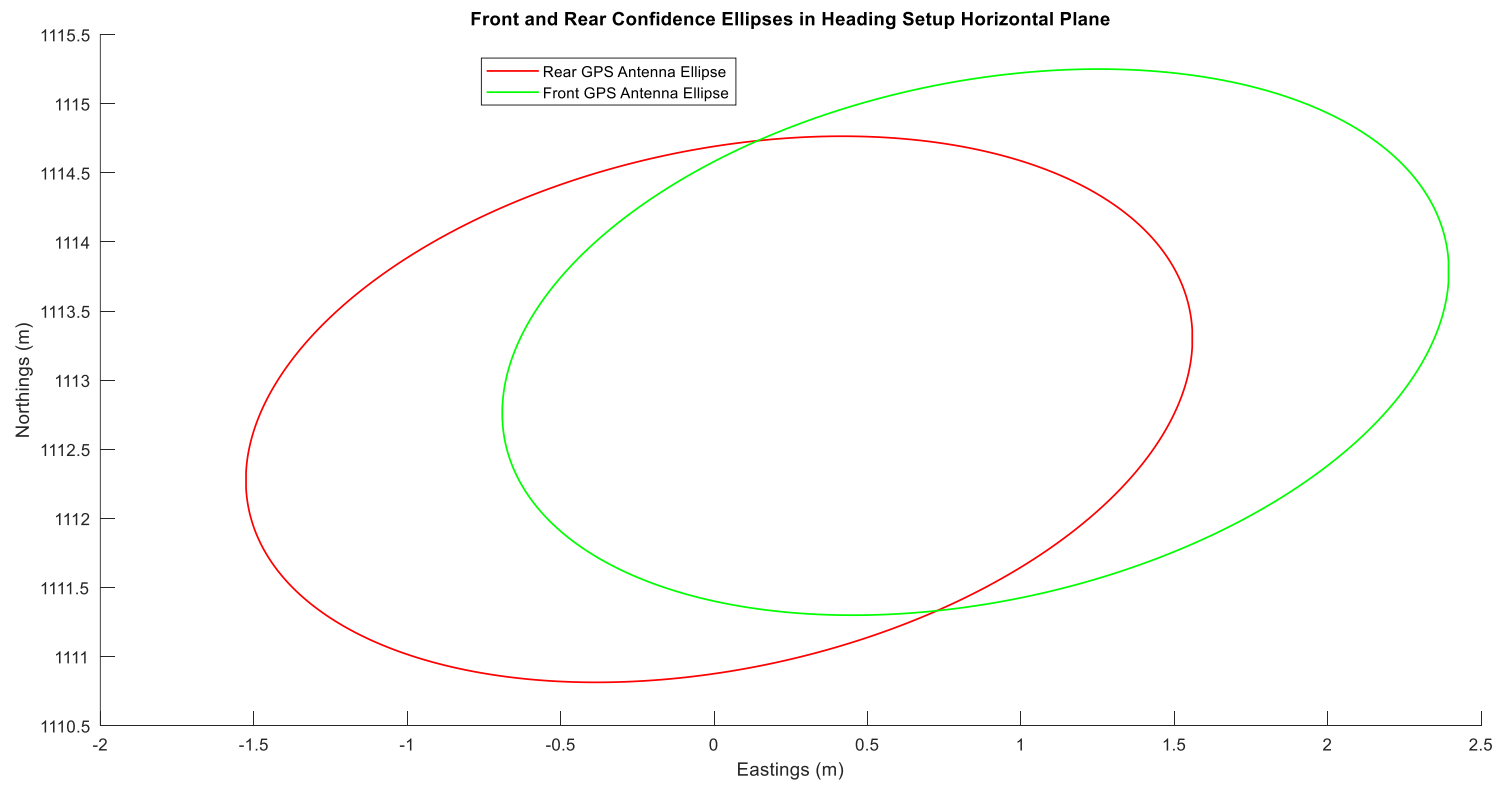

Figure 74: Propagated 95\% Confidence Ellipses for Front and Rear GPS Antennas in Simulated Data Set B-1 
Table 103: Mean Global Error Statistics of Unfused Pitch for Simulation Set B-1

\begin{tabular}{|c|c|c|c|}
\hline Method & $\begin{array}{c}\text { Measured Standard } \\
\text { Deviation } \\
\text { (degrees) }\end{array}$ & $\begin{array}{c}\text { Estimated } \\
\text { Standard } \\
\text { Deviation } \\
\text { (degrees) }\end{array}$ & $\begin{array}{c}\text { Mean RMS } \\
\text { Error } \\
\text { (degrees) }\end{array}$ \\
\hline Single Point GPS (Pseudoranges) & 34.603 & 121.283 & 28.382 \\
\hline Single Point GPS (Carrier Phase) & 32.445 & 0.156 & 25.695 \\
\hline $\begin{array}{c}\text { Single Difference GPS } \\
\text { (Pseudoranges) }\end{array}$ & 37.475 & 88.682 & 31.4 \\
\hline $\begin{array}{c}\text { Single Difference GPS (Carrier } \\
\text { Phase) }\end{array}$ & 36.746 & 0.102 & 31.41 \\
\hline $\begin{array}{c}\text { Double Difference GPS } \\
\text { (Pseudoranges) }\end{array}$ & 36.496 & 63.396 & 28.925 \\
\hline $\begin{array}{c}\text { Double Difference GPS (Carrier } \\
\text { Phase) }\end{array}$ & 36.134 & 0.126 & 29.682 \\
\hline IMU Measurement & 0.007 & - & 0.006 \\
\hline
\end{tabular}

Table 104: Mean Global Error Statistics of Unfused Heading for Simulation Set B-1

\begin{tabular}{|c|c|c|c|}
\hline Method & $\begin{array}{c}\text { Measured Standard } \\
\text { Deviation } \\
\text { (degrees) }\end{array}$ & $\begin{array}{c}\text { Estimated } \\
\text { Standard } \\
\text { Deviation } \\
\text { (degrees) }\end{array}$ & $\begin{array}{c}\text { Mean RMS } \\
\text { Error } \\
\text { (degrees) }\end{array}$ \\
\hline Single Point GPS (Pseudoranges) & 94.459 & 531.372 & 68.048 \\
\hline Single Point GPS (Carrier Phase) & 100.135 & 0.437 & 68.413 \\
\hline $\begin{array}{c}\text { Single Difference GPS } \\
\text { (Pseudoranges) }\end{array}$ & 83.104 & 338.806 & 68.928 \\
\hline $\begin{array}{c}\text { Single Difference GPS (Carrier } \\
\text { Phase) }\end{array}$ & 89.304 & 0.298 & 68.102 \\
\hline $\begin{array}{c}\text { Double Difference GPS } \\
\text { (Pseudoranges) }\end{array}$ & 104.337 & 419.144 & 66.08 \\
\hline $\begin{array}{c}\text { Double Difference GPS (Carrier } \\
\text { Phase) }\end{array}$ & 103.95 & 2.527 & 66.694 \\
\hline IMU Measurement & 0.305 & - & 0.244 \\
\hline
\end{tabular}


Table 105: Mean Global Error Statistics of Fused Pitch for Simulation Set B-1

\begin{tabular}{|c|c|c|c|}
\hline Method & $\begin{array}{c}\text { Measured Standard } \\
\text { Deviation } \\
\text { (degrees) }\end{array}$ & $\begin{array}{c}\text { Estimated } \\
\text { Standard } \\
\text { Deviation } \\
\text { (degrees) }\end{array}$ & $\begin{array}{c}\text { Mean RMS } \\
\text { Error } \\
\text { (degrees) }\end{array}$ \\
\hline Single Point GPS (Pseudoranges) & 0.05 & 0.199 & 0.015 \\
\hline Single Point GPS (Carrier Phase) & 4.354 & 0.058 & 2.631 \\
\hline $\begin{array}{c}\text { Single Difference GPS } \\
\text { (Pseudoranges) }\end{array}$ & 0.985 & 0.198 & 0.109 \\
\hline $\begin{array}{c}\text { Single Difference GPS (Carrier } \\
\text { Phase) }\end{array}$ & 7.104 & 0.044 & 4.17 \\
\hline $\begin{array}{c}\text { Double Difference GPS } \\
\text { (Pseudoranges) }\end{array}$ & 0.021 & 0.2 & 0.013 \\
\hline $\begin{array}{c}\text { Double Difference GPS (Carrier } \\
\text { Phase) }\end{array}$ & 7.46 & 0.051 & 3.774 \\
\hline IMU Measurement & 0.007 & - & 0.006 \\
\hline
\end{tabular}

Table 106: Mean Global Error Statistics of Fused Heading for Simulation Set B-1

\begin{tabular}{|c|c|c|c|}
\hline Method & $\begin{array}{c}\text { Measured Standard } \\
\text { Deviation } \\
\text { (degrees) }\end{array}$ & $\begin{array}{c}\text { Estimated } \\
\text { Standard } \\
\text { Deviation } \\
\text { (degrees) }\end{array}$ & $\begin{array}{c}\text { Mean RMS } \\
\text { Error } \\
\text { (degrees) }\end{array}$ \\
\hline Single Point GPS (Pseudoranges) & 1.837 & 8.187 & 0.552 \\
\hline Single Point GPS (Carrier Phase) & 66.438 & 0.186 & 51.033 \\
\hline $\begin{array}{c}\text { Single Difference GPS } \\
\text { (Pseudoranges) }\end{array}$ & 5.049 & 8.229 & 1.014 \\
\hline $\begin{array}{c}\text { Single Difference GPS (Carrier } \\
\text { Phase) }\end{array}$ & 68.512 & 0.134 & 49.084 \\
\hline $\begin{array}{c}\text { Double Difference GPS } \\
\text { (Pseudoranges) }\end{array}$ & 1.761 & 8.177 & 0.654 \\
\hline $\begin{array}{c}\text { Double Difference GPS (Carrier } \\
\text { Phase) }\end{array}$ & 59.456 & 0.355 & 39.253 \\
\hline IMU Measurement & 0.305 & - & 0.244 \\
\hline
\end{tabular}


Table 107: Success Rates of Reaching Angular Specifications of Unfused Pitch for Simulation Data Set B-1

\begin{tabular}{|c|c|c|c|c|c|c|c|c|}
\hline & \multicolumn{7}{|c|}{ Portion of Trials in Which Target Specification was reached } \\
\hline & $\pm 0.5^{\circ}$ & $\pm 1^{\circ}$ & $\pm 2^{\circ}$ & $\pm 5^{\circ}$ & $\pm 10^{\circ}$ & $\pm 15^{\circ}$ & $\pm 20^{\circ}$ & $\pm 25^{\circ}$ \\
\hline Method & 59 & 59 & 60 & 62 & 66 & 73 & 77 & 79 \\
\hline $\begin{array}{c}\text { Single Point GPS } \\
\text { (Pseudoranges) }\end{array}$ & 60 & 60 & 60 & 63 & 71 & 76 & 76 & 79 \\
\hline $\begin{array}{c}\text { Single Point GPS (Carrier } \\
\text { Phase) }\end{array}$ & 59 & 60 & 62 & 66 & 70 & 71 & 76 & 78 \\
\hline $\begin{array}{c}\text { Single Difference GPS } \\
\text { (Pseudoranges) }\end{array}$ & 63 & 63 & 63 & 64 & 67 & 74 & 78 & 81 \\
\hline $\begin{array}{c}\text { Single Difference GPS } \\
\text { (Carrier Phase) }\end{array}$ & 56 & 59 & 60 & 64 & 68 & 78 & 81 & 84 \\
\hline $\begin{array}{c}\text { Double Difference GPS } \\
\text { (Pseudoranges) }\end{array}$ & 58 & 58 & 59 & 61 & 68 & 76 & 79 & 83 \\
\hline $\begin{array}{c}\text { Double Difference GPS } \\
\text { (Carrier Phase) }\end{array}$ & 100 & 100 & 100 & 100 & 100 & 100 & 100 & 100 \\
\hline IMU Measured & & & & & & & & \\
\hline
\end{tabular}

Table 108: Success Rates of Reaching Angular Specifications of Unfused Heading for Simulation Data Set B-1

\begin{tabular}{|c|c|c|c|c|c|c|c|c|}
\hline & \multicolumn{7}{|c|}{ Portion of Trials in Which Target Specification was reached } \\
\hline Method & $\pm 0.5^{\circ}$ & $\pm 1^{\circ}$ & $\pm 2^{\circ}$ & $\pm 5^{\circ}$ & $\pm 10^{\circ}$ & $\pm 15^{\circ}$ & $\pm 20^{\circ}$ & $\pm 25^{\circ}$ \\
\hline $\begin{array}{c}\text { Single Point GPS } \\
\text { (Pseudoranges) }\end{array}$ & 46 & 46 & 46 & 49 & 52 & 54 & 57 & 60 \\
\hline $\begin{array}{c}\text { Single Point GPS (Carrier } \\
\text { Phase) }\end{array}$ & 50 & 50 & 52 & 52 & 57 & 57 & 60 & 60 \\
\hline $\begin{array}{c}\text { Single Difference GPS } \\
\text { (Pseudoranges) }\end{array}$ & 46 & 46 & 47 & 52 & 54 & 56 & 58 & 59 \\
\hline $\begin{array}{c}\text { Single Difference GPS } \\
\text { (Carrier Phase) }\end{array}$ & 46 & 46 & 46 & 48 & 52 & 52 & 56 & 58 \\
\hline $\begin{array}{c}\text { Double Difference GPS } \\
\text { (Pseudoranges) }\end{array}$ & 58 & 58 & 59 & 59 & 62 & 65 & 66 & 68 \\
\hline $\begin{array}{c}\text { Double Difference GPS } \\
\text { (Carrier Phase) }\end{array}$ & 58 & 58 & 59 & 60 & 60 & 61 & 64 & 66 \\
\hline IMU Measured & 95 & 100 & 100 & 100 & 100 & 100 & 100 & 100 \\
\hline
\end{tabular}


Table 109: Success Rates of Reaching Angular Specifications of Fused Pitch for Simulation Data Set B-1

\begin{tabular}{|c|c|c|c|c|c|c|c|c|}
\hline & \multicolumn{7}{|c|}{ Portion of Trials in Which Target Specification was reached } \\
\hline Method & $\pm 0.5^{\circ}$ & $\pm 1^{\circ}$ & $\pm 2^{\circ}$ & $\pm 5^{\circ}$ & $\pm 10^{\circ}$ & $\pm 15^{\circ}$ & $\pm 20^{\circ}$ & $\pm 25^{\circ}$ \\
\hline $\begin{array}{c}\text { Single Point GPS } \\
\text { (Pseudoranges) }\end{array}$ & 100 & 100 & 100 & 100 & 100 & 100 & 100 & 100 \\
\hline $\begin{array}{c}\text { Single Point GPS (Carrier } \\
\text { Phase) }\end{array}$ & 70 & 86 & 91 & 97 & 99 & 100 & 100 & 100 \\
\hline $\begin{array}{c}\text { Single Difference GPS } \\
\text { (Pseudoranges) }\end{array}$ & 99 & 99 & 99 & 99 & 100 & 100 & 100 & 100 \\
\hline $\begin{array}{c}\text { Single Difference GPS } \\
\text { (Carrier Phase) }\end{array}$ & 68 & 76 & 86 & 91 & 97 & 98 & 98 & 98 \\
\hline $\begin{array}{c}\text { Double Difference GPS } \\
\text { (Pseudoranges) }\end{array}$ & 100 & 100 & 100 & 100 & 100 & 100 & 100 & 100 \\
\hline $\begin{array}{c}\text { Double Difference GPS } \\
\text { (Carrier Phase) }\end{array}$ & 69 & 76 & 88 & 96 & 98 & 100 & 100 & 100 \\
\hline IMU Measured & 100 & 100 & 100 & 100 & 100 & 100 & 100 & 100 \\
\hline
\end{tabular}

Table 110: Success Rates of Reaching Angular Specifications of Fused Heading for Simulation Data Set B-1

\begin{tabular}{|c|c|c|c|c|c|c|c|c|}
\hline & \multicolumn{7}{|c|}{ Portion of Trials in Which Target Specification was reached } \\
\hline & $\pm 0.5^{\circ}$ & $\pm 1^{\circ}$ & $\pm 2^{\circ}$ & $\pm 5^{\circ}$ & $\pm 10^{\circ}$ & $\pm 15^{\circ}$ & $\pm 20^{\circ}$ & $\pm 25^{\circ}$ \\
\hline Method & 85 & 95 & 99 & 100 & 100 & 100 & 100 & 100 \\
\hline $\begin{array}{c}\text { Single Point GPS } \\
\text { (Pseudoranges) }\end{array}$ & 39 & 39 & 40 & 48 & 51 & 55 & 58 & 58 \\
\hline $\begin{array}{c}\text { Single Point GPS (Carrier } \\
\text { Phase) }\end{array}$ & 87 & 98 & 98 & 99 & 99 & 99 & 99 & 99 \\
\hline $\begin{array}{c}\text { Single Difference GPS } \\
\text { (Pseudoranges) }\end{array}$ & 38 & 38 & 38 & 45 & 50 & 54 & 57 & 57 \\
\hline $\begin{array}{c}\text { Single Difference GPS } \\
\text { (Carrier Phase) }\end{array}$ & 75 & 94 & 98 & 99 & 99 & 99 & 100 & 100 \\
\hline $\begin{array}{c}\text { Double Difference GPS } \\
\text { (Pseudoranges) }\end{array}$ & 48 & 50 & 51 & 52 & 58 & 61 & 62 & 63 \\
\hline $\begin{array}{c}\text { Double Difference GPS } \\
\text { (Carrier Phase) }\end{array}$ & 95 & 100 & 100 & 100 & 100 & 100 & 100 & 100 \\
\hline IMU Measured & & & & & & & & \\
\hline
\end{tabular}


Table 111: Pitch / Heading Correlation Coefficients for Each Computation Method in Simulation Data Set B-1

\begin{tabular}{|c|c|c|}
\hline & \multicolumn{2}{|c|}{ Pitch / Heading Correlation Coefficient } \\
\hline Method & Unfused & Fused \\
\hline $\begin{array}{c}\text { Single Point GPS } \\
\text { (Pseudoranges) }\end{array}$ & 0.080141 & 0.000005 \\
\hline $\begin{array}{c}\text { Single Difference GPS } \\
\text { (Pseudoranges) }\end{array}$ & -0.93829 & -0.00998 \\
\hline $\begin{array}{c}\text { Double Difference GPS } \\
\text { (Pseudoranges) }\end{array}$ & 0.981793 & 0.00015 \\
\hline
\end{tabular}

\section{Set B-2: 3 m Dual Antenna Separation}
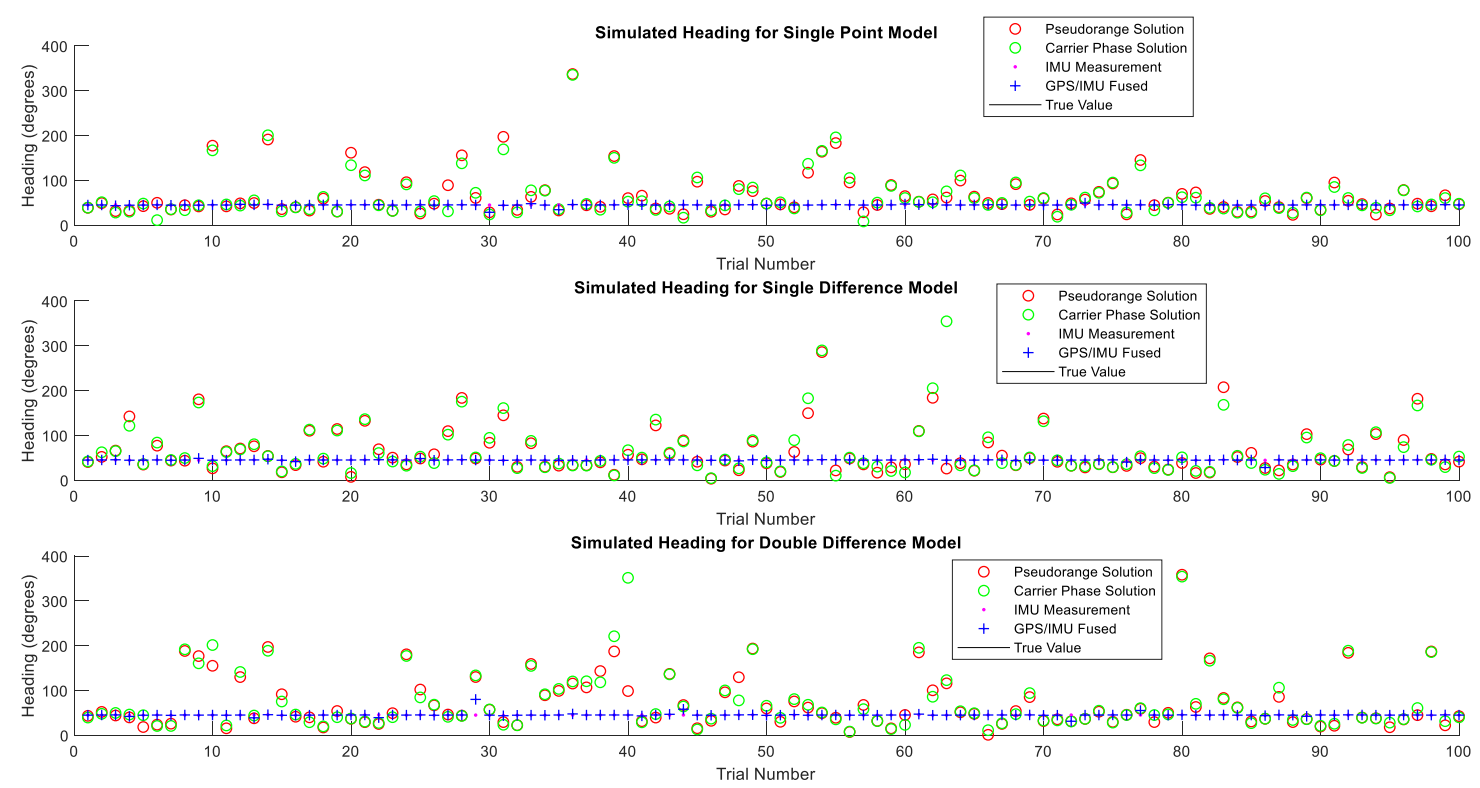

Figure 75: Computed and Measured Headings for Simulation Set B-2 


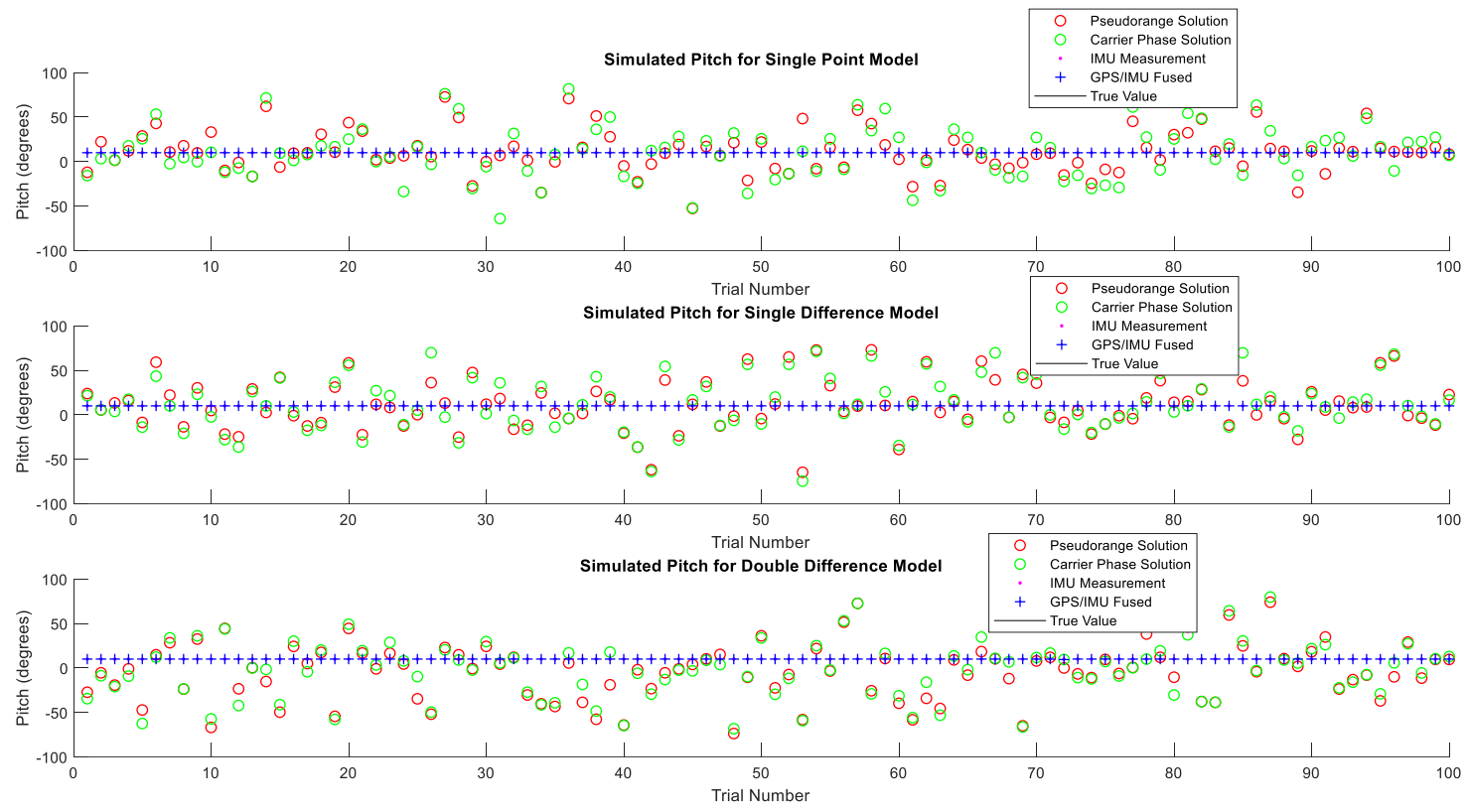

Figure 76: Computed and Measured Pitch for Simulation Set B-2 


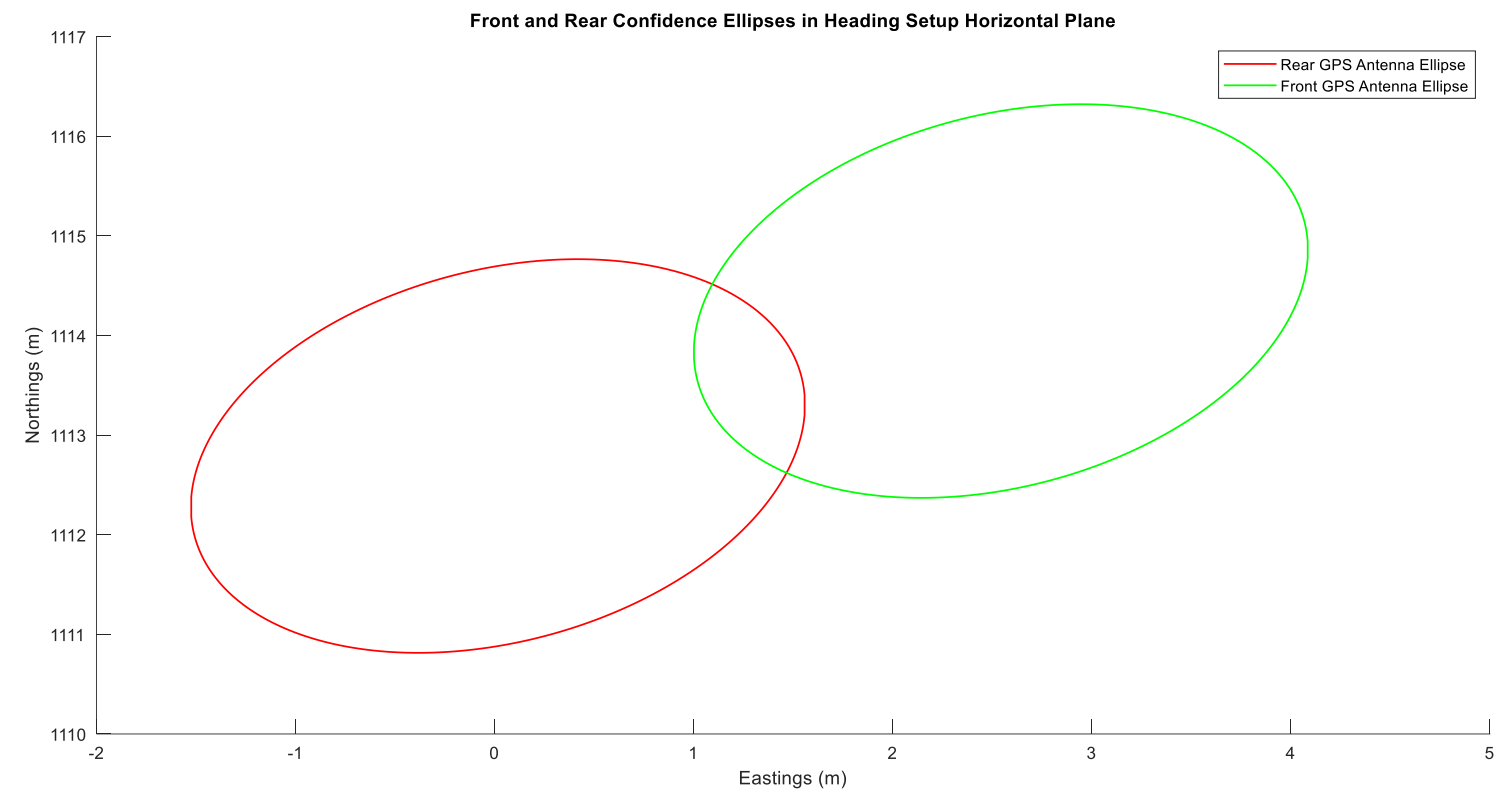

Figure 77: Propagated 95\% Confidence Ellipses for Front and Rear GPS Antennas in Simulated Data Set B-2

Table 112: Mean Global Error Statistics of Unfused Pitch for Simulation Set B-2

\begin{tabular}{|c|c|c|c|}
\hline Method & $\begin{array}{c}\text { Measured Standard } \\
\text { Deviation } \\
\text { (degrees) }\end{array}$ & $\begin{array}{c}\text { Estimated } \\
\text { Standard } \\
\text { Deviation } \\
\text { (degrees) }\end{array}$ & $\begin{array}{c}\text { Mean RMS } \\
\text { Error } \\
\text { (degrees) }\end{array}$ \\
\hline Single Point GPS (Pseudoranges) & 23.486 & 81.661 & 17.29 \\
\hline Single Point GPS (Carrier Phase) & 29.048 & 0.094 & 23.031 \\
\hline $\begin{array}{c}\text { Single Difference GPS } \\
\text { (Pseudoranges) }\end{array}$ & 27.737 & 67.485 & 21.473 \\
\hline $\begin{array}{c}\text { Single Difference GPS (Carrier } \\
\text { Phase) }\end{array}$ & 30.238 & 0.071 & 23.913 \\
\hline $\begin{array}{c}\text { Double Difference GPS } \\
\text { (Pseudoranges) }\end{array}$ & 31.053 & 51.166 & 26.163 \\
\hline $\begin{array}{c}\text { Double Difference GPS (Carrier } \\
\text { Phase) }\end{array}$ & 31.336 & 0.094 & 25.91 \\
\hline IMU Measurement & 0.008 & - & 0.006 \\
\hline
\end{tabular}


Table 113: Mean Global Error Statistics of Unfused Heading for Simulation Set B-2

\begin{tabular}{|c|c|c|c|}
\hline Method & $\begin{array}{c}\text { Measured Standard } \\
\text { Deviation } \\
\text { (degrees) }\end{array}$ & $\begin{array}{c}\text { Estimated } \\
\text { Standard } \\
\text { Deviation } \\
\text { (degrees) }\end{array}$ & $\begin{array}{c}\text { Mean RMS } \\
\text { Error } \\
\text { (degrees) }\end{array}$ \\
\hline Single Point GPS (Pseudoranges) & 48.586 & 307.582 & 26.949 \\
\hline Single Point GPS (Carrier Phase) & 48.17 & 0.902 & 27.213 \\
\hline $\begin{array}{c}\text { Single Difference GPS } \\
\text { (Pseudoranges) }\end{array}$ & 49.4 & 254.488 & 30.983 \\
\hline $\begin{array}{c}\text { Single Difference GPS (Carrier } \\
\text { Phase) }\end{array}$ & 57.415 & 0.336 & 31.994 \\
\hline $\begin{array}{c}\text { Double Difference GPS } \\
\text { (Pseudoranges) }\end{array}$ & 59.328 & 289.133 & 37.515 \\
\hline $\begin{array}{c}\text { Double Difference GPS (Carrier } \\
\text { Phase) }\end{array}$ & 65.969 & 0.427 & 37.35 \\
\hline IMU Measurement & 0.29 & - & 0.227 \\
\hline
\end{tabular}

Table 114: Mean Global Error Statistics of Fused Pitch for Simulation Set B-2

\begin{tabular}{|c|c|c|c|}
\hline Method & $\begin{array}{c}\text { Measured Standard } \\
\text { Deviation } \\
\text { (degrees) }\end{array}$ & $\begin{array}{c}\text { Estimated } \\
\text { Standard } \\
\text { Deviation } \\
\text { (degrees) }\end{array}$ & $\begin{array}{c}\text { Mean RMS } \\
\text { Error } \\
\text { (degrees) }\end{array}$ \\
\hline Single Point GPS (Pseudoranges) & 0.036 & 0.199 & 0.011 \\
\hline Single Point GPS (Carrier Phase) & 3.872 & 0.042 & 2.59 \\
\hline $\begin{array}{c}\text { Single Difference GPS } \\
\text { (Pseudoranges) }\end{array}$ & 0.027 & 0.199 & 0.013 \\
\hline $\begin{array}{c}\text { Single Difference GPS (Carrier } \\
\text { Phase) }\end{array}$ & 4.733 & 0.032 & 3.762 \\
\hline $\begin{array}{c}\text { Double Difference GPS } \\
\text { (Pseudoranges) }\end{array}$ & 0.056 & 0.197 & 0.018 \\
\hline $\begin{array}{c}\text { Double Difference GPS (Carrier } \\
\text { Phase) }\end{array}$ & 4.172 & 0.043 & 2.686 \\
\hline IMU Measurement & 0.008 & - & 0.006 \\
\hline
\end{tabular}


Table 115: Mean Global Error Statistics of Fused Heading for Simulation Set B-2

\begin{tabular}{|c|c|c|c|}
\hline Method & $\begin{array}{c}\text { Measured Standard } \\
\text { Deviation } \\
\text { (degrees) }\end{array}$ & $\begin{array}{c}\text { Estimated } \\
\text { Standard } \\
\text { Deviation } \\
\text { (degrees) }\end{array}$ & $\begin{array}{c}\text { Mean RMS } \\
\text { Error } \\
\text { (degrees) }\end{array}$ \\
\hline Single Point GPS (Pseudoranges) & 2.085 & 7.141 & 0.66 \\
\hline Single Point GPS (Carrier Phase) & 36.851 & 0.188 & 16.656 \\
\hline $\begin{array}{c}\text { Single Difference GPS } \\
\text { (Pseudoranges) }\end{array}$ & 1.907 & 6.87 & 0.646 \\
\hline $\begin{array}{c}\text { Single Difference GPS (Carrier } \\
\text { Phase) }\end{array}$ & 40.769 & 0.151 & 23.42 \\
\hline $\begin{array}{c}\text { Double Difference GPS } \\
\text { (Pseudoranges) }\end{array}$ & 4.257 & 7.051 & 1.208 \\
\hline $\begin{array}{c}\text { Double Difference GPS (Carrier } \\
\text { Phase) }\end{array}$ & 33.634 & 0.194 & 20.362 \\
\hline IMU Measurement & 0.29 & - & 0.227 \\
\hline
\end{tabular}

Table 116: Success Rates of Reaching Angular Specifications of Unfused Pitch for Simulation Data Set B-2

\begin{tabular}{|c|c|c|c|c|c|c|c|c|}
\hline & \multicolumn{7}{|c|}{ Portion of Trials in Which Target Specification was reached } \\
\hline Method & $\pm 0.5^{\circ}$ & $\pm 1^{\circ}$ & $\pm 2^{\circ}$ & $\pm 5^{\circ}$ & $\pm 10^{\circ}$ & $\pm 15^{\circ}$ & $\pm 20^{\circ}$ & $\pm 25^{\circ}$ \\
\hline $\begin{array}{c}\text { Single Point GPS } \\
\text { (Pseudoranges) }\end{array}$ & 51 & 54 & 59 & 63 & 75 & 79 & 81 & 86 \\
\hline $\begin{array}{c}\text { Single Point GPS (Carrier } \\
\text { Phase) }\end{array}$ & 52 & 52 & 53 & 55 & 64 & 68 & 80 & 84 \\
\hline $\begin{array}{c}\text { Single Difference GPS } \\
\text { (Pseudoranges) }\end{array}$ & 52 & 52 & 55 & 62 & 68 & 72 & 76 & 79 \\
\hline $\begin{array}{c}\text { Single Difference GPS } \\
\text { (Carrier Phase) }\end{array}$ & 51 & 51 & 55 & 57 & 65 & 69 & 73 & 76 \\
\hline $\begin{array}{c}\text { Double Difference GPS } \\
\text { (Pseudoranges) }\end{array}$ & 70 & 71 & 73 & 76 & 82 & 87 & 89 & 91 \\
\hline $\begin{array}{c}\text { Double Difference GPS } \\
\text { (Carrier Phase) }\end{array}$ & 67 & 68 & 70 & 73 & 79 & 82 & 87 & 92 \\
\hline IMU Measured & 100 & 100 & 100 & 100 & 100 & 100 & 100 & 100 \\
\hline
\end{tabular}


Table 117: Success Rates of Reaching Angular Specifications of Unfused Heading for Simulation Data Set B-2

\begin{tabular}{|c|c|c|c|c|c|c|c|c|}
\hline & \multicolumn{7}{|c|}{ Portion of Trials in Which Target Specification was reached } \\
\hline Method & $\pm 0.5^{\circ}$ & $\pm 1^{\circ}$ & $\pm 2^{\circ}$ & $\pm 5^{\circ}$ & $\pm 10^{\circ}$ & $\pm 15^{\circ}$ & $\pm 20^{\circ}$ & $\pm 25^{\circ}$ \\
\hline $\begin{array}{c}\text { Single Point GPS } \\
\text { (Pseudoranges) }\end{array}$ & 43 & 43 & 47 & 56 & 60 & 64 & 71 & 74 \\
\hline $\begin{array}{c}\text { Single Point GPS (Carrier } \\
\text { Phase) }\end{array}$ & 44 & 44 & 46 & 52 & 61 & 63 & 73 & 73 \\
\hline $\begin{array}{c}\text { Single Difference GPS } \\
\text { (Pseudoranges) }\end{array}$ & 52 & 53 & 54 & 60 & 65 & 68 & 71 & 74 \\
\hline $\begin{array}{c}\text { Single Difference GPS } \\
\text { (Carrier Phase) }\end{array}$ & 51 & 52 & 53 & 56 & 66 & 66 & 71 & 73 \\
\hline $\begin{array}{c}\text { Double Difference GPS } \\
\text { (Pseudoranges) }\end{array}$ & 49 & 50 & 51 & 55 & 60 & 63 & 66 & 69 \\
\hline $\begin{array}{c}\text { Double Difference GPS } \\
\text { (Carrier Phase) }\end{array}$ & 49 & 49 & 52 & 57 & 60 & 62 & 66 & 69 \\
\hline IMU Measured & 98 & 100 & 100 & 100 & 100 & 100 & 100 & 100 \\
\hline
\end{tabular}

Table 118: Success Rates of Reaching Angular Specifications of Fused Pitch for Simulation Data Set B-2

\begin{tabular}{|c|c|c|c|c|c|c|c|c|}
\hline & \multicolumn{7}{|c|}{ Portion of Trials in Which Target Specification was reached } \\
\hline Method & $\pm 0.5^{\circ}$ & $\pm 1^{\circ}$ & $\pm 2^{\circ}$ & $\pm 5^{\circ}$ & $\pm 10^{\circ}$ & $\pm 15^{\circ}$ & $\pm 20^{\circ}$ & $\pm 25^{\circ}$ \\
\hline $\begin{array}{c}\text { Single Point GPS } \\
\text { (Pseudoranges) }\end{array}$ & 100 & 100 & 100 & 100 & 100 & 100 & 100 & 100 \\
\hline $\begin{array}{c}\text { Single Point GPS (Carrier } \\
\text { Phase) }\end{array}$ & 54 & 79 & 92 & 97 & 98 & 99 & 99 & 100 \\
\hline $\begin{array}{c}\text { Single Difference GPS } \\
\text { (Pseudoranges) }\end{array}$ & 100 & 100 & 100 & 100 & 100 & 100 & 100 & 100 \\
\hline $\begin{array}{c}\text { Single Difference GPS } \\
\text { (Carrier Phase) }\end{array}$ & 57 & 70 & 86 & 94 & 99 & 100 & 100 & 100 \\
\hline $\begin{array}{c}\text { Double Difference GPS } \\
\text { (Pseudoranges) }\end{array}$ & 100 & 100 & 100 & 100 & 100 & 100 & 100 & 100 \\
\hline $\begin{array}{c}\text { Double Difference GPS } \\
\text { (Carrier Phase) }\end{array}$ & 78 & 86 & 92 & 98 & 98 & 99 & 99 & 99 \\
\hline IMU Measured & 100 & 100 & 100 & 100 & 100 & 100 & 100 & 100 \\
\hline
\end{tabular}


Table 119: Success Rates of Reaching Angular Specifications of Fused Heading for Simulation Data Set B-2

\begin{tabular}{|c|c|c|c|c|c|c|c|c|}
\hline & \multicolumn{7}{|c|}{ Portion of Trials in Which Target Specification was reached } \\
\hline & $\pm 0.5^{\circ}$ & $\pm 1^{\circ}$ & $\pm 2^{\circ}$ & $\pm 5^{\circ}$ & $\pm 10^{\circ}$ & $\pm 15^{\circ}$ & $\pm 20^{\circ}$ & $\pm 25^{\circ}$ \\
\hline Method & 89 & 94 & 97 & 100 & 100 & 100 & 100 & 100 \\
\hline $\begin{array}{c}\text { Single Point GPS } \\
\text { (Pseudoranges) }\end{array}$ & 45 & 47 & 48 & 57 & 73 & 83 & 87 & 90 \\
\hline $\begin{array}{c}\text { Single Point GPS (Carrier } \\
\text { Phase) }\end{array}$ & 92 & 96 & 98 & 100 & 100 & 100 & 100 & 100 \\
\hline $\begin{array}{c}\text { Single Difference GPS } \\
\text { (Pseudoranges) }\end{array}$ & 50 & 52 & 53 & 60 & 70 & 77 & 82 & 84 \\
\hline $\begin{array}{c}\text { Single Difference GPS } \\
\text { (Carrier Phase) }\end{array}$ & 91 & 94 & 95 & 97 & 97 & 99 & 99 & 99 \\
\hline $\begin{array}{c}\text { Double Difference GPS } \\
\text { (Pseudoranges) }\end{array}$ & 48 & 50 & 54 & 58 & 67 & 73 & 79 & 81 \\
\hline $\begin{array}{c}\text { Double Difference GPS } \\
\text { (Carrier Phase) }\end{array}$ & 98 & 100 & 100 & 100 & 100 & 100 & 100 & 100 \\
\hline
\end{tabular}

Table 120: Pitch / Heading Correlation Coefficients for Each Computation Method in Simulation Data Set B-2

\begin{tabular}{|c|c|c|}
\hline & \multicolumn{2}{|c|}{ Pitch / Heading Correlation Coefficient } \\
\hline Method & Unfused & Fused \\
\hline $\begin{array}{c}\text { Single Point GPS } \\
\text { (Pseudoranges) }\end{array}$ & 0.870646 & 0.010325 \\
\hline $\begin{array}{c}\text { Single Difference GPS } \\
\text { (Pseudoranges) }\end{array}$ & 0.748203 & 0.000944 \\
\hline $\begin{array}{c}\text { Double Difference GPS } \\
\text { (Pseudoranges) }\end{array}$ & 0.819718 & 0.016298 \\
\hline
\end{tabular}




\section{Set B-3: 5 m Dual Antenna Separation}

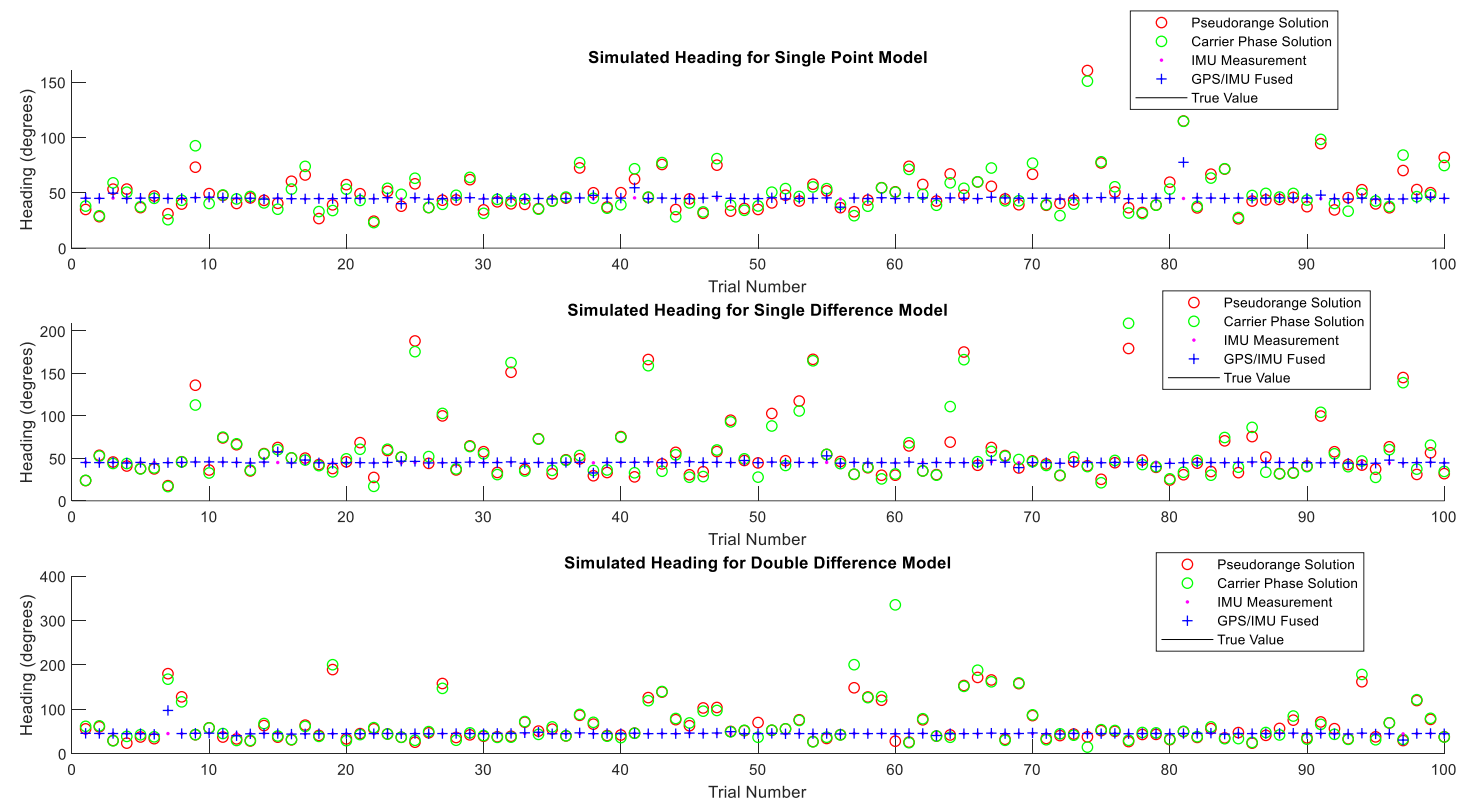

Figure 78: Computed and Measured Headings for Simulation Set B-3 


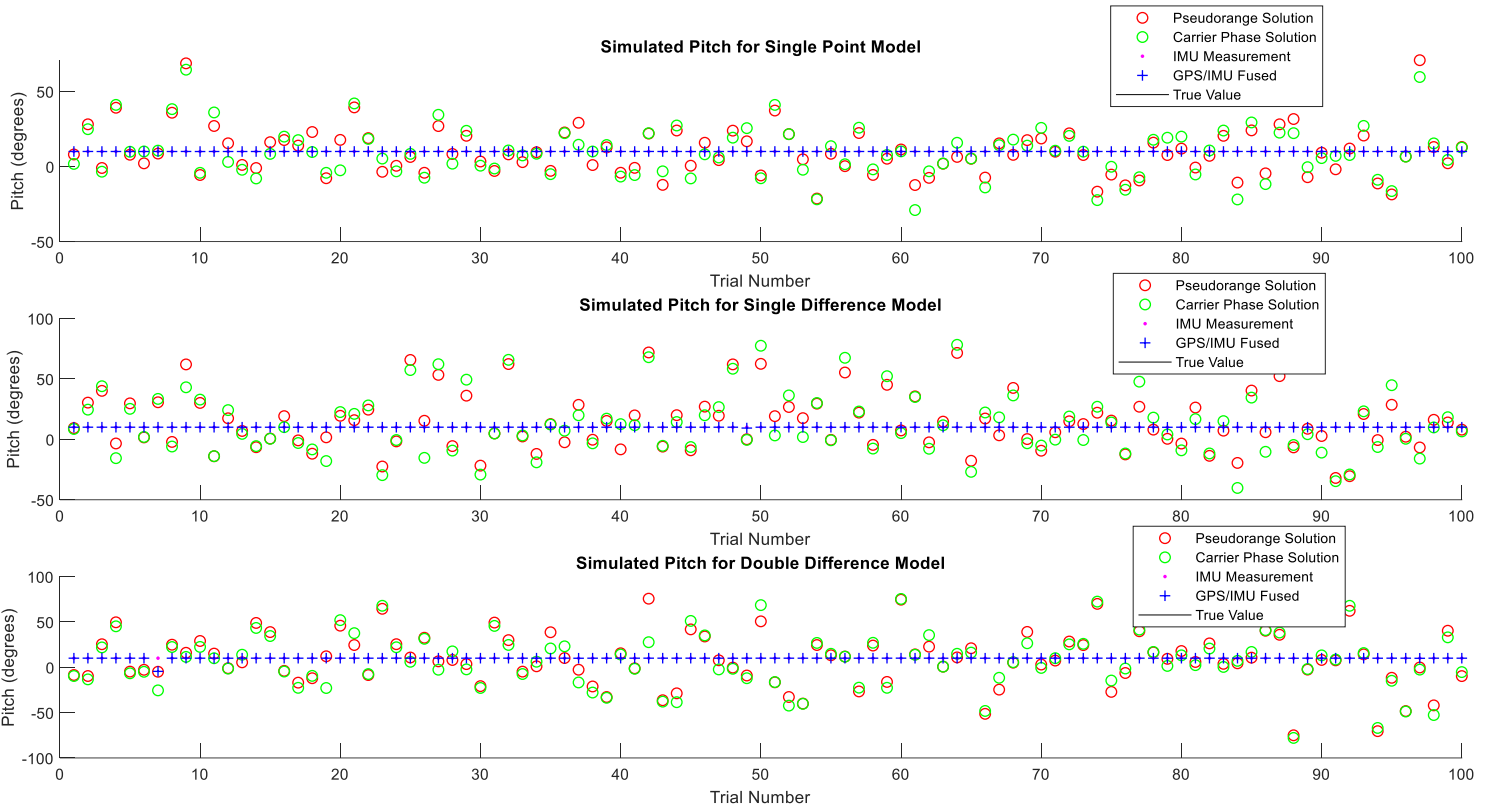

Figure 79: Computed and Measured Pitch for Simulation Set B-3

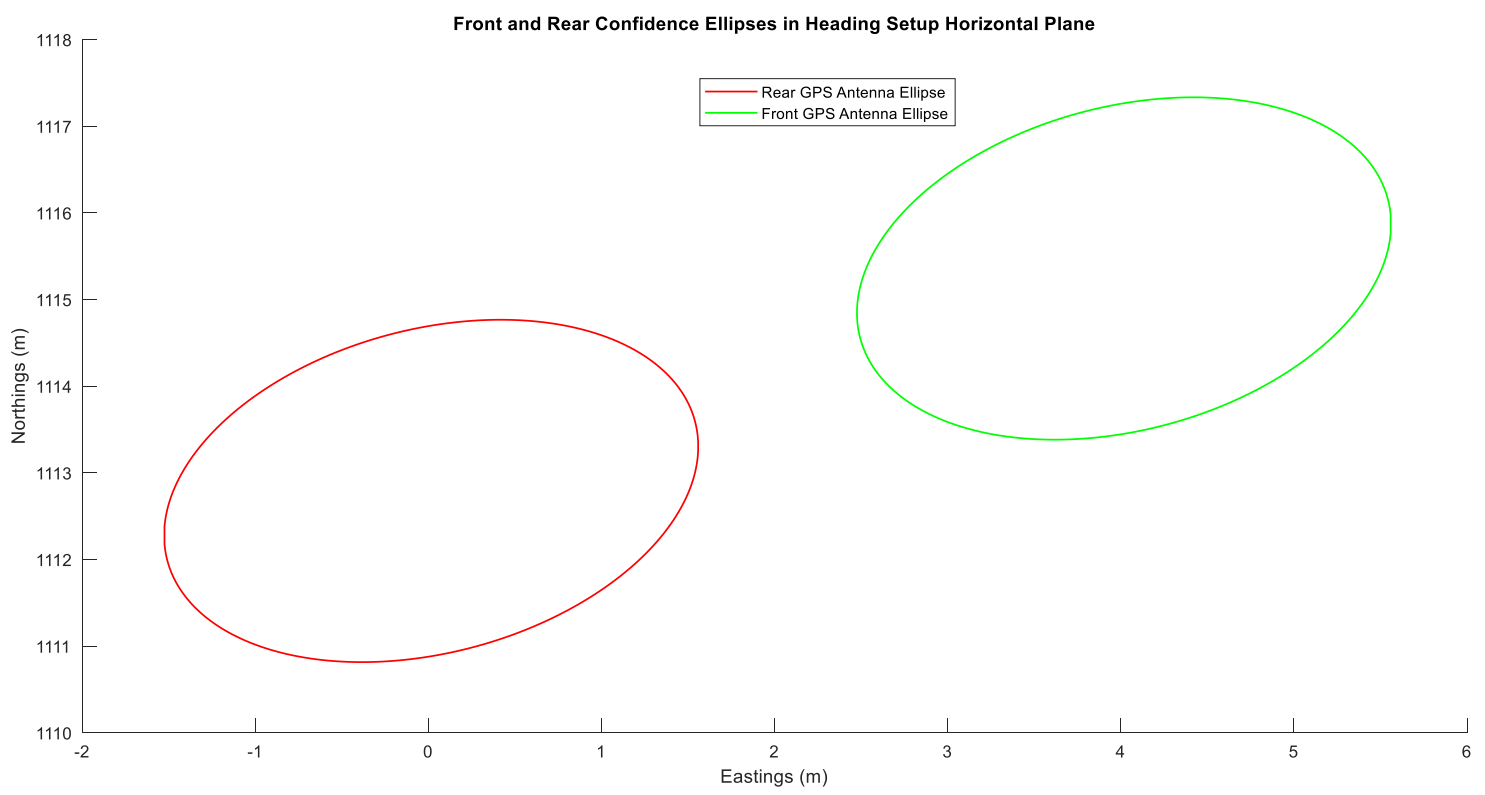

Figure 80: Propagated 95\% Confidence Ellipses for Front and Rear GPS Antennas in Simulated Data Set B-3 
Table 121: Mean Global Error Statistics of Unfused Pitch for Simulation Set B-3

\begin{tabular}{|c|c|c|c|}
\hline Method & $\begin{array}{c}\text { Measured Standard } \\
\text { Deviation } \\
\text { (degrees) }\end{array}$ & $\begin{array}{c}\text { Estimated } \\
\text { Standard } \\
\text { Deviation } \\
\text { (degrees) }\end{array}$ & $\begin{array}{c}\text { Mean RMS } \\
\text { Error } \\
\text { (degrees) }\end{array}$ \\
\hline Single Point GPS (Pseudoranges) & 15.844 & 42.771 & 12.058 \\
\hline Single Point GPS (Carrier Phase) & 16.467 & 0.044 & 12.563 \\
\hline $\begin{array}{c}\text { Single Difference GPS } \\
\text { (Pseudoranges) }\end{array}$ & 22.756 & 43.824 & 17.798 \\
\hline $\begin{array}{c}\text { Single Difference GPS (Carrier } \\
\text { Phase) }\end{array}$ & 25.581 & 0.054 & 20.098 \\
\hline $\begin{array}{c}\text { Double Difference GPS } \\
\text { (Pseudoranges) }\end{array}$ & 28.967 & 57.048 & 21.966 \\
\hline $\begin{array}{c}\text { Double Difference GPS (Carrier } \\
\text { Phase) }\end{array}$ & 29.548 & 0.111 & 22.877 \\
\hline IMU Measurement & 0.008 & - & 0.006 \\
\hline
\end{tabular}

Table 122: Mean Global Error Statistics of Unfused Heading for Simulation Set B-3

\begin{tabular}{|c|c|c|c|}
\hline Method & $\begin{array}{c}\text { Measured Standard } \\
\text { Deviation } \\
\text { (degrees) }\end{array}$ & $\begin{array}{c}\text { Estimated } \\
\text { Standard } \\
\text { Deviation } \\
\text { (degrees) }\end{array}$ & $\begin{array}{c}\text { Mean RMS } \\
\text { Error } \\
\text { (degrees) }\end{array}$ \\
\hline Single Point GPS (Pseudoranges) & 18.776 & 95.456 & 11.997 \\
\hline Single Point GPS (Carrier Phase) & 19.408 & 0.067 & 12.766 \\
\hline $\begin{array}{c}\text { Single Difference GPS } \\
\text { (Pseudoranges) }\end{array}$ & 36.572 & 116.748 & 21.849 \\
\hline $\begin{array}{c}\text { Single Difference GPS (Carrier } \\
\text { Phase) }\end{array}$ & 36.94 & 0.097 & 22.508 \\
\hline $\begin{array}{c}\text { Double Difference GPS } \\
\text { (Pseudoranges) }\end{array}$ & 40.825 & 179.035 & 27.003 \\
\hline $\begin{array}{c}\text { Double Difference GPS (Carrier } \\
\text { Phase) }\end{array}$ & 50.691 & 0.354 & 28.424 \\
\hline IMU Measurement & 0.351 & - & 0.277 \\
\hline
\end{tabular}


Table 123: Mean Global Error Statistics of Fused Pitch for Simulation Set B-3

\begin{tabular}{|c|c|c|c|}
\hline Method & $\begin{array}{c}\text { Measured Standard } \\
\text { Deviation } \\
\text { (degrees) }\end{array}$ & $\begin{array}{c}\text { Estimated } \\
\text { Standard } \\
\text { Deviation } \\
\text { (degrees) }\end{array}$ & $\begin{array}{c}\text { Mean RMS } \\
\text { Error } \\
\text { (degrees) }\end{array}$ \\
\hline Single Point GPS (Pseudoranges) & 0.013 & 0.2 & 0.009 \\
\hline Single Point GPS (Carrier Phase) & 3.291 & 0.021 & 2.708 \\
\hline $\begin{array}{c}\text { Single Difference GPS } \\
\text { (Pseudoranges) }\end{array}$ & 0.072 & 0.199 & 0.017 \\
\hline $\begin{array}{c}\text { Single Difference GPS (Carrier } \\
\text { Phase) }\end{array}$ & 4.907 & 0.025 & 3.801 \\
\hline $\begin{array}{c}\text { Double Difference GPS } \\
\text { (Pseudoranges) }\end{array}$ & 1.455 & 0.198 & 0.157 \\
\hline $\begin{array}{c}\text { Double Difference GPS (Carrier } \\
\text { Phase) }\end{array}$ & 5.16 & 0.045 & 2.938 \\
\hline IMU Measurement & 0.008 & - & 0.006 \\
\hline
\end{tabular}

Table 124: Mean Global Error Statistics of Fused Heading for Simulation Set B-3

\begin{tabular}{|c|c|c|c|}
\hline Method & $\begin{array}{c}\text { Measured Standard } \\
\text { Deviation } \\
\text { (degrees) }\end{array}$ & $\begin{array}{c}\text { Estimated } \\
\text { Standard } \\
\text { Deviation } \\
\text { (degrees) }\end{array}$ & $\begin{array}{c}\text { Mean RMS } \\
\text { Error } \\
\text { (degrees) }\end{array}$ \\
\hline Single Point GPS (Pseudoranges) & 3.584 & 5.469 & 0.954 \\
\hline Single Point GPS (Carrier Phase) & 13.924 & 0.033 & 9.924 \\
\hline $\begin{array}{c}\text { Single Difference GPS } \\
\text { (Pseudoranges) }\end{array}$ & 2.214 & 6.111 & 0.894 \\
\hline $\begin{array}{c}\text { Single Difference GPS (Carrier } \\
\text { Phase) }\end{array}$ & 29.077 & 0.045 & 17.246 \\
\hline $\begin{array}{c}\text { Double Difference GPS } \\
\text { (Pseudoranges) }\end{array}$ & 5.512 & 6.568 & 1.209 \\
\hline $\begin{array}{c}\text { Double Difference GPS (Carrier } \\
\text { Phase) }\end{array}$ & 29.718 & 0.157 & 15.133 \\
\hline IMU Measurement & 0.351 & - & 0.277 \\
\hline
\end{tabular}


Table 125: Success Rates of Reaching Angular Specifications of Unfused Pitch for Simulation Data Set B-3

\begin{tabular}{|c|c|c|c|c|c|c|c|c|}
\hline & \multicolumn{7}{|c|}{ Portion of Trials in Which Target Specification was reached } \\
\hline & $\pm 0.5^{\circ}$ & $\pm 1^{\circ}$ & $\pm 2^{\circ}$ & $\pm 5^{\circ}$ & $\pm 10^{\circ}$ & $\pm 15^{\circ}$ & $\pm 20^{\circ}$ & $\pm 25^{\circ}$ \\
\hline Method & 58 & 58 & 61 & 65 & 76 & 88 & 93 & 94 \\
\hline $\begin{array}{c}\text { Single Point GPS } \\
\text { (Pseudoranges) }\end{array}$ & 57 & 61 & 61 & 67 & 77 & 86 & 92 & 93 \\
\hline $\begin{array}{c}\text { Single Point GPS (Carrier } \\
\text { Phase) }\end{array}$ & 50 & 50 & 50 & 55 & 69 & 73 & 81 & 84 \\
\hline $\begin{array}{c}\text { Single Difference GPS } \\
\text { (Pseudoranges) }\end{array}$ & 52 & 52 & 54 & 58 & 67 & 74 & 78 & 83 \\
\hline $\begin{array}{c}\text { Single Difference GPS } \\
\text { (Carrier Phase) }\end{array}$ & 54 & 57 & 58 & 62 & 67 & 74 & 80 & 82 \\
\hline $\begin{array}{c}\text { Double Difference GPS } \\
\text { (Pseudoranges) }\end{array}$ & 53 & 53 & 55 & 62 & 67 & 75 & 81 & 84 \\
\hline $\begin{array}{c}\text { Double Difference GPS } \\
\text { (Carrier Phase) }\end{array}$ & 100 & 100 & 100 & 100 & 100 & 100 & 100 & 100 \\
\hline IMU Measured & & & & & & & & \\
\hline
\end{tabular}

Table 126: Success Rates of Reaching Angular Specifications of Unfused Heading for Simulation Data Set B-3

\begin{tabular}{|c|c|c|c|c|c|c|c|c|}
\hline & \multicolumn{7}{|c|}{ Portion of Trials in Which Target Specification was reached } \\
\hline & $\pm 0.5^{\circ}$ & $\pm 1^{\circ}$ & $\pm 2^{\circ}$ & $\pm 5^{\circ}$ & $\pm 10^{\circ}$ & $\pm 15^{\circ}$ & $\pm 20^{\circ}$ & $\pm 25^{\circ}$ \\
\hline Method & 53 & 56 & 56 & 62 & 74 & 81 & 84 & 88 \\
\hline $\begin{array}{c}\text { Single Point GPS } \\
\text { (Pseudoranges) }\end{array}$ & 50 & 50 & 56 & 65 & 76 & 81 & 84 & 84 \\
\hline $\begin{array}{c}\text { Single Point GPS (Carrier } \\
\text { Phase) }\end{array}$ & 49 & 52 & 55 & 60 & 67 & 74 & 79 & 82 \\
\hline $\begin{array}{c}\text { Single Difference GPS } \\
\text { (Pseudoranges) }\end{array}$ & 48 & 49 & 52 & 59 & 69 & 73 & 78 & 81 \\
\hline $\begin{array}{c}\text { Single Difference GPS } \\
\text { (Carrier Phase) }\end{array}$ & 49 & 49 & 51 & 54 & 59 & 67 & 71 & 74 \\
\hline $\begin{array}{c}\text { Double Difference GPS } \\
\text { (Pseudoranges) }\end{array}$ & 50 & 51 & 53 & 58 & 62 & 65 & 70 & 74 \\
\hline $\begin{array}{c}\text { Double Difference GPS } \\
\text { (Carrier Phase) }\end{array}$ & 93 & 99 & 100 & 100 & 100 & 100 & 100 & 100 \\
\hline IMU Measured & & & & & & & & \\
\hline
\end{tabular}


Table 127: Success Rates of Reaching Angular Specifications of Fused Pitch for Simulation Data Set B-3

\begin{tabular}{|c|c|c|c|c|c|c|c|c|}
\hline & \multicolumn{7}{|c|}{ Portion of Trials in Which Target Specification was reached } \\
\hline & $\pm 0.5^{\circ}$ & $\pm 1^{\circ}$ & $\pm 2^{\circ}$ & $\pm 5^{\circ}$ & $\pm 10^{\circ}$ & $\pm 15^{\circ}$ & $\pm 20^{\circ}$ & $\pm 25^{\circ}$ \\
\hline Method & 100 & 100 & 100 & 100 & 100 & 100 & 100 & 100 \\
\hline $\begin{array}{c}\text { Single Point GPS } \\
\text { (Pseudoranges) }\end{array}$ & 62 & 77 & 98 & 100 & 100 & 100 & 100 & 100 \\
\hline $\begin{array}{c}\text { Single Point GPS (Carrier } \\
\text { Phase) }\end{array}$ & 100 & 100 & 100 & 100 & 100 & 100 & 100 & 100 \\
\hline $\begin{array}{c}\text { Single Difference GPS } \\
\text { (Pseudoranges) }\end{array}$ & 57 & 66 & 86 & 94 & 98 & 99 & 100 & 100 \\
\hline $\begin{array}{c}\text { Single Difference GPS } \\
\text { (Carrier Phase) }\end{array}$ & 100 & 100 & 100 & 100 & 100 & 100 & 100 & 100 \\
\hline $\begin{array}{c}\text { Double Difference GPS } \\
\text { (Pseudoranges) }\end{array}$ & 67 & 77 & 92 & 97 & 99 & 100 & 100 & 100 \\
\hline $\begin{array}{c}\text { Double Difference GPS } \\
\text { (Carrier Phase) }\end{array}$ & 100 & 100 & 100 & 100 & 100 & 100 & 100 & 100 \\
\hline IMU Measured & & & & & & & & \\
\hline
\end{tabular}

Table 128: Success Rates of Reaching Angular Specifications of Fused Heading for Simulation Data Set B-3

\begin{tabular}{|c|c|c|c|c|c|c|c|c|}
\hline & \multicolumn{7}{|c|}{ Portion of Trials in Which Target Specification was reached } \\
\hline & $\pm 0.5^{\circ}$ & $\pm 1^{\circ}$ & $\pm 2^{\circ}$ & $\pm 5^{\circ}$ & $\pm 10^{\circ}$ & $\pm 15^{\circ}$ & $\pm 20^{\circ}$ & $\pm 25^{\circ}$ \\
\hline Method & 82 & 93 & 95 & 98 & 99 & 99 & 99 & 99 \\
\hline $\begin{array}{c}\text { Single Point GPS } \\
\text { (Pseudoranges) }\end{array}$ & 50 & 53 & 56 & 67 & 78 & 87 & 90 & 93 \\
\hline $\begin{array}{c}\text { Single Point GPS (Carrier } \\
\text { Phase) }\end{array}$ & 80 & 92 & 94 & 98 & 99 & 100 & 100 & 100 \\
\hline $\begin{array}{c}\text { Single Difference GPS } \\
\text { (Pseudoranges) }\end{array}$ & 48 & 50 & 52 & 59 & 74 & 81 & 85 & 89 \\
\hline $\begin{array}{c}\text { Single Difference GPS } \\
\text { (Carrier Phase) }\end{array}$ & 82 & 91 & 97 & 99 & 99 & 99 & 99 & 99 \\
\hline $\begin{array}{c}\text { Double Difference GPS } \\
\text { (Pseudoranges) }\end{array}$ & 50 & 51 & 54 & 60 & 73 & 79 & 86 & 88 \\
\hline $\begin{array}{c}\text { Double Difference GPS } \\
\text { (Carrier Phase) }\end{array}$ & 93 & 99 & 100 & 100 & 100 & 100 & 100 & 100 \\
\hline IMU Measured & & & & & & & & \\
\hline
\end{tabular}


Table 129: Pitch / Heading Correlation Coefficients for Each Computation Method in Simulation Data Set B-3

\begin{tabular}{|c|c|c|}
\hline & \multicolumn{2}{|c|}{ Pitch / Heading Correlation Coefficient } \\
\hline Method & Unfused & Fused \\
\hline $\begin{array}{c}\text { Single Point GPS } \\
\text { (Pseudoranges) }\end{array}$ & 0.21306 & 0.001037 \\
\hline $\begin{array}{c}\text { Single Difference GPS } \\
\text { (Pseudoranges) }\end{array}$ & 0.945381 & 0.050411 \\
\hline $\begin{array}{c}\text { Double Difference GPS } \\
\text { (Pseudoranges) }\end{array}$ & -0.91592 & -0.02784 \\
\hline
\end{tabular}

\section{Set B-4: 10 m Dual Antenna Separation}

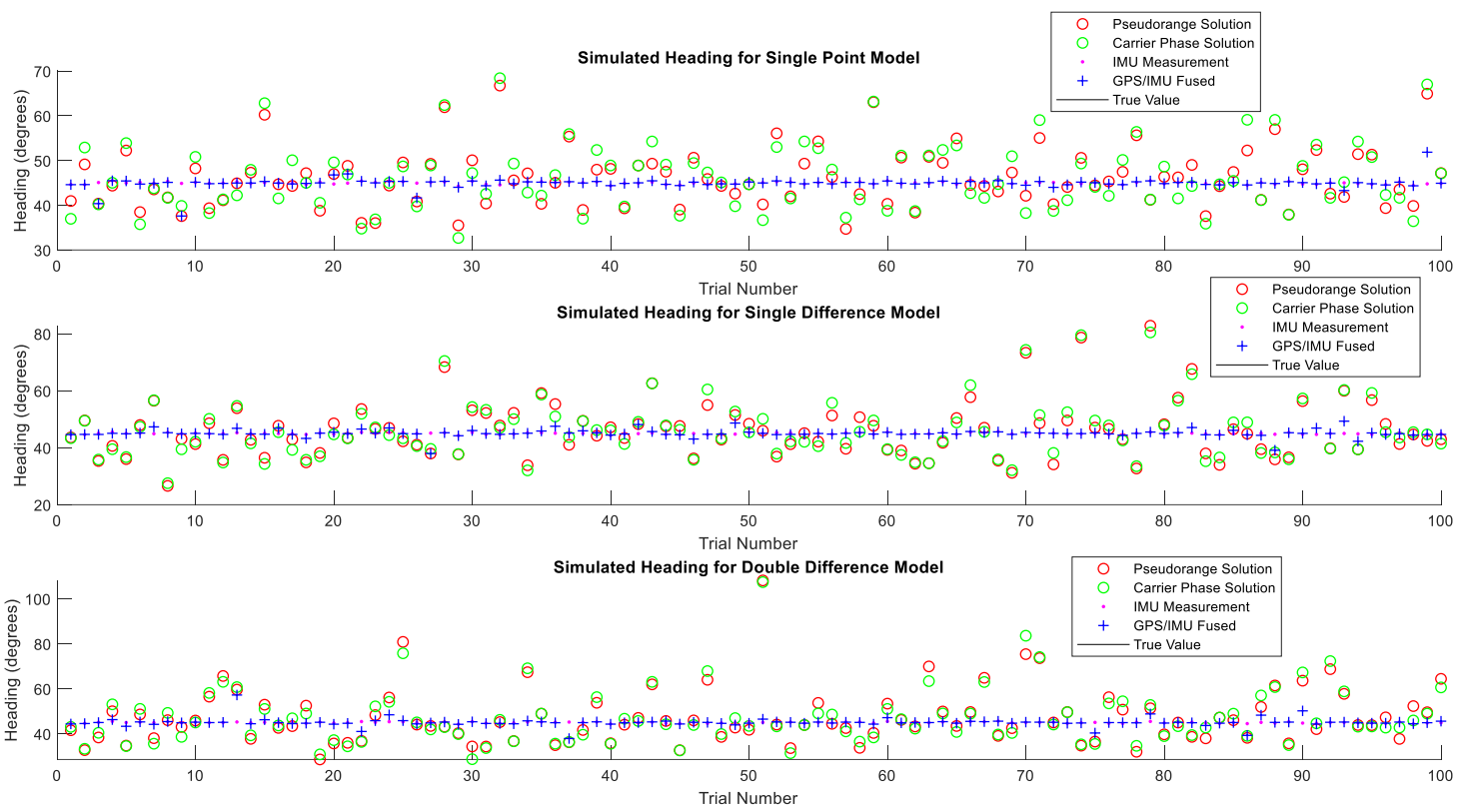

Figure 81: Computed and Measured Headings for Simulation Set B-4 


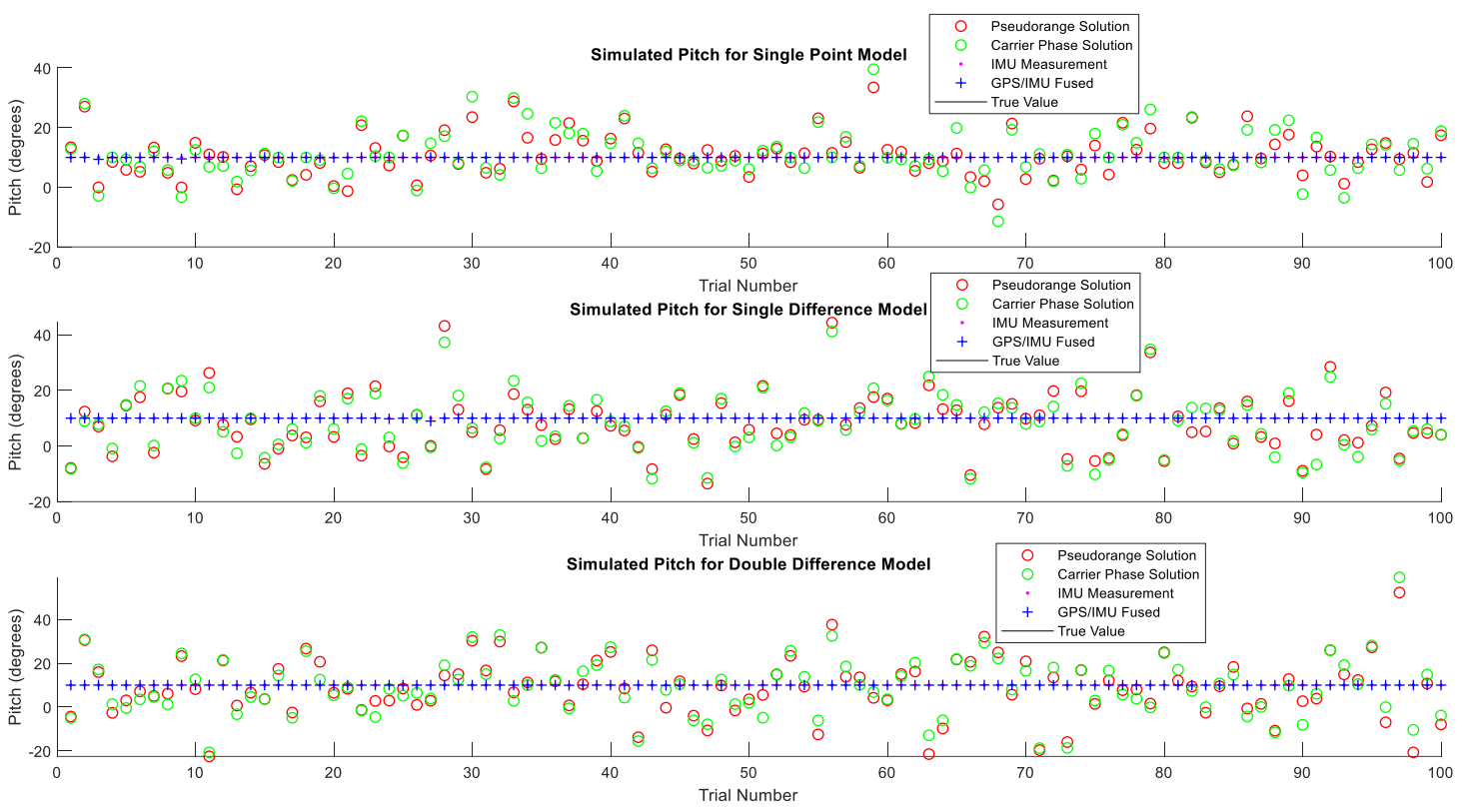

Figure 82: Computed and Measured Pitch for Simulation Set B-4

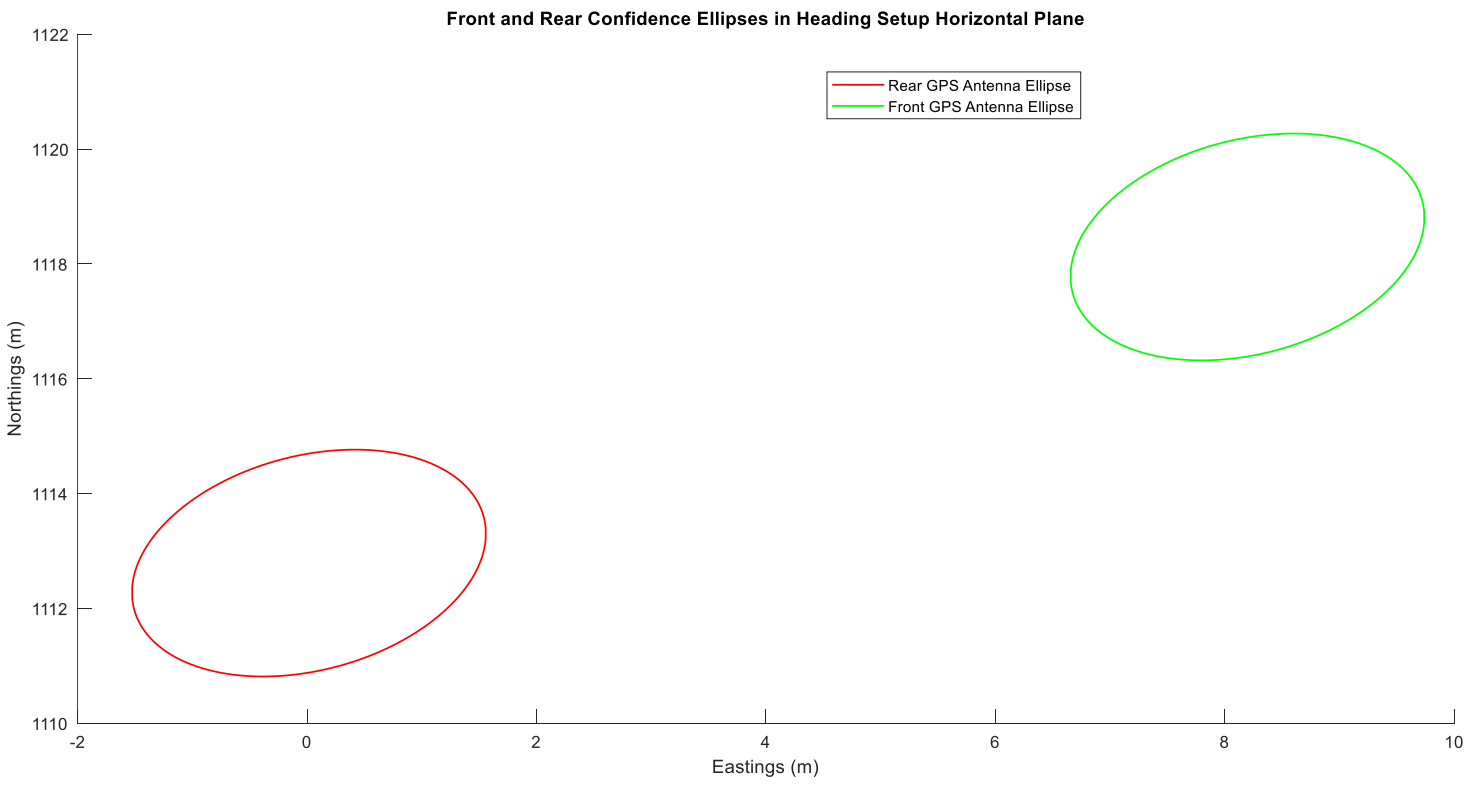

Figure 83: Propagated 95\% Confidence Ellipses for Front and Rear GPS Antennas in Simulated Data Set B-4 
Table 130: Mean Global Error Statistics of Unfused Pitch for Simulation Set B-4

\begin{tabular}{|c|c|c|c|}
\hline Method & $\begin{array}{c}\text { Measured Standard } \\
\text { Deviation } \\
\text { (degrees) }\end{array}$ & $\begin{array}{c}\text { Estimated } \\
\text { Standard } \\
\text { Deviation } \\
\text { (degrees) }\end{array}$ & $\begin{array}{c}\text { Mean RMS } \\
\text { Error } \\
\text { (degrees) }\end{array}$ \\
\hline Single Point GPS (Pseudoranges) & 7.083 & 19.072 & 5.376 \\
\hline Single Point GPS (Carrier Phase) & 8.144 & 0.018 & 6.007 \\
\hline $\begin{array}{c}\text { Single Difference GPS } \\
\text { (Pseudoranges) }\end{array}$ & 10.461 & 17.47 & 8.288 \\
\hline $\begin{array}{c}\text { Single Difference GPS (Carrier } \\
\text { Phase) }\end{array}$ & 10.753 & 0.016 & 8.799 \\
\hline $\begin{array}{c}\text { Double Difference GPS } \\
\text { (Pseudoranges) }\end{array}$ & 13.366 & 17.167 & 10.322 \\
\hline $\begin{array}{c}\text { Double Difference GPS (Carrier } \\
\text { Phase) }\end{array}$ & 13.266 & 0.033 & 10.497 \\
\hline IMU Measurement & 0.007 & - & 0.006 \\
\hline
\end{tabular}

Table 131: Mean Global Error Statistics of Unfused Heading for Simulation Set B-4

\begin{tabular}{|c|c|c|c|}
\hline Method & $\begin{array}{c}\text { Measured Standard } \\
\text { Deviation } \\
\text { (degrees) }\end{array}$ & $\begin{array}{c}\text { Estimated } \\
\text { Standard } \\
\text { Deviation } \\
\text { (degrees) }\end{array}$ & $\begin{array}{c}\text { Mean RMS } \\
\text { Error } \\
\text { (degrees) }\end{array}$ \\
\hline Single Point GPS (Pseudoranges) & 6.45 & 21.464 & 4.957 \\
\hline Single Point GPS (Carrier Phase) & 7.372 & 0.015 & 5.877 \\
\hline $\begin{array}{c}\text { Single Difference GPS } \\
\text { (Pseudoranges) }\end{array}$ & 9.91 & 27.881 & 7.3 \\
\hline $\begin{array}{c}\text { Single Difference GPS (Carrier } \\
\text { Phase) }\end{array}$ & 10.055 & 0.021 & 7.436 \\
\hline $\begin{array}{c}\text { Double Difference GPS } \\
\text { (Pseudoranges) }\end{array}$ & 12.247 & 26.039 & 8.498 \\
\hline $\begin{array}{c}\text { Double Difference GPS (Carrier } \\
\text { Phase) }\end{array}$ & 12.374 & 0.049 & 8.636 \\
\hline IMU Measurement & 0.278 & - & 0.228 \\
\hline
\end{tabular}


Table 132: Mean Global Error Statistics of Fused Pitch for Simulation Set B-4

\begin{tabular}{|c|c|c|c|}
\hline Method & $\begin{array}{c}\text { Measured Standard } \\
\text { Deviation } \\
\text { (degrees) }\end{array}$ & $\begin{array}{c}\text { Estimated } \\
\text { Standard } \\
\text { Deviation } \\
\text { (degrees) }\end{array}$ & $\begin{array}{c}\text { Mean RMS } \\
\text { Error } \\
\text { (degrees) }\end{array}$ \\
\hline Single Point GPS (Pseudoranges) & 0.084 & 0.197 & 0.02 \\
\hline Single Point GPS (Carrier Phase) & 3.027 & 0.009 & 2.072 \\
\hline $\begin{array}{c}\text { Single Difference GPS } \\
\text { (Pseudoranges) }\end{array}$ & 0.109 & 0.197 & 0.023 \\
\hline $\begin{array}{c}\text { Single Difference GPS (Carrier } \\
\text { Phase) }\end{array}$ & 4.306 & 0.008 & 3.754 \\
\hline $\begin{array}{c}\text { Double Difference GPS } \\
\text { (Pseudoranges) }\end{array}$ & 0.018 & 0.2 & 0.011 \\
\hline $\begin{array}{c}\text { Double Difference GPS (Carrier } \\
\text { Phase) }\end{array}$ & 3.094 & 0.016 & 2.427 \\
\hline IMU Measurement & 0.007 & - & 0.006 \\
\hline
\end{tabular}

Table 133: Mean Global Error Statistics of Fused Heading for Simulation Set B-4

\begin{tabular}{|c|c|c|c|}
\hline Method & $\begin{array}{c}\text { Measured Standard } \\
\text { Deviation } \\
\text { (degrees) }\end{array}$ & $\begin{array}{c}\text { Estimated } \\
\text { Standard } \\
\text { Deviation } \\
\text { (degrees) }\end{array}$ & $\begin{array}{c}\text { Mean RMS } \\
\text { Error } \\
\text { (degrees) }\end{array}$ \\
\hline Single Point GPS (Pseudoranges) & 1.246 & 3.898 & 0.517 \\
\hline Single Point GPS (Carrier Phase) & 6.94 & 0.008 & 5.561 \\
\hline $\begin{array}{c}\text { Single Difference GPS } \\
\text { (Pseudoranges) }\end{array}$ & 1.364 & 3.531 & 0.714 \\
\hline $\begin{array}{c}\text { Single Difference GPS (Carrier } \\
\text { Phase) }\end{array}$ & 8.566 & 0.01 & 6.724 \\
\hline $\begin{array}{c}\text { Double Difference GPS } \\
\text { (Pseudoranges) }\end{array}$ & 1.912 & 4.268 & 0.852 \\
\hline $\begin{array}{c}\text { Double Difference GPS (Carrier } \\
\text { Phase) }\end{array}$ & 10.611 & 0.025 & 7.445 \\
\hline IMU Measurement & 0.278 & - & 0.228 \\
\hline
\end{tabular}


Table 134: Success Rates of Reaching Angular Specifications of Unfused Pitch for Simulation Data Set B-4

\begin{tabular}{|c|c|c|c|c|c|c|c|c|}
\hline & \multicolumn{7}{|c|}{ Portion of Trials in Which Target Specification was reached } \\
\hline & $\pm 0.5^{\circ}$ & $\pm 1^{\circ}$ & $\pm 2^{\circ}$ & $\pm 5^{\circ}$ & $\pm 10^{\circ}$ & $\pm 15^{\circ}$ & $\pm 20^{\circ}$ & $\pm 25^{\circ}$ \\
\hline Method & 54 & 56 & 64 & 78 & 88 & 97 & 99 & 100 \\
\hline $\begin{array}{c}\text { Single Point GPS } \\
\text { (Pseudoranges) }\end{array}$ & 59 & 60 & 63 & 75 & 87 & 95 & 98 & 99 \\
\hline $\begin{array}{c}\text { Single Point GPS (Carrier } \\
\text { Phase) }\end{array}$ & 60 & 62 & 64 & 75 & 91 & 95 & 97 & 98 \\
\hline $\begin{array}{c}\text { Single Difference GPS } \\
\text { (Pseudoranges) }\end{array}$ & 59 & 59 & 61 & 73 & 87 & 97 & 97 & 98 \\
\hline $\begin{array}{c}\text { Single Difference GPS } \\
\text { (Carrier Phase) }\end{array}$ & 56 & 57 & 60 & 71 & 78 & 88 & 95 & 98 \\
\hline $\begin{array}{c}\text { Double Difference GPS } \\
\text { (Pseudoranges) }\end{array}$ & 56 & 57 & 57 & 68 & 81 & 88 & 95 & 99 \\
\hline $\begin{array}{c}\text { Double Difference GPS } \\
\text { (Carrier Phase) }\end{array}$ & 100 & 100 & 100 & 100 & 100 & 100 & 100 & 100 \\
\hline IMU Measured & & & & & & & & \\
\hline
\end{tabular}

Table 135: Success Rates of Reaching Angular Specifications of Unfused Heading for Simulation Data Set B-4

\begin{tabular}{|c|c|c|c|c|c|c|c|c|}
\hline & \multicolumn{7}{|c|}{ Portion of Trials in Which Target Specification was reached } \\
\hline Method & $\pm 0.5^{\circ}$ & $\pm 1^{\circ}$ & $\pm 2^{\circ}$ & $\pm 5^{\circ}$ & $\pm 10^{\circ}$ & $\pm 15^{\circ}$ & $\pm 20^{\circ}$ & $\pm 25^{\circ}$ \\
\hline $\begin{array}{c}\text { Single Point GPS } \\
\text { (Pseudoranges) }\end{array}$ & 51 & 53 & 57 & 78 & 90 & 95 & 99 & 100 \\
\hline $\begin{array}{c}\text { Single Point GPS (Carrier } \\
\text { Phase) }\end{array}$ & 54 & 54 & 56 & 72 & 90 & 95 & 98 & 100 \\
\hline $\begin{array}{c}\text { Single Difference GPS } \\
\text { (Pseudoranges) }\end{array}$ & 51 & 52 & 56 & 75 & 85 & 93 & 94 & 97 \\
\hline $\begin{array}{c}\text { Single Difference GPS } \\
\text { (Carrier Phase) }\end{array}$ & 54 & 56 & 59 & 73 & 84 & 90 & 94 & 96 \\
\hline $\begin{array}{c}\text { Double Difference GPS } \\
\text { (Pseudoranges) }\end{array}$ & 55 & 58 & 61 & 72 & 81 & 86 & 92 & 96 \\
\hline $\begin{array}{c}\text { Double Difference GPS } \\
\text { (Carrier Phase) }\end{array}$ & 54 & 56 & 61 & 72 & 81 & 85 & 92 & 95 \\
\hline IMU Measured & 99 & 100 & 100 & 100 & 100 & 100 & 100 & 100 \\
\hline
\end{tabular}


Table 136: Success Rates of Reaching Angular Specifications of Fused Pitch for Simulation Data Set B-4

\begin{tabular}{|c|c|c|c|c|c|c|c|c|}
\hline & \multicolumn{7}{|c|}{ Portion of Trials in Which Target Specification was reached } \\
\hline & $\pm 0.5^{\circ}$ & $\pm 1^{\circ}$ & $\pm 2^{\circ}$ & $\pm 5^{\circ}$ & $\pm 10^{\circ}$ & $\pm 15^{\circ}$ & $\pm 20^{\circ}$ & $\pm 25^{\circ}$ \\
\hline Method & 100 & 100 & 100 & 100 & 100 & 100 & 100 & 100 \\
\hline $\begin{array}{c}\text { Single Point GPS } \\
\text { (Pseudoranges) }\end{array}$ & 62 & 69 & 92 & 100 & 100 & 100 & 100 & 100 \\
\hline $\begin{array}{c}\text { Single Point GPS (Carrier } \\
\text { Phase) }\end{array}$ & 100 & 100 & 100 & 100 & 100 & 100 & 100 & 100 \\
\hline $\begin{array}{c}\text { Single Difference GPS } \\
\text { (Pseudoranges) }\end{array}$ & 61 & 66 & 94 & 100 & 100 & 100 & 100 & 100 \\
\hline $\begin{array}{c}\text { Single Difference GPS } \\
\text { (Carrier Phase) }\end{array}$ & 100 & 100 & 100 & 100 & 100 & 100 & 100 & 100 \\
\hline $\begin{array}{c}\text { Double Difference GPS } \\
\text { (Pseudoranges) }\end{array}$ & 61 & 74 & 91 & 100 & 100 & 100 & 100 & 100 \\
\hline $\begin{array}{c}\text { Double Difference GPS } \\
\text { (Carrier Phase) }\end{array}$ & 100 & 100 & 100 & 100 & 100 & 100 & 100 & 100 \\
\hline IMU Measured & & & & & & & & \\
\hline
\end{tabular}

Table 137: Success Rates of Reaching Angular Specifications of Fused Heading for Simulation Data Set B-4

\begin{tabular}{|c|c|c|c|c|c|c|c|c|}
\hline & \multicolumn{7}{|c|}{ Portion of Trials in Which Target Specification was reached } \\
\hline Method & $\pm 0.5^{\circ}$ & $\pm 1^{\circ}$ & $\pm 2^{\circ}$ & $\pm 5^{\circ}$ & $\pm 10^{\circ}$ & $\pm 15^{\circ}$ & $\pm 20^{\circ}$ & $\pm 25^{\circ}$ \\
\hline $\begin{array}{c}\text { Single Point GPS } \\
\text { (Pseudoranges) }\end{array}$ & 96 & 97 & 98 & 99 & 100 & 100 & 100 & 100 \\
\hline $\begin{array}{c}\text { Single Point GPS (Carrier } \\
\text { Phase) }\end{array}$ & 54 & 54 & 58 & 73 & 91 & 96 & 98 & 100 \\
\hline $\begin{array}{c}\text { Single Difference GPS } \\
\text { (Pseudoranges) }\end{array}$ & 82 & 87 & 94 & 100 & 100 & 100 & 100 & 100 \\
\hline $\begin{array}{c}\text { Single Difference GPS } \\
\text { (Carrier Phase) }\end{array}$ & 54 & 56 & 61 & 76 & 85 & 92 & 98 & 99 \\
\hline $\begin{array}{c}\text { Double Difference GPS } \\
\text { (Pseudoranges) }\end{array}$ & 85 & 91 & 94 & 98 & 99 & 100 & 100 & 100 \\
\hline $\begin{array}{c}\text { Double Difference GPS } \\
\text { (Carrier Phase) }\end{array}$ & 54 & 57 & 61 & 72 & 83 & 90 & 94 & 98 \\
\hline IMU Measured & 99 & 100 & 100 & 100 & 100 & 100 & 100 & 100 \\
\hline
\end{tabular}


Table 138: Pitch / Heading Correlation Coefficients for Each Computation Method in Simulation Data Set B-4

\begin{tabular}{|c|c|c|}
\hline & \multicolumn{2}{|c|}{ Pitch / Heading Correlation Coefficient } \\
\hline Method & Unfused & Fused \\
\hline $\begin{array}{c}\text { Single Point GPS } \\
\text { (Pseudoranges) }\end{array}$ & 0.775919 & 0.012938 \\
\hline $\begin{array}{c}\text { Single Difference GPS } \\
\text { (Pseudoranges) }\end{array}$ & 0.910818 & 0.129777 \\
\hline $\begin{array}{c}\text { Double Difference GPS } \\
\text { (Pseudoranges) }\end{array}$ & -0.66525 & -0.02701 \\
\hline
\end{tabular}

\section{Set B-5: 30 m Dual Antenna Separation}

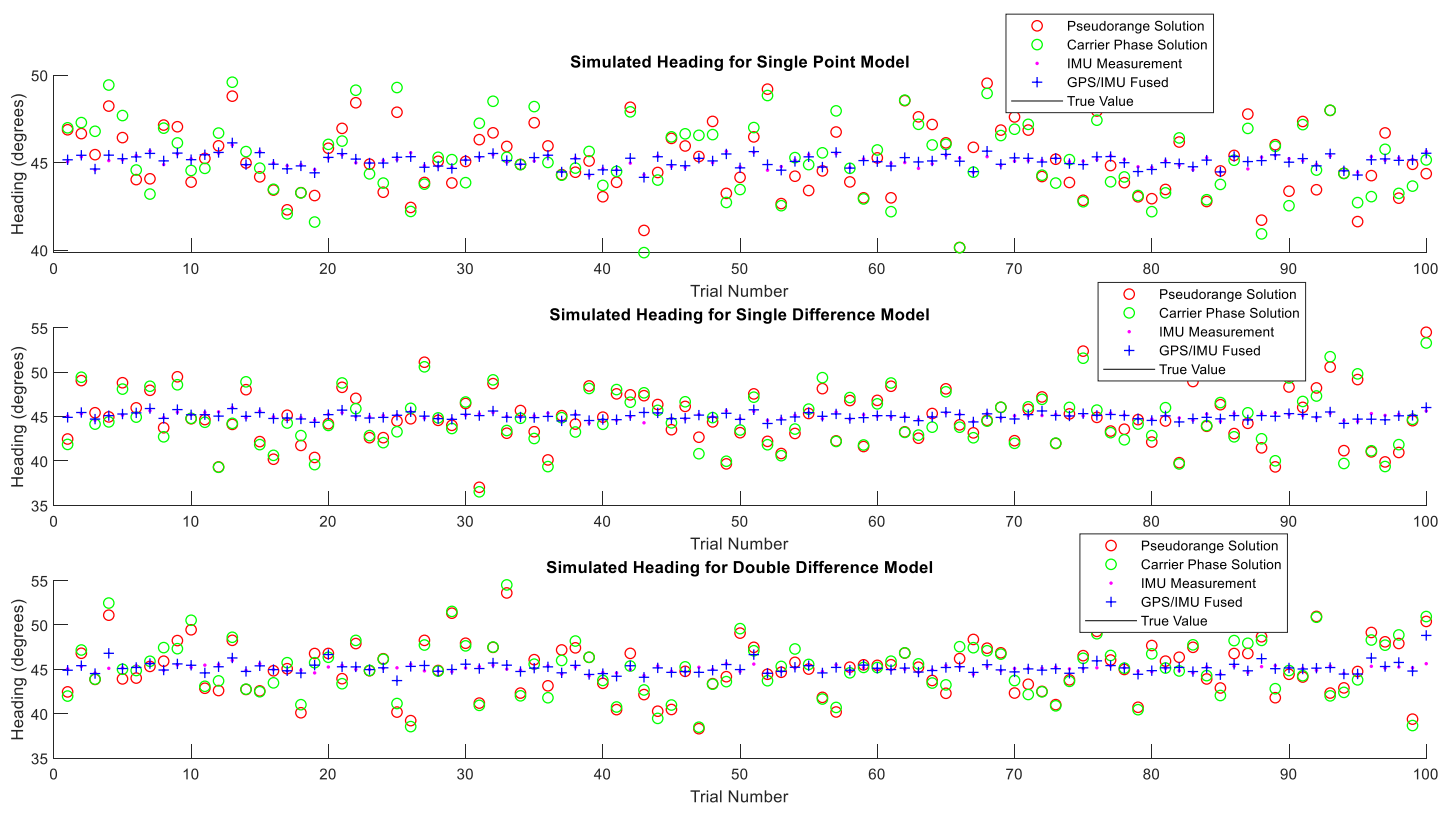

Figure 84: Computed and Measured Headings for Simulation Set B-5 


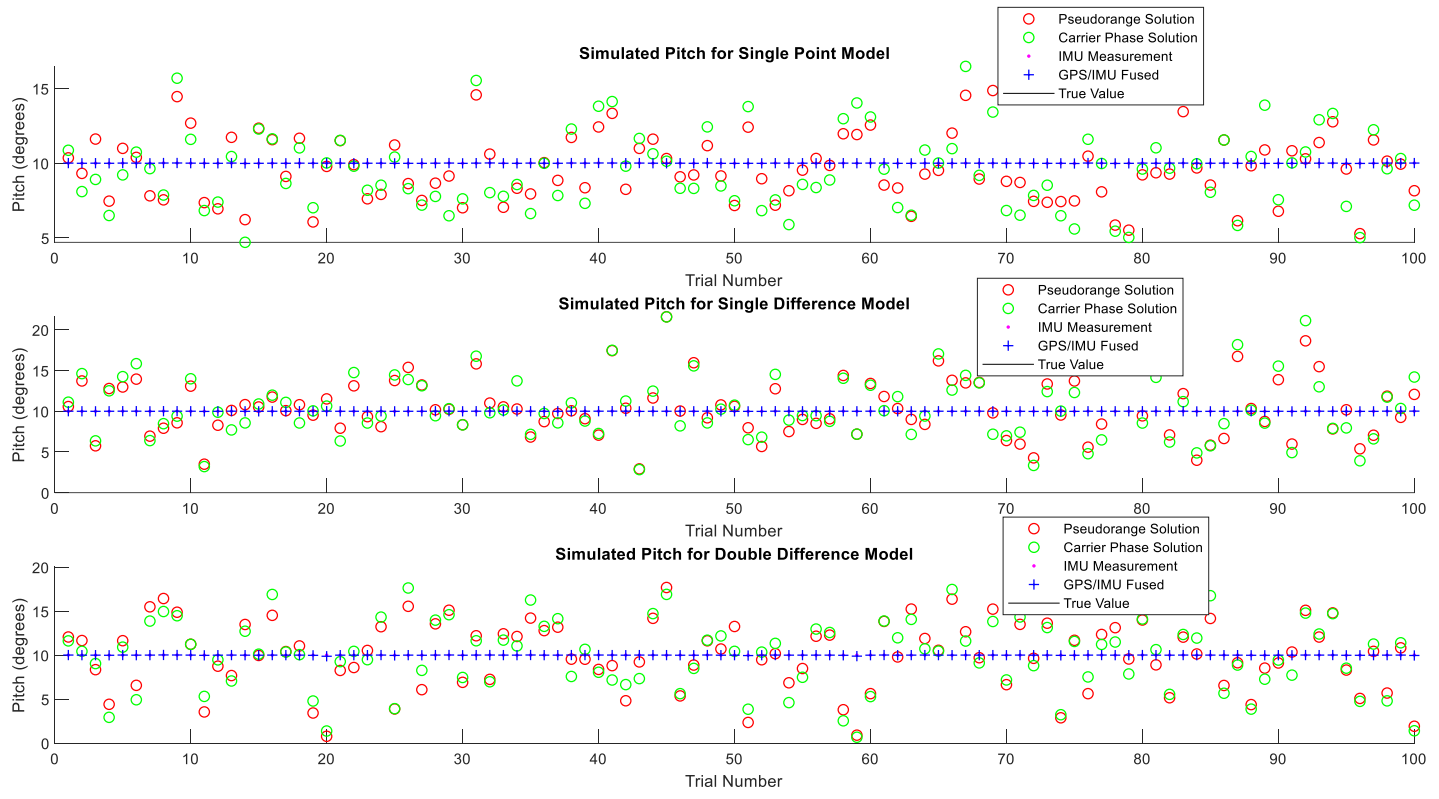

Figure 85: Computed and Measured Pitch for Simulation Set B-5

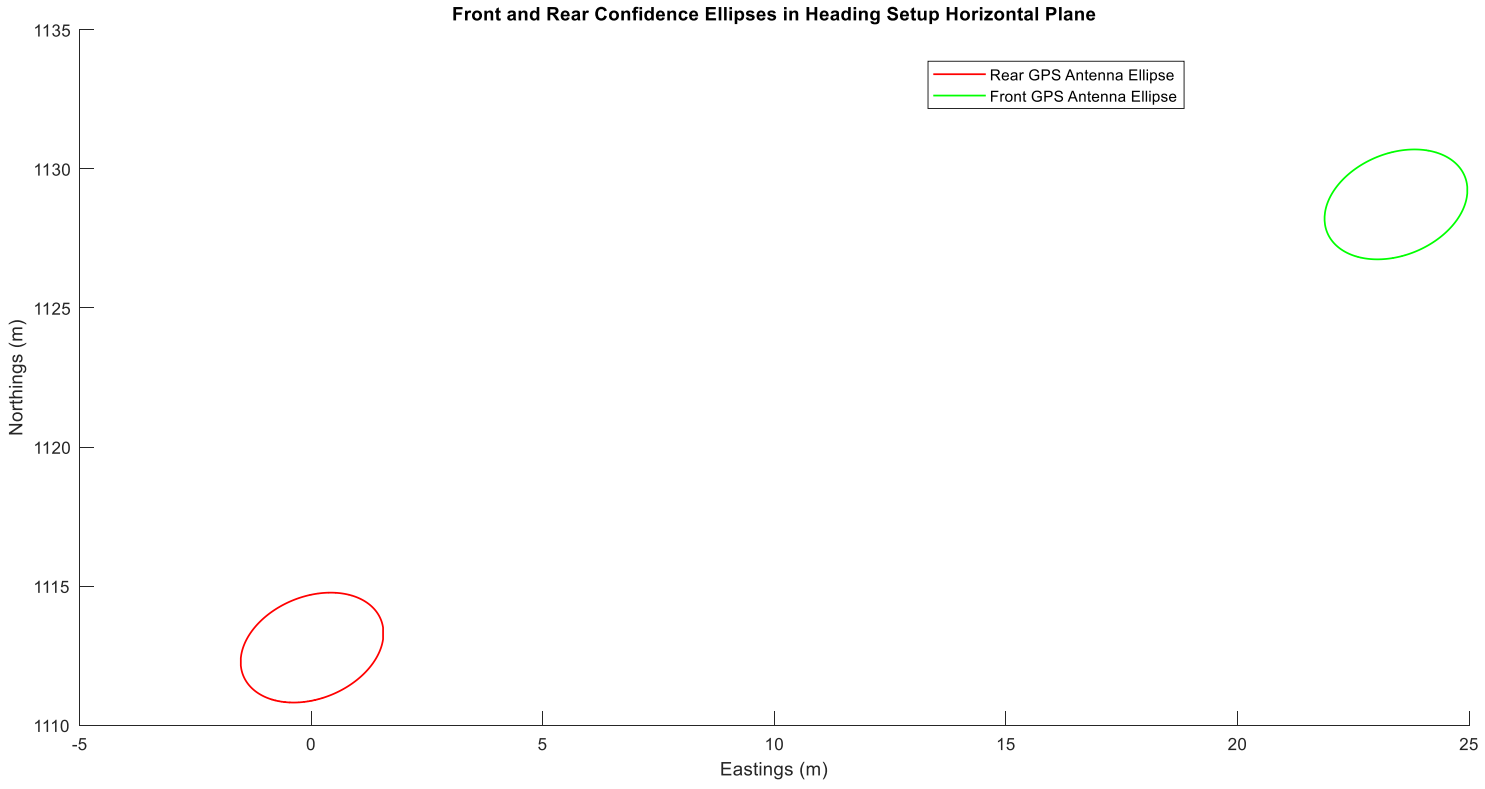

Figure 86: Propagated 95\% Confidence Ellipses for Front and Rear GPS Antennas in Simulated Data Set B-5 
Table 139: Mean Global Error Statistics of Unfused Pitch for Simulation Set B-5

\begin{tabular}{|c|c|c|c|}
\hline Method & $\begin{array}{c}\text { Measured Standard } \\
\text { Deviation } \\
\text { (degrees) }\end{array}$ & $\begin{array}{c}\text { Estimated } \\
\text { Standard } \\
\text { Deviation } \\
\text { (degrees) }\end{array}$ & $\begin{array}{c}\text { Mean RMS } \\
\text { Error } \\
\text { (degrees) }\end{array}$ \\
\hline Single Point GPS (Pseudoranges) & 2.158 & 6.313 & 1.806 \\
\hline Single Point GPS (Carrier Phase) & 2.621 & 0.004 & 2.181 \\
\hline $\begin{array}{c}\text { Single Difference GPS } \\
\text { (Pseudoranges) }\end{array}$ & 3.506 & 6.75 & 2.736 \\
\hline $\begin{array}{c}\text { Single Difference GPS (Carrier } \\
\text { Phase) }\end{array}$ & 3.81 & 0.005 & 2.992 \\
\hline $\begin{array}{c}\text { Double Difference GPS } \\
\text { (Pseudoranges) }\end{array}$ & 3.836 & 5.085 & 3.108 \\
\hline $\begin{array}{c}\text { Double Difference GPS (Carrier } \\
\text { Phase) }\end{array}$ & 3.923 & 0.01 & 3.194 \\
\hline IMU Measurement & 0.006 & - & 0.005 \\
\hline
\end{tabular}

Table 140: Mean Global Error Statistics of Unfused Heading for Simulation Set B-5

\begin{tabular}{|c|c|c|c|}
\hline Method & $\begin{array}{c}\text { Measured Standard } \\
\text { Deviation } \\
\text { (degrees) }\end{array}$ & $\begin{array}{c}\text { Estimated } \\
\text { Standard } \\
\text { Deviation } \\
\text { (degrees) }\end{array}$ & $\begin{array}{c}\text { Mean RMS } \\
\text { Error } \\
\text { (degrees) }\end{array}$ \\
\hline Single Point GPS (Pseudoranges) & 1.978 & 5.088 & 1.638 \\
\hline Single Point GPS (Carrier Phase) & 2.144 & 0.003 & 1.752 \\
\hline $\begin{array}{c}\text { Single Difference GPS } \\
\text { (Pseudoranges) }\end{array}$ & 3.088 & 6.22 & 2.446 \\
\hline $\begin{array}{c}\text { Single Difference GPS (Carrier } \\
\text { Phase) }\end{array}$ & 3.181 & 0.003 & 2.584 \\
\hline $\begin{array}{c}\text { Double Difference GPS } \\
\text { (Pseudoranges) }\end{array}$ & 2.973 & 4.278 & 2.387 \\
\hline $\begin{array}{c}\text { Double Difference GPS (Carrier } \\
\text { Phase) }\end{array}$ & 3.08 & 0.009 & 2.44 \\
\hline IMU Measurement & 0.343 & - & 0.287 \\
\hline
\end{tabular}


Table 141: Mean Global Error Statistics of Fused Pitch for Simulation Set B-5

\begin{tabular}{|c|c|c|c|}
\hline Method & $\begin{array}{c}\text { Measured Standard } \\
\text { Deviation } \\
\text { (degrees) }\end{array}$ & $\begin{array}{c}\text { Estimated } \\
\text { Standard } \\
\text { Deviation } \\
\text { (degrees) }\end{array}$ & $\begin{array}{c}\text { Mean RMS } \\
\text { Error } \\
\text { (degrees) }\end{array}$ \\
\hline Single Point GPS (Pseudoranges) & 0.007 & 0.2 & 0.006 \\
\hline Single Point GPS (Carrier Phase) & 1.598 & 0.002 & 1.356 \\
\hline $\begin{array}{c}\text { Single Difference GPS } \\
\text { (Pseudoranges) }\end{array}$ & 0.008 & 0.2 & 0.006 \\
\hline $\begin{array}{c}\text { Single Difference GPS (Carrier } \\
\text { Phase) }\end{array}$ & 2.174 & 0.003 & 1.719 \\
\hline $\begin{array}{c}\text { Double Difference GPS } \\
\text { (Pseudoranges) }\end{array}$ & 0.02 & 0.199 & 0.01 \\
\hline $\begin{array}{c}\text { Double Difference GPS (Carrier } \\
\text { Phase) }\end{array}$ & 2.076 & 0.005 & 1.496 \\
\hline IMU Measurement & 0.006 & - & 0.005 \\
\hline
\end{tabular}

Table 142: Mean Global Error Statistics of Fused Heading for Simulation Set B-5

\begin{tabular}{|c|c|c|c|}
\hline Method & $\begin{array}{c}\text { Measured Standard } \\
\text { Deviation } \\
\text { (degrees) }\end{array}$ & $\begin{array}{c}\text { Estimated } \\
\text { Standard } \\
\text { Deviation } \\
\text { (degrees) }\end{array}$ & $\begin{array}{c}\text { Mean RMS } \\
\text { Error } \\
\text { (degrees) }\end{array}$ \\
\hline Single Point GPS (Pseudoranges) & 0.358 & 1.266 & 0.299 \\
\hline Single Point GPS (Carrier Phase) & 2.126 & 0.001 & 1.737 \\
\hline $\begin{array}{c}\text { Single Difference GPS } \\
\text { (Pseudoranges) }\end{array}$ & 0.366 & 1.588 & 0.289 \\
\hline $\begin{array}{c}\text { Single Difference GPS (Carrier } \\
\text { Phase) }\end{array}$ & 3.143 & 0.002 & 2.556 \\
\hline $\begin{array}{c}\text { Double Difference GPS } \\
\text { (Pseudoranges) }\end{array}$ & 0.638 & 1.353 & 0.426 \\
\hline $\begin{array}{c}\text { Double Difference GPS (Carrier } \\
\text { Phase) }\end{array}$ & 2.993 & 0.004 & 2.373 \\
\hline IMU Measurement & 0.343 & - & 0.287 \\
\hline
\end{tabular}


Table 143: Success Rates of Reaching Angular Specifications of Unfused Pitch for Simulation Data Set B-5

\begin{tabular}{|c|c|c|c|c|c|c|c|c|}
\hline & \multicolumn{7}{|c|}{ Portion of Trials in Which Target Specification was reached } \\
\hline & $\pm 0.5^{\circ}$ & $\pm 1^{\circ}$ & $\pm 2^{\circ}$ & $\pm 5^{\circ}$ & $\pm 10^{\circ}$ & $\pm 15^{\circ}$ & $\pm 20^{\circ}$ & $\pm 25^{\circ}$ \\
\hline Method & 68 & 73 & 87 & 100 & 100 & 100 & 100 & 100 \\
\hline $\begin{array}{c}\text { Single Point GPS } \\
\text { (Pseudoranges) }\end{array}$ & 68 & 74 & 82 & 96 & 100 & 100 & 100 & 100 \\
\hline $\begin{array}{c}\text { Single Point GPS (Carrier } \\
\text { Phase) }\end{array}$ & 56 & 63 & 69 & 88 & 99 & 100 & 100 & 100 \\
\hline $\begin{array}{c}\text { Single Difference GPS } \\
\text { (Pseudoranges) }\end{array}$ & 57 & 60 & 68 & 89 & 98 & 100 & 100 & 100 \\
\hline $\begin{array}{c}\text { Single Difference GPS } \\
\text { (Carrier Phase) }\end{array}$ & 53 & 57 & 64 & 91 & 100 & 100 & 100 & 100 \\
\hline $\begin{array}{c}\text { Double Difference GPS } \\
\text { (Pseudoranges) }\end{array}$ & 52 & 56 & 71 & 94 & 100 & 100 & 100 & 100 \\
\hline $\begin{array}{c}\text { Double Difference GPS } \\
\text { (Carrier Phase) }\end{array}$ & 100 & 100 & 100 & 100 & 100 & 100 & 100 & 100 \\
\hline IMU Measured & & & & & & & & \\
\hline
\end{tabular}

Table 144: Success Rates of Reaching Angular Specifications of Unfused Heading for Simulation Data Set B-5

\begin{tabular}{|c|c|c|c|c|c|c|c|c|}
\hline & \multicolumn{7}{|c|}{ Portion of Trials in Which Target Specification was reached } \\
\hline Method & $\pm 0.5^{\circ}$ & $\pm 1^{\circ}$ & $\pm 2^{\circ}$ & $\pm 5^{\circ}$ & $\pm 10^{\circ}$ & $\pm 15^{\circ}$ & $\pm 20^{\circ}$ & $\pm 25^{\circ}$ \\
\hline $\begin{array}{c}\text { Single Point GPS } \\
\text { (Pseudoranges) }\end{array}$ & 59 & 65 & 80 & 100 & 100 & 100 & 100 & 100 \\
\hline $\begin{array}{c}\text { Single Point GPS (Carrier } \\
\text { Phase) }\end{array}$ & 56 & 62 & 79 & 100 & 100 & 100 & 100 & 100 \\
\hline $\begin{array}{c}\text { Single Difference GPS } \\
\text { (Pseudoranges) }\end{array}$ & 64 & 67 & 75 & 96 & 100 & 100 & 100 & 100 \\
\hline $\begin{array}{c}\text { Single Difference GPS } \\
\text { (Carrier Phase) }\end{array}$ & 61 & 66 & 77 & 95 & 100 & 100 & 100 & 100 \\
\hline $\begin{array}{c}\text { Double Difference GPS } \\
\text { (Pseudoranges) }\end{array}$ & 55 & 60 & 75 & 95 & 100 & 100 & 100 & 100 \\
\hline $\begin{array}{c}\text { Double Difference GPS } \\
\text { (Carrier Phase) }\end{array}$ & 55 & 63 & 71 & 94 & 100 & 100 & 100 & 100 \\
\hline IMU Measured & 91 & 99 & 100 & 100 & 100 & 100 & 100 & 100 \\
\hline
\end{tabular}


Table 145: Success Rates of Reaching Angular Specifications of Fused Pitch for Simulation Data Set B-5

\begin{tabular}{|c|c|c|c|c|c|c|c|c|}
\hline & \multicolumn{7}{|c|}{ Portion of Trials in Which Target Specification was reached } \\
\hline Method & $\pm 0.5^{\circ}$ & $\pm 1^{\circ}$ & $\pm 2^{\circ}$ & $\pm 5^{\circ}$ & $\pm 10^{\circ}$ & $\pm 15^{\circ}$ & $\pm 20^{\circ}$ & $\pm 25^{\circ}$ \\
\hline $\begin{array}{c}\text { Single Point GPS } \\
\text { (Pseudoranges) }\end{array}$ & 100 & 100 & 100 & 100 & 100 & 100 & 100 & 100 \\
\hline $\begin{array}{c}\text { Single Point GPS (Carrier } \\
\text { Phase) }\end{array}$ & 71 & 82 & 94 & 100 & 100 & 100 & 100 & 100 \\
\hline $\begin{array}{c}\text { Single Difference GPS } \\
\text { (Pseudoranges) }\end{array}$ & 100 & 100 & 100 & 100 & 100 & 100 & 100 & 100 \\
\hline $\begin{array}{c}\text { Single Difference GPS } \\
\text { (Carrier Phase) }\end{array}$ & 59 & 67 & 80 & 100 & 100 & 100 & 100 & 100 \\
\hline $\begin{array}{c}\text { Double Difference GPS } \\
\text { (Pseudoranges) }\end{array}$ & 100 & 100 & 100 & 100 & 100 & 100 & 100 & 100 \\
\hline $\begin{array}{c}\text { Double Difference GPS } \\
\text { (Carrier Phase) }\end{array}$ & 61 & 74 & 95 & 100 & 100 & 100 & 100 & 100 \\
\hline IMU Measured & 100 & 100 & 100 & 100 & 100 & 100 & 100 & 100 \\
\hline
\end{tabular}

Table 146: Success Rates of Reaching Angular Specifications of Fused Heading for Simulation Data Set B-5

\begin{tabular}{|c|c|c|c|c|c|c|c|c|}
\hline & \multicolumn{7}{|c|}{ Portion of Trials in Which Target Specification was reached } \\
\hline & $\pm 0.5^{\circ}$ & $\pm 1^{\circ}$ & $\pm 2^{\circ}$ & $\pm 5^{\circ}$ & $\pm 10^{\circ}$ & $\pm 15^{\circ}$ & $\pm 20^{\circ}$ & $\pm 25^{\circ}$ \\
\hline Method & 89 & 99 & 100 & 100 & 100 & 100 & 100 & 100 \\
\hline $\begin{array}{c}\text { Single Point GPS } \\
\text { (Pseudoranges) }\end{array}$ & 56 & 63 & 80 & 100 & 100 & 100 & 100 & 100 \\
\hline $\begin{array}{c}\text { Single Point GPS (Carrier } \\
\text { Phase) }\end{array}$ & 90 & 99 & 100 & 100 & 100 & 100 & 100 & 100 \\
\hline $\begin{array}{c}\text { Single Difference GPS } \\
\text { (Pseudoranges) }\end{array}$ & 61 & 67 & 77 & 95 & 100 & 100 & 100 & 100 \\
\hline $\begin{array}{c}\text { Single Difference GPS } \\
\text { (Carrier Phase) }\end{array}$ & 84 & 93 & 99 & 100 & 100 & 100 & 100 & 100 \\
\hline $\begin{array}{c}\text { Double Difference GPS } \\
\text { (Pseudoranges) }\end{array}$ & 91 & 63 & 71 & 94 & 100 & 100 & 100 & 100 \\
\hline $\begin{array}{c}\text { Double Difference GPS } \\
\text { (Carrier Phase) }\end{array}$ & 59 & 100 & 100 & 100 & 100 & 100 & 100 \\
\hline IMU Measured & 91 & & & & & & & \\
\hline
\end{tabular}


Table 147: Pitch / Heading Correlation Coefficients for Each Computation Method in Simulation Data Set B-5

\begin{tabular}{|c|c|c|}
\hline & \multicolumn{2}{|c|}{ Pitch / Heading Correlation Coefficient } \\
\hline Method & Unfused & Fused \\
\hline $\begin{array}{c}\text { Single Point GPS } \\
\text { (Pseudoranges) }\end{array}$ & 0.88543 & 0.136893 \\
\hline $\begin{array}{c}\text { Single Difference GPS } \\
\text { (Pseudoranges) }\end{array}$ & 0.770276 & 0.057302 \\
\hline $\begin{array}{c}\text { Double Difference GPS } \\
\text { (Pseudoranges) }\end{array}$ & 0.800147 & 0.401223 \\
\hline
\end{tabular}

\section{Set B-6: 50 m Dual Antenna Separation}

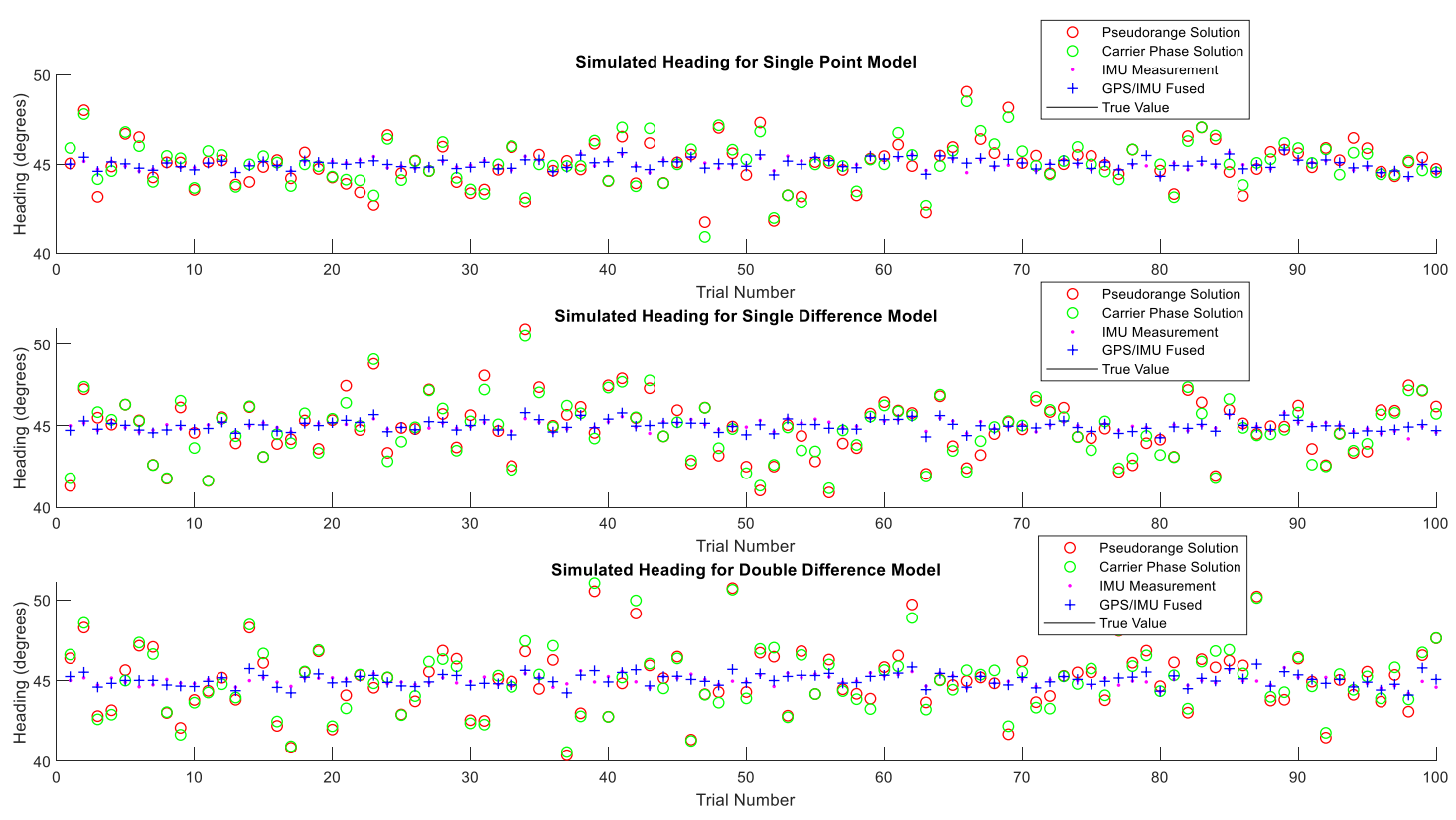

Figure 87: Computed and Measured Headings for Simulation Set B-6 


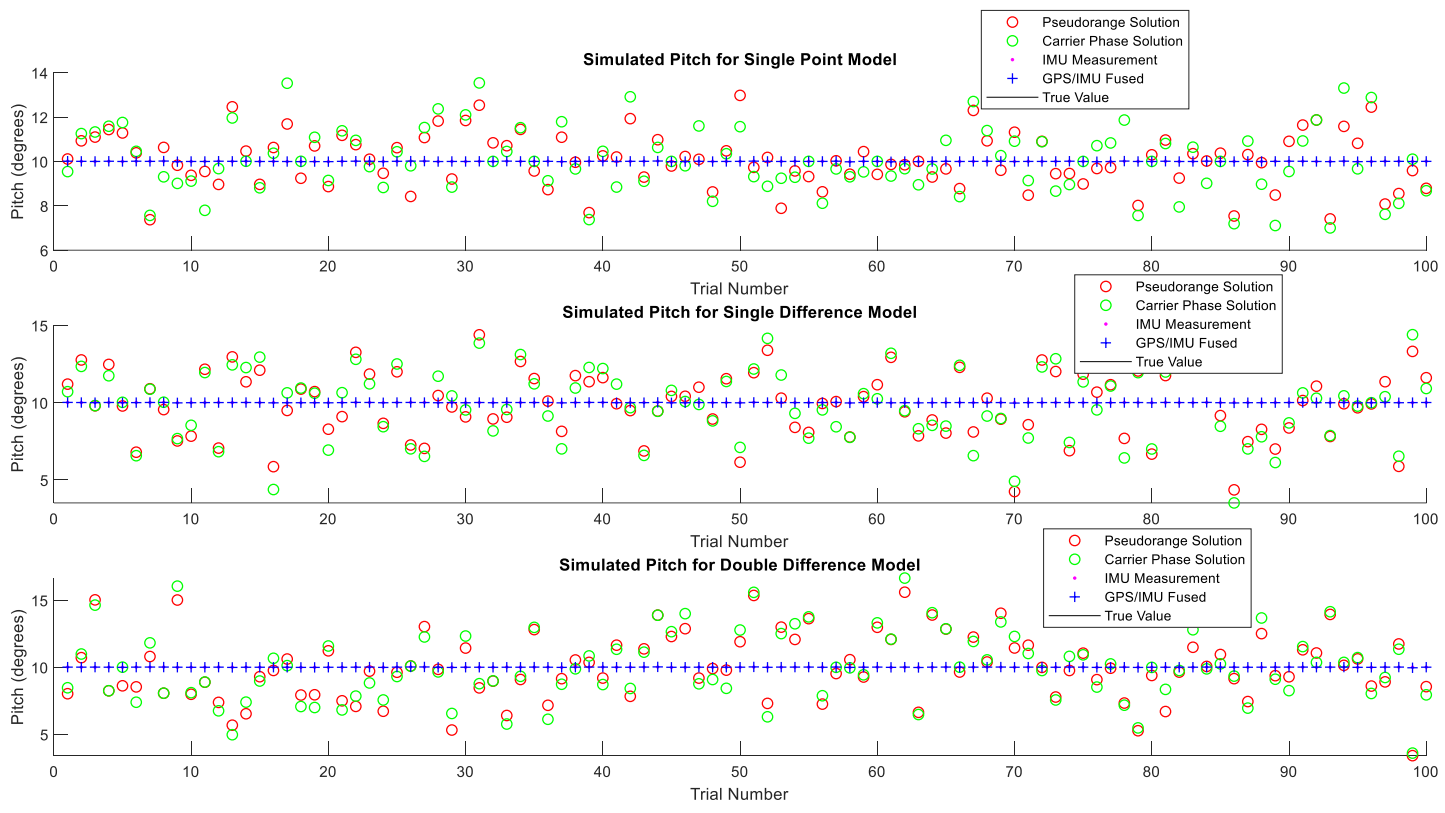

Figure 88: Computed and Measured Pitch for Simulation Set B-6 


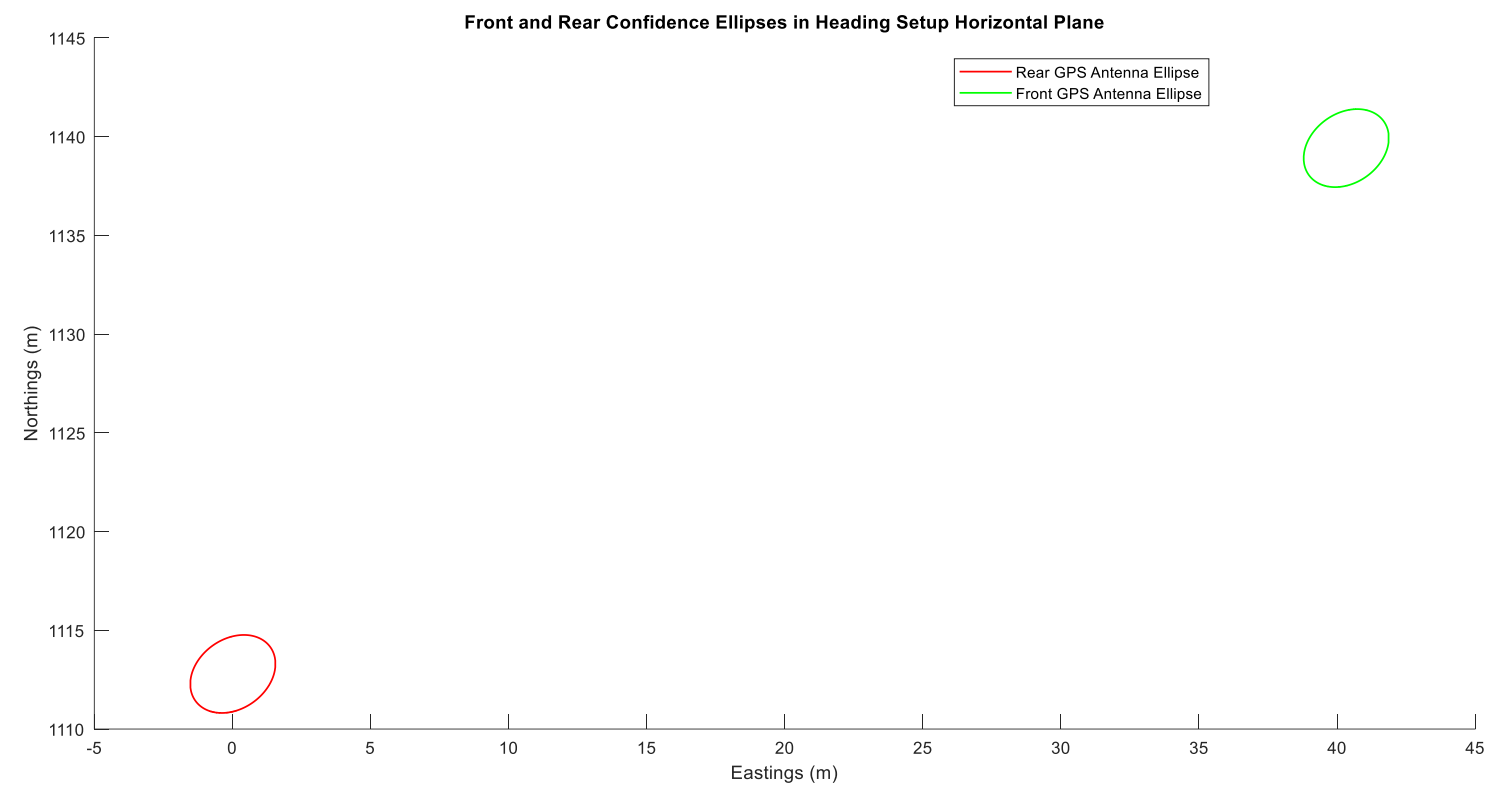

Figure 89: Propagated 95\% Confidence Ellipses for Front and Rear GPS Antennas in Simulated Data Set B-6

Table 148: Mean Global Error Statistics of Unfused Pitch for Simulation Set B-6

\begin{tabular}{|c|c|c|c|}
\hline Method & $\begin{array}{c}\text { Measured Standard } \\
\text { Deviation } \\
\text { (degrees) }\end{array}$ & $\begin{array}{c}\text { Estimated } \\
\text { Standard } \\
\text { Deviation } \\
\text { (degrees) }\end{array}$ & $\begin{array}{c}\text { Mean RMS } \\
\text { Error } \\
\text { (degrees) }\end{array}$ \\
\hline Single Point GPS (Pseudoranges) & 1.234 & 3.995 & 0.981 \\
\hline Single Point GPS (Carrier Phase) & 1.461 & 0.003 & 1.149 \\
\hline $\begin{array}{c}\text { Single Difference GPS } \\
\text { (Pseudoranges) }\end{array}$ & 2.144 & 3.897 & 1.757 \\
\hline $\begin{array}{c}\text { Single Difference GPS (Carrier } \\
\text { Phase) }\end{array}$ & 2.338 & 0.003 & 1.9 \\
\hline $\begin{array}{c}\text { Double Difference GPS } \\
\text { (Pseudoranges) }\end{array}$ & 2.396 & 3.1 & 1.893 \\
\hline $\begin{array}{c}\text { Double Difference GPS (Carrier } \\
\text { Phase) }\end{array}$ & 2.551 & 0.006 & 2.028 \\
\hline IMU Measurement & 0.007 & - & 0.005 \\
\hline
\end{tabular}


Table 149: Mean Global Error Statistics of Unfused Heading for Simulation Set B-6

\begin{tabular}{|c|c|c|c|}
\hline Method & $\begin{array}{c}\text { Measured Standard } \\
\text { Deviation } \\
\text { (degrees) }\end{array}$ & $\begin{array}{c}\text { Estimated } \\
\text { Standard } \\
\text { Deviation } \\
\text { (degrees) }\end{array}$ & $\begin{array}{c}\text { Mean RMS } \\
\text { Error } \\
\text { (degrees) }\end{array}$ \\
\hline Single Point GPS (Pseudoranges) & 1.332 & 3.286 & 0.986 \\
\hline Single Point GPS (Carrier Phase) & 1.306 & 0.002 & 0.964 \\
\hline $\begin{array}{c}\text { Single Difference GPS } \\
\text { (Pseudoranges) }\end{array}$ & 1.825 & 3.245 & 1.446 \\
\hline $\begin{array}{c}\text { Single Difference GPS (Carrier } \\
\text { Phase) }\end{array}$ & 1.785 & 0.002 & 1.426 \\
\hline $\begin{array}{c}\text { Double Difference GPS } \\
\text { (Pseudoranges) }\end{array}$ & 2.01 & 2.423 & 1.545 \\
\hline $\begin{array}{c}\text { Double Difference GPS (Carrier } \\
\text { Phase) }\end{array}$ & 2.057 & 0.005 & 1.604 \\
\hline IMU Measurement & 0.3 & - & 0.24 \\
\hline
\end{tabular}

Table 150: Mean Global Error Statistics of Fused Pitch for Simulation Set B-6

\begin{tabular}{|c|c|c|c|}
\hline Method & $\begin{array}{c}\text { Measured Standard } \\
\text { Deviation } \\
\text { (degrees) }\end{array}$ & $\begin{array}{c}\text { Estimated } \\
\text { Standard } \\
\text { Deviation } \\
\text { (degrees) }\end{array}$ & $\begin{array}{c}\text { Mean RMS } \\
\text { Error } \\
\text { (degrees) }\end{array}$ \\
\hline Single Point GPS (Pseudoranges) & 0.007 & 0.199 & 0.005 \\
\hline Single Point GPS (Carrier Phase) & 1.033 & 0.001 & 0.816 \\
\hline $\begin{array}{c}\text { Single Difference GPS } \\
\text { (Pseudoranges) }\end{array}$ & 0.008 & 0.199 & 0.007 \\
\hline $\begin{array}{c}\text { Single Difference GPS (Carrier } \\
\text { Phase) }\end{array}$ & 1.735 & 0.001 & 1.383 \\
\hline $\begin{array}{c}\text { Double Difference GPS } \\
\text { (Pseudoranges) }\end{array}$ & 0.009 & 0.199 & 0.007 \\
\hline $\begin{array}{c}\text { Double Difference GPS (Carrier } \\
\text { Phase) }\end{array}$ & 1.391 & 0.003 & 1.093 \\
\hline IMU Measurement & 0.007 & - & 0.005 \\
\hline
\end{tabular}


Table 151: Mean Global Error Statistics of Fused Heading for Simulation Set B-6

\begin{tabular}{|c|c|c|c|}
\hline Method & $\begin{array}{c}\text { Measured Standard } \\
\text { Deviation } \\
\text { (degrees) }\end{array}$ & $\begin{array}{c}\text { Estimated } \\
\text { Standard } \\
\text { Deviation } \\
\text { (degrees) }\end{array}$ & $\begin{array}{c}\text { Mean RMS } \\
\text { Error } \\
\text { (degrees) }\end{array}$ \\
\hline Single Point GPS (Pseudoranges) & 0.294 & 0.826 & 0.233 \\
\hline Single Point GPS (Carrier Phase) & 1.299 & 0.001 & 0.959 \\
\hline $\begin{array}{c}\text { Single Difference GPS } \\
\text { (Pseudoranges) }\end{array}$ & 0.333 & 0.82 & 0.263 \\
\hline $\begin{array}{c}\text { Single Difference GPS (Carrier } \\
\text { Phase) }\end{array}$ & 1.774 & 0.001 & 1.418 \\
\hline $\begin{array}{c}\text { Double Difference GPS } \\
\text { (Pseudoranges) }\end{array}$ & 0.387 & 0.766 & 0.317 \\
\hline $\begin{array}{c}\text { Double Difference GPS (Carrier } \\
\text { Phase) }\end{array}$ & 2.027 & 0.002 & 1.58 \\
\hline IMU Measurement & 0.3 & - & 0.24 \\
\hline
\end{tabular}

Table 152: Success Rates of Reaching Angular Specifications of Unfused Pitch for Simulation Data Set B-6

\begin{tabular}{|c|c|c|c|c|c|c|c|c|}
\hline & \multicolumn{7}{|c|}{ Portion of Trials in Which Target Specification was reached } \\
\hline & $\pm 0.5^{\circ}$ & $\pm 1^{\circ}$ & $\pm 2^{\circ}$ & $\pm 5^{\circ}$ & $\pm 10^{\circ}$ & $\pm 15^{\circ}$ & $\pm 20^{\circ}$ & $\pm 25^{\circ}$ \\
\hline Method & 65 & 79 & 94 & 100 & 100 & 100 & 100 & 100 \\
\hline $\begin{array}{c}\text { Single Point GPS } \\
\text { (Pseudoranges) }\end{array}$ & 66 & 77 & 92 & 100 & 100 & 100 & 100 & 100 \\
\hline $\begin{array}{c}\text { Single Point GPS (Carrier } \\
\text { Phase) }\end{array}$ & 60 & 65 & 82 & 100 & 100 & 100 & 100 & 100 \\
\hline $\begin{array}{c}\text { Single Difference GPS } \\
\text { (Pseudoranges) }\end{array}$ & 57 & 68 & 80 & 100 & 100 & 100 & 100 & 100 \\
\hline $\begin{array}{c}\text { Single Difference GPS } \\
\text { (Carrier Phase) }\end{array}$ & 61 & 68 & 80 & 96 & 100 & 100 & 100 & 100 \\
\hline $\begin{array}{c}\text { Double Difference GPS } \\
\text { (Pseudoranges) }\end{array}$ & 62 & 69 & 77 & 97 & 100 & 100 & 100 & 100 \\
\hline $\begin{array}{c}\text { Double Difference GPS } \\
\text { (Carrier Phase) }\end{array}$ & 100 & 100 & 100 & 100 & 100 & 100 & 100 & 100 \\
\hline
\end{tabular}


Table 153: Success Rates of Reaching Angular Specifications of Unfused Heading for Simulation Data Set B-6

\begin{tabular}{|c|c|c|c|c|c|c|c|c|}
\hline & \multicolumn{7}{|c|}{ Portion of Trials in Which Target Specification was reached } \\
\hline & $\pm 0.5^{\circ}$ & $\pm 1^{\circ}$ & $\pm 2^{\circ}$ & $\pm 5^{\circ}$ & $\pm 10^{\circ}$ & $\pm 15^{\circ}$ & $\pm 20^{\circ}$ & $\pm 25^{\circ}$ \\
\hline Method & 67 & 82 & 93 & 100 & 100 & 100 & 100 & 100 \\
\hline $\begin{array}{c}\text { Single Point GPS } \\
\text { (Pseudoranges) }\end{array}$ & 65 & 79 & 92 & 100 & 100 & 100 & 100 & 100 \\
\hline $\begin{array}{c}\text { Single Point GPS (Carrier } \\
\text { Phase) }\end{array}$ & 63 & 75 & 87 & 99 & 100 & 100 & 100 & 100 \\
\hline $\begin{array}{c}\text { Single Difference GPS } \\
\text { (Pseudoranges) }\end{array}$ & 63 & 77 & 88 & 99 & 100 & 100 & 100 & 100 \\
\hline $\begin{array}{c}\text { Single Difference GPS } \\
\text { (Carrier Phase) }\end{array}$ & 58 & 68 & 89 & 97 & 100 & 100 & 100 & 100 \\
\hline $\begin{array}{c}\text { Double Difference GPS } \\
\text { (Pseudoranges) }\end{array}$ & 60 & 71 & 87 & 97 & 100 & 100 & 100 & 100 \\
\hline $\begin{array}{c}\text { Double Difference GPS } \\
\text { (Carrier Phase) }\end{array}$ & 95 & 100 & 100 & 100 & 100 & 100 & 100 & 100 \\
\hline IMU Measured & & & & & & & & \\
\hline
\end{tabular}

Table 154: Success Rates of Reaching Angular Specifications of Fused Pitch for Simulation Data Set B-6

\begin{tabular}{|c|c|c|c|c|c|c|c|c|}
\hline & \multicolumn{7}{|c|}{ Portion of Trials in Which Target Specification was reached } \\
\hline & $\pm 0.5^{\circ}$ & $\pm 1^{\circ}$ & $\pm 2^{\circ}$ & $\pm 5^{\circ}$ & $\pm 10^{\circ}$ & $\pm 15^{\circ}$ & $\pm 20^{\circ}$ & $\pm 25^{\circ}$ \\
\hline Method & 100 & 100 & 100 & 100 & 100 & 100 & 100 & 100 \\
\hline $\begin{array}{c}\text { Single Point GPS } \\
\text { (Pseudoranges) }\end{array}$ & 69 & 82 & 97 & 100 & 100 & 100 & 100 & 100 \\
\hline $\begin{array}{c}\text { Single Point GPS (Carrier } \\
\text { Phase) }\end{array}$ & 100 & 100 & 100 & 100 & 100 & 100 & 100 & 100 \\
\hline $\begin{array}{c}\text { Single Difference GPS } \\
\text { (Pseudoranges) }\end{array}$ & 62 & 74 & 93 & 100 & 100 & 100 & 100 & 100 \\
\hline $\begin{array}{c}\text { Single Difference GPS } \\
\text { (Carrier Phase) }\end{array}$ & 100 & 100 & 100 & 100 & 100 & 100 & 100 & 100 \\
\hline $\begin{array}{c}\text { Double Difference GPS } \\
\text { (Pseudoranges) }\end{array}$ & 69 & 78 & 96 & 100 & 100 & 100 & 100 & 100 \\
\hline $\begin{array}{c}\text { Double Difference GPS } \\
\text { (Carrier Phase) }\end{array}$ & 100 & 100 & 100 & 100 & 100 & 100 & 100 & 100 \\
\hline IMU Measured & & & & & & & & \\
\hline
\end{tabular}


Table 155: Success Rates of Reaching Angular Specifications of Fused Heading for Simulation Data Set B-6

\begin{tabular}{|c|c|c|c|c|c|c|c|c|}
\hline & \multicolumn{7}{|c|}{ Portion of Trials in Which Target Specification was reached } \\
\hline & $\pm 0.5^{\circ}$ & $\pm 1^{\circ}$ & $\pm 2^{\circ}$ & $\pm 5^{\circ}$ & $\pm 10^{\circ}$ & $\pm 15^{\circ}$ & $\pm 20^{\circ}$ & $\pm 25^{\circ}$ \\
\hline Method & 95 & 100 & 100 & 100 & 100 & 100 & 100 & 100 \\
\hline $\begin{array}{c}\text { Single Point GPS } \\
\text { (Pseudoranges) }\end{array}$ & 65 & 79 & 93 & 100 & 100 & 100 & 100 & 100 \\
\hline $\begin{array}{c}\text { Single Point GPS (Carrier } \\
\text { Phase) }\end{array}$ & 92 & 100 & 100 & 100 & 100 & 100 & 100 & 100 \\
\hline $\begin{array}{c}\text { Single Difference GPS } \\
\text { (Pseudoranges) }\end{array}$ & 63 & 77 & 88 & 99 & 100 & 100 & 100 & 100 \\
\hline $\begin{array}{c}\text { Single Difference GPS } \\
\text { (Carrier Phase) }\end{array}$ & 88 & 99 & 100 & 100 & 100 & 100 & 100 & 100 \\
\hline $\begin{array}{c}\text { Double Difference GPS } \\
\text { (Pseudoranges) }\end{array}$ & 60 & 71 & 87 & 97 & 100 & 100 & 100 & 100 \\
\hline $\begin{array}{c}\text { Double Difference GPS } \\
\text { (Carrier Phase) }\end{array}$ & 95 & 100 & 100 & 100 & 100 & 100 & 100 & 100 \\
\hline IMU Measured & & & & & & & & \\
\hline
\end{tabular}

Table 156: Pitch / Heading Correlation Coefficients for Each Computation Method in Simulation Data Set B-6

\begin{tabular}{|c|c|c|}
\hline & Pitch / Heading Correlation Coefficient \\
\hline Method & Unfused & Fused \\
\hline $\begin{array}{c}\text { Single Point GPS } \\
\text { (Pseudoranges) }\end{array}$ & 0.879492 & 0.205581 \\
\hline $\begin{array}{c}\text { Single Difference GPS } \\
\text { (Pseudoranges) }\end{array}$ & 0.849702 & 0.14371 \\
\hline $\begin{array}{c}\text { Double Difference GPS } \\
\text { (Pseudoranges) }\end{array}$ & 0.774141 & 0.172438 \\
\hline
\end{tabular}




\section{Set B-7: 70 m Dual Antenna Separation}

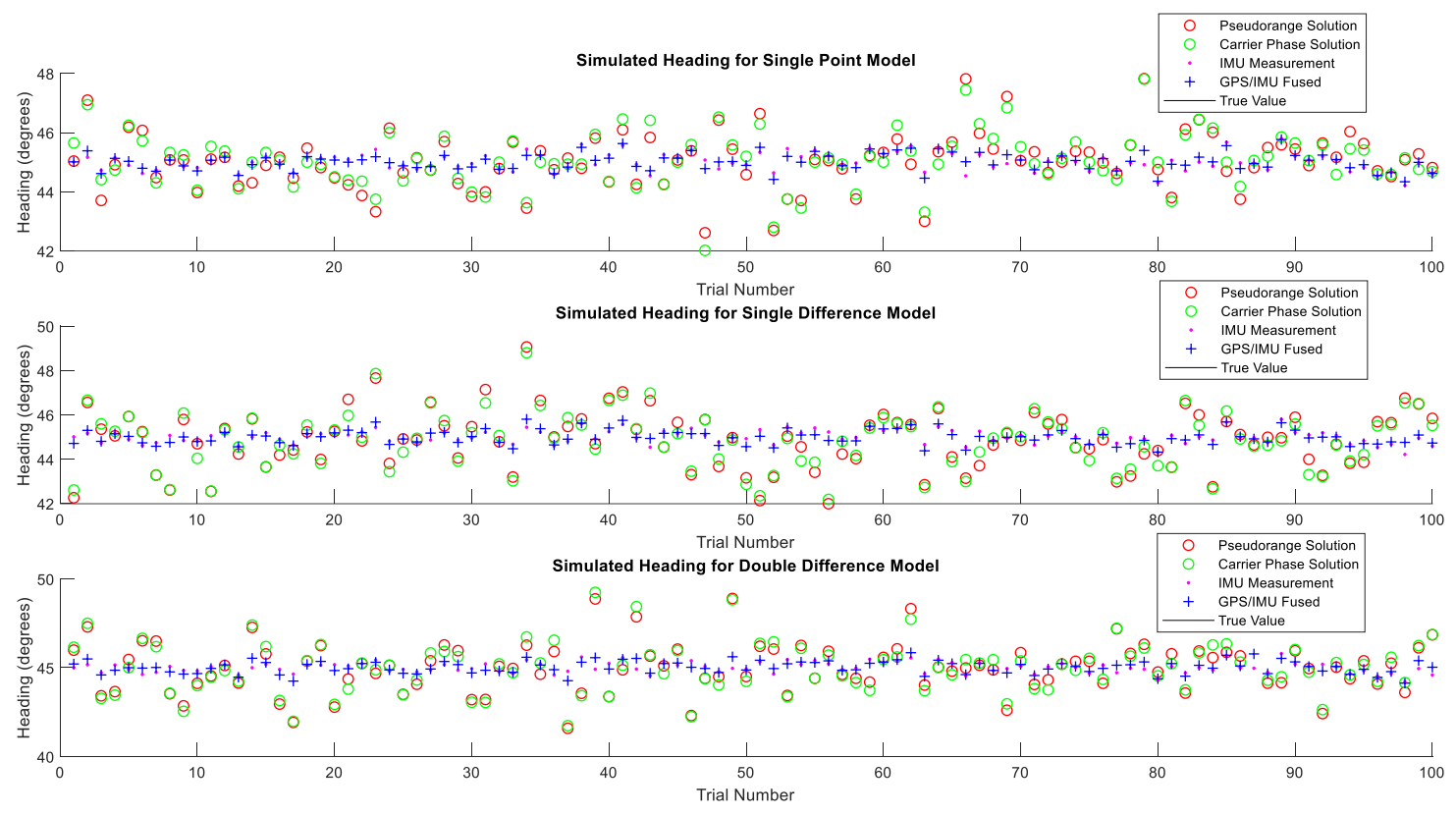

Figure 90: Computed and Measured Headings for Simulation Set B-7 


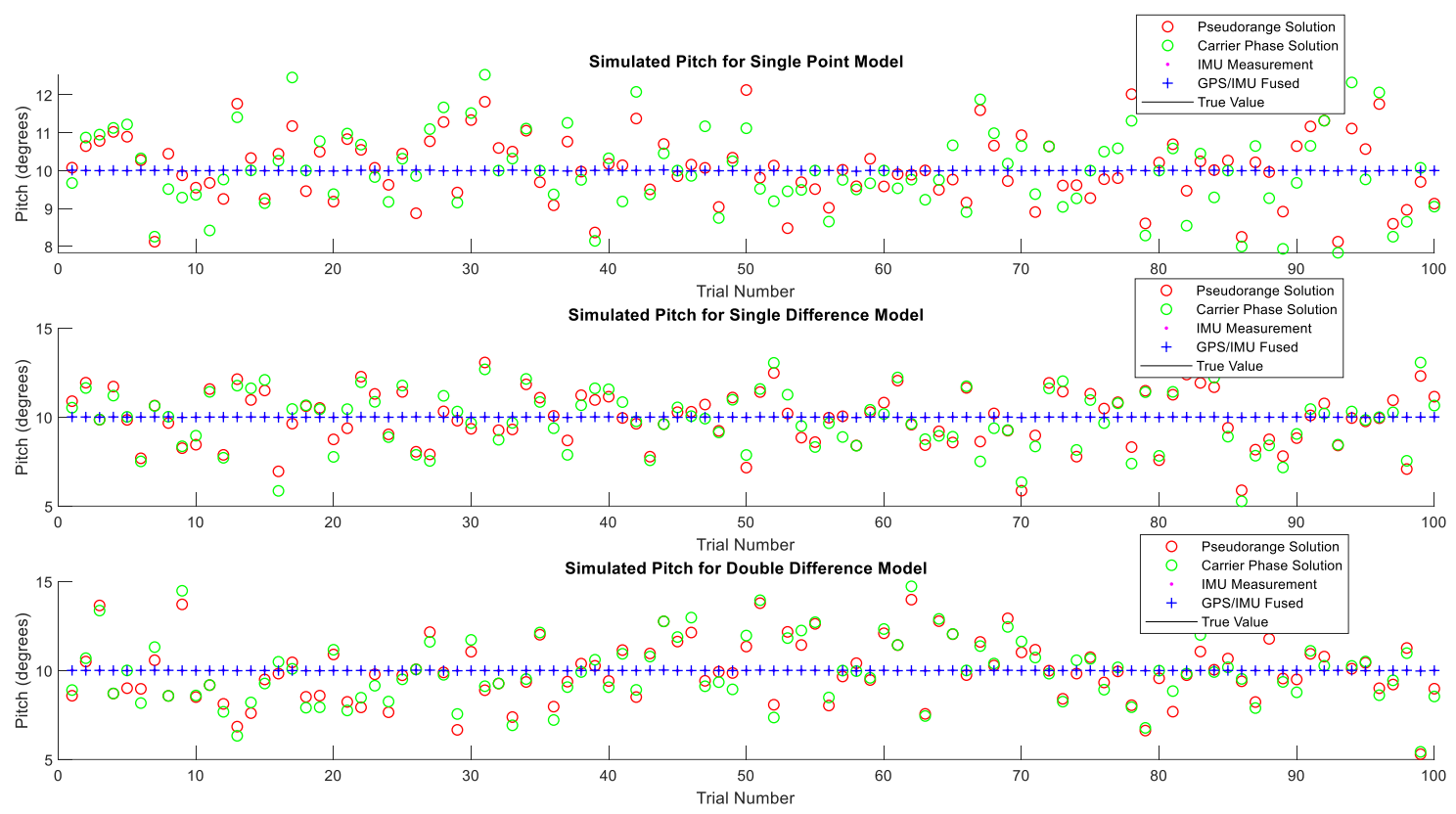

Figure 91: Computed and Measured Pitch for Simulation Set B-7

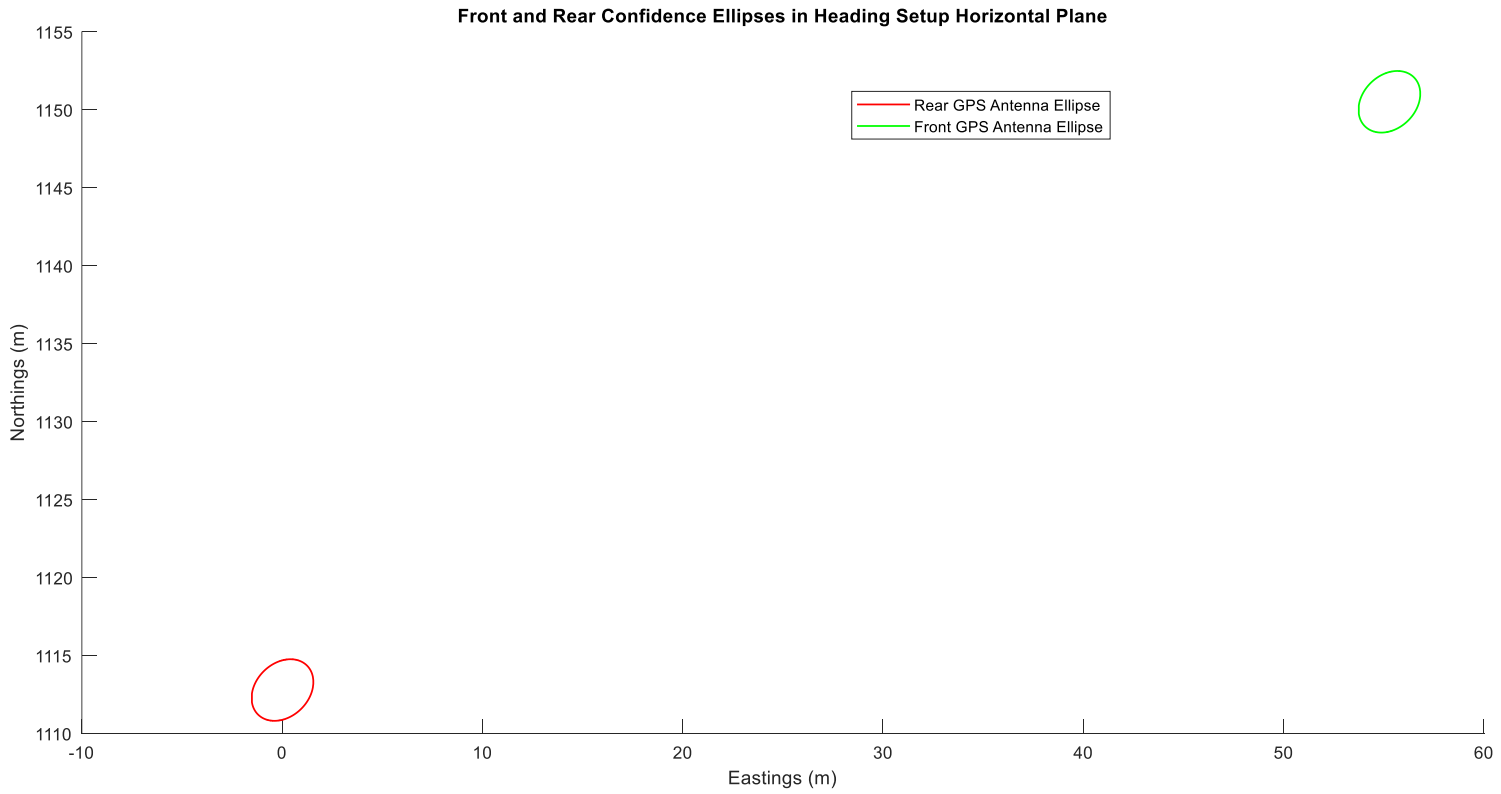

Figure 92: Propagated 95\% Confidence Ellipses for Front and Rear GPS Antennas in Simulated Data Set B-7 
Table 157: Mean Global Error Statistics of Unfused Pitch for Simulation Set B-7

\begin{tabular}{|c|c|c|c|}
\hline Method & $\begin{array}{c}\text { Measured Standard } \\
\text { Deviation } \\
\text { (degrees) }\end{array}$ & $\begin{array}{c}\text { Estimated } \\
\text { Standard } \\
\text { Deviation } \\
\text { (degrees) }\end{array}$ & $\begin{array}{c}\text { Mean RMS } \\
\text { Error } \\
\text { (degrees) }\end{array}$ \\
\hline Single Point GPS (Pseudoranges) & 0.88 & 2.849 & 0.699 \\
\hline Single Point GPS (Carrier Phase) & 1.041 & 0.002 & 0.819 \\
\hline $\begin{array}{c}\text { Single Difference GPS } \\
\text { (Pseudoranges) }\end{array}$ & 1.534 & 2.8 & 1.257 \\
\hline $\begin{array}{c}\text { Single Difference GPS (Carrier } \\
\text { Phase) }\end{array}$ & 1.674 & 0.002 & 1.359 \\
\hline $\begin{array}{c}\text { Double Difference GPS } \\
\text { (Pseudoranges) }\end{array}$ & 1.716 & 2.236 & 1.355 \\
\hline $\begin{array}{c}\text { Double Difference GPS (Carrier } \\
\text { Phase) }\end{array}$ & 1.828 & 0.004 & 1.451 \\
\hline IMU Measurement & 0.007 & - & 0.005 \\
\hline
\end{tabular}

Table 158: Mean Global Error Statistics of Unfused Heading for Simulation Set B-7

\begin{tabular}{|c|c|c|c|}
\hline Method & $\begin{array}{c}\text { Measured Standard } \\
\text { Deviation } \\
\text { (degrees) }\end{array}$ & $\begin{array}{c}\text { Estimated } \\
\text { Standard } \\
\text { Deviation } \\
\text { (degrees) }\end{array}$ & $\begin{array}{c}\text { Mean RMS } \\
\text { Error } \\
\text { (degrees) }\end{array}$ \\
\hline Single Point GPS (Pseudoranges) & 0.947 & 2.323 & 0.702 \\
\hline Single Point GPS (Carrier Phase) & 0.929 & 0.001 & 0.686 \\
\hline $\begin{array}{c}\text { Single Difference GPS } \\
\text { (Pseudoranges) }\end{array}$ & 1.304 & 2.29 & 1.035 \\
\hline $\begin{array}{c}\text { Single Difference GPS (Carrier } \\
\text { Phase) }\end{array}$ & 1.276 & 0.001 & 1.021 \\
\hline $\begin{array}{c}\text { Double Difference GPS } \\
\text { (Pseudoranges) }\end{array}$ & 1.434 & 1.715 & 1.104 \\
\hline $\begin{array}{c}\text { Double Difference GPS (Carrier } \\
\text { Phase) }\end{array}$ & 1.467 & 0.003 & 1.146 \\
\hline IMU Measurement & 0.3 & - & 0.24 \\
\hline
\end{tabular}


Table 159: Mean Global Error Statistics of Fused Pitch for Simulation Set B-7

\begin{tabular}{|c|c|c|c|}
\hline Method & $\begin{array}{c}\text { Measured Standard } \\
\text { Deviation } \\
\text { (degrees) }\end{array}$ & $\begin{array}{c}\text { Estimated } \\
\text { Standard } \\
\text { Deviation } \\
\text { (degrees) }\end{array}$ & $\begin{array}{c}\text { Mean RMS } \\
\text { Error } \\
\text { (degrees) }\end{array}$ \\
\hline Single Point GPS (Pseudoranges) & 0.007 & 0.199 & 0.005 \\
\hline Single Point GPS (Carrier Phase) & 0.804 & 0.001 & 0.634 \\
\hline $\begin{array}{c}\text { Single Difference GPS } \\
\text { (Pseudoranges) }\end{array}$ & 0.008 & 0.198 & 0.007 \\
\hline $\begin{array}{c}\text { Single Difference GPS (Carrier } \\
\text { Phase) }\end{array}$ & 1.327 & 0.001 & 1.067 \\
\hline $\begin{array}{c}\text { Double Difference GPS } \\
\text { (Pseudoranges) }\end{array}$ & 0.008 & 0.197 & 0.007 \\
\hline $\begin{array}{c}\text { Double Difference GPS (Carrier } \\
\text { Phase) }\end{array}$ & 1.121 & 0.002 & 0.888 \\
\hline IMU Measurement & 0.007 & - & 0.005 \\
\hline
\end{tabular}

Table 160: Mean Global Error Statistics of Fused Heading for Simulation Set B-7

\begin{tabular}{|c|c|c|c|}
\hline Method & $\begin{array}{c}\text { Measured Standard } \\
\text { Deviation } \\
\text { (degrees) }\end{array}$ & $\begin{array}{c}\text { Estimated } \\
\text { Standard } \\
\text { Deviation } \\
\text { (degrees) }\end{array}$ & $\begin{array}{c}\text { Mean RMS } \\
\text { Error } \\
\text { (degrees) }\end{array}$ \\
\hline Single Point GPS (Pseudoranges) & 0.283 & 0.591 & 0.223 \\
\hline Single Point GPS (Carrier Phase) & 0.926 & 0.001 & 0.684 \\
\hline $\begin{array}{c}\text { Single Difference GPS } \\
\text { (Pseudoranges) }\end{array}$ & 0.322 & 0.584 & 0.255 \\
\hline $\begin{array}{c}\text { Single Difference GPS (Carrier } \\
\text { Phase) }\end{array}$ & 1.271 & 0.001 & 1.017 \\
\hline $\begin{array}{c}\text { Double Difference GPS } \\
\text { (Pseudoranges) }\end{array}$ & 0.352 & 0.545 & 0.289 \\
\hline $\begin{array}{c}\text { Double Difference GPS (Carrier } \\
\text { Phase) }\end{array}$ & 1.451 & 0.002 & 1.134 \\
\hline IMU Measurement & 0.3 & - & 0.24 \\
\hline
\end{tabular}


Table 161: Success Rates of Reaching Angular Specifications of Unfused Pitch for Simulation Data Set B-7

\begin{tabular}{|c|c|c|c|c|c|c|c|c|}
\hline & \multicolumn{7}{|c|}{ Portion of Trials in Which Target Specification was reached } \\
\hline & $\pm 0.5^{\circ}$ & $\pm 1^{\circ}$ & $\pm 2^{\circ}$ & $\pm 5^{\circ}$ & $\pm 10^{\circ}$ & $\pm 15^{\circ}$ & $\pm 20^{\circ}$ & $\pm 25^{\circ}$ \\
\hline Method & 69 & 85 & 98 & 100 & 100 & 100 & 100 & 100 \\
\hline $\begin{array}{c}\text { Single Point GPS } \\
\text { (Pseudoranges) }\end{array}$ & 68 & 82 & 95 & 100 & 100 & 100 & 100 & 100 \\
\hline $\begin{array}{c}\text { Single Point GPS (Carrier } \\
\text { Phase) }\end{array}$ & 61 & 72 & 93 & 100 & 100 & 100 & 100 & 100 \\
\hline $\begin{array}{c}\text { Single Difference GPS } \\
\text { (Pseudoranges) }\end{array}$ & 62 & 74 & 90 & 100 & 100 & 100 & 100 & 100 \\
\hline $\begin{array}{c}\text { Single Difference GPS } \\
\text { (Carrier Phase) }\end{array}$ & 65 & 73 & 85 & 100 & 100 & 100 & 100 & 100 \\
\hline $\begin{array}{c}\text { Double Difference GPS } \\
\text { (Pseudoranges) }\end{array}$ & 64 & 73 & 85 & 100 & 100 & 100 & 100 & 100 \\
\hline $\begin{array}{c}\text { Double Difference GPS } \\
\text { (Carrier Phase) }\end{array}$ & 100 & 100 & 100 & 100 & 100 & 100 & 100 & 100 \\
\hline IMU Measured & & & & & & & & \\
\hline
\end{tabular}

Table 162: Success Rates of Reaching Angular Specifications of Unfused Heading for Simulation Data Set B-7

\begin{tabular}{|c|c|c|c|c|c|c|c|c|}
\hline & \multicolumn{7}{|c|}{ Portion of Trials in Which Target Specification was reached } \\
\hline & $\pm 0.5^{\circ}$ & $\pm 1^{\circ}$ & $\pm 2^{\circ}$ & $\pm 5^{\circ}$ & $\pm 10^{\circ}$ & $\pm 15^{\circ}$ & $\pm 20^{\circ}$ & $\pm 25^{\circ}$ \\
\hline Method & 75 & 86 & 96 & 100 & 100 & 100 & 100 & 100 \\
\hline $\begin{array}{c}\text { Single Point GPS } \\
\text { (Pseudoranges) }\end{array}$ & 69 & 87 & 98 & 100 & 100 & 100 & 100 & 100 \\
\hline $\begin{array}{c}\text { Single Point GPS (Carrier } \\
\text { Phase) }\end{array}$ & 66 & 84 & 96 & 100 & 100 & 100 & 100 & 100 \\
\hline $\begin{array}{c}\text { Single Difference GPS } \\
\text { (Pseudoranges) }\end{array}$ & 67 & 84 & 98 & 100 & 100 & 100 & 100 & 100 \\
\hline $\begin{array}{c}\text { Single Difference GPS } \\
\text { (Carrier Phase) }\end{array}$ & 64 & 79 & 92 & 100 & 100 & 100 & 100 & 100 \\
\hline $\begin{array}{c}\text { Double Difference GPS } \\
\text { (Pseudoranges) }\end{array}$ & 65 & 76 & 92 & 100 & 100 & 100 & 100 & 100 \\
\hline $\begin{array}{c}\text { Double Difference GPS } \\
\text { (Carrier Phase) }\end{array}$ & 95 & 100 & 100 & 100 & 100 & 100 & 100 & 100 \\
\hline IMU Measured & & & & & & & & \\
\hline
\end{tabular}


Table 163: Success Rates of Reaching Angular Specifications of Fused Pitch for Simulation Data Set B-7

\begin{tabular}{|c|c|c|c|c|c|c|c|c|}
\hline & \multicolumn{7}{|c|}{ Portion of Trials in Which Target Specification was reached } \\
\hline & $\pm 0.5^{\circ}$ & $\pm 1^{\circ}$ & $\pm 2^{\circ}$ & $\pm 5^{\circ}$ & $\pm 10^{\circ}$ & $\pm 15^{\circ}$ & $\pm 20^{\circ}$ & $\pm 25^{\circ}$ \\
\hline Method & 100 & 100 & 100 & 100 & 100 & 100 & 100 & 100 \\
\hline $\begin{array}{c}\text { Single Point GPS } \\
\text { (Pseudoranges) }\end{array}$ & 75 & 91 & 100 & 100 & 100 & 100 & 100 & 100 \\
\hline $\begin{array}{c}\text { Single Point GPS (Carrier } \\
\text { Phase) }\end{array}$ & 100 & 100 & 100 & 100 & 100 & 100 & 100 & 100 \\
\hline $\begin{array}{c}\text { Single Difference GPS } \\
\text { (Pseudoranges) }\end{array}$ & 66 & 77 & 96 & 100 & 100 & 100 & 100 & 100 \\
\hline $\begin{array}{c}\text { Single Difference GPS } \\
\text { (Carrier Phase) }\end{array}$ & 100 & 100 & 100 & 100 & 100 & 100 & 100 & 100 \\
\hline $\begin{array}{c}\text { Double Difference GPS } \\
\text { (Pseudoranges) }\end{array}$ & 71 & 80 & 97 & 100 & 100 & 100 & 100 & 100 \\
\hline $\begin{array}{c}\text { Double Difference GPS } \\
\text { (Carrier Phase) }\end{array}$ & 100 & 100 & 100 & 100 & 100 & 100 & 100 & 100 \\
\hline IMU Measured & & & & & & & & \\
\hline
\end{tabular}

Table 164: Success Rates of Reaching Angular Specifications of Fused Heading for Simulation Data Set B-7

\begin{tabular}{|c|c|c|c|c|c|c|c|c|}
\hline & \multicolumn{7}{|c|}{ Portion of Trials in Which Target Specification was reached } \\
\hline & $\pm 0.5^{\circ}$ & $\pm 1^{\circ}$ & $\pm 2^{\circ}$ & $\pm 5^{\circ}$ & $\pm 10^{\circ}$ & $\pm 15^{\circ}$ & $\pm 20^{\circ}$ & $\pm 25^{\circ}$ \\
\hline Method & 96 & 100 & 100 & 100 & 100 & 100 & 100 & 100 \\
\hline $\begin{array}{c}\text { Single Point GPS } \\
\text { (Pseudoranges) }\end{array}$ & 69 & 87 & 98 & 100 & 100 & 100 & 100 & 100 \\
\hline $\begin{array}{c}\text { Single Point GPS (Carrier } \\
\text { Phase) }\end{array}$ & 92 & 100 & 100 & 100 & 100 & 100 & 100 & 100 \\
\hline $\begin{array}{c}\text { Single Difference GPS } \\
\text { (Pseudoranges) }\end{array}$ & 68 & 84 & 98 & 100 & 100 & 100 & 100 & 100 \\
\hline $\begin{array}{c}\text { Single Difference GPS } \\
\text { (Carrier Phase) }\end{array}$ & 91 & 100 & 100 & 100 & 100 & 100 & 100 & 100 \\
\hline $\begin{array}{c}\text { Double Difference GPS } \\
\text { (Pseudoranges) }\end{array}$ & 65 & 76 & 92 & 100 & 100 & 100 & 100 & 100 \\
\hline $\begin{array}{c}\text { Double Difference GPS } \\
\text { (Carrier Phase) }\end{array}$ & 95 & 100 & 100 & 100 & 100 & 100 & 100 & 100 \\
\hline IMU Measured & & & & & & & & \\
\hline
\end{tabular}


Table 165: Pitch / Heading Correlation Coefficients for Each Computation Method in Simulation Data Set B-7

\begin{tabular}{|c|c|c|}
\hline & \multicolumn{2}{|c|}{ Pitch / Heading Correlation Coefficient } \\
\hline Method & Unfused & Fused \\
\hline $\begin{array}{c}\text { Single Point GPS } \\
\text { (Pseudoranges) }\end{array}$ & 0.876989 & 0.26835 \\
\hline $\begin{array}{c}\text { Single Difference GPS } \\
\text { (Pseudoranges) }\end{array}$ & 0.855939 & 0.209688 \\
\hline $\begin{array}{c}\text { Double Difference GPS } \\
\text { (Pseudoranges) }\end{array}$ & 0.778497 & 0.229756 \\
\hline
\end{tabular}

\section{Set B-8: 90 m Dual Antenna Separation}

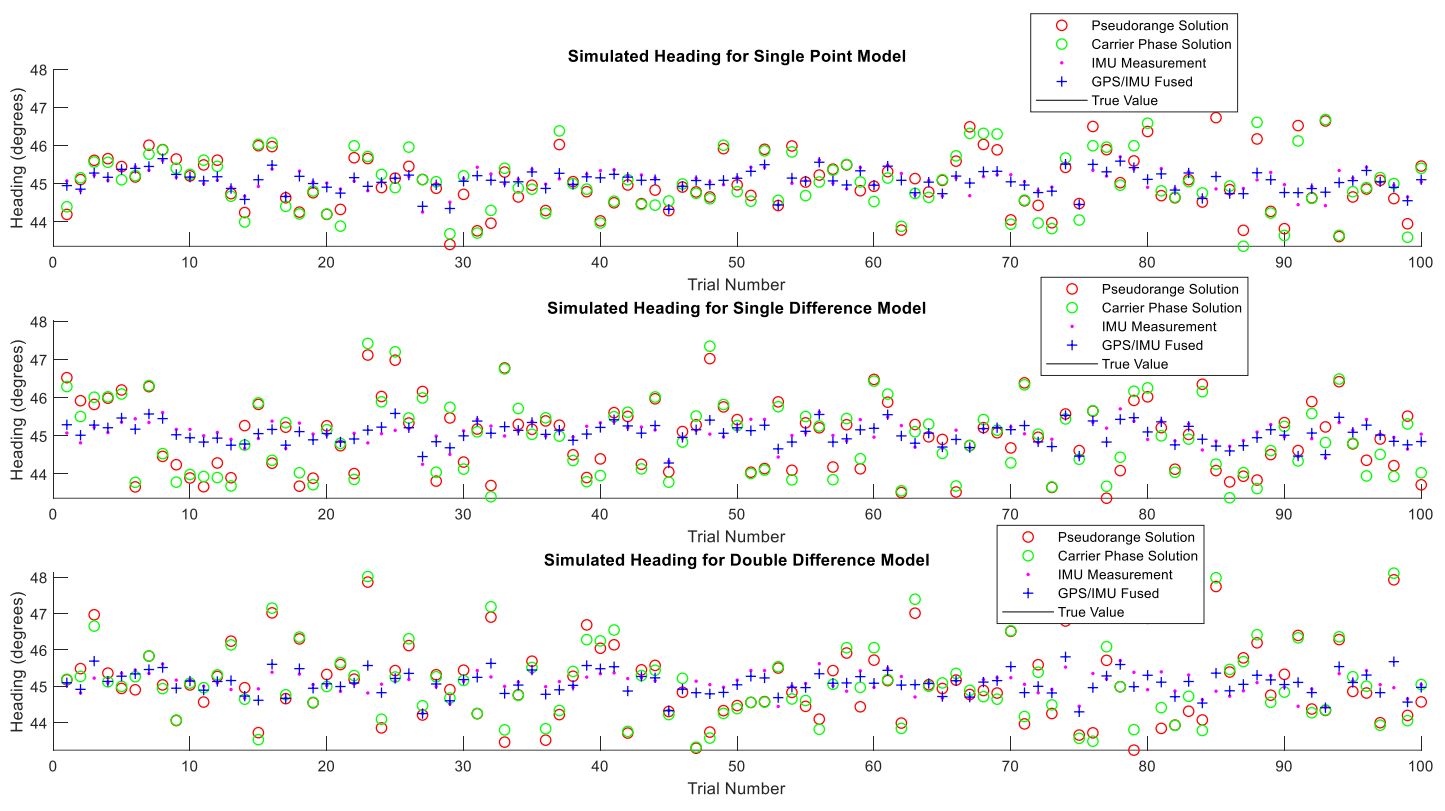

Figure 93: Computed and Measured Headings for Simulation Set B-8 


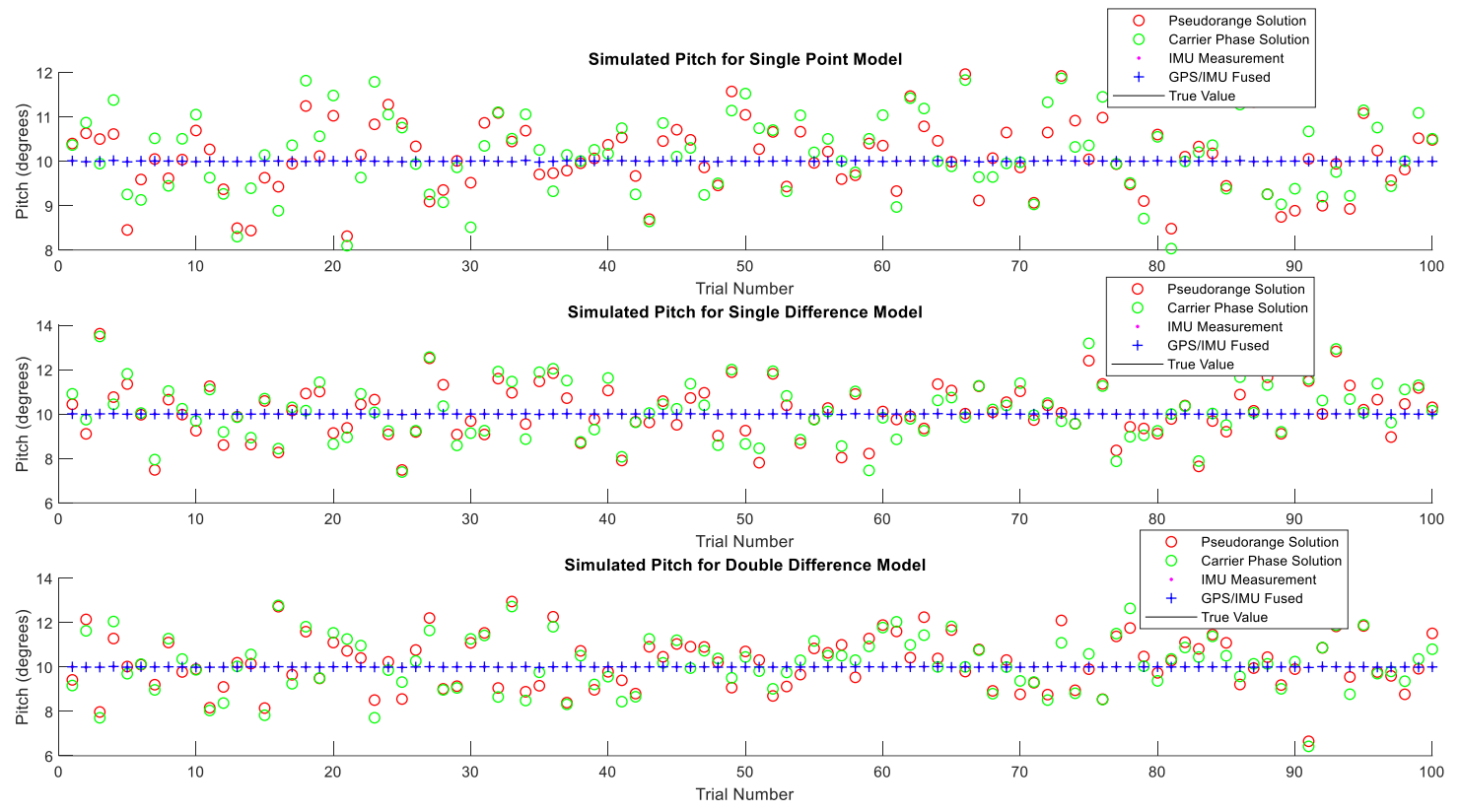

Figure 94: Computed and Measured Pitch for Simulation Set B-8

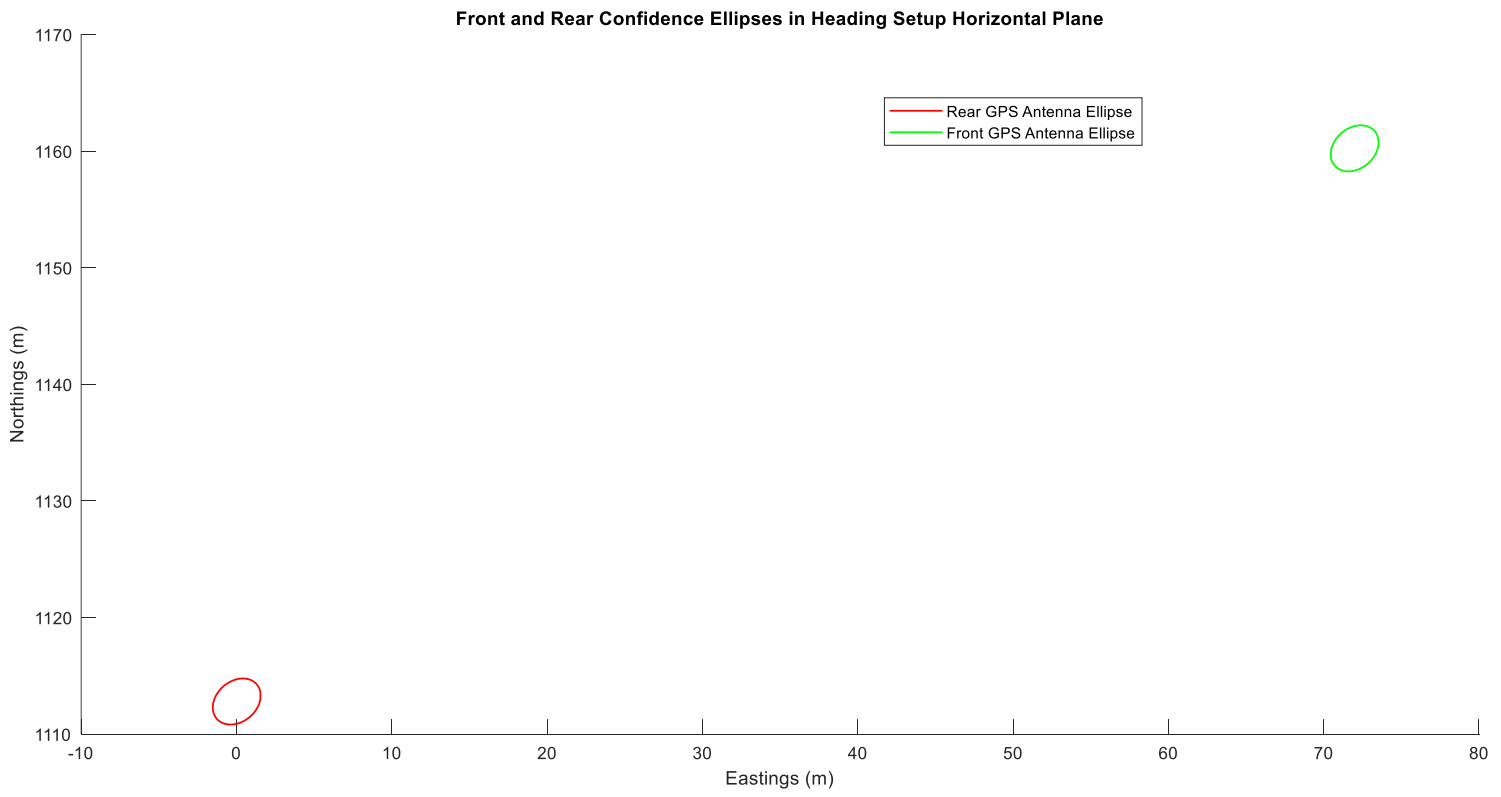

Figure 95: Propagated 95\% Confidence Ellipses for Front and Rear GPS Antennas in Simulated Data Set B-8 
Table 166: Mean Global Error Statistics of Unfused Pitch for Simulation Set B-8

\begin{tabular}{|c|c|c|c|}
\hline Method & $\begin{array}{c}\text { Measured Standard } \\
\text { Deviation } \\
\text { (degrees) }\end{array}$ & $\begin{array}{c}\text { Estimated } \\
\text { Standard } \\
\text { Deviation } \\
\text { (degrees) }\end{array}$ & $\begin{array}{c}\text { Mean RMS } \\
\text { Error } \\
\text { (degrees) }\end{array}$ \\
\hline Single Point GPS (Pseudoranges) & 0.79 & 2.217 & 0.634 \\
\hline Single Point GPS (Carrier Phase) & 0.885 & 0.002 & 0.73 \\
\hline $\begin{array}{c}\text { Single Difference GPS } \\
\text { (Pseudoranges) }\end{array}$ & 1.213 & 2.217 & 0.968 \\
\hline $\begin{array}{c}\text { Single Difference GPS (Carrier } \\
\text { Phase) }\end{array}$ & 1.278 & 0.002 & 1.023 \\
\hline $\begin{array}{c}\text { Double Difference GPS } \\
\text { (Pseudoranges) }\end{array}$ & 1.193 & 1.784 & 0.989 \\
\hline $\begin{array}{c}\text { Double Difference GPS (Carrier } \\
\text { Phase) }\end{array}$ & 1.225 & 0.004 & 0.987 \\
\hline IMU Measurement & 0.007 & - & 0.006 \\
\hline
\end{tabular}

Table 167: Mean Global Error Statistics of Unfused Heading for Simulation Set B-8

\begin{tabular}{|c|c|c|c|}
\hline Method & $\begin{array}{c}\text { Measured Standard } \\
\text { Deviation } \\
\text { (degrees) }\end{array}$ & $\begin{array}{c}\text { Estimated } \\
\text { Standard } \\
\text { Deviation } \\
\text { (degrees) }\end{array}$ & $\begin{array}{c}\text { Mean RMS } \\
\text { Error } \\
\text { (degrees) }\end{array}$ \\
\hline Single Point GPS (Pseudoranges) & 0.74 & 1.808 & 0.594 \\
\hline Single Point GPS (Carrier Phase) & 0.8 & 0.001 & 0.634 \\
\hline $\begin{array}{c}\text { Single Difference GPS } \\
\text { (Pseudoranges) }\end{array}$ & 0.927 & 1.827 & 0.782 \\
\hline $\begin{array}{c}\text { Single Difference GPS (Carrier } \\
\text { Phase) }\end{array}$ & 0.972 & 0.001 & 0.826 \\
\hline $\begin{array}{c}\text { Double Difference GPS } \\
\text { (Pseudoranges) }\end{array}$ & 1.032 & 1.377 & 0.808 \\
\hline $\begin{array}{c}\text { Double Difference GPS (Carrier } \\
\text { Phase) }\end{array}$ & 1.053 & 0.003 & 0.799 \\
\hline IMU Measurement & 0.293 & - & 0.237 \\
\hline
\end{tabular}


Table 168: Mean Global Error Statistics of Fused Pitch for Simulation Set B-8

\begin{tabular}{|c|c|c|c|}
\hline Method & $\begin{array}{c}\text { Measured Standard } \\
\text { Deviation } \\
\text { (degrees) }\end{array}$ & $\begin{array}{c}\text { Estimated } \\
\text { Standard } \\
\text { Deviation } \\
\text { (degrees) }\end{array}$ & $\begin{array}{c}\text { Mean RMS } \\
\text { Error } \\
\text { (degrees) }\end{array}$ \\
\hline Single Point GPS (Pseudoranges) & 0.008 & 0.197 & 0.006 \\
\hline Single Point GPS (Carrier Phase) & 0.721 & 0.001 & 0.594 \\
\hline $\begin{array}{c}\text { Single Difference GPS } \\
\text { (Pseudoranges) }\end{array}$ & 0.008 & 0.197 & 0.006 \\
\hline $\begin{array}{c}\text { Single Difference GPS (Carrier } \\
\text { Phase) }\end{array}$ & 1.033 & 0.001 & 0.829 \\
\hline $\begin{array}{c}\text { Double Difference GPS } \\
\text { (Pseudoranges) }\end{array}$ & 0.008 & 0.195 & 0.007 \\
\hline $\begin{array}{c}\text { Double Difference GPS (Carrier } \\
\text { Phase) }\end{array}$ & 0.816 & 0.002 & 0.654 \\
\hline IMU Measurement & 0.007 & - & 0.006 \\
\hline
\end{tabular}

Table 169: Mean Global Error Statistics of Fused Heading for Simulation Set B-8

\begin{tabular}{|c|c|c|c|}
\hline Method & $\begin{array}{c}\text { Measured Standard } \\
\text { Deviation } \\
\text { (degrees) }\end{array}$ & $\begin{array}{c}\text { Estimated } \\
\text { Standard } \\
\text { Deviation } \\
\text { (degrees) }\end{array}$ & $\begin{array}{c}\text { Mean RMS } \\
\text { Error } \\
\text { (degrees) }\end{array}$ \\
\hline Single Point GPS (Pseudoranges) & 0.278 & 0.469 & 0.225 \\
\hline Single Point GPS (Carrier Phase) & 0.798 & 0 & 0.632 \\
\hline $\begin{array}{c}\text { Single Difference GPS } \\
\text { (Pseudoranges) }\end{array}$ & 0.277 & 0.475 & 0.229 \\
\hline $\begin{array}{c}\text { Single Difference GPS (Carrier } \\
\text { Phase) }\end{array}$ & 0.969 & 0 & 0.824 \\
\hline $\begin{array}{c}\text { Double Difference GPS } \\
\text { (Pseudoranges) }\end{array}$ & 0.32 & 0.444 & 0.258 \\
\hline $\begin{array}{c}\text { Double Difference GPS (Carrier } \\
\text { Phase) }\end{array}$ & 1.045 & 0.001 & 0.793 \\
\hline IMU Measurement & 0.293 & - & 0.237 \\
\hline
\end{tabular}


Table 170: Success Rates of Reaching Angular Specifications of Unfused Pitch for Simulation Data Set B-8

\begin{tabular}{|c|c|c|c|c|c|c|c|c|}
\hline & \multicolumn{7}{|c|}{ Portion of Trials in Which Target Specification was reached } \\
\hline & $\pm 0.5^{\circ}$ & $\pm 1^{\circ}$ & $\pm 2^{\circ}$ & $\pm 5^{\circ}$ & $\pm 10^{\circ}$ & $\pm 15^{\circ}$ & $\pm 20^{\circ}$ & $\pm 25^{\circ}$ \\
\hline Method & 73 & 93 & 99 & 100 & 100 & 100 & 100 & 100 \\
\hline $\begin{array}{c}\text { Single Point GPS } \\
\text { (Pseudoranges) }\end{array}$ & 65 & 88 & 98 & 100 & 100 & 100 & 100 & 100 \\
\hline $\begin{array}{c}\text { Single Point GPS (Carrier } \\
\text { Phase) }\end{array}$ & 64 & 80 & 97 & 100 & 100 & 100 & 100 & 100 \\
\hline $\begin{array}{c}\text { Single Difference GPS } \\
\text { (Pseudoranges) }\end{array}$ & 62 & 80 & 96 & 100 & 100 & 100 & 100 & 100 \\
\hline $\begin{array}{c}\text { Single Difference GPS } \\
\text { (Carrier Phase) }\end{array}$ & 60 & 76 & 96 & 100 & 100 & 100 & 100 & 100 \\
\hline $\begin{array}{c}\text { Double Difference GPS } \\
\text { (Pseudoranges) }\end{array}$ & 58 & 71 & 95 & 100 & 100 & 100 & 100 & 100 \\
\hline $\begin{array}{c}\text { Double Difference GPS } \\
\text { (Carrier Phase) }\end{array}$ & 100 & 100 & 100 & 100 & 100 & 100 & 100 & 100 \\
\hline
\end{tabular}

Table 171: Success Rates of Reaching Angular Specifications of Unfused Heading for Simulation Data Set B-8

\begin{tabular}{|c|c|c|c|c|c|c|c|c|}
\hline & \multicolumn{7}{|c|}{ Portion of Trials in Which Target Specification was reached } \\
\hline Method & $\pm 0.5^{\circ}$ & $\pm 1^{\circ}$ & $\pm 2^{\circ}$ & $\pm 5^{\circ}$ & $\pm 10^{\circ}$ & $\pm 15^{\circ}$ & $\pm 20^{\circ}$ & $\pm 25^{\circ}$ \\
\hline $\begin{array}{c}\text { Single Point GPS } \\
\text { (Pseudoranges) }\end{array}$ & 78 & 94 & 98 & 100 & 100 & 100 & 100 & 100 \\
\hline $\begin{array}{c}\text { Single Point GPS (Carrier } \\
\text { Phase) }\end{array}$ & 81 & 94 & 99 & 100 & 100 & 100 & 100 & 100 \\
\hline $\begin{array}{c}\text { Single Difference GPS } \\
\text { (Pseudoranges) }\end{array}$ & 69 & 83 & 97 & 100 & 100 & 100 & 100 & 100 \\
\hline $\begin{array}{c}\text { Single Difference GPS } \\
\text { (Carrier Phase) }\end{array}$ & 64 & 81 & 97 & 100 & 100 & 100 & 100 & 100 \\
\hline $\begin{array}{c}\text { Double Difference GPS } \\
\text { (Pseudoranges) }\end{array}$ & 63 & 81 & 97 & 100 & 100 & 100 & 100 & 100 \\
\hline $\begin{array}{c}\text { Double Difference GPS } \\
\text { (Carrier Phase) }\end{array}$ & 68 & 81 & 94 & 100 & 100 & 100 & 100 & 100 \\
\hline IMU Measured & 92 & 100 & 100 & 100 & 100 & 100 & 100 & 100 \\
\hline
\end{tabular}


Table 172: Success Rates of Reaching Angular Specifications of Fused Pitch for Simulation Data Set B-8

\begin{tabular}{|c|c|c|c|c|c|c|c|c|}
\hline & \multicolumn{7}{|c|}{ Portion of Trials in Which Target Specification was reached } \\
\hline & $\pm 0.5^{\circ}$ & $\pm 1^{\circ}$ & $\pm 2^{\circ}$ & $\pm 5^{\circ}$ & $\pm 10^{\circ}$ & $\pm 15^{\circ}$ & $\pm 20^{\circ}$ & $\pm 25^{\circ}$ \\
\hline Method & 100 & 100 & 100 & 100 & 100 & 100 & 100 & 100 \\
\hline $\begin{array}{c}\text { Single Point GPS } \\
\text { (Pseudoranges) }\end{array}$ & 75 & 91 & 100 & 100 & 100 & 100 & 100 & 100 \\
\hline $\begin{array}{c}\text { Single Point GPS (Carrier } \\
\text { Phase) }\end{array}$ & 100 & 100 & 100 & 100 & 100 & 100 & 100 & 100 \\
\hline $\begin{array}{c}\text { Single Difference GPS } \\
\text { (Pseudoranges) }\end{array}$ & 72 & 87 & 99 & 100 & 100 & 100 & 100 & 100 \\
\hline $\begin{array}{c}\text { Single Difference GPS } \\
\text { (Carrier Phase) }\end{array}$ & 100 & 100 & 100 & 100 & 100 & 100 & 100 & 100 \\
\hline $\begin{array}{c}\text { Double Difference GPS } \\
\text { (Pseudoranges) }\end{array}$ & 65 & 90 & 100 & 100 & 100 & 100 & 100 & 100 \\
\hline $\begin{array}{c}\text { Double Difference GPS } \\
\text { (Carrier Phase) }\end{array}$ & 100 & 100 & 100 & 100 & 100 & 100 & 100 & 100 \\
\hline
\end{tabular}

Table 173: Success Rates of Reaching Angular Specifications of Fused Heading for Simulation Data Set B-8

\begin{tabular}{|c|c|c|c|c|c|c|c|c|}
\hline & \multicolumn{7}{|c|}{ Portion of Trials in Which Target Specification was reached } \\
\hline Method & $\pm 0.5^{\circ}$ & $\pm 1^{\circ}$ & $\pm 2^{\circ}$ & $\pm 5^{\circ}$ & $\pm 10^{\circ}$ & $\pm 15^{\circ}$ & $\pm 20^{\circ}$ & $\pm 25^{\circ}$ \\
\hline $\begin{array}{c}\text { Single Point GPS } \\
\text { (Pseudoranges) }\end{array}$ & 92 & 100 & 100 & 100 & 100 & 100 & 100 & 100 \\
\hline $\begin{array}{c}\text { Single Point GPS (Carrier } \\
\text { Phase) }\end{array}$ & 81 & 94 & 99 & 100 & 100 & 100 & 100 & 100 \\
\hline $\begin{array}{c}\text { Single Difference GPS } \\
\text { (Pseudoranges) }\end{array}$ & 94 & 100 & 100 & 100 & 100 & 100 & 100 & 100 \\
\hline $\begin{array}{c}\text { Single Difference GPS } \\
\text { (Carrier Phase) }\end{array}$ & 64 & 81 & 97 & 100 & 100 & 100 & 100 & 100 \\
\hline $\begin{array}{c}\text { Double Difference GPS } \\
\text { (Pseudoranges) }\end{array}$ & 92 & 100 & 100 & 100 & 100 & 100 & 100 & 100 \\
\hline $\begin{array}{c}\text { Double Difference GPS } \\
\text { (Carrier Phase) }\end{array}$ & 68 & 81 & 94 & 100 & 100 & 100 & 100 & 100 \\
\hline IMU Measured & 92 & 100 & 100 & 100 & 100 & 100 & 100 & 100 \\
\hline
\end{tabular}


Table 174: Pitch / Heading Correlation Coefficients for Each Computation Method in Simulation Data Set B-8

\begin{tabular}{|c|c|c|}
\hline & \multicolumn{2}{|c|}{ Pitch / Heading Correlation Coefficient } \\
\hline Method & Unfused & Fused \\
\hline $\begin{array}{c}\text { Single Point GPS } \\
\text { (Pseudoranges) }\end{array}$ & 0.863665 & 0.28378 \\
\hline $\begin{array}{c}\text { Single Difference GPS } \\
\text { (Pseudoranges) }\end{array}$ & 0.876758 & 0.3122 \\
\hline $\begin{array}{c}\text { Double Difference GPS } \\
\text { (Pseudoranges) }\end{array}$ & 0.776088 & 0.238242 \\
\hline
\end{tabular}

\section{Appendix C: 3 m Pseudorange Noise Standard}

\section{Deviation Pitch and Heading Simulation Results}

\section{Set C-1 1 m Dual Antenna Separation}

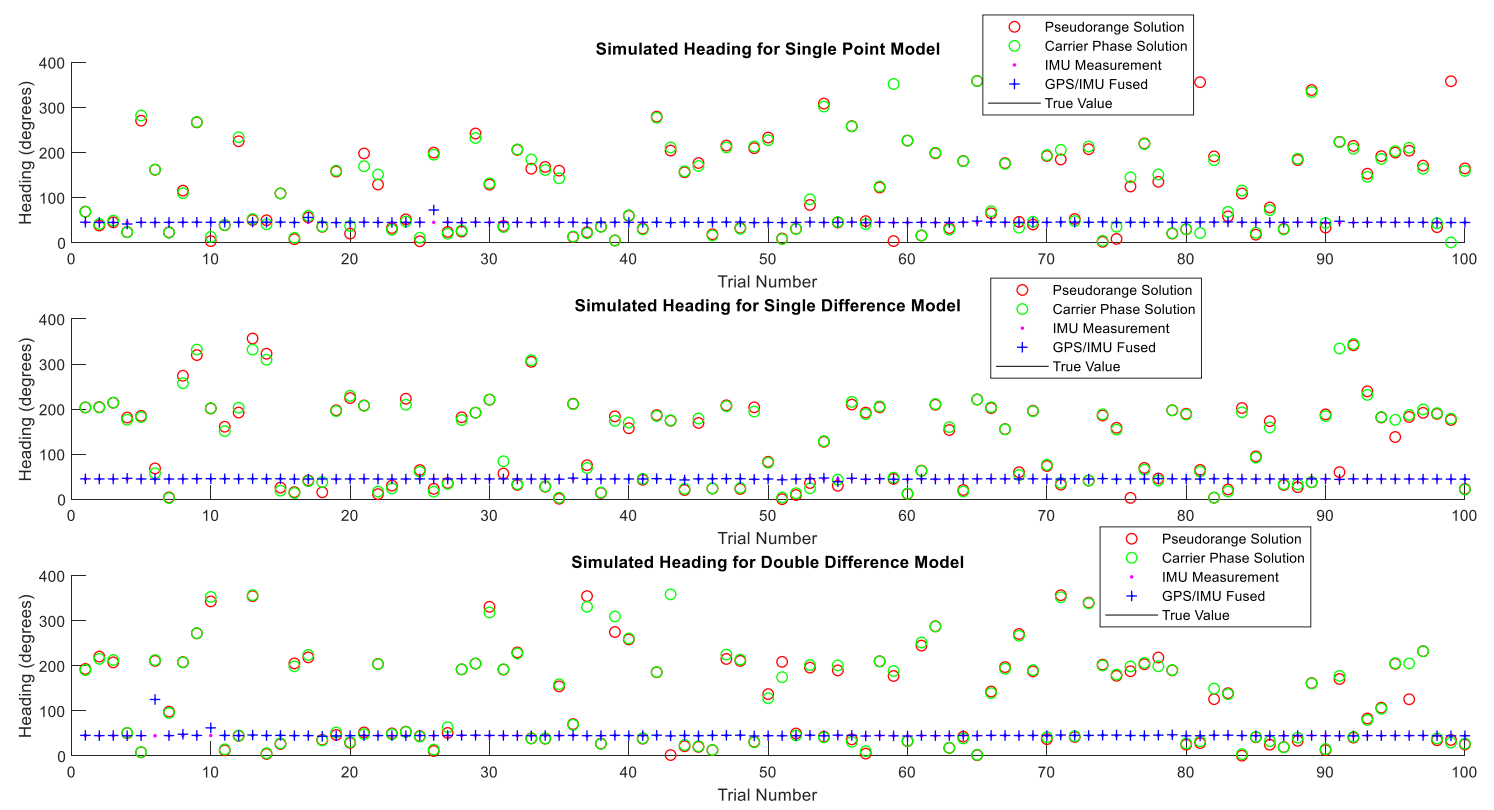

Figure 96: Computed and Measured Headings for Simulation Set C-1 


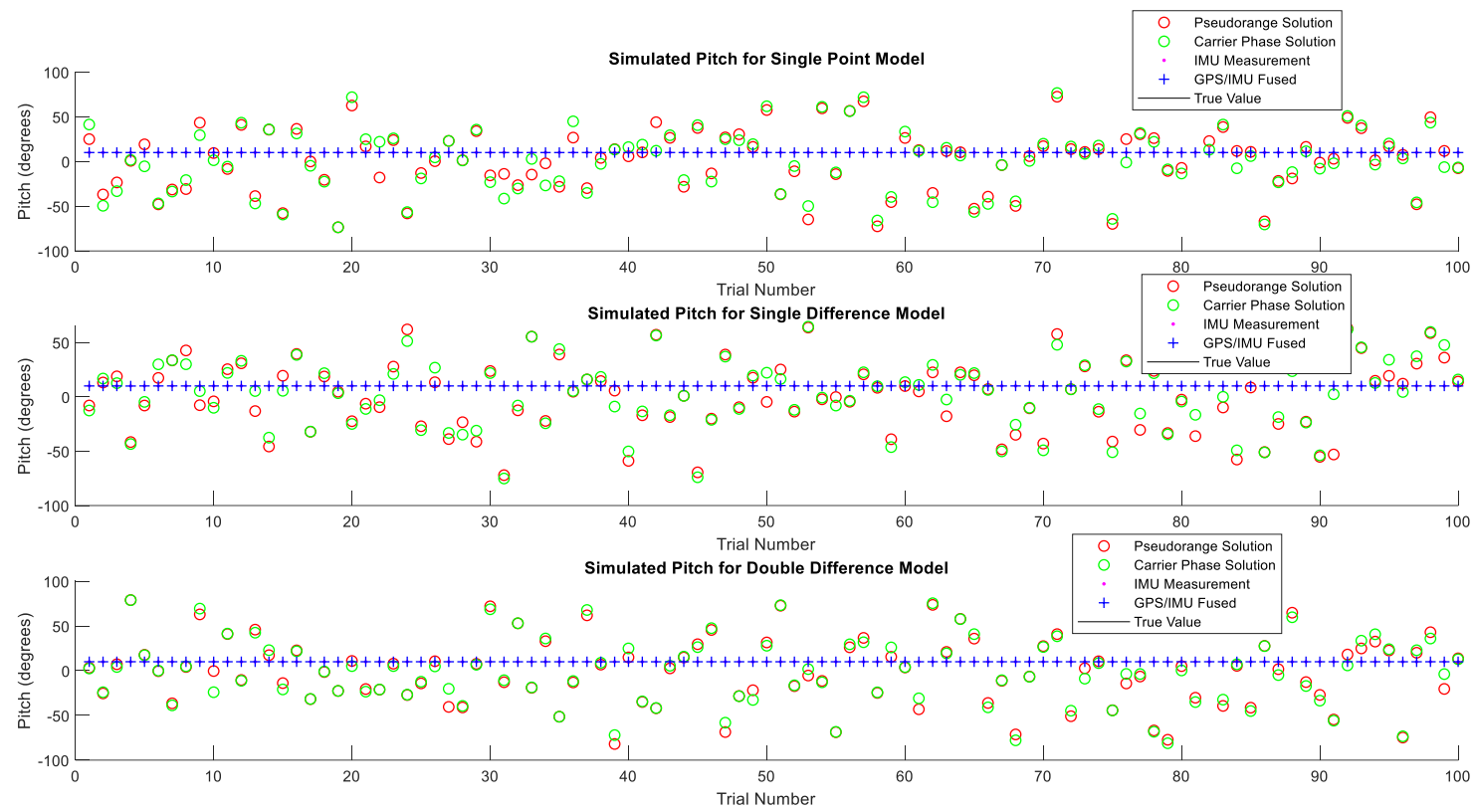

Figure 97: Computed and Measured Headings for Simulation Set C-1 


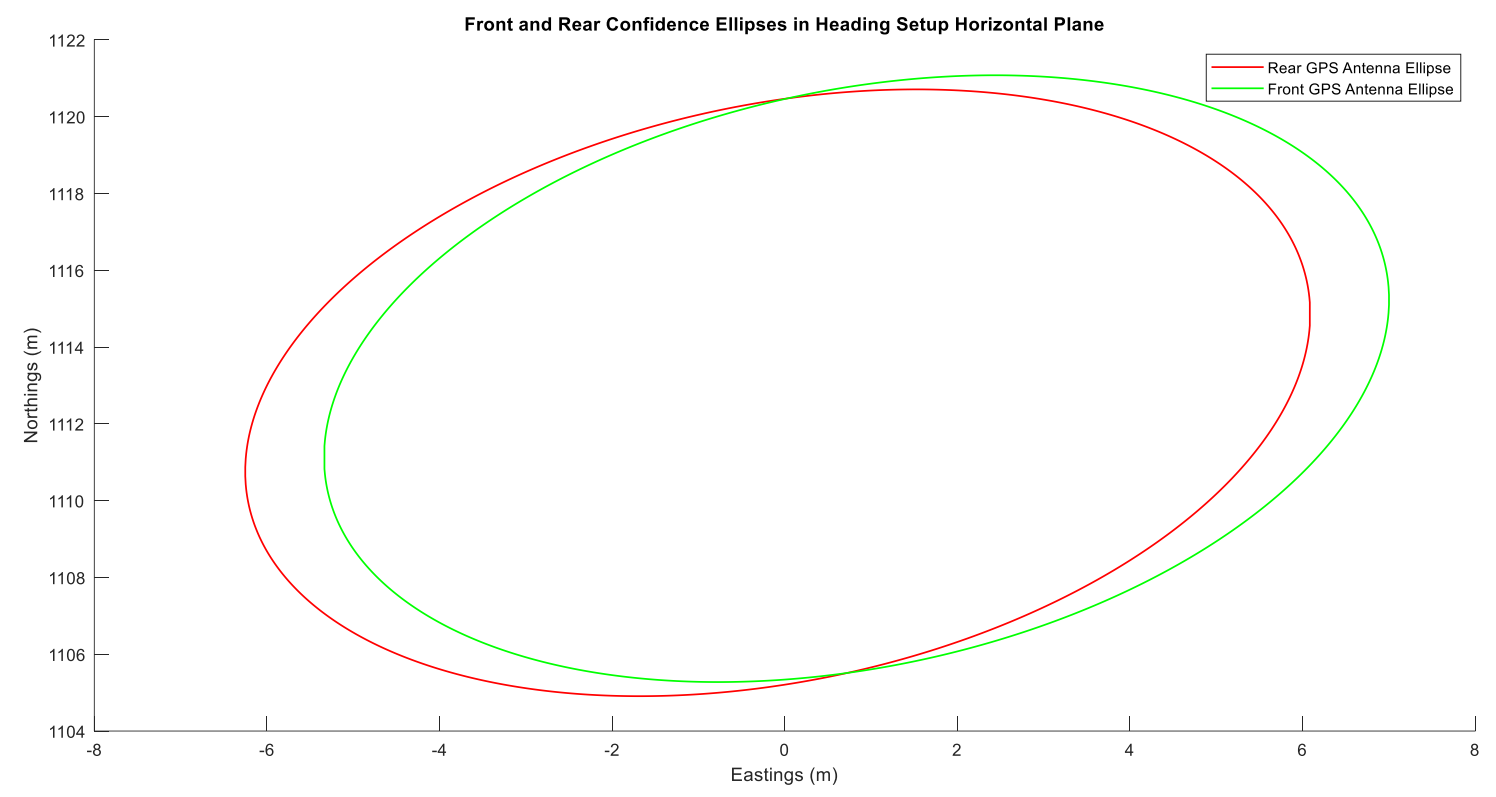

Figure 98: Propagated 95\% Confidence Ellipses for Front and Rear GPS Antennas in Simulated Data Set C-1

Table 175: Mean Global Error Statistics of Unfused Pitch for Simulation Set C-1

\begin{tabular}{|c|c|c|c|}
\hline Method & $\begin{array}{c}\text { Measured Standard } \\
\text { Deviation } \\
\text { (degrees) }\end{array}$ & $\begin{array}{c}\text { Estimated } \\
\text { Standard } \\
\text { Deviation } \\
\text { (degrees) }\end{array}$ & $\begin{array}{c}\text { Mean RMS } \\
\text { Error } \\
\text { (degrees) }\end{array}$ \\
\hline Single Point GPS (Pseudoranges) & 34.368 & 317.315 & 27.65 \\
\hline Single Point GPS (Carrier Phase) & 35.132 & 0.163 & 29.164 \\
\hline $\begin{array}{c}\text { Single Difference GPS } \\
\text { (Pseudoranges) }\end{array}$ & 32.066 & 191.936 & 26.756 \\
\hline $\begin{array}{c}\text { Single Difference GPS (Carrier } \\
\text { Phase) }\end{array}$ & 31.344 & 0.111 & 25.78 \\
\hline $\begin{array}{c}\text { Double Difference GPS } \\
\text { (Pseudoranges) }\end{array}$ & 37.743 & 116.399 & 31.284 \\
\hline $\begin{array}{c}\text { Double Difference GPS (Carrier } \\
\text { Phase) }\end{array}$ & 37.634 & 0.135 & 31.608 \\
\hline IMU Measurement & 0.006 & - & 0.005 \\
\hline
\end{tabular}


Table 176: Mean Global Error Statistics of Unfused Heading for Simulation Set C-1

\begin{tabular}{|c|c|c|c|}
\hline Method & $\begin{array}{c}\text { Measured Standard } \\
\text { Deviation } \\
\text { (degrees) }\end{array}$ & $\begin{array}{c}\text { Estimated } \\
\text { Standard } \\
\text { Deviation } \\
\text { (degrees) }\end{array}$ & $\begin{array}{c}\text { Mean RMS } \\
\text { Error } \\
\text { (degrees) }\end{array}$ \\
\hline Single Point GPS (Pseudoranges) & 96.937 & 2166.759 & 73.757 \\
\hline Single Point GPS (Carrier Phase) & 93.557 & 0.573 & 73.619 \\
\hline $\begin{array}{c}\text { Single Difference GPS } \\
\text { (Pseudoranges) }\end{array}$ & 95.091 & 959.116 & 84.036 \\
\hline $\begin{array}{c}\text { Single Difference GPS (Carrier } \\
\text { Phase) }\end{array}$ & 97.803 & 0.352 & 84.817 \\
\hline $\begin{array}{c}\text { Double Difference GPS } \\
\text { (Pseudoranges) }\end{array}$ & 102.56 & 581.813 & 76.231 \\
\hline $\begin{array}{c}\text { Double Difference GPS (Carrier } \\
\text { Phase) }\end{array}$ & 104.158 & 0.587 & 76.725 \\
\hline IMU Measurement & 0.34 & - & 0.264 \\
\hline
\end{tabular}

Table 177: Mean Global Error Statistics of Fused Pitch for Simulation Set C-1

\begin{tabular}{|c|c|c|c|}
\hline Method & $\begin{array}{c}\text { Measured Standard } \\
\text { Deviation } \\
\text { (degrees) }\end{array}$ & $\begin{array}{c}\text { Estimated } \\
\text { Standard } \\
\text { Deviation } \\
\text { (degrees) }\end{array}$ & $\begin{array}{c}\text { Mean RMS } \\
\text { Error } \\
\text { (degrees) }\end{array}$ \\
\hline Single Point GPS (Pseudoranges) & 0.023 & 0.2 & 0.008 \\
\hline Single Point GPS (Carrier Phase) & 3.506 & 0.059 & 2.449 \\
\hline $\begin{array}{c}\text { Single Difference GPS } \\
\text { (Pseudoranges) }\end{array}$ & 0.025 & 0.2 & 0.009 \\
\hline $\begin{array}{c}\text { Single Difference GPS (Carrier } \\
\text { Phase) }\end{array}$ & 5.641 & 0.044 & 3.392 \\
\hline $\begin{array}{c}\text { Double Difference GPS } \\
\text { (Pseudoranges) }\end{array}$ & 0.013 & 0.2 & 0.009 \\
\hline $\begin{array}{c}\text { Double Difference GPS (Carrier } \\
\text { Phase) }\end{array}$ & 9.263 & 0.052 & 4.428 \\
\hline IMU Measurement & 0.006 & - & 0.005 \\
\hline
\end{tabular}


Table 178: Mean Global Error Statistics of Fused Heading for Simulation Set C-1

\begin{tabular}{|c|c|c|c|}
\hline Method & $\begin{array}{c}\text { Measured Standard } \\
\text { Deviation } \\
\text { (degrees) }\end{array}$ & $\begin{array}{c}\text { Estimated } \\
\text { Standard } \\
\text { Deviation } \\
\text { (degrees) }\end{array}$ & $\begin{array}{c}\text { Mean RMS } \\
\text { Error } \\
\text { (degrees) }\end{array}$ \\
\hline Single Point GPS (Pseudoranges) & 2.989 & 8.513 & 0.729 \\
\hline Single Point GPS (Carrier Phase) & 66.968 & 0.203 & 47.943 \\
\hline $\begin{array}{c}\text { Single Difference GPS } \\
\text { (Pseudoranges) }\end{array}$ & 0.823 & 8.644 & 0.417 \\
\hline $\begin{array}{c}\text { Single Difference GPS (Carrier } \\
\text { Phase) }\end{array}$ & 78.956 & 0.142 & 65.154 \\
\hline $\begin{array}{c}\text { Double Difference GPS } \\
\text { (Pseudoranges) }\end{array}$ & 8.182 & 8.481 & 1.347 \\
\hline $\begin{array}{c}\text { Double Difference GPS (Carrier } \\
\text { Phase) }\end{array}$ & 59.943 & 0.245 & 48.695 \\
\hline IMU Measurement & 0.34 & - & 0.264 \\
\hline
\end{tabular}

Table 179: Success Rates of Reaching Angular Specifications of Unfused Pitch for Simulation Data Set C-1

\begin{tabular}{|c|c|c|c|c|c|c|c|c|}
\hline & \multicolumn{7}{|c|}{ Portion of Trials in Which Target Specification was reached } \\
\hline Method & $\pm 0.5^{\circ}$ & $\pm 1^{\circ}$ & $\pm 2^{\circ}$ & $\pm 5^{\circ}$ & $\pm 10^{\circ}$ & $\pm 15^{\circ}$ & $\pm 20^{\circ}$ & $\pm 25^{\circ}$ \\
\hline $\begin{array}{c}\text { Single Point GPS } \\
\text { (Pseudoranges) }\end{array}$ & 55 & 57 & 60 & 64 & 70 & 74 & 80 & 83 \\
\hline $\begin{array}{c}\text { Single Point GPS (Carrier } \\
\text { Phase) }\end{array}$ & 58 & 58 & 61 & 63 & 70 & 77 & 80 & 83 \\
\hline $\begin{array}{c}\text { Single Difference GPS } \\
\text { (Pseudoranges) }\end{array}$ & 58 & 58 & 58 & 64 & 72 & 77 & 82 & 87 \\
\hline $\begin{array}{c}\text { Single Difference GPS } \\
\text { (Carrier Phase) }\end{array}$ & 56 & 57 & 57 & 60 & 66 & 76 & 81 & 86 \\
\hline $\begin{array}{c}\text { Double Difference GPS } \\
\text { (Pseudoranges) }\end{array}$ & 61 & 64 & 64 & 67 & 71 & 76 & 80 & 83 \\
\hline $\begin{array}{c}\text { Double Difference GPS } \\
\text { (Carrier Phase) }\end{array}$ & 65 & 65 & 65 & 66 & 69 & 74 & 80 & 82 \\
\hline IMU Measured & 100 & 100 & 100 & 100 & 100 & 100 & 100 & 100 \\
\hline
\end{tabular}


Table 180: Success Rates of Reaching Angular Specifications of Unfused Heading for Simulation Data Set C-1

\begin{tabular}{|c|c|c|c|c|c|c|c|c|}
\hline & \multicolumn{7}{|c|}{ Portion of Trials in Which Target Specification was reached } \\
\hline Method & $\pm 0.5^{\circ}$ & $\pm 1^{\circ}$ & $\pm 2^{\circ}$ & $\pm 5^{\circ}$ & $\pm 10^{\circ}$ & $\pm 15^{\circ}$ & $\pm 20^{\circ}$ & $\pm 25^{\circ}$ \\
\hline $\begin{array}{c}\text { Single Point GPS } \\
\text { (Pseudoranges) }\end{array}$ & 48 & 48 & 49 & 51 & 54 & 57 & 58 & 59 \\
\hline $\begin{array}{c}\text { Single Point GPS (Carrier } \\
\text { Phase) }\end{array}$ & 49 & 50 & 52 & 54 & 55 & 56 & 57 & 60 \\
\hline $\begin{array}{c}\text { Single Difference GPS } \\
\text { (Pseudoranges) }\end{array}$ & 41 & 41 & 42 & 42 & 42 & 44 & 47 & 50 \\
\hline $\begin{array}{c}\text { Single Difference GPS } \\
\text { (Carrier Phase) }\end{array}$ & 43 & 43 & 43 & 44 & 45 & 46 & 49 & 51 \\
\hline $\begin{array}{c}\text { Double Difference GPS } \\
\text { (Pseudoranges) }\end{array}$ & 53 & 53 & 54 & 56 & 60 & 60 & 60 & 60 \\
\hline $\begin{array}{c}\text { Double Difference GPS } \\
\text { (Carrier Phase) }\end{array}$ & 54 & 54 & 54 & 56 & 59 & 59 & 60 & 61 \\
\hline IMU Measured & 95 & 100 & 100 & 100 & 100 & 100 & 100 & 100 \\
\hline
\end{tabular}

Table 181: Success Rates of Reaching Angular Specifications of Fused Pitch for Simulation Data Set C-1

\begin{tabular}{|c|c|c|c|c|c|c|c|c|}
\hline & \multicolumn{7}{|c|}{ Portion of Trials in Which Target Specification was reached } \\
\hline & $\pm 0.5^{\circ}$ & $\pm 1^{\circ}$ & $\pm 2^{\circ}$ & $\pm 5^{\circ}$ & $\pm 10^{\circ}$ & $\pm 15^{\circ}$ & $\pm 20^{\circ}$ & $\pm 25^{\circ}$ \\
\hline Method & 100 & 100 & 100 & 100 & 100 & 100 & 100 & 100 \\
\hline $\begin{array}{c}\text { Single Point GPS } \\
\text { (Pseudoranges) }\end{array}$ & 73 & 84 & 92 & 98 & 100 & 100 & 100 & 100 \\
\hline $\begin{array}{c}\text { Single Point GPS (Carrier } \\
\text { Phase) }\end{array}$ & 100 & 100 & 100 & 100 & 100 & 100 & 100 & 100 \\
\hline $\begin{array}{c}\text { Single Difference GPS } \\
\text { (Pseudoranges) }\end{array}$ & 63 & 80 & 89 & 94 & 99 & 100 & 100 & 100 \\
\hline $\begin{array}{c}\text { Single Difference GPS } \\
\text { (Carrier Phase) }\end{array}$ & 100 & 100 & 100 & 100 & 100 & 100 & 100 & 100 \\
\hline $\begin{array}{c}\text { Double Difference GPS } \\
\text { (Pseudoranges) }\end{array}$ & 69 & 79 & 85 & 93 & 96 & 97 & 99 & 99 \\
\hline $\begin{array}{c}\text { Double Difference GPS } \\
\text { (Carrier Phase) }\end{array}$ & 100 & 100 & 100 & 100 & 100 & 100 & 100 & 100 \\
\hline IMU Measured & & & & & & & & \\
\hline
\end{tabular}


Table 182: Success Rates of Reaching Angular Specifications of Fused Heading for Simulation Data Set C-1

\begin{tabular}{|c|c|c|c|c|c|c|c|c|}
\hline & \multicolumn{7}{|c|}{ Portion of Trials in Which Target Specification was reached } \\
\hline & $\pm 0.5^{\circ}$ & $\pm 1^{\circ}$ & $\pm 2^{\circ}$ & $\pm 5^{\circ}$ & $\pm 10^{\circ}$ & $\pm 15^{\circ}$ & $\pm 20^{\circ}$ & $\pm 25^{\circ}$ \\
\hline Method & 89 & 96 & 96 & 98 & 98 & 99 & 99 & 99 \\
\hline $\begin{array}{c}\text { Single Point GPS } \\
\text { (Pseudoranges) }\end{array}$ & 41 & 42 & 44 & 48 & 53 & 57 & 60 & 60 \\
\hline $\begin{array}{c}\text { Single Point GPS (Carrier } \\
\text { Phase) }\end{array}$ & 88 & 96 & 99 & 100 & 100 & 100 & 100 & 100 \\
\hline $\begin{array}{c}\text { Single Difference GPS } \\
\text { (Pseudoranges) }\end{array}$ & 38 & 38 & 40 & 42 & 45 & 49 & 51 & 52 \\
\hline $\begin{array}{c}\text { Single Difference GPS } \\
\text { (Carrier Phase) }\end{array}$ & 81 & 94 & 97 & 98 & 98 & 98 & 99 & 99 \\
\hline $\begin{array}{c}\text { Double Difference GPS } \\
\text { (Pseudoranges) }\end{array}$ & 40 & 40 & 41 & 47 & 51 & 51 & 52 & 52 \\
\hline $\begin{array}{c}\text { Double Difference GPS } \\
\text { (Carrier Phase) }\end{array}$ & 95 & 100 & 100 & 100 & 100 & 100 & 100 & 100 \\
\hline IMU Measured & & & & & & & & \\
\hline
\end{tabular}

Table 183: Pitch / Heading Correlation Coefficients for Each Computation Method in Simulation Data Set C-1

\begin{tabular}{|c|c|c|}
\hline & Pitch / Heading Correlation Coefficient \\
\hline Method & Unfused & Fused \\
\hline $\begin{array}{c}\text { Single Point GPS } \\
\text { (Pseudoranges) }\end{array}$ & 0.995489 & 0.001466 \\
\hline $\begin{array}{c}\text { Single Difference GPS } \\
\text { (Pseudoranges) }\end{array}$ & 0.950991 & 0.002704 \\
\hline $\begin{array}{c}\text { Double Difference GPS } \\
\text { (Pseudoranges) }\end{array}$ & 0.916941 & 0.003229 \\
\hline
\end{tabular}




\section{Set C-2 3 m Dual Antenna Separation}

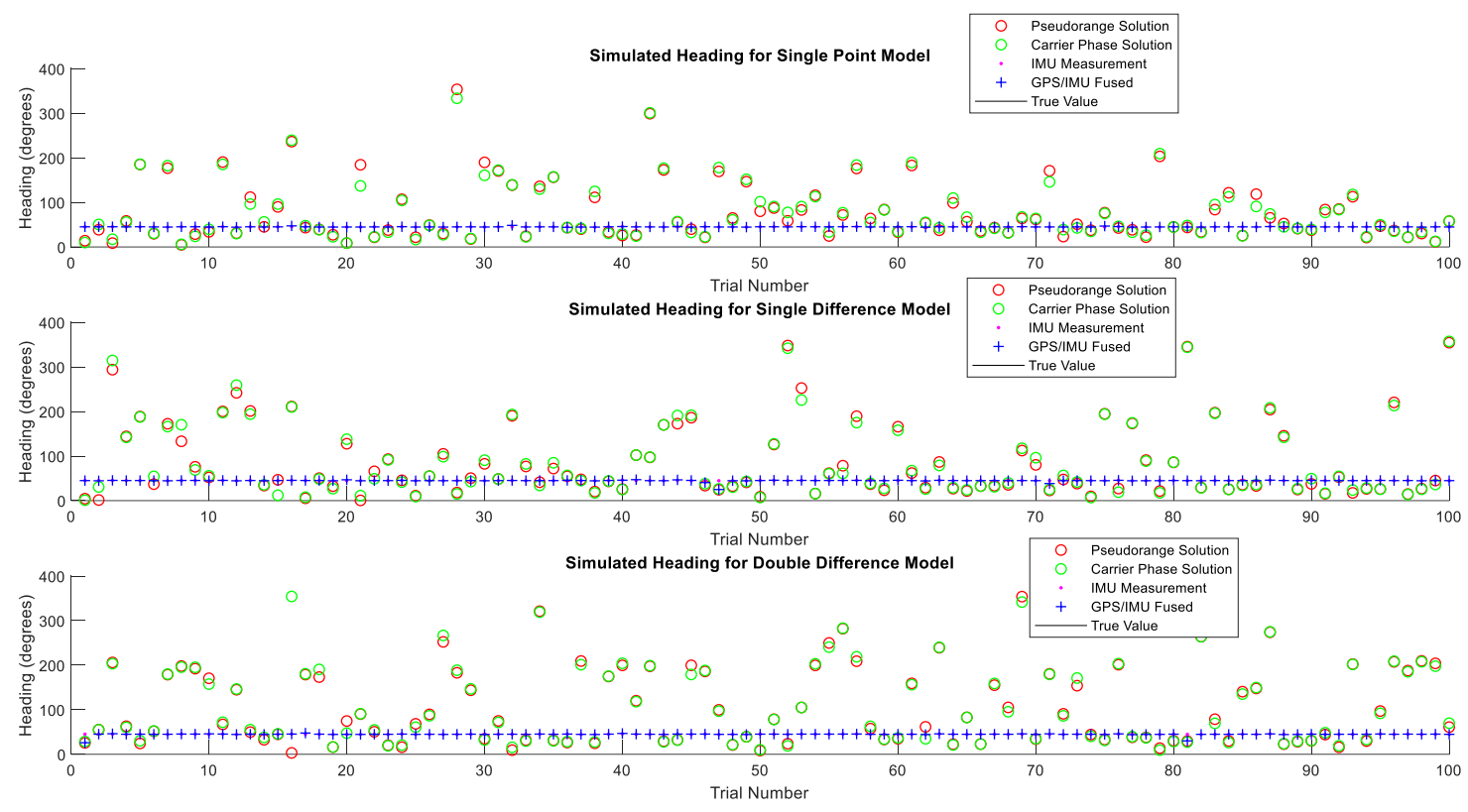

Figure 99: Computed and Measured Headings for Simulation Set C-2 


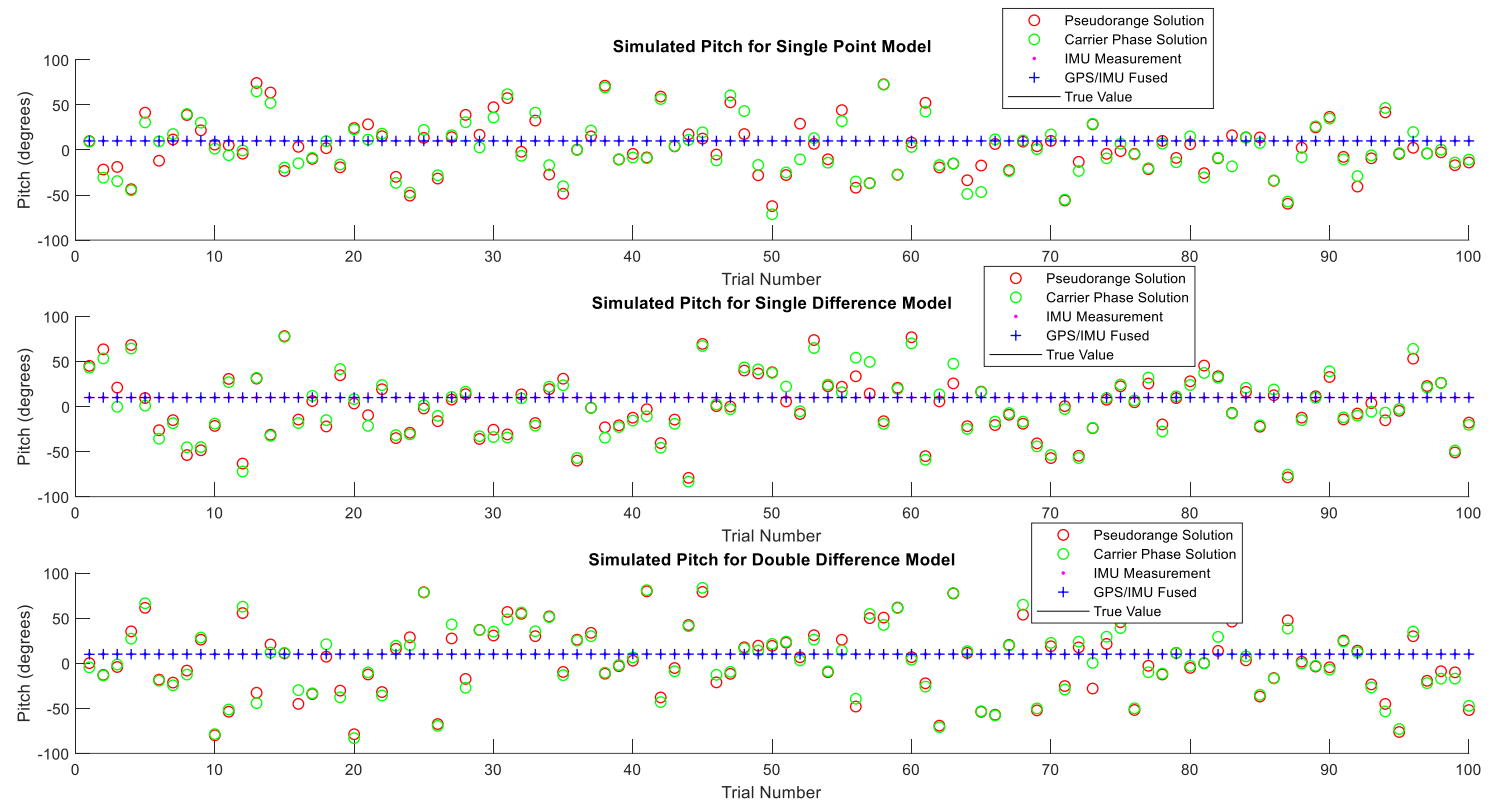

Figure 100: Computed and Measured Pitch for Simulation Set C-2

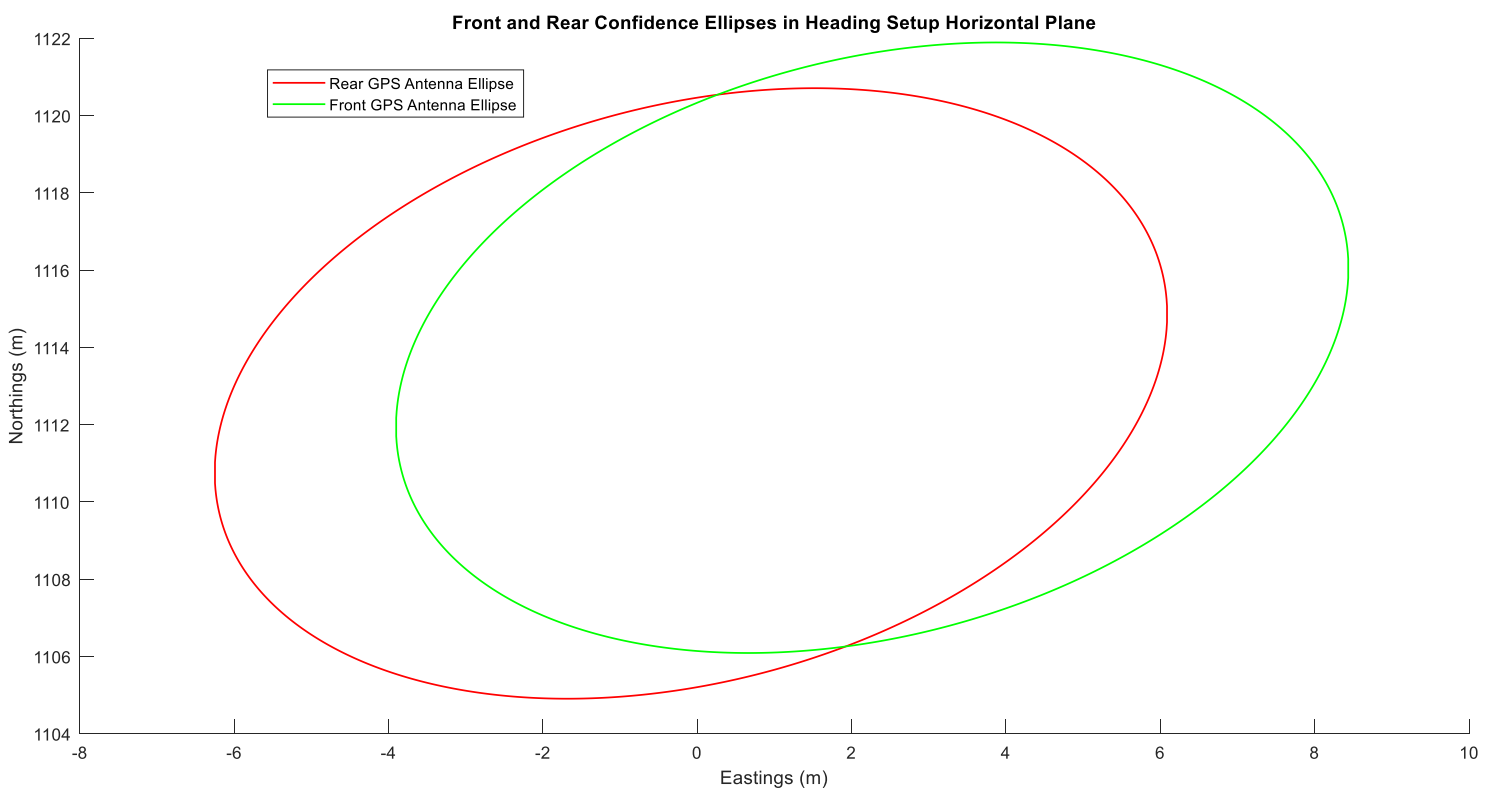

Figure 101: Propagated 95\% Confidence Ellipses for Front and Rear GPS Antennas in Simulated Data Set C-2 
Table 184: Mean Global Error Statistics of Unfused Pitch for Simulation Set C-2

\begin{tabular}{|c|c|c|c|}
\hline Method & $\begin{array}{c}\text { Measured Standard } \\
\text { Deviation } \\
\text { (degrees) }\end{array}$ & $\begin{array}{c}\text { Estimated } \\
\text { Standard } \\
\text { Deviation } \\
\text { (degrees) }\end{array}$ & $\begin{array}{c}\text { Mean RMS } \\
\text { Error } \\
\text { (degrees) }\end{array}$ \\
\hline Single Point GPS (Pseudoranges) & 29.784 & 234.755 & 24.357 \\
\hline Single Point GPS (Carrier Phase) & 29.828 & 0.125 & 25.185 \\
\hline $\begin{array}{c}\text { Single Difference GPS } \\
\text { (Pseudoranges) }\end{array}$ & 33.737 & 164.225 & 27.97 \\
\hline $\begin{array}{c}\text { Single Difference GPS (Carrier } \\
\text { Phase) }\end{array}$ & 34.755 & 0.113 & 29.312 \\
\hline $\begin{array}{c}\text { Double Difference GPS } \\
\text { (Pseudoranges) }\end{array}$ & 37.695 & 182.592 & 30.948 \\
\hline $\begin{array}{c}\text { Double Difference GPS (Carrier } \\
\text { Phase) }\end{array}$ & 38.317 & 0.159 & 31.498 \\
\hline IMU Measurement & 0.007 & - & 0.005 \\
\hline
\end{tabular}

Table 185: Mean Global Error Statistics of Unfused Heading for Simulation Set C-2

\begin{tabular}{|c|c|c|c|}
\hline Method & $\begin{array}{c}\text { Measured Standard } \\
\text { Deviation } \\
\text { (degrees) }\end{array}$ & $\begin{array}{c}\text { Estimated } \\
\text { Standard } \\
\text { Deviation } \\
\text { (degrees) }\end{array}$ & $\begin{array}{c}\text { Mean RMS } \\
\text { Error } \\
\text { (degrees) }\end{array}$ \\
\hline Single Point GPS (Pseudoranges) & 65.73 & 1780.105 & 41.604 \\
\hline Single Point GPS (Carrier Phase) & 64.01 & 2.032 & 41.466 \\
\hline $\begin{array}{c}\text { Single Difference GPS } \\
\text { (Pseudoranges) }\end{array}$ & 82.914 & 1066.18 & 49.601 \\
\hline $\begin{array}{c}\text { Single Difference GPS (Carrier } \\
\text { Phase) }\end{array}$ & 83.239 & 1.977 & 49.891 \\
\hline $\begin{array}{c}\text { Double Difference GPS } \\
\text { (Pseudoranges) }\end{array}$ & 85.076 & 1734.718 & 65.706 \\
\hline $\begin{array}{c}\text { Double Difference GPS (Carrier } \\
\text { Phase) }\end{array}$ & 88.017 & 0.807 & 65.345 \\
\hline IMU Measurement & 0.289 & - & 0.229 \\
\hline
\end{tabular}


Table 186: Mean Global Error Statistics of Fused Pitch for Simulation Set C-2

\begin{tabular}{|c|c|c|c|}
\hline Method & $\begin{array}{c}\text { Measured Standard } \\
\text { Deviation } \\
\text { (degrees) }\end{array}$ & $\begin{array}{c}\text { Estimated } \\
\text { Standard } \\
\text { Deviation } \\
\text { (degrees) }\end{array}$ & $\begin{array}{c}\text { Mean RMS } \\
\text { Error } \\
\text { (degrees) }\end{array}$ \\
\hline Single Point GPS (Pseudoranges) & 0.007 & 0.2 & 0.006 \\
\hline Single Point GPS (Carrier Phase) & 3.158 & 0.048 & 2.479 \\
\hline $\begin{array}{c}\text { Single Difference GPS } \\
\text { (Pseudoranges) }\end{array}$ & 0.02 & 0.2 & 0.009 \\
\hline $\begin{array}{c}\text { Single Difference GPS (Carrier } \\
\text { Phase) }\end{array}$ & 6.199 & 0.044 & 3.475 \\
\hline $\begin{array}{c}\text { Double Difference GPS } \\
\text { (Pseudoranges) }\end{array}$ & 0.034 & 0.199 & 0.015 \\
\hline $\begin{array}{c}\text { Double Difference GPS (Carrier } \\
\text { Phase) }\end{array}$ & 9.625 & 0.056 & 4.75 \\
\hline IMU Measurement & 0.007 & - & 0.005 \\
\hline
\end{tabular}

Table 187: Mean Global Error Statistics of Fused Heading for Simulation Set C-2

\begin{tabular}{|c|c|c|c|}
\hline Method & $\begin{array}{c}\text { Measured Standard } \\
\text { Deviation } \\
\text { (degrees) }\end{array}$ & $\begin{array}{c}\text { Estimated } \\
\text { Standard } \\
\text { Deviation } \\
\text { (degrees) }\end{array}$ & $\begin{array}{c}\text { Mean RMS } \\
\text { Error } \\
\text { (degrees) }\end{array}$ \\
\hline Single Point GPS (Pseudoranges) & 0.545 & 8.179 & 0.316 \\
\hline Single Point GPS (Carrier Phase) & 39.345 & 0.326 & 25.653 \\
\hline $\begin{array}{c}\text { Single Difference GPS } \\
\text { (Pseudoranges) }\end{array}$ & 2.203 & 8.485 & 0.592 \\
\hline $\begin{array}{c}\text { Single Difference GPS (Carrier } \\
\text { Phase) }\end{array}$ & 63.207 & 0.275 & 37.751 \\
\hline $\begin{array}{c}\text { Double Difference GPS } \\
\text { (Pseudoranges) }\end{array}$ & 2.466 & 8.392 & 0.646 \\
\hline $\begin{array}{c}\text { Double Difference GPS (Carrier } \\
\text { Phase) }\end{array}$ & 45.794 & 0.314 & 32.907 \\
\hline IMU Measurement & 0.289 & - & 0.229 \\
\hline
\end{tabular}


Table 188: Success Rates of Reaching Angular Specifications of Unfused Pitch for Simulation Data Set C-2

\begin{tabular}{|c|c|c|c|c|c|c|c|c|}
\hline & \multicolumn{7}{|c|}{ Portion of Trials in Which Target Specification was reached } \\
\hline Method & $\pm 0.5^{\circ}$ & $\pm 1^{\circ}$ & $\pm 2^{\circ}$ & $\pm 5^{\circ}$ & $\pm 10^{\circ}$ & $\pm 15^{\circ}$ & $\pm 20^{\circ}$ & $\pm 25^{\circ}$ \\
\hline $\begin{array}{c}\text { Single Point GPS } \\
\text { (Pseudoranges) }\end{array}$ & 66 & 66 & 67 & 72 & 78 & 81 & 84 & 85 \\
\hline $\begin{array}{c}\text { Single Point GPS (Carrier } \\
\text { Phase) }\end{array}$ & 65 & 65 & 68 & 70 & 77 & 80 & 82 & 87 \\
\hline $\begin{array}{c}\text { Single Difference GPS } \\
\text { (Pseudoranges) }\end{array}$ & 62 & 62 & 63 & 67 & 71 & 77 & 81 & 88 \\
\hline $\begin{array}{c}\text { Single Difference GPS } \\
\text { (Carrier Phase) }\end{array}$ & 60 & 61 & 63 & 64 & 69 & 78 & 80 & 83 \\
\hline $\begin{array}{c}\text { Double Difference GPS } \\
\text { (Pseudoranges) }\end{array}$ & 55 & 55 & 58 & 60 & 66 & 70 & 78 & 81 \\
\hline $\begin{array}{c}\text { Double Difference GPS } \\
\text { (Carrier Phase) }\end{array}$ & 54 & 54 & 57 & 61 & 65 & 71 & 78 & 79 \\
\hline IMU Measured & 100 & 100 & 100 & 100 & 100 & 100 & 100 & 100 \\
\hline
\end{tabular}

Table 189: Success Rates of Reaching Angular Specifications of Unfused Heading for Simulation Data Set C-2

\begin{tabular}{|c|c|c|c|c|c|c|c|c|}
\hline & \multicolumn{7}{|c|}{ Portion of Trials in Which Target Specification was reached } \\
\hline Method & $\pm 0.5^{\circ}$ & $\pm 1^{\circ}$ & $\pm 2^{\circ}$ & $\pm 5^{\circ}$ & $\pm 10^{\circ}$ & $\pm 15^{\circ}$ & $\pm 20^{\circ}$ & $\pm 25^{\circ}$ \\
\hline $\begin{array}{c}\text { Single Point GPS } \\
\text { (Pseudoranges) }\end{array}$ & 51 & 51 & 52 & 53 & 56 & 61 & 65 & 66 \\
\hline $\begin{array}{c}\text { Single Point GPS (Carrier } \\
\text { Phase) }\end{array}$ & 48 & 48 & 49 & 53 & 55 & 60 & 62 & 64 \\
\hline $\begin{array}{c}\text { Single Difference GPS } \\
\text { (Pseudoranges) }\end{array}$ & 51 & 52 & 53 & 56 & 62 & 62 & 64 & 65 \\
\hline $\begin{array}{c}\text { Single Difference GPS } \\
\text { (Carrier Phase) }\end{array}$ & 53 & 53 & 53 & 57 & 60 & 63 & 65 & 67 \\
\hline $\begin{array}{c}\text { Double Difference GPS } \\
\text { (Pseudoranges) }\end{array}$ & 47 & 47 & 47 & 48 & 50 & 52 & 55 & 57 \\
\hline $\begin{array}{c}\text { Double Difference GPS } \\
\text { (Carrier Phase) }\end{array}$ & 46 & 47 & 47 & 49 & 52 & 53 & 56 & 58 \\
\hline IMU Measured & 96 & 100 & 100 & 100 & 100 & 100 & 100 & 100 \\
\hline
\end{tabular}


Table 190: Success Rates of Reaching Angular Specifications of Fused Pitch for Simulation Data Set C-2

\begin{tabular}{|c|c|c|c|c|c|c|c|c|}
\hline & \multicolumn{7}{|c|}{ Portion of Trials in Which Target Specification was reached } \\
\hline & $\pm 0.5^{\circ}$ & $\pm 1^{\circ}$ & $\pm 2^{\circ}$ & $\pm 5^{\circ}$ & $\pm 10^{\circ}$ & $\pm 15^{\circ}$ & $\pm 20^{\circ}$ & $\pm 25^{\circ}$ \\
\hline Method & 100 & 100 & 100 & 100 & 100 & 100 & 100 & 100 \\
\hline $\begin{array}{c}\text { Single Point GPS } \\
\text { (Pseudoranges) }\end{array}$ & 75 & 83 & 91 & 97 & 100 & 100 & 100 & 100 \\
\hline $\begin{array}{c}\text { Single Point GPS (Carrier } \\
\text { Phase) }\end{array}$ & 100 & 100 & 100 & 100 & 100 & 100 & 100 & 100 \\
\hline $\begin{array}{c}\text { Single Difference GPS } \\
\text { (Pseudoranges) }\end{array}$ & 67 & 82 & 91 & 96 & 98 & 99 & 100 & 100 \\
\hline $\begin{array}{c}\text { Single Difference GPS } \\
\text { (Carrier Phase) }\end{array}$ & 100 & 100 & 100 & 100 & 100 & 100 & 100 & 100 \\
\hline $\begin{array}{c}\text { Double Difference GPS } \\
\text { (Pseudoranges) }\end{array}$ & 67 & 79 & 88 & 96 & 96 & 96 & 96 & 97 \\
\hline $\begin{array}{c}\text { Double Difference GPS } \\
\text { (Carrier Phase) }\end{array}$ & 100 & 100 & 100 & 100 & 100 & 100 & 100 & 100 \\
\hline IMU Measured & & & & & & & & \\
\hline
\end{tabular}

Table 191: Success Rates of Reaching Angular Specifications of Fused Heading for Simulation Data Set C-2

\begin{tabular}{|c|c|c|c|c|c|c|c|c|}
\hline & \multicolumn{7}{|c|}{ Portion of Trials in Which Target Specification was reached } \\
\hline Method & $\pm 0.5^{\circ}$ & $\pm 1^{\circ}$ & $\pm 2^{\circ}$ & $\pm 5^{\circ}$ & $\pm 10^{\circ}$ & $\pm 15^{\circ}$ & $\pm 20^{\circ}$ & $\pm 25^{\circ}$ \\
\hline $\begin{array}{c}\text { Single Point GPS } \\
\text { (Pseudoranges) }\end{array}$ & 91 & 97 & 98 & 100 & 100 & 100 & 100 & 100 \\
\hline $\begin{array}{c}\text { Single Point GPS (Carrier } \\
\text { Phase) }\end{array}$ & 46 & 47 & 48 & 56 & 65 & 71 & 75 & 77 \\
\hline $\begin{array}{c}\text { Single Difference GPS } \\
\text { (Pseudoranges) }\end{array}$ & 90 & 97 & 99 & 100 & 100 & 100 & 100 & 100 \\
\hline $\begin{array}{c}\text { Single Difference GPS } \\
\text { (Carrier Phase) }\end{array}$ & 51 & 52 & 53 & 60 & 69 & 73 & 74 & 75 \\
\hline $\begin{array}{c}\text { Double Difference GPS } \\
\text { (Pseudoranges) }\end{array}$ & 89 & 97 & 99 & 100 & 100 & 100 & 100 & 100 \\
\hline $\begin{array}{c}\text { Double Difference GPS } \\
\text { (Carrier Phase) }\end{array}$ & 37 & 38 & 40 & 46 & 55 & 61 & 65 & 69 \\
\hline IMU Measured & 96 & 100 & 100 & 100 & 100 & 100 & 100 & 100 \\
\hline
\end{tabular}


Table 192: Pitch / Heading Correlation Coefficients for Each Computation Method in Simulation Data Set C-2

\begin{tabular}{|c|c|c|}
\hline & \multicolumn{2}{|c|}{ Pitch / Heading Correlation Coefficient } \\
\hline Method & Unfused & Fused \\
\hline $\begin{array}{c}\text { Single Point GPS } \\
\text { (Pseudoranges) }\end{array}$ & -0.83668 & -0.00099 \\
\hline $\begin{array}{c}\text { Single Difference GPS } \\
\text { (Pseudoranges) }\end{array}$ & -0.92067 & -0.00024 \\
\hline $\begin{array}{c}\text { Double Difference GPS } \\
\text { (Pseudoranges) }\end{array}$ & -0.32977 & $-1.7 \times 10^{-5}$ \\
\hline
\end{tabular}

\section{Set C-3 5 m Dual Antenna Separation}

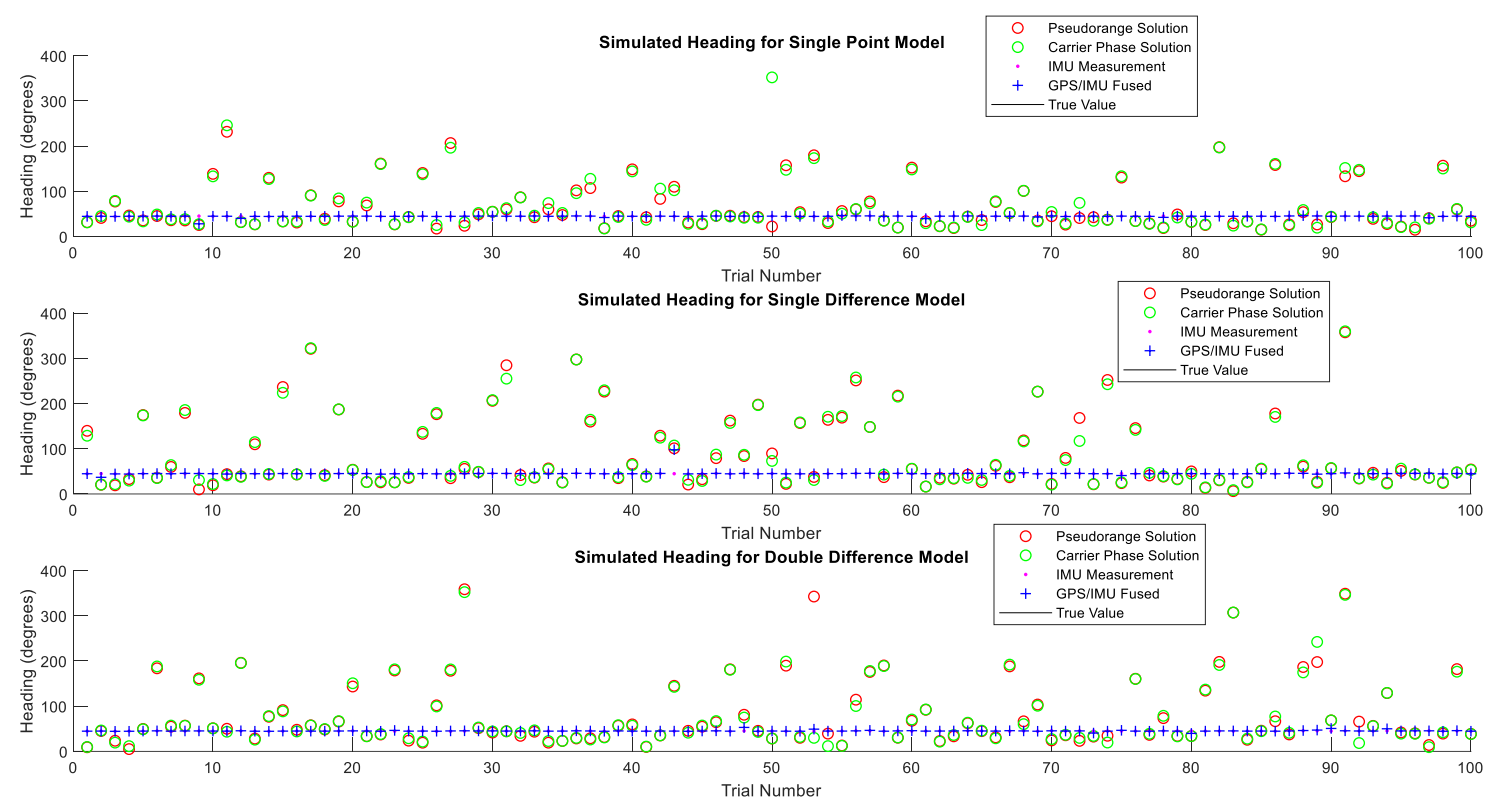

Figure 102: Computed and Measured Headings for Simulation Set C-3 


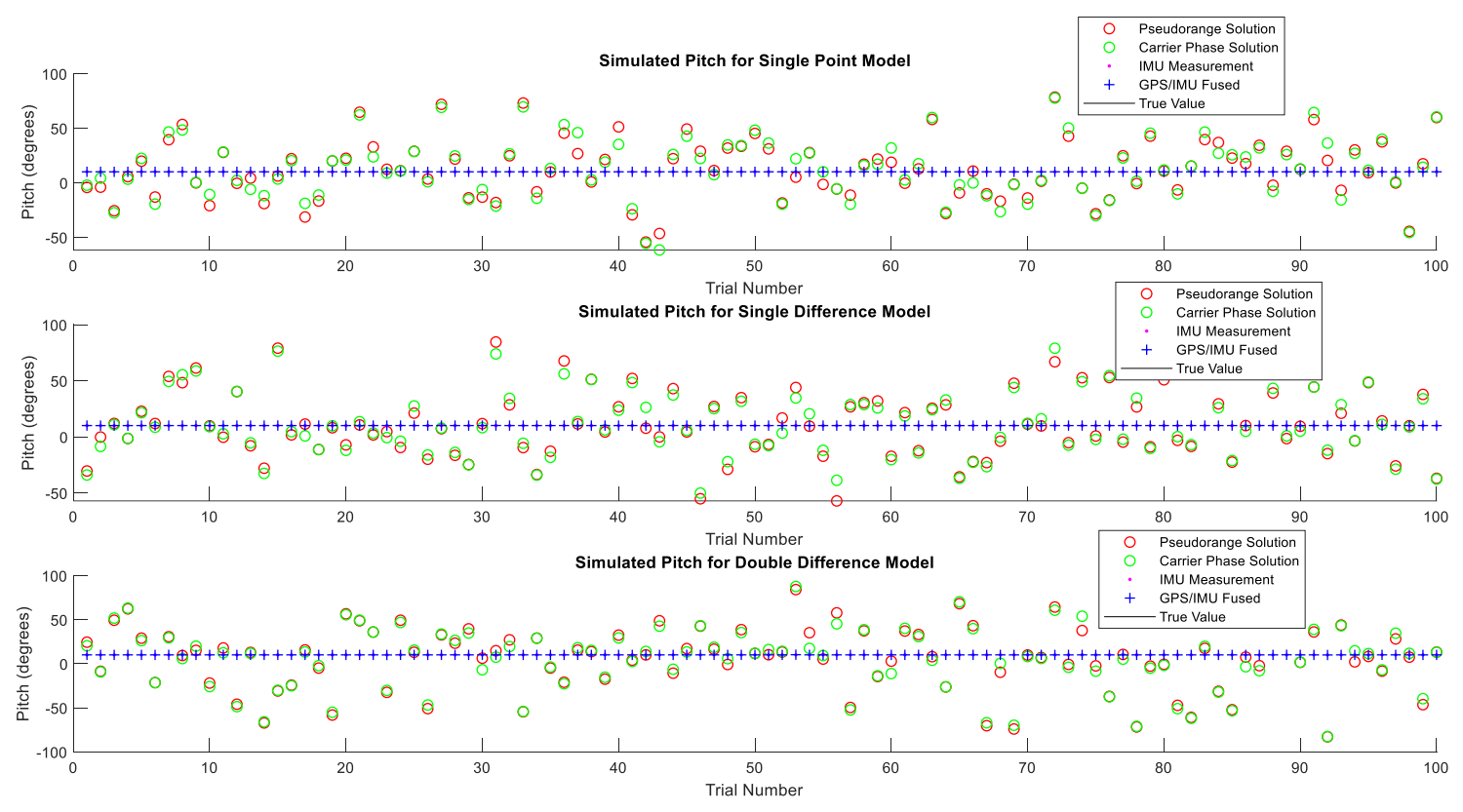

Figure 103: Computed and Measured Pitch for Simulation Set C-3

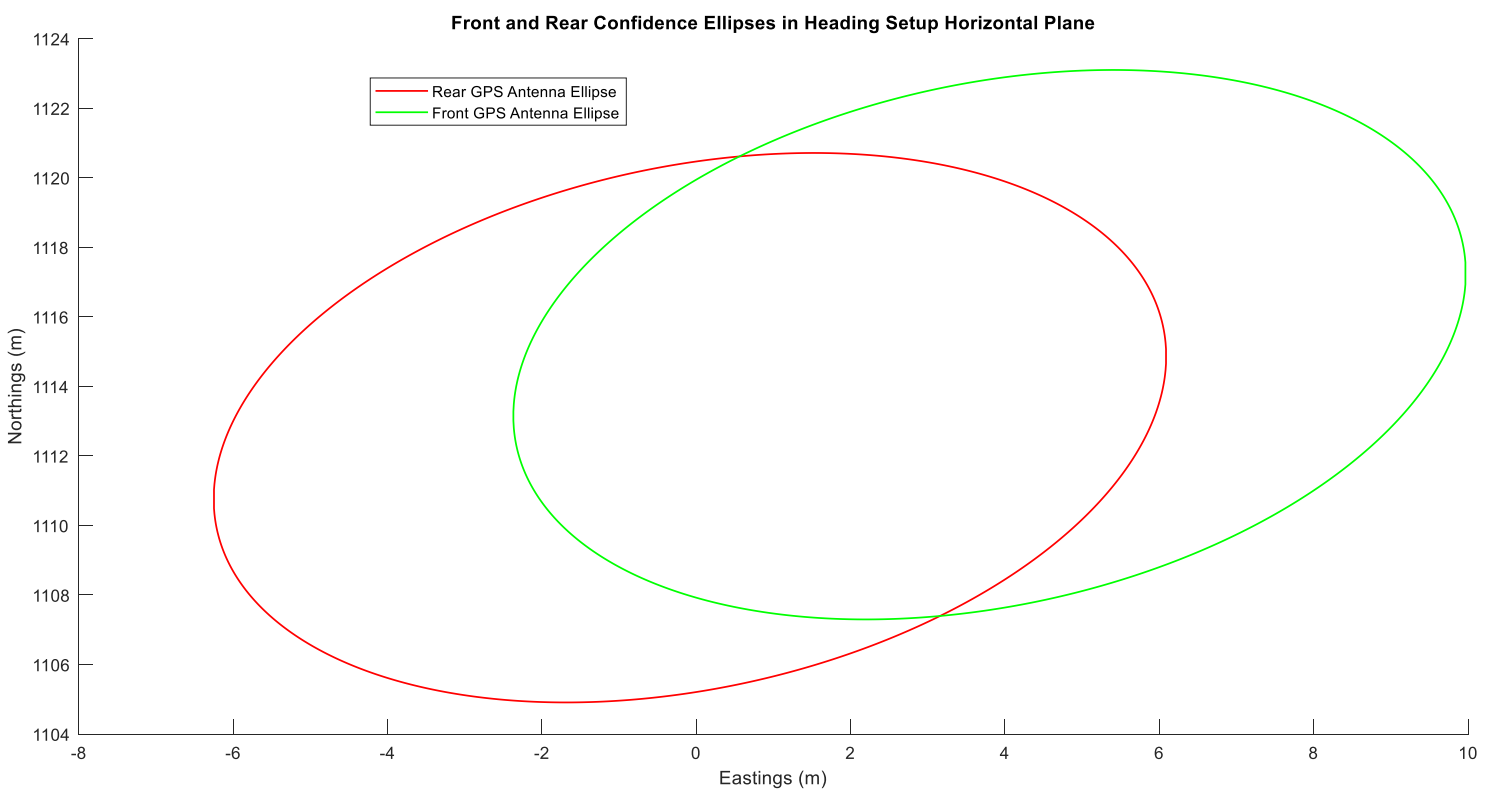

Figure 104: Propagated 95\% Confidence Ellipses for Front and Rear GPS Antennas in Simulated Data Set C-3 
Table 193: Mean Global Error Statistics of Unfused Pitch for Simulation Set C-3

\begin{tabular}{|c|c|c|c|}
\hline Method & $\begin{array}{c}\text { Measured Standard } \\
\text { Deviation } \\
\text { (degrees) }\end{array}$ & $\begin{array}{c}\text { Estimated } \\
\text { Standard } \\
\text { Deviation } \\
\text { (degrees) }\end{array}$ & $\begin{array}{c}\text { Mean RMS } \\
\text { Error } \\
\text { (degrees) }\end{array}$ \\
\hline Single Point GPS (Pseudoranges) & 26.931 & 223.215 & 21.626 \\
\hline Single Point GPS (Carrier Phase) & 27.681 & 0.129 & 22.343 \\
\hline $\begin{array}{c}\text { Single Difference GPS } \\
\text { (Pseudoranges) }\end{array}$ & 29.725 & 159.458 & 23.437 \\
\hline $\begin{array}{c}\text { Single Difference GPS (Carrier } \\
\text { Phase) }\end{array}$ & 29.075 & 0.103 & 23.445 \\
\hline $\begin{array}{c}\text { Double Difference GPS } \\
\text { (Pseudoranges) }\end{array}$ & 36.4 & 103.456 & 27.756 \\
\hline $\begin{array}{c}\text { Double Difference GPS (Carrier } \\
\text { Phase) }\end{array}$ & 36.192 & 0.108 & 27.871 \\
\hline IMU Measurement & 0.007 & - & 0.006 \\
\hline
\end{tabular}

Table 194: Mean Global Error Statistics of Unfused Heading for Simulation Set C-3

\begin{tabular}{|c|c|c|c|}
\hline Method & $\begin{array}{c}\text { Measured Standard } \\
\text { Deviation } \\
\text { (degrees) }\end{array}$ & $\begin{array}{c}\text { Estimated } \\
\text { Standard } \\
\text { Deviation } \\
\text { (degrees) }\end{array}$ & $\begin{array}{c}\text { Mean RMS } \\
\text { Error } \\
\text { (degrees) }\end{array}$ \\
\hline Single Point GPS (Pseudoranges) & 48.704 & 870.824 & 32.053 \\
\hline Single Point GPS (Carrier Phase) & 56.576 & 0.343 & 33.057 \\
\hline $\begin{array}{c}\text { Single Difference GPS } \\
\text { (Pseudoranges) }\end{array}$ & 82.843 & 594.777 & 48.459 \\
\hline $\begin{array}{c}\text { Single Difference GPS (Carrier } \\
\text { Phase) }\end{array}$ & 81.585 & 0.202 & 48.019 \\
\hline $\begin{array}{c}\text { Double Difference GPS } \\
\text { (Pseudoranges) }\end{array}$ & 80.516 & 547.863 & 40.76 \\
\hline $\begin{array}{c}\text { Double Difference GPS (Carrier } \\
\text { Phase) }\end{array}$ & 77.333 & 0.435 & 40.52 \\
\hline IMU Measurement & 0.316 & - & 0.267 \\
\hline
\end{tabular}


Table 195: Mean Global Error Statistics of Fused Pitch for Simulation Set C-3

\begin{tabular}{|c|c|c|c|}
\hline Method & $\begin{array}{c}\text { Measured Standard } \\
\text { Deviation } \\
\text { (degrees) }\end{array}$ & $\begin{array}{c}\text { Estimated } \\
\text { Standard } \\
\text { Deviation } \\
\text { (degrees) }\end{array}$ & $\begin{array}{c}\text { Mean RMS } \\
\text { Error } \\
\text { (degrees) }\end{array}$ \\
\hline Single Point GPS (Pseudoranges) & 0.011 & 0.2 & 0.007 \\
\hline Single Point GPS (Carrier Phase) & 3.276 & 0.049 & 2.35 \\
\hline $\begin{array}{c}\text { Single Difference GPS } \\
\text { (Pseudoranges) }\end{array}$ & 0.018 & 0.2 & 0.009 \\
\hline $\begin{array}{c}\text { Single Difference GPS (Carrier } \\
\text { Phase) }\end{array}$ & 5.25 & 0.043 & 3.373 \\
\hline $\begin{array}{c}\text { Double Difference GPS } \\
\text { (Pseudoranges) }\end{array}$ & 0.026 & 0.2 & 0.011 \\
\hline $\begin{array}{c}\text { Double Difference GPS (Carrier } \\
\text { Phase) }\end{array}$ & 10.853 & 0.046 & 4.683 \\
\hline IMU Measurement & 0.007 & - & 0.006 \\
\hline
\end{tabular}

Table 196: Mean Global Error Statistics of Fused Heading for Simulation Set C-3

\begin{tabular}{|c|c|c|c|}
\hline Method & $\begin{array}{c}\text { Measured Standard } \\
\text { Deviation } \\
\text { (degrees) }\end{array}$ & $\begin{array}{c}\text { Estimated } \\
\text { Standard } \\
\text { Deviation } \\
\text { (degrees) }\end{array}$ & $\begin{array}{c}\text { Mean RMS } \\
\text { Error } \\
\text { (degrees) }\end{array}$ \\
\hline Single Point GPS (Pseudoranges) & 1.91 & 7.903 & 0.645 \\
\hline Single Point GPS (Carrier Phase) & 34.519 & 0.142 & 19.706 \\
\hline $\begin{array}{c}\text { Single Difference GPS } \\
\text { (Pseudoranges) }\end{array}$ & 5.395 & 7.918 & 0.987 \\
\hline $\begin{array}{c}\text { Single Difference GPS (Carrier } \\
\text { Phase) }\end{array}$ & 63.32 & 0.089 & 33.647 \\
\hline $\begin{array}{c}\text { Double Difference GPS } \\
\text { (Pseudoranges) }\end{array}$ & 1.436 & 7.993 & 0.659 \\
\hline $\begin{array}{c}\text { Double Difference GPS (Carrier } \\
\text { Phase) }\end{array}$ & 44.332 & 0.195 & 27.166 \\
\hline IMU Measurement & 0.316 & - & 0.267 \\
\hline
\end{tabular}


Table 197: Success Rates of Reaching Angular Specifications of Unfused Pitch for Simulation Data Set C-3

\begin{tabular}{|c|c|c|c|c|c|c|c|c|}
\hline & \multicolumn{7}{|c|}{ Portion of Trials in Which Target Specification was reached } \\
\hline Method & $\pm 0.5^{\circ}$ & $\pm 1^{\circ}$ & $\pm 2^{\circ}$ & $\pm 5^{\circ}$ & $\pm 10^{\circ}$ & $\pm 15^{\circ}$ & $\pm 20^{\circ}$ & $\pm 25^{\circ}$ \\
\hline $\begin{array}{c}\text { Single Point GPS } \\
\text { (Pseudoranges) }\end{array}$ & 46 & 48 & 50 & 53 & 60 & 70 & 76 & 82 \\
\hline $\begin{array}{c}\text { Single Point GPS (Carrier } \\
\text { Phase) }\end{array}$ & 46 & 47 & 49 & 52 & 57 & 67 & 76 & 80 \\
\hline $\begin{array}{c}\text { Single Difference GPS } \\
\text { (Pseudoranges) }\end{array}$ & 54 & 55 & 61 & 62 & 63 & 67 & 75 & 78 \\
\hline $\begin{array}{c}\text { Single Difference GPS } \\
\text { (Carrier Phase) }\end{array}$ & 55 & 56 & 57 & 60 & 62 & 66 & 74 & 80 \\
\hline $\begin{array}{c}\text { Double Difference GPS } \\
\text { (Pseudoranges) }\end{array}$ & 53 & 53 & 54 & 60 & 67 & 69 & 73 & 77 \\
\hline $\begin{array}{c}\text { Double Difference GPS } \\
\text { (Carrier Phase) }\end{array}$ & 49 & 49 & 53 & 60 & 69 & 70 & 75 & 79 \\
\hline IMU Measured & 100 & 100 & 100 & 100 & 100 & 100 & 100 & 100 \\
\hline
\end{tabular}

Table 198: Success Rates of Reaching Angular Specifications of Unfused Heading for Simulation Data Set C-3

\begin{tabular}{|c|c|c|c|c|c|c|c|c|}
\hline & \multicolumn{7}{|c|}{ Portion of Trials in Which Target Specification was reached } \\
\hline Method & $\pm 0.5^{\circ}$ & $\pm 1^{\circ}$ & $\pm 2^{\circ}$ & $\pm 5^{\circ}$ & $\pm 10^{\circ}$ & $\pm 15^{\circ}$ & $\pm 20^{\circ}$ & $\pm 25^{\circ}$ \\
\hline $\begin{array}{c}\text { Single Point GPS } \\
\text { (Pseudoranges) }\end{array}$ & 58 & 59 & 61 & 64 & 68 & 71 & 73 & 74 \\
\hline $\begin{array}{c}\text { Single Point GPS (Carrier } \\
\text { Phase) }\end{array}$ & 57 & 57 & 59 & 60 & 67 & 68 & 71 & 71 \\
\hline $\begin{array}{c}\text { Single Difference GPS } \\
\text { (Pseudoranges) }\end{array}$ & 58 & 58 & 59 & 62 & 66 & 71 & 73 & 74 \\
\hline $\begin{array}{c}\text { Single Difference GPS } \\
\text { (Carrier Phase) }\end{array}$ & 58 & 58 & 59 & 61 & 64 & 69 & 73 & 73 \\
\hline $\begin{array}{c}\text { Double Difference GPS } \\
\text { (Pseudoranges) }\end{array}$ & 51 & 52 & 52 & 56 & 60 & 65 & 67 & 73 \\
\hline $\begin{array}{c}\text { Double Difference GPS } \\
\text { (Carrier Phase) }\end{array}$ & 53 & 54 & 56 & 58 & 60 & 67 & 69 & 72 \\
\hline IMU Measured & 94 & 100 & 100 & 100 & 100 & 100 & 100 & 100 \\
\hline
\end{tabular}


Table 199: Success Rates of Reaching Angular Specifications of Fused Pitch for Simulation Data Set C-3

\begin{tabular}{|c|c|c|c|c|c|c|c|c|}
\hline & \multicolumn{7}{|c|}{ Portion of Trials in Which Target Specification was reached } \\
\hline & $\pm 0.5^{\circ}$ & $\pm 1^{\circ}$ & $\pm 2^{\circ}$ & $\pm 5^{\circ}$ & $\pm 10^{\circ}$ & $\pm 15^{\circ}$ & $\pm 20^{\circ}$ & $\pm 25^{\circ}$ \\
\hline Method & 100 & 100 & 100 & 100 & 100 & 100 & 100 & 100 \\
\hline $\begin{array}{c}\text { Single Point GPS } \\
\text { (Pseudoranges) }\end{array}$ & 61 & 78 & 94 & 98 & 99 & 99 & 100 & 100 \\
\hline $\begin{array}{c}\text { Single Point GPS (Carrier } \\
\text { Phase) }\end{array}$ & 100 & 100 & 100 & 100 & 100 & 100 & 100 & 100 \\
\hline $\begin{array}{c}\text { Single Difference GPS } \\
\text { (Pseudoranges) }\end{array}$ & 62 & 81 & 91 & 94 & 97 & 98 & 99 & 99 \\
\hline $\begin{array}{c}\text { Single Difference GPS } \\
\text { (Carrier Phase) }\end{array}$ & 100 & 100 & 100 & 100 & 100 & 100 & 100 & 100 \\
\hline $\begin{array}{c}\text { Double Difference GPS } \\
\text { (Pseudoranges) }\end{array}$ & 67 & 76 & 89 & 95 & 97 & 98 & 98 & 98 \\
\hline $\begin{array}{c}\text { Double Difference GPS } \\
\text { (Carrier Phase) }\end{array}$ & 100 & 100 & 100 & 100 & 100 & 100 & 100 & 100 \\
\hline
\end{tabular}

Table 200: Success Rates of Reaching Angular Specifications of Fused Heading for Simulation Data Set C-3

\begin{tabular}{|c|c|c|c|c|c|c|c|c|}
\hline & \multicolumn{7}{|c|}{ Portion of Trials in Which Target Specification was reached } \\
\hline Method & $\pm 0.5^{\circ}$ & $\pm 1^{\circ}$ & $\pm 2^{\circ}$ & $\pm 5^{\circ}$ & $\pm 10^{\circ}$ & $\pm 15^{\circ}$ & $\pm 20^{\circ}$ & $\pm 25^{\circ}$ \\
\hline $\begin{array}{c}\text { Single Point GPS } \\
\text { (Pseudoranges) }\end{array}$ & 93 & 98 & 100 & 100 & 100 & 100 & 100 & 100 \\
\hline $\begin{array}{c}\text { Single Point GPS (Carrier } \\
\text { Phase) }\end{array}$ & 56 & 57 & 59 & 63 & 70 & 77 & 79 & 85 \\
\hline $\begin{array}{c}\text { Single Difference GPS } \\
\text { (Pseudoranges) }\end{array}$ & 92 & 97 & 99 & 99 & 99 & 99 & 99 & 99 \\
\hline $\begin{array}{c}\text { Single Difference GPS } \\
\text { (Carrier Phase) }\end{array}$ & 52 & 52 & 53 & 55 & 65 & 70 & 73 & 74 \\
\hline $\begin{array}{c}\text { Double Difference GPS } \\
\text { (Pseudoranges) }\end{array}$ & 84 & 91 & 95 & 98 & 100 & 100 & 100 & 100 \\
\hline $\begin{array}{c}\text { Double Difference GPS } \\
\text { (Carrier Phase) }\end{array}$ & 48 & 50 & 52 & 58 & 65 & 69 & 74 & 77 \\
\hline IMU Measured & 94 & 100 & 100 & 100 & 100 & 100 & 100 & 100 \\
\hline
\end{tabular}


Table 201: Pitch / Heading Correlation Coefficients for Each Computation Method in Simulation Data Set C-3

\begin{tabular}{|c|c|c|}
\hline & \multicolumn{2}{|c|}{ Pitch / Heading Correlation Coefficient } \\
\hline Method & Unfused & Fused \\
\hline $\begin{array}{c}\text { Single Point GPS } \\
\text { (Pseudoranges) }\end{array}$ & -0.4922 & $-8.8 \times 10^{-5}$ \\
\hline $\begin{array}{c}\text { Single Difference GPS } \\
\text { (Pseudoranges) }\end{array}$ & -0.60894 & $-9.4 \times 10^{-5}$ \\
\hline $\begin{array}{c}\text { Double Difference GPS } \\
\text { (Pseudoranges) }\end{array}$ & 0.822186 & 0.00113 \\
\hline
\end{tabular}

\section{Set C-4 10 m Dual Antenna Separation}
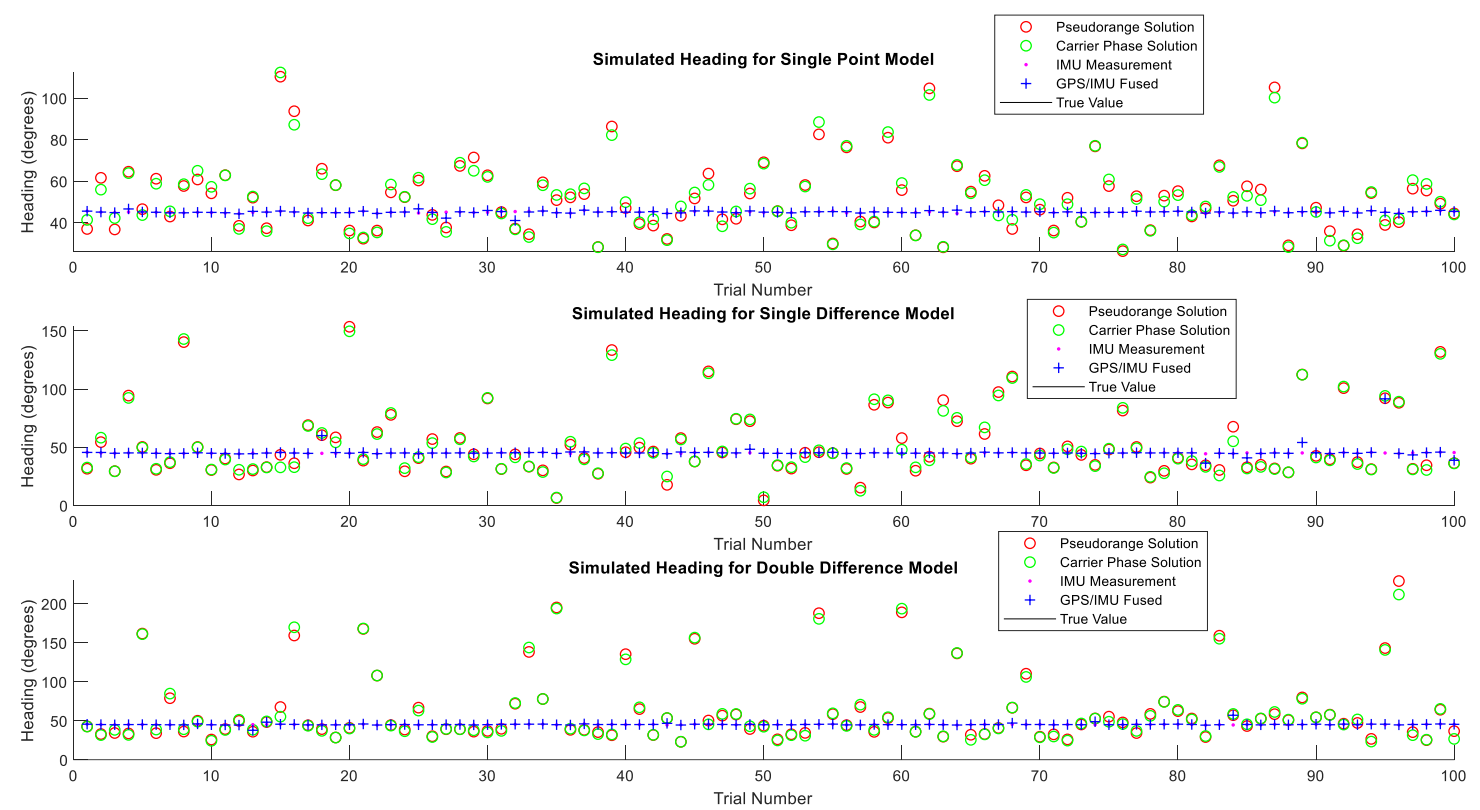

Figure 105: Computed and Measured Headings for Simulation Set C-4 


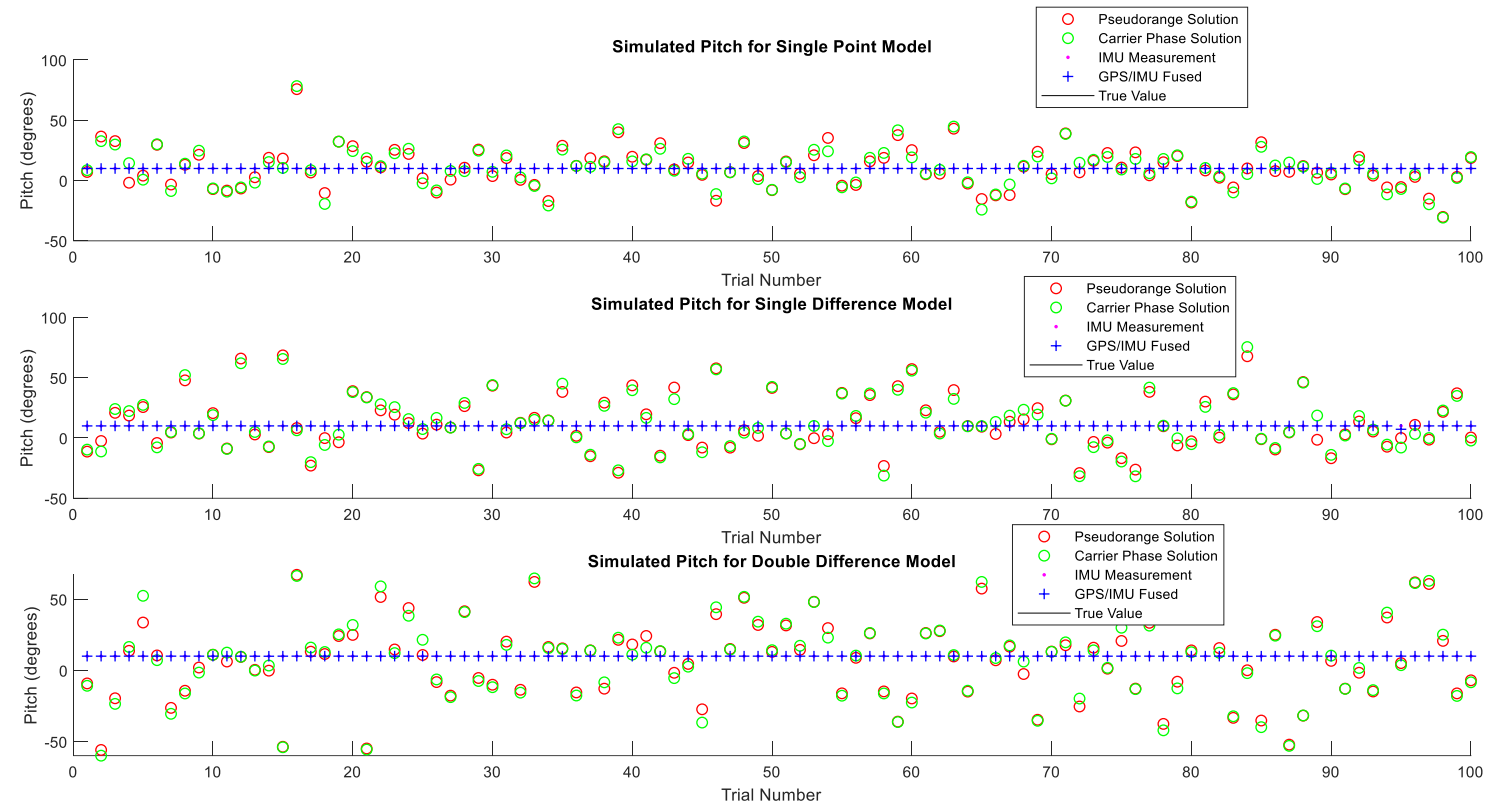

Figure 106: Computed and Measured Pitch for Simulation Set C-4

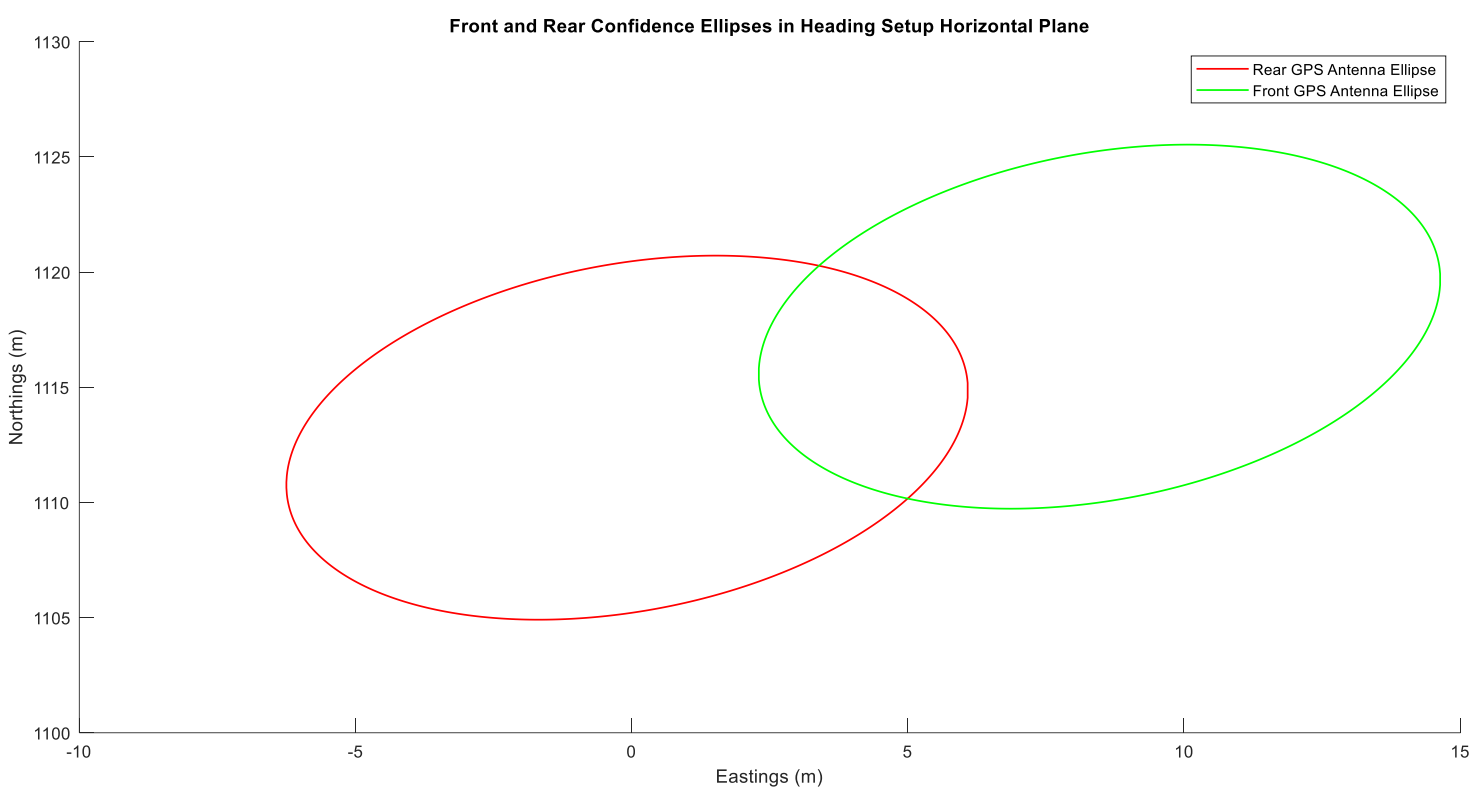

Figure 107: Propagated 95\% Confidence Ellipses for Front and Rear GPS Antennas in Simulated Data Set C-4 
Table 202: Mean Global Error Statistics of Unfused Pitch for Simulation Set C-4

\begin{tabular}{|c|c|c|c|}
\hline Method & $\begin{array}{c}\text { Measured Standard } \\
\text { Deviation } \\
\text { (degrees) }\end{array}$ & $\begin{array}{c}\text { Estimated } \\
\text { Standard } \\
\text { Deviation } \\
\text { (degrees) }\end{array}$ & $\begin{array}{c}\text { Mean RMS } \\
\text { Error } \\
\text { (degrees) }\end{array}$ \\
\hline Single Point GPS (Pseudoranges) & 16.345 & 89.422 & 12.72 \\
\hline Single Point GPS (Carrier Phase) & 16.598 & 0.048 & 12.615 \\
\hline $\begin{array}{c}\text { Single Difference GPS } \\
\text { (Pseudoranges) }\end{array}$ & 22.023 & 81.832 & 17.422 \\
\hline $\begin{array}{c}\text { Single Difference GPS (Carrier } \\
\text { Phase) }\end{array}$ & 22.286 & 0.052 & 17.699 \\
\hline $\begin{array}{c}\text { Double Difference GPS } \\
\text { (Pseudoranges) }\end{array}$ & 27.019 & 95.913 & 21.088 \\
\hline $\begin{array}{c}\text { Double Difference GPS (Carrier } \\
\text { Phase) }\end{array}$ & 28.275 & 0.09 & 21.95 \\
\hline IMU Measurement & 0.007 & - & 0.005 \\
\hline
\end{tabular}

Table 203: Mean Global Error Statistics of Unfused Heading for Simulation Set C-4

\begin{tabular}{|c|c|c|c|}
\hline Method & $\begin{array}{c}\text { Measured Standard } \\
\text { Deviation } \\
\text { (degrees) }\end{array}$ & $\begin{array}{c}\text { Estimated } \\
\text { Standard } \\
\text { Deviation } \\
\text { (degrees) }\end{array}$ & $\begin{array}{c}\text { Mean RMS } \\
\text { Error } \\
\text { (degrees) }\end{array}$ \\
\hline Single Point GPS (Pseudoranges) & 16.952 & 230.015 & 13.286 \\
\hline Single Point GPS (Carrier Phase) & 16.68 & 0.126 & 13.175 \\
\hline $\begin{array}{c}\text { Single Difference GPS } \\
\text { (Pseudoranges) }\end{array}$ & 29.232 & 838.467 & 20.336 \\
\hline $\begin{array}{c}\text { Single Difference GPS (Carrier } \\
\text { Phase) }\end{array}$ & 28.991 & 0.867 & 20.34 \\
\hline $\begin{array}{c}\text { Double Difference GPS } \\
\text { (Pseudoranges) }\end{array}$ & 44.332 & 248.361 & 26.771 \\
\hline $\begin{array}{c}\text { Double Difference GPS (Carrier } \\
\text { Phase) }\end{array}$ & 43.974 & 0.225 & 26.889 \\
\hline IMU Measurement & 0.315 & - & 0.252 \\
\hline
\end{tabular}


Table 204: Mean Global Error Statistics of Fused Pitch for Simulation Set C-4

\begin{tabular}{|c|c|c|c|}
\hline Method & $\begin{array}{c}\text { Measured Standard } \\
\text { Deviation } \\
\text { (degrees) }\end{array}$ & $\begin{array}{c}\text { Estimated } \\
\text { Standard } \\
\text { Deviation } \\
\text { (degrees) }\end{array}$ & $\begin{array}{c}\text { Mean RMS } \\
\text { Error } \\
\text { (degrees) }\end{array}$ \\
\hline Single Point GPS (Pseudoranges) & 0.007 & 0.201 & 0.006 \\
\hline Single Point GPS (Carrier Phase) & 3.901 & 0.023 & 2.578 \\
\hline $\begin{array}{c}\text { Single Difference GPS } \\
\text { (Pseudoranges) }\end{array}$ & 0.279 & 0.198 & 0.035 \\
\hline $\begin{array}{c}\text { Single Difference GPS (Carrier } \\
\text { Phase) }\end{array}$ & 4.112 & 0.024 & 3.38 \\
\hline $\begin{array}{c}\text { Double Difference GPS } \\
\text { (Pseudoranges) }\end{array}$ & 0.018 & 0.2 & 0.008 \\
\hline $\begin{array}{c}\text { Double Difference GPS (Carrier } \\
\text { Phase) }\end{array}$ & 2.744 & 0.042 & 1.996 \\
\hline IMU Measurement & 0.007 & - & 0.005 \\
\hline
\end{tabular}

Table 205: Mean Global Error Statistics of Fused Heading for Simulation Set C-4

\begin{tabular}{|c|c|c|c|}
\hline Method & $\begin{array}{c}\text { Measured Standard } \\
\text { Deviation } \\
\text { (degrees) }\end{array}$ & $\begin{array}{c}\text { Estimated } \\
\text { Standard } \\
\text { Deviation } \\
\text { (degrees) }\end{array}$ & $\begin{array}{c}\text { Mean RMS } \\
\text { Error } \\
\text { (degrees) }\end{array}$ \\
\hline Single Point GPS (Pseudoranges) & 0.652 & 6.958 & 0.38 \\
\hline Single Point GPS (Carrier Phase) & 13.212 & 0.059 & 10.381 \\
\hline $\begin{array}{c}\text { Single Difference GPS } \\
\text { (Pseudoranges) }\end{array}$ & 5.123 & 6.373 & 1.21 \\
\hline $\begin{array}{c}\text { Single Difference GPS (Carrier } \\
\text { Phase) }\end{array}$ & 18.57 & 0.221 & 13.449 \\
\hline $\begin{array}{c}\text { Double Difference GPS } \\
\text { (Pseudoranges) }\end{array}$ & 1.536 & 7.811 & 0.563 \\
\hline $\begin{array}{c}\text { Double Difference GPS (Carrier } \\
\text { Phase) }\end{array}$ & 25.235 & 0.107 & 15.388 \\
\hline IMU Measurement & 0.315 & - & 0.252 \\
\hline
\end{tabular}


Table 206: Success Rates of Reaching Angular Specifications of Unfused Pitch for Simulation Data Set C-4

\begin{tabular}{|c|c|c|c|c|c|c|c|c|}
\hline & \multicolumn{7}{|c|}{ Portion of Trials in Which Target Specification was reached } \\
\hline & $\pm 0.5^{\circ}$ & $\pm 1^{\circ}$ & $\pm 2^{\circ}$ & $\pm 5^{\circ}$ & $\pm 10^{\circ}$ & $\pm 15^{\circ}$ & $\pm 20^{\circ}$ & $\pm 25^{\circ}$ \\
\hline Method & 52 & 55 & 56 & 60 & 75 & 82 & 88 & 93 \\
\hline $\begin{array}{c}\text { Single Point GPS } \\
\text { (Pseudoranges) }\end{array}$ & 51 & 51 & 54 & 61 & 77 & 85 & 91 & 95 \\
\hline $\begin{array}{c}\text { Single Point GPS (Carrier } \\
\text { Phase) }\end{array}$ & 55 & 56 & 57 & 62 & 68 & 74 & 77 & 80 \\
\hline $\begin{array}{c}\text { Single Difference GPS } \\
\text { (Pseudoranges) }\end{array}$ & 54 & 54 & 54 & 57 & 67 & 72 & 78 & 83 \\
\hline $\begin{array}{c}\text { Single Difference GPS } \\
\text { (Carrier Phase) }\end{array}$ & 50 & 52 & 53 & 62 & 70 & 77 & 82 & 87 \\
\hline $\begin{array}{c}\text { Double Difference GPS } \\
\text { (Pseudoranges) }\end{array}$ & 48 & 50 & 51 & 62 & 71 & 75 & 81 & 86 \\
\hline $\begin{array}{c}\text { Double Difference GPS } \\
\text { (Carrier Phase) }\end{array}$ & 100 & 100 & 100 & 100 & 100 & 100 & 100 & 100 \\
\hline IMU Measured & & & & & & & & \\
\hline
\end{tabular}

Table 207: Success Rates of Reaching Angular Specifications of Unfused Heading for Simulation Data Set C-4

\begin{tabular}{|c|c|c|c|c|c|c|c|c|}
\hline & \multicolumn{7}{|c|}{ Portion of Trials in Which Target Specification was reached } \\
\hline Method & $\pm 0.5^{\circ}$ & $\pm 1^{\circ}$ & $\pm 2^{\circ}$ & $\pm 5^{\circ}$ & $\pm 10^{\circ}$ & $\pm 15^{\circ}$ & $\pm 20^{\circ}$ & $\pm 25^{\circ}$ \\
\hline $\begin{array}{c}\text { Single Point GPS } \\
\text { (Pseudoranges) }\end{array}$ & 40 & 41 & 45 & 48 & 64 & 75 & 84 & 89 \\
\hline $\begin{array}{c}\text { Single Point GPS (Carrier } \\
\text { Phase) }\end{array}$ & 43 & 43 & 43 & 48 & 63 & 76 & 86 & 90 \\
\hline $\begin{array}{c}\text { Single Difference GPS } \\
\text { (Pseudoranges) }\end{array}$ & 58 & 60 & 61 & 63 & 69 & 74 & 77 & 79 \\
\hline $\begin{array}{c}\text { Single Difference GPS } \\
\text { (Carrier Phase) }\end{array}$ & 57 & 57 & 59 & 67 & 71 & 75 & 77 & 79 \\
\hline $\begin{array}{c}\text { Double Difference GPS } \\
\text { (Pseudoranges) }\end{array}$ & 51 & 51 & 52 & 57 & 65 & 74 & 77 & 81 \\
\hline $\begin{array}{c}\text { Double Difference GPS } \\
\text { (Carrier Phase) }\end{array}$ & 51 & 52 & 53 & 56 & 65 & 73 & 76 & 79 \\
\hline IMU Measured & 92 & 100 & 100 & 100 & 100 & 100 & 100 & 100 \\
\hline
\end{tabular}


Table 208: Success Rates of Reaching Angular Specifications of Fused Pitch for Simulation Data Set C-5

\begin{tabular}{|c|c|c|c|c|c|c|c|c|}
\hline & \multicolumn{7}{|c|}{ Portion of Trials in Which Target Specification was reached } \\
\hline & $\pm 0.5^{\circ}$ & $\pm 1^{\circ}$ & $\pm 2^{\circ}$ & $\pm 5^{\circ}$ & $\pm 10^{\circ}$ & $\pm 15^{\circ}$ & $\pm 20^{\circ}$ & $\pm 25^{\circ}$ \\
\hline Method & 100 & 100 & 100 & 100 & 100 & 100 & 100 & 100 \\
\hline $\begin{array}{c}\text { Single Point GPS } \\
\text { (Pseudoranges) }\end{array}$ & 58 & 76 & 99 & 99 & 99 & 99 & 99 & 100 \\
\hline $\begin{array}{c}\text { Single Point GPS (Carrier } \\
\text { Phase) }\end{array}$ & 100 & 100 & 100 & 100 & 100 & 100 & 100 & 100 \\
\hline $\begin{array}{c}\text { Single Difference GPS } \\
\text { (Pseudoranges) }\end{array}$ & 56 & 66 & 90 & 97 & 100 & 100 & 100 & 100 \\
\hline $\begin{array}{c}\text { Single Difference GPS } \\
\text { (Carrier Phase) }\end{array}$ & 100 & 100 & 100 & 100 & 100 & 100 & 100 & 100 \\
\hline $\begin{array}{c}\text { Double Difference GPS } \\
\text { (Pseudoranges) }\end{array}$ & 60 & 75 & 91 & 97 & 99 & 100 & 100 & 100 \\
\hline $\begin{array}{c}\text { Double Difference GPS } \\
\text { (Carrier Phase) }\end{array}$ & 100 & 100 & 100 & 100 & 100 & 100 & 100 & 100 \\
\hline IMU Measured & & & & & & & & \\
\hline
\end{tabular}

Table 209: Success Rates of Reaching Angular Specifications of Fused Heading for Simulation Data Set C-5

\begin{tabular}{|c|c|c|c|c|c|c|c|c|}
\hline & \multicolumn{7}{|c|}{ Portion of Trials in Which Target Specification was reached } \\
\hline Method & $\pm 0.5^{\circ}$ & $\pm 1^{\circ}$ & $\pm 2^{\circ}$ & $\pm 5^{\circ}$ & $\pm 10^{\circ}$ & $\pm 15^{\circ}$ & $\pm 20^{\circ}$ & $\pm 25^{\circ}$ \\
\hline $\begin{array}{c}\text { Single Point GPS } \\
\text { (Pseudoranges) }\end{array}$ & 85 & 98 & 100 & 100 & 100 & 100 & 100 & 100 \\
\hline $\begin{array}{c}\text { Single Point GPS (Carrier } \\
\text { Phase) }\end{array}$ & 43 & 43 & 43 & 51 & 70 & 86 & 91 & 94 \\
\hline $\begin{array}{c}\text { Single Difference GPS } \\
\text { (Pseudoranges) }\end{array}$ & 85 & 96 & 96 & 97 & 98 & 99 & 99 & 99 \\
\hline $\begin{array}{c}\text { Single Difference GPS } \\
\text { (Carrier Phase) }\end{array}$ & 58 & 58 & 61 & 70 & 78 & 84 & 87 & 89 \\
\hline $\begin{array}{c}\text { Double Difference GPS } \\
\text { (Pseudoranges) }\end{array}$ & 81 & 95 & 97 & 99 & 99 & 100 & 100 & 100 \\
\hline $\begin{array}{c}\text { Double Difference GPS } \\
\text { (Carrier Phase) }\end{array}$ & 51 & 52 & 54 & 61 & 73 & 80 & 82 & 87 \\
\hline IMU Measured & 92 & 100 & 100 & 100 & 100 & 100 & 100 & 100 \\
\hline
\end{tabular}


Table 210: Pitch / Heading Correlation Coefficients for Each Computation Method in Simulation Data Set C-4

\begin{tabular}{|c|c|c|}
\hline & \multicolumn{2}{|c|}{ Pitch / Heading Correlation Coefficient } \\
\hline Method & Unfused & Fused \\
\hline $\begin{array}{c}\text { Single Point GPS } \\
\text { (Pseudoranges) }\end{array}$ & 0.785031 & 0.000681 \\
\hline $\begin{array}{c}\text { Single Difference GPS } \\
\text { (Pseudoranges) }\end{array}$ & 0.945713 & 0.4263 \\
\hline $\begin{array}{c}\text { Double Difference GPS } \\
\text { (Pseudoranges) }\end{array}$ & -0.92185 & -0.00346 \\
\hline
\end{tabular}

\section{Set C-5 30 m Dual Antenna Separation}

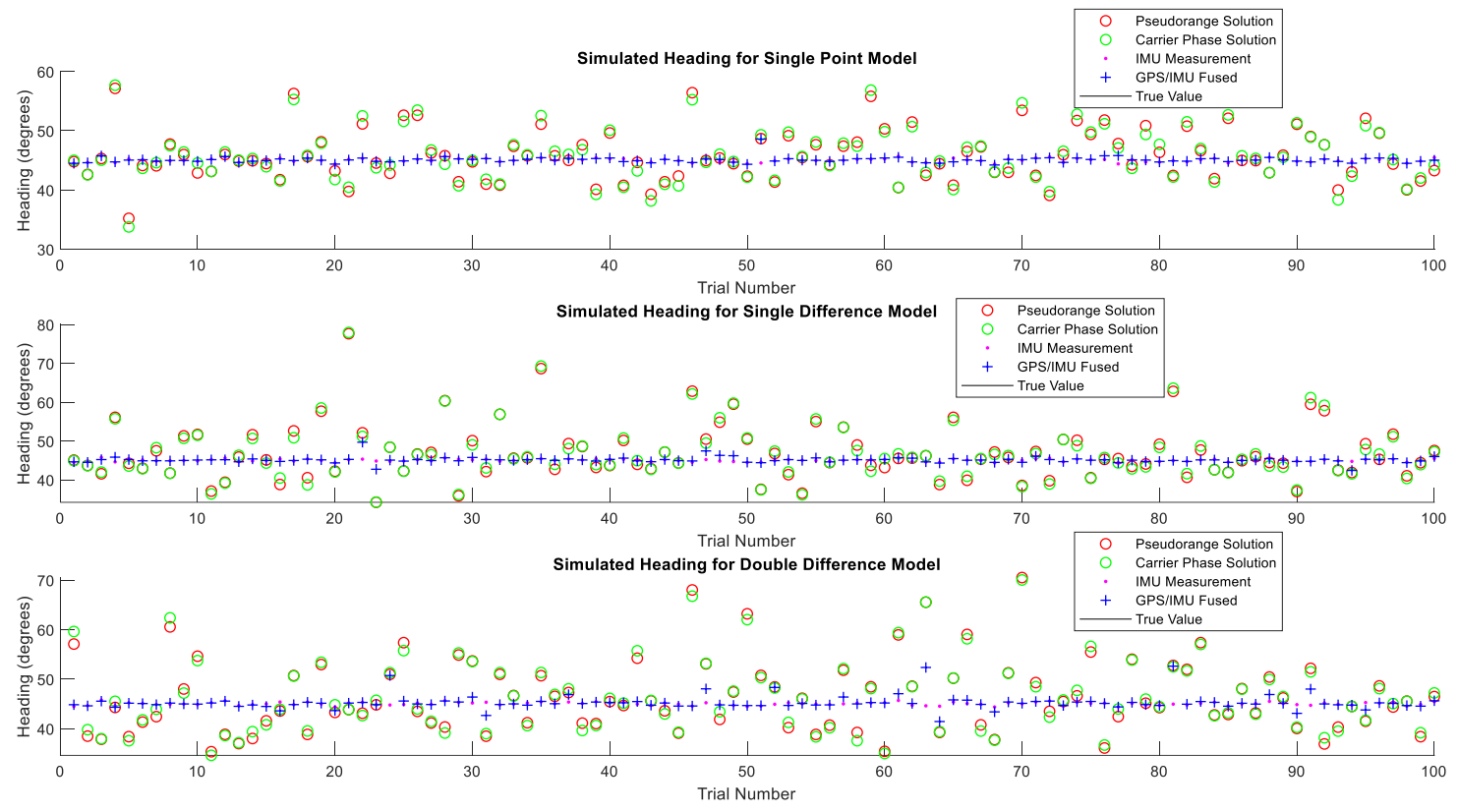

Figure 108: Computed and Measured Headings for Simulation Set C-5 


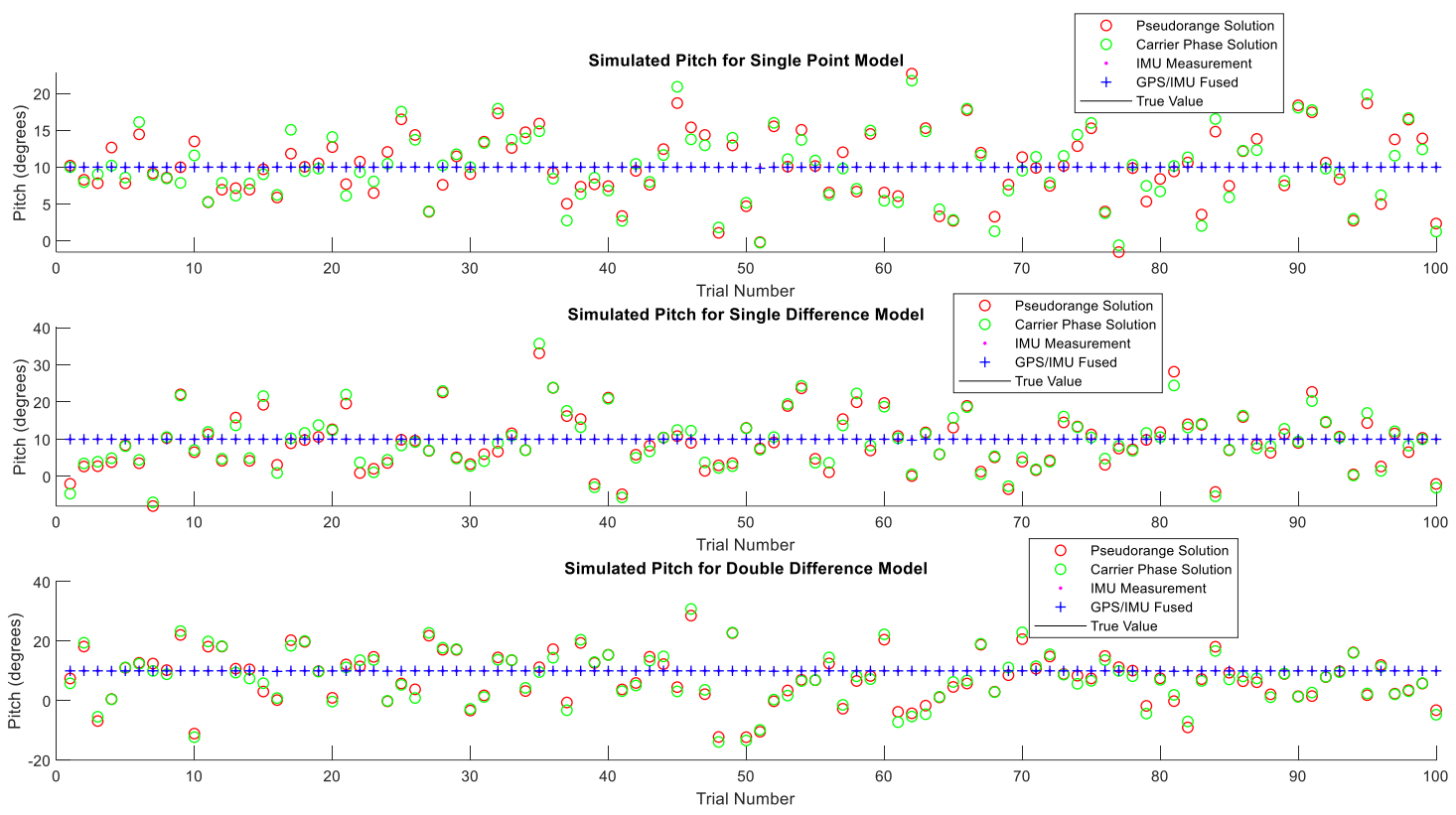

Figure 109: Computed and Measured Pitch for Simulation Set C-5

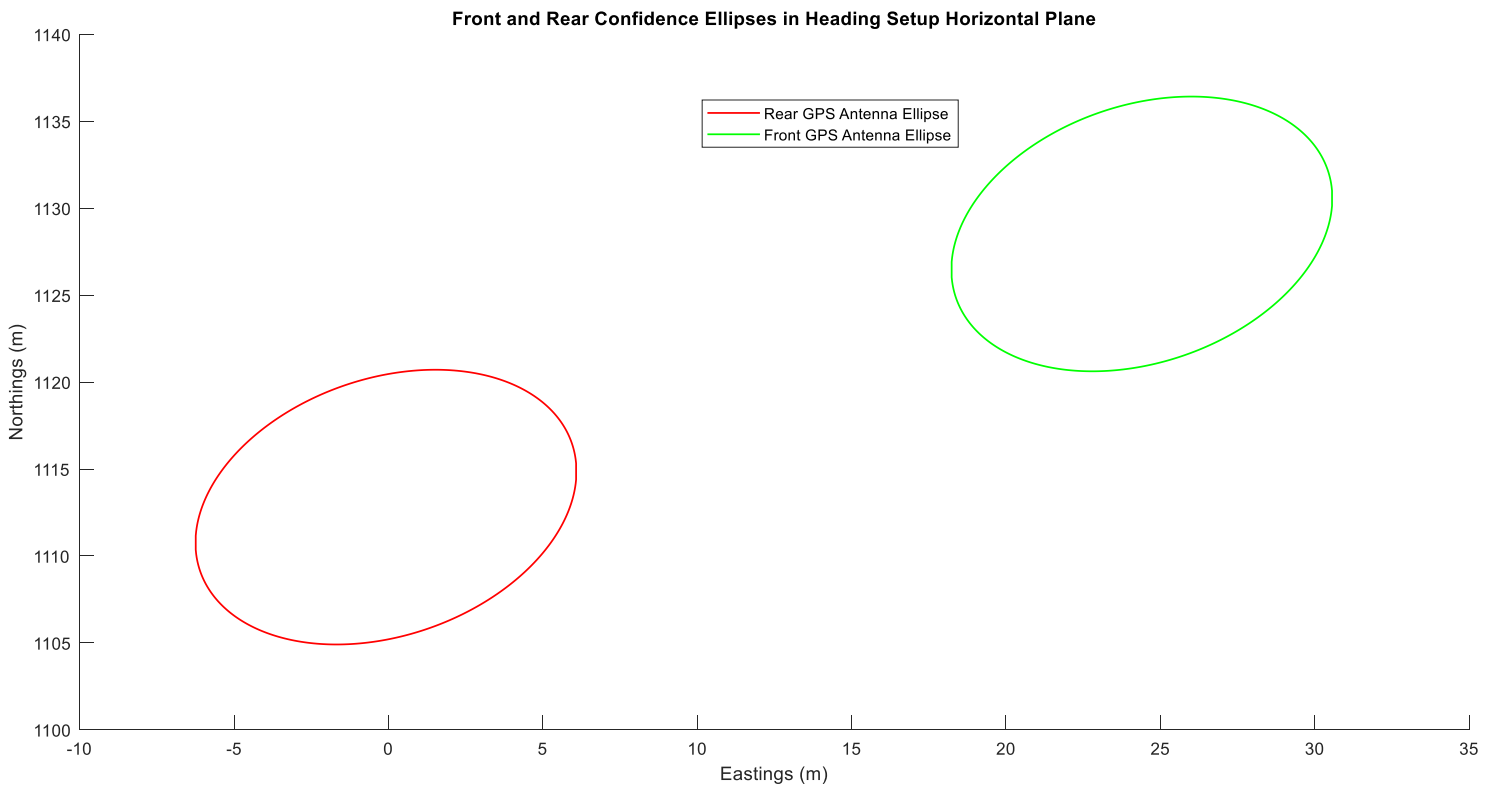

Figure 110: Propagated 95\% Confidence Ellipses for Front and Rear GPS Antennas in Simulated Data Set C-5 
Table 211: Mean Global Error Statistics of Unfused Pitch for Simulation Set C-5

\begin{tabular}{|c|c|c|c|}
\hline Method & $\begin{array}{c}\text { Measured Standard } \\
\text { Deviation } \\
\text { (degrees) }\end{array}$ & $\begin{array}{c}\text { Estimated } \\
\text { Standard } \\
\text { Deviation } \\
\text { (degrees) }\end{array}$ & $\begin{array}{c}\text { Mean RMS } \\
\text { Error } \\
\text { (degrees) }\end{array}$ \\
\hline Single Point GPS (Pseudoranges) & 4.691 & 25.361 & 3.786 \\
\hline Single Point GPS (Carrier Phase) & 4.849 & 0.01 & 3.834 \\
\hline $\begin{array}{c}\text { Single Difference GPS } \\
\text { (Pseudoranges) }\end{array}$ & 7.441 & 23.58 & 5.87 \\
\hline $\begin{array}{c}\text { Single Difference GPS (Carrier } \\
\text { Phase) }\end{array}$ & 7.591 & 0.01 & 5.939 \\
\hline $\begin{array}{c}\text { Double Difference GPS } \\
\text { (Pseudoranges) }\end{array}$ & 8.398 & 18.793 & 6.91 \\
\hline $\begin{array}{c}\text { Double Difference GPS (Carrier } \\
\text { Phase) }\end{array}$ & 8.739 & 0.019 & 7.173 \\
\hline IMU Measurement & 0.007 & - & 0.005 \\
\hline
\end{tabular}

Table 212: Mean Global Error Statistics of Unfused Heading for Simulation Set C-5

\begin{tabular}{|c|c|c|c|}
\hline Method & $\begin{array}{c}\text { Measured Standard } \\
\text { Deviation } \\
\text { (degrees) }\end{array}$ & $\begin{array}{c}\text { Estimated } \\
\text { Standard } \\
\text { Deviation } \\
\text { (degrees) }\end{array}$ & $\begin{array}{c}\text { Mean RMS } \\
\text { Error } \\
\text { (degrees) }\end{array}$ \\
\hline Single Point GPS (Pseudoranges) & 4.247 & 23.916 & 3.281 \\
\hline Single Point GPS (Carrier Phase) & 4.413 & 0.007 & 3.439 \\
\hline $\begin{array}{c}\text { Single Difference GPS } \\
\text { (Pseudoranges) }\end{array}$ & 7.089 & 27.081 & 5.033 \\
\hline $\begin{array}{c}\text { Single Difference GPS (Carrier } \\
\text { Phase) }\end{array}$ & 7.155 & 0.011 & 5.032 \\
\hline $\begin{array}{c}\text { Double Difference GPS } \\
\text { (Pseudoranges) }\end{array}$ & 7.3 & 19.968 & 5.652 \\
\hline $\begin{array}{c}\text { Double Difference GPS (Carrier } \\
\text { Phase) }\end{array}$ & 7.277 & 0.02 & 5.647 \\
\hline IMU Measurement & 0.312 & - & 0.255 \\
\hline
\end{tabular}


Table 213: Mean Global Error Statistics of Fused Pitch for Simulation Set C-5

\begin{tabular}{|c|c|c|c|}
\hline Method & $\begin{array}{c}\text { Measured Standard } \\
\text { Deviation } \\
\text { (degrees) }\end{array}$ & $\begin{array}{c}\text { Estimated } \\
\text { Standard } \\
\text { Deviation } \\
\text { (degrees) }\end{array}$ & $\begin{array}{c}\text { Mean RMS } \\
\text { Error } \\
\text { (degrees) }\end{array}$ \\
\hline Single Point GPS (Pseudoranges) & 0.017 & 0.2 & 0.007 \\
\hline Single Point GPS (Carrier Phase) & 2.65 & 0.005 & 1.877 \\
\hline $\begin{array}{c}\text { Single Difference GPS } \\
\text { (Pseudoranges) }\end{array}$ & 0.027 & 0.199 & 0.009 \\
\hline $\begin{array}{c}\text { Single Difference GPS (Carrier } \\
\text { Phase) }\end{array}$ & 3.719 & 0.005 & 2.947 \\
\hline $\begin{array}{c}\text { Double Difference GPS } \\
\text { (Pseudoranges) }\end{array}$ & 0.029 & 0.198 & 0.013 \\
\hline $\begin{array}{c}\text { Double Difference GPS (Carrier } \\
\text { Phase) }\end{array}$ & 3.141 & 0.009 & 2.595 \\
\hline IMU Measurement & 0.007 & - & 0.005 \\
\hline
\end{tabular}

Table 214: Mean Global Error Statistics of Fused Heading for Simulation Set C-5

\begin{tabular}{|c|c|c|c|}
\hline Method & $\begin{array}{c}\text { Measured Standard } \\
\text { Deviation } \\
\text { (degrees) }\end{array}$ & $\begin{array}{c}\text { Estimated } \\
\text { Standard } \\
\text { Deviation } \\
\text { (degrees) }\end{array}$ & $\begin{array}{c}\text { Mean RMS } \\
\text { Error } \\
\text { (degrees) }\end{array}$ \\
\hline Single Point GPS (Pseudoranges) & 0.477 & 4.412 & 0.288 \\
\hline Single Point GPS (Carrier Phase) & 4.29 & 0.004 & 3.351 \\
\hline $\begin{array}{c}\text { Single Difference GPS } \\
\text { (Pseudoranges) }\end{array}$ & 0.739 & 3.921 & 0.408 \\
\hline $\begin{array}{c}\text { Single Difference GPS (Carrier } \\
\text { Phase) }\end{array}$ & 6.32 & 0.005 & 4.703 \\
\hline $\begin{array}{c}\text { Double Difference GPS } \\
\text { (Pseudoranges) }\end{array}$ & 1.492 & 3.82 & 0.751 \\
\hline $\begin{array}{c}\text { Double Difference GPS (Carrier } \\
\text { Phase) }\end{array}$ & 6.582 & 0.01 & 5.23 \\
\hline IMU Measurement & 0.312 & - & 0.255 \\
\hline
\end{tabular}


Table 215: Success Rates of Reaching Angular Specifications of Unfused Pitch for Simulation Data Set C-5

\begin{tabular}{|c|c|c|c|c|c|c|c|c|}
\hline & \multicolumn{7}{|c|}{ Portion of Trials in Which Target Specification was reached } \\
\hline & $\pm 0.5^{\circ}$ & $\pm 1^{\circ}$ & $\pm 2^{\circ}$ & $\pm 5^{\circ}$ & $\pm 10^{\circ}$ & $\pm 15^{\circ}$ & $\pm 20^{\circ}$ & $\pm 25^{\circ}$ \\
\hline Method & 56 & 60 & 64 & 84 & 99 & 100 & 100 & 100 \\
\hline $\begin{array}{c}\text { Single Point GPS } \\
\text { (Pseudoranges) }\end{array}$ & 59 & 60 & 69 & 85 & 98 & 100 & 100 & 100 \\
\hline $\begin{array}{c}\text { Single Point GPS (Carrier } \\
\text { Phase) }\end{array}$ & 61 & 65 & 72 & 81 & 92 & 98 & 99 & 100 \\
\hline $\begin{array}{c}\text { Single Difference GPS } \\
\text { (Pseudoranges) }\end{array}$ & 59 & 63 & 67 & 81 & 89 & 99 & 99 & 99 \\
\hline $\begin{array}{c}\text { Single Difference GPS } \\
\text { (Carrier Phase) }\end{array}$ & 61 & 64 & 69 & 81 & 93 & 99 & 100 & 100 \\
\hline $\begin{array}{c}\text { Double Difference GPS } \\
\text { (Pseudoranges) }\end{array}$ & 65 & 65 & 70 & 81 & 92 & 99 & 99 & 100 \\
\hline $\begin{array}{c}\text { Double Difference GPS } \\
\text { (Carrier Phase) }\end{array}$ & 100 & 100 & 100 & 100 & 100 & 100 & 100 & 100 \\
\hline IMU Measured & & & & & & & & \\
\hline
\end{tabular}

Table 216: Success Rates of Reaching Angular Specifications of Unfused Heading for Simulation Data Set C-5

\begin{tabular}{|c|c|c|c|c|c|c|c|c|}
\hline & \multicolumn{7}{|c|}{ Portion of Trials in Which Target Specification was reached } \\
\hline Method & $\pm 0.5^{\circ}$ & $\pm 1^{\circ}$ & $\pm 2^{\circ}$ & $\pm 5^{\circ}$ & $\pm 10^{\circ}$ & $\pm 15^{\circ}$ & $\pm 20^{\circ}$ & $\pm 25^{\circ}$ \\
\hline $\begin{array}{c}\text { Single Point GPS } \\
\text { (Pseudoranges) }\end{array}$ & 54 & 62 & 66 & 82 & 96 & 100 & 100 & 100 \\
\hline $\begin{array}{c}\text { Single Point GPS (Carrier } \\
\text { Phase) }\end{array}$ & 51 & 56 & 65 & 83 & 96 & 100 & 100 & 100 \\
\hline $\begin{array}{c}\text { Single Difference GPS } \\
\text { (Pseudoranges) }\end{array}$ & 50 & 57 & 60 & 73 & 88 & 95 & 98 & 99 \\
\hline $\begin{array}{c}\text { Single Difference GPS } \\
\text { (Carrier Phase) }\end{array}$ & 49 & 52 & 62 & 76 & 86 & 94 & 98 & 99 \\
\hline $\begin{array}{c}\text { Double Difference GPS } \\
\text { (Pseudoranges) }\end{array}$ & 53 & 55 & 61 & 70 & 89 & 95 & 97 & 99 \\
\hline $\begin{array}{c}\text { Double Difference GPS } \\
\text { (Carrier Phase) }\end{array}$ & 48 & 55 & 59 & 71 & 87 & 95 & 97 & 99 \\
\hline IMU Measured & 94 & 100 & 100 & 100 & 100 & 100 & 100 & 100 \\
\hline
\end{tabular}


Table 217: Success Rates of Reaching Angular Specifications of Fused Pitch for Simulation Data Set C-5

\begin{tabular}{|c|c|c|c|c|c|c|c|c|}
\hline & \multicolumn{7}{|c|}{ Portion of Trials in Which Target Specification was reached } \\
\hline & $\pm 0.5^{\circ}$ & $\pm 1^{\circ}$ & $\pm 2^{\circ}$ & $\pm 5^{\circ}$ & $\pm 10^{\circ}$ & $\pm 15^{\circ}$ & $\pm 20^{\circ}$ & $\pm 25^{\circ}$ \\
\hline Method & 100 & 100 & 100 & 100 & 100 & 100 & 100 & 100 \\
\hline $\begin{array}{c}\text { Single Point GPS } \\
\text { (Pseudoranges) }\end{array}$ & 61 & 72 & 91 & 100 & 100 & 100 & 100 & 100 \\
\hline $\begin{array}{c}\text { Single Point GPS (Carrier } \\
\text { Phase) }\end{array}$ & 100 & 100 & 100 & 100 & 100 & 100 & 100 & 100 \\
\hline $\begin{array}{c}\text { Single Difference GPS } \\
\text { (Pseudoranges) }\end{array}$ & 63 & 72 & 86 & 100 & 100 & 100 & 100 & 100 \\
\hline $\begin{array}{c}\text { Single Difference GPS } \\
\text { (Carrier Phase) }\end{array}$ & 100 & 100 & 100 & 100 & 100 & 100 & 100 & 100 \\
\hline $\begin{array}{c}\text { Double Difference GPS } \\
\text { (Pseudoranges) }\end{array}$ & 70 & 81 & 94 & 100 & 100 & 100 & 100 & 100 \\
\hline $\begin{array}{c}\text { Double Difference GPS } \\
\text { (Carrier Phase) }\end{array}$ & 100 & 100 & 100 & 100 & 100 & 100 & 100 & 100 \\
\hline IMU Measured & & & & & & & & \\
\hline
\end{tabular}

Table 218: Success Rates of Reaching Angular Specifications of Fused Heading for Simulation Data Set C-5

\begin{tabular}{|c|c|c|c|c|c|c|c|c|}
\hline & \multicolumn{7}{|c|}{ Portion of Trials in Which Target Specification was reached } \\
\hline & $\pm 0.5^{\circ}$ & $\pm 1^{\circ}$ & $\pm 2^{\circ}$ & $\pm 5^{\circ}$ & $\pm 10^{\circ}$ & $\pm 15^{\circ}$ & $\pm 20^{\circ}$ & $\pm 25^{\circ}$ \\
\hline Method & 93 & 99 & 99 & 100 & 100 & 100 & 100 & 100 \\
\hline $\begin{array}{c}\text { Single Point GPS } \\
\text { (Pseudoranges) }\end{array}$ & 51 & 56 & 65 & 83 & 98 & 100 & 100 & 100 \\
\hline $\begin{array}{c}\text { Single Point GPS (Carrier } \\
\text { Phase) }\end{array}$ & 87 & 95 & 98 & 100 & 100 & 100 & 100 & 100 \\
\hline $\begin{array}{c}\text { Single Difference GPS } \\
\text { (Pseudoranges) }\end{array}$ & 49 & 52 & 62 & 76 & 87 & 96 & 99 & 100 \\
\hline $\begin{array}{c}\text { Single Difference GPS } \\
\text { (Carrier Phase) }\end{array}$ & 81 & 89 & 93 & 97 & 100 & 100 & 100 & 100 \\
\hline $\begin{array}{c}\text { Double Difference GPS } \\
\text { (Pseudoranges) }\end{array}$ & 48 & 55 & 60 & 73 & 88 & 96 & 99 & 100 \\
\hline $\begin{array}{c}\text { Double Difference GPS } \\
\text { (Carrier Phase) }\end{array}$ & 94 & 100 & 100 & 100 & 100 & 100 & 100 & 100 \\
\hline IMU Measured & & & & & & & & \\
\hline
\end{tabular}


Table 219: Pitch / Heading Correlation Coefficients for Each Computation Method in Simulation Data Set C-5

\begin{tabular}{|c|c|c|}
\hline & \multicolumn{2}{|c|}{ Pitch / Heading Correlation Coefficient } \\
\hline Method & Unfused & Fused \\
\hline $\begin{array}{c}\text { Single Point GPS } \\
\text { (Pseudoranges) }\end{array}$ & 0.914066 & 0.147612 \\
\hline $\begin{array}{c}\text { Single Difference GPS } \\
\text { (Pseudoranges) }\end{array}$ & -0.90226 & -0.14099 \\
\hline $\begin{array}{c}\text { Double Difference GPS } \\
\text { (Pseudoranges) }\end{array}$ & -0.85897 & -0.09175 \\
\hline
\end{tabular}

\section{Set C-6 50 m Dual Antenna Separation}

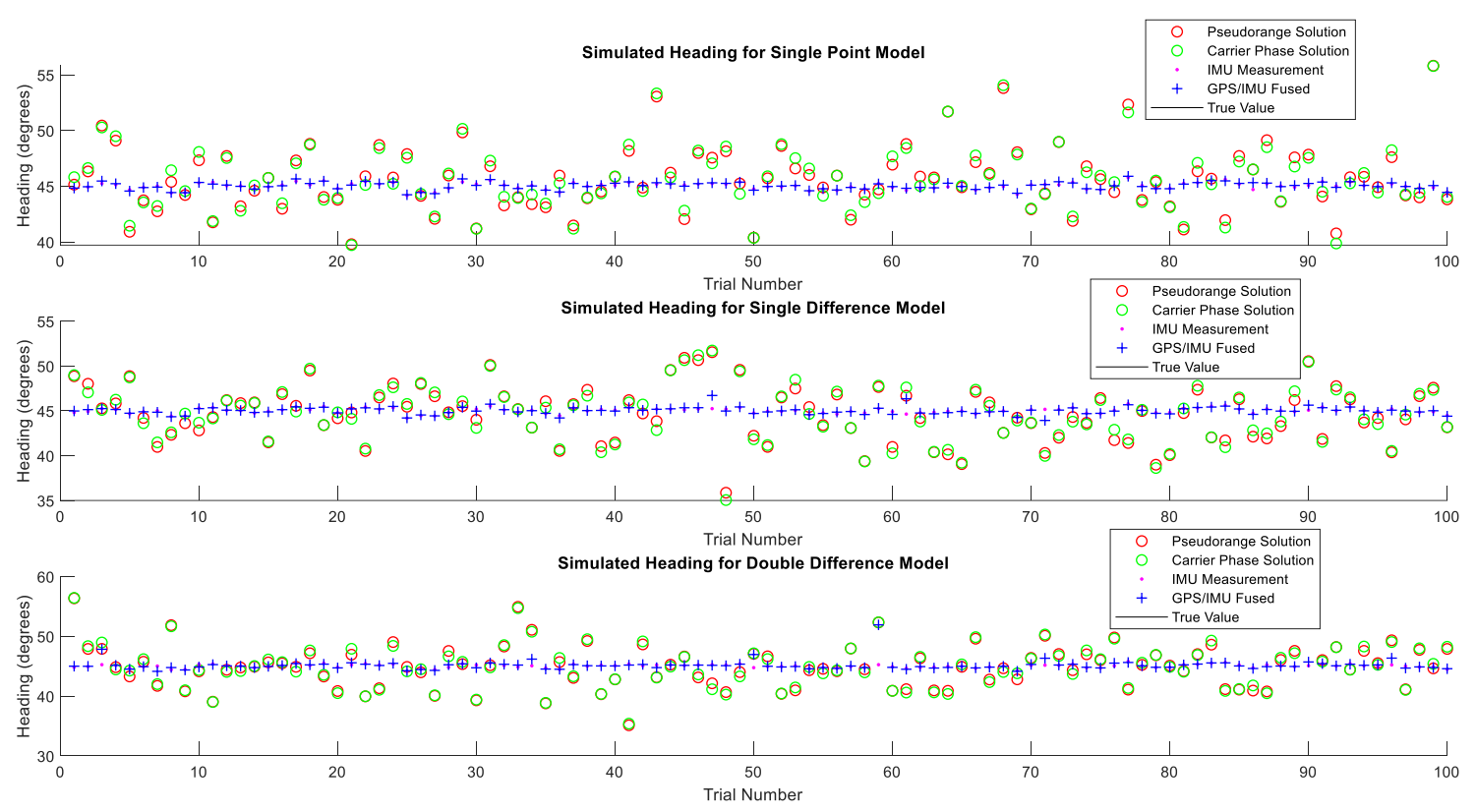

Figure 111: Computed and Measured Headings for Simulation Set C-6 


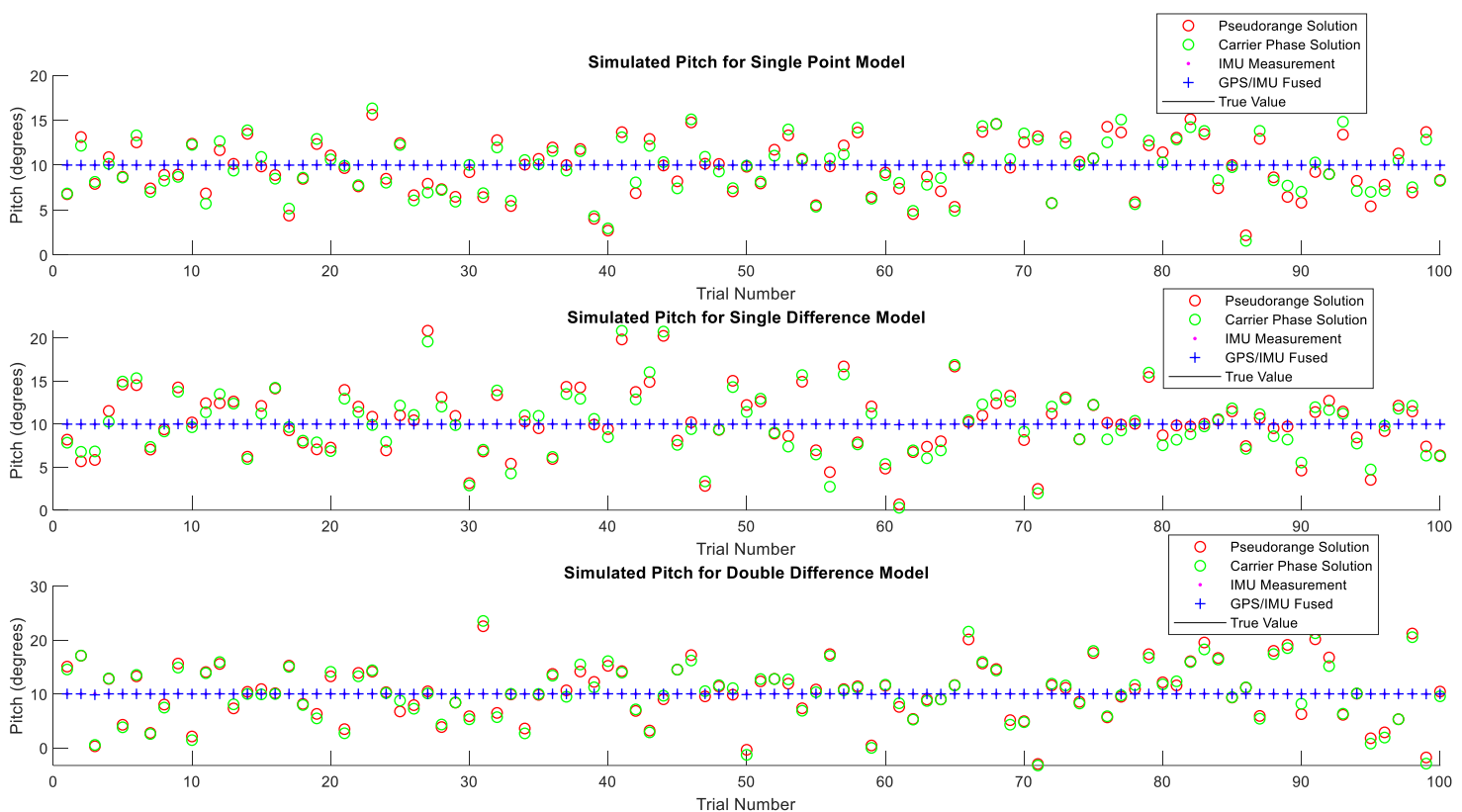

Figure 112: Computed and Measured Pitch for Simulation Set C-6

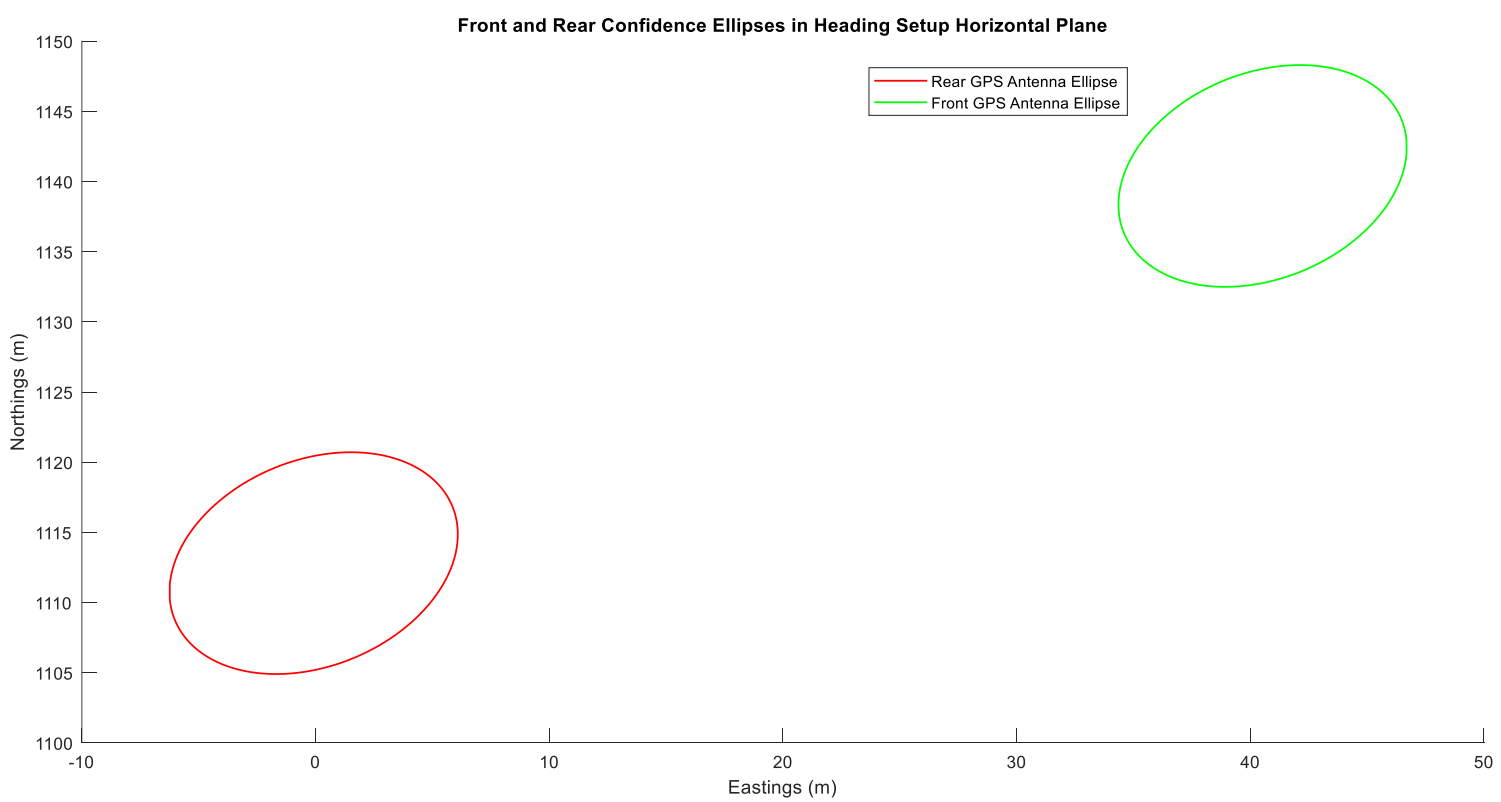

Figure 113: Propagated 95\% Confidence Ellipses for Front and Rear GPS Antennas in Simulated Data Set C-6 
Table 220: Mean Global Error Statistics of Unfused Pitch for Simulation Set C-6

\begin{tabular}{|c|c|c|c|}
\hline Method & $\begin{array}{c}\text { Measured Standard } \\
\text { Deviation } \\
\text { (degrees) }\end{array}$ & $\begin{array}{c}\text { Estimated } \\
\text { Standard } \\
\text { Deviation } \\
\text { (degrees) }\end{array}$ & $\begin{array}{c}\text { Mean RMS } \\
\text { Error } \\
\text { (degrees) }\end{array}$ \\
\hline Single Point GPS (Pseudoranges) & 3.001 & 15.258 & 2.5 \\
\hline Single Point GPS (Carrier Phase) & 3.011 & 0.006 & 2.49 \\
\hline $\begin{array}{c}\text { Single Difference GPS } \\
\text { (Pseudoranges) }\end{array}$ & 3.722 & 15.697 & 2.86 \\
\hline $\begin{array}{c}\text { Single Difference GPS (Carrier } \\
\text { Phase) }\end{array}$ & 3.783 & 0.006 & 2.963 \\
\hline $\begin{array}{c}\text { Double Difference GPS } \\
\text { (Pseudoranges) }\end{array}$ & 5.312 & 12.479 & 4.219 \\
\hline $\begin{array}{c}\text { Double Difference GPS (Carrier } \\
\text { Phase) }\end{array}$ & 5.452 & 0.012 & 4.267 \\
\hline IMU Measurement & 0.008 & - & 0.006 \\
\hline
\end{tabular}

Table 221: Mean Global Error Statistics of Unfused Heading for Simulation Set C-6

\begin{tabular}{|c|c|c|c|}
\hline Method & $\begin{array}{c}\text { Measured Standard } \\
\text { Deviation } \\
\text { (degrees) }\end{array}$ & $\begin{array}{c}\text { Estimated } \\
\text { Standard } \\
\text { Deviation } \\
\text { (degrees) }\end{array}$ & $\begin{array}{c}\text { Mean RMS } \\
\text { Error } \\
\text { (degrees) }\end{array}$ \\
\hline Single Point GPS (Pseudoranges) & 2.909 & 12.756 & 2.244 \\
\hline Single Point GPS (Carrier Phase) & 2.912 & 0.004 & 2.234 \\
\hline $\begin{array}{c}\text { Single Difference GPS } \\
\text { (Pseudoranges) }\end{array}$ & 3.046 & 14.389 & 2.482 \\
\hline $\begin{array}{c}\text { Single Difference GPS (Carrier } \\
\text { Phase) }\end{array}$ & 3.092 & 0.004 & 2.51 \\
\hline $\begin{array}{c}\text { Double Difference GPS } \\
\text { (Pseudoranges) }\end{array}$ & 3.633 & 11.547 & 2.789 \\
\hline $\begin{array}{c}\text { Double Difference GPS (Carrier } \\
\text { Phase) }\end{array}$ & 3.673 & 0.012 & 2.881 \\
\hline IMU Measurement & 0.304 & - & 0.239 \\
\hline
\end{tabular}


Table 222: Mean Global Error Statistics of Fused Pitch for Simulation Set C-6

\begin{tabular}{|c|c|c|c|}
\hline Method & $\begin{array}{c}\text { Measured Standard } \\
\text { Deviation } \\
\text { (degrees) }\end{array}$ & $\begin{array}{c}\text { Estimated } \\
\text { Standard } \\
\text { Deviation } \\
\text { (degrees) }\end{array}$ & $\begin{array}{c}\text { Mean RMS } \\
\text { Error } \\
\text { (degrees) }\end{array}$ \\
\hline Single Point GPS (Pseudoranges) & 0.008 & 0.2 & 0.006 \\
\hline Single Point GPS (Carrier Phase) & 1.856 & 0.003 & 1.467 \\
\hline $\begin{array}{c}\text { Single Difference GPS } \\
\text { (Pseudoranges) }\end{array}$ & 0.01 & 0.2 & 0.007 \\
\hline $\begin{array}{c}\text { Single Difference GPS (Carrier } \\
\text { Phase) }\end{array}$ & 2.264 & 0.003 & 1.696 \\
\hline $\begin{array}{c}\text { Double Difference GPS } \\
\text { (Pseudoranges) }\end{array}$ & 0.025 & 0.199 & 0.011 \\
\hline $\begin{array}{c}\text { Double Difference GPS (Carrier } \\
\text { Phase) }\end{array}$ & 2.768 & 0.006 & 1.868 \\
\hline IMU Measurement & 0.008 & - & 0.006 \\
\hline
\end{tabular}

Table 223: Mean Global Error Statistics of Fused Heading for Simulation Set C-6

\begin{tabular}{|c|c|c|c|}
\hline Method & $\begin{array}{c}\text { Measured Standard } \\
\text { Deviation } \\
\text { (degrees) }\end{array}$ & $\begin{array}{c}\text { Estimated } \\
\text { Standard } \\
\text { Deviation } \\
\text { (degrees) }\end{array}$ & $\begin{array}{c}\text { Mean RMS } \\
\text { Error } \\
\text { (degrees) }\end{array}$ \\
\hline Single Point GPS (Pseudoranges) & 0.318 & 2.966 & 0.253 \\
\hline Single Point GPS (Carrier Phase) & 2.869 & 0.002 & 2.206 \\
\hline $\begin{array}{c}\text { Single Difference GPS } \\
\text { (Pseudoranges) }\end{array}$ & 0.402 & 3.206 & 0.29 \\
\hline $\begin{array}{c}\text { Single Difference GPS (Carrier } \\
\text { Phase) }\end{array}$ & 3.06 & 0.002 & 2.483 \\
\hline $\begin{array}{c}\text { Double Difference GPS } \\
\text { (Pseudoranges) }\end{array}$ & 0.859 & 3.192 & 0.409 \\
\hline $\begin{array}{c}\text { Double Difference GPS (Carrier } \\
\text { Phase) }\end{array}$ & 3.545 & 0.006 & 2.781 \\
\hline IMU Measurement & 0.304 & - & 0.239 \\
\hline
\end{tabular}


Table 224: Success Rates of Reaching Angular Specifications of Unfused Pitch for Simulation Data Set C-6

\begin{tabular}{|c|c|c|c|c|c|c|c|c|}
\hline & \multicolumn{7}{|c|}{ Portion of Trials in Which Target Specification was reached } \\
\hline & $\pm 0.5^{\circ}$ & $\pm 1^{\circ}$ & $\pm 2^{\circ}$ & $\pm 5^{\circ}$ & $\pm 10^{\circ}$ & $\pm 15^{\circ}$ & $\pm 20^{\circ}$ & $\pm 25^{\circ}$ \\
\hline Method & 60 & 65 & 72 & 98 & 100 & 100 & 100 & 100 \\
\hline $\begin{array}{c}\text { Single Point GPS } \\
\text { (Pseudoranges) }\end{array}$ & 58 & 68 & 73 & 97 & 100 & 100 & 100 & 100 \\
\hline $\begin{array}{c}\text { Single Point GPS (Carrier } \\
\text { Phase) }\end{array}$ & 56 & 59 & 67 & 93 & 98 & 100 & 100 & 100 \\
\hline $\begin{array}{c}\text { Single Difference GPS } \\
\text { (Pseudoranges) }\end{array}$ & 54 & 57 & 70 & 91 & 98 & 100 & 100 & 100 \\
\hline $\begin{array}{c}\text { Single Difference GPS } \\
\text { (Carrier Phase) }\end{array}$ & 50 & 55 & 64 & 79 & 96 & 100 & 100 & 100 \\
\hline $\begin{array}{c}\text { Double Difference GPS } \\
\text { (Pseudoranges) }\end{array}$ & 51 & 53 & 64 & 80 & 96 & 100 & 100 & 100 \\
\hline $\begin{array}{c}\text { Double Difference GPS } \\
\text { (Carrier Phase) }\end{array}$ & 100 & 100 & 100 & 100 & 100 & 100 & 100 & 100 \\
\hline IMU Measured & & & & & & & & \\
\hline
\end{tabular}

Table 225: Success Rates of Reaching Angular Specifications of Unfused Heading for Simulation Data Set C-6

\begin{tabular}{|c|c|c|c|c|c|c|c|c|}
\hline & \multicolumn{7}{|c|}{ Portion of Trials in Which Target Specification was reached } \\
\hline & $\pm 0.5^{\circ}$ & $\pm 1^{\circ}$ & $\pm 2^{\circ}$ & $\pm 5^{\circ}$ & $\pm 10^{\circ}$ & $\pm 15^{\circ}$ & $\pm 20^{\circ}$ & $\pm 25^{\circ}$ \\
\hline Method & 48 & 62 & 72 & 94 & 99 & 100 & 100 & 100 \\
\hline $\begin{array}{c}\text { Single Point GPS } \\
\text { (Pseudoranges) }\end{array}$ & 51 & 60 & 69 & 93 & 99 & 100 & 100 & 100 \\
\hline $\begin{array}{c}\text { Single Point GPS (Carrier } \\
\text { Phase) }\end{array}$ & 58 & 65 & 80 & 95 & 100 & 100 & 100 & 100 \\
\hline $\begin{array}{c}\text { Single Difference GPS } \\
\text { (Pseudoranges) }\end{array}$ & 58 & 65 & 76 & 96 & 100 & 100 & 100 & 100 \\
\hline $\begin{array}{c}\text { Single Difference GPS } \\
\text { (Carrier Phase) }\end{array}$ & 59 & 63 & 72 & 93 & 99 & 100 & 100 & 100 \\
\hline $\begin{array}{c}\text { Double Difference GPS } \\
\text { (Pseudoranges) }\end{array}$ & 56 & 60 & 73 & 93 & 99 & 100 & 100 & 100 \\
\hline $\begin{array}{c}\text { Double Difference GPS } \\
\text { (Carrier Phase) }\end{array}$ & 97 & 100 & 100 & 100 & 100 & 100 & 100 & 100 \\
\hline IMU Measured & & & & & & & & \\
\hline
\end{tabular}


Table 226: Success Rates of Reaching Angular Specifications of Fused Pitch for Simulation Data Set C-6

\begin{tabular}{|c|c|c|c|c|c|c|c|c|}
\hline & \multicolumn{7}{|c|}{ Portion of Trials in Which Target Specification was reached } \\
\hline & $\pm 0.5^{\circ}$ & $\pm 1^{\circ}$ & $\pm 2^{\circ}$ & $\pm 5^{\circ}$ & $\pm 10^{\circ}$ & $\pm 15^{\circ}$ & $\pm 20^{\circ}$ & $\pm 25^{\circ}$ \\
\hline Method & 100 & 100 & 100 & 100 & 100 & 100 & 100 & 100 \\
\hline $\begin{array}{c}\text { Single Point GPS } \\
\text { (Pseudoranges) }\end{array}$ & 68 & 74 & 95 & 100 & 100 & 100 & 100 & 100 \\
\hline $\begin{array}{c}\text { Single Point GPS (Carrier } \\
\text { Phase) }\end{array}$ & 100 & 100 & 100 & 100 & 100 & 100 & 100 & 100 \\
\hline $\begin{array}{c}\text { Single Difference GPS } \\
\text { (Pseudoranges) }\end{array}$ & 56 & 70 & 90 & 100 & 100 & 100 & 100 & 100 \\
\hline $\begin{array}{c}\text { Single Difference GPS } \\
\text { (Carrier Phase) }\end{array}$ & 100 & 100 & 100 & 100 & 100 & 100 & 100 & 100 \\
\hline $\begin{array}{c}\text { Double Difference GPS } \\
\text { (Pseudoranges) }\end{array}$ & 57 & 69 & 92 & 100 & 100 & 100 & 100 & 100 \\
\hline $\begin{array}{c}\text { Double Difference GPS } \\
\text { (Carrier Phase) }\end{array}$ & 100 & 100 & 100 & 100 & 100 & 100 & 100 & 100 \\
\hline IMU Measured & & & & & & & & \\
\hline
\end{tabular}

Table 227: Success Rates of Reaching Angular Specifications of Fused Heading for Simulation Data Set C-6

\begin{tabular}{|c|c|c|c|c|c|c|c|c|}
\hline & \multicolumn{7}{|c|}{ Portion of Trials in Which Target Specification was reached } \\
\hline & $\pm 0.5^{\circ}$ & $\pm 1^{\circ}$ & $\pm 2^{\circ}$ & $\pm 5^{\circ}$ & $\pm 10^{\circ}$ & $\pm 15^{\circ}$ & $\pm 20^{\circ}$ & $\pm 25^{\circ}$ \\
\hline Method & 96 & 100 & 100 & 100 & 100 & 100 & 100 & 100 \\
\hline $\begin{array}{c}\text { Single Point GPS } \\
\text { (Pseudoranges) }\end{array}$ & 52 & 60 & 69 & 93 & 99 & 100 & 100 & 100 \\
\hline $\begin{array}{c}\text { Single Point GPS (Carrier } \\
\text { Phase) }\end{array}$ & 94 & 98 & 100 & 100 & 100 & 100 & 100 & 100 \\
\hline $\begin{array}{c}\text { Single Difference GPS } \\
\text { (Pseudoranges) }\end{array}$ & 58 & 65 & 77 & 96 & 100 & 100 & 100 & 100 \\
\hline $\begin{array}{c}\text { Single Difference GPS } \\
\text { (Carrier Phase) }\end{array}$ & 87 & 94 & 98 & 99 & 100 & 100 & 100 & 100 \\
\hline $\begin{array}{c}\text { Double Difference GPS } \\
\text { (Pseudoranges) }\end{array}$ & 56 & 60 & 73 & 93 & 99 & 100 & 100 & 100 \\
\hline $\begin{array}{c}\text { Double Difference GPS } \\
\text { (Carrier Phase) }\end{array}$ & 97 & 100 & 100 & 100 & 100 & 100 & 100 & 100 \\
\hline IMU Measured & & & & & & & & \\
\hline
\end{tabular}


Table 228: Pitch / Heading Correlation Coefficients for Each Computation Method in Simulation Data Set C-6

\begin{tabular}{|c|c|c|}
\hline & \multicolumn{2}{|c|}{ Pitch / Heading Correlation Coefficient } \\
\hline Method & Unfused & Fused \\
\hline $\begin{array}{c}\text { Single Point GPS } \\
\text { (Pseudoranges) }\end{array}$ & 0.887863 & 0.059522 \\
\hline $\begin{array}{c}\text { Single Difference GPS } \\
\text { (Pseudoranges) }\end{array}$ & 0.901028 & 0.08013 \\
\hline $\begin{array}{c}\text { Double Difference GPS } \\
\text { (Pseudoranges) }\end{array}$ & 0.748957 & 0.029468 \\
\hline
\end{tabular}

\section{Set C-7 70 m Dual Antenna Separation}

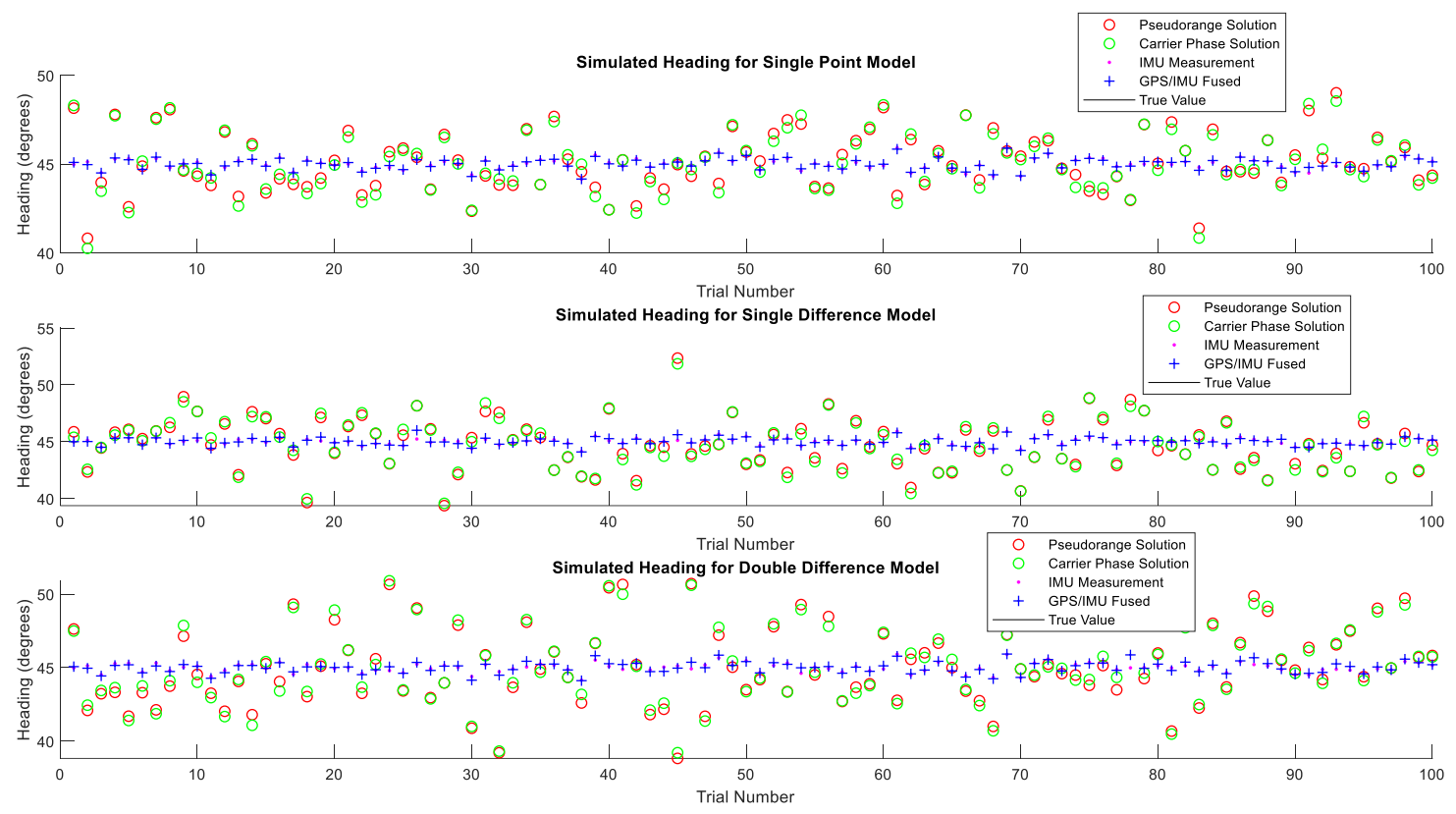

Figure 114: Computed and Measured Headings for Simulation Set C-7 


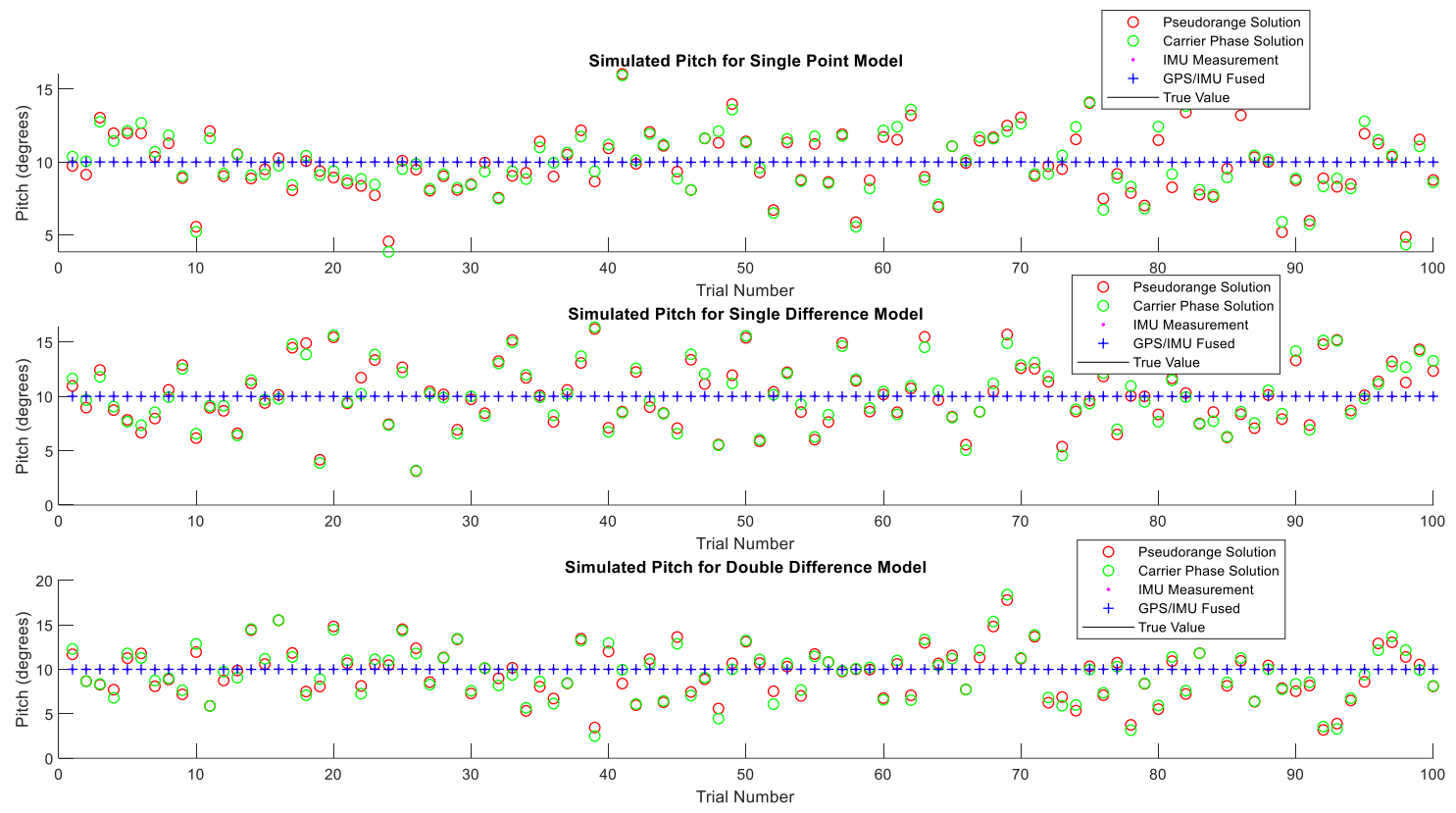

Figure 115: Computed and Measured Pitch for Simulation Set C-7

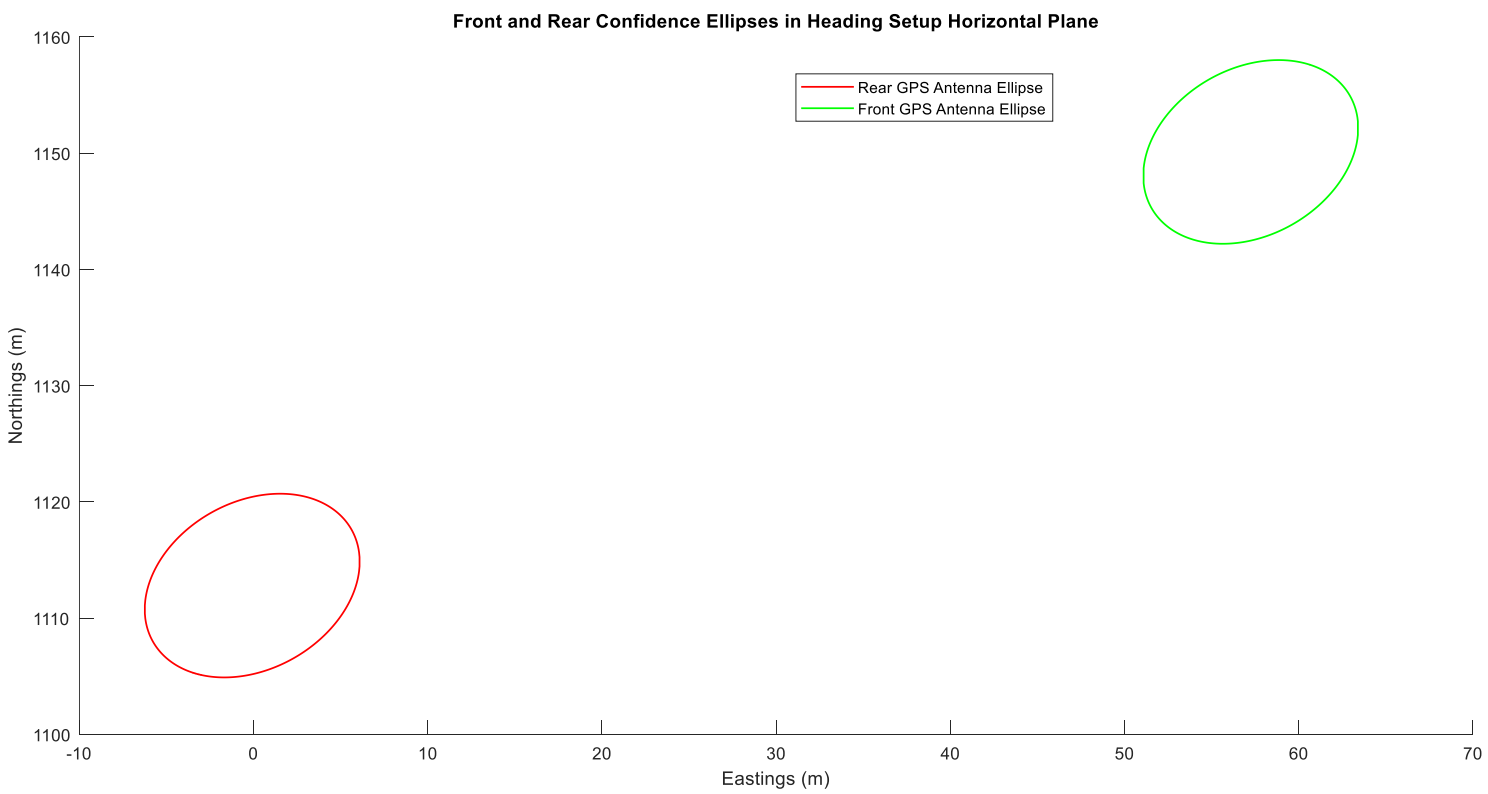

Figure 116: Propagated 95\% Confidence Ellipses for Front and Rear GPS Antennas in Simulated Data Set C-7 
Table 229: Mean Global Error Statistics of Unfused Pitch for Simulation Set C-7

\begin{tabular}{|c|c|c|c|}
\hline Method & $\begin{array}{c}\text { Measured Standard } \\
\text { Deviation } \\
\text { (degrees) }\end{array}$ & $\begin{array}{c}\text { Estimated } \\
\text { Standard } \\
\text { Deviation } \\
\text { (degrees) }\end{array}$ & $\begin{array}{c}\text { Mean RMS } \\
\text { Error } \\
\text { (degrees) }\end{array}$ \\
\hline Single Point GPS (Pseudoranges) & 2.113 & 11.093 & 1.701 \\
\hline Single Point GPS (Carrier Phase) & 2.202 & 0.004 & 1.76 \\
\hline $\begin{array}{c}\text { Single Difference GPS } \\
\text { (Pseudoranges) }\end{array}$ & 2.875 & 11.265 & 2.335 \\
\hline $\begin{array}{c}\text { Single Difference GPS (Carrier } \\
\text { Phase) }\end{array}$ & 2.896 & 0.004 & 2.345 \\
\hline $\begin{array}{c}\text { Double Difference GPS } \\
\text { (Pseudoranges) }\end{array}$ & 2.87 & 8.581 & 2.361 \\
\hline $\begin{array}{c}\text { Double Difference GPS (Carrier } \\
\text { Phase) }\end{array}$ & 2.97 & 0.009 & 2.397 \\
\hline IMU Measurement & 0.007 & - & 0.006 \\
\hline
\end{tabular}

Table 230: Mean Global Error Statistics of Unfused Heading for Simulation Set C-7

\begin{tabular}{|c|c|c|c|}
\hline Method & $\begin{array}{c}\text { Measured Standard } \\
\text { Deviation } \\
\text { (degrees) }\end{array}$ & $\begin{array}{c}\text { Estimated } \\
\text { Standard } \\
\text { Deviation } \\
\text { (degrees) }\end{array}$ & $\begin{array}{c}\text { Mean RMS } \\
\text { Error } \\
\text { (degrees) }\end{array}$ \\
\hline Single Point GPS (Pseudoranges) & 1.632 & 9.1 & 1.33 \\
\hline Single Point GPS (Carrier Phase) & 1.694 & 0.003 & 1.364 \\
\hline $\begin{array}{c}\text { Single Difference GPS } \\
\text { (Pseudoranges) }\end{array}$ & 2.345 & 9.897 & 1.905 \\
\hline $\begin{array}{c}\text { Single Difference GPS (Carrier } \\
\text { Phase) }\end{array}$ & 2.345 & 0.003 & 1.915 \\
\hline $\begin{array}{c}\text { Double Difference GPS } \\
\text { (Pseudoranges) }\end{array}$ & 2.598 & 6.653 & 2.075 \\
\hline $\begin{array}{c}\text { Double Difference GPS (Carrier } \\
\text { Phase) }\end{array}$ & 2.582 & 0.007 & 2.074 \\
\hline IMU Measurement & 0.327 & - & 0.257 \\
\hline
\end{tabular}


Table 231: Mean Global Error Statistics of Fused Pitch for Simulation Set C-7

\begin{tabular}{|c|c|c|c|}
\hline Method & $\begin{array}{c}\text { Measured Standard } \\
\text { Deviation } \\
\text { (degrees) }\end{array}$ & $\begin{array}{c}\text { Estimated } \\
\text { Standard } \\
\text { Deviation } \\
\text { (degrees) }\end{array}$ & $\begin{array}{c}\text { Mean RMS } \\
\text { Error } \\
\text { (degrees) }\end{array}$ \\
\hline Single Point GPS (Pseudoranges) & 0.007 & 0.2 & 0.006 \\
\hline Single Point GPS (Carrier Phase) & 1.469 & 0.002 & 1.142 \\
\hline $\begin{array}{c}\text { Single Difference GPS } \\
\text { (Pseudoranges) }\end{array}$ & 0.007 & 0.2 & 0.006 \\
\hline $\begin{array}{c}\text { Single Difference GPS (Carrier } \\
\text { Phase) }\end{array}$ & 1.869 & 0.002 & 1.487 \\
\hline $\begin{array}{c}\text { Double Difference GPS } \\
\text { (Pseudoranges) }\end{array}$ & 0.008 & 0.2 & 0.006 \\
\hline $\begin{array}{c}\text { Double Difference GPS (Carrier } \\
\text { Phase) }\end{array}$ & 1.493 & 0.004 & 1.15 \\
\hline IMU Measurement & 0.007 & - & 0.006 \\
\hline
\end{tabular}

Table 232: Mean Global Error Statistics of Fused Heading for Simulation Set C-7

\begin{tabular}{|c|c|c|c|}
\hline Method & $\begin{array}{c}\text { Measured Standard } \\
\text { Deviation } \\
\text { (degrees) }\end{array}$ & $\begin{array}{c}\text { Estimated } \\
\text { Standard } \\
\text { Deviation } \\
\text { (degrees) }\end{array}$ & $\begin{array}{c}\text { Mean RMS } \\
\text { Error } \\
\text { (degrees) }\end{array}$ \\
\hline Single Point GPS (Pseudoranges) & 0.321 & 2.19 & 0.255 \\
\hline Single Point GPS (Carrier Phase) & 1.68 & 0.001 & 1.353 \\
\hline $\begin{array}{c}\text { Single Difference GPS } \\
\text { (Pseudoranges) }\end{array}$ & 0.347 & 2.355 & 0.272 \\
\hline $\begin{array}{c}\text { Single Difference GPS (Carrier } \\
\text { Phase) }\end{array}$ & 2.326 & 0.001 & 1.899 \\
\hline $\begin{array}{c}\text { Double Difference GPS } \\
\text { (Pseudoranges) }\end{array}$ & 0.377 & 1.996 & 0.299 \\
\hline $\begin{array}{c}\text { Double Difference GPS (Carrier } \\
\text { Phase) }\end{array}$ & 2.525 & 0.003 & 2.03 \\
\hline IMU Measurement & 0.327 & - & 0.257 \\
\hline
\end{tabular}


Table 233: Success Rates of Reaching Angular Specifications of Unfused Pitch for Simulation Data Set C-7

\begin{tabular}{|c|c|c|c|c|c|c|c|c|}
\hline & \multicolumn{7}{|c|}{ Portion of Trials in Which Target Specification was reached } \\
\hline & $\pm 0.5^{\circ}$ & $\pm 1^{\circ}$ & $\pm 2^{\circ}$ & $\pm 5^{\circ}$ & $\pm 10^{\circ}$ & $\pm 15^{\circ}$ & $\pm 20^{\circ}$ & $\pm 25^{\circ}$ \\
\hline Method & 63 & 66 & 88 & 99 & 100 & 100 & 100 & 100 \\
\hline $\begin{array}{c}\text { Single Point GPS } \\
\text { (Pseudoranges) }\end{array}$ & 62 & 65 & 83 & 99 & 100 & 100 & 100 & 100 \\
\hline $\begin{array}{c}\text { Single Point GPS (Carrier } \\
\text { Phase) }\end{array}$ & 59 & 63 & 74 & 93 & 100 & 100 & 100 & 100 \\
\hline $\begin{array}{c}\text { Single Difference GPS } \\
\text { (Pseudoranges) }\end{array}$ & 58 & 62 & 72 & 95 & 100 & 100 & 100 & 100 \\
\hline $\begin{array}{c}\text { Single Difference GPS } \\
\text { (Carrier Phase) }\end{array}$ & 58 & 70 & 83 & 98 & 100 & 100 & 100 & 100 \\
\hline $\begin{array}{c}\text { Double Difference GPS } \\
\text { (Pseudoranges) }\end{array}$ & 60 & 65 & 80 & 97 & 100 & 100 & 100 & 100 \\
\hline $\begin{array}{c}\text { Double Difference GPS } \\
\text { (Carrier Phase) }\end{array}$ & 100 & 100 & 100 & 100 & 100 & 100 & 100 & 100 \\
\hline
\end{tabular}

Table 234: Success Rates of Reaching Angular Specifications of Unfused Heading for Simulation Data Set C-7

\begin{tabular}{|c|c|c|c|c|c|c|c|c|}
\hline & \multicolumn{7}{|c|}{ Portion of Trials in Which Target Specification was reached } \\
\hline & $\pm 0.5^{\circ}$ & $\pm 1^{\circ}$ & $\pm 2^{\circ}$ & $\pm 5^{\circ}$ & $\pm 10^{\circ}$ & $\pm 15^{\circ}$ & $\pm 20^{\circ}$ & $\pm 25^{\circ}$ \\
\hline Method & 62 & 71 & 85 & 100 & 100 & 100 & 100 & 100 \\
\hline $\begin{array}{c}\text { Single Point GPS } \\
\text { (Pseudoranges) }\end{array}$ & 62 & 71 & 86 & 100 & 100 & 100 & 100 & 100 \\
\hline $\begin{array}{c}\text { Single Point GPS (Carrier } \\
\text { Phase) }\end{array}$ & 59 & 70 & 83 & 98 & 100 & 100 & 100 & 100 \\
\hline $\begin{array}{c}\text { Single Difference GPS } \\
\text { (Pseudoranges) }\end{array}$ & 62 & 71 & 80 & 98 & 100 & 100 & 100 & 100 \\
\hline $\begin{array}{c}\text { Single Difference GPS } \\
\text { (Carrier Phase) }\end{array}$ & 61 & 67 & 75 & 96 & 100 & 100 & 100 & 100 \\
\hline $\begin{array}{c}\text { Double Difference GPS } \\
\text { (Pseudoranges) }\end{array}$ & 59 & 68 & 75 & 97 & 100 & 100 & 100 & 100 \\
\hline $\begin{array}{c}\text { Double Difference GPS } \\
\text { (Carrier Phase) }\end{array}$ & 95 & 100 & 100 & 100 & 100 & 100 & 100 & 100 \\
\hline IMU Measured & & & & & & & & \\
\hline
\end{tabular}


Table 235: Success Rates of Reaching Angular Specifications of Fused Pitch for Simulation Data Set C-7

\begin{tabular}{|c|c|c|c|c|c|c|c|c|}
\hline & \multicolumn{7}{|c|}{ Portion of Trials in Which Target Specification was reached } \\
\hline & $\pm 0.5^{\circ}$ & $\pm 1^{\circ}$ & $\pm 2^{\circ}$ & $\pm 5^{\circ}$ & $\pm 10^{\circ}$ & $\pm 15^{\circ}$ & $\pm 20^{\circ}$ & $\pm 25^{\circ}$ \\
\hline Method & 100 & 100 & 100 & 100 & 100 & 100 & 100 & 100 \\
\hline $\begin{array}{c}\text { Single Point GPS } \\
\text { (Pseudoranges) }\end{array}$ & 65 & 78 & 95 & 100 & 100 & 100 & 100 & 100 \\
\hline $\begin{array}{c}\text { Single Point GPS (Carrier } \\
\text { Phase) }\end{array}$ & 100 & 100 & 100 & 100 & 100 & 100 & 100 & 100 \\
\hline $\begin{array}{c}\text { Single Difference GPS } \\
\text { (Pseudoranges) }\end{array}$ & 60 & 69 & 84 & 100 & 100 & 100 & 100 & 100 \\
\hline $\begin{array}{c}\text { Single Difference GPS } \\
\text { (Carrier Phase) }\end{array}$ & 100 & 100 & 100 & 100 & 100 & 100 & 100 & 100 \\
\hline $\begin{array}{c}\text { Double Difference GPS } \\
\text { (Pseudoranges) }\end{array}$ & 69 & 84 & 97 & 100 & 100 & 100 & 100 & 100 \\
\hline $\begin{array}{c}\text { Double Difference GPS } \\
\text { (Carrier Phase) }\end{array}$ & 100 & 100 & 100 & 100 & 100 & 100 & 100 & 100 \\
\hline IMU Measured & & & & & & & & \\
\hline
\end{tabular}

Table 236: Success Rates of Reaching Angular Specifications of Fused Heading for Simulation Data Set C-7

\begin{tabular}{|c|c|c|c|c|c|c|c|c|}
\hline & \multicolumn{7}{|c|}{ Portion of Trials in Which Target Specification was reached } \\
\hline & $\pm 0.5^{\circ}$ & $\pm 1^{\circ}$ & $\pm 2^{\circ}$ & $\pm 5^{\circ}$ & $\pm 10^{\circ}$ & $\pm 15^{\circ}$ & $\pm 20^{\circ}$ & $\pm 25^{\circ}$ \\
\hline Method & 96 & 100 & 100 & 100 & 100 & 100 & 100 & 100 \\
\hline $\begin{array}{c}\text { Single Point GPS } \\
\text { (Pseudoranges) }\end{array}$ & 62 & 71 & 86 & 100 & 100 & 100 & 100 & 100 \\
\hline $\begin{array}{c}\text { Single Point GPS (Carrier } \\
\text { Phase) }\end{array}$ & 94 & 99 & 100 & 100 & 100 & 100 & 100 & 100 \\
\hline $\begin{array}{c}\text { Single Difference GPS } \\
\text { (Pseudoranges) }\end{array}$ & 62 & 71 & 80 & 98 & 100 & 100 & 100 & 100 \\
\hline $\begin{array}{c}\text { Single Difference GPS } \\
\text { (Carrier Phase) }\end{array}$ & 92 & 100 & 100 & 100 & 100 & 100 & 100 & 100 \\
\hline $\begin{array}{c}\text { Double Difference GPS } \\
\text { (Pseudoranges) }\end{array}$ & 59 & 68 & 75 & 97 & 100 & 100 & 100 & 100 \\
\hline $\begin{array}{c}\text { Double Difference GPS } \\
\text { (Carrier Phase) }\end{array}$ & 95 & 100 & 100 & 100 & 100 & 100 & 100 & 100 \\
\hline IMU Measured & & & & & & & & \\
\hline
\end{tabular}


Table 237: Pitch / Heading Correlation Coefficients for Each Computation Method in Simulation Data Set C-7

\begin{tabular}{|c|c|c|}
\hline & \multicolumn{2}{|c|}{ Pitch / Heading Correlation Coefficient } \\
\hline Method & Unfused & Fused \\
\hline $\begin{array}{c}\text { Single Point GPS } \\
\text { (Pseudoranges) }\end{array}$ & 0.882005 & 0.070036 \\
\hline $\begin{array}{c}\text { Single Difference GPS } \\
\text { (Pseudoranges) }\end{array}$ & 0.854762 & 0.050123 \\
\hline $\begin{array}{c}\text { Double Difference GPS } \\
\text { (Pseudoranges) }\end{array}$ & 0.799174 & 0.072105 \\
\hline
\end{tabular}

\section{Set C-8 90 m Dual Antenna Separation}

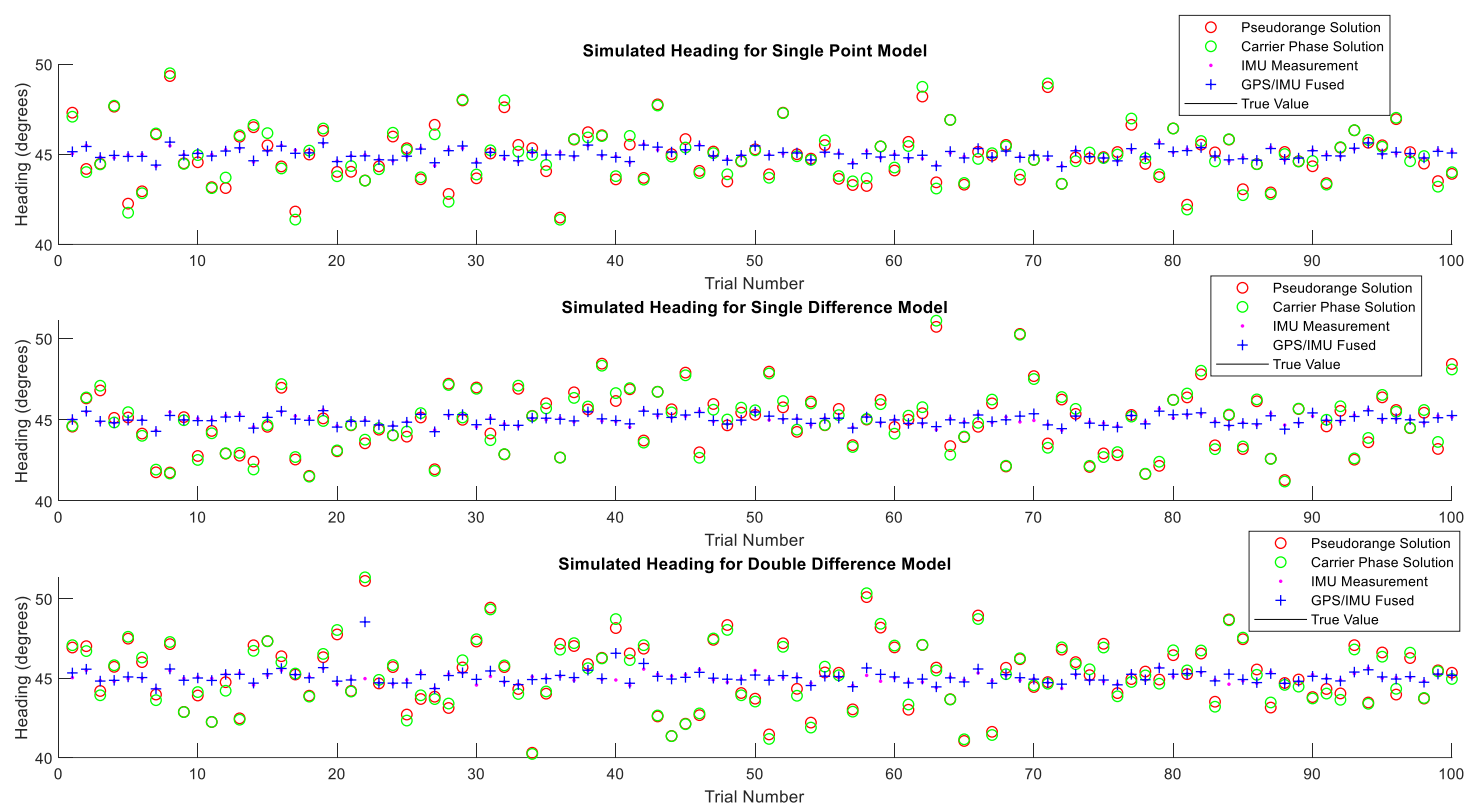

Figure 117: Computed and Measured Headings for Simulation Set C-8 


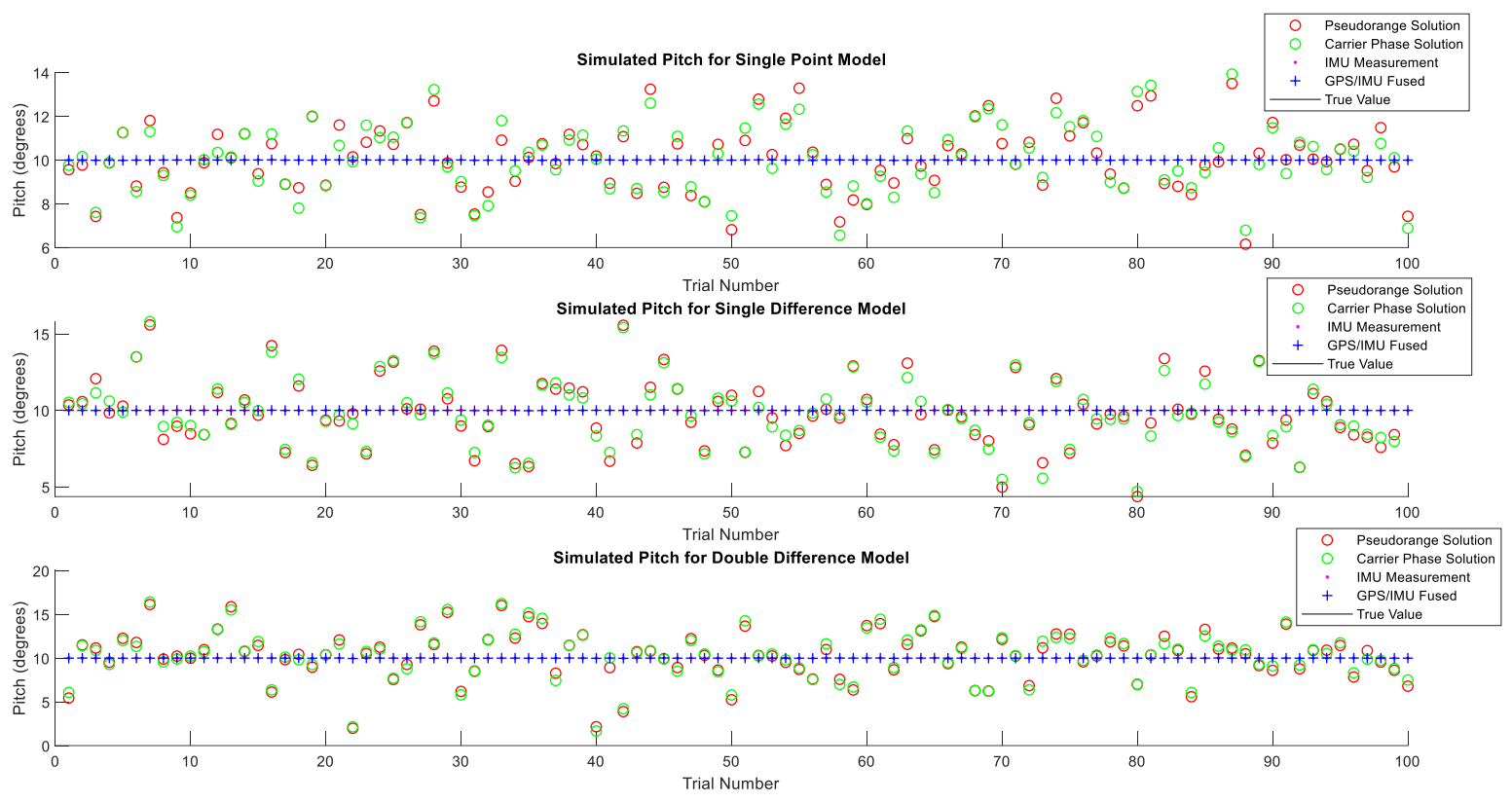

Figure 118: Computed and Measured Pitch for Simulation Set C-8

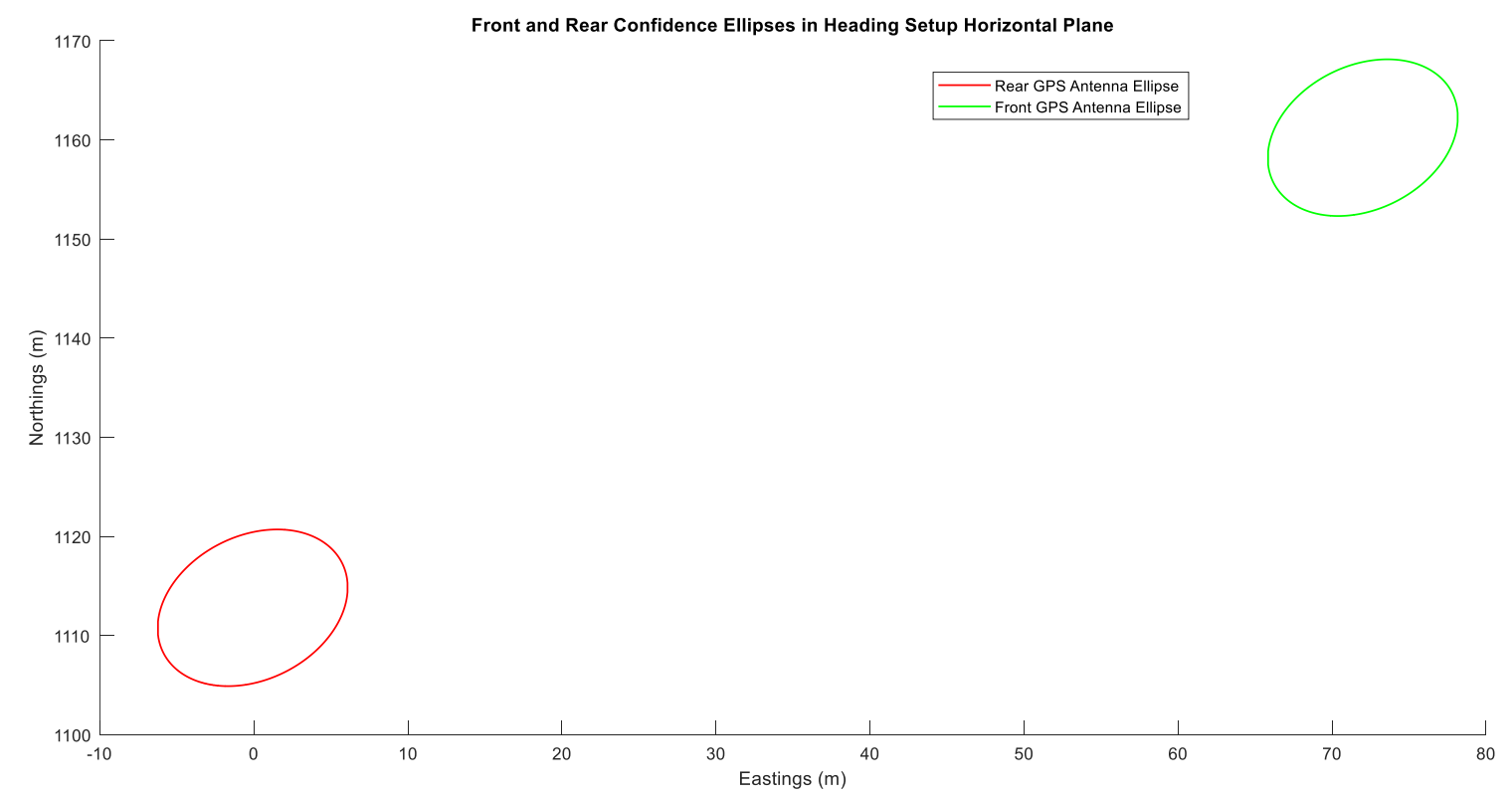

Figure 119: Propagated 95\% Confidence Ellipses for Front and Rear GPS Antennas in Simulated Data Set C-8 
Table 238: Mean Global Error Statistics of Unfused Pitch for Simulation Set C-8

\begin{tabular}{|c|c|c|c|}
\hline Method & $\begin{array}{c}\text { Measured Standard } \\
\text { Deviation } \\
\text { (degrees) }\end{array}$ & $\begin{array}{c}\text { Estimated } \\
\text { Standard } \\
\text { Deviation } \\
\text { (degrees) }\end{array}$ & $\begin{array}{c}\text { Mean RMS } \\
\text { Error } \\
\text { (degrees) }\end{array}$ \\
\hline Single Point GPS (Pseudoranges) & 1.542 & 8.795 & 1.23 \\
\hline Single Point GPS (Carrier Phase) & 1.58 & 0.003 & 1.288 \\
\hline $\begin{array}{c}\text { Single Difference GPS } \\
\text { (Pseudoranges) }\end{array}$ & 2.261 & 8.6 & 1.804 \\
\hline $\begin{array}{c}\text { Single Difference GPS (Carrier } \\
\text { Phase) }\end{array}$ & 2.177 & 0.003 & 1.738 \\
\hline $\begin{array}{c}\text { Double Difference GPS } \\
\text { (Pseudoranges) }\end{array}$ & 2.766 & 7.102 & 2.139 \\
\hline $\begin{array}{c}\text { Double Difference GPS (Carrier } \\
\text { Phase) }\end{array}$ & 2.779 & 0.007 & 2.132 \\
\hline IMU Measurement & 0.007 & - & 0.005 \\
\hline
\end{tabular}

Table 239: Mean Global Error Statistics of Unfused Heading for Simulation Set C-8

\begin{tabular}{|c|c|c|c|}
\hline Method & $\begin{array}{c}\text { Measured Standard } \\
\text { Deviation } \\
\text { (degrees) }\end{array}$ & $\begin{array}{c}\text { Estimated } \\
\text { Standard } \\
\text { Deviation } \\
\text { (degrees) }\end{array}$ & $\begin{array}{c}\text { Mean RMS } \\
\text { Error } \\
\text { (degrees) }\end{array}$ \\
\hline Single Point GPS (Pseudoranges) & 1.504 & 7.291 & 1.178 \\
\hline Single Point GPS (Carrier Phase) & 1.564 & 0.002 & 1.195 \\
\hline $\begin{array}{c}\text { Single Difference GPS } \\
\text { (Pseudoranges) }\end{array}$ & 1.909 & 7.126 & 1.517 \\
\hline $\begin{array}{c}\text { Single Difference GPS (Carrier } \\
\text { Phase) }\end{array}$ & 1.936 & 0.002 & 1.544 \\
\hline $\begin{array}{c}\text { Double Difference GPS } \\
\text { (Pseudoranges) }\end{array}$ & 2.054 & 5.781 & 1.68 \\
\hline $\begin{array}{c}\text { Double Difference GPS (Carrier } \\
\text { Phase) }\end{array}$ & 2.104 & 0.006 & 1.726 \\
\hline IMU Measurement & 0.301 & - & 0.24 \\
\hline
\end{tabular}


Table 240: Mean Global Error Statistics of Fused Pitch for Simulation Set C-8

\begin{tabular}{|c|c|c|c|}
\hline Method & $\begin{array}{c}\text { Measured Standard } \\
\text { Deviation } \\
\text { (degrees) }\end{array}$ & $\begin{array}{c}\text { Estimated } \\
\text { Standard } \\
\text { Deviation } \\
\text { (degrees) }\end{array}$ & $\begin{array}{c}\text { Mean RMS } \\
\text { Error } \\
\text { (degrees) }\end{array}$ \\
\hline Single Point GPS (Pseudoranges) & 0.006 & 0.2 & 0.005 \\
\hline Single Point GPS (Carrier Phase) & 1.099 & 0.002 & 0.892 \\
\hline $\begin{array}{c}\text { Single Difference GPS } \\
\text { (Pseudoranges) }\end{array}$ & 0.007 & 0.2 & 0.006 \\
\hline $\begin{array}{c}\text { Single Difference GPS (Carrier } \\
\text { Phase) }\end{array}$ & 1.515 & 0.002 & 1.214 \\
\hline $\begin{array}{c}\text { Double Difference GPS } \\
\text { (Pseudoranges) }\end{array}$ & 0.008 & 0.2 & 0.006 \\
\hline $\begin{array}{c}\text { Double Difference GPS (Carrier } \\
\text { Phase) }\end{array}$ & 1.584 & 0.004 & 1.109 \\
\hline IMU Measurement & 0.007 & - & 0.005 \\
\hline
\end{tabular}

Table 241: Mean Global Error Statistics of Fused Heading for Simulation Set C-8

\begin{tabular}{|c|c|c|c|}
\hline Method & $\begin{array}{c}\text { Measured Standard } \\
\text { Deviation } \\
\text { (degrees) }\end{array}$ & $\begin{array}{c}\text { Estimated } \\
\text { Standard } \\
\text { Deviation } \\
\text { (degrees) }\end{array}$ & $\begin{array}{c}\text { Mean RMS } \\
\text { Error } \\
\text { (degrees) }\end{array}$ \\
\hline Single Point GPS (Pseudoranges) & 0.294 & 1.776 & 0.234 \\
\hline Single Point GPS (Carrier Phase) & 1.554 & 0.001 & 1.187 \\
\hline $\begin{array}{c}\text { Single Difference GPS } \\
\text { (Pseudoranges) }\end{array}$ & 0.299 & 1.738 & 0.239 \\
\hline $\begin{array}{c}\text { Single Difference GPS (Carrier } \\
\text { Phase) }\end{array}$ & 1.924 & 0.001 & 1.534 \\
\hline $\begin{array}{c}\text { Double Difference GPS } \\
\text { (Pseudoranges) }\end{array}$ & 0.491 & 1.786 & 0.295 \\
\hline $\begin{array}{c}\text { Double Difference GPS (Carrier } \\
\text { Phase) }\end{array}$ & 2.071 & 0.003 & 1.697 \\
\hline IMU Measurement & 0.301 & - & 0.24 \\
\hline
\end{tabular}


Table 242: Success Rates of Reaching Angular Specifications of Unfused Pitch for Simulation Data Set C-8

\begin{tabular}{|c|c|c|c|c|c|c|c|c|}
\hline & \multicolumn{7}{|c|}{ Portion of Trials in Which Target Specification was reached } \\
\hline & $\pm 0.5^{\circ}$ & $\pm 1^{\circ}$ & $\pm 2^{\circ}$ & $\pm 5^{\circ}$ & $\pm 10^{\circ}$ & $\pm 15^{\circ}$ & $\pm 20^{\circ}$ & $\pm 25^{\circ}$ \\
\hline Method & 60 & 75 & 89 & 100 & 100 & 100 & 100 & 100 \\
\hline $\begin{array}{c}\text { Single Point GPS } \\
\text { (Pseudoranges) }\end{array}$ & 59 & 69 & 91 & 100 & 100 & 100 & 100 & 100 \\
\hline $\begin{array}{c}\text { Single Point GPS (Carrier } \\
\text { Phase) }\end{array}$ & 65 & 72 & 82 & 98 & 100 & 100 & 100 & 100 \\
\hline $\begin{array}{c}\text { Single Difference GPS } \\
\text { (Pseudoranges) }\end{array}$ & 62 & 73 & 84 & 98 & 100 & 100 & 100 & 100 \\
\hline $\begin{array}{c}\text { Single Difference GPS } \\
\text { (Carrier Phase) }\end{array}$ & 50 & 60 & 75 & 96 & 100 & 100 & 100 & 100 \\
\hline $\begin{array}{c}\text { Double Difference GPS } \\
\text { (Pseudoranges) }\end{array}$ & 50 & 60 & 75 & 95 & 100 & 100 & 100 & 100 \\
\hline $\begin{array}{c}\text { Double Difference GPS } \\
\text { (Carrier Phase) }\end{array}$ & 100 & 100 & 100 & 100 & 100 & 100 & 100 & 100 \\
\hline IMU Measured & & & & & & & & \\
\hline
\end{tabular}

Table 243: Success Rates of Reaching Angular Specifications of Unfused Heading for Simulation Data Set C-8

\begin{tabular}{|c|c|c|c|c|c|c|c|c|}
\hline & \multicolumn{7}{|c|}{ Portion of Trials in Which Target Specification was reached } \\
\hline Method & $\pm 0.5^{\circ}$ & $\pm 1^{\circ}$ & $\pm 2^{\circ}$ & $\pm 5^{\circ}$ & $\pm 10^{\circ}$ & $\pm 15^{\circ}$ & $\pm 20^{\circ}$ & $\pm 25^{\circ}$ \\
\hline $\begin{array}{c}\text { Single Point GPS } \\
\text { (Pseudoranges) }\end{array}$ & 68 & 80 & 91 & 100 & 100 & 100 & 100 & 100 \\
\hline $\begin{array}{c}\text { Single Point GPS (Carrier } \\
\text { Phase) }\end{array}$ & 71 & 77 & 90 & 100 & 100 & 100 & 100 & 100 \\
\hline $\begin{array}{c}\text { Single Difference GPS } \\
\text { (Pseudoranges) }\end{array}$ & 64 & 72 & 90 & 98 & 100 & 100 & 100 & 100 \\
\hline $\begin{array}{c}\text { Single Difference GPS } \\
\text { (Carrier Phase) }\end{array}$ & 62 & 73 & 87 & 98 & 100 & 100 & 100 & 100 \\
\hline $\begin{array}{c}\text { Double Difference GPS } \\
\text { (Pseudoranges) }\end{array}$ & 54 & 62 & 77 & 98 & 100 & 100 & 100 & 100 \\
\hline $\begin{array}{c}\text { Double Difference GPS } \\
\text { (Carrier Phase) }\end{array}$ & 55 & 63 & 80 & 98 & 100 & 100 & 100 & 100 \\
\hline IMU Measured & 95 & 100 & 100 & 100 & 100 & 100 & 100 & 100 \\
\hline
\end{tabular}


Table 244: Success Rates of Reaching Angular Specifications of Fused Pitch for Simulation Data Set C-8

\begin{tabular}{|c|c|c|c|c|c|c|c|c|}
\hline & \multicolumn{7}{|c|}{ Portion of Trials in Which Target Specification was reached } \\
\hline & $\pm 0.5^{\circ}$ & $\pm 1^{\circ}$ & $\pm 2^{\circ}$ & $\pm 5^{\circ}$ & $\pm 10^{\circ}$ & $\pm 15^{\circ}$ & $\pm 20^{\circ}$ & $\pm 25^{\circ}$ \\
\hline Method & 100 & 100 & 100 & 100 & 100 & 100 & 100 & 100 \\
\hline $\begin{array}{c}\text { Single Point GPS } \\
\text { (Pseudoranges) }\end{array}$ & 65 & 83 & 98 & 100 & 100 & 100 & 100 & 100 \\
\hline $\begin{array}{c}\text { Single Point GPS (Carrier } \\
\text { Phase) }\end{array}$ & 100 & 100 & 100 & 100 & 100 & 100 & 100 & 100 \\
\hline $\begin{array}{c}\text { Single Difference GPS } \\
\text { (Pseudoranges) }\end{array}$ & 70 & 80 & 94 & 100 & 100 & 100 & 100 & 100 \\
\hline $\begin{array}{c}\text { Single Difference GPS } \\
\text { (Carrier Phase) }\end{array}$ & 100 & 100 & 100 & 100 & 100 & 100 & 100 & 100 \\
\hline $\begin{array}{c}\text { Double Difference GPS } \\
\text { (Pseudoranges) }\end{array}$ & 62 & 79 & 94 & 100 & 100 & 100 & 100 & 100 \\
\hline $\begin{array}{c}\text { Double Difference GPS } \\
\text { (Carrier Phase) }\end{array}$ & 100 & 100 & 100 & 100 & 100 & 100 & 100 & 100 \\
\hline IMU Measured & & & & & & & & \\
\hline
\end{tabular}

Table 245: Success Rates of Reaching Angular Specifications of Fused Heading for Simulation Data Set C-8

\begin{tabular}{|c|c|c|c|c|c|c|c|c|}
\hline & \multicolumn{7}{|c|}{ Portion of Trials in Which Target Specification was reached } \\
\hline & $\pm 0.5^{\circ}$ & $\pm 1^{\circ}$ & $\pm 2^{\circ}$ & $\pm 5^{\circ}$ & $\pm 10^{\circ}$ & $\pm 15^{\circ}$ & $\pm 20^{\circ}$ & $\pm 25^{\circ}$ \\
\hline Method & 94 & 100 & 100 & 100 & 100 & 100 & 100 & 100 \\
\hline $\begin{array}{c}\text { Single Point GPS } \\
\text { (Pseudoranges) }\end{array}$ & 71 & 78 & 90 & 100 & 100 & 100 & 100 & 100 \\
\hline $\begin{array}{c}\text { Single Point GPS (Carrier } \\
\text { Phase) }\end{array}$ & 94 & 100 & 100 & 100 & 100 & 100 & 100 & 100 \\
\hline $\begin{array}{c}\text { Single Difference GPS } \\
\text { (Pseudoranges) }\end{array}$ & 62 & 74 & 87 & 98 & 100 & 100 & 100 & 100 \\
\hline $\begin{array}{c}\text { Single Difference GPS } \\
\text { (Carrier Phase) }\end{array}$ & 89 & 98 & 99 & 100 & 100 & 100 & 100 & 100 \\
\hline $\begin{array}{c}\text { Double Difference GPS } \\
\text { (Pseudoranges) }\end{array}$ & 55 & 63 & 81 & 98 & 100 & 100 & 100 & 100 \\
\hline $\begin{array}{c}\text { Double Difference GPS } \\
\text { (Carrier Phase) }\end{array}$ & 95 & 100 & 100 & 100 & 100 & 100 & 100 & 100 \\
\hline IMU Measured & & & & & & & & \\
\hline
\end{tabular}


Table 246: Pitch / Heading Correlation Coefficients for Each Computation Method in Simulation Data Set C-8

\begin{tabular}{|c|c|c|}
\hline & \multicolumn{2}{|c|}{ Pitch / Heading Correlation Coefficient } \\
\hline Method & Unfused & Fused \\
\hline $\begin{array}{c}\text { Single Point GPS } \\
\text { (Pseudoranges) }\end{array}$ & 0.89194 & 0.121567 \\
\hline $\begin{array}{c}\text { Single Difference GPS } \\
\text { (Pseudoranges) }\end{array}$ & 0.81833 & 0.049476 \\
\hline $\begin{array}{c}\text { Double Difference GPS } \\
\text { (Pseudoranges) }\end{array}$ & 0.815788 & 0.096819 \\
\hline
\end{tabular}




\section{Appendix D: Summary of Global Trends Across}

\section{all Partial Attitude Simulation Data Sets}

The highest performing GPS attitude method across all simulations was the single point pseudorange solution. Therefore, this section will examine error and success metrics for the pseudorange single point method across each simulation series.

\section{Series A Summary}
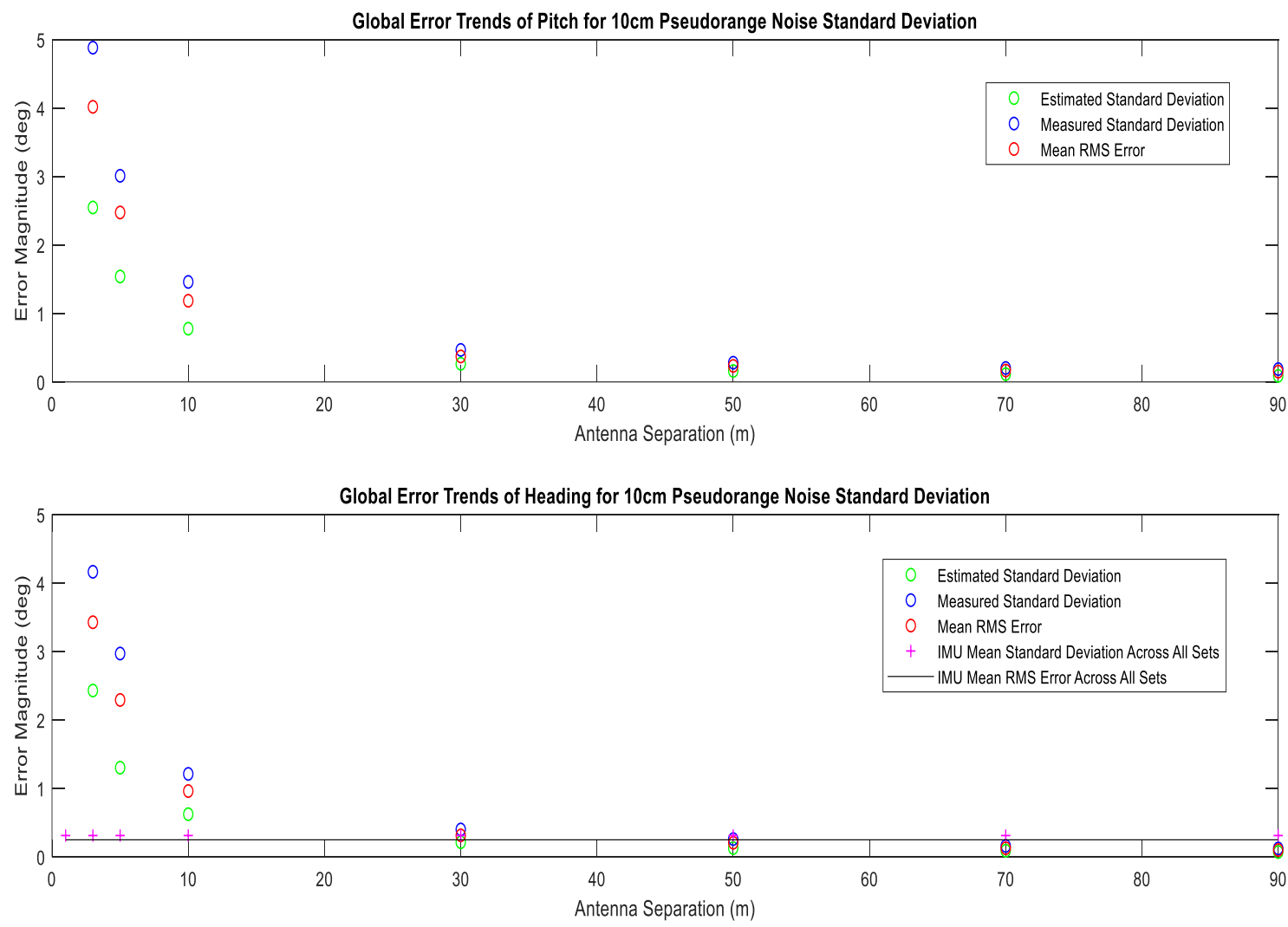

Figure 120: Global Error and Noise Trends for Pseudorange Solutions Across All Series A Simulation Data Sets 

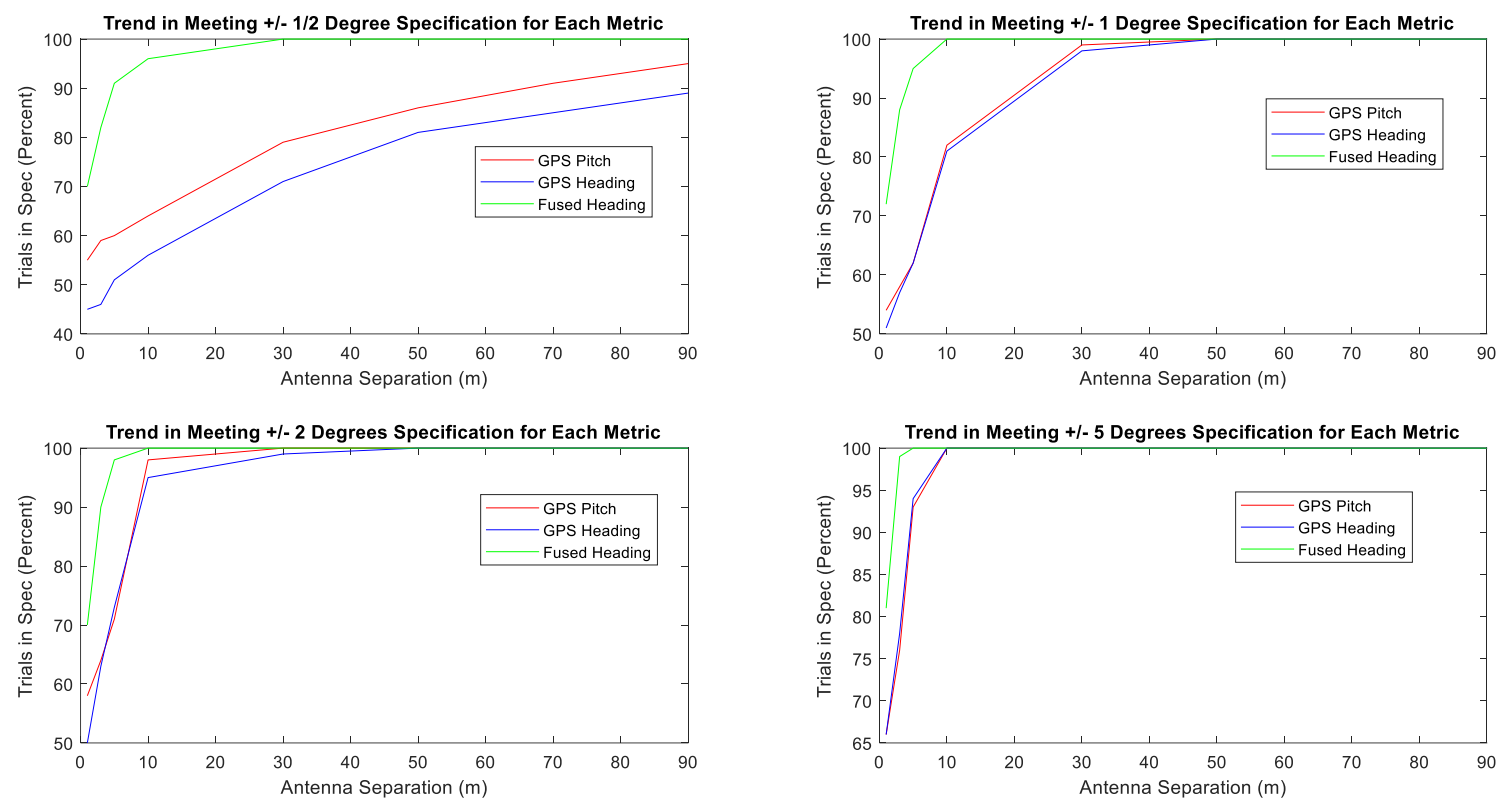

Figure 121: Series A Success Levels in Meeting Angular Specifications Between 1/2 Degree and 5 Degrees
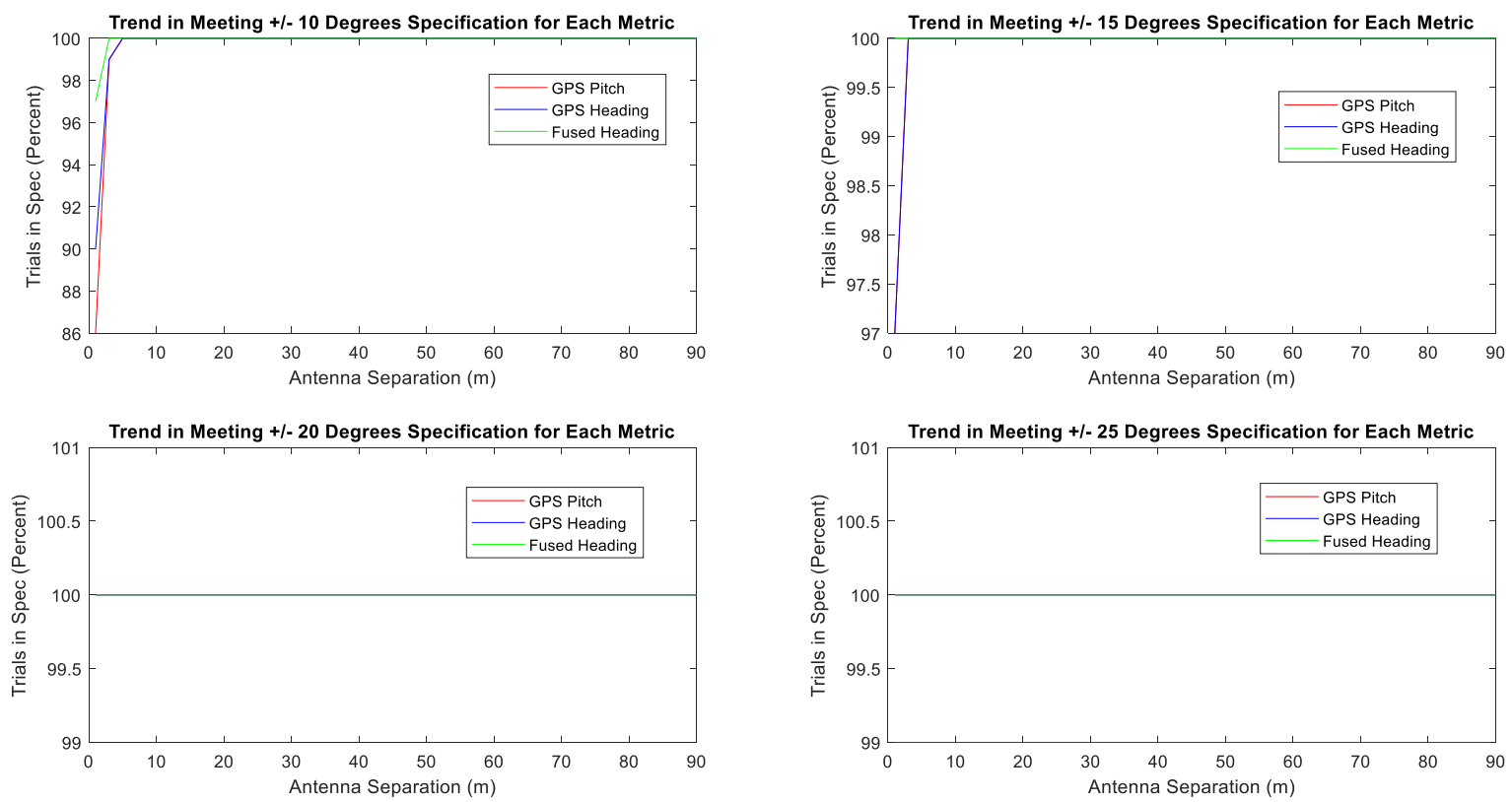

Figure 122: Series A Success Levels in Meeting Angular Specifications Between 10 Degrees and 25 Degrees 


\section{Series B Summary}
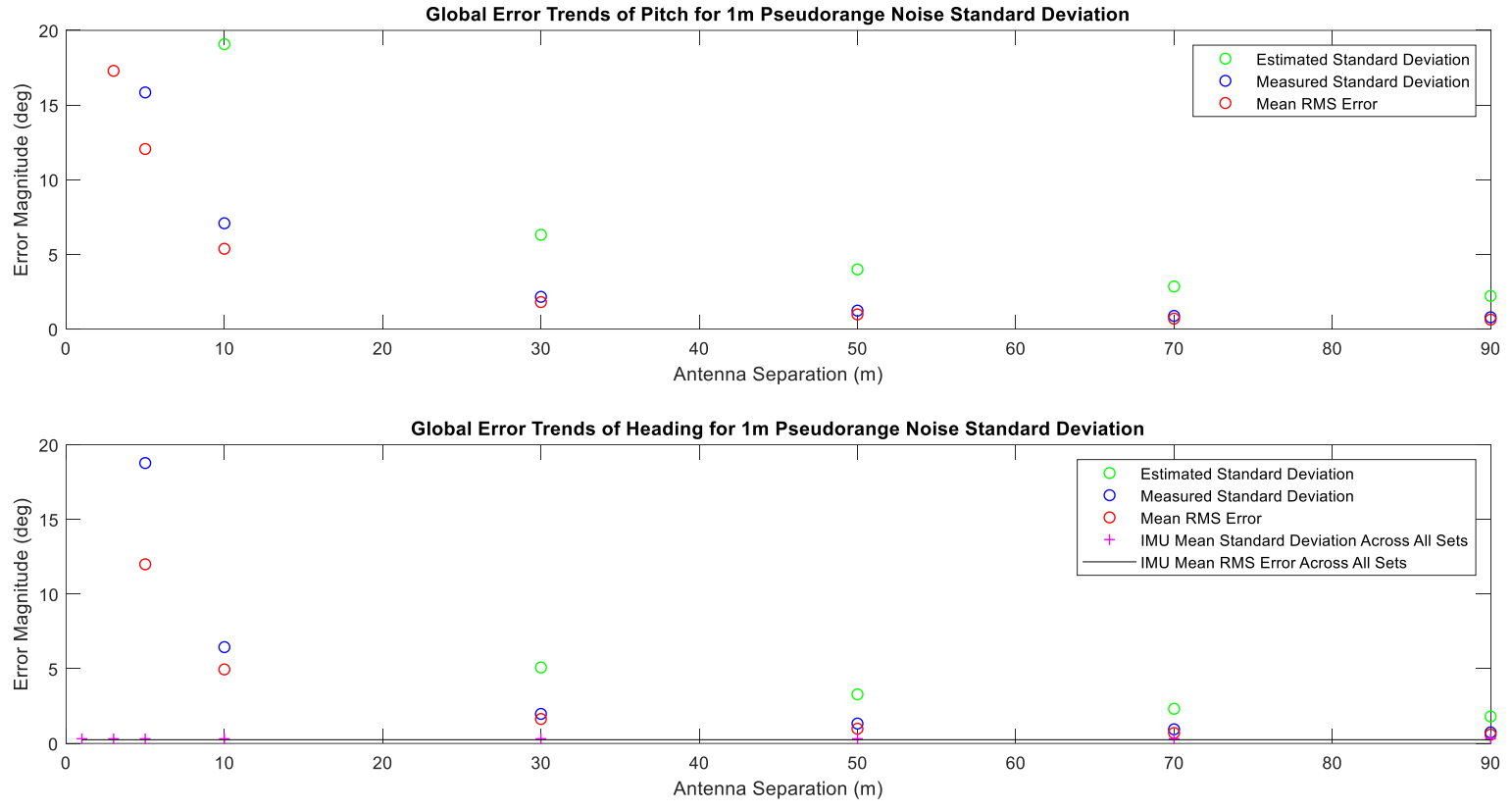

Figure 123: Global Error and Noise Trends for Pseudorange Solutions Across All Series B Simulation Data Sets 

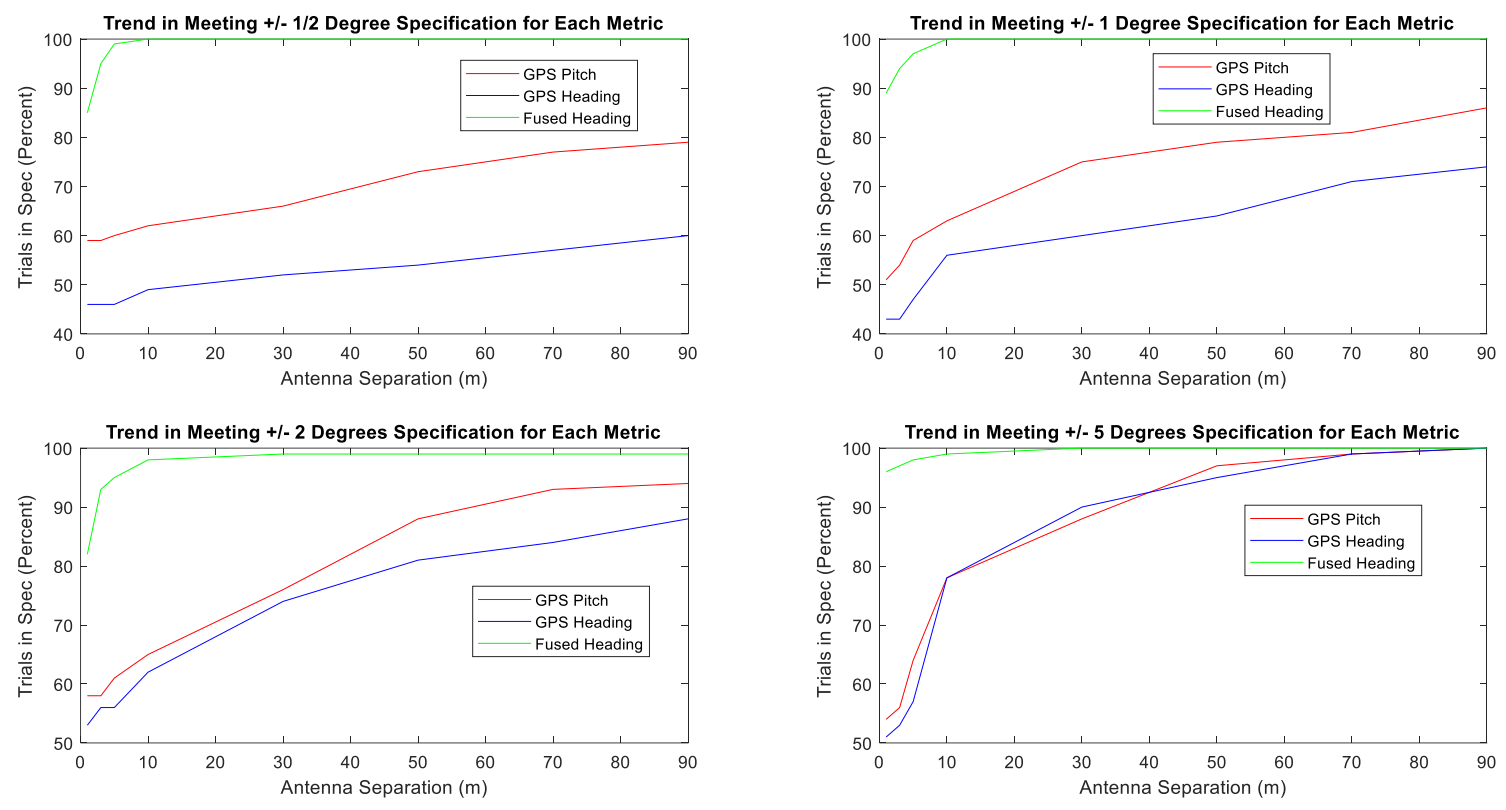

Figure 124: Series B Success Levels in Meeting Angular Specifications Between 1/2 Degree and 5 Degrees
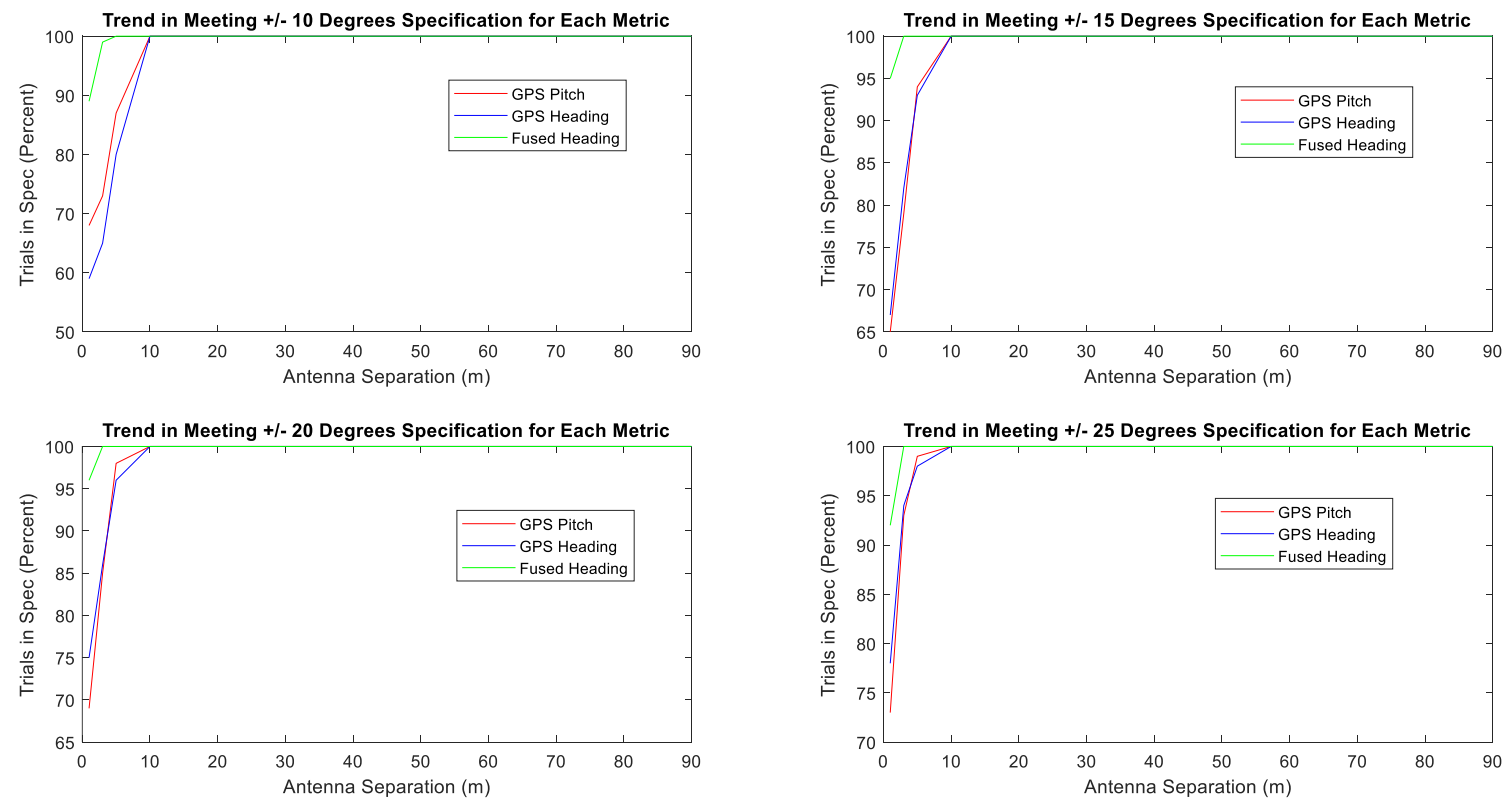

Figure 125: Series B Success Levels in Meeting Angular Specifications Between 10 Degrees and 25 Degrees 


\section{Series C Summary}
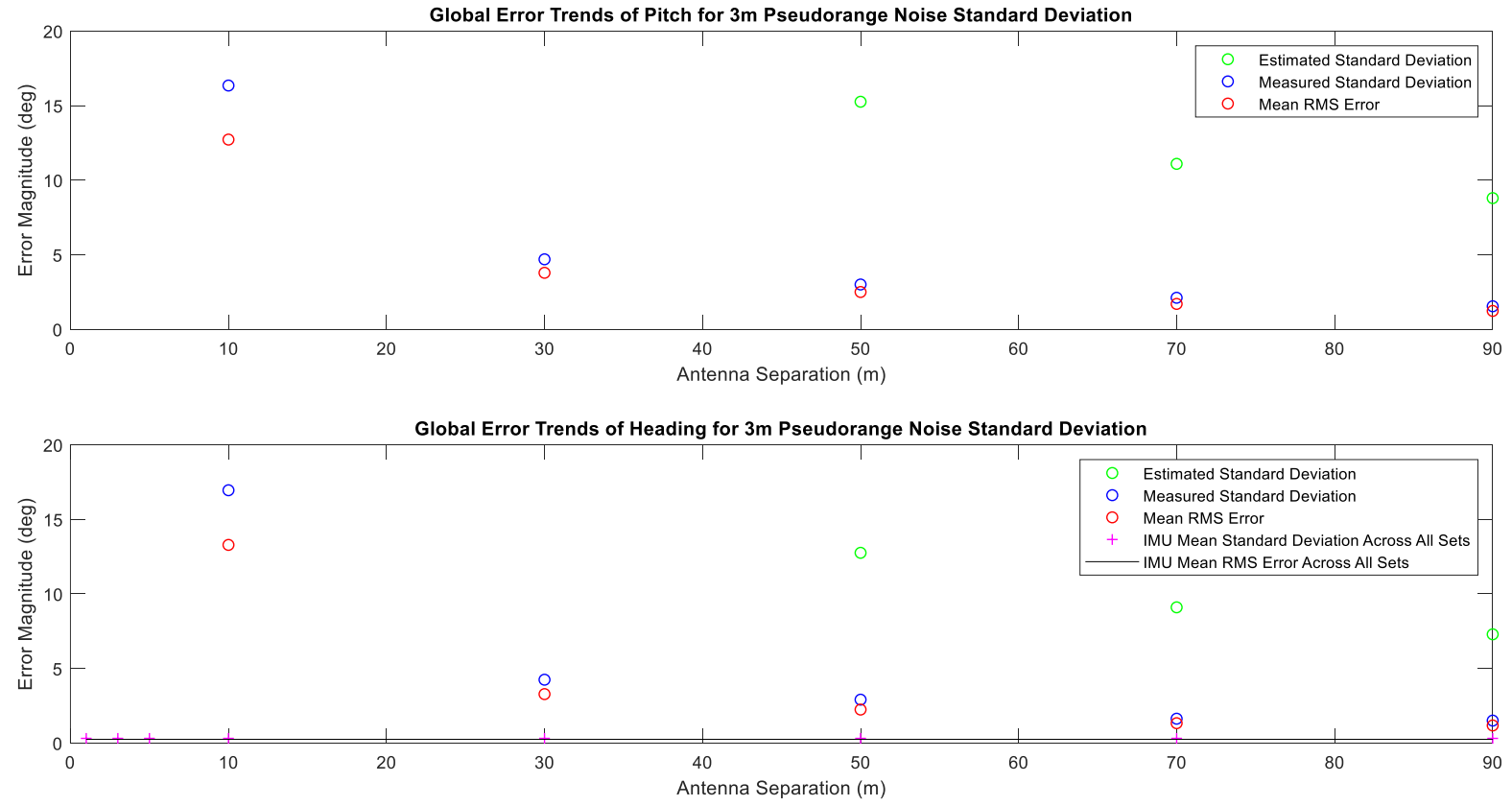

Figure 126: Global Error and Noise Trends for Pseudorange Solutions Across All Series C Simulation Data Sets 

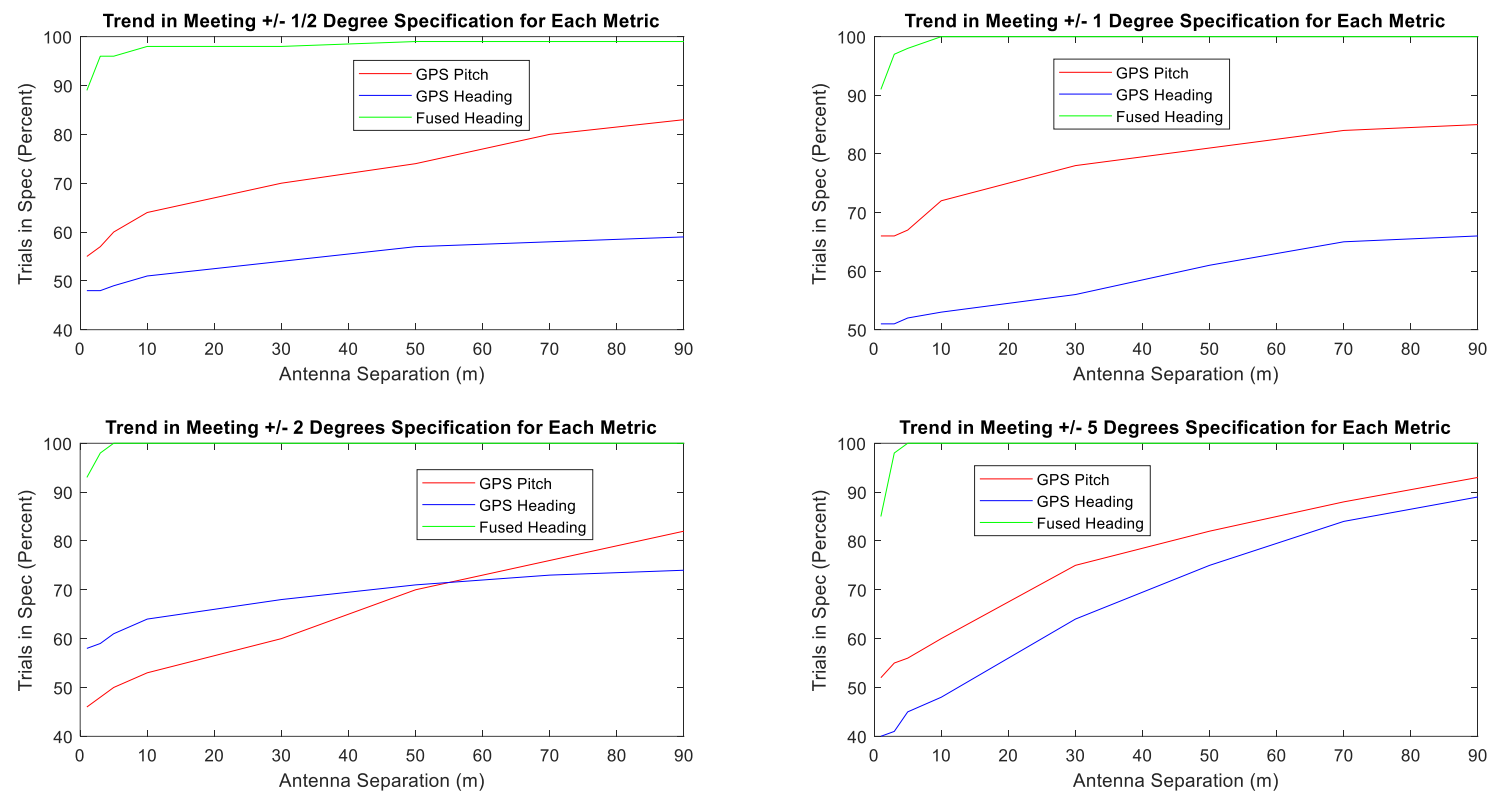

Figure 127: Series C Success Levels in Meeting Angular Specifications Between 1/2 Degree and 5 Degrees
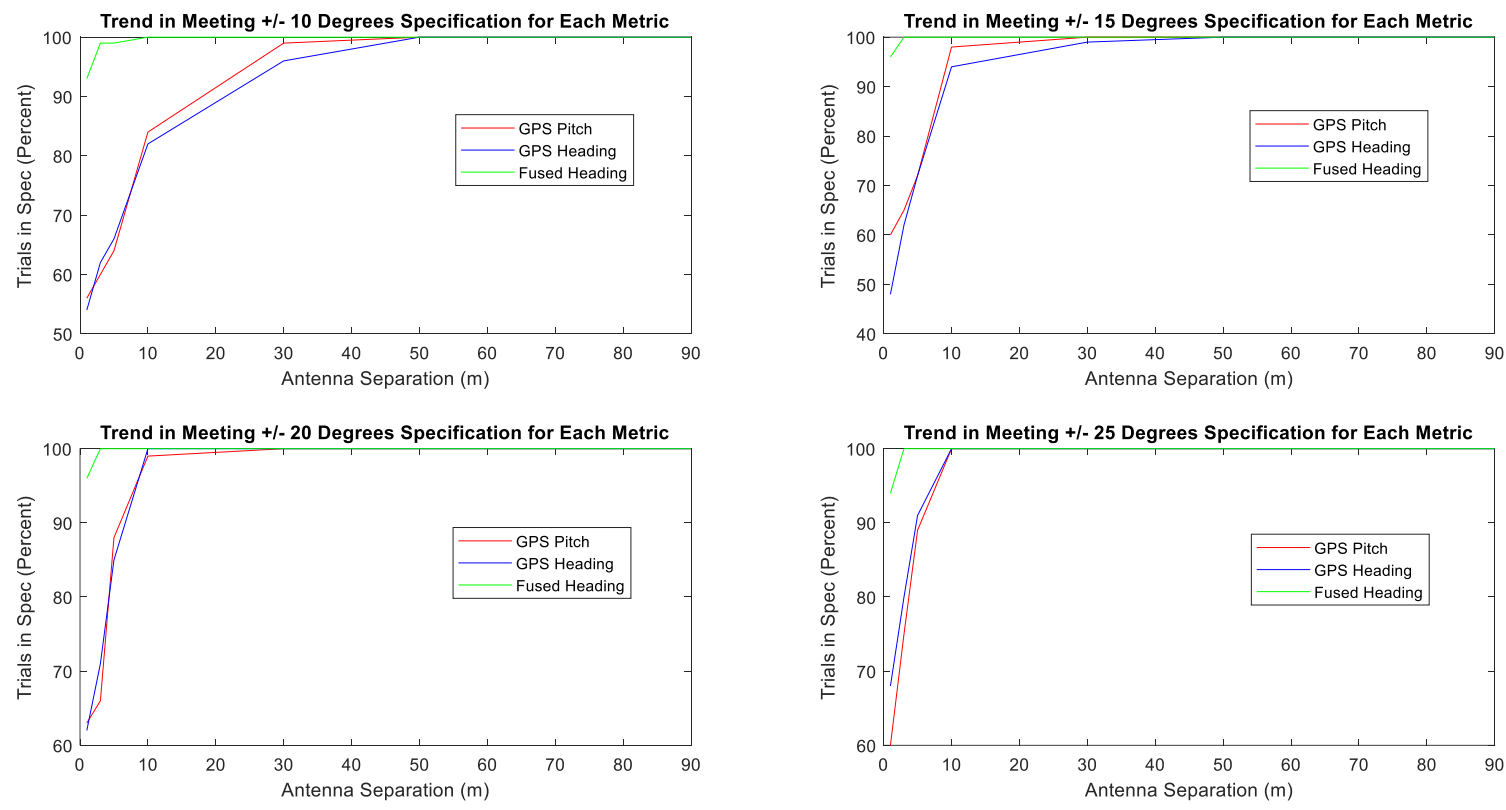

Figure 128: Series C Success Levels in Meeting Angular Specifications Between 10 Degrees and 25 Degrees 
